\title{
Gefügeabhängigkeit technischer Gesteinseigenschaften
}

Dissertation zur Erlangung des Doktorgrades

Mathematisch-Naturwissenschaftliche Fakultäten der

Georg-August-Universität zu Göttingen

vorgelegt von

Daniel Strohmeyer

aus Walsrode

Göttingen 2003 
Referent: $\quad$ Prof. Dr. S. Siegesmund

Korreferent: $\quad$ Prof. Dr. K.-H. Nitsch

Tag der Disputation: 03.11.2003 


\section{Kurzfassung}

Naturwerksteine sind aufgrund ihrer häufig komplexen geologischen Vergangenheit ein heterogener und anisotroper Werkstoff, dessen Verwendung eine gute Kenntnis der technischen Eigenschaften erfordert. Im Rahmen dieser Studie wurden 20 niedrigporöse Kristallingesteine unterschiedlicher mineralogischer Zusammensetzung (Granitoide, Gabbros, Peridotit, Quarzit, Marmor) und Gesteinsgefüge hinsichtlich der Gefügeabhängigkeit der technischen Gesteinseigenschaften untersucht. Die Proben lassen sich nach ihrer makroskopischen Gefügeanisotropie in drei Makrogefüge-Gruppen einteilen. Für 14 der Gesteine wurden die Glimmertexturen bestimmt. Die maximale Intensität $\left(\mathrm{I}_{\max }\right)$ steigt analog zur zunehmenden Gefügeanisotropie an und erlaubt die Definition von $\mathrm{I}_{\max }-$ Werten für die Makrogefüge-Gruppen I/II/III. Des Weiteren wurden die Rissdichte der offenen Mikrorisse, deren bevorzugte Orientierung sowie die Korngröße berücksichtigt. Die technischen Eigenschaften kapillare Wasseraufnahme, Druck-, Spaltzug-, Biege-, Abriebfestigkeit, die EModuln und die thermische Dehnung wurden mindestens in drei senkrecht aufeinander stehenden Richtungen ermittelt und ergänzt durch die skalaren Indexeigenschaften Dichte und Porosität. Sowohl die Mittelwerte (arithmetisches Mittel aus den drei HauptgefügeRichtungen - x, y, z-Richtung) als auch die Anisotropie der technischen Kennwerte konnten zu den Gefügemerkmalen in Beziehung gesetzt werden.

Im Grundsatz korreliert die makroskopische Gefügeanisotropie positiv mit der Anisotropie der technischen Eigenschaften. Die Mittelwerte der Gesteinsfestigkeiten (Druck-, Spaltzug-, Biege-, Abriebfestigkeit) hängen hauptsächlich von den mechanischen Eigenschaften des Mineralbestands und der Mikrorissdichte ab, aber auch die Korngröße spielt eine Rolle. Die Anisotropie wird durch die Textur der gesteinsbildenden Minerale und die bevorzugte Orientierung der offenen Mikrorisse bestimmt. Gleiches gilt für den statischen E-Modul, wobei hier die elastischen Eigenschaften des Mineralbestands einen stärkeren Einfluss haben. Diese sind neben der Rissdichte auch für die Mittelwerte des dynamischen E-Moduls entscheidend. Dessen Anisotropie ergibt sich aus der räumlichen Anordnung der offenen Mikrorisse und der Textur der Minerale. Die kapillare Wasseraufnahme, gekennzeichnet durch den w-Wert, ist vom Mineralbestand unabhängig und wird durch den Volumenanteil der Kapillarporen und deren Verteilung im Gesteinsgefüge dominiert. Im Gegensatz dazu besteht bezüglich der thermischen Dehnung, die zu einem großen Teil von den thermischen Dehnungseigenschaften der gesteinsbildenden Minerale abhängt, nur ein untergeordneter Einfluss der Mikrorisse durch den Prozess der Risspufferung. Von größerer Bedeutung ist in diesem Zusammenhang die thermisch induzierte Rissbildung, die beim Kalzit-Marmor zu deutlich erhöhten thermischen Ausdehnungskoeffizienten führt.

Aus den Gefügeabhängigkeiten der einzelnen technischen Kennwerte und den Prozessen bei deren experimenteller Bestimmung folgen Regeln für die Kreuzkorrelation der gesteinstechnischen Parameter untereinander. Eine gute Korrelation der (richtungsunabhängigen) Mittelwerte ist dann zu erwarten, wenn die technischen Kennwerte in möglichst gleicher Weise von den gleichen Gefügemerkmalen abhängen, die technischen Parameter eine gute Korrelation in Bezug auf den Mineralbestand aufweisen und die Prozesse während der experimentellen Bestimmung der Eigenschaften ähnlich sind. Bei der richtungsabhängigen Betrachtung muss für ein repräsentatives Verhältnis zusätzlich die Raumlage der Prozesszonen bei der Ermittlung der technischen Kenngrößen identisch sein.

Die Verwendung insbesondere von Gesteinen mit einer hohen Gefügeanisotropie erfordert eine richtungsabhängige Analyse der technischen Kennwerte. Die Anisotropie der mechanischen Eigenschaften kann 80\% und die der thermischen Dehnung 70\% erreichen, aber auch die kapillare Wasseraufnahme und die E-Moduln weisen z.T. ein deutlich 
anisotropes Verhalten auf, welches bei der bautechnischen Anwendung und der Schadensanalyse Beachtung finden muss.

\section{Abstract}

By virtue of their usually complex geological past, natural stones represent a heterogeneous and anisotropic material. Their use demands good knowledge of their technical properties. In this study, 20 low-porosity crystalline rocks of different mineralogical compositions and rock fabrics (granitoids, gabbros, peridotite, quartzite, marble) were investigated with respect to the fabric dependency of the technical stone properties. The samples could be classified into three macrostructural groups according to their macroscopic fabric anisotropy. The mica texture was determined in 14 of the stones. The maximum intensity $\left(\mathrm{I}_{\max }\right)$ increased proportionately to increasing fabric anisotropy, enabling definition of $\mathrm{I}_{\max }$ for macrofabric groups $\mathrm{I} / \mathrm{II} / \mathrm{III}$. Additionally, the crack density of the microcracks, their preferred orientation and the grain size were taken into account. The technical properties capillary water absorption, compression strength, tensile strength, flexural strength, abrasive resistance, E-modulus and thermal expansion were determined in at least in three directions perpendicular to each other and supplemented by the scalar index characteristics density and porosity. Relationships to the fabric characteristics were obtained both for the means (arithmetic mean from the three main fabric directions $-\mathrm{x}, \mathrm{y}, \mathrm{z}$ axes) and the anisotropy of the technical characteristic values.

In principle, the macroscopic fabric anisotropy shows a positive correlation with that of the technical properties. The means of the rock strengths (compression strength, tensile strength, flexural strength, abrasive resistance) mainly depend on the mechanical properties of the mineralogical composition and microcrack density, while grain size also plays a part. The anisotropy is determined by the texture of the rock-forming minerals and the preferred orientation of open microcracks. The same applies to the statically determined E-modulus, where the elastic properties of the mineralogical composition have a strong influence. Alongside the crack density, these properties are also crucial for the means of the dynamic Emodulus. Its anisotropy is a product of the spatial arrangement of the open microcracks and the texture of the minerals. The capillary water absorption, characterized by the w-value, is not dependent on mineralogical composition, but is dominated by the volume percentage of the capillary pores and their distribution in the rock fabric. In contrast, with regard to thermal expansion, which to a large extent depends on the thermal expansion properties of the rockforming minerals, the influence of microcracks through crack buffering is less significant. In this context, thermally induced crack formation, which can lead to appreciably elevated thermal coefficients of expansion in calcite marble, is of greater importance.

Rules for the cross-correlations of the technical stone parameters to each other can be derived from fabric dependencies of the individual technical characteristics and the processes used for their experimental determination. A good correlation of the (direction-independent) means can be expected when a) the technical characteristics show the most similar type of dependency on the same fabric characteristics, b) the technical parameter show a good correlation with respect to the rock forming minerals and c) the processes during the experimental determination of properties are similar. From a direction-dependent viewpoint, to achieve a representative relationship, the spatial position of the process zones must additionally be identical when determining technical characteristics.

The use of stones, particularly those with a high fabric anisotropy, requires a directionally dependent analysis of the technical characteristics values. The anisotropy of the mechanical properties can reach $80 \%$ and that of thermal expansion $70 \%$. But also the capillary water absorption and the E-modules can sometimes exhibit markedly anisotropic behavior, a factor that must be accounted for in construction engineering applications and damage analysis. 


\section{$\underline{\text { II. Inhaltsverzeichnis }}$}

\section{Inhaltsverzeichnis}

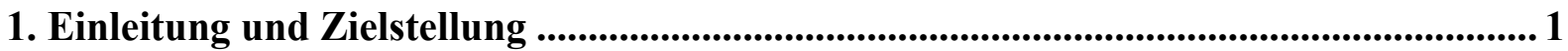

2. Methoden und Terminologie ................................................................................4

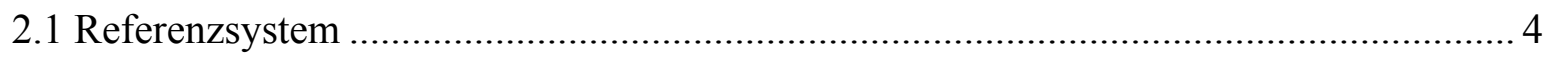

2.1.1 Probenreferenzsystem und Darstellung von Polfiguren und Isolinienplots .............. 4

2.2 Chemische und mineralogische Zusammensetzung .................................................. 5

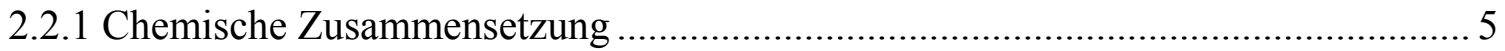

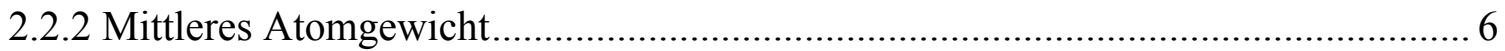

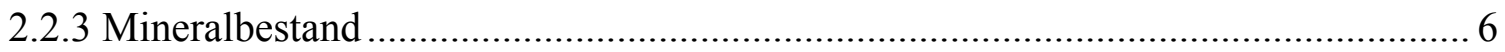

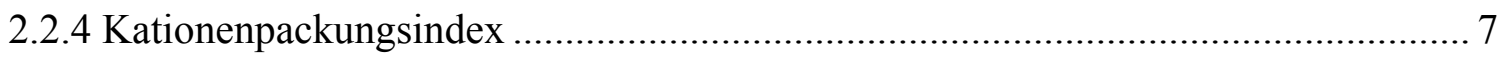

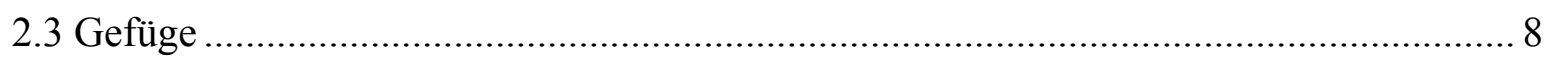

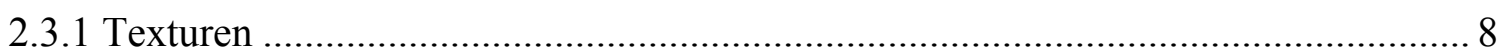

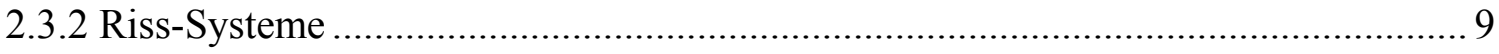

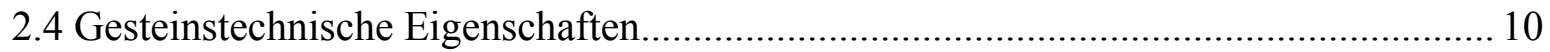

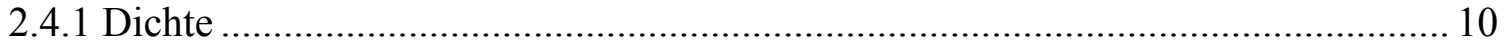

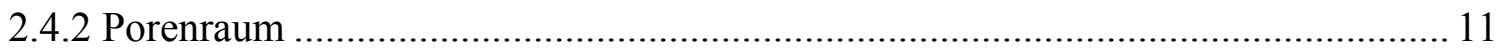

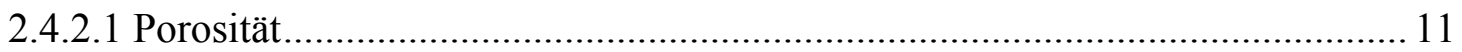

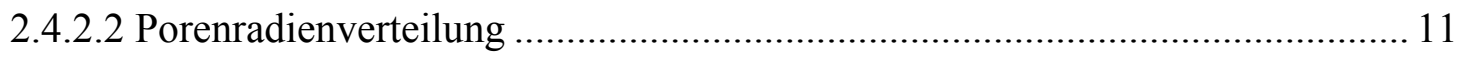

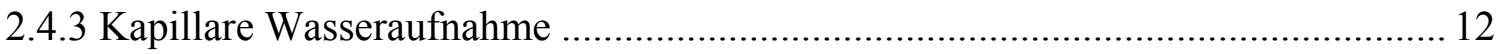

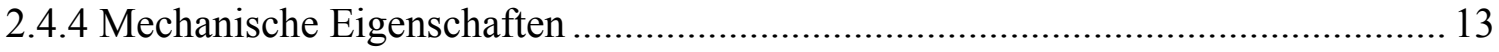

2.4.4.1 Mittlere Härte und Spaltbarkeitsindex ............................................................ 14

2.4.4.2 Druck-, Spalt-, Biege- und Abriebfestigkeit ................................................... 14

2.4.4.3 Richtungsdefinition für die Festigkeitsparameter .......................................... 15

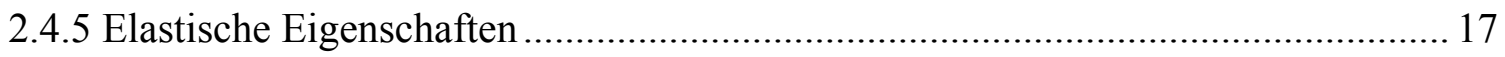

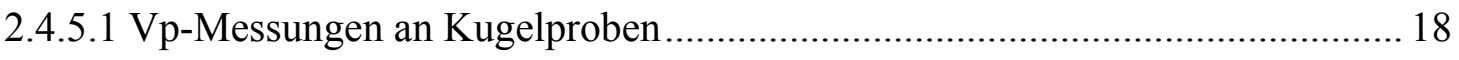

2.4.5.2 Dynamischer E-Modul und Poisson-Zahl ......................................................... 19

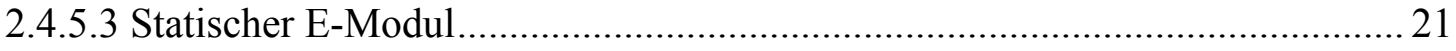

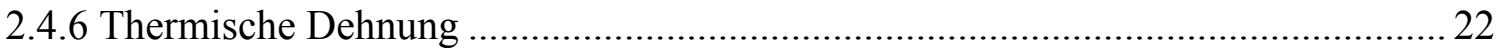

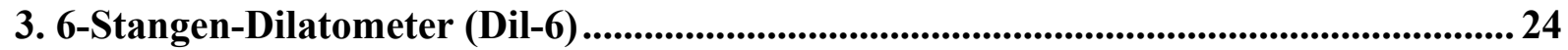

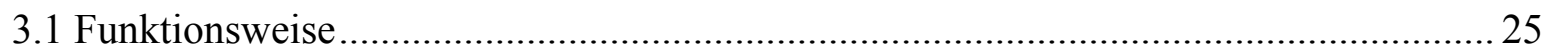

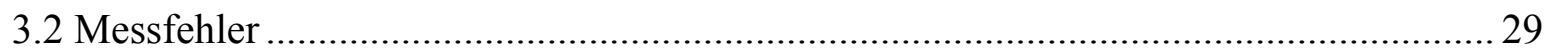

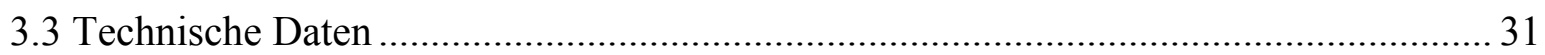




\section{$\underline{\text { II. Inhaltsverzeichnis }}$}

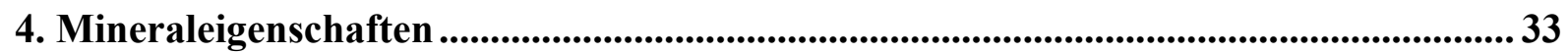

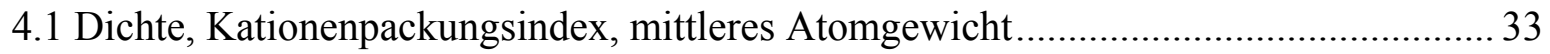

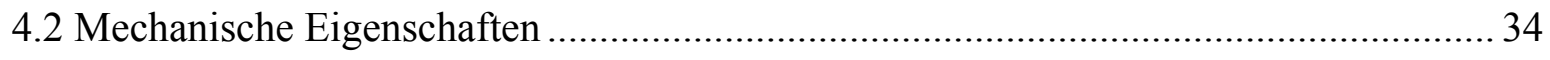

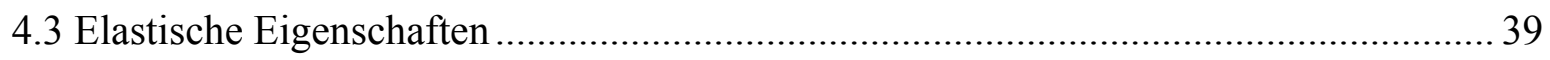

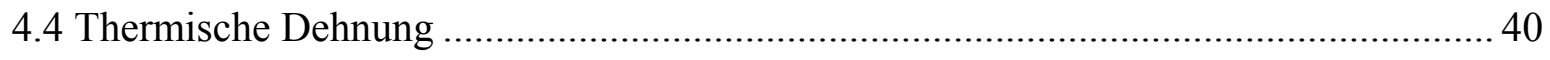

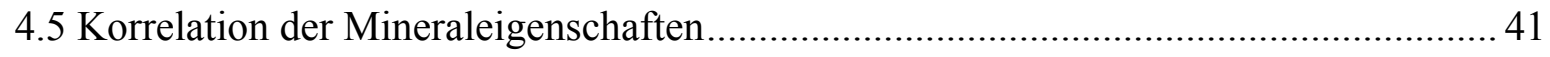

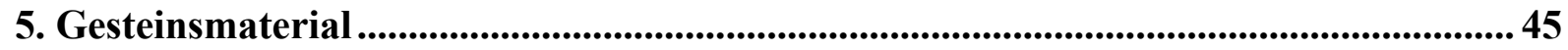

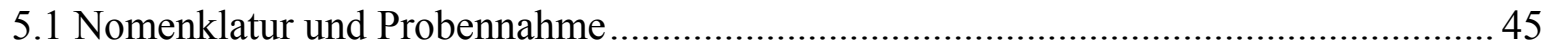

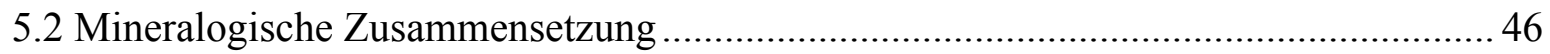

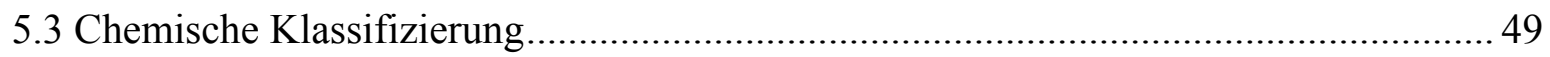

5.4 Verknüpfung und Zusammenstellung mineralogischer und chemischer Daten............. 50

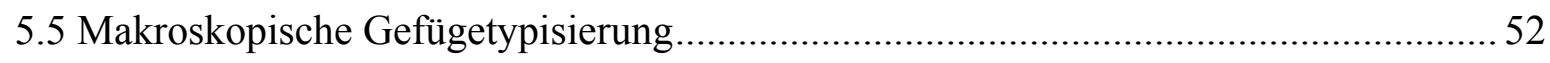

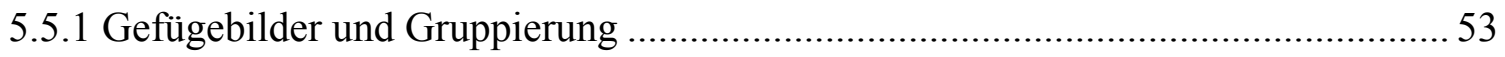

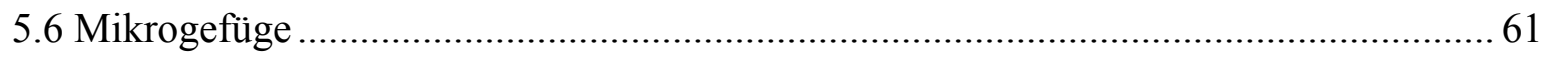

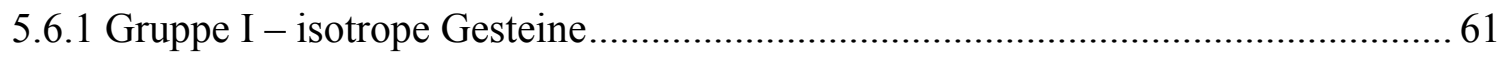

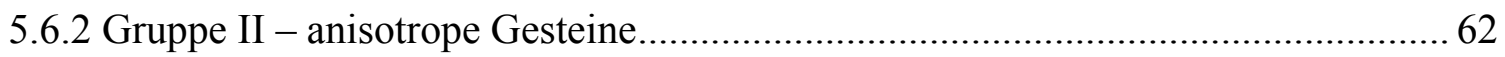

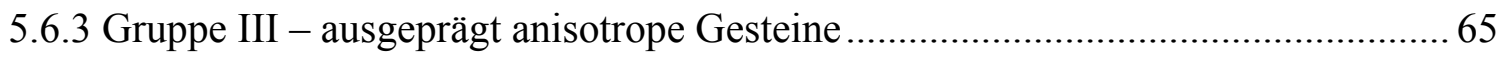

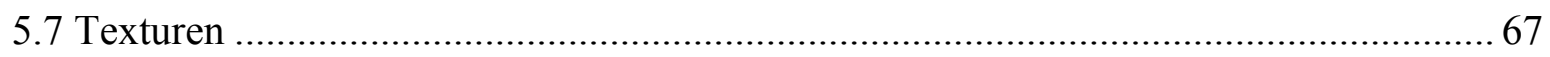

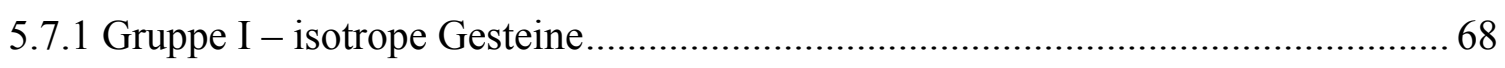

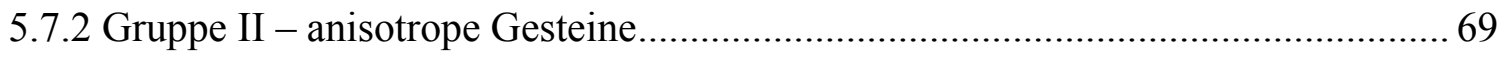

5.7.3 Gruppe III - ausgeprägt anisotrope Gesteine .................................................... 72

5.8 Korrelation von makroskopischem Erscheinungsbild und Textur ................................ 73

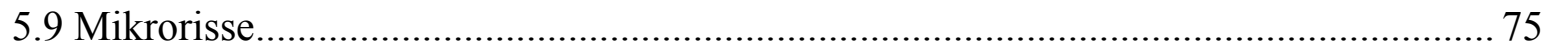

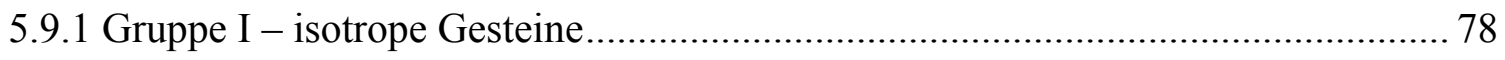

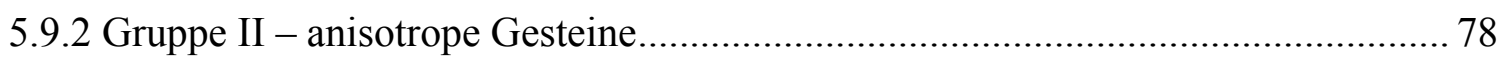

5.9.3 Gruppe III - ausgeprägt anisotrope Gesteine .................................................. 80

5.9.4 Gruppierung nach der Rissdichte offener Mikrorisse ......................................... 81

5.9.5 Beispiele für offene Mikrorisse in den untersuchten Gesteinen ............................ 82

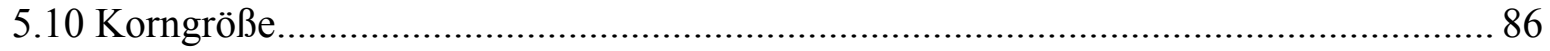

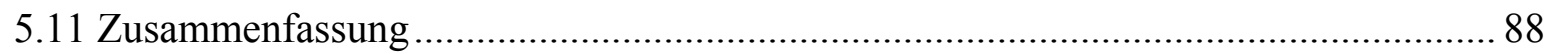

6. Technische Gesteinseigenschaften ..........................................................................................90

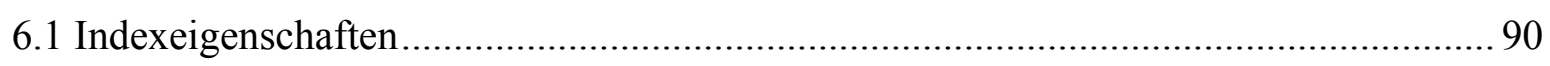

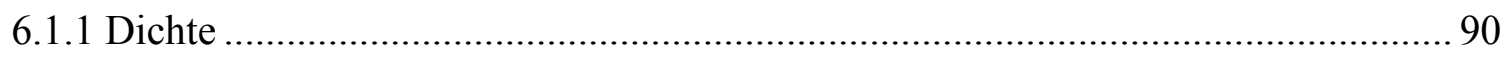

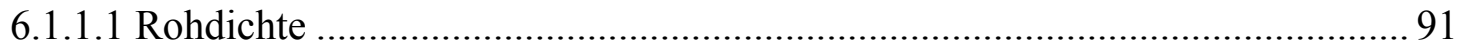




\section{$\underline{\text { II. Inhaltsverzeichnis }}$}

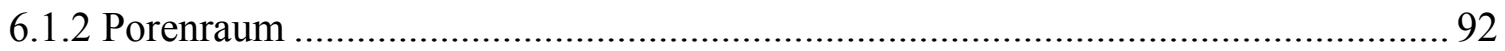

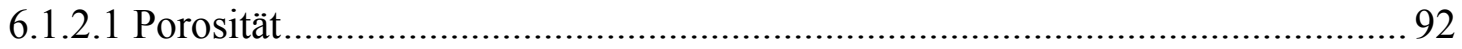

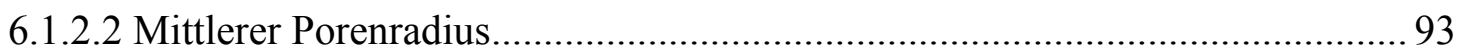

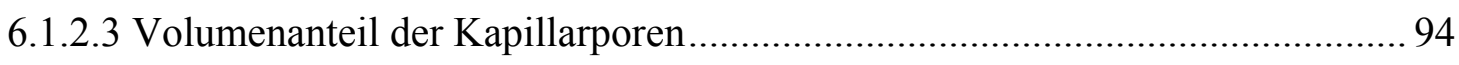

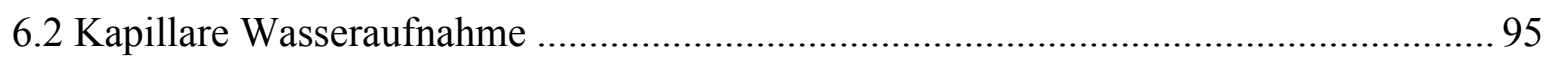

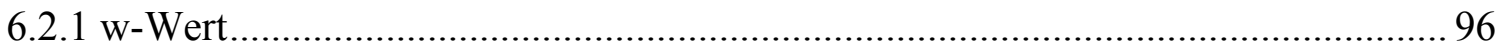

6.2.2 w-Werte und Porenraumeigenschaften ................................................................. 97

6.3 Druck-, Zug-, Biege- und Abriebfestigkeit ............................................................... 100

6.3.1 Ergebnisse in Relation zum makroskopischen Erscheinungsbild ......................... 102

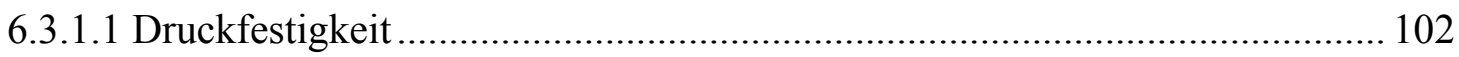

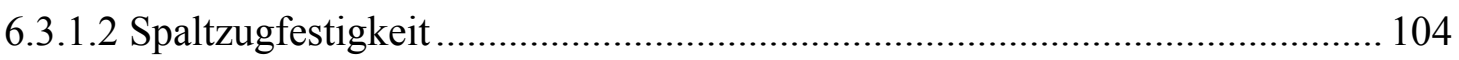

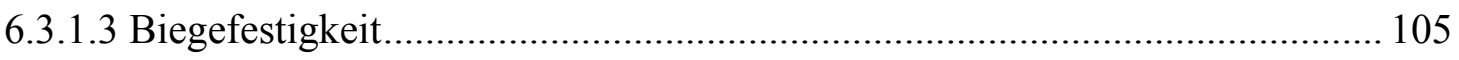

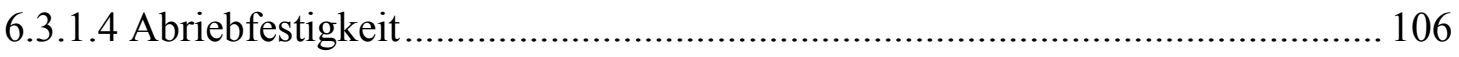

6.3.2 Gesteinsfestigkeiten und mineralogische Zusammensetzung ............................. 107

6.3.3 Beziehung zwischen der Anisotropie der Gesteinsfestigkeiten und der

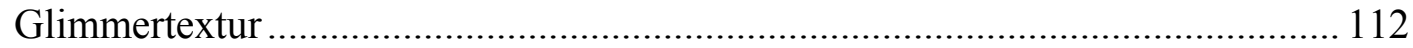

6.3.4 Gesteinsfestigkeiten, offene Mikroriss-Systeme und Korngröße ......................... 115

6.3.5 Bevorzugt aktivierte Gefügeelemente beim Spaltzug- und Abriebversuch ........... 118

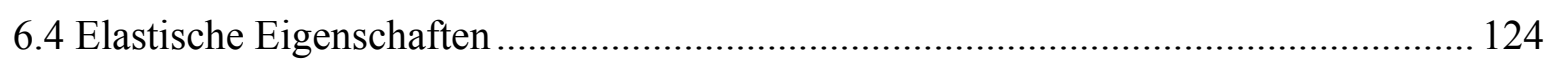

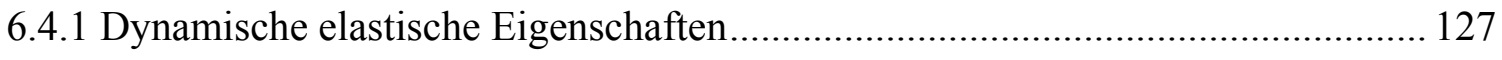

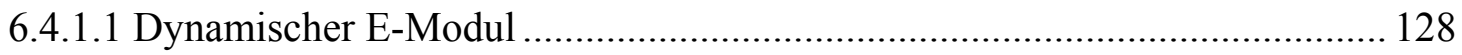

6.4.1.2 Dynamisch ermittelte Poisson-Zahl ............................................................. 129

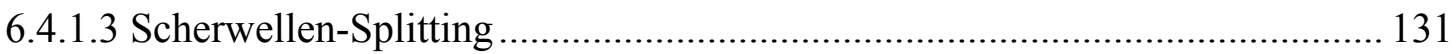

6.4.1.4 Anisotropie des dynamischen E-Moduls.................................................... 133

6.4.1.5 Dynamischer E-Modul, offene Mikrorisse und Mineralbestand..................... 136

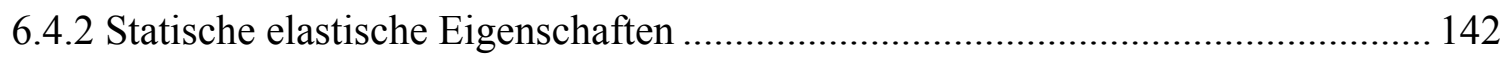

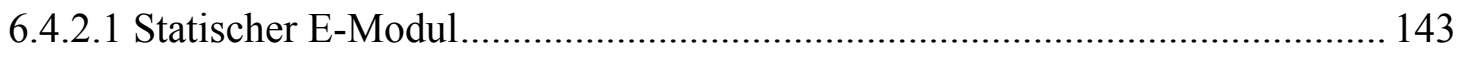

6.4.2.2 Statischer E-Modul, Mineralbestand und offene Mikrorisse ........................ 145

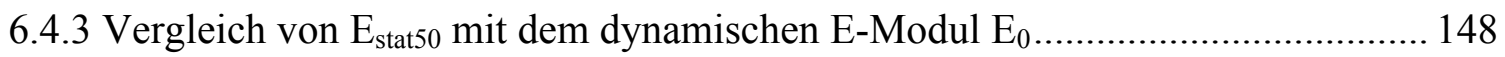

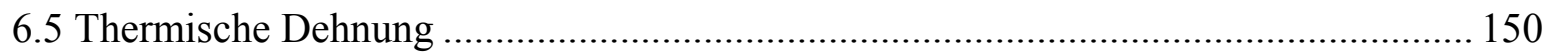

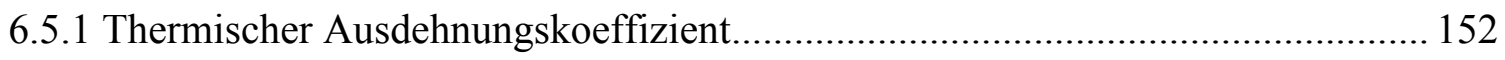

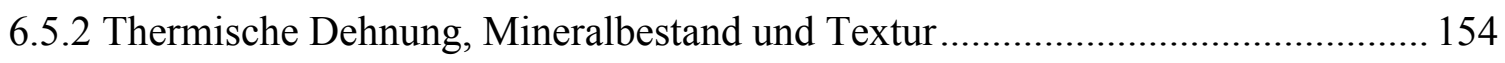

6.5.3 Risspufferung und thermisch induzierte Rissbildung ..................................... 157

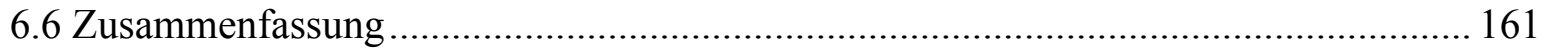




\section{Inhaltsverzeichnis}

7. Richtungsabhängigkeit technischer Gesteinseigenschaften an Fallbeispielen 167

7.1 Sesia-Lanzo-Mylonite 167

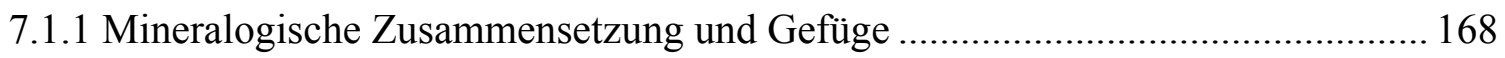

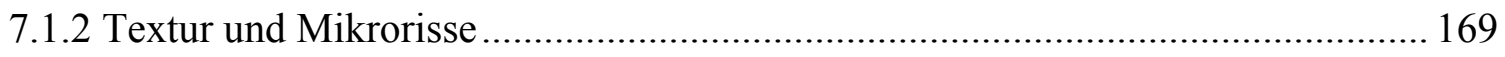

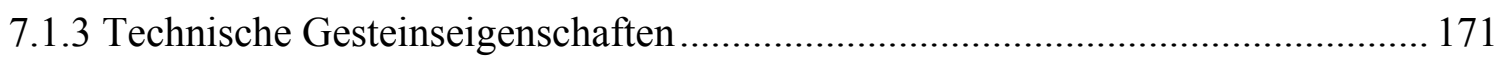

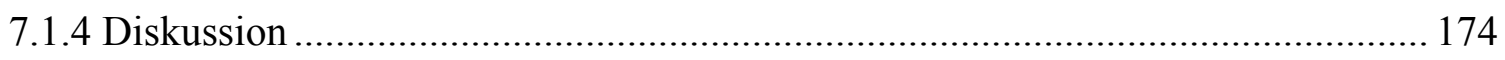

7.1.4.1 Mechanische und statische elastische Eigenschaften ................................. 174

7.1.4.2 Dynamischer E-Modul und thermische Dehnung ....................................... 175

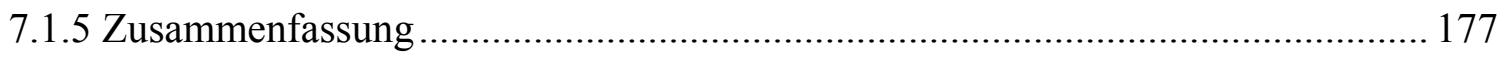

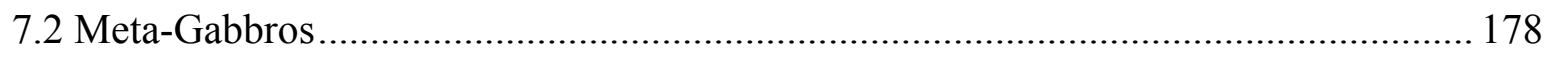

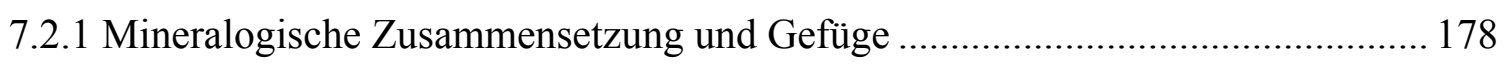

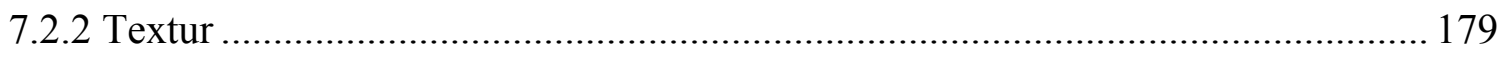

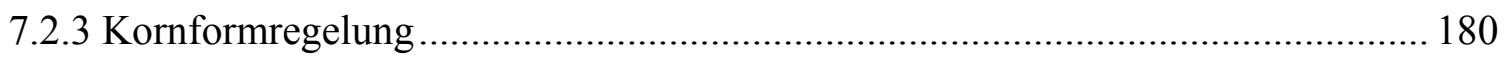

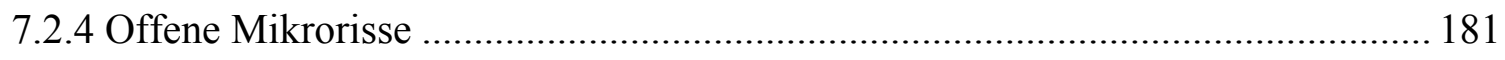

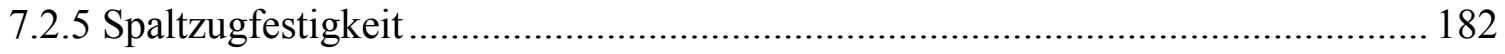

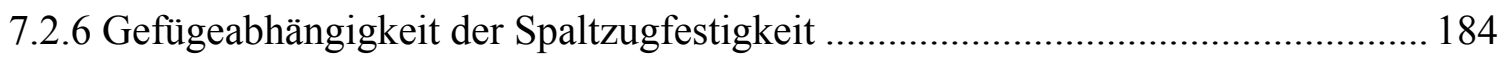

8. Korrelation der technischen Gesteinseigenschaften ................................................ 189

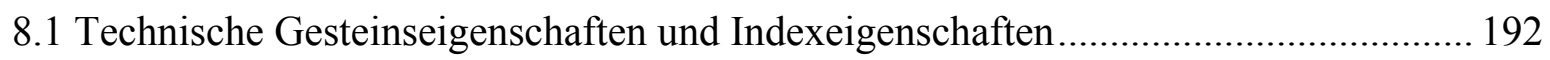

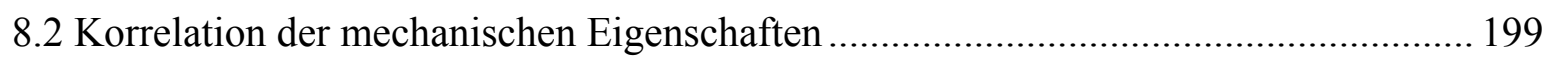

8.2.1 Versagensmodelle und Festigkeitsprognosen .................................................. 202

8.3 Elastische, mechanische Eigenschaften, thermische Dehnung .................................. 208

8.3.1 Dynamischer E-Modul, mechanische Kennwerte, thermische Dehnung.............. 209

8.3.2 Statischer E-Modul, mechanische Kennwerte, thermische Dehnung ................... 212

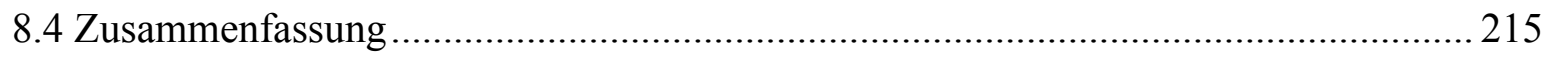

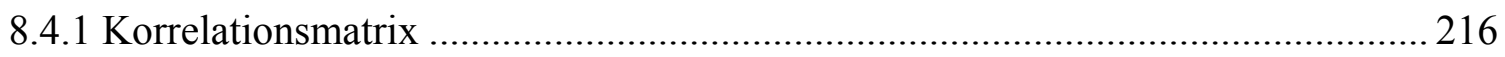

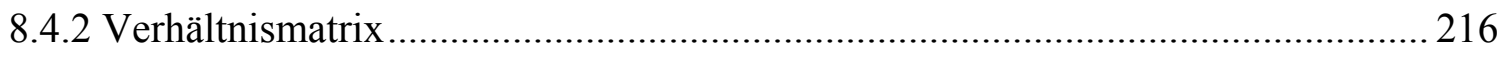

9. Zusammenfassung ........................................................................................................................ 218

9.1 Charakterisierung und Gruppierung der Proben .................................................. 220

9.2 Technische Gesteinseigenschaften, mineralogische Zusammensetzung und Gefüge.. 224

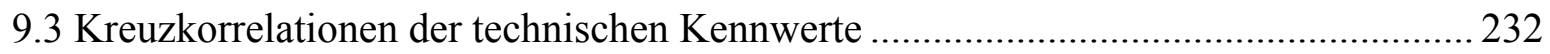

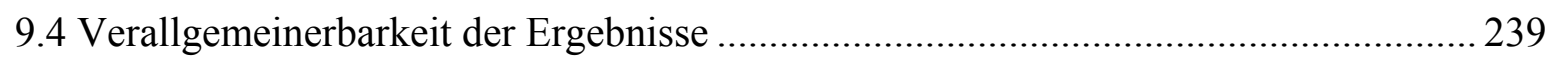

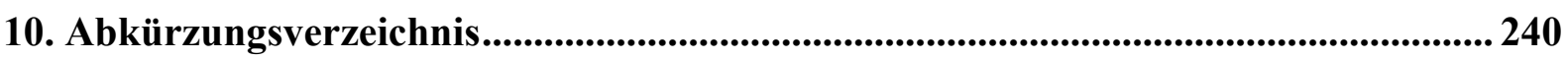

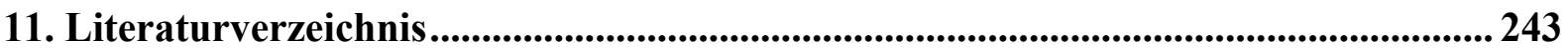




\section{Inhaltsverzeichnis}

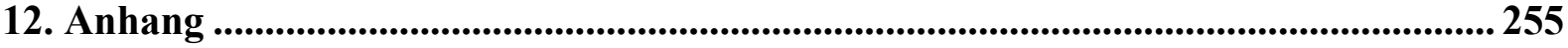

Anhang I.1: Chemische Zusammensetzung und mittlere Atommasse............................ 256

Anhang I.2: Mineralogische Zusammensetzung und Kationenpackungsindex ................ 259

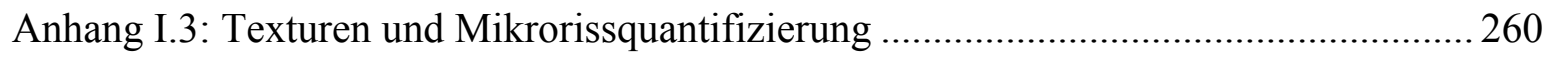

Anhang I.4: Dichte, Porosität, kapillare Wasseraufnahme ............................................ 261

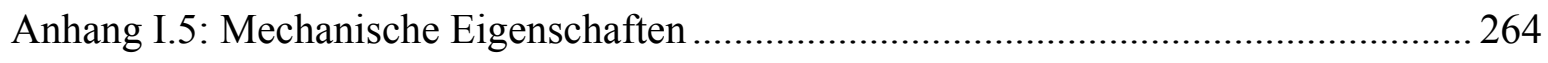

Anhang I.6: Dynamische elastische Eigenschaften........................................................ 268

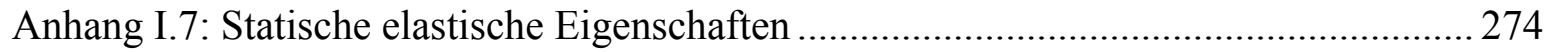

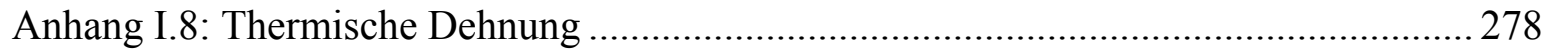

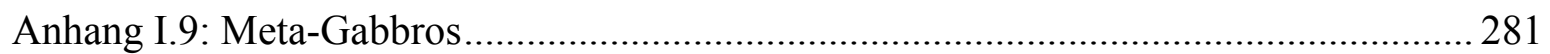

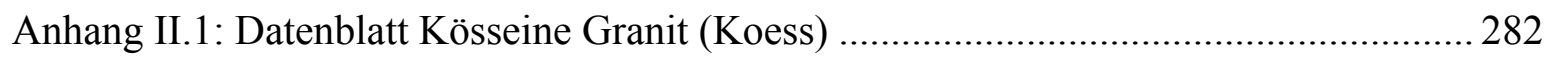

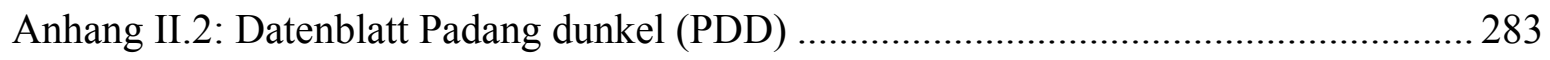

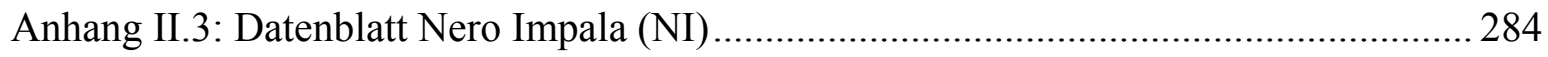

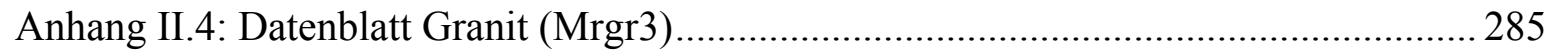

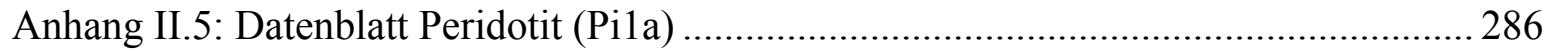

Anhang II.6: Datenblatt Rosa Estremoz (Rek)................................................................. 287

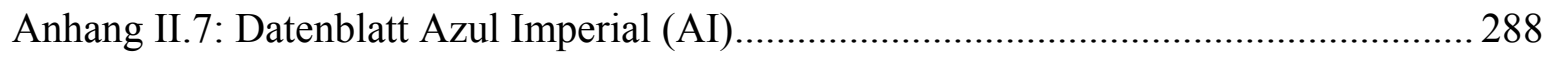

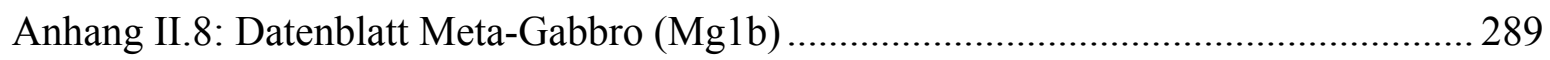

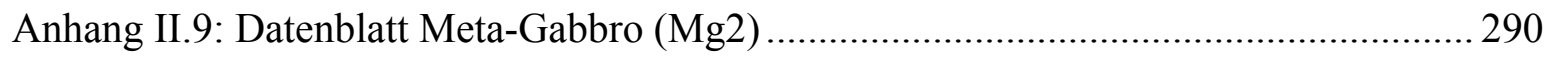

Anhang II.10: Datenblatt granodioritischer Protomylonit (Iv1) ...................................... 291

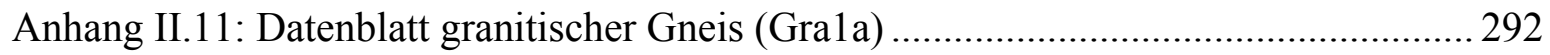

Anhang II.12: Datenblatt granitischer Protomylonit (Mrmy5) ......................................... 293

Anhang II.13: Datenblatt granodioritischer Gneis (Be1) .................................................. 294

Anhang II.14: Datenblatt granodioritischer Mylonit (Iv1b).......................................... 295

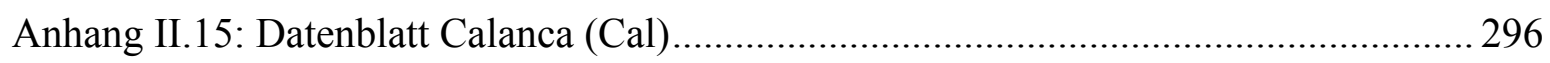

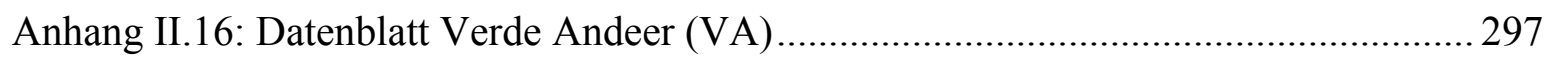

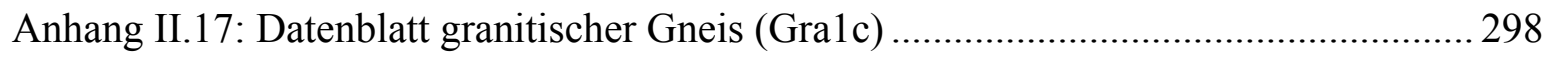

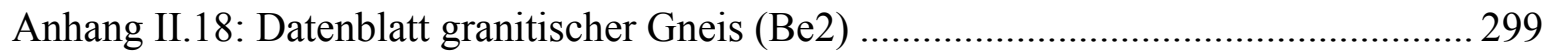

Anhang II.19: Datenblatt granodioritischer Ultramylonit (Iv2) ...................................... 300

Anhang II.20: Datenblatt granitischer Ultramylonit (Gra3a) ............................................ 301

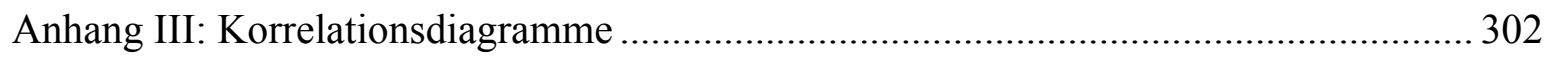

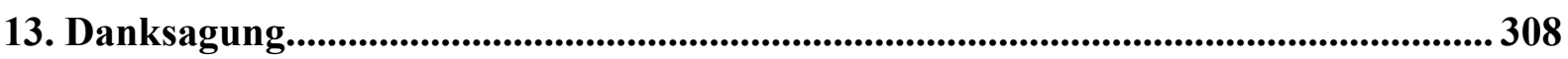

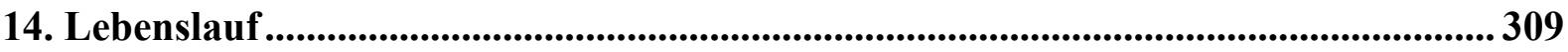




\section{Einleitung und Zielstellung}

Naturwerksteine in ihrer Einzigartigkeit und Beständigkeit finden eine breite bautechnische und künstlerische Anwendung. Sowohl für Fassaden, als auch für Bodenbeläge im Innen- und Außenbereich, als Dekorstein und für Skulpturen und Denkmäler sind Naturwerksteine besonders dort gefragt, wo Exklusivität, Nachhaltigkeit und unverwechselbares Design die Werkstoffauswahl bestimmen. Jedes Gestein ist aufgrund seiner häufig komplexen geologischen Geschichte von Kristallisation aus der Schmelze bzw. Sedimentation und anschließender Diagenese sowie Metamorphose und Deformation in Zeiträumen von Jahrmillionen einzigartig. Die resultierende chemische und mineralogische Zusammensetzung und das Gesteinsgefüge charakterisieren den Werkstoff Naturwerkstein und bedingen von Heterogenität und Anisotropie geprägte Gebrauchseigenschaften. Daraus ergeben sich für die verschiedenen Gesteine eine Vielzahl von Unterschieden in Bezug auf die technische Verwendbarkeit außerhalb der rein ästhetischen Betrachtungsweise. Dieser Umstand erfordert tiefgreifende Kenntnisse über das Gesteinsmaterial und dessen Eignung für eine bestimmte Verwendung, um die angemessene und dem Zweck gerechte Auswahl treffen zu können. Die technische Analyse erfolgt zumeist nach den aktuellen europäischen (EN) und deutschen Normen (DIN). Ausgehend von einer Produktnorm, in der die Anforderungen im Hinblick auf die spätere Verwendung festgelegt sind, wird auf entsprechende Prüfnormen verwiesen, die Details zur experimentellen Ermittlung der gesteinstechnischen Parameter enthalten. Das Prozedere erfordert jedoch keine zusammenfassende Interpretation und Empfehlung, welche die spezifischen Eigenarten des Materials berücksichtigt, so dass die abschließende Beurteilung und Auswahl des Materials dem jeweiligen Entscheidungsträger (z.B. Bauherr, Architekt, Bauingenieur) und der Verbau häufig der ausführenden Baufirma überlassen bleibt. Unabhängig von den DIN EN Normen existieren eine Reihe von Fachbüchern und Artikeln, die sich mit den Besonderheiten des uneinheitlichen Materials Naturwerkstein auseinandersetzen. Zu nennen sind in diesem Zusammenhang Hirschwald (1912), Peschel (1983), Reinsch (1991), Winkler (1994) und Müller (2001), die neben Informationen zur Gesteinsgenese auch Datenkompilationen $\mathrm{zu}$ den technischen Eigenschaften der unterschiedlichen Gesteinstypen anbieten. Doch auch hier werden die komplexen Bezüge zu der mineralogischen Zusammensetzung und der Gefügeausprägung weitestgehend vermieden und lediglich in Ansätzen von Peschel (1983) und Winkler (1994) aufgezeigt. Mit diesem sehr wichtigen Problem beschäftigen sich in intensiverer Form die Studien von Schuh (1987); Fitzner (1988) und Weiss (1992). Des Weiteren beinhalten die ZDGG (Zeitschrift der 
deutschen geologischen Gesellschaft)-Sonderbände 150 (1999)/152 (2001) und die Geological Society of London, Special Publication 205 (2002) eine große Anzahl von stateof-the-art-Publikationen zum Thema der technischen Gesteinseigenschaften und dem Verwitterungsverhalten, bei denen die Abhängigkeit der technischen Parameter von der mineralogischen Komposition und den Gefügeeigenschaften eine zentrale Stellung einnimmt. An dieser Stelle setzt die vorliegende Arbeit an, in deren Rahmen 20 niedrigporöse Magmatite und Metamorphite hinsichtlich der Anisotropie ihrer technisch-physikalischen Eigenschaften untersucht wurden.

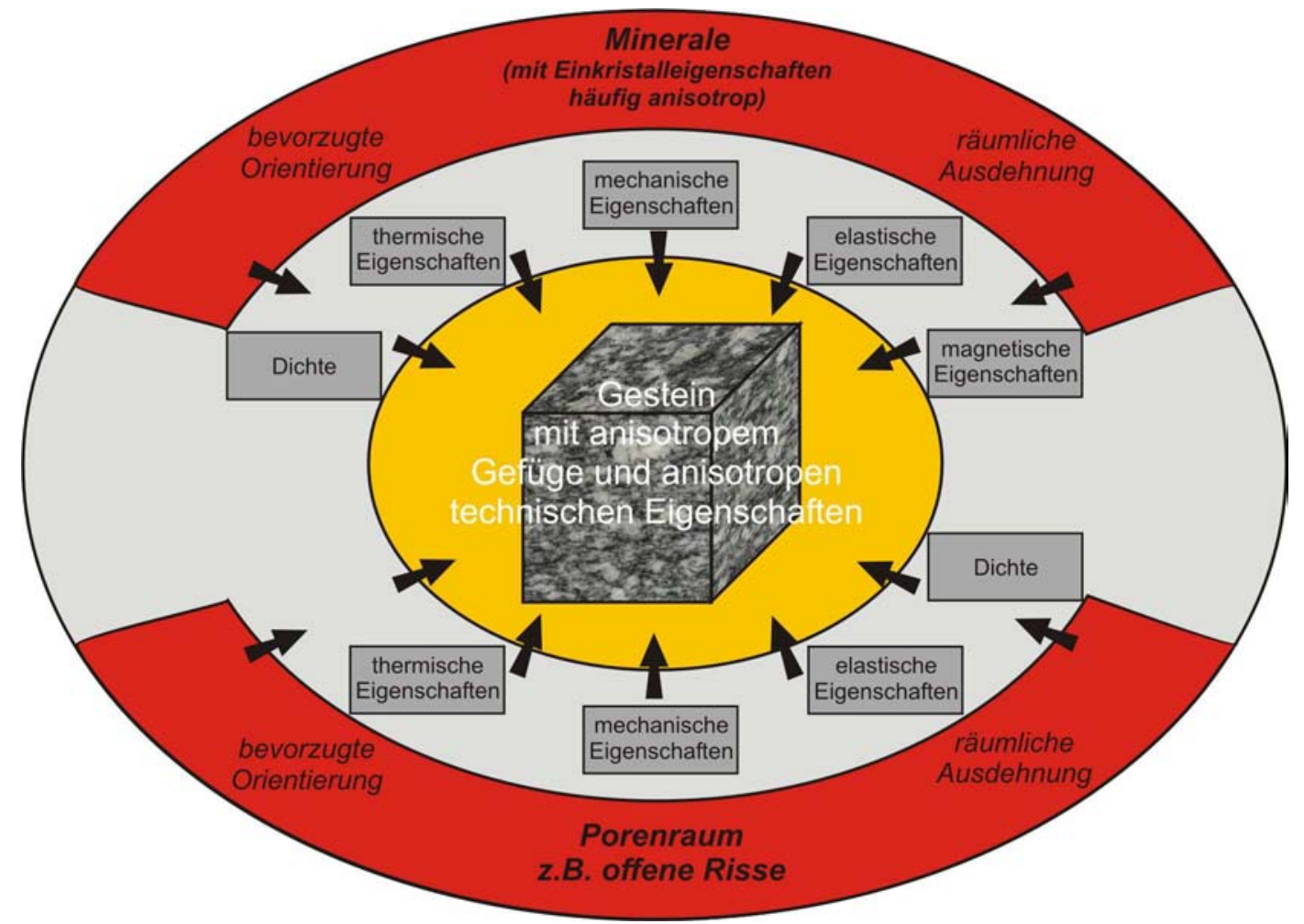

Abb.1-1: Zusammenhang zwischen der mineralogischer Zusammensetzung, dem Gefüge und den technischen Gesteinseigenschaften. Details siehe Text.

Basierend auf dem Wissen aus der Gesteinsmechanik und den Materialwissenschaften folgt die Studie einem Grundkonzept: Gesteine bestehen aus Mineralen, die in einem Kornverband miteinander verbunden sind und einem Porenraum. Die unterschiedlichen Mineralkomponenten haben (häufig anisotrope) physikalische Eigenschaften, die sie gemäß ihrer Volumenanteile und ihrer räumlichen Anordnung in das Gestein einbringen und deren Einfluss von den Eigenschaften des Porenraums überlagert wird. Es resultiert ein Gestein mit je nach Zusammensetzung und Ausbildung des Gefüges unterschiedlichen und ggf. anisotropen technischen Gebrauchseigenschaften (Abb.1-1).

Die verschiedenen gesteinstechnischen Parameter hängen dabei in völlig unterschiedlicher Intensität von bestimmten Gefügemerkmalen und Eigenschaften des Mineralbestands ab. Der 


\section{$\underline{\text { 1. Einleitung und Zielstellung }}$}

Schwerpunkt der vorliegenden Untersuchungen liegt auf der detaillierten Analyse unterschiedlicher Gefügekomponenten, wie z.B. der Textur der gesteinsbildenden Minerale oder Mikrorissen und deren Bedeutung für die unterschiedlichen gesteinstechnischen Parameter nebst deren Richtungsabhängigkeit. Es gilt insbesondere festzustellen welche Eigenschaft wie stark von welchen Gefügemerkmalen abhängt und die Ergebnisse durch theoretische Erklärungsmodelle zu untermauern und wenn möglich eine Quantifizierung vorzunehmen. Um die avisierten Ziele zu erreichen wurden Methoden und Fachwissen aus den Bereichen der Geowissenschaften, der Materialwissenschaften und der Physik miteinander verknüpft und zu einer möglichst umfassenden Betrachtung aller Einflussgrößen herangezogen. 


\section{Methoden und Terminologie}

Bei der Untersuchung der gesteinstechnischen Eigenschaften wurde größtenteils auf deutsche und europäische Normen (DIN EN) zurückgegriffen. Ausnahmen und Abweichungen von den Regelwerken sind in den jeweiligen Unterkapiteln dargestellt.

Die Charakterisierung sowohl der chemischen und mineralogischen Zusammensetzung als auch der Gesteinsgefüge erfolgte mit Hilfe etablierter Methoden aus dem Bereich der Geowissenschaften. Hauptziel ist hier die Quantifizierung und die Reduktion teilweise komplizierter Sachverhalte auf aussagekräftige Parameter. Grundsätzlich wird für alle Untersuchungen eine Normalverteilung der Messwerte angenommen und diese charakterisiert durch den arithmetischen Mittelwert und die einfache Standardabweichung $(\sigma)$ ausgehend von einer Stichprobe. Abweichungen von diesem Schema werden im jeweiligen Kapitel erläutert. Ist die gesuchte Größe eine Funktion verschiedener Grundgrößen, so wird der Fehler (F) mittels Fehlerfortpflanzungsgesetz aus den Werten der Grundgrößen und deren jeweiliger $1-\sigma$-Fehler bzw. absoluter Fehler berechnet.

\subsection{Referenzsystem}

Zur Interpretation von Gefügen und ihrer Korrelation mit resultierenden z.T. stark anisotropen technischen Gesteinseigenschaften ist die Verwendung eines einheitlichen Referenzkoordinatensystems unerlässlich. Hierdurch wird die Orientierungsgleichheit der Proben unterschiedlicher Untersuchungsmethoden und damit der Vergleich von Gefügen mit Richtungsdaten der gesteinstechnischen Eigenschaften gewährleistet.

\subsubsection{Probenreferenzsystem und Darstellung von Polfiguren und Isolinienplots}

Alle Proben wurden nach ihrer makroskopisch sichtbaren Foliation und ihrem makroskopisch sichtbaren Linear zu einem kartesischen x-, y-, z-Koordinatensystem orientiert. Waren keine signifikanten Gefügemerkmale zu erkennen, so erfolgte die Referenzierung nach praktischen Gesichtspunkten (z.B. Probengeometrie). 
(a)

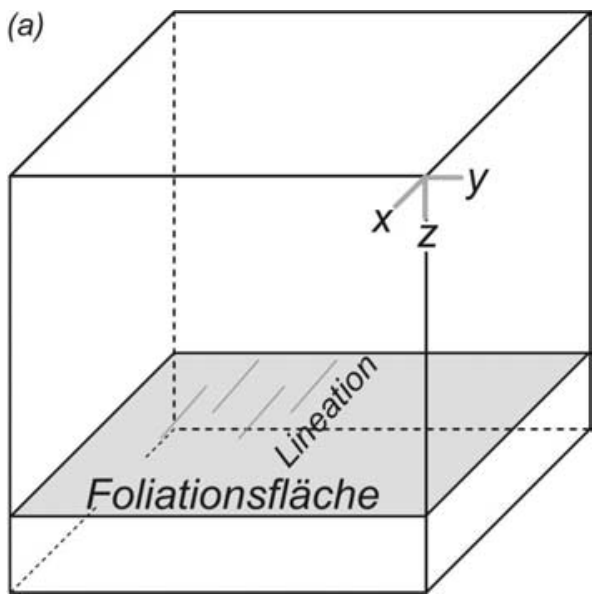

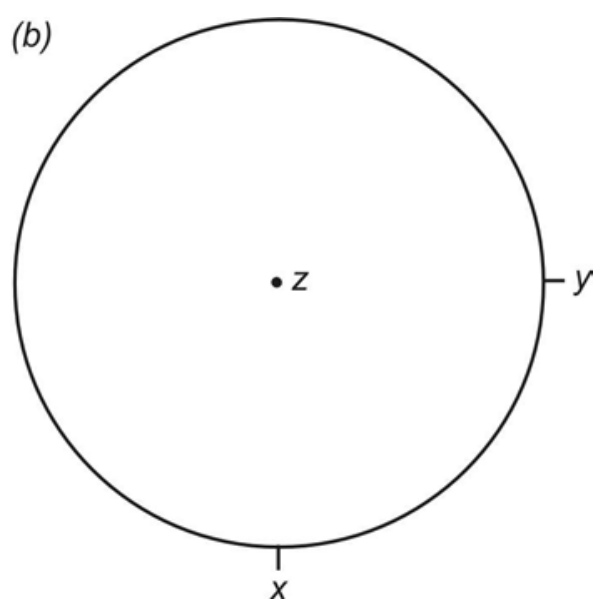

Abb.2-1: (a) Orientierung des Referenzsystems zu den makroskopischen Gefügeelementen Foliation und Lineation. (b) Darstellung von Polfiguren und Isolinienplots.

Die Lineation verläuft parallel zur x-Achse, und die Foliation ist parallel zur x-y-Ebene orientiert. Alle Polfiguren und Isolinienplots sind in Form von Schmidt-Netz-Projektionen (untere Halbkugel) und in gleicher Orientierung in Bezug auf das Referenzkoordinatensystem (x, y, z) dargestellt (Abb.2-1).

\subsection{Chemische und mineralogische Zusammensetzung}

Bezüglich der chemischen Zusammensetzung werden alle Hauptelement-Daten in Gew.\% der jeweiligen Elementoxide angegeben. Die Volumenanteile der Mineralphasen werden in Vol.\% angegeben. Dabei finden nur Komponenten mit Anteilen $>1$ Vol.\% Berücksichtigung. Deren Anteile sind auf 100\% normiert. Die akzessorischen Phasen mit $<1$ Vol.\% sind in ihrer Summe mit nicht mehr als 5\% am Gesamtvolumen beteiligt.

\subsubsection{Chemische Zusammensetzung}

Die chemische Zusammensetzung wurde mit Hilfe der Röntgen-Fluoreszenz-Analyse (RFA) an einem sequentiellen Röntgenspektrometer der Firma Philips (Typ PW 1480) ermittelt. Angegeben werden die Ergebnisse in Gew.\% der Oxide der Elemente Si, Ti, Al, $\mathrm{Fe}^{3+}, \mathrm{Mn}$, $\mathrm{Mg}, \mathrm{Ca}, \mathrm{Na}, \mathrm{K}, \mathrm{P}$. Zusätzlich wurde titrimetrisch die $\mathrm{Fe}^{2+}-$ Konzentration für die Proben PDD, Koess, Gra1a, Gra1c, Gra3a, VA, Cal, Be1, Be2, NI, MrGr3 und MrMy5 (Probennomenklatur siehe Anhang II) bestimmt. Für die RFA-Analysen wurden zwischen $6 \mathrm{~kg}$ und $11 \mathrm{~kg}$ des jeweiligen Probenmaterials verwendet. Unter Berücksichtigung der in den untersuchten Gesteinen vorkommenden Minerale mit ihren Korngrößen und Volumenanteilen kann der 
Fehler aufgrund unrepräsentativer Probenmengen auf besser als 0,06 Gew.\% absolut abgeschätzt werden (Lafitte 1953; Heinrichs \& Herrmann 1990).

\subsubsection{Mittleres Atomgewicht}

Um stellvertretend für die komplexe chemische Zusammensetzung einen Parameter zu erhalten, wurde nach folgendem Zusammenhang das mittlere Atomgewicht $\left(\mathrm{M}_{\mathrm{G}}\right)$ der Gesteine bestimmt:

$$
M_{G}=\left(\sum_{i=1}^{n} x_{i} / M_{i}\right)^{-1}
$$

$M_{G}-$ mittleres Atomgewicht. $x_{i}-$ Anteile des i-ten Oxids [Gew.\%]. $M_{i}-$ mittleres Atomgewicht des $i-$ ten Oxids; aus Siegesmund (1989).

\subsubsection{Mineralbestand}

Für die Quantifizierung des Mineralbestands wurden unterschiedliche Methoden angewendet. Die Phasenanteile der Proben Be1, Be2, MrMy5, Cal, Rek und AI wurden mit Hilfe des Programms MONA V3.01 (Metzner \& Grimmeisen 1990; www.unimarburg.de/geowissenschaften/mo-desc.htm) ermittelt. Hier werden aus chemischen Gesamtgesteinsanalysen sowie der chemischen Zusammensetzung der Minerale die Phasenanteile in Gew.\% berechnet. Diese Daten wurden anschließend über das Verhältnis von Mineraldichte zur Gesamtgesteinsdichte in Vol.\% umgerechnet (Heinrichs und Herrmann 1990).

Die Quantifizierung des Mineralbestands der Proben MrGr3, PDD, NI, Koess, VA, Gra1a, Gra1c, Gra3a, Pila fand mittels der röntgenographischen Rietveld-Methode (Young 1995) statt. Die Untersuchungen wurden an einem Röntgendiffraktometer der Firma Phillips durchgeführt. Die in Gew.\% angegebenen Werte wurden, wie bereits beschrieben, in Vol.\% umgerechnet.

Die Proben Iv1, Iv1b und Iv2 werden bezüglich ihres Mineralbestands als identisch angesehen. Stellvertretend für die Probenserie wurde für Iv1b per Mikrosonden MAP- und anschließender Phasenanalyse an drei Dünnschliffen aus den Schnittlagen yz, xz und xy der quantitative Mineralbestand ermittelt. Die Untersuchungen wurden an einer Elektronenstrahlmikrosonde des Typ JEOL JXA 8900RL durchgeführt. 
Der Mineralbestand der Meta-Gabbros von Anzola geht auf bereits von Dornbusch (1995) publizierte Daten zurück. Es handelt sich dabei um den arithmetischen Mittelwert der Dornbusch-Proben DT 46, DT47 und DT48.

\subsubsection{Kationenpackungsindex}

Innerhalb des untersuchten Probensatzes kommen 17 verschiedene Minerale mit variierenden Volumenanteilen vor. Als übergeordneter Wert, der sowohl die unterschiedlichen Volumenanteile der Phasen, als auch in Ansätzen die Bindungen und damit gewisse Charakteristika der Minerale selbst einbezieht, wurde der Kationenpackungsindex $\left(\mathrm{k}_{\mathrm{G}}\right)$ (Buntebarth 1982; Rybach \& Buntebarth 1982) berechnet:

$$
k_{G}=\sum_{i=1}^{n} p_{i} * k_{M i}=\sum_{i=1}^{n} p_{i} * \frac{c_{i}}{V_{M, i}} \quad\left[\mathrm{~mol} / \mathrm{cm}^{3}\right]
$$

$k_{G}$ - Kationenpackungsindex des Gesteins $\left[\mathrm{mol} / \mathrm{cm}^{3}\right] ; k_{M i}-$ Kationenpackungsindex der i-ten Phase $\left[\mathrm{mol} / \mathrm{cm}^{3}\right] ; p_{i}-$ Volumenanteil der i-ten Phase; $c_{i}$ - Anzahl der Kationen je Formeleinheit der i-ten Phase; $V_{M, i}-$ Molarvolumen der i-ten Phase $\left[\mathrm{cm}^{3} / \mathrm{mol}\right]$.

Tab.2-1: Kationen je Formeleinheit (c), Molarvolumen $\left(V_{M}\right)$ und Kationenpackungsindex $\left(k_{M}\right)$ der Minerale zur Berechnung des Gesteinskationenpackungsindex $\left(k_{G}\right)$.

\begin{tabular}{|c|c|c|c|c|}
\hline Mineral & Formel & c & $\mathrm{V}_{\mathrm{M}}\left[\mathrm{cm}^{3} / \mathrm{mol}\right]$ & $\mathrm{k}_{\mathrm{M}}\left[\mathrm{mol} / \mathrm{cm}^{3}\right]$ \\
\hline a-Quarz & $\mathrm{SiO}_{2}$ & & & $0,0441^{*}$ \\
\hline Kalifeldspat (Orthoklas) & $\mathrm{KAISi}_{3} \mathrm{O}_{8}$ & & & $0,0458^{*}$ \\
\hline Plagioklas (Albit) & $\mathrm{NaAISi}_{3} \mathrm{O}_{8}$ & & & $0,0499^{*}$ \\
\hline Plagioklas (Anorthit) & $\mathrm{CaAl}_{2} \mathrm{Si}_{2} \mathrm{O}_{8}$ & & & $0,0497^{*}$ \\
\hline Muskovit & $\mathrm{KAl}_{2}(\mathrm{OH}, \mathrm{F})_{2}\left[\mathrm{AlSi}_{3} \mathrm{O}_{10}\right]$ & & & $0,0498^{*}$ \\
\hline Annite & $\mathrm{KFe}_{3}\left(\mathrm{AlSi}_{3}\right) \mathrm{O}_{10}(\mathrm{OH})_{2}$ & 8 & 152,63 & $0,0524^{* *}$ \\
\hline Phlogopit & $\mathrm{KMg}_{3}\left(\mathrm{AISi}_{3}\right) \mathrm{O}_{10}(\mathrm{OH})_{2}$ & 8 & 146,87 & $0,0545^{* *}$ \\
\hline Chlorit & $\left(\mathrm{Mg}_{2} \mathrm{Al}\right)\left(\mathrm{AlSi}_{3}\right) \mathrm{O}_{10}(\mathrm{OH})_{2}$ & 7 & 210,57 & $0,0332^{* *}$ \\
\hline Granat (Grossular) & $\mathrm{Ca}_{3} \mathrm{Al}_{2} \mathrm{Si}_{3} \mathrm{O}_{12}$ & & & $0,0639^{*}$ \\
\hline Granat (Almandin) & $\mathrm{Fe}_{3} \mathrm{Al}_{2} \mathrm{Si}_{3} \mathrm{O}_{12}$ & & & $0,0694^{*}$ \\
\hline Granat (Pyrop) & $\mathrm{Mg}_{3} \mathrm{Al}_{2} \mathrm{Si}_{3} \mathrm{O}_{12}$ & & & $0,0706^{*}$ \\
\hline Epidot/Klinozoisit & $\mathrm{Ca}_{2} \mathrm{Al}_{3} \mathrm{Si}_{3} \mathrm{O}_{12}(\mathrm{OH})$ & 8 & 136,83 & $0,0585^{\star \star}$ \\
\hline Dumortierit & $\mathrm{Al}_{6,5-7}\left(\mathrm{BO}_{3}\right)\left(\mathrm{SiO}_{4}\right)_{3}(\mathrm{O}, \mathrm{OH})_{3}$ & & & $0,0550^{* * *}$ \\
\hline Calcit & $\mathrm{CaCO}_{3}$ & 2 & 36,9257 & $0,0542^{* *}$ \\
\hline Hornblende (Pargasit) & $\mathrm{NaCa}_{2} \mathrm{FeMg}_{4} \mathrm{Al}_{2} \mathrm{Si}_{6} \mathrm{O}_{22}(\mathrm{OH})_{2}$ & 16 & 274,9 & $0,0582^{\star \star}$ \\
\hline Klinopyroxen (Diopsid) & $\mathrm{CaMgSi}_{2} \mathrm{O}_{6}$ & & & $0,0605^{*}$ \\
\hline Klinopyroxen (Hedenbergit) & $\mathrm{CaFeSi}_{2} \mathrm{O}_{6}$ & & & $0,0606^{*}$ \\
\hline Orthopyroxen(Enstatit) & $\mathrm{MgSiO}_{3}$ & & & $0,0637^{*}$ \\
\hline Olivin $\left(\mathrm{Fo}_{90} \mathrm{Fa}_{10}\right)$ & $(\mathrm{Fe}, \mathrm{Mg})_{2} \mathrm{SiO}_{4}$ & & & $0,0680^{*}$ \\
\hline Magnetit & $\mathrm{Fe}_{3} \mathrm{O}_{4}$ & & & 0,0674 * \\
\hline Spinell & $\mathrm{MgAl}_{2} \mathrm{O}_{4}$ & & & $0,0755^{*}$ \\
\hline Hercynit & $\mathrm{FeAl}_{2} \mathrm{O}_{4}$ & & & 0,0735 * \\
\hline Hämatit & $\mathrm{Fe}_{2} \mathrm{O}_{3}$ & 2 & 30,388 & $0,0658^{* *}$ \\
\hline
\end{tabular}

*Daten aus Buntebarth 1982; **Werte errechnet mit $V_{M}$ aus Smyth \& McCormick 1995; ***abgeschätzt nach der Dichte von Dumortierit (3,39 $\left.\mathrm{g} / \mathrm{cm}^{3}-w w w . w e b m i n e r a l . c o m\right)$. 
Die für die Berechnung verwendeten Mineralparameter (c, $\mathrm{V}_{\mathrm{M}}, \mathrm{k}_{\mathrm{M}}$ ) sind in Tab.2-1 zusammengefasst.

\subsection{Gefüge}

Für die Durchführung lichtmikroskopischer Untersuchungen (Korngefüge, Risse, Glimmertexturen) wurden Dünnschliffe senkrecht zu den Richtungen x, y und z angefertigt. Ziel aller Gefügeuntersuchungen ist zum ersten die Beschreibung, zum zweiten aber auch die Umsetzung der Beobachtungen in quantifizierbare Größen, was zu einer Gruppierung der Proben nach bestimmten Gefügemerkmalen führt.

\subsubsection{Texturen}

An 19 der 20 Gesteine wurden Texturuntersuchungen durchgeführt. Zur Ermittlung der Glimmertexturen (Biotit und/oder Muskovit) wurde die Raumlage der Glimmer (001)-Flächen in drei senkrecht zueinander orientierten Dünnschliffen am Universal-Drehtisch-Mikroskop (U-Tisch) vermessen. Die Daten der 109 bis 212 gemessenen Einzelkörner wurden in die Schnittlage xy (Fläche senkrecht zu z) rotiert und mit dem Programm STEREONETT VS 2.4.6 (Duyster, J.) als Belegungsdichte-Diagramm (Schmidt-Netz untere Halbkugel) dargestellt. Mit Hilfe des Programms ARIANE2 (Adam 1982) erfolgte die Bestimmung der Eigenvektoren und der zugehörigen Eigenwerte (s1, s2 und s3) sowie dem daraus ableitbaren Symmetrieparameter $\mathrm{k}_{\mathrm{W}}$ (Woodcock 1977) und dem Wert für die Regelungsschärfe $\mathrm{c}_{\mathrm{W}}$ (Woodcock 1977). In Anlehnung an Jelinek (1981) wurde der dimensionslose Formfaktor T nach folgender Gleichung ermittelt:

$$
T=\frac{\ln (s 3 / s 1)-\ln (s 2 / s 1)}{\ln (s 3 / s 1)+\ln (s 2 / s 1)}
$$

mit $\mathrm{s} 1 \leq \mathrm{s} 2 \leq \mathrm{s} 3$

$T$ - Formfaktor. s1, s2, s3 - Eigenwerte der Eigenvektoren.

Die Texturen von Quarz, Plagioklas, Kalzit, Hornblende, Klinopyroxen, Orthopyroxen und Olivin konnten unter Einsatz des Neutronen-Beugungs-Verfahren nach dem time-of-flightPrinzip (Feldmann 1989) sowie einer anschließenden quantitativen Texturanalyse bestimmt werden. Die Dimensionen der zylindrischen Proben betrugen D40 mm*40 mm. Die Neutronen-Beugungs-Messungen wurden am Flugzeitdiffraktometer (SKAT) am Vereinigten Institut für Kernforschung in Dubna/Russland durchgeführt (Ullemeyer et al. 1998). Die 
Auswertung der Messdaten erfolgte mittels der Software GEOTOF und des Software-Pakets BEARTEX (von Matthies, S., Wenk, H.R. \& Kunze, K.). Nicht experimentell ermittelbare Polfiguren wurden dabei über die orientation distribution funktion (ODF) berechnet. Die Polfiguren für die unterschiedlichen Minerale und die verschiedenen kristallographischen Elemente wurden mit dem Programm PFPLOT im xy-Schnitt (Schmidt-Netz untere Halbkugel) dargestellt. Mathematische Grundlagen zur quantitativen Texturanalyse liefert z.B. Bunge (1985,1987). Einige der speziellen Probleme bei der Texturanalyse geologischer Materialien behandeln Leiss \& Ullemeyer (1999) sowie Leiss (2002).

\subsubsection{Riss-Systeme}

Die Riss-Systeme innerhalb der Gesteine konnten lichtoptisch erfasst und gemäß ihrer Ausbildung bestimmten Gruppen zugeordnet werden. Die Unterteilung erfolgt zunächst nach Mineralphasen, dann nach der Lage des Risses zum Korn/Korngefüge und schließlich nach Risstyp. Die Benennung richtet sich nach den Nomenklaturen von Kranz (1983), Vollbrecht et al. (1994) und Blenkinsop (2000).

\section{Mineralphase:}

z.B. Plagioklas, Quarz, Biotit, u.s.w.

\section{Lage:}
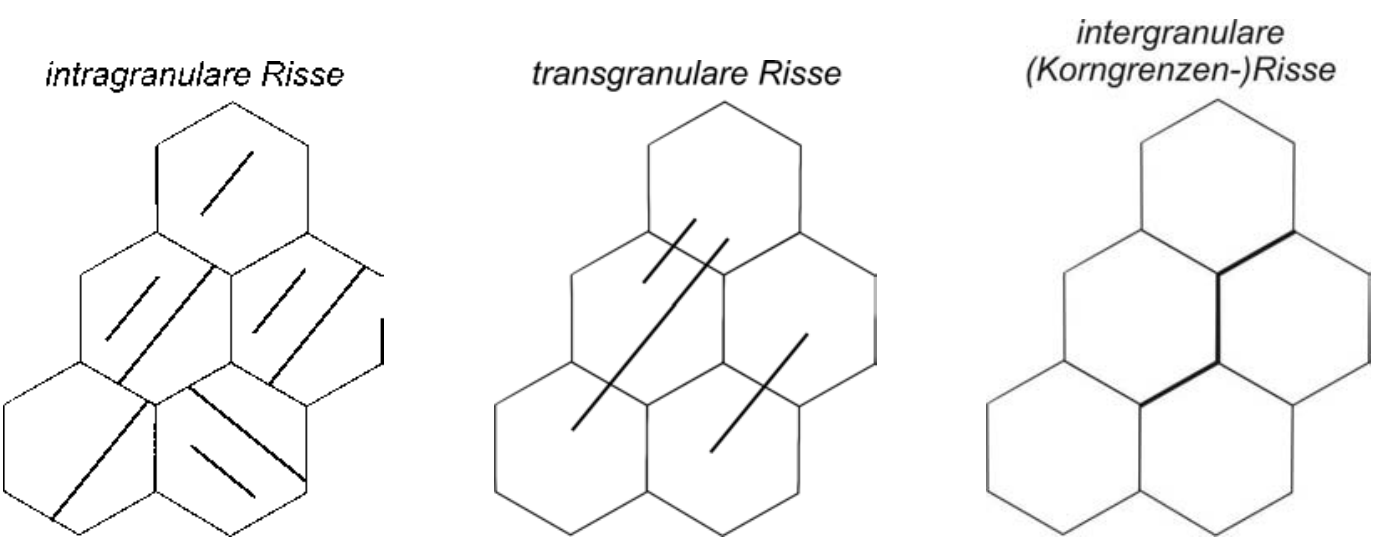

Abb.2-2: Klassifikation der Risslagen.

Risstyp: Offener Riss (z.B. Spaltflächenriss), verheilter Riss, mineralisierter Riss

Aussagen über die Rissdichte und eine mögliche Vorzugsorientierung der offenen RissSysteme werden anhand der Ergebnisse von Vp-Ultraschallmessungen an trockenen und wassergesättigten Kugelproben getroffen (vgl. Kap.2.4.5.1). 


\subsection{Gesteinstechnische Eigenschaften}

Bereits während der Präparation für die nachfolgend beschriebenen gesteinstechnischen Untersuchungen fand die repräsentative Probenauswahl eine besondere Beachtung. Proben mit makroskopischen Rissen sowie eindeutigen Anzeichen von Alterationen und Proben aus besonders inhomogenen Bereichen wurden von vornherein aussortiert. Das Ziel ist, eine möglichst geringe Streuung der Messwerte aufgrund falscher (nicht repräsentativer) Probennahme zu erreichen. Die angewendeten Normen sehen eine Probentrocknung bei $70^{\circ} \mathrm{C}$ (z.B. DIN EN 1926) bis $110^{\circ} \mathrm{C}$ (z.B. DIN 52108) vor. Insbesondere bei Marmoren (Zeisig et al. 2002), aber auch bei Graniten und Gneisen kann eine thermische Vorschädigung und eine damit verbundene Rissbildung und Veränderung der technischen Eigenschaften nicht ausgeschlossen werden. Daher wurden alle Untersuchungen nach mindestens 48-stündiger Trocknung bei lediglich $30^{\circ} \mathrm{C}$ bis zur Gewichtskonstanz durchgeführt.

\subsubsection{Dichte}

Bezüglich der Gesteinsdichte wird zwischen der Matrixdichte $\varrho_{M}$ (mittlere Dichte der Mineralbestandteile ohne Poren) und der Rohdichte $Q_{\text {Roh }}$ (mittlere Dichte von Mineralbestandteilen und Poren) unterschieden. Beide Parameter wurden mit Hilfe der Auftriebswägung nach dem Archimedes-Prinzip (z.B. Monicard 1980) an würfelförmigen Proben mit Kantenlängen zwischen $60 \mathrm{~mm}$ und $100 \mathrm{~mm}$ bestimmt. Die Proben wurden trocken und wassergesättigt an der Raumluft sowie wassergesättigt unter Wasser gewogen. Nach der Beziehung

$$
\rho_{M}=\frac{m_{t}}{m_{t}-m_{a u}} \quad\left[\mathrm{~g} / \mathrm{cm}^{3}\right]
$$

$\varrho_{M}-$ Matrixdichte $\left[\mathrm{g} / \mathrm{cm}^{3}\right] ; m_{t}-$ Masse trocken [g]; $m_{a u}-$ Masse Auftrieb wassergesättigt [g].

wird die Matrixdichte und nach der Beziehung

$$
\rho_{\text {Roh }}=\frac{m_{t}}{m_{n}-m_{a u}} \quad\left[\mathrm{~g} / \mathrm{cm}^{3}\right]
$$

$\varrho_{\text {Roh }}$ - Rohdichte $\left[g / \mathrm{cm}^{3}\right] ; m_{t}-$ Masse trocken $[g] ; m_{n}$ - Masse wassergesättigt $[g] ; m_{a u}-$ Masse Auftrieb wassergesättigt [g].

die Rohdichte bestimmt. Bei der Anwendung der beschriebenen Methode bleibt der abgeschlossene Porenraum unberücksichtigt, da er nicht wassergesättigt wird. Durch die 
resultierenden zu niedrigen Auftriebs- und Nassgewichte wird die Matrixdichte tendenziell unterschätzt. Unter der Annahme von maximalen Porositäten von 2 Vol.\% und dem Zusammenhang

$$
\rho_{M}=\rho_{\text {Roh }} * \frac{V_{\text {ges }}}{V_{\text {ges }}-\phi} \quad\left[\mathrm{g} / \mathrm{cm}^{3}\right]
$$

$\varrho_{M}-$ Matrixdichte $\left[\mathrm{g} / \mathrm{cm}^{3}\right] ; \varrho_{\text {Roh }}-$ Rohdichte $\left[\mathrm{g} / \mathrm{cm}^{3}\right] ; V_{\text {ges }}-$ Gesamtvolumen des Gesteins [Vol.\%]; $\phi-$ Porosität [Vol.\%].

ergeben sich maximal 2\% relativ zu kleine Matrixdichten, und das auch nur im Fall von 100\% Anteil des abgeschlossenen Porenraums an der Porosität. Eine Berechnung der Matrixdichte aus den Volumenanteilen der einzelnen Minerale und deren Einkristalldichten führt bei der z.T. sehr komplexen mineralogischen Zusammensetzung der Proben sowie der variierenden chemischen Zusammensetzung der Minerale zu deutlich größeren Fehlern und wird folglich nicht durchgeführt.

\subsubsection{Porenraum}

\subsubsection{Porosität}

Die Porosität ( $\phi)$ wurde nach der unter Kap.2.4.1 beschriebenen Auftriebswägung ermittelt. Aus den Werten für $\mathrm{m}_{\mathrm{t}}, \mathrm{m}_{\mathrm{n}}$ und $\mathrm{m}_{\mathrm{au}}$ kann mit der Gleichung

$$
\phi=\frac{m_{n}-m_{t}}{m_{n}-m_{a u}} * 100 \quad[\text { Vol. } \%]
$$

$\phi$ - Porosität [Vol.\%]; $m_{n}$ - Masse wassergesättigt [g]; $m_{t}-$ Masse trocken [g]; $m_{a u}-$ Masse Auftrieb wassergesättigt [g].

die Porosität berechnet werden. Es handelt sich hierbei um die effektive Porosität, die den von außen durch Flüssigkeiten und Gase zugänglichen Porenraum umfasst. Wird im Weiteren Verlauf dieser Arbeit von Porosität gesprochen, so ist stets die effektive Porosität gemeint.

\subsubsection{Porenradienverteilung}

Zur Bestimmung der Porenradienverteilung wurde Hg unter zunehmendem Druck (0 bar bis 4000 bar) in evakuierte Proben mit dem Durchmesser 12,5 mm und der Länge 25-30 mm gepresst. Unter der Annahme röhrenförmiger Poren sowie der Oberflächenspannung von $\mathrm{Hg}$ 
$(\sigma=480 \mathrm{mN} / \mathrm{m})$ und dem Randwinkel zwischen Hg und Luft $\left(\mathrm{v}=141,3^{\circ}\right)$ wird jedem Druck $(p)$ ein Porenradius (r) zugeordnet (Doveton 1987).

$$
r=\frac{2 * \sigma * \cos v}{p} \quad[\mu \mathrm{m}]
$$

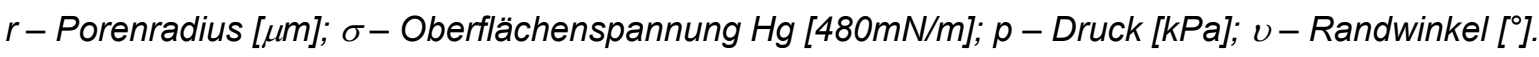

Es handelt sich hierbei um einen Poreneintritts- oder Porenhalsradius. Das HgIntrusionsvolumen je Druckbereich $(\Delta \mathrm{p})$ im Verhältnis zum Gesamtvolumen der Probe ergibt den Volumenanteil der Poren für das entsprechende Porenradienintervall. Aus den Daten der Porenradienverteilung wird nach der Gleichung

$$
r_{m}=10^{\sum_{i=1}^{n}\left(\frac{\log a i+\log b i)_{*}}{2} \phi a b, i / \phi\right.}
$$

$r_{m}$ - mittlerer Porenradius [ $\mu \mathrm{m}$ ]; $a_{i}$ - untere Grenze eines Porenradienintervalls [ $\mu \mathrm{m}$ ] $b_{i}$ - obere Grenze des i-ten Porenradienintervalls [ $\mu \mathrm{m}] ; \phi_{a b, i}-$ Porosität des i-ten Porenradienintervalls mit den Grenzen $a_{i} b_{i}[$ Vol.\%]; $\phi-$ Gesamtporosität des Gesteins [Vol.\%].

der mittlere Porenradius $\left(r_{\mathrm{m}}\right)$ berechnet. Im Porenradienbereich von $0,1 \mu \mathrm{m}$ bis $1 \mathrm{~mm}$ findet kapillares Saugen statt (Snethlage 1984; Klopfer 1985). Der Anteil des Kapillarporenvolumens am Gesamtgesteinsvolumen lässt sich mit der Gleichung

$$
\phi_{r k a p}=\sum_{i=0,1}^{1000} \phi_{i}[\mu \mathrm{m}]
$$

$\phi_{\text {rkap }}$ - Volumenanteil der Kapillarporen [Vol.\%]; $\phi_{i}$ - Volumenanteil des i-ten Porenradienintervalls zwischen $0,1 \mu \mathrm{m}$ und $1 \mathrm{~mm}$ [Vol.\%].

bestimmen.

\subsubsection{Kapillare Wasseraufnahme}

Untersuchungen zur kapillaren Saugfähigkeit der Gesteine wurden in mindestens zwei orthogonalen Richtungen durchgeführt. Dabei wurden jene gewählt, welche potenziell Maxima bzw. Minima der kapillaren Wasseraufnahme aufweisen. Der Saugversuch wurde in Anlehnung an prEN 1925 an trockenen Gesteinswürfeln mit Kantenlängen zwischen 60 mm und $100 \mathrm{~mm}$ durchgeführt. Diese wurden an eine Unterflurwaage und mit einer Seite ca. 3 $\mathrm{mm}$ unter die Wasserlinie eines Wasserbeckens gehängt. Ein Maß für die kapillare 
Saugfähigkeit eines Gesteins ist der Wasseraufnahmekoeffizient (w), welcher aus der Dauer des Versuchs, Fläche des Probenkörpers und der Masse des aufgenommenen Wassers nach

$(\mathrm{G} .2-11) \quad w=\frac{\Delta H_{2} O}{\sqrt{\Delta t}} * \frac{1}{A} \quad\left[\mathrm{~kg} / \mathrm{m}^{2} * \sqrt{\mathrm{t}}\right]$

w - Wasseraufnahmekoeffizient $\left[\mathrm{kg} / \mathrm{m}_{2}{ }^{*} / \mathrm{h}\right] ; \Delta \mathrm{H}_{2} \mathrm{O}$ - Masse des aufgenommenen Wassers im Zeitintervall $\Delta t$ [kg]; $\Delta t$ - Zeitintervall [Std]; A - Fläche der Probenwürfelseite $\left[\mathrm{m}^{2}\right]$.

ermittelt werden (Snethlage 1985) kann. Weitere Ansätze zur Methode liefern Koch \& Siegesmund (2001). Dem w-Wert aus der Messung einer Würfelprobenseite wird die Richtung zugeordnet, auf der die Würfelfläche senkrecht steht. Beispiel: Die Seite des Würfels, welche der xy-Ebene des Referenzkoordinatensystems (vgl. Kap. 2.1.1) entspricht, hängt im Wasser - der entsprechende w-Wert wird damit der z-Richtung zugeordnet.

\subsubsection{Mechanische Eigenschaften}

Als skalare Größen wurden die mittlere Härte des Gesteins und der Spaltbarkeitsindex aus der mineralogischen Zusammensetzung und den physikalischen Eigenschaften der Minerale ermittelt.

Die mechanischen Eigenschaften (im Rahmen dieser Studie ermittelt: Einaxiale Druck-, Spaltzug-, Biege- und Abriebfestigkeit) der Gesteine sind vektorielle Größen und damit richtungsabhängig und möglicherweise anisotrop. Daher wurden diese mechanischen Kenngrößen in mindestens drei senkrecht aufeinander stehenden Raumrichtungen bestimmt. Alle Druck-, Spalt-, und Biegezugversuche wurden mit einer Klasse 1 Universalprüfmaschine der Firma Walter \& Bai durchgeführt; die Angabe der Werte erfolgt in MPa. Die Charakterisierung der beim Spaltzug- und Abriebtest entstandenen Bruch- bzw. Abriebflächen fand mittels rasterelektronenmikroskopischer Untersuchungen an einem FEREM der Firma Leo Gemini statt. 


\subsubsection{Mittlere Härte und Spaltbarkeitsindex}

Die mittlere Härte eines Gesteins ergibt sich aus dem Modalbestand und den Einkristalldaten (Kap.4.2) nach

$$
H_{G}=\sum_{i=1}^{n} H_{M i} * x_{i}
$$

$H_{G}-$ mittlere Härte des Gesteins [HV]. $H_{M i}-$ Härte der i-ten Mineralphase [HV] (vgl. Kap.4.2). $x_{i}-$ Volumenanteil der i-ten Mineralphase.

Der Spaltbarkeitsindex basiert ebenfalls auf der Komposition des Gesteins und den Eigenschaften der Minerale. Der Spaltbarkeitsindex wurde als Kenngröße für die mechanische Stabilität entwickelt. Die Quantifizierung erfolgt nach:

$$
I_{S p G}=\sum_{i=1}^{n} I_{S p M_{i}} * x_{i}
$$

$I_{S p G}$ - Spaltbarkeitsindex des Gesteins. I I $I_{S M i}$ - Spaltbarkeitsindex der i-ten Mineralphase (vgl. Kap.4.2). $x_{i}$-Volumenanteil der i-ten Mineralphase.

Die Berechnungsgrundlagen für den Spaltbarkeitsindex $\left(\mathrm{I}_{\mathrm{SpM}}\right)$ der Minerale werden im Einzelnen in Kap.4.2 dargelegt.

\subsubsection{Druck-, Spalt-, Biege- und Abriebfestigkeit}

Die einaxiale Druckfestigkeit (R) wurde nach DIN EN 1926 an zylindrischen Proben mit dem Durchmesser und der Höhe von 50 mm ermittelt. Laut DIN EN 1926 ist dieses Probenformat zum Zweck der Qualitätsüberwachung zugelassen, standardmäßig werden jedoch Probenwürfel mit der Kantenlänge 70 mm bzw. Zylinder mit Durchmesser und Höhe von 70 mm empfohlen. Für die im Rahmen dieser Arbeit untersuchten niedrigporösen Kristallingesteine ist weder durch die kleineren Probendurchmesser (Stephenson \& Traindafilidis 1974), noch durch die um $40^{\circ} \mathrm{C}$ geringere Trocknungstemperatur (Rohowski 2001) eine signifikante Änderung der Druckfestigkeiten zu erwarten.

In Anlehnung an die Empfehlung Nr. 10 des Arbeitskreises 19 „Versuchstechnik Fels“ (Gartung 1985) und an die DIN 22024 wurden die Spaltzugversuche (Brazil-Test) durchgeführt. Der Probendurchmesser betrug $40 \mathrm{~mm}$ und die Probenhöhe $20 \mathrm{~mm}$. Als Belastungszunahme wurden $30 \mathrm{~N} / \mathrm{s}$ gewählt. Die Spaltzugfestigkeit $\left(\beta_{\mathrm{SZ}}\right)$ wurde nach der Gleichung 
(G.2-14) $\quad \beta_{S Z}=\frac{2 * F_{\max }}{\Pi * d^{*} h} \quad\left[\mathrm{~N} / \mathrm{mm}^{2}\right][\mathrm{MPa}]$

$\beta_{\text {SZ }}$ - Spaltzugfestigkeit [MPa]; $F_{\text {max }}-$ Maximalkraft [N]; $d$-Probendurchmesser [mm]; $h$ - Probenhöhe [mm]

errechnet.

Die Biegefestigkeit unter Mittellinienlast $\left(\mathrm{R}_{\mathrm{tf}}\right)$ wurde nach DIN EN 12372 an quaderförmigen Proben mit den Dimensionen $25 \mathrm{~mm} * 50 \mathrm{~mm} * 150 \mathrm{~mm}$ (Höhe*Breite*Länge) ermittelt. Aus der Maximalkraft $\left(F_{\max }\right)$, dem Auflagerabstand (l) sowie der Probenbreite (b) und der Probenhöhe (h) wurde mit

$$
R_{t f}=\frac{3 * F_{\max } * l}{2 * b^{*} h^{2}} \quad\left[\mathrm{~N} / \mathrm{mm}^{2}\right][\mathrm{MPa}]
$$

$R_{t f}-$ Biegefestigkeit unter Mittellinienlast [MPa]; $F_{\max }-$ Maximalkraft [N]; I - Auflagerabstand [mm]; $b$ Probenbreite $[\mathrm{mm}] ; \mathrm{h}$ - Probenhöhe $[\mathrm{mm}]$.

die Biegefestigkeit berechnet.

Zur Charakterisierung des Abrasionsverhaltens der Gesteine wurde die Abriebfestigkeit nach DIN 52108 (Böhme-Verfahren) bestimmt. Die Angabe der Werte erfolgt als Volumenverlust $\left(\mathrm{W}_{\mathrm{V}}\right)$ in $\mathrm{cm}^{3}$. Relativ höhere Werte entsprechen niedrigen Abriebfestigkeiten und umgekehrt.

\subsubsection{Richtungsdefinition für die Festigkeitsparameter}

Um die Richtungsabhängigkeit und Anisotropie der mechanischen Eigenschaften mit den Gefügeelementen der Gesteine in Relation setzen zu können, muss die Probenorientierung und damit auch die Orientierung der Kraftvektoren und der Bruchzonen in Bezug auf das Referenzkoordinatensystem definiert werden. Die Zuordnung eines Messwertes zu einer Richtung erfolgt prinzipiell nach den Hauptachsen des kartesischen Referenzsystems. Im Fall von Messwerten, die schiefwinklig zum Referenzsystem ermittelt wurden, wird die Zuordnung und Nomenklatur im jeweiligen Kapitel erläutert. 


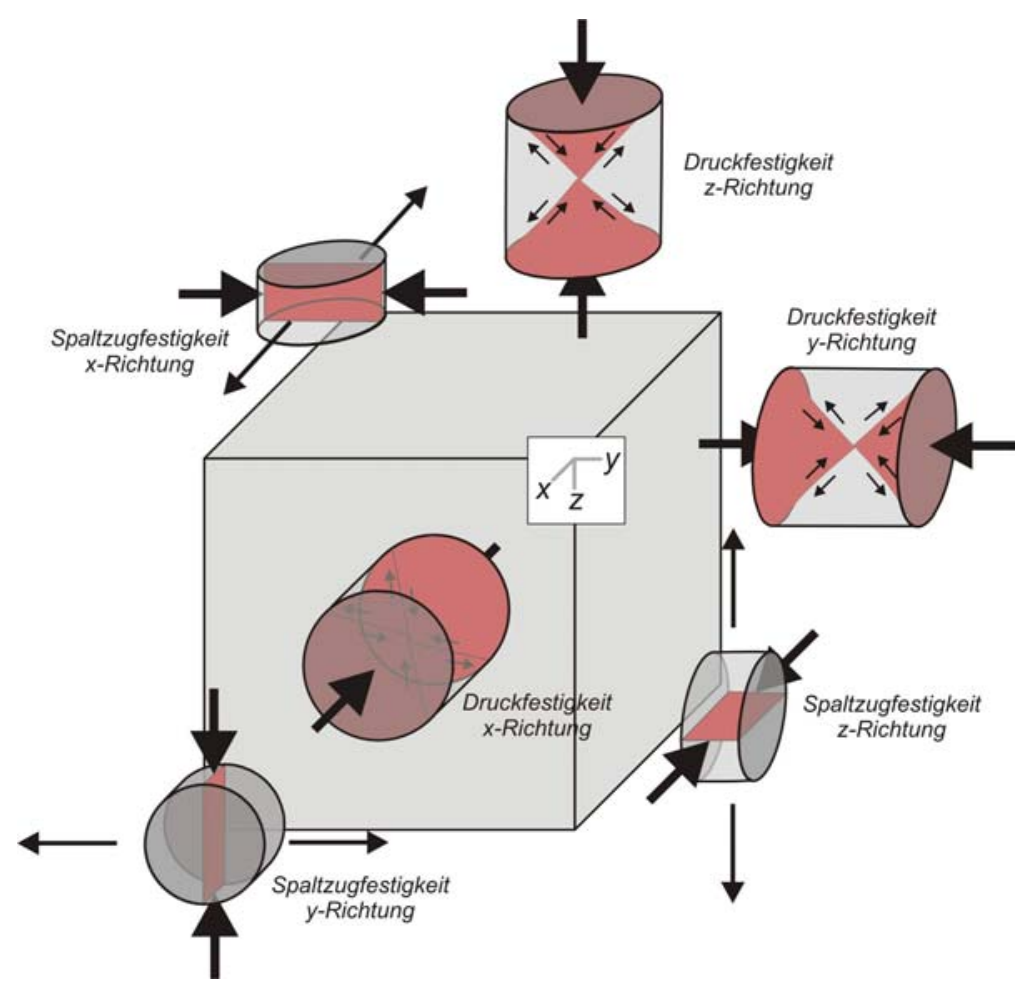

Abb.2-3: Orientierung der Druckfestigkeits- und Spaltzugfestigkeitsprüfkörper in Bezug auf des Referenzkoordinatensystem; dicke Pfeile markieren den Vektor der aufgebrachten Kraft während des Versuchs; dünne Pfeile markieren Vektoren der Kräfte, die zum Bruch der Probe führen; rote Bereiche zeigen die potenzielle Lage, der beim Versuch entstehenden Bruchflächen; der Typ des Prüfkörpers und die zugeordnete Richtung sind gekennzeichnet.

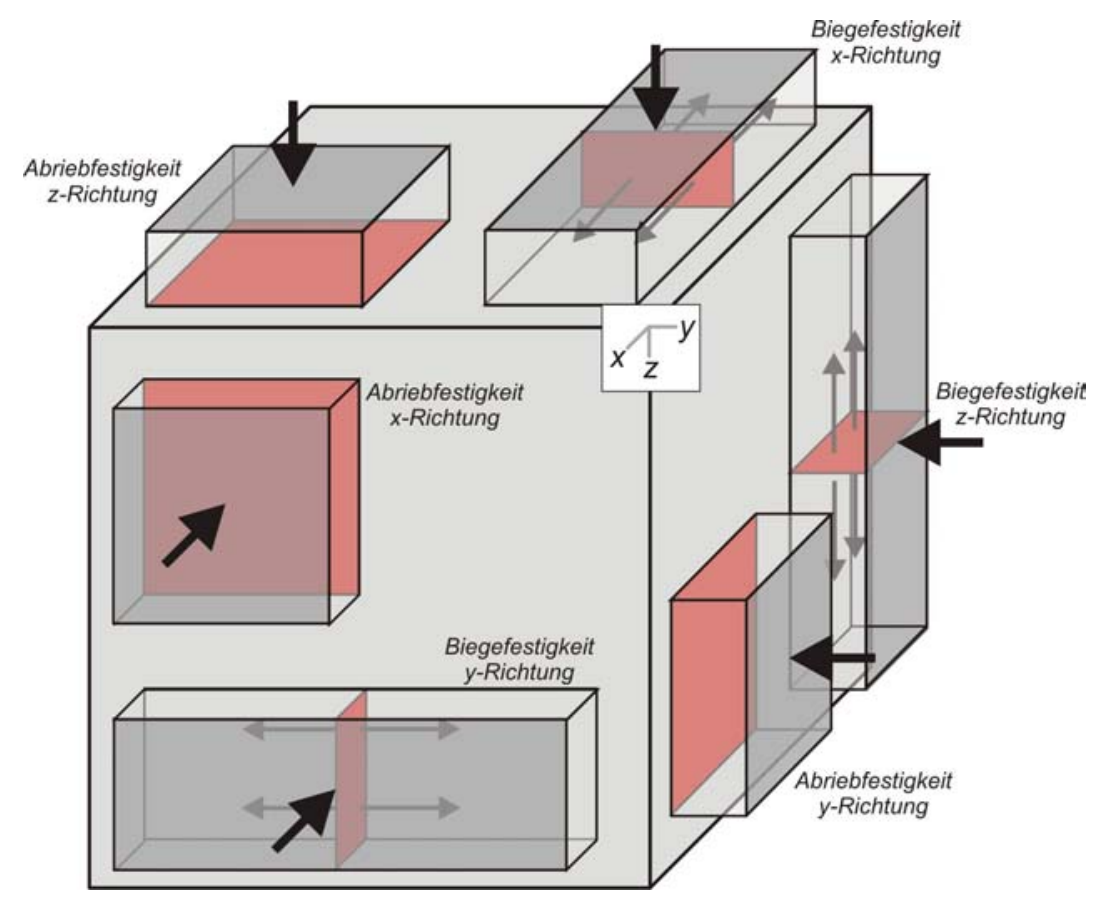

Abb.2-4: Orientierung der Biegefestigkeits- und Abriebprüfkörper in Bezug auf das Referenzkoordinatensystem; dicke Pfeile markieren den Vektor der aufgebrachten Kraft während des Versuchs; dünne Pfeile markieren Vektoren der Kräfte, die zum Bruch der Probe führen; rote Bereiche zeigen die potenzielle Lage der beim Versuch entstehenden Bruchflächen/Abriebflächen; der Typ des Prüfkörpers und die zugeordnete Richtung sind gekennzeichnet. 
Druckfestigkeit: Die Druckfestigkeitsprüfkörper wurden je nach der Lage der Zylinderachse den Richtungen x, y oder z zugeordnet (Abb.2-3). Zusammen mit dem Probennamen werden die zugehörigen Druckfestigkeitswerte z.B. als PDDx-Richtung oder PDDx (Probe Granodiorit - x-Richtung) bezeichnet.

Spaltzugfestigkeit: Die Spaltzugfestigkeitsprüfkörper wurden nach der Richtung des Vektors der beim Versuch entstehenden Zugkräfte den Richtungen x, y oder z zugeordnet (Abb.2-3). Zusammen mit dem Probennamen werden die zugehörigen Spaltzugfestigkeitswerte z.B. als PDDx-Richtung oder PDDx (Probe Granodiorit - x-Richtung) bezeichnet. Geht man ausschließlich von den Hauptrichtungen des orthogonalen Koordinatensystems aus, so existieren für jede Richtung der Spaltzugfestigkeiten zwei unterschiedliche Möglichkeiten, Proben zu deren Bestimmung zu gewinnen. Beispiel: Die Spaltzugfestigkeit der y-Richtung lässt sich auch am Prüfkörper der x-Richtung durch eine 90-Drehung (nur der Körper wird gedreht; die Kraftvektoren bleiben stationär) um die Zylinderachse ermitteln. Um eine eindeutige Orientierung der Gefügeelemente des Gesteins zu den beim Versuch auftretenden Kräften zu gewährleisten, wurden die Proben immer nach dem gleichen Schema (Abb.2-3) entnommen und getestet.

Biegefestigkeit: Bei der Biegefestigkeit besteht ebenfalls die Möglichkeit, die Festigkeitswerte für eine Richtung aus zwei unterschiedlich orientierten Probekörpern zu ermitteln (ohne schiefwinklig zum Koordinatensystem entnommene Proben). Analog zu den Spaltzugfestigkeitsprüfkörpern wurden auch die Proben für die Biegefestigkeit immer in der gleichen Orientierung hergestellt und getestet (Abb.2-4). Die Zuordnung der Biegefestigkeitsprüfkörper erfolgt nach der Raumlage der Vektoren der beim Versuch auftretenden Zugkräfte. Zusammen mit dem Probennamen werden die zugehörigen Biegezugfestigkeitswerte z.B. als PDDx-Richtung oder PDDx (Granodiorit - x-Richtung) bezeichnet.

Abriebfestigkeit: Die Abriebfestigkeitsprüfkörper wurden gemäß der Richtung des Vektors der beim Versuch aufgebrachten Anpresskräfte bezeichnet (Abb.2-4). Da die Probe während des Versuchs um die Achse dieses Vektors gedreht wird, ist keine weitere Definition der Orientierung notwendig.

\subsubsection{Elastische Eigenschaften}

Die elastischen Eigenschaften als vektorielle und damit richtungsabhängige Größen wurden in mindestens drei senkrecht aufeinander stehenden Raumrichtungen ermittelt. Abweichungen werden im jeweiligen Kapitel erläutert. 


\subsubsection{Vp-Messungen an Kugelproben}

Um die dreidimensionale Verteilung der offenen Mikrorisse zu bestimmen, wurden an Kugeln mit einem Durchmesser von $50 \mathrm{~mm}$ die Kompressionswellengeschwindigkeiten (Vp) in 192 Richtungen ermittelt. Aus den Richtungsdaten wird die räumliche Verteilung der Vp im Gestein berechnet und in Form eines Isolinienplots im Schmidt-Netz dargestellt. Die Daten wurden nach dem Durchschallungsverfahren mit einer Wellenfrequenz von $1 \mathrm{MHz}$ bestimmt. Die Kugelproben wurden jeweils wassergesättigt und trocken gemessen. Bei der unter Vakuum wassergesättigten Probe ist der Einfluss des offenen Porenraums (z.B. offene Risse) auf die Vp unterdrückt. Dieser Effekt ist bedingt durch die höhere Vp in Wasser (ca. 1,5 km/s) gegenüber der in Luft (ca. $0,3 \mathrm{~km} / \mathrm{s}$ ) sowie der besseren Ankopplung zweier durch einen Riss getrennten Gesteinsflächen durch das Wasser. Die Messung der wassergesättigten Kugel erbringt also annähernd die intrinsische $\mathrm{Vp}$ des Gesteins, welche durch die mineralogische Zusammensetzung und die räumliche Anordnung der z.T. anisotropen Kristalle bestimmt wird (Siegesmund et al. 1993; Siegesmund 1996).
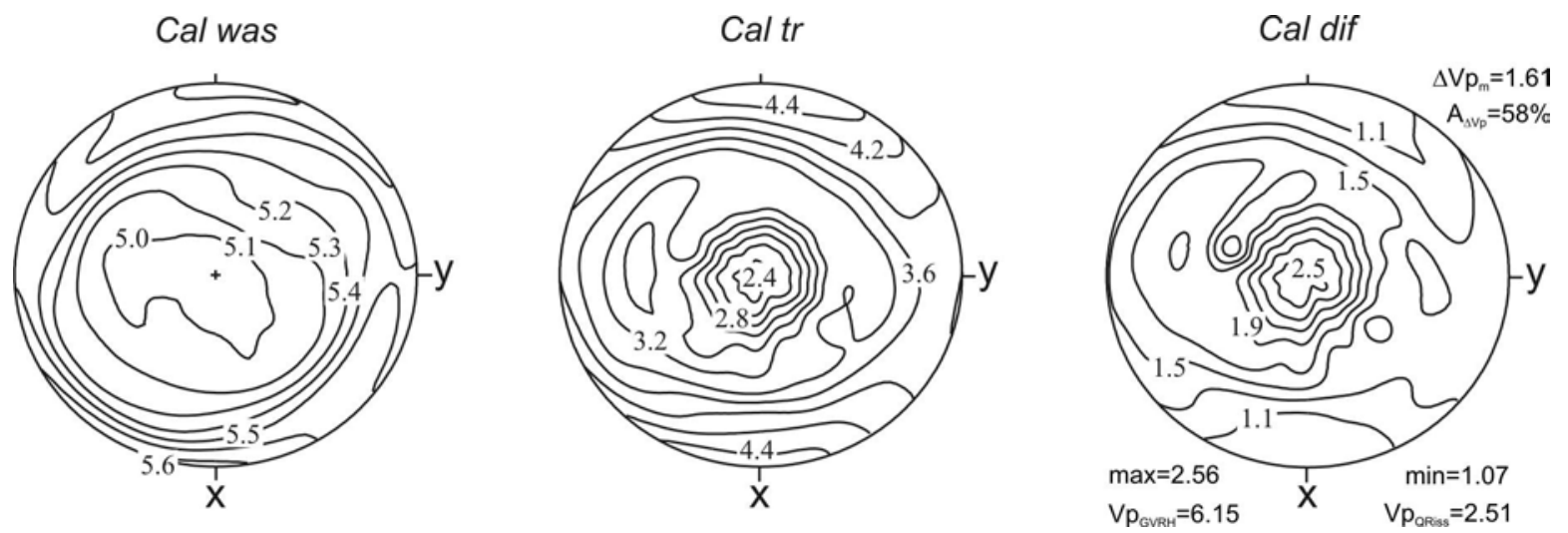

Abb.2-5: Isolinienplot der $V p$ [km/s] von wassergesättigter (Cal was), trockener (Cal tr) Messung sowie der Differenzisolinienplot (Cal dif) mit $\Delta V p_{m}$ - mittlere $\Delta V p$ des Differenzisolinienplot; $A_{\Delta V_{p}}-$ Anisotropie der $\Delta V p$ des Differenzisolinienplot; $V p_{G V R H}$ - Voigt-Reuss-Hill-Mittel der Vp des Gesteins (vgl. G.2-17); V $p_{\text {QRiss }}$ - Parameter zur Rissquantifizierung (siehe Text; vgl. G.2-16). Am Beispiel des granodioritischen Gneises Calanca (Cal).

Bei der Trocken-Messung werden die Vp sowohl durch die intrinsischen Eigenschaften als auch durch den Porenraum beeinflusst. Die Differenz aus der Wassergesättigt-Messung und der Trocken-Messung $\left(\mathrm{Vp}_{\mathrm{was}}-\mathrm{Vp}_{\mathrm{tr}}=\Delta \mathrm{Vp}\right)$ birgt eine Aussage über Rissdichte und die rissbedingte Anisotropie des Gesteins und damit über die bevorzugte Orientierung der offenen und von außen zugänglichen Riss-Systeme (Schild et al. 2001). Höhere $\Delta V$ p bedeuten relativ höhere Dichten von offenen Rissen mit einer bevorzugten Orientierung senkrecht zum $\Delta \mathrm{Vp}$ Wert (Abb.2-5). Diese Methode ist vergleichbar $\mathrm{zu}$ der Vp-Messung an trockenen Gesteinsproben unter variierendem Umschließungsdruck (Meglis et al. 1996; Duerrast et al. 1999; Rasolofosaon et al. 2000). Es ist darauf hinzuweisen, dass sowohl der abgeschlossene 
Porenraum als auch mineralisierte Risse nicht wassergesättigt und damit durch diese Methode nicht erfasst werden können. Um auch den abgeschlossenen Porenraum zu berücksichtigen, wurde nach der Gleichung

$$
\begin{array}{lll}
V p_{\text {QRiss }}=V p_{\text {GVRH }}-V p_{\text {trockm }} & \text { mit } & \\
V p_{\text {GVRH }}=\sum_{i=1}^{n} V p_{\text {MVRH } i} * x_{i} & & {[\mathrm{~km} / \mathrm{s}]}
\end{array}
$$

$V p_{Q R i s s}$ - Parameter zur Rissquantifizierung (Details siehe Text). $V p_{G V R H}$ - nach Voigt-Reuss-Hill (Hill 1952) gemittelte $V p$ für ein polykristallines Aggregat. $V p_{\text {trockm }}-$ Mittelwert der $V p$ aus der Ultraschallmessung der trockenen Kugel. Vp $p_{M V R H i}$ - Vp (Voigt-Reuss-Hill-Mittel) der i-ten Mineralphase. $x_{i}$ - Volumenanteil der i-ten Mineralphase.

ein richtungsunabhängiger Parameter zur Rissquantifizierung ermittelt. Dabei ist $\mathrm{Vp}_{\mathrm{GVRH}}$ die mittlere intrinsische Kompressionswellengeschwindigkeit, basierend auf dem Modalbestand des jeweiligen Gesteins und den Einkristallgeschwindigkeiten als Voigt-Reuss-Hill-Mittel für ein polykristallines Aggregat (G.2-17). V $p_{\text {trockm }}$ ist der Mittelwert der Kompressionswellengeschwindigkeiten aus der trockenen Messung der Gesteinskugel (Abb.2-5).

Um die Anisotropie der offenen Mikroriss-Systeme in Verbindung mit der Rissdichte zu quantifizieren und damit die wirksame Anisotropie der Mikrorisse zu beschreiben, wurde nach

$$
A_{\text {Riss }}=A_{\Delta V p} * V p_{Q R i s s}
$$

$[\% * \mathrm{~km} / \mathrm{s}]$

$A_{R i s s}-$ Rissanisotropiefaktor; $A_{\Delta V_{p}}-$ Anisotropie der $V p$ aus dem Differenzisolinienplot $\left(A_{\Delta V p}=\left(\Delta V p_{\max }{ }^{-}\right.\right.$ $\left.\left.\Delta V p_{\min }\right) / \Delta V p_{\max }{ }^{*} 100\right)[\%] ; V p_{\text {Qriss }}-$ Rissquantifizierungsparameter $[\mathrm{km} / \mathrm{s}]$.

der Rissanisotropiefaktor $\left(\mathrm{A}_{\text {Riss }}\right)$ bestimmt.

\subsubsection{Dynamischer E-Modul und Poisson-Zahl}

An zylindrischen Proben mit dem Durchmesser 30 mm und der Länge 30-35 mm wurden die $\mathrm{Vp}$ sowie die Scherwellengeschwindigkeiten $\left(\mathrm{Vs}_{1}, \quad \mathrm{Vs}_{2}\right)$ zweier mit ihren Polarisationsrichtungen senkrecht aufeinander stehenden Scherwellen $\left(S_{1}, S_{2}\right)$ bestimmt. Die Probenzylinder wurden längs ihrer Zylinderachsen mit einer Frequenz von $1 \mathrm{MHz}$ durchschallt. Für jede Gesteinsprobe wurden die Untersuchungen parallel zu den Achsen x, y, $z$ des Referenzsystems und unter schrittweise zunehmendem Druck im Druckbereich von 2 MPa bis $400 \mathrm{MPa}$ durchgeführt. Aus den Daten wurden für jede der 9 Druckstufen nach 


$$
v=\left(V s-\frac{V p^{2}}{2}\right) /\left(V s^{2}-V p^{2}\right)
$$

$v$ - Poisson-Zahl (Verhältnis von Querdehnung zu Längsdehnung); Vs - Scherwellengeschwindigkeit $[\mathrm{m} / \mathrm{s}] ; \mathrm{Vp}$ - Kompressionswellengeschwindigkeit [m/s]

die Poisson-Zahl (v) und nach

$$
E=2 * V s^{2} * \rho_{R o h} *(1+v) \quad[\mathrm{Pa}]
$$

E - dynamisch bestimmter E-Modul [Pa]; Vs - Scherwellengeschwindigkeit [m/s]; $\rho_{\text {Roh }}-$ Rohdichte des Gesteins $\left[\mathrm{g} / \mathrm{cm}^{3}\right] ; v$ - Poisson-Zahl.

der dynamische E-Modul (E) ermittelt.

Standardmäßig wurde die mittlere Geschwindigkeit aus der schnelleren Scherwelle $\left(\mathrm{Vs}_{1}\right)$ und der langsameren Scherwelle $\left(\mathrm{Vs}_{2}\right)$ für die Berechnungen verwendet. Unterschiede und Besonderheiten, die sich aufgrund unterschiedlicher Vs von $\mathrm{S}_{1}$ und $\mathrm{S}_{2}$ (Scherwellen-Splitting) ergeben, werden in Kap.6.4.1.3 erläutert. Für detaillierte Informationen zur Methode siehe Jahns (1995) und Brosch et al. (2000).

Zur Bestimmung von Poisson-Zahl und E-Modul unter $0 \mathrm{MPa}$ Umschließungsdruck wurden die Vp und Vs nach dem Durchschallungsverfahren mit $0,5 \mathrm{MHz}$ an trockenen Würfeln der Kantenlängen $60 \mathrm{~mm}$ bis $100 \mathrm{~mm}$ ebenfalls parallel zu den Achsen x, y und z des Referenzsystems ermittelt. Zusätzlich wurden aus den Vp- und Vs- über Druckdiagrammen der druckabhängigen Messungen (siehe oben) die Vp- und Vs-Werte für $\mathrm{p}=0 \mathrm{MPa}$ Umschließungsdruck durch Interpolation bestimmt. Die Berechnung der Parameter $v$ und E erfolgte nach G.2-19 und G.2-20. Die Werte wurden gemäß der Raumlage der Durchschallungsachse den Achsen des Referenzsystems zugeordnet. Beispiel: Der Probekörper PDD wurde in x-Richtung durchschallt. Die ermittelten Parameter werden folglich mit PDDx oder PDDx-Richtung indiziert. 


\section{Methoden und Terminologie}

\subsubsection{Statischer E-Modul}

Der statische E-Modul $\left(\mathrm{E}_{\text {stat }}\right)$ wurde aus der Spannungs-Dehnungs-Kurve des einaxialen Druckversuchs (vgl. Kap. 2.4.4.2) nach der Gleichung

$(\mathrm{G} .2-21) \quad E_{\text {stat }}=\frac{d \sigma}{d \varepsilon}$

E - statisch bestimmter E-Modul [Pa]; $d \sigma / d \varepsilon-1$.Ableitung der Spannungs ( $\sigma$ )-Dehnungs ( $\varepsilon$ )-Funktion oder Steigung einer Tangente an die Spannungs-Dehnungskurve bei gegebenem $\sigma$.

gemäß Abb.2-6 für Spannungen von 10\%, 30\%, 50\%, 70\% bzw. 90\% der Bruchspannung bestimmt. Es handelt sich hierbei um den Tangenten-Modulus (Brown 1981).

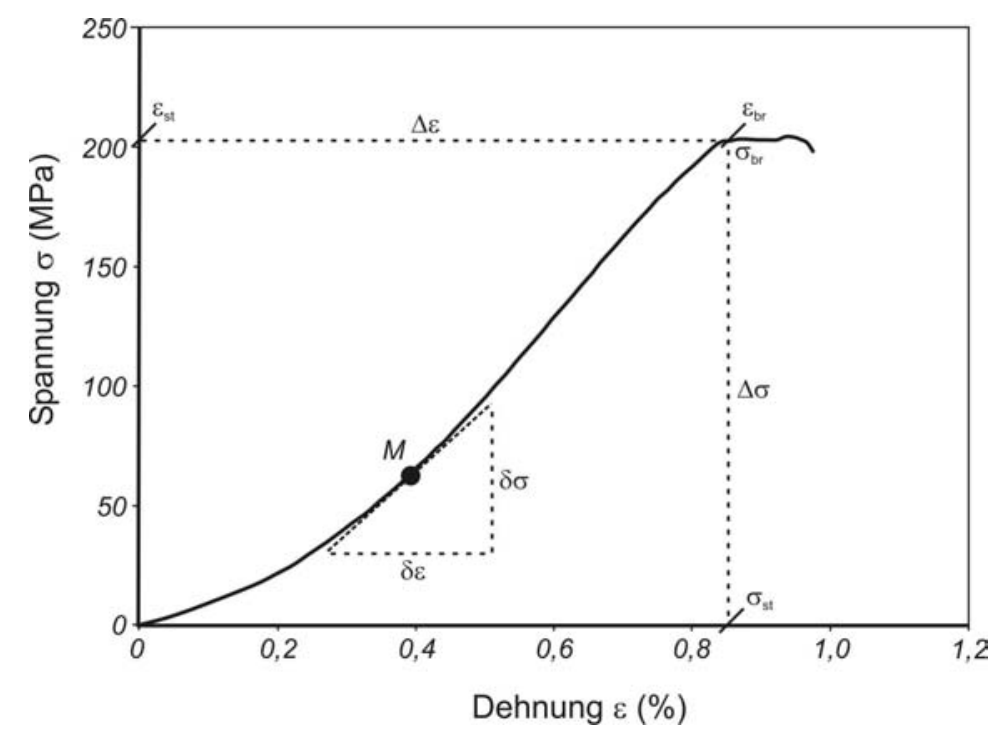

Abb.2-6: Beispielhaftes Spannungs-Dehnungs-Diagramm aus dem einaxialen Druckversuch und die Ermittlung des statischen E-Moduls nach der Tangenten- und der Sekanten-Methode (siehe Text).

Ergänzend wurde der statische E-Modul als Sekanten-Modulus ( $\mathrm{E}_{\text {stats }}-$ Bruchmodul) unter Verwendung der Gleichung

$$
E_{\text {stats }}=\frac{\sigma_{b r}-\sigma_{s t}}{\varepsilon_{b r}-\varepsilon_{s t}}=\frac{\Delta \sigma}{\Delta \varepsilon}
$$

$E_{\text {stats }}$ - statischer E-Modul als Sekantenmodul (Bruchmodul) über den gesamten Druckbereich des Versuchs [Pa]; $\sigma_{b r}-$ Spannung bei Bruch [Pa]; $\sigma_{s t}-$ Spannung zu Beginn des Druckversuchs [Pa]; $\varepsilon_{b r}$ - Dehnung bei Bruch; $\varepsilon_{s t}$-Dehnung zu Beginn des Druckversuchs.

berechnet (Brown 1981-Abb.2-6).

Die Zuordnung zu den Richtungen des Referenzsystems erfolgt nach der Orientierung der Druckzylinder (vgl. Kap.2.4.4.3). 


\subsubsection{Thermische Dehnung}

Die Messungen wurden mit einem eigens entwickelten 6-Stangen-Dilatometer (Kap.3) an zylindrischen Proben mit dem Durchmesser $15 \mathrm{~mm}$ und der Länge $50 \mathrm{~mm}$ durchgeführt. Jeweils drei Proben sowie ein Standard und zwei Dummys (zur Messung der Probentemperatur) durchliefen ein Messprogramm mit dem in $\boldsymbol{A b} \boldsymbol{b} . \mathbf{2 - 7}$ dargestellten Temperaturverlauf. Gemessen wurde dabei die Längenänderung $(\Delta \mathrm{l})$ der Zylinder parallel zu ihrer Längsachse in Abhängigkeit von der Temperatur (T).
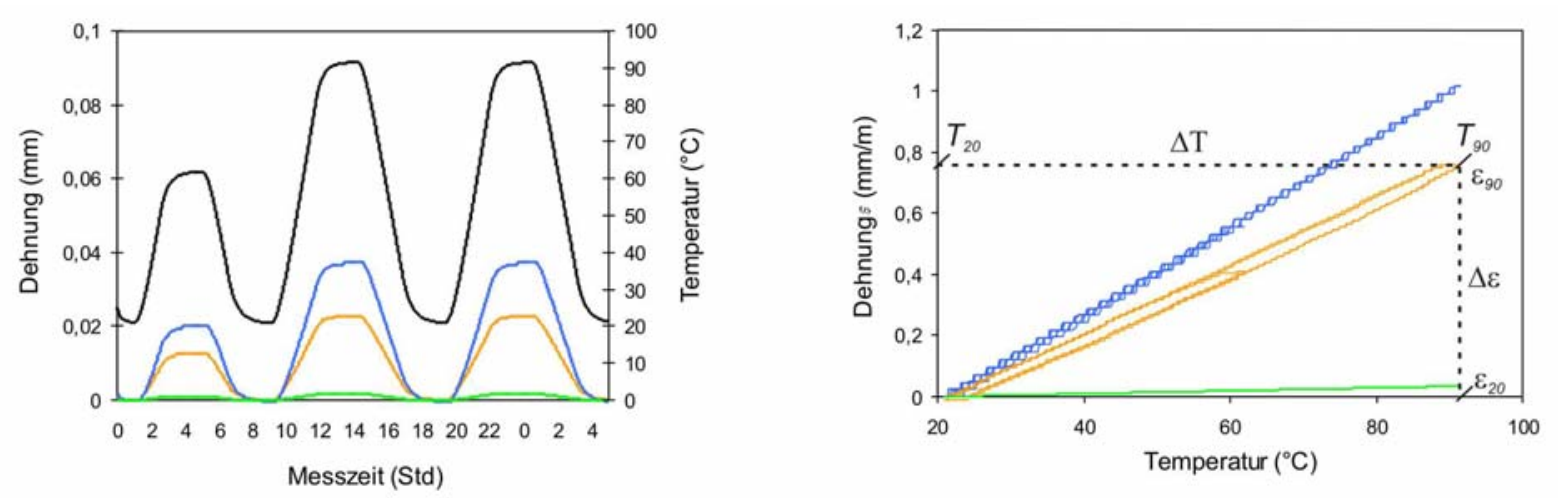

Abb.2-7: Links: Temperaturverlauf (schwarz) und die Dehnung dreier Proben (farbig) während des Messprogramms. Rechts: Die selben Dehnungswerte im Dehnungs-Temperatur-Diagramm; $\left.\Delta T{ }^{\circ} \mathrm{C}\right]$ und $\Delta \varepsilon[\mathrm{mm} / \mathrm{m}]$ zur Berechnung des linearen Ausdehnungskoeffizienten $\alpha_{20-90}$ nach (G.2-23) sind indiziert.

Als arithmetisches Mittel aus den Werten der aufsteigenden und absteigenden Rampen (beim Kalzit-Marmor (Rek) wurde nur die erste aufsteigende $90^{\circ} \mathrm{C}$-Rampe berücksichtigt) im Temperaturintervall von $20^{\circ} \mathrm{C}$ bis $90^{\circ} \mathrm{C}$ (Nennwerte: Die tatsächlichen Werte können um bis $\mathrm{zu} 5^{\circ} \mathrm{C}$ abweichen) wurde mit der Gleichung

$$
\alpha_{20-90}=\frac{l_{90}-l_{20}}{\Delta T^{*} L}=\frac{\Delta \varepsilon}{\Delta T} / 1000
$$

$\alpha_{20-90}$ - linearer Ausdehnungskoeffizient [1/K]; $I_{90}$ - Länge der Probe bei $90^{\circ} \mathrm{C}[\mathrm{mm}] ; I_{20}-$ Länge der Probe bei $20^{\circ} \mathrm{C}[\mathrm{mm}] ;$ L - Länge der Probe vor dem Versuch [mm]; $\Delta \varepsilon$ - Dehnung im Temperaturbereich von $20^{\circ} \mathrm{C}$ bis $90^{\circ} \mathrm{C}[\mathrm{mm} / \mathrm{m}] ; \Delta T-$ Temperaturdifferenz $\left[{ }^{\circ} \mathrm{C}\right]$.

der Ausdehnungskoeffizient $\left(\alpha_{20-90}\right)$ berechnet. Der $\alpha$-Wert eines Probenzylinders mit der Zylinderachse parallel zu x, y, z wird mit x, y, z bzw. x-, y-, z-Richtung indiziert. Unter der Annahme einer orthorhombischen Symmetrie wird der Volumenausdehnungskoeffizient $\left(\alpha_{\mathrm{VG}}\right)$ mit Hilfe der drei Ausdehnungskoeffizienten für die Richtungen $\mathrm{x}, \mathrm{y}, \mathrm{z}$ des Referenzsystems nach 
(G.2-24)

$$
\alpha_{V G}=\alpha_{x}+\alpha_{y}+\alpha_{z} \quad[1 / \mathrm{K}]
$$

$\alpha_{V G}-$ Volumenausdehnungskoeffizient des Gesteins. $\alpha_{x}, \alpha_{y}, \alpha_{z}-$ Ausdehnungskoeffizient parallel zu $x, y, z$. Berechnung nach Barron (1998).

bestimmt. Die Werte gelten für den jeweilige Temperaturbereich der Messungen von x-, yund z-Richtung.

Zusätzlich kann der Volumenausdehnungskoeffizient des Gesteins theoretisch aus dem Modalbestand und den Volumenausdehnungskoeffizienten der gesteinsbildenden Minerale ermittelt werden:

$$
\alpha_{V G T}=\sum_{i=1}^{n} \alpha_{V M i} * x_{i}
$$

$\alpha_{V G T}$ - theoretisch ermittelter Volumenausdehnungskoeffizient des Gesteins [1/K]. $\alpha_{V M}$ Volumenausdehnungskoeffizient der i-ten Mineralphase $[1 / K] . \quad x_{i}-$ Volumenanteil der i-ten Mineralphase.

Analog dazu wird die maximal mögliche Anisotropie des Gesteins, also sein Anisotropiepotential, wie folgt berechnet.

$$
A_{a G \max }=\sum_{i=1}^{n} A_{\alpha M i} * x_{i}
$$

$A_{\alpha G \max }$ - maximal mögliche Anisotropie der thermischen Dehnung (Anisotropiepotential) [\%]. A $\alpha_{M i}-$ Anisotropie der thermischen Dehnung der i-ten Mineralphase [\%]. $x_{i}$ - Volumenanteil der i-ten Mineralphase. 


\section{6-Stangen-Dilatometer (Dil-6)}

Das BMBF-Projekt „Naturwerksteine: Gefüge und technische Gesteinseigenschaften“ beinhaltete die Entwicklung eines Messgeräts zur Untersuchung der Richtungsabhängigkeit der thermischen Dehnung von Naturwerksteinen. Insbesondere sollten die mitteleuropäischen Umweltbedingungen simuliert, darüber hinaus aber auch extremere Temperaturbereiche zur Verdeutlichung von Prinzipien der thermischen Dehnung und der Schädigung von Naturwerksteinen durch thermische Verwitterung erreicht werden.

Es sollten Proben mit dem Durchmesser von $15 \mathrm{~mm}$ und einer Länge von $100 \mathrm{~mm}$ in einem Temperaturbereich von $-20^{\circ} \mathrm{C}$ bis $150^{\circ} \mathrm{C}$ unter trockenen wie nassen (Kammer mit Wasser geflutet- Temperaturbereich von $+5^{\circ} \mathrm{C}$ bis $90^{\circ} \mathrm{C}$ ) Bedingungen messbar sein. Um bei den avisierten Langzeitversuchen (1Tag bis zu mehreren Wochen) eine möglichst effektive Messzeitnutzung zu erreichen, müssen sechs Proben simultan gemessen werden können. Zur Erfüllung der beschriebenen Messanforderungen kommt nur ein Stangen-Dilatometer in einem horizontalen Aufbau mit einer Längenänderungsmessung mittels inkrementaler Messtaster in Frage. Auf diese Weise kann ein räumlich begrenzter Probenrahmen realisiert und damit eine auf die Probenzahl von 6 bezogene kleine Probenkammer mit einer akzeptablen Temperaturhomogenität gewährleistet werden. Aus demselben Grund wurde der horizontale dem vertikalen Aufbau vorgezogen. Letzterer führt durch erhöhte Konvektion zu einer schlechteren Temperaturhomogenität in der Kammer (Gaal 1998). Geräte dieser Bauart sind auf dem Markt nur in Versionen mit maximal zwei Messplätzen erhältlich und daher nicht ausreichend für die geforderten Anwendungskriterien. Andere Methoden der Längenänderungsmessung, wie z.B. die Interferometrie (Hahn 1998), 2-Mikroskop-Technik (Taylor et al. 1998), kapazitive Messung (Swenson 1998) oder laseroptische Techniken ermöglichen zwar eine im Vergleich zu den inkrementalen Messtastern höhere Auflösung und Genauigkeit, sind jedoch in Verbindung mit einer flutbaren Kammer von möglichst geringen räumlichen Ausmaßen technisch nicht umsetzbar. Völlig andere Konzepte zur Untersuchung des thermischen Dehnungsverhaltens sind die Röntgen-Diffraktometrie (McKinstry et al. 1998) und volumetrische Verfahren (Ruffino 1998), bei denen mit Probenpulvern gearbeitet wird, weshalb jedoch eine richtungsabhängige Bestimmung von Dehnungseigenschaften im bestehenden Mineralverband des Gesteins nicht möglich ist.

Bei der Temperierung der Kammer konnte auf die kostenintensive Verwendung von flüssigem Stickstoff als Kühlmittel verzichtet und der geforderte Temperaturbereich über einen Umwälzthermostaten mit einem Silikonöl als energieübertragendem Medium abgedeckt 


\section{6-Stangen-Dilatometer (Dil-6)}

werden. Die Betriebskosten des Dilatometers beschränken sich also auf den Stromverbrauch (Umwälzthermostat-3 kW; PC`s und Elektronik ca. 0,7 kW), die Wartung sowie die unter bestimmten Messbedingungen benötigten Druckluft- und Wassermengen.

\subsection{Funktionsweise}

Das Dil-6 besteht aus einer temperierbaren und flutbaren Probenkammer sowie einem Referenzrahmen aus Quarzglas und Aluminium, in den sechs Schubstangen und sechs Messtaster zur Dehnungsübertragung und -messung integriert sind. Die maximal sechs Proben werden in der verschiebbaren Probenkammer temperiert, dabei wird die Dehnung der Proben in Relation zum Referenzrahmen simultan $\mathrm{zu}$ den Temperaturwerten der drei Temperatursensoren (T1, T2, T3) erfasst und abgespeichert. Die Messwerteerfassungselektronik der Firma IBR und die Software DasyLab Vs 5.6 erlauben dabei eine Abtastrate von bis zu $3000 \mathrm{~Hz}$. Die Temperatur kann sowohl in der Kammer (Lufttemperatur in der Kammer) als auch in einer mit einer Bohrung versehenden DummyProbe ermittelt werden. Auch die Erfassung der Temperatur in einem Kammer-Medium (z.B. Wasser) ist mit Hilfe von wasserdichten Temperatursensoren möglich. Die Proben werden durch die Federkraft (0,3-1 N) der inkrementalen Messtaster in ihrer Halterung fixiert. Diese Kraft wird bei geneigtem Aufbau durch die aus der Neigung und der Gewichtskraft der Schubstangen resultierenden Kraft verstärkt. Durch die speziell für dieses Gerät entwickelte Lagerung des gesamten Probenrahmens auf Blattfedern und die flexible Schubstangenaufhängung (Abb.3-1) weist das Dil-6 eine hohe Resistenz gegen Erschütterungen auf. 

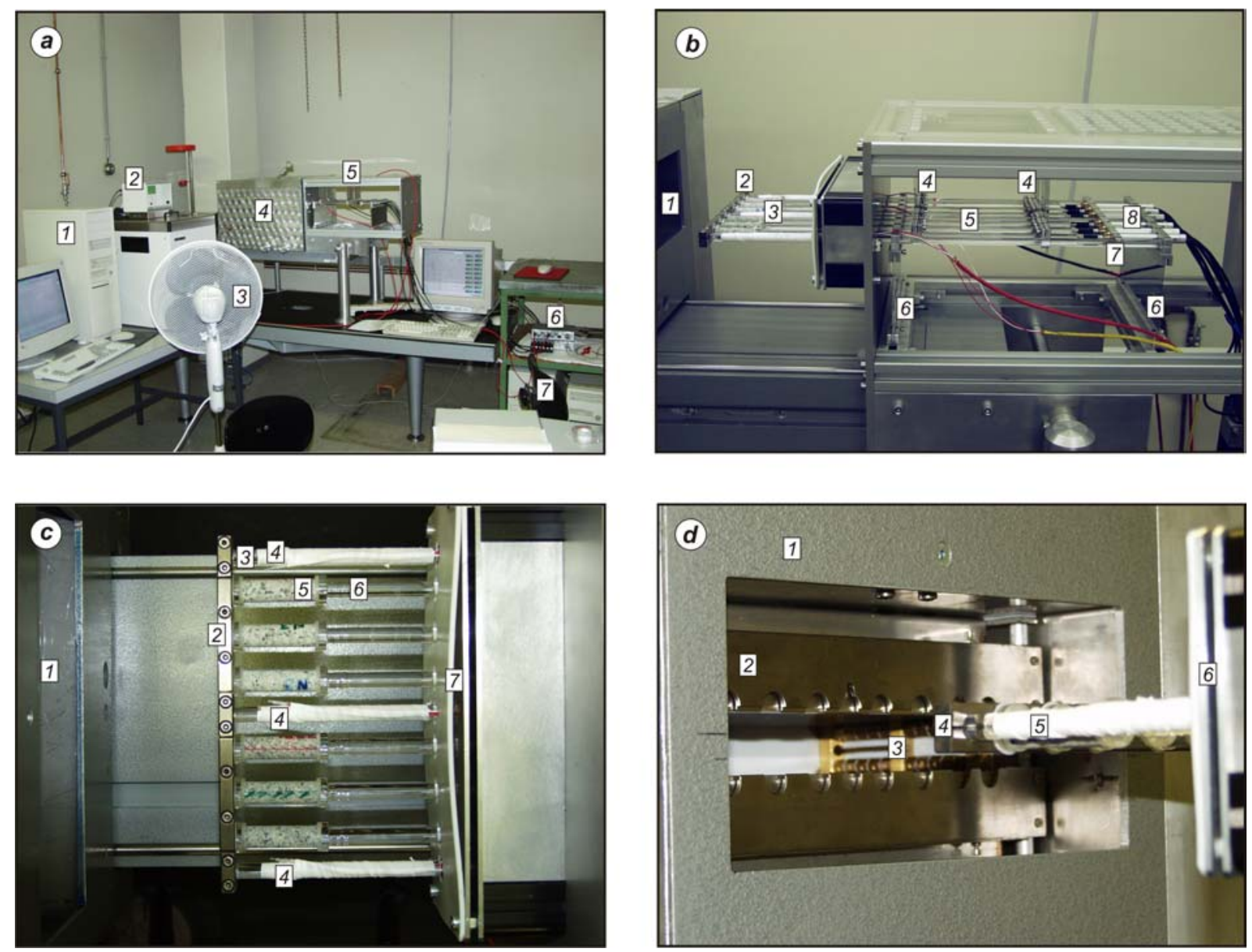

Abb.3-1: 6-Stangen-Dilatometer. (a) Laborübersicht; (1) PC zur Steuerung des Umwälzthermostaten; (2) Umwälzthermostat (Haake F8-C50); (3) Ventilator; (4) Kammer (nur Außenverkleidung sichtbar); (5) hinterer Teil des Dilatometers mit inkr. Messtastern und Schubstangenaufhängung; (6) Messwerterfassungselektronik (IBR-it De8/Da8); (7) PC zur Messwerterfassung. (b) Dilatometer hinterer Teil, Seitenansicht; (1) Kammer; (2) Referenzrahmenquerstrebe A aus Invar; (3) Proben, Schubstangen (Qz-Glas) und Stangen des Referenzrahmens (Qz-Glas-umwickelte Stangen); (4) flexible Schubstangenaufhängung; (5) Schubstangen und Stangen des Referenzrahmens; (6) Blattfederbefestigung des Referenzrahmens; (7) Querstrebe C zur Befestigung der Messtaster; (8) inkrementale Messtaster (Heidenhain ST1271). (c) Dilatometer, Aufsicht; (1) Kammer; (2) Querstrebe A; (3) Stange des Referenzrahmens; (4) Pt-100 Widerstandsthermometer; (5) Probe; (6) Schubstange; (7) Kammerabdichtung. (d) Dilatometer-Kammer. (1) Kammer außen; (2) Shutter Probenkammer; (3) Düsenleiste für Umluft; (4) Querstrebe A; (5) Stange des Referenzrahmens; (6) Kammerabdichtung. 
Die Energieübertragung auf die Proben kann beim Dil-6 auf drei unterschiedliche Arten erfolgen. In allen drei Fällen wird ein Teil der Energie über die von Silikonöl (vom Umwälzthermostaten) durchflossenen Kupfer-Wärmetauscherplatten in der Kammer umgesetzt. Der Energielieferant ist der Umwälzthermostat.

\section{Messung trocken und ohne Umluft}

Über die Kupfer-Wärmetauscherplatten wird die Luft in der Kammer erhitzt/abgekühlt. Die Energieübertragung auf die Probe findet durch die Wärmeleitung der Luft und in geringem Maße über konvektiven Transport aufgrund von Luftzirkulation in der Kammer statt. Auch die Energieübertragung von den Kupfer-Platten auf die Probe durch Wärmestrahlung ist nicht zu vernachlässigen. Bei dieser Versuchsmodifikation liegt der gesamte Aufbau in der Horizontalen (Abb.3-1).

\section{Messung trocken mit Umluft}

Zusätzlich zur Energieübertragung mittels Wärmetauscherplatten wird equivalent zur Vorlauftemperatur des Silikonöls temperierte Luft über Düsenleisten (Abb.3-1) in die Kammer geblasen. Der Druck, mit dem die Luft in die Kammer eingebracht wird, ist zwischen 0,1 bar und 5 bar variabel. Durch die Umluft wird die Energie hauptsächlich konvektiv auf die Proben übertragen, wobei auch hier der Anteil der Wärmestrahlung nicht zu vernachlässigen ist. Während der Kühlrampen wird durch die ständige Zufuhr von ggf. vorgetrockneter Luft die Bildung von Kondenswasser auf den Proben verhindert.

\section{Messung nass (geflutete Kammer)}

Der gesamte Aufbau kann um ca. $25^{\circ}$ gegen die Horizontale geneigt und die Kammer mit einem flüssigen Medium geflutet werden (Abb.3-3). Die Energieübertragung hängt damit von der Wärmeleitfähigkeit des Mediums, von der Absorptionsfähigkeit des Mediums gegenüber der Wärmestrahlung der Kupfer-Wärmetauscherplatten und der Konvektion des Mediums in der Kammer ab. Gegenüber den Messarten 1 und 2 kann hier nur in einem Temperaturbereich gemessen werden, in dem das Medium in flüssiger Form vorliegt und nicht siedet. 


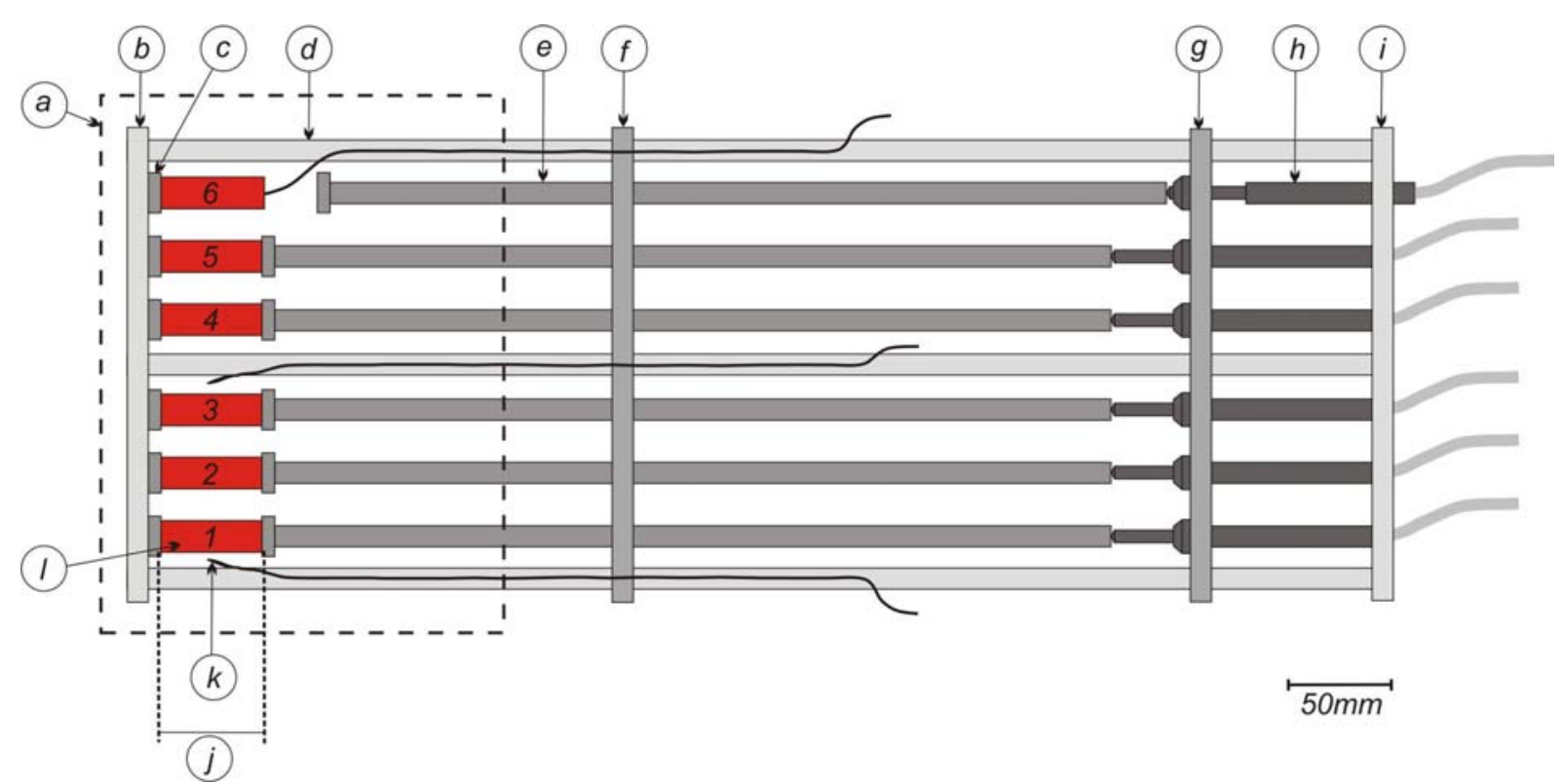

Abb.3-2: Schema zum Messprinzip des Dilatometers, Aufsicht. Teile, die zum Referenzrahmen gehören, sind in hellgrau gehalten. (1-6) Messplätze des Dilatometers; Platz 6 ist mit Dummy zur Temperaturmessung belegt. (a) Ausmaße und Lage der inneren, temperierbaren und flutbaren Kammer. (b) Querstrebe A (Invar). (c) Probenhalter-Gegenlager. (d) Stange des Referenzrahmens (Qz-Glas). (e) Schubstange (Qz-Glas). (f) Querstrebe B zur Referenzrahmenhalterung (Aluminium). (g) Querstrebe C zur Messtasterhalterung (Aluminium). (h) Inkrementaler Messtaster (Heidenhain ST1271). (i) Querstrebe $D$ zur Halterung des Referenzrahmens und zum Referenzrahmen (Aluminium). (j) Probenlänge für Korrektur der Dehnung des Referenzrahmens. (k) Pt-100 Widerstandthermometer. (I) Probe.

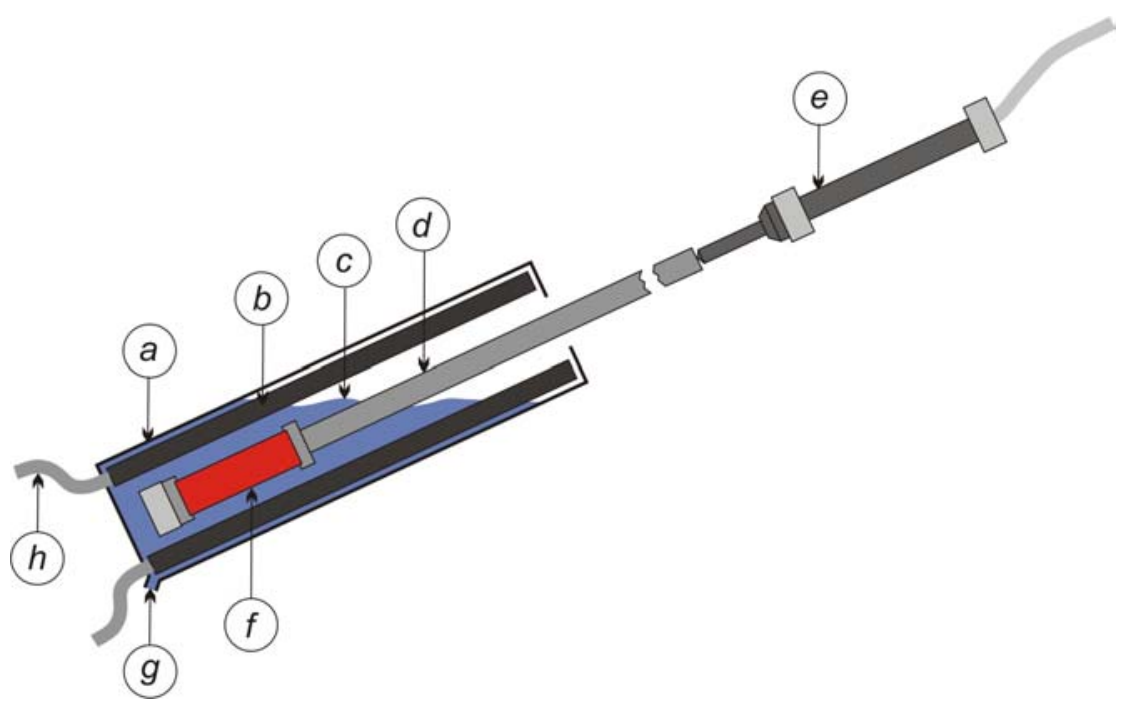

Abb.3-3: Schema Dilatometer, Seitenansicht im Schnitt, Maßstab 1:4. Kammer ist $25^{\circ}$ zur Horizontalen geneigt und geflutet. (a) Außenwand der inneren Kammer. (b) Wärmetauscher-Platten (Kupfer). (c) Flüssigkeitsspiegel. (d) Schubstange (Qz-Glas). (e) Inkrementaler Messtaster. (f) Probe. (g) Kammer-Einlass/Auslass. (h) Vorlauf/Rücklauf der Wärmetauscher-Platten.

Bauartbedingt ist eine Kalibrierung des Dilatometers mit Hilfe von Qz-Glas-Proben notwendig. Diese Proben sollten möglichst dieselben Dimensionen wie die später zu messenden Materialien haben und dasselbe Messprogramm durchlaufen. Während der 
gesamten Kalibrierungssmessung muss die Dehnung 0,0000 mm betragen, weil sowohl der Referenzrahmen als auch die Proben aus dem gleichen Material sind und sich damit der probenlängenäquivalente Abschnitt des Referenzrahmens in der gleichen Weise ausdehnt wie die Probe selbst (Abb.3-2). Ist das nicht der Fall, so können geometrische Effekte aufgrund von Verwindungen des Rahmens die Ursache sein, und die Daten müssen nachträglich Messplatzweise um die entsprechenden Abweichungsbeträge korrigiert werden. Zusätzlich muss zu den Dehnungsbeträgen für alle Messplätze die Dehnung von Quarzglas-Proben (die Werte werden aus der Länge der Probe und dem Ausdehnungskoeffizienten $\alpha_{\mathrm{QzG}}=0,5^{*} 10^{-6}$ $1 / \mathrm{K}$ für den Temperaturverlauf berechnet) addiert werden. Aufgrund der Konstruktion des Dil-6 kann die thermische Dehnung von Materialien mit einem Ausdehnungskoeffizienten gleich dem von Qz-Glas nur theoretisch bestimmt, aber nicht direkt gemessen werden. Im Endeffekt ist die gemessene Dehnung jeder Probe die Differenz zwischen der Dehnung der Probe und der Dehnung des Referenzrahmenabschnitts mit äquivalenter Länge (Abb.3-2). Es gilt also für die tatsächliche Dehnung/Längenänderung der Probe:

$$
\Delta L_{\text {tat }}=\Delta L_{\text {gem }}+\Delta L_{Q z G}-\Delta L_{K a l} \quad[\mathrm{~mm}]
$$

$\Delta L_{\text {tat }}$ - tatsächliche Längenänderung der Probe; $\Delta L_{\text {gem }}$ - gemessene Längenänderung; $\Delta L_{Q z G}-$ theoretische Längenänderung einer Qz-Glas-Probe mit gesteinsprobenäquivalenter Länge und einem Ausdehnungskoeffizienten $\alpha=0,5^{\star} 10^{-6} 1 / \mathrm{K}$ bei jeweiligem Messprogramm; $\Delta L_{\text {Kal }}-$ aus der Kalibration mit Qz-Glasproben ermittelte Abweichung von der Null-Ausdehnung.

\subsection{Messfehler}

Die Messung der thermischen Dehnung im Dil-6 ist mit einem gerätebedingten Fehler behaftet, der bei der Betrachtung und Interpretation der Messdaten berücksichtigt werden muss. Im Folgenden werden die Fehlerquellen benannt und beispielhaft deren Einfluss auf die Genauigkeit des Ausdehnungskoeffizienten für den Temperaturbereich von $20^{\circ} \mathrm{C}$ bis $90^{\circ} \mathrm{C}$ dargelegt.

Fehlerquellen:

- Temperaturinhomogenität in der Probenkammer

siehe $\boldsymbol{A b b . 3 - 4}$

- Probeneinbau

$+/-0,002 \mathrm{~mm}$

- Fehler der Temperaturwerte

$+/-0,1 \mathrm{~K}$

- Fehler der Dehnungswerte

$+/-0,001 \mathrm{~mm}$

- Verwindung des Referenzrahmens während der Messung

Fehler aus

Kalibration 
- Fehler der Längenmessung (Ausgangslänge L der Probe) $\quad 0,1 \mathrm{~mm}$

Die Fehler gehen nach dem Fehlerfortpflanzungsgesetz gemäss der folgenden Gleichung in die Berechnung ein.

$$
\alpha_{20-90}=\frac{l_{90}-l_{20}}{\left(T_{90}-T_{20}\right) * L}
$$

$\alpha_{20-90}$ - linearer Ausdehnungskoeffizient [1/K]; $I_{90}$ - Länge der Probe bei $90^{\circ} \mathrm{C}[\mathrm{mm}] ; I_{20}-$ Länge der Probe bei $20^{\circ} \mathrm{C}[\mathrm{mm}] ; T_{90}$ - fehlerbehaftete Temperatur $90^{\circ} \mathrm{C}$; $T_{20}-$ fehlerbehaftete Temperatur $20^{\circ} \mathrm{C}$; $L$ - Länge der Probe vor dem Versuch [mm].

$\mathrm{L}_{90}$ und $\mathrm{L}_{20}$ sind dabei mit einem Fehler von $+/-0,001 \mathrm{~mm}$ behaftet (messtasterbedingter Fehler der Dehnungswerte). Die Fehler für die Temperaturwerte $\mathrm{T}_{90}$ und $\mathrm{T}_{20}$ werden nach $\boldsymbol{A} \boldsymbol{b} \boldsymbol{b}$.3-4 ermittelt. $\mathrm{T}_{90}$ und $\mathrm{T}_{20}$ sind hier die arithmetischen Mittelwerte der Temperaturwerte an den Temperatursensoren T1 bis T3. Ausgehend von dem Temperaturprofil in $\boldsymbol{A b b}$.3-4 mit $\mathrm{T} 2<\mathrm{T} 1 \approx \mathrm{T} 3$ ergibt sich der durch die Temperaturinhomogenität bedingte Fehler der Temperaturwerte nach $\mathrm{F}_{\mathrm{T}}=\Delta \mathrm{T} / 2$ mit $\Delta \mathrm{T}$ als maximaler Temperaturdifferenz zwischen $\mathrm{T} 1$ und $\mathrm{T} 3 \mathrm{zu}$ einer gegebenen Temperatur.
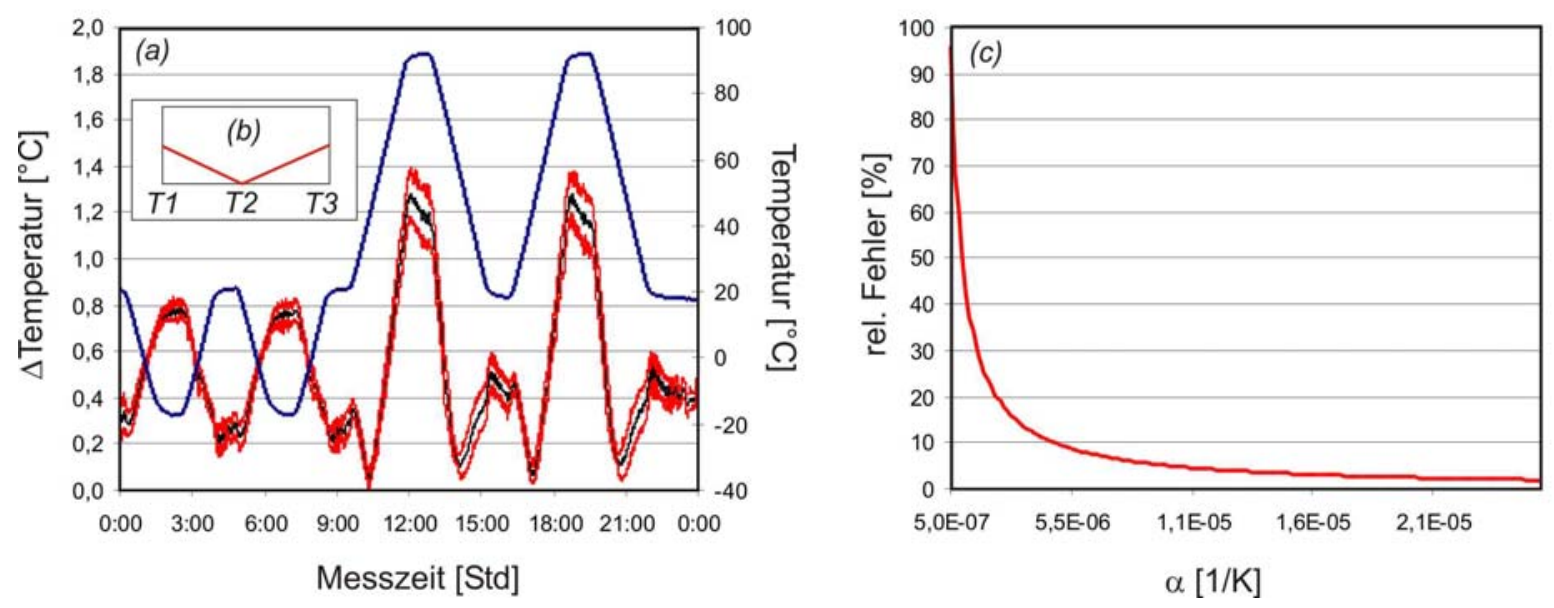

Abb.3-4: (a) Temperaturhomogenität in der Probenkammer. Blaue Kurve zeigt die Temperatur während der Messung als arithmetisches Mittel der drei Temperatursensoren T1, T2, T3. Schwarze Kurve zeigt den Verlauf der maximalen Temperaturdifferenz zwischen den Sensoren T1 bis T3 als Mittelwert aus 8 Messungen. Rote Kurven: Schwarze Kurve +/-1- $\sigma$-Fehler aus den 8 Messungen (b) Schema zum Temperaturprofil über die drei Pt-100 Temperatursensoren in der Kammer; T2 zeigt durchweg die niedrigste Temperatur. (c) Relativer Fehler in Abhängigkeit vom Ausdehnungskoeffizienten $\alpha$ des untersuchten Materials. Relativer Fehler bedingt durch den internen Gerätefehler $\left(F_{\alpha 20-90}=0,48^{*} 10^{-6} 1 / \mathrm{K}\right.$ für den Temperaturbereich von $20^{\circ} \mathrm{C}$ bis $\left.90^{\circ} \mathrm{C}\right)$ aus den Messungen des Aluminium-Standards.

Der Fehler der Temperaturmessung von +/-0,1 K aufgrund der Sensorgenauigkeit ist dagegen vernachlässigbar klein und findet keine Berücksichtigung. Die Länge L der Probe hat einen Messfehler von $+/-0,1 \mathrm{~mm}$. Unter den gegebenen Bedingungen und der Annahme eines 
Ausdehnungskoeffizienten von $\alpha_{A L 20-90}=23,8^{*} 10^{-6} 1 / \mathrm{K}$ für Aluminium ergibt sich für die $\alpha_{A L 20-90}$ ein Größtfehler $F_{\alpha A L 20-90}=+-0,9 * 10^{-6} 1 / K$, was einem relativen Größtfehler von ca. 4\% entspricht.

Um dem komplexen experimentellen Aufbau Rechnung zu tragen und alle möglichen Fehlerquellen inklusive eventueller systematischer Fehler einzubeziehen, wurde zusätzlich reines Aluminium $\left(99,99\right.$ Gew.\% Al) mit einem bekannten Ausdehnungskoeffizienten $\alpha_{\mathrm{Al} 20-}$ $100=23,8 * 10^{-6} 1 / \mathrm{K}$ (Heinzler et al. 1997) bzw. $\alpha_{\mathrm{Al}}=24,0^{*} 10^{-6} 1 / \mathrm{K}$ als Standardmaterial gemessen. An 8 verschiedenen Messtagen wurde 29-mal für Aluminiumproben des Durchmessers $15 \mathrm{~mm}$ und der Länge $50 \mathrm{~mm}$ auf den Messplätzen 1 (8-mal), 2 (7), 4 (7) und 5 (7) die thermische Dehnung bestimmt und aus den aufsteigenden sowie absteigenden Rampen (Abb.2-7) 98-mal der Ausdehnungskoeffizient $\alpha_{\mathrm{Al20-90}}$ berechnet.

Ergebnisse:

$\underline{\text { Mittelwert }}$

$$
\alpha_{\mathrm{A} 120-90}=23,7 * 10^{-6} 1 / \mathrm{K}
$$

$\underline{\text { Standardabweichung vom Mittelwert }}$

$\alpha_{\mathrm{A} 120-90}=0,48 * 10^{-6} 1 / \mathrm{K}$

Der Mittelwert liegt im Bereich der Literaturangaben, so dass ein systematischer Fehler ausgeschlossen werden kann. Der $1-\sigma$-Fehler der Mittelwertes ist als Gerätefehler (Laborreproduzierbarkeit) zu verstehen, der als absoluter Wert für jedes untersuchte Material gilt und damit je nach Ausdehnungskoeffizienten des Materials einen entsprechenden relativen Fehler verursacht $(\boldsymbol{A b b} .3-4)$.

\subsection{Technische Daten}

Dilatometer-Typ: Horizontales 6-Stangen-Dilatometer (pushrod dilatometer)

Probengrößen: $\quad \max . \mathrm{D} 15 \mathrm{~mm} * 100 \mathrm{~mm}$ bei trockener Messung max. D15 mm*50 mm bei geneigter und gefluteter Kammer

Temperaturbereich: $\quad-20$ bis $160^{\circ} \mathrm{C}$ bei trockener Messung

5 bis $90^{\circ} \mathrm{C}$ bei mit Wasser gefluteter Kammer

Heizrate: $\quad \max$. ca. $1^{\circ} \mathrm{C} / \mathrm{min}$ $\max$. ca. $2^{\circ} \mathrm{C} / \mathrm{min}$ $\max$. ca. $1^{\circ} \mathrm{C} / \mathrm{min}$ im Temperaturbereich von -20 bis $0^{\circ} \mathrm{C}$ im Temperaturbereich von 0 bis $160^{\circ} \mathrm{C}$ mit Wasser geflutete Kammer

Umwälzthermostat: ThermoHaake F8-C50 


\section{6-Stangen-Dilatometer (Dil-6)}

Messprogramme: 6 Segmente mit bis zu 89 Zyklen oder 24 Segmente mit bis zu 8 Zyklen über internen Programmgeber des Umwälzthermostaten 99 Segmente mit bis zu 99 Zyklen über PC-Steuerung mittels Software ThermStar95 (Fa. ThermoHaake)

$\underline{\text { Medium }}$

(Umwälzthermostat): Synthetisches Silikonöl SIL180 (Fa. ThermoHaake) für den Einsatz im Temperaturbereich von $-40^{\circ} \mathrm{C}$ bis $180^{\circ} \mathrm{C}$

Messtaster: $\quad 6 *$ inkrementaler Messtaster Typ ST1271 (Fa. Heidenhain)

Auflösung: $\quad 0,0005 \mathrm{~mm}$

Genauigkeit: $\quad 0,001 \mathrm{~mm}$

Temperaturfühler: $\quad 3 *$ Pt-100 Widerstandsthermometer Klasse B für den Einsatz im Temperaturbereich von $-50^{\circ} \mathrm{C}$ bis $200^{\circ} \mathrm{C}$

Auflösung: $\quad 0,1^{\circ} \mathrm{C}$

Genauigkeit: $\quad 0,1^{\circ} \mathrm{C}$

Elektronik: $\quad$ IBR-it De8 (Fa. IBR) für die 6 inkrementalen Messtaster IBR-it Da8 (Fa. IBR) für die 3 Pt-100 Widerstandsthermometer

Frequenz/Abtastrate: $\max .3000 \mathrm{~Hz}$

Software: $\quad$ DasyLab VS 5.6 


\section{Mineraleigenschaften}

Es ist $\mathrm{zu}$ vermuten, dass die Gesamtgesteinseigenschaften maßgeblich durch die Eigenschaften ihrer Minerale beeinflusst werden. Insbesondere skalare Größen wie die Dichte, der Kationenpackungsindex und das mittlere Atomgewicht dürften aufgrund der niedrigen Porositäten $(\leq 1$ Vol.\%) fast ausschließlich durch die entsprechenden Eigenschaften des Mineralbestands bestimmt werden. Im Hinblick auf die mechanischen, elastischen und thermischen Eigenschaften spielen zudem die Mikroriss-Systeme eine Rolle und müssen berücksichtigt werden. Der Einfluss von offenen Rissen auf die mechanische Stabilität der einzelnen Minerale wird in Kap.4.2 erörtert. Für die Abhängigkeit der elastischen und thermischen Eigenschaften von Mikrorissen liegen keine Einkristalldaten, sondern lediglich Untersuchungen an Gesteinen vor. Folglich werden diese Zusammenhänge in Verbindung mit dem Einfluss von offenen Rissen auf die Gesamtgesteinseigenschaften behandelt (Kap.6.4).

In diesem Kapitel werden die 17 innerhalb des Probensatzes vorkommenden Minerale mit einem Volumenanteil von $>1 \%$ bezüglich der Dichte, des Kationenpackungsindex, des mittleren Atomgewichts sowie der mechanischen, elastischen und thermischen Dehnungseigenschaften charakterisiert. Um eine spätere Interpretation im Zusammenhang mit den Gesamtgesteinseigenschaften zu erlauben, werden die entsprechenden Einkristallkennwerte für Druck- und Temperaturbedingungen verwendet, die denen der gesteinstechnischen Untersuchungen nahe kommen. Nicht für alle Minerale sind Literaturdaten zur vollständigen Beschreibung aller Eigenschaften existent. Soweit möglich, wird bei mehreren Mineralen mit struktureller und chemischer Ähnlichkeit zumindest eines stellvertretend charakterisiert. Mischkristallreihen sind meist durch die Eigenschaften ihrer Endglieder beschrieben. Die entsprechenden Daten werden in der weiteren Interpretation als obere und untere Grenzwerte betrachtet.

\subsection{Dichte, Kationenpackungsindex, mittleres Atomgewicht}

Die richtungsunabhängigen Größen Dichte, Kationenpackungsindex und mittlere Atommassen sowie die chemischen Formeln der Minerale sind inklusive der zugehörigen Referenzen in Tab.4-1 zusammengestellt. Die Dichte bewegt sich im Bereich von 2,571 g/ $\mathrm{cm}^{3}$ (Kalifeldspat) bis 5,255 g/ $\mathrm{cm}^{3}$ (Hämatit). Der Kationenpackungsindex beträgt $0,0441 \mathrm{~mol} / \mathrm{cm}^{3}$ für Quarz und $0,0755 \mathrm{~mol} / \mathrm{cm}^{3}$ für Spinell. Das niedrigste mittlere Atomgewicht weist mit 19,72 Dumortierit, das höchste mit 33,08 Magnetit auf. 
Tab.4-1: Minerale: Chemische Formel, Dichte $\rho$, Kationenpackungsindex $k_{M}$, mittleres Atomgewicht M.

\begin{tabular}{|c|c|c|c|c|}
\hline Mineral & Formel & $\begin{array}{l}\text { Dichte } \\
{\left[\mathrm{g} / \mathrm{cm}^{3}\right]}\end{array}$ & $\mathrm{k}_{\mathrm{M}}\left[\mathrm{mol} / \mathrm{cm}^{3}\right]$ & $\mathrm{M}^{* \star * * * *}$ \\
\hline Kalifeldspat & $\mathrm{KAISi}_{3} \mathrm{O}_{8}$ & $2,571^{* * * *}$ & $0,0458^{*}$ & 21,40 \\
\hline Albit & $\mathrm{NaAISi}{ }_{3} \mathrm{O}_{8}$ & $2,621^{* * * *}$ & $0,0499^{*}$ & 20,17 \\
\hline Chlorit & $\left(\mathrm{Mg}_{2} \mathrm{Al}\right)\left(\mathrm{AlSi}_{3}\right) \mathrm{O}_{10}(\mathrm{OH})_{2}$ & $2,640^{* * * *}$ & $0,0332^{* *}$ & 19,85 \\
\hline Tief-Quarz & $\mathrm{SiO}_{2}$ & $2,648^{* * * *}$ & $0,0441^{*}$ & 20,03 \\
\hline Kalzit & $\mathrm{CaCO}_{3}$ & $2,7106^{* * * *}$ & $0,0542^{* *}$ & 20,02 \\
\hline Anorthit & $\mathrm{CaAl}_{2} \mathrm{Si}_{2} \mathrm{O}_{8}$ & $2,765^{* * * *}$ & $0,0497^{*}$ & 21,40 \\
\hline Muskovit & $\mathrm{KAl}_{2}(\mathrm{OH}, \mathrm{F})_{2}\left[\mathrm{AISi}_{3} \mathrm{O}_{10}\right]$ & $2,834^{* * * *}$ & $0,0498^{*}$ & 20,96 \\
\hline Phlogopit & $\mathrm{KMg}_{3}\left(\mathrm{AISi}_{3}\right) \mathrm{O}_{10}(\mathrm{OH})_{2}$ & $2,872^{* * * *}$ & $0,0545^{* *}$ & 20,86 \\
\hline Pargasit & $\mathrm{NaCa}_{2} \mathrm{FeMg}_{4} \mathrm{Al}_{2} \mathrm{Si}_{6} \mathrm{O}_{22}(\mathrm{OH})_{2}$ & $3,165^{* * * *}$ & $0,0582^{* *}$ & $20,58^{* * * * * * *}$ \\
\hline Enstatit & $\mathrm{MgSiO}_{3}$ & $3,204^{* * * *}$ & $0,0637^{*}$ & 20,08 \\
\hline Annite & $\mathrm{KFe}_{3}\left(\mathrm{AISi}_{3}\right) \mathrm{O}_{10}(\mathrm{OH})_{2}$ & $3,215^{\star * * *}$ & $0,0524^{* *}$ & 23,27 \\
\hline Diopsid & $\mathrm{CaMgSi}_{2} \mathrm{O}_{6}$ & $3,279 * * * *$ & $0,0605^{*}$ & 21,65 \\
\hline Klinozoisit & $\mathrm{Ca}_{2} \mathrm{Al}_{3} \mathrm{Si}_{3} \mathrm{O}_{12}(\mathrm{OH})$ & $3,321^{* * * *}$ & $0,0585^{\star *}$ & $21,64^{* * * * * * *}$ \\
\hline Olivin(Fo $\left.{ }_{90} \mathrm{Fa}_{10}\right)$ & $(\mathrm{Fe}, \mathrm{Mg})_{2} \mathrm{SiO}_{4}$ & $3,34^{*}$ & $0,0680^{*}$ & 21,00 \\
\hline Dumortierit & $\mathrm{Al}_{6,5-7}\left(\mathrm{BO}_{3}\right)\left(\mathrm{SiO}_{4}\right)_{3}(\mathrm{O}, \mathrm{OH})_{3}$ & $3,39^{* * * * *}$ & $0,0550^{* * *}$ & $19,72^{* * * * * * *}$ \\
\hline Pyrop & $\mathrm{Mg}_{3} \mathrm{Al}_{2} \mathrm{Si}_{3} \mathrm{O}_{12}$ & $3,565^{* * * *}$ & $0,0706^{*}$ & 20,15 \\
\hline Spinell & $\mathrm{MgAl}_{2} \mathrm{O}_{4}$ & $3,578^{* * * *}$ & $0,0755^{*}$ & 20,32 \\
\hline Grossular & $\mathrm{Ca}_{3} \mathrm{Al}_{2} \mathrm{Si}_{3} \mathrm{O}_{12}$ & $3,600 * * * *$ & $0,0639^{*}$ & 22,52 \\
\hline Hedenbergit & $\mathrm{CaFeSi}_{2} \mathrm{O}_{6}$ & $3,656^{* * * *}$ & $0,0606^{*}$ & $24,81^{* * * * * * *}$ \\
\hline Almandin & $\mathrm{Fe}_{3} \mathrm{Al}_{2} \mathrm{Si}_{3} \mathrm{O}_{12}$ & $4,312^{* * * *}$ & $0,0694^{*}$ & 24,88 \\
\hline Hercynit & $\mathrm{FeAl}_{2} \mathrm{O}_{4}$ & $4,256^{* * * *}$ & $0,0735^{*}$ & $24,83^{* * * * * * *}$ \\
\hline Magnetit & $\mathrm{Fe}_{3} \mathrm{O}_{4}$ & $5,200^{* * * *}$ & $0,0674^{*}$ & $33,08^{* * * * * * *}$ \\
\hline Hämatit & $\mathrm{Fe}_{2} \mathrm{O}_{3}$ & $5,255^{\star * * *}$ & $0,0658^{* *}$ & $31,94^{* * * * * * *}$ \\
\hline
\end{tabular}

Daten nach Dichte sortiert. * Daten aus Buntebarth (1982). ** Werte errechnet mit $V_{M}$ aus Smyth \& McCormick (1995). ${ }^{* * *}$ Abgeschätzt nach der Dichte von Dumortierit (3,39 $\mathrm{g} / \mathrm{cm}^{3}-$ www.webmineral.com). ${ }^{* * * *}$ Aus Smyth \& McCormick (1995). ${ }^{* * * *}$ www.webmineral.com. ${ }^{* * * * * *}$ Aus Gebrande (1982).****** Errechnet gemäss chemischer Formel mit relativen Atommassen aus Holleman \& Wiberg (1995).

\subsection{Mechanische Eigenschaften}

Die mechanische Stabilität eines Minerals - es wird zunächst die Zugbelastung betrachtet - ist meist anisotrop und kann mit Hilfe der spezifischen Bruchflächenenergie $(\gamma)$ sowie der Bruchzähigkeit $\left(\mathrm{K}_{\mathrm{IC}}\right)$ abgeschätzt werden. $\gamma\left[\mathrm{J} / \mathrm{m}^{2}\right]$ ist ein Maß für die aufgewendete Energie, die zur Bildung einer neuen Rissflächeneinheit notwendig ist, und $\mathrm{K}_{\mathrm{IC}}\left[\mathrm{MPa}^{*} \mathrm{~m}^{1 / 2}\right]$ quantifiziert die Resistenz eines Minerals gegenüber der Rissausbreitung, also die Empfindlichkeit gegenüber Zugspannungen bei bereits bestehenden offenen Rissen. Beide Parameter weisen häufig eine Richtungsabhängigkeit auf und werden daher mit einem Bezug auf die kristallographischen Elemente des jeweiligen Minerals angegeben. Lediglich für die Minerale Quarz, Orthoklas, Mikroklin, Muskovit, Kalzit, Olivin und Spinell liegen sowohl $\mathrm{K}_{\mathrm{IC}}$-Werte als auch $\gamma$-Werte vor und somit nur für einige der innerhalb des Probensatzes vorkommenden Minerale. Zudem erstrecken sich die Daten nur über einen geringen Teil des kristallographischen Inventars (Tab.4-3), was eine Einschätzung der Anisotropie des mechanischen Verhaltens bei vielen Mineralen verhindert. $\mathrm{K}_{\mathrm{IIC}}-\mathrm{Werte}$, die eine Aussage in 
Bezug auf die mechanische Empfindlichkeit gegenüber Scherspannungen erlauben, sind nur rudimentär vorhanden. Insgesamt ist die Datenbasis $\mathrm{zu}$ klein, um eine vollständige Charakterisierung des Mineralbestands bezüglich seiner mechanischen Eigenschaften zu ermöglichen. Es kommt erschwerend hinzu, dass die Werte unterschiedlicher Autoren (z.B. für Quarz: Hartley \& Wilshaw 1973, Atkinson 1979, Atkinson \& Avdis 1980, Norton \& Atkinson 1981, Meredith \& Atkinson 1982) teilweise uneinheitlich ausfallen oder in jüngerer Literatur bereits angezweifelt werden (z.B. für Orthoklas: Brace \& Walsh 1962 angezweifelt in Smith \& Brown 1988).

Aus diesem Grund wurde im Rahmen dieser Arbeit ein Spaltbarkeitsindex $\left(\mathrm{I}_{\mathrm{Sp}}\right)$ definiert, der geeignet erscheint, die mechanische Stabilität (im Sinne von Resistenz gegenüber Zugbeanspruchung bei bestehenden Rissen) mit einfachen Mitteln zu erfassen. Der Spaltbarkeitsindex basiert auf folgenden Annahmen:

1. Es gibt keine Minerale ohne Gitterstörungen und Risse im Gestein. Daher ist die Bruchzähigkeit $\left(\mathrm{K}_{\mathrm{IC}}\right)$ der ausschlaggebende Parameter bei der Betrachtung der mechanischen Stabilität unter den vorherrschenden Versuchsbedingungen.

2. Die Bruchzähigkeit $\left(\mathrm{K}_{\mathrm{IC}}\right)$ korreliert negativ mit der Spaltbarkeit. Kristallographische Flächen mit einer hohen Spaltbarkeit zeigen tendenziell niedrige Bruchzähigkeitswerte (Tab.4-3); die stellvertretende Verwendung von Spaltbarkeiten als ein Bewertungskriterium für die mechanischen Eigenschaften ist daher möglich.

3. Je mehr Spaltflächen mit einer hohen Spaltbarkeit in einem Mineral ausgebildet sind, desto schwächer ist das Mineral gegenüber mechanischer Beanspruchung.

4. Je stärker der Unterschied in den Spaltbarkeiten eines Minerals ist, desto stärker ist die Anisotropie gegenüber mechanischer Belastung.

Unter Berücksichtigung dieser Theoreme wird der Spaltbarkeitsindex der Minerale $\left(\mathrm{I}_{\mathrm{SpM}}\right)$ wie folgt definiert:

(G.4-1) $\quad \mathrm{I}_{\mathrm{SpM}}=\mathrm{a}+\mathrm{b}+\mathrm{c}$ mit a, b, c

a, b, c-Spaltbarkeitswerte kristallographischer Flächen eines Minerals. 
Sind nicht drei, sondern zwei oder nur eine Spaltbarkeit ausgebildet, oder in der Literatur angegeben, so gilt:

$$
\mathrm{I}_{\mathrm{SpM}}=\mathrm{a}+\mathrm{b}+\mathrm{e} \text { mit } \mathrm{e}=1 \text { wenn } b>1 \text {; mit } e=0 \text { wenn } b \leq 1
$$

bzw.

(G.4-3) $\quad \mathrm{I}_{\mathrm{SpM}}=\mathrm{a}+\mathrm{e}_{1}+\mathrm{e}_{2}$ mit $e_{1}=1$ und $e_{2}=0$ wenn $a>1 ;$ mit $e_{1}=0$ und $e_{2}=0$ wenn $a \leq 0$

a, $b$-Spaltbarkeitswerte kristallographischer Flächen. e, $e_{1}, e_{2}-$ ergänzte Spaltbarkeitswerte.

Im Falle von Mineralen, deren Spaltbarkeit als annähernd isotrop angesehen werden kann und für die nur ein Spaltbarkeitswert angegeben ist (z.B. Granat), gilt:

(G.4-4) $\quad \mathrm{I}_{\mathrm{SPM}}=\mathrm{a}_{\mathrm{I}}+\mathrm{a}_{\mathrm{I}}+\mathrm{a}_{\mathrm{I}}$ mit

$a_{1}$ - richtungsunabhängig angegebener Spaltbarkeitswert.

Die Anisotropie der Spaltbarkeit $\left(\mathrm{A}_{\mathrm{ISpM}}\right)$ ist definiert als:

(G.4-5) $\quad \mathrm{A}_{\mathrm{ISpM}}=\mathrm{Sp}_{\max }-\mathrm{Sp}_{\min }$

$S p_{\max }-$ maximaler Spaltbarkeitswert. $S p_{\min }-$ minimaler Spaltbarkeitswert.

Zur Berechnung wird die Spaltbarkeitsskala nach Rösler (1991) verwendet, die Spaltbarkeiten von 5 (höchst vollkommen) bis 0 (ohne) unterscheidet. In Tab.4-2 ist diese Skala im Vergleich mit der nach Tröger (1982) und Deer et al. (1992) verwendeten Nomenklatur dargestellt. Mindestens drei Angaben für die Spaltbarkeit sind notwendig, um den Spaltbarkeitsindex gemäss G.4-1 zu quantifizieren. Da nur sehr selten drei Werte der Spaltbarkeit für unterschiedliche Flächen eines Minerals angegeben sind, werden die fehlenden Werte nach den Vorschriften aus G.4-2/G.4-3 ergänzt.

Tab.4-2: Spaltbarkeitsskalen

\begin{tabular}{|l|c|c|}
\hline nach Rösler (1991) & nach Tröger (1982) & nach Deer et al. (1992) \\
\hline 5 (höchst vollkommen) & vv (sehr vollkommen) & perfect \\
\hline 4 (vollkommen) & v (vollkommen) & perfect \\
\hline 3 (gut, mittel) & g (gut) & mood \\
\hline 2 (deutlich, mäßig, unvollkommen) & $\mathrm{d}$ (deutlich) & poor/weak \\
\hline 1 (angedeutet, undeutlich, sehr unv.) & sch (schlecht) & none \\
\hline 0 (ohne) & & \\
\hline
\end{tabular}


Die Anisotropie ergibt sich aus der Differenz von maximalem und minimalem Spaltbarkeitswert (G.4-5). Minerale mit hohen $\mathrm{I}_{\mathrm{SpM}^{-}}$Werten sind mechanisch schwächer gegenüber Zugbelastungen als solche mit niedrigen $\mathrm{I}_{\mathrm{SpM}^{-}}$Werten.

Eine weitere Möglichkeit zur Beschreibung der mechanischen Mineraleigenschaften ist die Härte. Weit verbreitet ist die Ritzhärte nach Mohs; Verwendung finden aber auch die Eindruckhärte nach Vickers, die Rückprallhärte nach Shore oder die Schleifhärte/Abrasionshärte nach Rosival/Kessler. Eine Übersicht zu den erwähnten Härteprüfverfahren und über die Korrelation der unterschiedlichen Härteskalen liefern z.B. Tertsch (1949), Peschel (1983), Czichos (1991) und Rösler (1991). Auch die Härte ist eine richtungsabhängige Eigenschaft (Rösler 1991), doch die Daten in der Literatur beschränken sich meist auf eine pauschale Bereichsangabe ohne eine Richtungsdefinition, so dass die Abschätzung der Härteanisotropie in der Regel nicht möglich ist. Der Versuch einer direkten Verknüpfung von Härte und anderen technischen Eigenschaften wurde am Beispiel der ShoreHärte und der Druckfestigkeit an Gesteinen unternommen (Peschel 1983). Hohe Shore-Härten zeigen hier eine Übereinstimmung mit hohen Druckfestigkeitswerten. Zwar existieren keine derartigen Untersuchungen für Minerale, doch kann auch hier eine positive Korrelation von Härte und anderen mechanischen Festigkeitskennwerten, wie z.B. der Druckfestigkeit und Zugfestigkeit, als Tendenz angenommen, wenngleich nicht quantifiziert werden. Im Rahmen dieser Studie wird die Ritzhärte nach Mohs verwendet. Um eine lineare Härte-Skala zu erhalten wird die Mohs-Härte der Minerale nach der Gleichung:

$$
\mathrm{H}_{\mathrm{M}}=0,7 * \mathrm{H}_{\mathrm{V}}{ }^{1 / 3}
$$

HM - Mohs-Härte; HV - Vickers-Härte; nach Picot \& Johan aus Rösler (1991).

in eine Vickers-Härte umgewandelt. Soweit in der Literatur Härtebereiche für ein Mineral angegeben sind, erfolgt die Berechnung aufgrund der mittleren Mohs-Härte. Die Kombination von Härte als komplexer Festigkeitseigenschaft (Peschel 1983) und dem Spaltbarkeitsindex als Maß für die Resistenz eines Minerals gegenüber Zugbeanspruchung bei bestehenden Rissen birgt die Möglichkeit einer allgemeinen Abschätzung der mechanischen Stabilität (z.B.

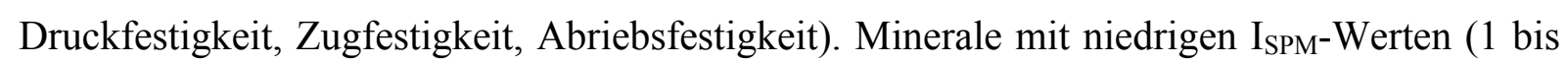
6) und hoher Härte ( $>5$ nach Mohs) können als mechanisch stark bezeichnet werden. Alle Daten zu den mechanischen Mineraleigenschaften wie Bruchzähigkeit, spezifische Bruchflächenenergie, Spaltbarkeit, Spaltbarkeitsindex, Anisotropie der Spaltbarkeit, MohsHärte und Vickers-Härte sowie die entsprechenden Referenzen sind in Tab.4-3 zusammengefasst. 


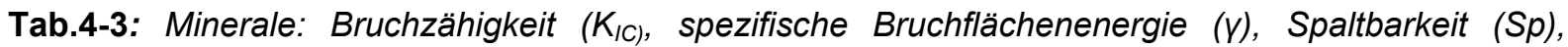
Spaltbarkeitsindex $\left(I_{S p M}\right)$. Anisotropie der Spaltbarkeit $A_{I S p M}$, Mohs-Härte $(H)$; Vickers-Härte $\left(H_{M}\right)$

\begin{tabular}{|c|c|c|c|c|c|c|c|}
\hline Mineral & $\mathrm{K}_{\mathrm{IC}}\left[\mathrm{MPa}^{*} \mathrm{~m}^{1 / 2}\right]$ & $\mathrm{V}\left[\mathrm{J} / \mathrm{m}^{2}\right]$ & $\mathrm{Sp}^{* * *}$ & $\underset{* \star \star * *}{I_{\mathrm{spM}}}$ & $\underset{* * \star \star * *}{\mathrm{~A}_{\text {ISPM }}}$ & H & $\underset{* * * * * *}{\mathrm{H}_{\mathrm{M}}[\mathrm{HV}]}$ \\
\hline Kalzit (10-11) & $0,19^{*}$ & $0,35^{* / * *}$ & 5 & & & & \\
\hline Kalzit (10-10) & & $0,38^{* *}$ & 5 & & & & \\
\hline Kalzit (0-110) & $0,2^{* *}$ & & 5 & 15 & 0 & 3 & 79 \\
\hline $\begin{array}{c}\text { Hornblende }(110) / \\
(1-10)\end{array}$ & & & 4/4/1(e) & 9 & 3 & 5,75 & 554 \\
\hline $\begin{array}{c}\text { Anorthit } \\
(001) /(010) /(110)\end{array}$ & & & $4 / 4 / 1$ & 9 & 3 & 6,25 & 712 \\
\hline Albit $(001) /(010) /(110)$ & & & $4 / 4 / 1$ & 9 & 3 & 6,25 & 712 \\
\hline Mikroklin (001) & $0,39^{*}$ & $3,2^{*}$ & 4 & & & & \\
\hline Mikroklin (010) & $0,39^{*}$ & $5,25^{*}$ & 3 & & & & \\
\hline Mikroklin (110) & $0,31^{*}$ & $3,74^{*}$ & 1(e) & 8 & 3 & 6 & 630 \\
\hline Orthoklas (001) & $1,3^{* *}$ & $7,77^{*}$ & 4 & & & & \\
\hline Orthoklas (010) & & & $3 / 1(e)$ & 8 & 3 & 6 & 630 \\
\hline Chlorit & & & $5 / 1 / 1(a)$ & 7 (a) & $4(a)$ & 2,25 & 33 \\
\hline Muskovit (001) & $0,21^{* *}$ & $0,38^{* / * *}$ & 5 & & & & \\
\hline Muskovit (110)/(010) & & & $1 / 1$ & 7 & 4 & 2,5 & 46 \\
\hline Biotit (001) & & & 5 & & & & \\
\hline Biotit (110)/ (010) & & & $1 / 1$ & 7 & 4 & 2,75 & 61 \\
\hline Enstatit $(210) /(-210) / \mathrm{e}$ & & & $2,5 / 2,5 / 1$ & 6 & 1,5 & 5,5 & 485 \\
\hline Diopsid $(110) /(1-10)$ & & & $\begin{array}{c}2,5 / 2,5 / 1 \\
(\mathrm{e})\end{array}$ & 6 & 1,5 & 5,5 & 485 \\
\hline $\begin{array}{c}\text { Hedenbergit }(110) / \\
(1-10)\end{array}$ & & & $\begin{array}{c}2,5 / 2,5 / 1(\mathrm{e} \\
)\end{array}$ & 6 & 1,5 & 5,75 & 554 \\
\hline $\begin{array}{c}\text { Epidot/Klinozoisit } \\
(001)\end{array}$ & & & $4 / 1(e) / 0(e)$ & 5 & 4 & 6,25 & 712 \\
\hline OlivinFo88Fa12 (010) & $0,59^{*}$ & $\underset{* *}{0,98 * / 8,63}$ & 2 & & & & \\
\hline OlivinFo88Fa12 (001) & $0,73^{*}$ & $\begin{array}{c}1,26^{*} / 12,0 \\
6^{* *}\end{array}$ & $1(\mathrm{e})$ & & & & \\
\hline OlivinFo88Fa12 (100) & & & 2 & 5 & 1 & 6,75 & 897 \\
\hline $\begin{array}{l}\text { Dumortierit } \\
(100) /(110)\end{array}$ & & & $3 / 1 / 1(e)$ & 5 & 2 & 8,5 & 1790 \\
\hline Spinell (111) & $1,27^{*}$ & $0,94^{*}$ & 2 & & & & \\
\hline Spinell & & & $1(\mathrm{e}) / 0(\mathrm{e})$ & 3 & 2 & 7,75 & 1357 \\
\hline Tief-Quarz & $\begin{array}{c}0,31-2,14 ;(0,46- \\
2,14(\text { für } 10-10))^{*} / * *\end{array}$ & $\begin{array}{c}0,41- \\
4,83^{* / * *}\end{array}$ & $0,5 / 0,5 / 0,5$ & 1,5 & 0 & 7 & 1000 \\
\hline Granat & & & $0,5 / 0,5 / 0,5$ & 1,5 & 0 & 7 & 1000 \\
\hline Hämatit & & & $1 / 0(e) / 0(e)$ & 1 & 1 & 5,5 & 485 \\
\hline Magnetit & & & $1 / 0(\mathrm{e}) / 0(\mathrm{e})$ & 1 & 1 & 7,75 & 1357 \\
\hline
\end{tabular}

Daten nach Spaltbarkeitsindex und in zweiter Linie nach Härte sortiert. *aus Atkinson \& Meredith (1987). ${ }^{* *}$ Aus Rummel (1982). ${ }^{* * *}$ Spaltbarkeiten aus Rösler (1991). ${ }^{* * * *}$ Berechnung siehe Text. *****Berechnung siehe Text. ${ }^{* * * * * *}$ Berechnet aus durchschnittlicher Mohshärte $\left(H_{M}\right)$ (aus Rösler 1991. www.webmineral.com) des jeweiligen Minerals nach $H_{M}=0,7^{*} H_{V}^{1 / 3}$ (Picot \& Johan aus Rösler 1991). (a) Aufgrund von strukturellen Ähnlichkeiten zu Muskovit und Biotit angenommene Werte. (e) Ergänzte Spaltbarkeiten (siehe Text). In Rummel (1982) und Atkinson \& Meredith (1987) sind Daten bereits kompiliert, einige der Originalreferenzen sind im Text zitiert.

Die Werte für den Spaltbarkeitsindex liegen zwischen 1 (z.B. Magnetit) und 15 (Kalzit). In Verbindung mit der geringen Härte von 3 (Mohs) bzw. 79 (Vickers) führt das zu dem Schluss, dass Kalzit potenziell das mechanisch schwächste der aufgeführten Minerale ist. Als Beispiele 
für mechanisch stabile Minerale können Olivin $\left(\mathrm{I}_{\mathrm{SpM}}=5, \mathrm{HV}=897\right)$ und Hedenbergit $\left(\mathrm{I}_{\mathrm{SpM}}=6\right.$, $\mathrm{HV}=554$ ) angesehen werden. Die mit $\mathrm{A}_{\mathrm{ISpM}}=4$ höchste Anisotropie weisen die Phyllosilikate auf. Der Quarz lässt sich bezüglich seiner mechanischen Eigenschaften nur schwer einschätzen. Der niedrige Spaltbarkeitsindex von $\mathrm{I}_{\mathrm{SpM}}=1,5$ und die hohe Härte von $\mathrm{HV}=1000$ deuten auf eine hohe mechanische Stabilität hin, jedoch sind die $\mathrm{K}_{\mathrm{IC}}$-Werte uneinheitlich und können diese Vermutung nicht zweifelsfrei bestätigen.

\subsection{Elastische Eigenschaften}

Zur Charakterisierung der elastischen Mineraleigenschaften werden die Voigt-Reuss-HillMittel des Schubmodul (K), des Schermodul (G), des E-Moduls (E) sowie der Kompressionswellengeschwindigkeiten Vp verwendet. Letztere werden zusätzlich nach $\mathrm{A}_{\mathrm{VpM}}=\left(\mathrm{V} \mathrm{p}_{\mathrm{Mmax}}-\mathrm{Vp}_{\mathrm{Mmin}}\right) / \mathrm{Vp}_{\mathrm{Mmax}} * 100$ zur Abschätzung der Anisotropie der elastischen Eigenschaften herangezogen.

Tab.4-4: Elastische Eigenschaften der Minerale: Schubmodul $\left(K_{M V R H}\right)$, Schermodul $\left(G_{M V R H}\right)$, Elastizitätsmodul $E_{M V R H}$ (berechnet aus $G_{M V R H}$ und $K_{M V R H}$ ), Kompressionswellengeschwindigkeit $\left(V p_{M V R H}\right)$, Anisotropie der Kompressionswellengeschwindigkeit $\left(A_{V P M}\right)$; $(V R H$ - Voigt-Reuss-Hill-Mittel).

\begin{tabular}{|c|c|c|c|c|c|}
\hline Mineral & $\begin{array}{c}\mathrm{K}_{\text {MVRH }} \\
\text { [GPa] }^{*}\end{array}$ & $\begin{array}{c}\mathbf{G}_{\text {MVRH }} \\
\text { [GPa }^{*}\end{array}$ & $\begin{array}{c}\mathrm{E}_{\mathrm{MVRH}} \\
\mathrm{GPPa}^{* *}\end{array}$ & $V p_{\text {MVRH }}[\mathbf{k m} / \mathbf{s}]^{* * *}$ & $A_{V p M}[\%]^{* * * *}$ \\
\hline Kalifeldspat (Mikroklin) & 55,4 & 28,1 & 72,1 & 5930 (Or) & 38 \\
\hline Albit & 56,9 & 28,6 & 73,5 & 5940 & 28 (Plag) \\
\hline Kalzit & 73,3 & 32 & 83,8 & 6540 & 26 \\
\hline Muskovit & 58,2 & 35,3 & 88,1 & 5810 & 44 \\
\hline Tief-Quarz & 37,8 & 44,3 & 95,6 & 6050 & 24 \\
\hline Anorthit & 84,2 & 39,9 & 103,4 & 7050 & \\
\hline Hornblende & $87-93,3$ & $43-49$ & 125,1 & 6810 & 24 \\
\hline Fayalit & 134 & 50,7 & 135,1 & & \\
\hline Hedenbergit & 120 & 61 & 156,5 & & \\
\hline Diopsid & $113-114$ & $64,9-67$ & 163,6 & 7700 & 25 \\
\hline Bronzit & 103,5 & 74,9 & 181,0 & 7360 & 16 \\
\hline Enstatit & 107,8 & 75,7 & 184,0 & 8080 & \\
\hline Forsterit & 129,5 & 81,1 & 201,3 & 8570 & \\
\hline Olivin (Fo90Fa10) & & & & 8420 & 22 \\
\hline Magnetit & 162,0 & 91,2 & 230,3 & & \\
\hline Pyrop & 172,8 & 92 & 234,4 & 8960 & $<1$ \\
\hline Grossular & 168,4 & 108,9 & 268,8 & 9310 & \\
\hline Almandin & & & & 8420 & \\
\hline Spinell & 197,9 & 108,5 & 275,2 & & \\
\hline
\end{tabular}

Daten nach E-Modul sortiert. Or - Orthoklas. Plag-Plagioklas. *Aus Bass (1995). **Berechnet nach $E_{M V R H}=9^{*} K_{M V R H}{ }^{*} G_{M V R H} /\left(3^{*} K_{M V R H}+G_{M V R H}\right)$ aus Gebrande (1982). ${ }^{* * *}$ Aus Gebrande (1982). ${ }^{* * * * B e r e c h n e t}$ nach $A_{V p M}=\left(V p_{M \max }-V p_{M \min }\right) / V p_{M \max }{ }^{*} 100$; Einkristallelastizitätskonstanten für: Olivin - Isaak (1992); Bronzit - Frisillo \& Barsch (1972); Diopsid - Levien et al. (1979); Hornblende - Aleksandrov \& Ryzhova (1961); Plagioklas - Aleksandrov et al. (1974); Quarz - McSkimin et al. (1965); Muskovit Vaughan \& Guggenheim (1986); Kalzit - Dandekar (1968); Granat - Bonczar et al. (1977). 
Mit $\mathrm{E}_{\mathrm{MVRH}}=234,4 \mathrm{GPa}$ und $\mathrm{E}_{\mathrm{MVRH}}=275 \mathrm{GPa}$ sind der Pyrop und der Spinell Beispiele für Minerale mit sehr hohen E-Moduln, bezogen auf ein untexturiertes, polykristallines Aggregat. Deutlich niedrigere durchschnittliche E-Moduln weisen die typischen Minerale der oberen Erdkruste wie z.B. Quarz $\left(\mathrm{E}_{\mathrm{MVRH}}=95,6 \mathrm{GPa}\right)$, Kalifeldspat $\left(\mathrm{E}_{\mathrm{MVRH}}=72,1 \mathrm{GPa}\right)$ und Muskovit $\left(\mathrm{E}_{\mathrm{MVRH}}=88,1 \mathrm{GPa}\right)$ auf. Letzterer zeigt mit $\mathrm{A}_{\mathrm{VpM}}=44 \%$ auch die höchste Anisotropie der Kompressionswellengeschwindigkeiten. Vergleichsweise niedrig ist die Anisotropie bei den Orthopyroxenen (z.B. Bronzit - 16\%) und bei Olivin (22\%). Alle Einkristalldaten sind inklusive der zugehörigen Referenzen in Tab.4-4 zusammengefasst.

\subsection{Thermische Dehnung}

Die Größenordnung der thermische Dehnung der gesteinsbildenden Minerale wird durch den Volumenausdehnungskoeffizienten $\left(\alpha_{\mathrm{VM}}\right)$ beschrieben; die Anisotropie dieser Eigenschaft wird aus den richtungsabhängigen Ausdehnungskoeffizienten $(\alpha)$ nach $\mathrm{A}_{\alpha \mathrm{M}}=\left(\alpha_{\mathrm{Mmax}}{ }^{-}\right.$ $\left.\alpha_{\text {Mmin }}\right) / \alpha_{\text {Mmax }} * 100$ bestimmt. Die Daten der temperaturabhängigen Größen beziehen sich auf einen Temperaturbereich von $20^{\circ} \mathrm{C}$ bis $90^{\circ} \mathrm{C}$ und sind in $\mathbf{T a b} .4-5$ zusammengestellt.

Tab.4-5: Thermische Ausdehnungskoeffizienten der Minerale: Thermischer Volumenausdehnungskoeffizient $\left(\alpha_{V M}\right)$, Thermischer Ausdehnungskoeffizient $\alpha_{M}$; Anisotropie der thermischen Dehnung $A_{\alpha M}$; alle Daten für $20^{\circ} \mathrm{C}$ bis $90^{\circ} \mathrm{C}$.

\begin{tabular}{|c|c|c|c|}
\hline Mineral & $\alpha_{\mathrm{VM}}[1 / \mathrm{K}]^{-6}$ & $\alpha_{M}[1 / K]^{-6}$ & $\mathrm{~A}_{\alpha M}[\%]^{\star \star \star \star \star}$ \\
\hline Kalzit a & & $-3,2^{*}$ & \\
\hline Kalzit c & & $15,8^{*}$ & \\
\hline Kalzit $\alpha_{v M}, A_{\alpha M}$ & $6,59^{*}$ & & 120 \\
\hline Albit low a & & $11,3^{*}$ & \\
\hline Albit low b & & $4,83^{*}$ & \\
\hline Albit low c, $A_{a M}$ & & $0,385^{*}$ & 97 \\
\hline Plagioklas Ab77An23 $\alpha_{V M}$ & $10,4^{*}$ & & \\
\hline Plagioklas Ab56An44 $\alpha_{V M}$ & $11,6^{*}$ & & \\
\hline Kalifeldspat Or88,3Ab9,3An2,4 $\alpha_{\mathrm{VM}}$ & $12,6^{*}$ & & \\
\hline Plagioklas Ab5An95 $\alpha_{\mathrm{VM}}$ & $14^{*}$ & & \\
\hline Mikroklin Or83,5Ab16,5 $\alpha_{\mathrm{VM}}$ & $15,8^{*}$ & & \\
\hline Almandin $\alpha_{V M}, \alpha_{M}$ & $16,9^{*}$ & $5,62^{* * * *}$ & \\
\hline Grossular $\alpha_{\mathrm{VM}}, \alpha_{\mathrm{M}}$ & $17,4^{*}$ & $5,79^{* * * *}$ & \\
\hline Spinell & $19,9^{*}$ & & \\
\hline Pyrop $\alpha_{v M}, \alpha_{M}$ & $20,7^{*}$ & $6,90^{* * * *}$ & \\
\hline Hornblende & $24,1^{*}$ & & \\
\hline Enstatit $\alpha_{\mathrm{VM}}$ & $25,0^{*}$ & & \\
\hline Hercynit & $27,0^{*}$ & & \\
\hline Fayalit a & & $5,93^{*}$ & \\
\hline Fayalit b & & $8,06^{*}$ & \\
\hline Fayalit c & & $10,4^{*}$ & \\
\hline Fayalit $\alpha_{v M}, A_{a M}$ & $27,1^{*}$ & & 43 \\
\hline Forsterit a & & $7,03^{*}$ & \\
\hline Forsterit b & & $10,4^{*}$ & \\
\hline Forsterit c & & $11,1^{*}$ & \\
\hline Forsterit $\alpha_{v M}, A_{a M}$ & $27,6^{*}$ & & 37 \\
\hline
\end{tabular}




\begin{tabular}{|c|c|c|c|}
\hline Mineral & $\alpha_{\mathrm{VM}}[1 / \mathrm{K}]$ & $\alpha_{M}[1 / K]$ & $\mathrm{A}_{\mathrm{\alpha M}}[\%]^{\star \star \star \star *}$ \\
\hline Hedenbergit a & & $7,2^{*}$ & \\
\hline Hedenbergit b & & $17,6^{*}$ & \\
\hline Hedenbergit c & & $6,0^{*}$ & \\
\hline Hedenbergit $\alpha_{V M}, A_{a M}$ & $29,8^{*}$ & & 66 \\
\hline Diopsid a & & $7,8^{*}$ & \\
\hline Diopsid b & & $20,5^{*}$ & \\
\hline Diopsid c & & $6,5^{*}$ & \\
\hline Diopsid $\alpha_{V M}, A_{a M}$ & $33,3^{*}$ & & 68 \\
\hline Muskovit a & & $9,9^{*}$ & \\
\hline Muskovit b & & $11,1^{*}$ & \\
\hline Muskovit c & & $13,8^{*}$ & \\
\hline Muskovit $\alpha_{\mathrm{VM}}, A_{\alpha M}$ & $35,4^{*}$ & & 28 \\
\hline Tief-Quarz c & & $9^{* *}$ & \\
\hline Tief-Quarz a & & $14^{* *}$ & \\
\hline Tief-Quarz $\alpha_{\mathrm{vM}}, A_{a M}$ & $37^{* * *}$ & & 36 \\
\hline Bronzit Mg0,8Fe0,2 a & & $16,4^{*}$ & \\
\hline Bronzit Mg0,8Fe0,2 b & & $14,5^{*}$ & \\
\hline Bronzit Mg0,8Fe0,2 c & & $16,8^{*}$ & \\
\hline Bronzit Mg0,8Fe0,2 $\alpha_{\mathrm{VM}}, \mathrm{A}_{a \mathrm{M}}$ & $44,7^{*}$ & & 2 \\
\hline
\end{tabular}

Daten sind nach Volumenausdehnungskoeffizienten sortiert. *Aus Fei (1995). **Aus Kleber (1990). ${ }^{* * *}$ Berechnet nach $\alpha_{V M}=2^{*} \alpha_{a M}+\alpha_{C M}$ für trigonale Symmetrie aus Barron (1998). **** Berechnet nach $\alpha_{V M}=3^{*} \alpha_{M}$ für kubische Symmetrie aus Barron (1998). ${ }^{* * * * *}$ Berechnet nach $A_{\alpha M}=\left(\alpha_{M \max }{ }^{-}\right.$ $\left.\alpha_{\text {Mmin }}\right) / \alpha_{\text {Mmax }} * 100$.

Den höchsten Volumenausdehnungskoeffizienten der betrachteten Minerale weist mit $\alpha_{\mathrm{VM}}=44,7 \mathrm{e}^{-6} 1 / \mathrm{K}$ der Bronzit, den niedrigsten mit $\alpha_{\mathrm{VM}}=6,59 \mathrm{e}^{-6} 1 / \mathrm{K}$ der Kalzit auf. Minerale mit einer extrem hohen Anisotropie der thermischen Dehnung sind Albit (97\%), der diesbezüglich stellvertretend für das thermische Dehnungsverhalten der Feldspäte angesehen werden kann (Smith \& Brown 1988), und der Kalzit, dessen Anisotropiewert von über 100\% sich aus einem negativen Ausdehnungskoeffizienten in Richtung der a-Achse ergibt.

\subsection{Korrelation der Mineraleigenschaften}

Die Abhängigkeiten der unterschiedlichen bereits behandelten technisch-physikalischen Mineralparameter liegen in der chemischen Zusammensetzung und der Kristallstruktur der Minerale begründet. Jedoch folgen die Verhältnisse nicht immer einem linearen Trend und sind zudem von Ausnahmen und Anomalien des Mineralverhaltens geprägt. In Abb.4-1 und Abb.4-2 sind einige wichtige Zusammenhänge für die vorkommenden Minerale dargestellt, ohne dass eine Quantifizierung stattfindet und Kausalitäten zur Mineralchemie sowie zur Kristallographie aufgezeigt werden. Es geht allein darum, deskriptiv eine Argumentationsgrundlage für die Korrelationen der gesteinstechnischen Parameter (Kap.8) zu liefern. Auch aus diesem Grund wurde auf die Angabe von Korrelationskoeffizienten verzichtet. Schwerpunktmäßig werden Besonderheiten der wichtigen gesteinbildenden 
Minerale herausgestellt. Diese weichen zum Teil deutlich von den generellen Trends ab und sind damit Faktoren, welche bei der Interpretation der Abhängigkeiten zwischen den gesteinstechnischen Eigenschaften berücksichtigt werden müssen. Zunächst wird die Dichteabhängigkeit der mechanischen, elastischen und thermischen Parameter betrachtet.

Zwischen der Dichte und dem Spaltbarkeitsindex besteht eine Antikorrelation, wobei die Minerale Quarz und Kalzit weit außerhalb des generellen Trends liegen. Der Spaltbarkeitsindex - als im Rahmen dieser Arbeit entwickelte praxisnahe und rein qualitative Größe - erlaubt die Abschätzung von Relationen der mechanischen Stabilität, lässt jedoch keine quantifizierbaren Rückschlüssen auf die tatsächlichen Bindungsenergien innerhalb des Kristallgitters zu. Somit ist eine Korrelation mit anderen physikalischen Eigenschaften ebenfalls qualitativ und in keinem Fall linear (Abb.4-1).
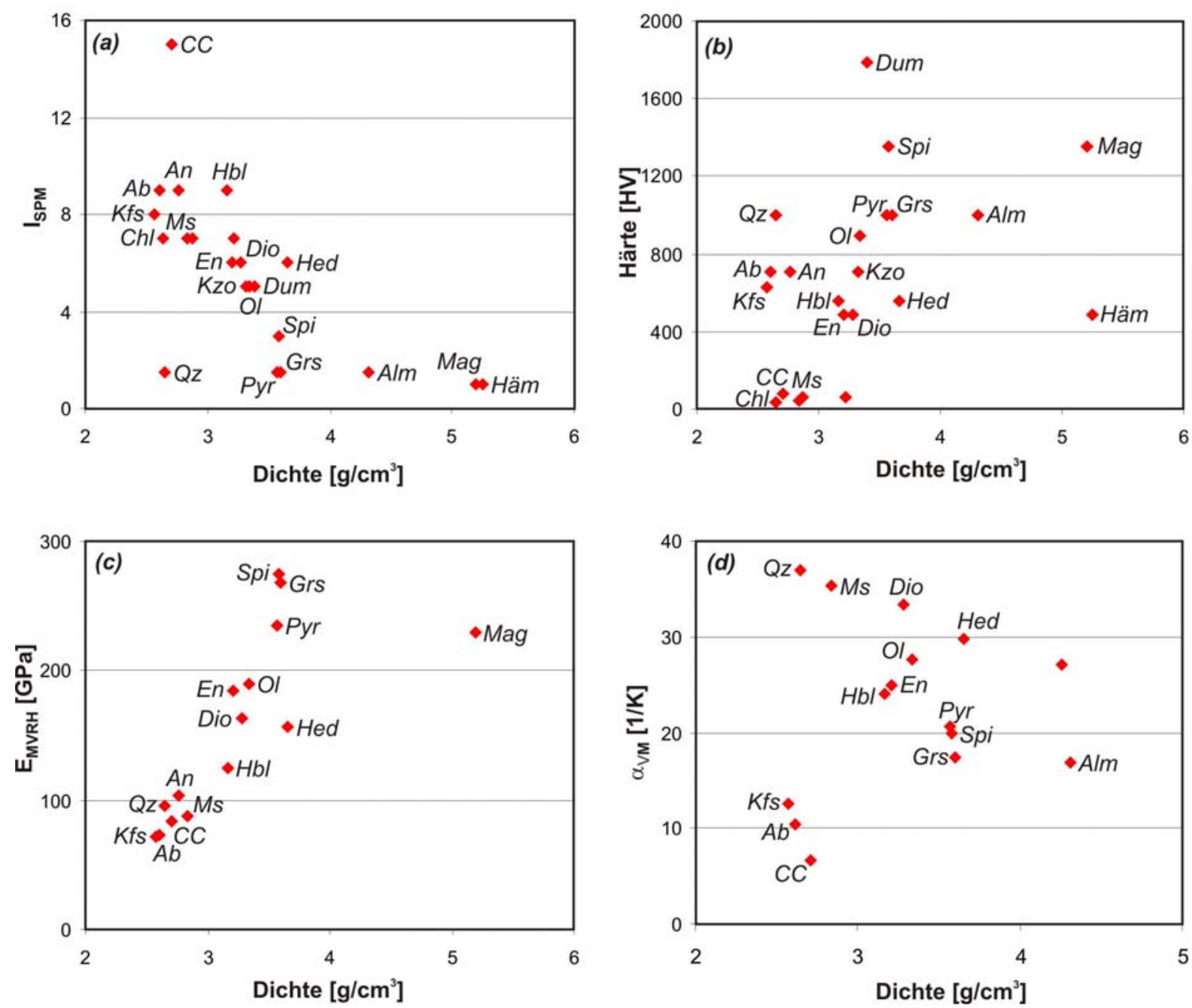

Abb.4-1: Technische Eigenschaften der gesteinsbildenden Minerale in Abhängigkeit von der Dichte. (a) Spaltbarkeitsindex (ISPM) vs. Dichte. (b) Härte vs. Dichte. (c) E-Modul $E_{M V R H}$ vs. Dichte. (d) Thermischer Volumenausdehnungskoeffizient $\left(\alpha_{V M}\right)$ vs. Dichte. Ab - Albit; An - Anorthit; $\mathrm{Hbl}-$ Hornblende; Kfs - Kalifeldspat; Ms - Muskovit; Chl - Chlorit; En - Enstatit; Dio - Diopsid; Hed Hedenbergit; Kzo - Klinozoisit; Dum - Dumortierit; OI - Olivin; Spi - Spinell; Grs - Grossular; Pyr Pyrop; Alm - Almandin; Mag - Magnetit; Häm - Hämatit; Qz - Quarz; CC - Kalzit. 
Minerale mit einer relativ höheren Dichte weisen tendenziell höhere Härten und auch höhere dynamische E-Moduln auf. Dieser Trend ist zwar deutlich ausgeprägt, doch besteht eine große Streuung innerhalb der Daten, und gerade wichtige gesteinsbildende Minerale wie Quarz, Albit und Kalifeldspat liegen außerhalb der Regressionsgeraden. Zwischen der Dichte und dem Volumenausdehnungskoeffizienten besteht eine Antikorrelation, wobei die Minerale Kalzit, Albit und Kalifeldspat mit außergewöhnlich niedrigen Volumenausdehnungskoeffizienten Besonderheiten darstellen.

Auch zwischen den mechanischen, elastischen und thermischen Eigenschaften bestehen einige signifikante Zusammenhänge. Eine generelle Antikorrelation besteht zwischen dem EModul ( $\left.\mathrm{E}_{\mathrm{MVRH}}\right)$ und dem Spaltbarkeitsindex ( $\left.\mathrm{I}_{\mathrm{SPM}}\right)$ sowie dem E-Modul und dem Volumenausdehnungskoeffizienten $\left(\alpha_{V M}\right)$.
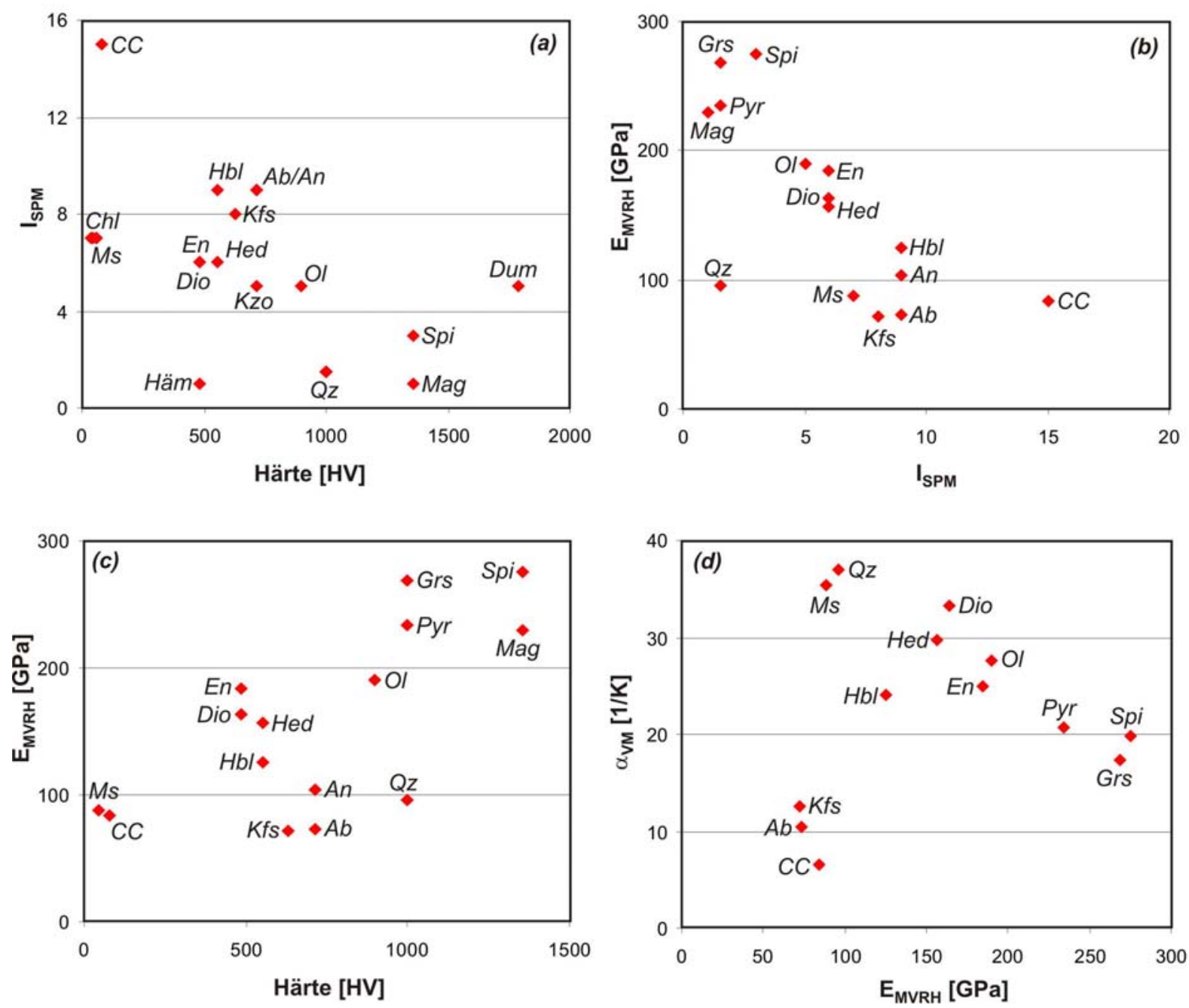

Abb.4-2: Korrelation der technischen Mineraleigenschaften. (a) Spaltbarkeitsindex (ISPM) vs. Härte. (b) E-Modul ( $\left.E_{M V R H}\right)$ vs. Spaltbarkeitsindex (ISPM). (c) E-Modul $E_{M V R H}$ vs. Härte. (d) Thermischer Volumenausdehnungskoeffizient $\left(\alpha_{V M}\right)$ vs. E-Modul $\left(E_{M V R H}\right)$. Ab - Albit; An - Anorthit; Hbl Hornblende; Kfs - Kalifeldspat; Ms - Muskovit; Chl - Chlorit; En - Enstatit; Dio - Diopsid; Hed Hedenbergit; Kzo - Klinozoisit; Dum - Dumortierit; Ol - Olivin; Spi - Spinell; Grs - Grossular; Pyr Pyrop; Alm - Almandin; Mag - Magnetit; Häm - Hämatit; Qz - Quarz; CC - Kalzit. 


\section{Mineraleigenschaften}

Außerhalb der Trends liegen im ersten Fall Quarz und im zweiten Fall Kalifeldspat, Kalzit und Albit (Abb.4-2). Bezüglich der Härte und dem Spaltbarkeitsindex ergibt sich ein uneinheitliches Bild. Zwar ist eine leichte Tendenz zwischen hohen Härten und niedrigen $\mathrm{I}_{\mathrm{SpM}}$-Werten zu erkennen, doch ist dieser Zusammenhang gerade für wichtige gesteinbildende Minerale nicht signifikant (z.B. Muskovit, Kalifeldspat, Enstatit, Olivin mit ähnlichen $\mathrm{I}_{\mathrm{SPM}^{-}}$ Werten aber sehr unterschiedlichen Härten). Die Härte korreliert linear mit dem dynamischen E-Modul (Abb.4-2). Auffällige Minerale sind hier Quarz und die Feldspäte mit tendenziell höheren Härten, als aufgrund der E-Moduln zu erwarten wäre.

Zusammenfassend lässt sich feststellen, dass zwischen den technisch-physikalischen Eigenschaften signifikante Zusammenhänge beobachtbar sind. Allerdings befinden sich gerade wichtige gesteinsbildende Minerale (z.B. Quarz, Feldspäte, Kalzit) in vielen Fällen außerhalb der generellen Trends, so dass die Ergebnisse für die Betrachtung der Korrelation der gesteinstechnischen Eigenschaften nicht pauschal angewendet werden können und einer detaillierten Betrachtung bedürfen. 


\section{Gesteinsmaterial}

\subsection{Nomenklatur und Probennahme}

Im Rahmen dieser Arbeit werden die fünf magmatischen Gesteine nach der Terminologie von Streckeisen $(1974$, 1980) benannt. Die 15 Metamorphite werden nach ihrem Hauptmineralbestand (z.B. Marmor (Anzahl = 1), Quarzit (1), Meta-Gabbro (2), MetaGranitoid (11)) bezeichnet, wobei die elf Proben der Meta-Granitoide gemäss ihres Deformationszustands nach dem Schema von Wise et al. (1984) als Mylonite bzw. Gneise eingeordnet (Abb.5-1) und zusätzlich durch einen vorangestellten petrographischen, den Mineralbestand wiederspiegelnden Begriff charakterisiert werden (Beispiele: granitischer Gneis/granodioritischer Mylonit).

Daraus ergibt sich eine Anzahl von fünf Myloniten (zwei Protomyloniten, ein Mylonit, zwei Ultramyloniten) sowie sechs Gneisen granitoider Zusammensetzung. Des Weiteren finden gefügespezifizierende Termini Anwendung, die auf der von Passchier \& Trouw (1998) verwendeten Nomenklatur basieren (z.B. polygonal/granoblastisch/interlobat, equigranular/inequigranular).

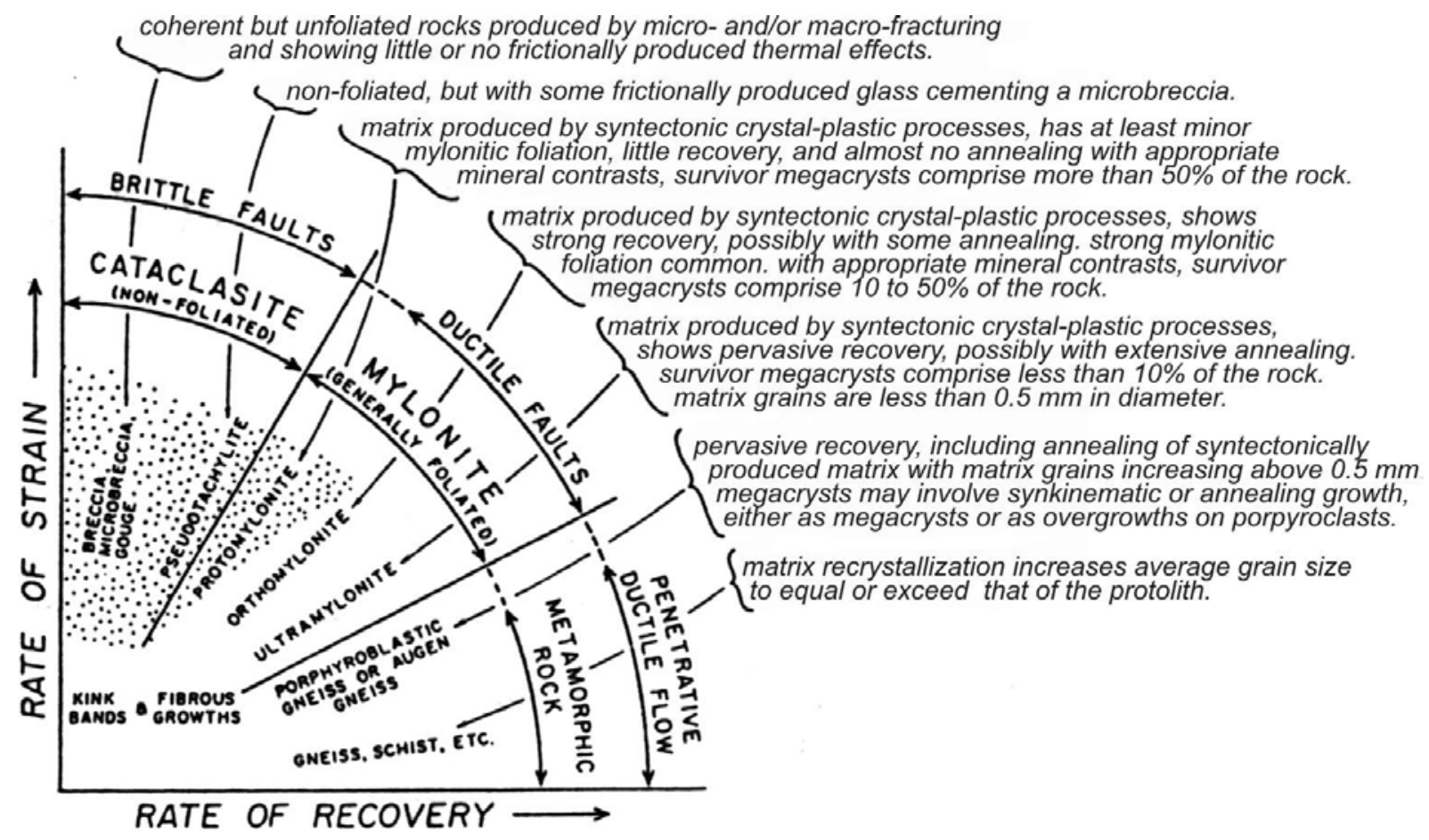

Abb.5-1: Nomenklatur störungsgebundener Gesteine (verändert nach Wise et al. 1984)

Neun der untersuchten Proben sind handelsübliche Naturwerksteine, deren Handelsnamen ebenfalls, aber niemals separat ohne eine im petrographischen Sinne korrekte Benennung Verwendung finden. Für jede Probe existiert ein Probenkürzel, welches insbesondere in 


\section{$\underline{\text { 5. Gesteinsmaterial }}$}

Diagrammen, aber auch im Text benutzt wird. Alle Probennamen, Probenkürzel und Handelnamen sind im Anhang II zusammengestellt.

Der aus insgesamt 20 Kristallingesteinen unterschiedlicher Herkunft bestehende Probensatz variiert deutlich sowohl in der chemischen und mineralogischen Zusammensetzungen als auch in den Gesteinsgefügen. Innerhalb dieser Gruppe befinden sich vier Serien zu je drei bzw. zwei Proben, die von ihrer Herkunft, der Genese und dem Mineralbestand ähnlich sind und sich durch unterschiedliche Deformationsgrade auszeichnen. Die Serie Iv besteht aus drei progressiv mylonitisch deformierten Meta-Granitoiden. Die granitische Serie (Gra) beinhaltet drei Proben, zwei granulitfaziell überprägte Gesteine und einen Ultramylonit. Zwei MetaGabbros $(\mathrm{Mg})$ sowie ein Granit und sein mylonitisiertes Äquivalent (Mr) bilden die Serien drei und vier. Einige dieser annähernd isochemen und isomineralogischen, progressiv deformierten Serien werden im Rahmen von Fallstudien (Kap.7) detailliert betrachtet. Alle Proben wurden entweder an Straßenaufschlüssen bzw. in Steinbrüchen genommen oder über Natursteinhändler bestellt. Es galt grundsätzlich möglichst ungestörte, unverwitterte und im Beobachtungsmaßstab von etwa $50 \mathrm{~cm} * 50 \mathrm{~cm} * 50 \mathrm{~cm}$ homogene Proben in einer Menge zu erhalten, welche die Durchführung aller Methoden an einer Ausgangsprobe ermöglichte. Bei den Geländeproben waren diese Qualitätskriterien nicht immer zu erfüllen.

\subsection{Mineralogische Zusammensetzung}

Innerhalb der 20 Proben treten 17 unterschiedliche Minerale mit einem Gehalt von mehr als 1 Vol.\% auf. Nach der im Rahmen dieser Arbeit verwendeten Terminologie von Streckeisen (1974, 1980) besteht die Gruppe der Magmatite aus zwei Graniten, einem Granodiorit, einem Gabbro-Norit und einem Peridotit (Abb.5-2). Die Metamorphite im allgemeinen Sinn (Murawski 1992) unterteilen sich in einen Quarzit, einen Marmor, zwei Meta-Gabbros und elf metamorph überprägte Magmatite und Sedimentite von granitischer bis granodioritischer Zusammensetzung. Letztere sowie der Quarzit haben einen Anteil von mafischen Komponenten unter 35 Vol.\% und gehören daher ausnahmslos zu den leukokraten Gesteinen. In diesem Punkt unterscheiden sie sich deutlich von den melanokraten Meta-Gabbros und dem ultramafischen Peridotit. 


\section{Gesteinsmaterial}
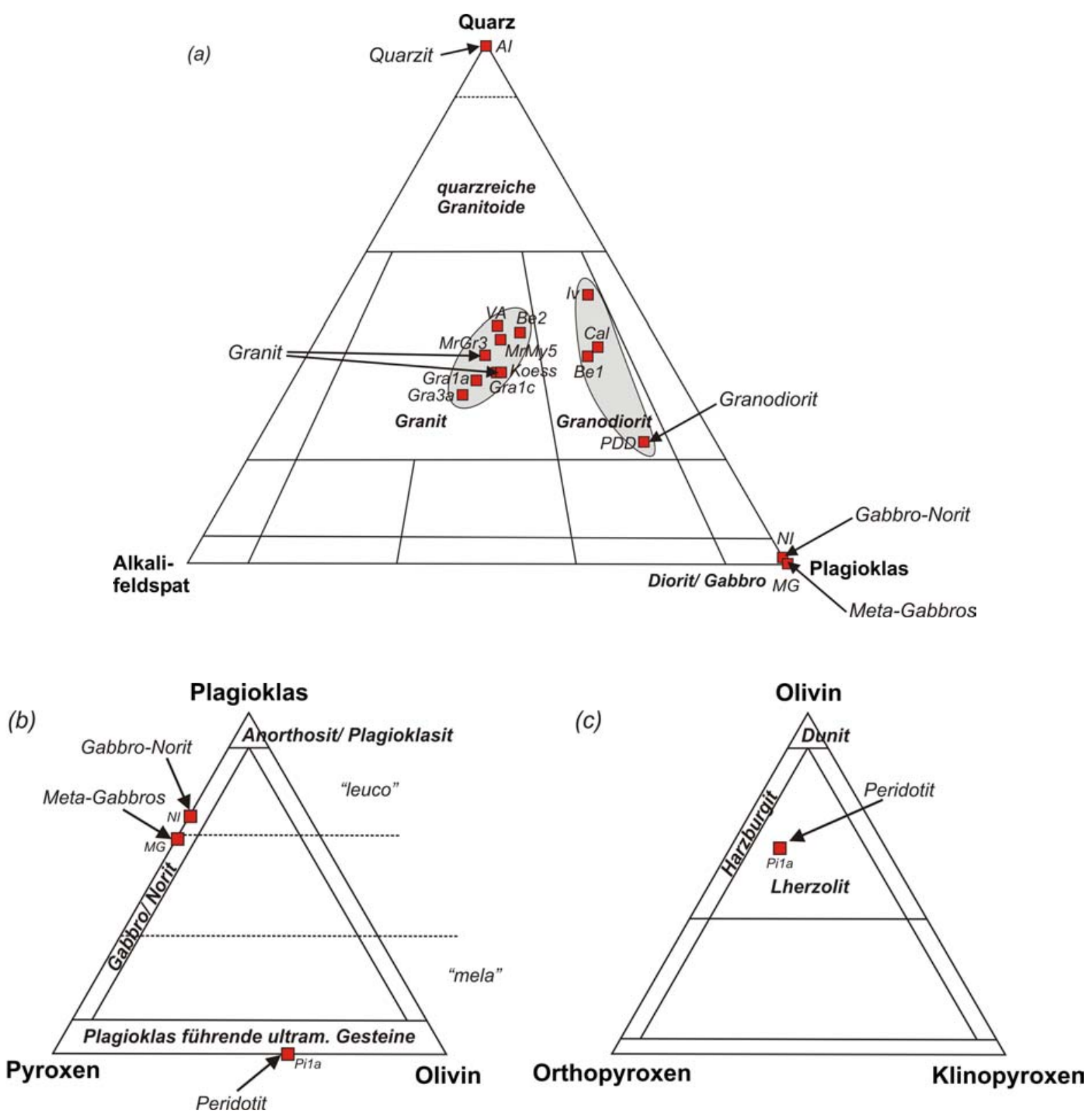

Abb.5-2: (a) Proben im Streckeisen-Diagramm. (b) Klassifikation der mafischen Gesteine. (c) Einordnung der ultramafischen Gesteine. $(a, b)$ nach Streckeisen $(1974,1982)$. (c) nach Harte (1983) Die Gruppen kristalliner Gesteine mit granitischer bzw. granodioritischer Zusammensetzung sind grau hinterlegt. Die Datenpunkte Iv und MG stehen stellvertretend für drei bzw. zwei Proben einer Serie. Die magmatischen Gesteine sowie der Quarzit und die Meta-Gabbros sind gesondert gekennzeichnet. Zusätzlich wurde ein Marmor untersucht.

Der untersuchte Marmor passt nicht in die, auf der mineralogischen Zusammensetzung basierenden Einteilungsmuster und muss daher gesondert betrachtet werden.

Der Kationenpackungsindex $\left(\mathrm{k}_{\mathrm{G}}\right)$ der Gesteine liegt zwischen 4,5 $\left(\mathrm{mol} / \mathrm{cm}^{3}\right)^{-2}$ für den Quarzit und 6,61 $\left(\mathrm{mol} / \mathrm{cm}^{3}\right)^{-2}$ für den Peridotit und reflektiert die Variationsbreite der mineralogischen Zusammensetzung (Buntebarth 1982; Rybach \& Buntebarth 1982). Eine weiterführende Diskussion findet in Kap.6.4.1.5 statt.

15 der untersuchten Gesteine und damit sämtliche der granitischen, granodioritischen und quarzitischen Proben enthalten Phyllosilikate in unterschiedlichen Anteilen. Die Phyllosilikate 


\section{$\underline{\text { 5. Gesteinsmaterial }}$}

Muskovit, Biotit und Chlorit sind bezüglich der meisten ihrer physikalischen Eigenschaften stark anisotrop und stehen in Kombination mit einer bevorzugten kristallographischen Orientierung im Verdacht, für die Anisotropie einer Reihe von petrophysikalischen Eigenschaften verantwortlich zu sein (z.B. Gottschalk et al. 1990; Siegesmund et al. 1995). Aus diesem Grund wurden die phyllosilikathaltigen Gesteine in einem modifizierten Streckeisen-Diagram dargestellt und charakterisiert (Abb.5-3). Die Gehalte liegen zwischen 4 Vol.\% für den granoblastischen Gneis (Grala) und 26 Vol.\% für den chloritisierten Orthogneis (VA). Die Werte sind direkt aus dem Diagramm ablesbar, weil alle Mineralkomponenten berücksichtigt wurden und daher keine Normierung stattgefunden hat.

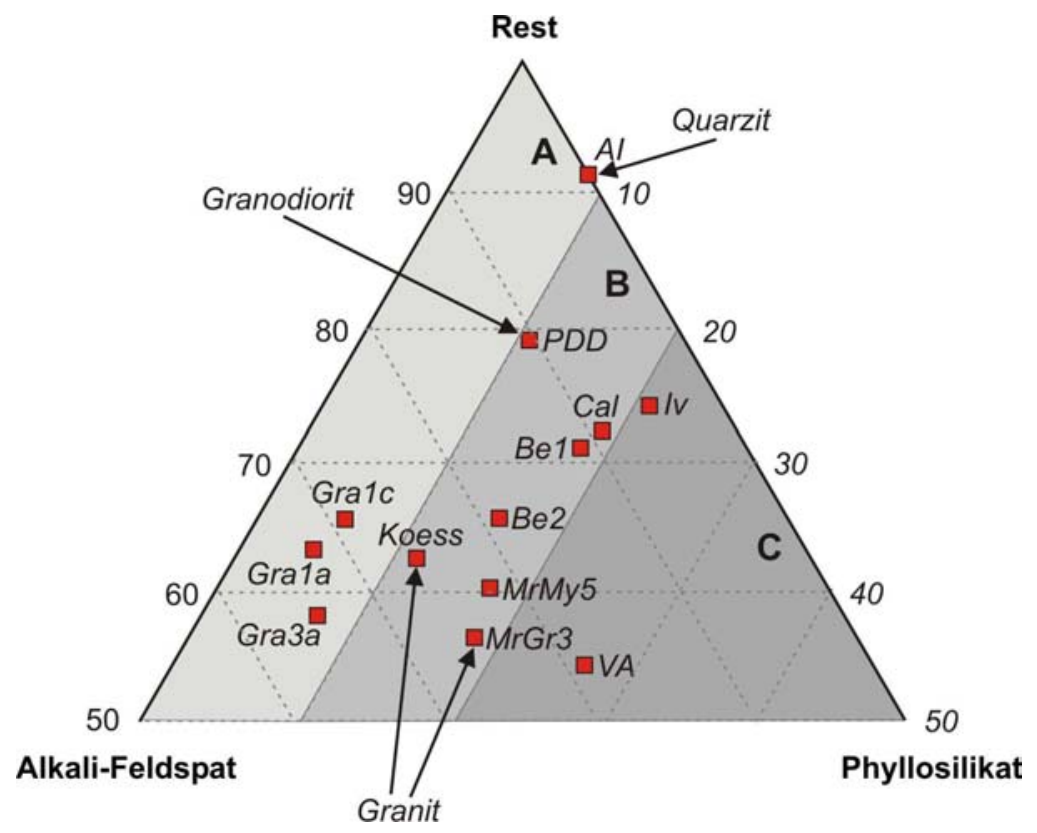

Abb.5-3: Modifiziertes Streckeisen-Diagramm. Dargestellt sind alle phyllosilikathaltigen Gesteine. Der Datenpunkt Iv steht stellvertretend für drei Proben einer Serie. Die Gruppen A, B, C beinhalten Proben mit Phyllosilikatgehalten <10 Vol.\%, 10-20 Vol.\%, bzw. >20 Vol.\%.

Nach einer Einteilung von Shea \& Kronenberg (1993) sind die Phyllosilikatgehalte der untersuchten Gesteine, mit Ausnahme der Probenserie Iv1/Iv1b/Iv2 sowie der bereits erwähnten Probe VA, als niedrig einzustufen. Die Grenze von den niedrigen zu den hohen Glimmergehalten liegt bei $20 \mathrm{Vol} \%$. Sie basiert auf qualitativen Beobachtungen zur Segregationsneigung von Glimmern in deformierten Gesteinen und dem mechanischen Deformationsverhalten im Laborversuch in Abhängigkeit vom Volumenanteil der Glimmer. Gesteine mit niedrigen Gehalten weisen eine geringere Tendenz zur Bildung penetrativer Lagen und ein mehr sprödes Bruchverhalten auf. In Erweiterung der von Shea \& Kronenberg (1993) getroffenen Einstufung wurden folgende Gruppen der phyllosilikathaltigen Gesteine gebildet (Abb.5-3): 


\section{$\underline{\text { 5. Gesteinsmaterial }}$}

Gruppe A - Gesteine mit sehr niedrigen Phyllosilikatgehalten ( $<10$ Vol.\%)

Gruppe B - Gesteine mit niedrigen Phyllosilikatgehalten (10-20 Vol.\%)

Gruppe C - Gesteine mit hohen Phyllosilikatgehalten (>20 Vol.\%)

Die Daten sind inklusive der Gruppeneinteilung in Tab.5-5/Kap.5.11 aufgeführt.

\subsection{Chemische Klassifizierung}

Die große Variationsbreite der mineralogischen Zusammensetzung spiegelt sich auch in der chemischen Zusammensetzung wieder (Daten aller Hauptelementzusammensetzungen in Anhang I.1). Die $\mathrm{SiO}_{2}$-Gehalte schwanken zwischen 0,4 Gew.\% für den Marmor und 91,4 Gew.\% für den Quarzit und variieren prinzipiell analog zum Volumenanteil der Minerale mit hohen $\mathrm{SiO}_{2}$-Gehalten (z.B. Quarz, Feldspäte) bzw. niedrigen $\mathrm{SiO}_{2}$-Gehalten (z.B. Hornblende, Pyroxen, Olivin). Nach der Einteilung gemäß der $\mathrm{SiO}_{2}$-Anteile werden zwei Proben den ultrabasischen, zwei Proben den basischen, zwei Proben den intermediären und 14 Proben den sauren Gesteinen zugerechnet. Der Marmor (0,4 Gew.\% $\left.\mathrm{SiO}_{2}\right)$ und der Quarzit (91,4 Gew.\% $\mathrm{SiO}_{2}$ ) liegen im Harker-Diagramm weit außerhalb des angedeuteten magmatischen Trends. Deutlich erkennbar gruppieren die nach Streckeisen $(1974,1980)$ als granitisch bzw. granodioritisch eingestuften Proben in dem für krustendominierter Derivate magmatischer Gesteine typischen sauren bis intermediären Bereich (Abb.5-4). Mit einem $\mathrm{SiO}_{2}-\mathrm{Gehalt}$ von 60 Gew.\% ist der Granodiorit der basischste Vertreter dieser beiden Gruppen. Der Peridotit kann nach seiner Hauptelementkomposition als Standard-Beispiel für einen Spinell-Lherzolit (Maaloe \& Aoki 1977) eingeordnet werden. Etwas ungewöhnlich dagegen sind die hohen $\mathrm{Na}_{2} \mathrm{O}+\mathrm{K}_{2} \mathrm{O}$-Werte des Meta-Gabbros $(\mathrm{Mg} 2)$, insbesondere gegenüber dem herkunftsgleichen Mglb. Betrachtet man den Mineralbestand dieser Gesteine, so könnte ein erhöhter Volumenanteil der ferro-pargasitischen bis magnesio-hastingsitischen Hornblende in der Probe MG2 für diesen Trend verantwortlich sein. Dieser Verdacht lässt sich allerdings nicht endgültig verifizieren, da für die beiden Proben dieser Serie keine separate Mineralquantifizierung durchgeführt wurde. 


\section{$\underline{\text { 5. Gesteinsmaterial }}$}

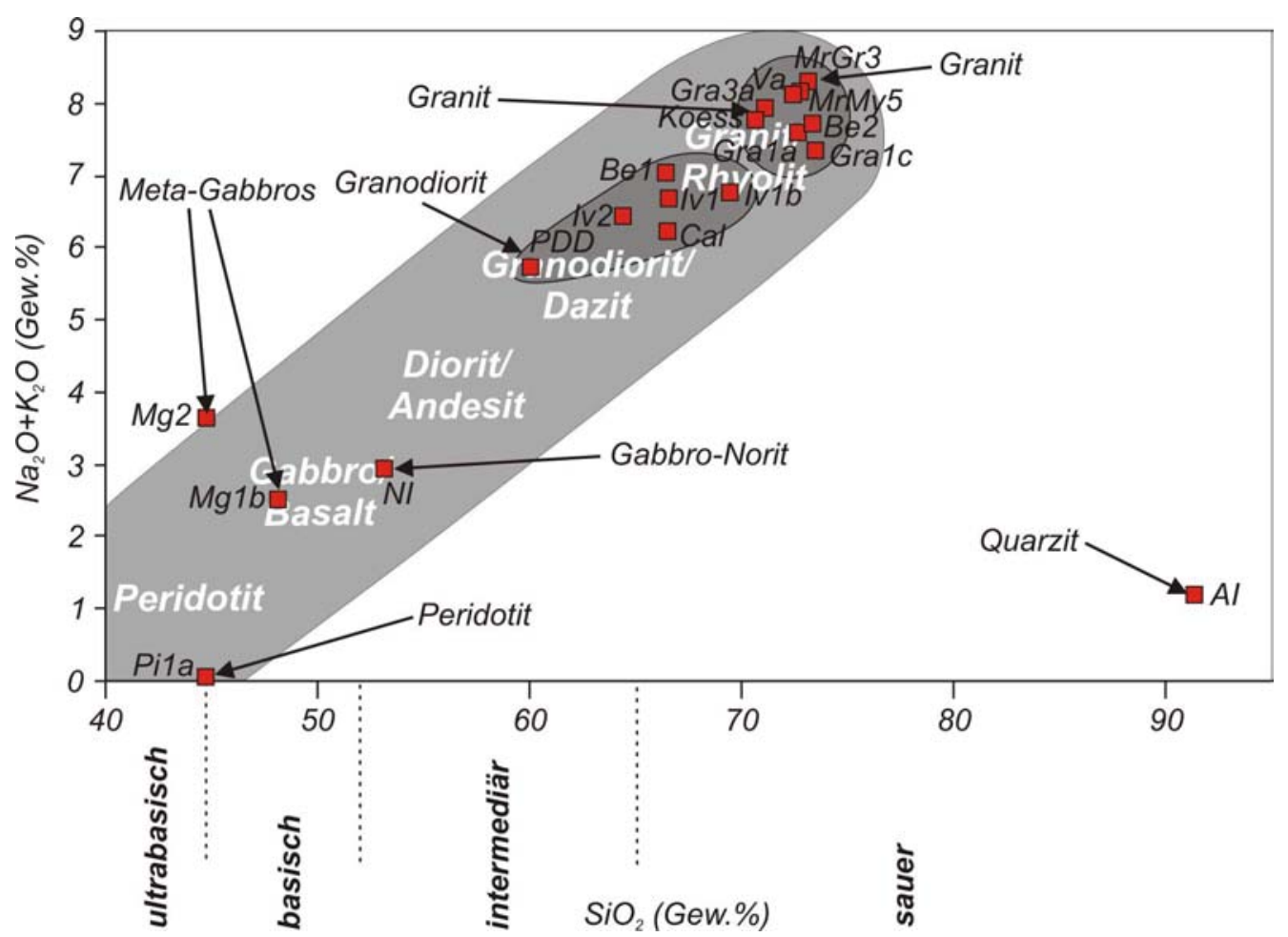

Abb.5-4: $\mathrm{Na}_{2} \mathrm{O}+\mathrm{K}_{2} \mathrm{O}-\mathrm{SiO}_{2}$-Diagramm; Lage der Proben ist mit roten Quadraten gekennzeichnet, die Proben sind mit Kürzeln benannt; die Magmatite sowie die Meta-Gabbros und der Quarzit sind einzeln hervorgehoben; die dunkelgrauen Bereiche bezeichnen die Gruppen granitischer und granodioritischer Zusammensetzung nach Streckeisen-Diagramm; der graue Bereich zeigt die Entwicklung der $\mathrm{Na}_{2} \mathrm{O}+\mathrm{K}_{2} \mathrm{O}$ zu SiO${ }_{2}$-Verhältnisse gemäss einer magmatischen Fraktionierung und deren Variationsbreite; Lage einiger Hauptgesteinstypen sind gekennzeichnet (verändert aus Hall (1996)).

Die aus den Hauptelementoxiden der Gesteine berechneten mittleren Atomgewichte bewegen sich zwischen 20,03 für den Kalzit-Marmor (Rek) und 22,11 für den Meta-Gabbro (MG2). Die Werte sind im Anhang I.1 zusammengefasst.

\subsection{Verknüpfung und Zusammenstellung mineralogischer und chemischer Daten}

Tab.5-1: Matrixdichte $\left(\rho_{M}\right)$, mittlere Atommasse $\left(M_{G}\right)$, Kationenpackungsindex $\left(k_{G}\right)$ und mineralogische Zusammensetzung

\begin{tabular}{|c|c|c|c|c|c|}
\hline & Probe & $\begin{array}{c}\rho_{\mathrm{M}} \\
\left(\mathrm{g} / \mathrm{cm}^{3}\right)\end{array}$ & $M_{G}$ & $\begin{array}{c}\mathbf{k}_{G} \\
\left(\mathrm{~mol} / \mathrm{cm}^{3}\right)^{-2}\end{array}$ & Mineralogische Zus. (Vol.\%) \\
\hline \multirow{6}{*}{ 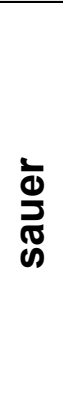 } & Quarzit (Al) & 2,69 & 20,31 & 4,50 & Qz87;Ms9;Dum4 \\
\hline & $\begin{array}{c}\text { granitischer Gneis } \\
(\mathrm{Be} 2)\end{array}$ & 2,67 & 20,81 & 4,70 & Qz38;Kfs19;Pl28;Ms7;Bt7;Chl1 \\
\hline & Granit (MrGr3) & 2,67 & 20,86 & 4,70 & Qz33;Kfs25;Pl24;Ms15;Bt3 \\
\hline & $\begin{array}{c}\text { granitischer } \\
\text { Protomylonit (MrMy5) }\end{array}$ & 2,67 & 20,87 & 4,69 & Qz36;Kfs22;PI25;Ms10;Bt6;Chl1 \\
\hline & $\begin{array}{c}\text { granitischer } \\
\text { Orthogneis (VA) }\end{array}$ & 2,71 & 20,94 & 4,64 & Qz34;Kfs19;Pl21;Ms22;Chl4 \\
\hline & $\begin{array}{c}\text { granitischer Gneis } \\
\text { (Gra1a) }\end{array}$ & 2,71 & 20,45 & 4,67 & Qz33;Kfs32;Pl28;Bt4;Gt2 \\
\hline
\end{tabular}




\section{$\underline{\text { 5. Gesteinsmaterial }}$}

\begin{tabular}{|c|c|c|c|c|c|}
\hline & Probe & $\begin{array}{c}\rho_{M} \\
\left(\mathrm{~g} / \mathrm{cm}^{3}\right)\end{array}$ & $M_{G}$ & $\begin{array}{c}\mathbf{k}_{\mathrm{G}} \\
\left(\mathrm{mol} / \mathrm{cm}^{3}\right)^{-2}\end{array}$ & Mineralogische Zus. (Vol.\%) \\
\hline & $\begin{array}{c}\text { granitischer Gneis } \\
\text { (Gra1c) }\end{array}$ & 2,69 & 20,55 & 4,75 & Qz34;Kfs28;PI30;Bt5;Gt3 \\
\hline & $\begin{array}{c}\text { granitischer } \\
\text { Ultramylonit (Gra3a) }\end{array}$ & 2,69 & 20,59 & 4,76 & Qz30;Kfs34;PI26;Bt7;Gt3 \\
\hline & Granit (Koess) & 2,68 & 20,77 & 4,71 & Qz33;Kfs26;Pl30;Bt10;Chl1 \\
\hline & $\begin{array}{l}\text { granodioritischer } \\
\text { Protomylonit (Iv1) }\end{array}$ & 2,79 & 21,25 & 4,79 & Qz36;Kfs5;PI28;Ms15;Chl6;Gt4;Ep6 \\
\hline & $\begin{array}{l}\text { granodioritischer } \\
\text { Mylonit (Iv1b) }\end{array}$ & 2,74 & 21,26 & 4,79 & Qz36;Kfs5;Pl28;Ms15;Chl6;Gt4;Ep6 \\
\hline & $\begin{array}{l}\text { granodioritischer } \\
\text { Ultramylonit (Iv2) }\end{array}$ & 2,75 & 21,39 & 4,79 & Qz36;Kfs5;Pl28;Ms15;Chl6;Gt4;Ep6 \\
\hline & $\begin{array}{c}\text { granodioritischer } \\
\text { Gneis (Be1) }\end{array}$ & 2,72 & 21,08 & 4,73 & Qz32;Kfs11;Pl38;Ms4;Bt13;Chl1 \\
\hline & $\begin{array}{l}\text { granodioritischer } \\
\text { Paragneis (Cal) }\end{array}$ & 2,73 & 20,74 & 4,80 & Qz34;Kfs9;PI38;Bt18;Chl1 \\
\hline \multirow{2}{*}{$-\dot{E}$} & Granodiorit (PDD) & 2,80 & 20,95 & 4,98 & Qz19;Kfs10;PI51;Bt11;Cpx7;Mag2 \\
\hline & Gabbro-Norit (NI) & 2,92 & 20,69 & 5,28 & Qz1;PI74;Cpx13;Opx12 \\
\hline \multirow{2}{*}{ فـ } & Meta-Gabbro (Mg1b) & 3,06 & 21,77 & 5,59 & $\mathrm{PI} 35 ; \mathrm{Hbl44;Cpx16;Opx5}$ \\
\hline & Meta-Gabbro (Mg2) & 3,09 & 22,11 & 5,59 & $\mathrm{Pl} 35 ; \mathrm{Hbl} 44 ; \mathrm{Cpx} 16 ; \mathrm{Opx5}$ \\
\hline \multirow{2}{*}{ ف } & Peridotit (Pi1a) & 3,34 & 20,28 & 6,61 & Cpx13;Opx26;Ol59;Spi2 \\
\hline & Marmor (Rek) & 2,72 & 20,03 & 5,44 & Cc98;Häm2 \\
\hline
\end{tabular}

Daten sind nach dem $\mathrm{SiO}_{2}$-Gehalt sortiert, Probenserien (Iv1-IV2;Gra1a-Gra3a;MG1b-MG2) wurden zusammengelassen und nach ihrem mittleren $\mathrm{SiO}_{2}$-Gehalt eingeordnet; i.m. - intermediär; $b$. basisch; u.b. - ultrabasisch; Qz - Quarz; Kfs - Kalifeldspat; Pl - Plagioklas; Ms - Muskovit; Bt - Biotit; Chl - Chlorit; Gt - Granat; Dum - Dumortierit; Ep - Epidot; Cpx - Klinopyroxen; Opx - Orthopyroxen; OI - Olivin; Mag - Magnetit; Spi - Spinell; Häm - Hämatit; Cc - Kalzit.

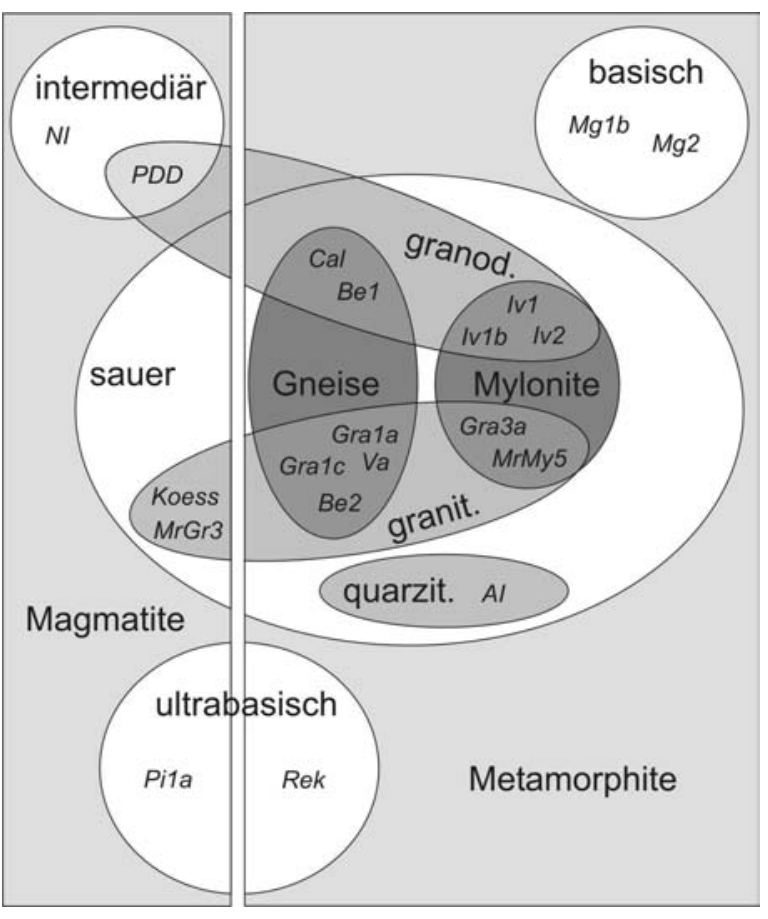

Aus $\boldsymbol{A} \boldsymbol{b} \boldsymbol{b} .5$-5 können die Eigenschaften der Gesteinsproben und ihre Gruppierung abgelesen werden. Der $\mathrm{SiO}_{2}$-Gehalt (sauer, intermediär, basisch, ultrabasisch), die mineralogische Zusammensetzung (z.B. quarzitisch, granodioritisch) und die Gefügetypisierung (Gneis, Mylonit) sind berücksichtigt. Zusätzlich lässt sich ersehen, welche Gesteine zu den Magmatiten und welche zu den Metamorphiten zählen.

Abb.5-5: Diagramm zur Einordnung der Gesteine nach der Mineralogie, Chemie sowie nach Gefügemerkmalen. Al-Quarzit. Be2, Gra1a, Gra1c, VA - granitische Gneise. Cal, Be1granodioritische Gneise. Gra3a, MrMy5 - granitischer Mylonit. Iv1, Iv1b, Iv2 - granodioritische Mylonite. Koess, MrGr3 - Granite. NI - Gabbro-Norit. PDD - Granodiorit. Mg1b, Mg2 - Meta-Gabbros. Pi1a - Peridotit. Rek - Kalzit-Marmor. 


\subsection{Makroskopische Gefügetypisierung}

Der Schwerpunkt dieser Arbeit liegt auf der Gefügeabhängigkeit und der damit verbundenen Anisotropie der gesteinstechnischen Parameter. Aus diesem Grund kommt der Charakterisierung der Proben nach dem Gefügeinventar eine bedeutsame Rolle zu. Zunächst werden die Gesteine nach ihrem makroskopischen Erscheinungsbild hinsichtlich ihrer Gefügeanisotropie katalogisiert. Um eine Einstufung möglichst objektiv vornehmen und die Ergebnisse auf andere Gesteine transferieren zu können, ist es unerlässlich, Gefüge-Kriterien zu definieren. Ohne Frage erfordert die akkurate Einordnung nach dem Makrogefüge eine eingehende Betrachtung an drei möglichst senkrecht zu den Gefügeachsen ausgerichteten und geschliffenen Flächen. Am bruchrauen Handstück können Anschnittseffekte zu einer falschen Einschätzung verleiten. Auch der Einfluss von farblichen Kontrasten innerhalb des Mineralbestands ist nicht zu unterschätzen. So führen Schwarz-Weiß-Kontraste (z.B. dunkle Hornblende zu weißem Plagioklas) zu einer Überschätzung der Gefügeanisotropie, wohingegen das menschliche Auge einheitlich aus dunklen Mineralen zusammengesetzte Gesteine bezüglich ihrer Anisotropie tendenziell unterschätzt.

(a)

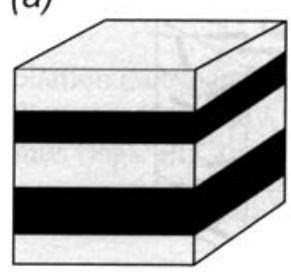

(d)

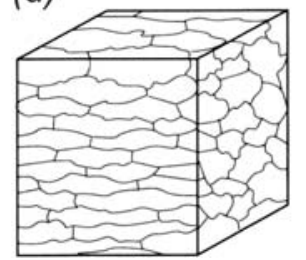

(b)

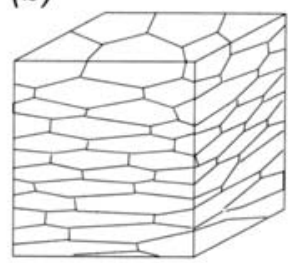

(e)

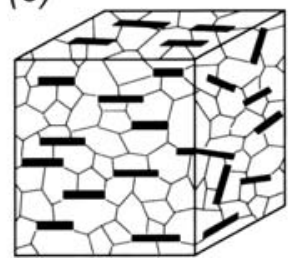

(c)

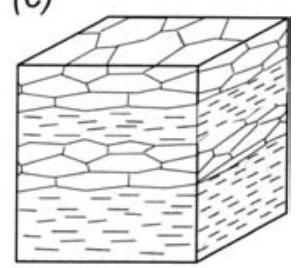

(f)

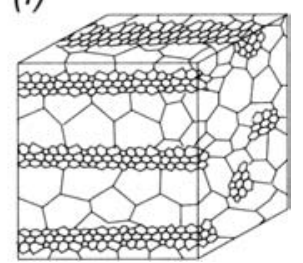

Abb.5-6: Schemata zu Gefügeelementen, die eine Foliation (a-c) bzw. eine Lineation (d-f) definieren. (a) Foliation durch lagigen Materialwechsel. (b) Foliation durch Kornformregelung. (c) Foliation durch Kombination von lagigem Materialwechsel, Kornformregelung xenomorpher Kristalle und Einregelung hypideomorpher bis ideomorpher Minerale. (d) Lineation durch Kornformregelung. (e) Lineation durch Einregelung hypideomorpher bis ideomorpher Minerale. (f) Lineation durch langgestreckte Mineralaggregate. Zusammengestellt aus Passchier \& Trouw (1998).

Nach Passchier und Trouw (1998) können Foliation und Lineation in einem Gestein auf die räumliche Anordnung unterschiedlicher Gefügeelemente zurückgeführt werden. Die Ausprägung der Foliation und der Lineation ist definiert durch die Intensität des Materialwechsels und den Grad der Kornformregelungen der gesteinsbildenden Minerale (Abb.5-6). Letzteres beinhaltet sowohl die bevorzugte Kornformorientierung xenomorph (z.B. Kalzit im Marmor) ausgebildeter, als auch die Einregelung von formanisotropen, ideomorph 


\section{Gesteinsmaterial}

bis hypideomorph (z.B. Glimmer in Meta-Granitoiden) ausgebildeten Mineralen. Es wird diesbezüglich nicht zwischen Mineralen und Mineralaggregaten unterschieden, was aufgrund des makroskopischen Befundes auch meist nicht möglich ist. Beide Elemente, Materialwechsel und Kornformregelung, treten nicht selten in Kombination auf, was den Eindruck der Anisotropie ggf. verstärken kann. Ist keine Foliation oder Lineation erkennbar, wird das Gesteinsgefüge als regellos/richtungslos körnig bezeichnet. Des Weiteren werden die Gesteine danach eingeordnet, ob die Foliation oder die Lineation den makroskopischen Eindruck bestimmen. Hier wird zwischen S-Typ (Foliation dominiert), S-L-Typ (Foliation und Linear gleichermaßen deutlich) und L-Typ (Lineation dominiert) unterschieden.

\subsubsection{Gefügebilder und Gruppierung}

Die folgenden Gefügebilder zeigen die Gesteine im y-z-Schnitt und im x-z-Schnitt, also senkrecht zur Foliation und zum Linear bzw. senkrecht zur Foliation und parallel zum Linear. Die Gesteine sind nach ihrer späteren Gruppenzugehörigkeit und innerhalb der Gruppen nach zunehmender Gefügeanisotropie geordnet. Rechts neben den Photos befinden sich der Probenname, das Kürzel, ggf. der Handelsname sowie die Index-Gefügeeigenschaften, die zur späteren Gruppenzuordnung geführt haben. Außerdem ist gekennzeichnet, ob es sich um einen S-, S-L- oder L-Typ handelt.

\section{Gruppe I: Isotrope Gesteine}
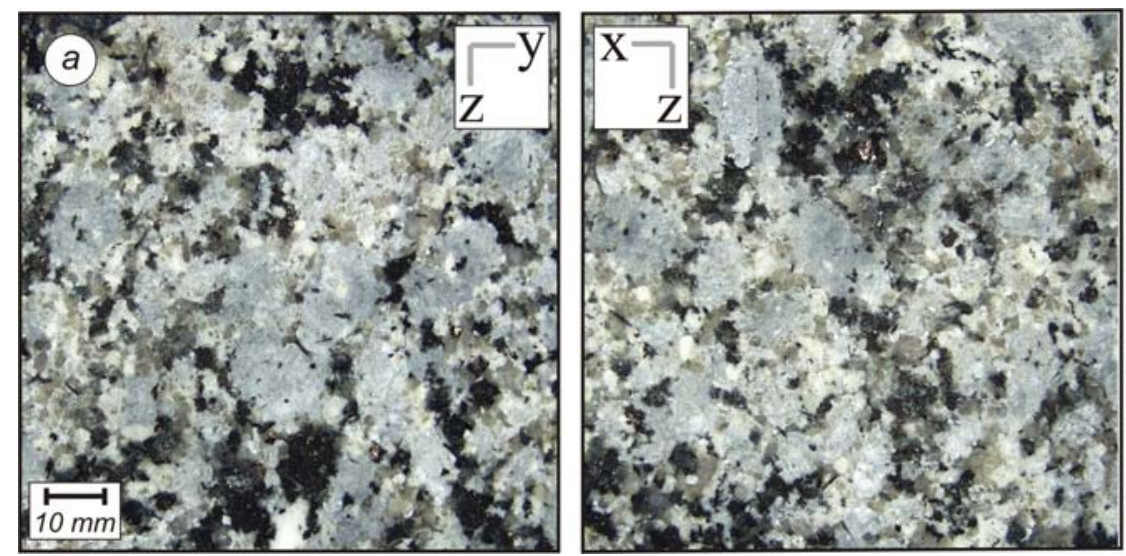

Granit (Koess)

Handelsname: Kösseine

Granit

- richtungslos körnig 

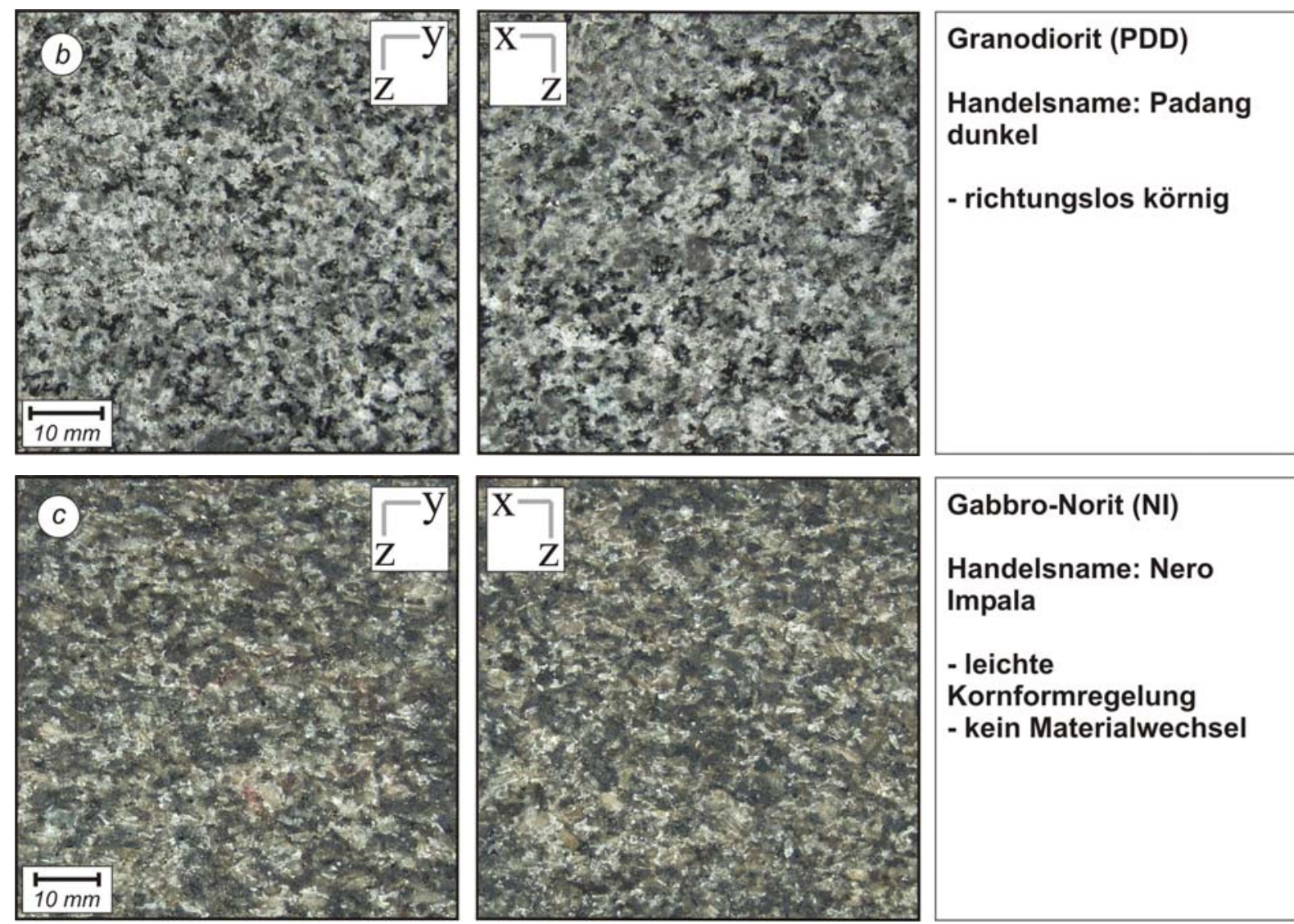

\section{Gabbro-Norit (NI) \\ Handelsname: Nero Impala \\ - leichte \\ Kornformregelung \\ - kein Materialwechsel}
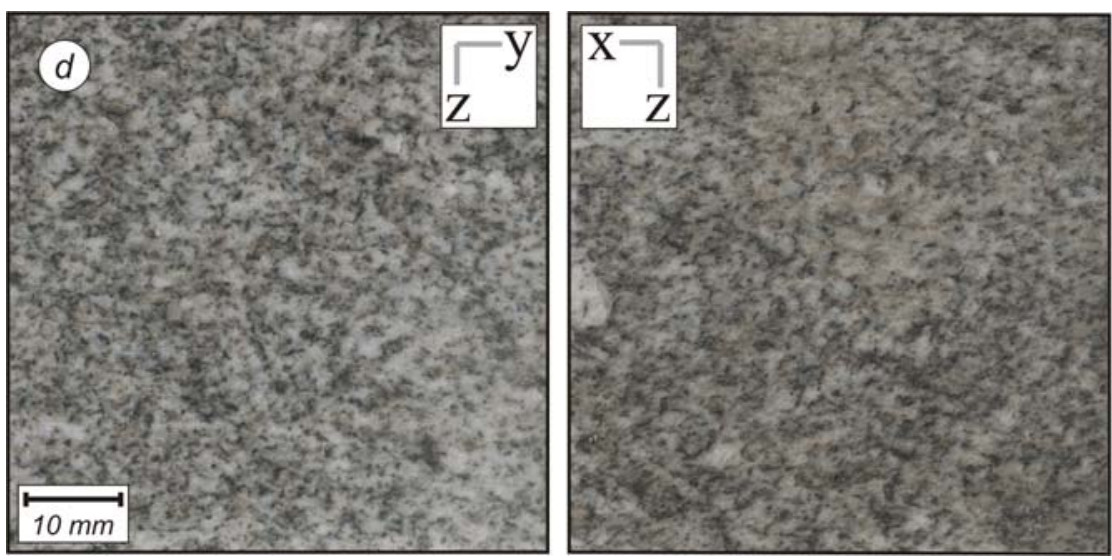

Granit (MrGr3)

Handelsname: ---

- leichte

Kornformregelung

- kein Materialwechsel

Abb.5-7: Gruppe I: Isotrope Gesteine

\section{Gruppe II: Anisotrope Gesteine}
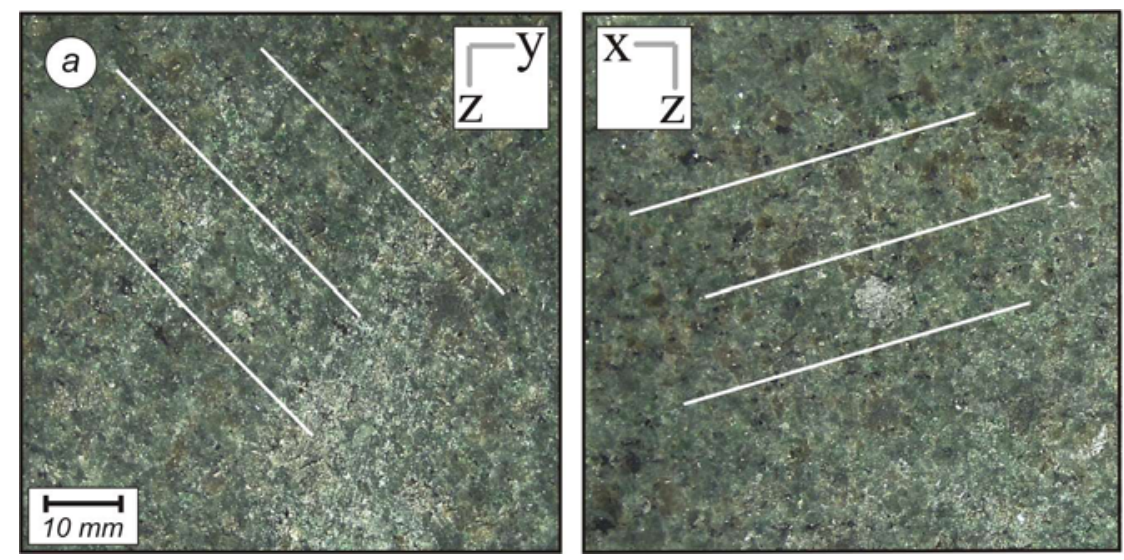

\section{Peridotit (Pi1a) \\ Handelsname: --- \\ - deutliche \\ Kornformregelung \\ - sehr schwacher \\ Materialwechsel \\ S-L-Typ \\ Lage der Foliation \\ angedeutet}




\section{$\underline{\text { 5. Gesteinsmaterial }}$}
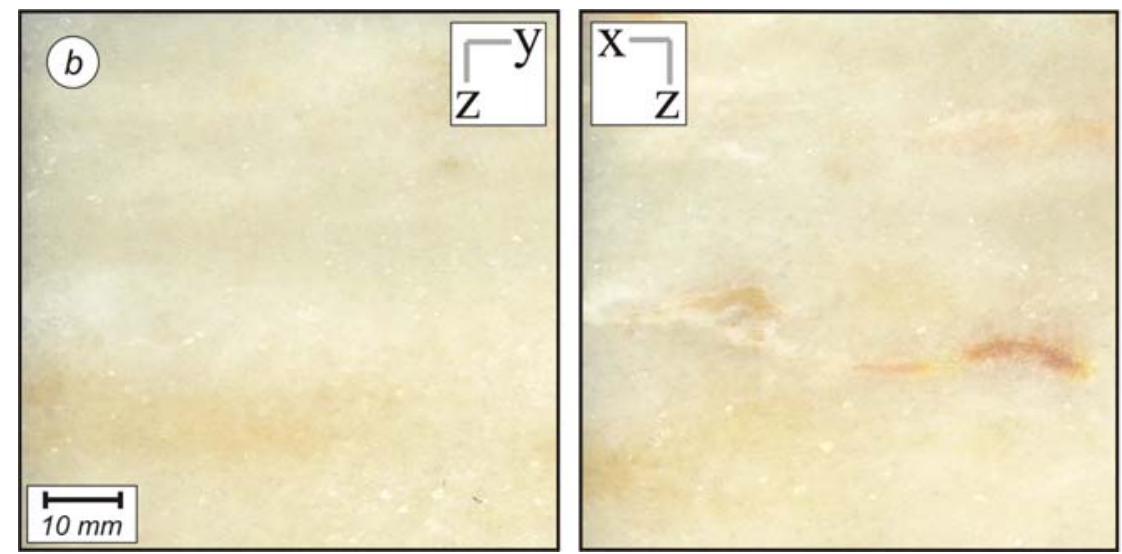

Kalzit-Marmor (Rek)

Handelsname: Rosa

Estremoz

- deutliche

Kornformregelung

- schwacher

Materialwechsel

S-Typ
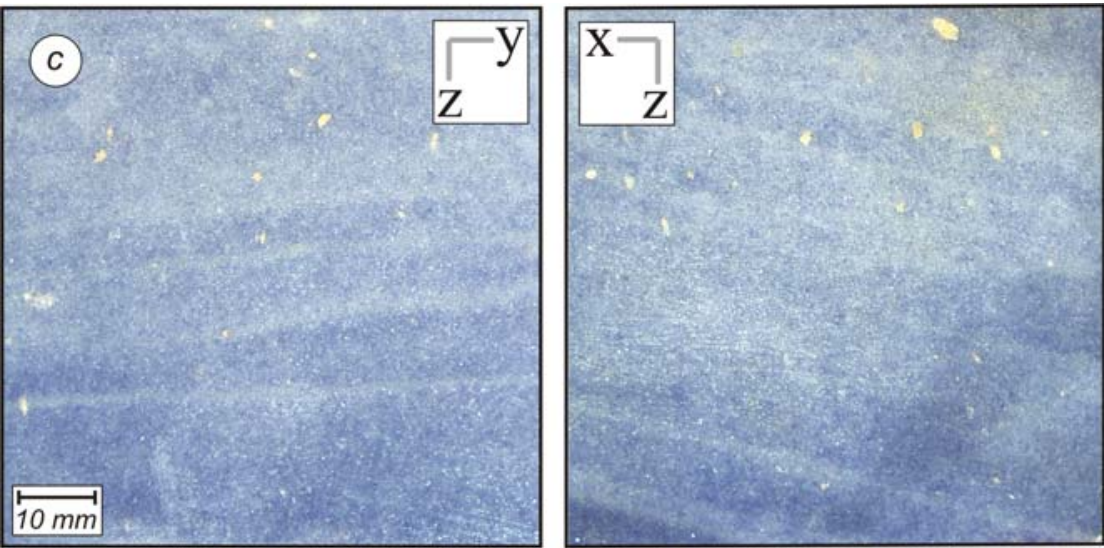

Quarzit (AI)

Handelsname: Azul Imperial

- schwache

Kornformregelung

- schwacher bis

deutlicher

Materialwechsel

S-Typ
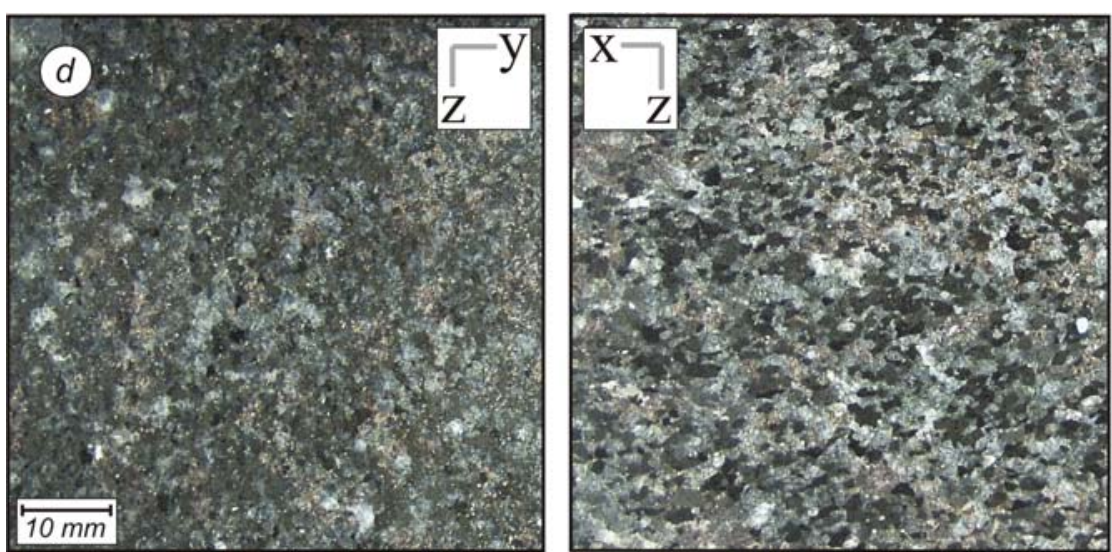

Meta-Gabbro (Mg1b)

Handelsname: ---

- deutliche

Kornformregelung

- sehr schwacher

Materialwechsel

L-Typ
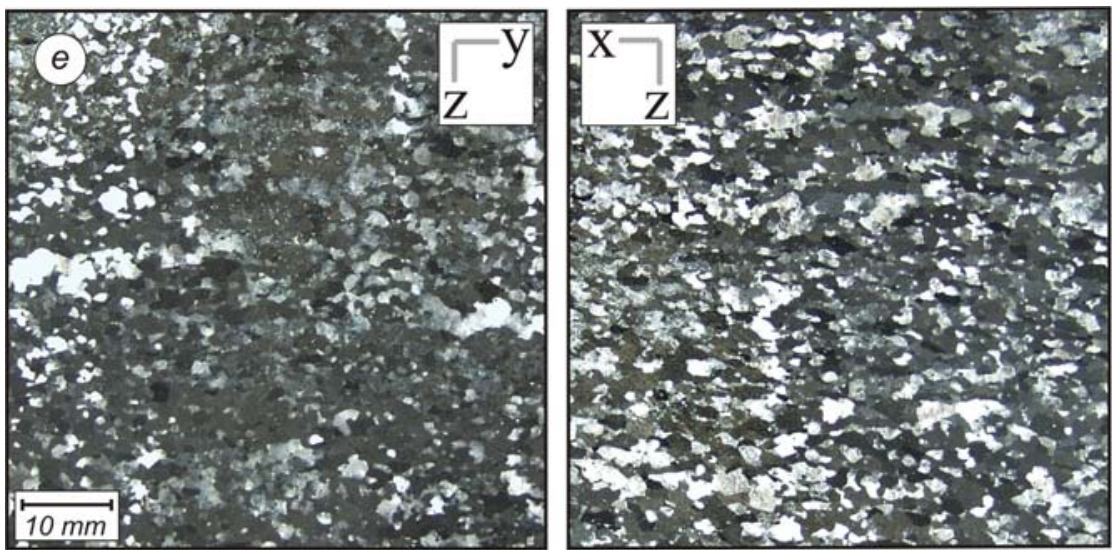

Meta-Gabbro (Mg2)

Handelsname: ---

- deutliche

Kornformregelung

- schwacher

Materialwechsel

S-L-Typ 

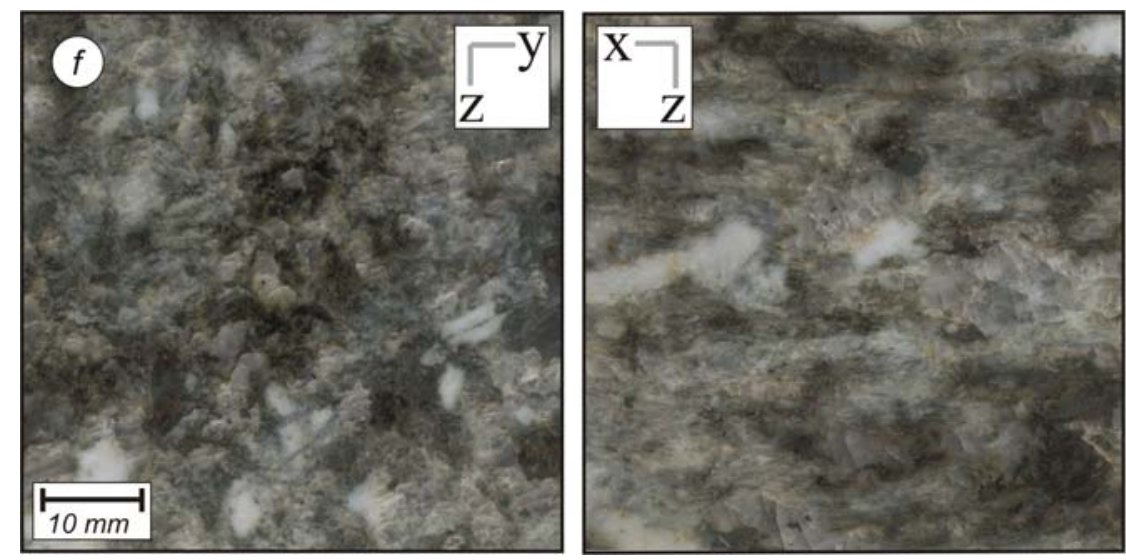

granodiorit. Protomylonit

(Iv1)

Handelsname: ---

- deutliche

Kornformregelung

- schwacher

Materialwechsel

L-Typ
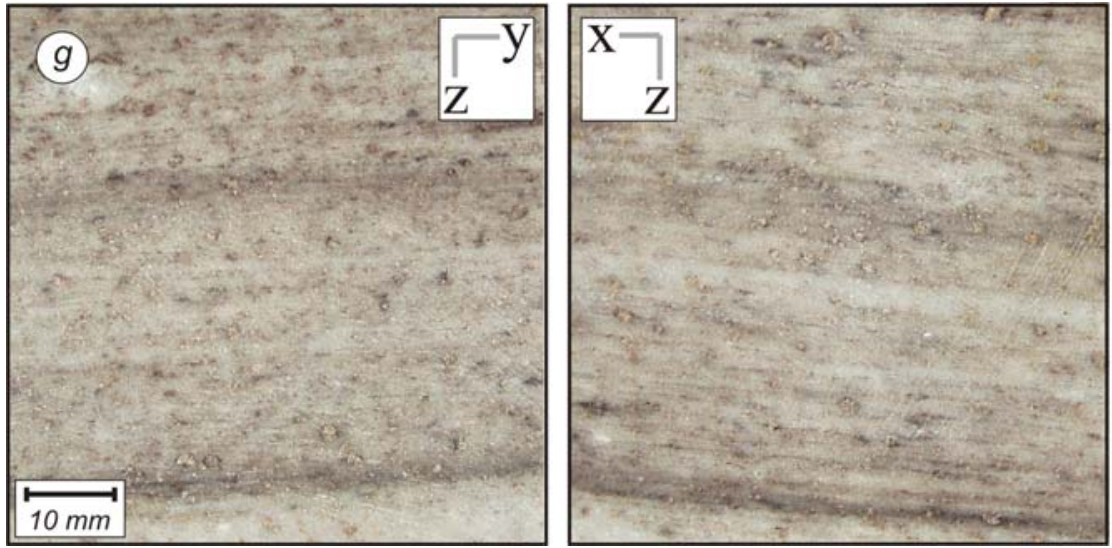

granit. Gneis (Gra1a)

Handelsname: ---

- schwache

Kornformregelung

- deutlicher

Materialwechsel

S-Typ

Abb.5-8: Gruppe II: Anisotrope Gesteine

\section{Gruppe III: Ausgeprägt anisotrope Gesteine}
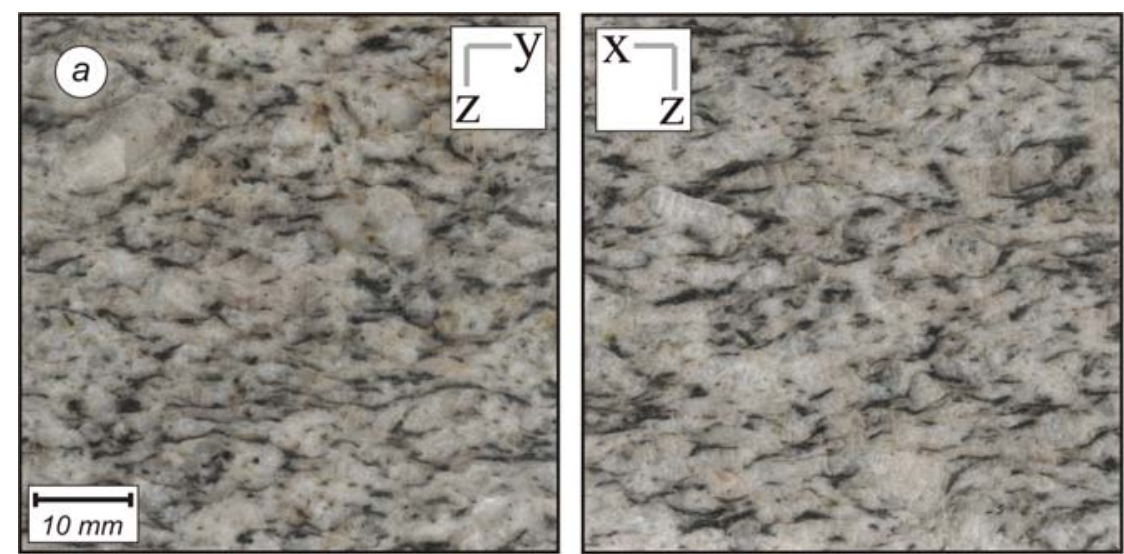

granit. Protomylonit

(MrMy5)

Handelsname : ---

- starke

Kornformregelung

- starker Materialwechsel

S-Typ
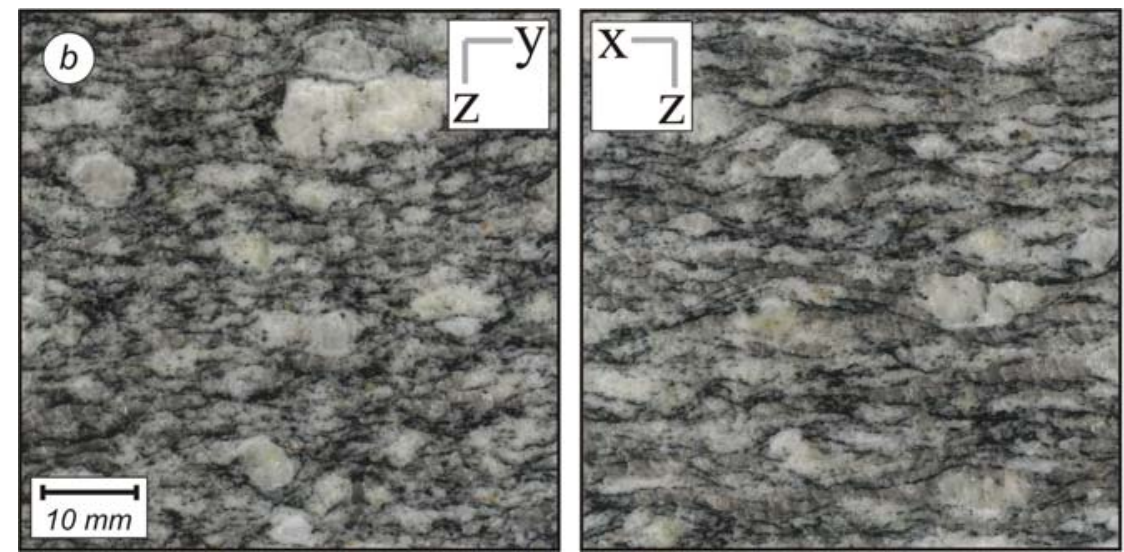

granod. Gneis (Be1)

Handelsname: Serizzo

Monte Rosa

- starke

Kornformregelung

- starker Materialwechsel

S-Typ 


\section{Gesteinsmaterial}
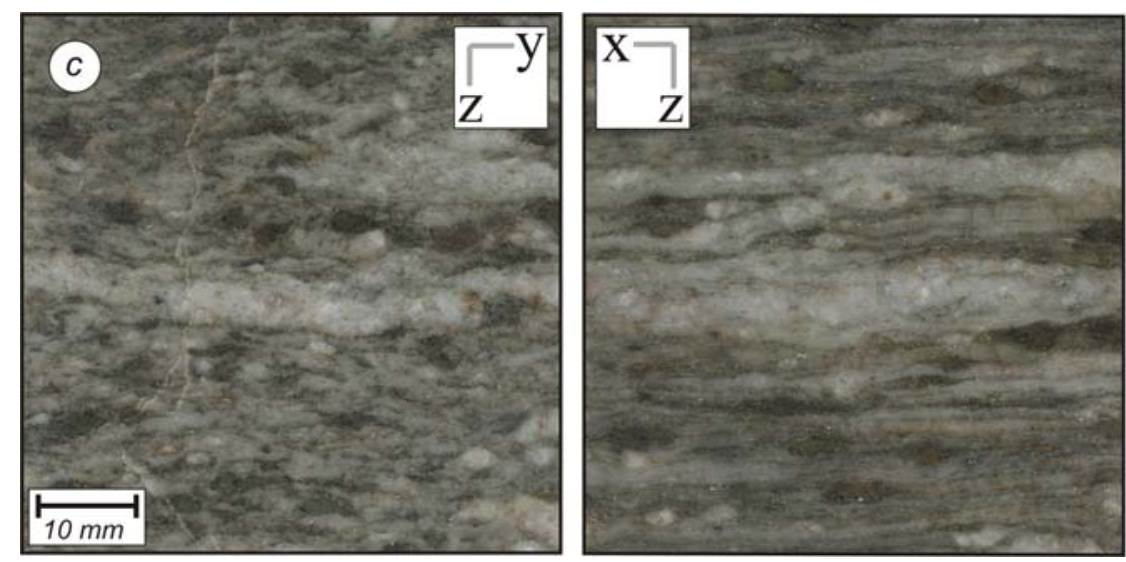

granod. Mylonit (Iv1b)

Handelsname: ---

- starke

Kornformregelung

- starker Materialwechsel

\section{S-L-Typ}
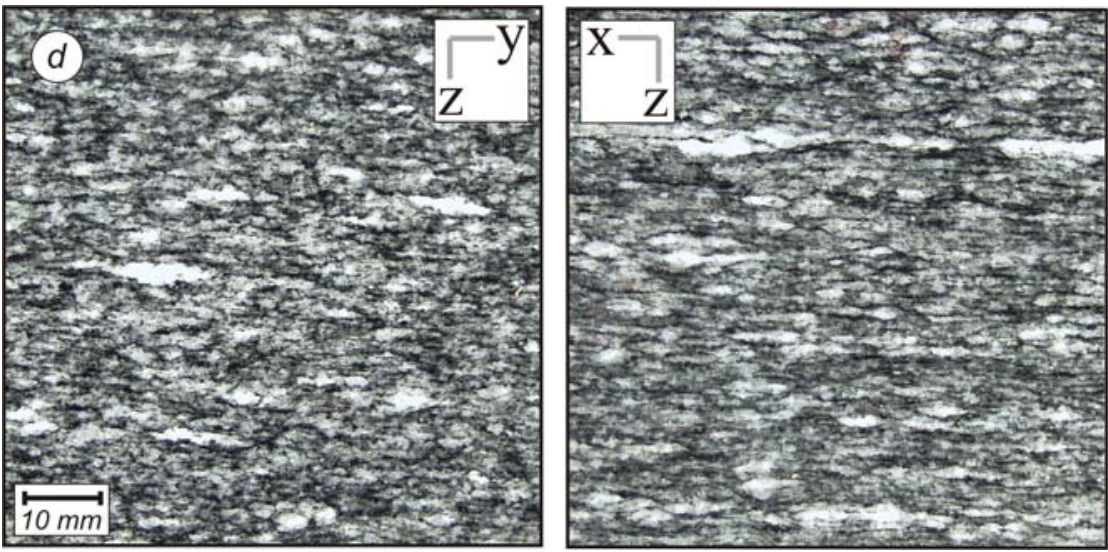

granod. Paragneis (Cal)

Handelsname: Calanca

- sehr starke

Kornformregelung

- starker Materialwechsel

S-L-Typ
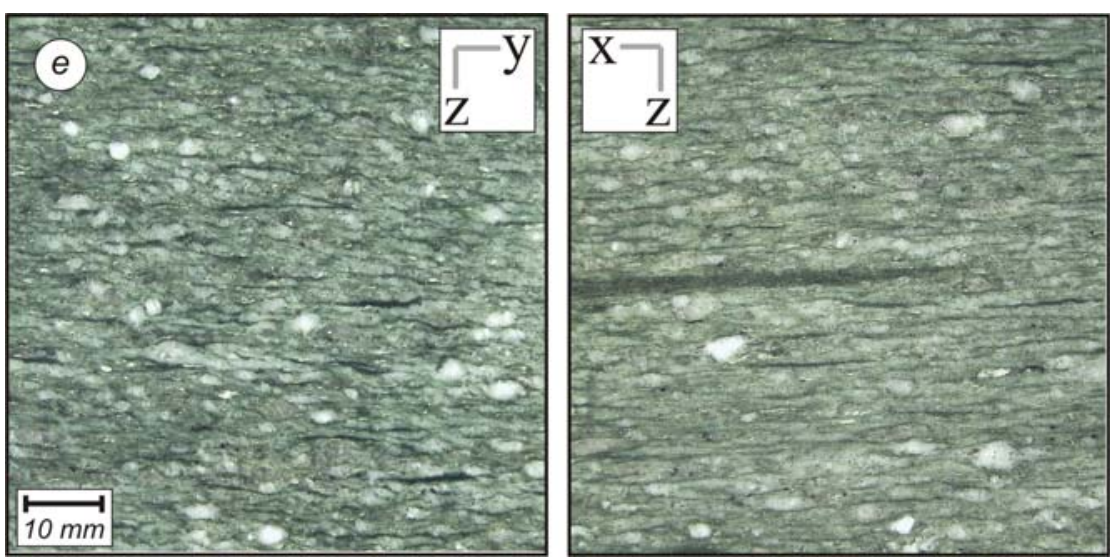

granit. Orthogneis (VA)

Handelsname: Verde

Andeer

- sehr starke

Kornformregelung

- starker Materialwechsel

S-Typ
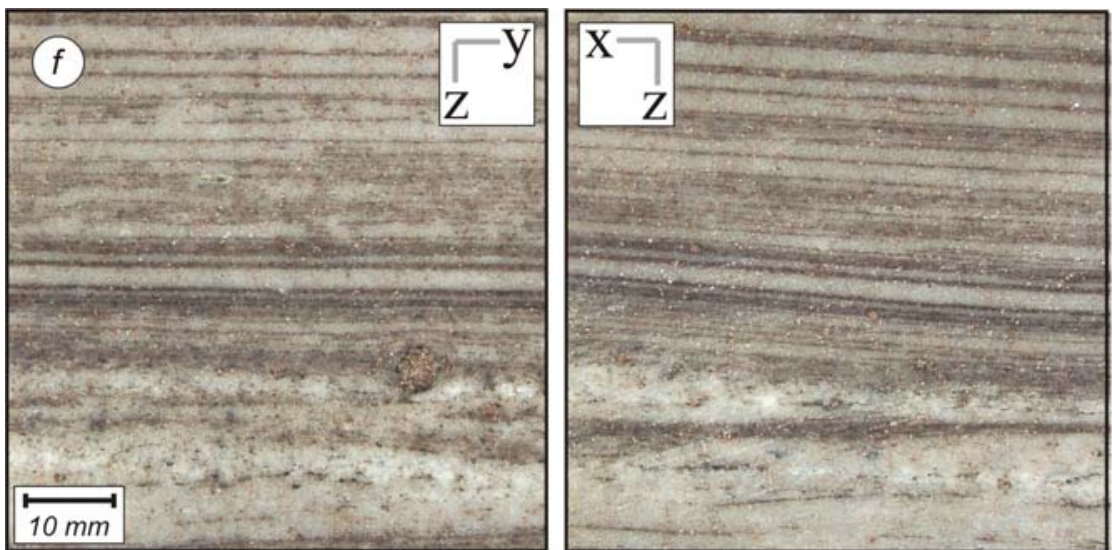

granit. Gneis (Gra1c)

Handelsname: -.-

- deutliche

Kornformregelung

- starker Materialwechsel

S-Typ 


\section{$\underline{\text { 5. Gesteinsmaterial }}$}
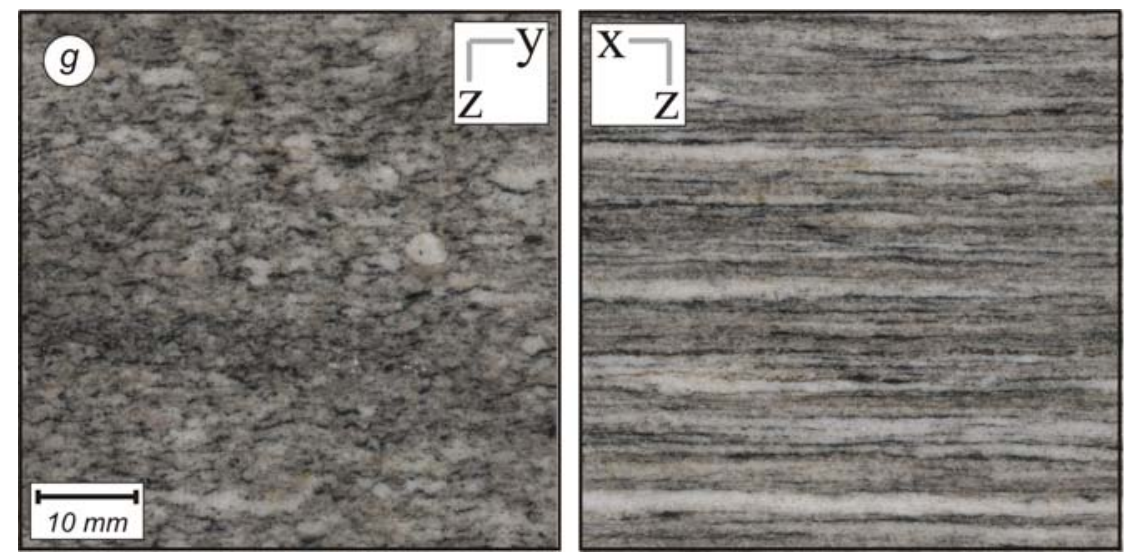

granit. Gneis (Be2)

Handelsname: Serizzo

Monte Rosa

- sehr starke

Kornformregelung

- starker Materialwechsel

S-L-Typ
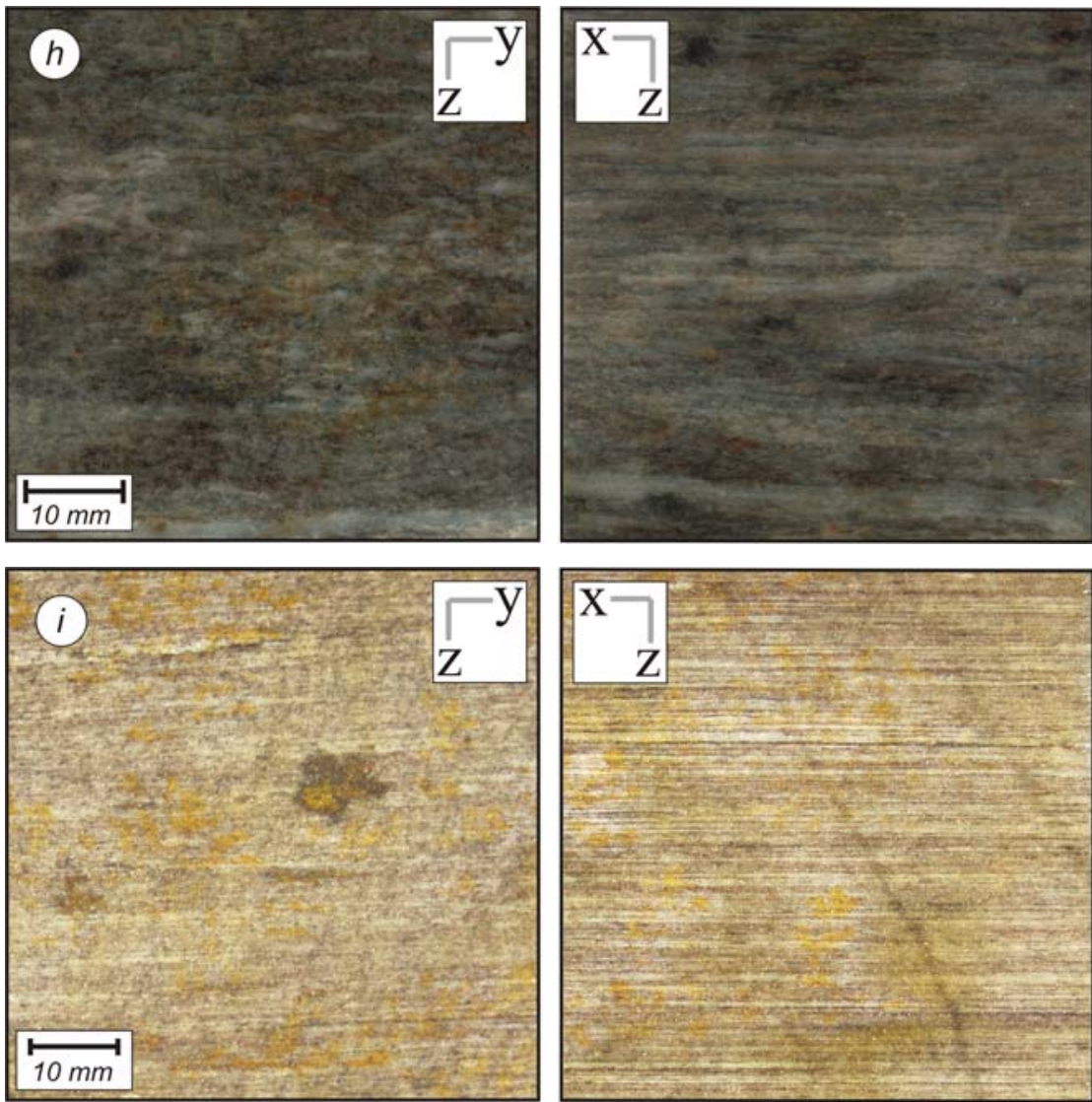

granod. Ultramylonit (Iv2)

Handelsname: ---

- sehr starke

Kornformregelung

- sehr starker

Materialwechsel

S-L-Typ

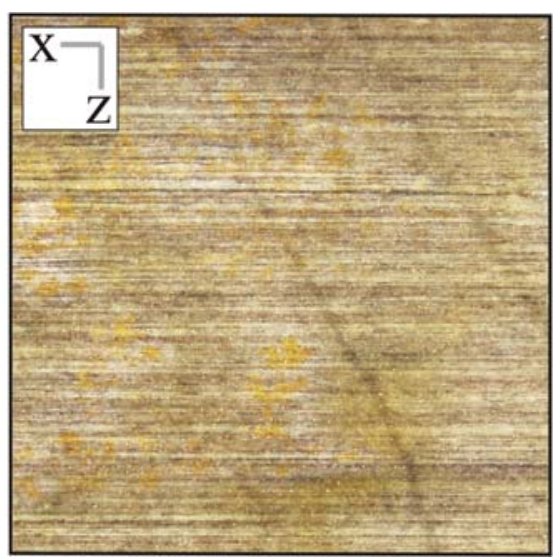

granit. Ultramylonit

(Gra3a)

Handelsname: ---

- sehr starke

Kornformregelung

- sehr starker

Materialwechsel

\section{S-L-Typ}

Abb.5-9: Gruppe III: Ausgeprägt anisotrope Gesteine.

Von richtungslos körnigen Magmatiten bis $\mathrm{zu}$ vollständig dynamisch rekristallisierten

Ultramyloniten decken die untersuchten Proben einen großen Bereich der in der Natur vorkommenden Korngefüge der kristallinen Gesteine ab.

Die gezeigten Gefügebilder führen unter Berücksichtigung der unter Kap.5.5 genannten Definitionen und Überlegungen zu einer Einteilung der untersuchten Gesteine in drei Gruppen (Tab.5-2): 


\section{$\underline{\text { 5. Gesteinsmaterial }}$}

Gruppe I: Isotrope Gesteine (isotrop wird hier im Sinne von quasi-isotrop und nicht im Sinne eines ideal isotropen Körpers verwendet)

Gruppe II: Anisotrope Gesteine

Gruppe III: Ausgeprägt anisotrope Gesteine

Die Gruppe I der isotropen Gesteine zeichnet sich durch richtungslos körnige bis leicht anisotrope Gefüge aus. Eine Foliation oder Lineation ist nicht oder nur sehr schwach ausgeprägt. Ein Materialwechsel kommt nicht vor. Diese Fraktion beinhaltet die Granite Koess und MrGr3 sowie den Granodiorit (PDD) und den Gabbro-Norit (NI) (Abb.5-7) und damit ausschließlich magmatische Gesteine ohne nennenswerte syn- oder postgenetische Deformationsgeschichte.

Tab.5-2: Gruppierung nach der Anisotropie des makroskopischen Gefügeeindrucks.

\begin{tabular}{|c|c|c|c|}
\hline & $\begin{array}{c}\text { Gruppe I - isotrope } \\
\text { Gesteine }\end{array}$ & $\begin{array}{c}\text { Gruppe II - anisotrope } \\
\text { Gesteine }\end{array}$ & $\begin{array}{c}\text { Gruppe III - ausgeprägt } \\
\text { anisotrope Gesteine }\end{array}$ \\
\hline $\begin{array}{c}\text { makrosk. } \\
\text { Gefüge- } \\
\text { merkmale der } \\
\text { Gruppen } \\
\text { (schematische } \\
\text { Beispiele) }\end{array}$ & & 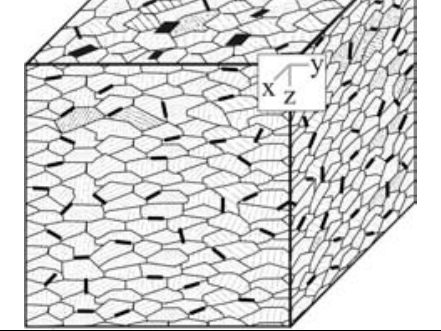 & $6 x^{\prime}$ \\
\hline $\begin{array}{l}\text { Definition der } \\
\text { Gruppen- } \\
\text { merkmale }\end{array}$ & $\begin{array}{l}\text { - Foliation und/oder } \\
\text { Lineation gar nicht oder } \\
\text { sehr schwach ausgeprägt } \\
\text { - Kornformregelung gar } \\
\text { nicht oder schwach } \\
\text { ausgeprägt } \\
\text { - kein Materialwechsel }\end{array}$ & $\begin{array}{l}\text { - Foliation und/oder Linear } \\
\text { deutlich zu erkennen } \\
\text { - Kornformregelung } \\
\text { schwach bis deutlich } \\
\text { ausgeprägt } \\
\text { - Materialwechsel schwach } \\
\text { bis deutlich ausgeprägt }\end{array}$ & $\begin{array}{c}\text { - Foliation und/oder } \\
\text { Linear dominieren das } \\
\text { Erscheinungsbild } \\
\text { - Kornformregelung stark } \\
\text { bis sehr stark ausgeprägt } \\
\text { - Materialwechsel stark } \\
\text { bis sehr stark ausgeprägt }\end{array}$ \\
\hline $\begin{array}{c}\text { zugehörige } \\
\text { Proben }\end{array}$ & $\begin{array}{c}\text { Gruppe I - isotrope } \\
\text { Gesteine }\end{array}$ & $\begin{array}{c}\text { Gruppe II - anisotrope } \\
\text { Gesteine }\end{array}$ & $\begin{array}{l}\text { Gruppe III - ausgeprägt } \\
\text { anisotrope Gesteine }\end{array}$ \\
\hline S-Typ & \multirow{3}{*}{$\begin{array}{c}\text { Granit (Koess) } \\
\text { Granodiorit (PDD) } \\
\text { Gabbro-Norit (NI) } \\
\text { Granit (MrGr3) }\end{array}$} & $\begin{array}{c}\text { Kalzit-Marmor (Rek) } \\
\text { granit. Gneis (Gra1a) } \\
\text { Quarzit (AI) }\end{array}$ & $\begin{array}{c}\text { granit. Protomylonit } \\
\text { (MrMy5) } \\
\text { granit. Gneis (Gra1c) } \\
\text { granod. Gneis (Be1) } \\
\text { granit. Orthogneis (VA) }\end{array}$ \\
\hline S-L-Typ & & $\begin{array}{c}\text { Meta-Gabbro (Mg2) } \\
\text { Peridotit (Pi1a) }\end{array}$ & $\begin{array}{l}\text { granod. Mylonit (Iv1b) } \\
\text { granod. Paragneis (Cal) } \\
\text { granod. Ultramylonit (Iv2) } \\
\text { granit. Ultramylonit } \\
\text { (Gra3a) granit. Gneis } \\
\text { (Be2) }\end{array}$ \\
\hline L-Typ & & $\begin{array}{c}\text { Meta-Gabbro (Mg1b) } \\
\text { granod. Protomylonit (lv1) }\end{array}$ & \\
\hline
\end{tabular}

Die Gruppe II der anisotropen Gesteine wird definiert durch deutlich anisotrope Gefüge, welche durch eine schwache bis deutliche Kornformregelung und/oder einen schwachen bis 


\section{$\underline{\text { 5. Gesteinsmaterial }}$}

deutlichen Materialwechsel bestimmt werden. Darin enthalten sind die Meta-Gabbros (Mg1b/Mg2), der Peridotit (Pi1a), der Kalzit-Marmor (Rek), der Quarzit (AI), der granitische Gneis (Gra1a) und der granodioritische Protomylonit (Iv1) (Abb.5-8), also ein magmatisches Gestein und sechs Metamorphite. Die Proben Grala, AI und Iv1 enthalten stark formanisotrope Phyllosilikate, allerdings mit geringer Vorzugsorientierung, so dass ihr Einfluss auf die makroskopische Gefügeanisotropie verhältnismäßig gering sein sollte. Die restlichen Proben dieser Gruppe sind aus Mineralen mit niedrigerem anisotropem Habitus zusammengesetzt. Die ausschließlich xenomorph ausgebildeten Kristalle weisen eine Kornformregelung auf, die den Gefügeeindruck bestimmt. Grundsätzlich ist der Materialwechsel in dieser Gruppe nur gering ausgeprägt, wobei die Probe Grala eine Ausnahme bildet. Sie weist einen deutlichen Lagenbau auf, welcher sich bei einem Blick mit der Lupe als nicht penetrativ erweist. Die Biotit-Kristalle sind in bestimmten Arealen angereichert, bilden aber keine vernetzten Lagen und sind isoliert. Bezüglich dieser Probe deutet der erste Eindruck auf ein stark anisotropes Gefüge hin, was durch eine eingehendere Betrachtung jedoch nicht bestätigt wird - daher die Einordnung in diese Gruppe.

Als Gefügetypen kommen sowohl der flächendominierte S-Typ (z.B. Kalzit-Marmor - Rek), als auch der S-L-Typ (z.B. Meta-Gabbro - Mg2) und der lineardominierte L-Typ (z.B. MetaGabbro - Mg1b) vor.

Die Gruppe III beinhaltet die ausgeprägt anisotropen Gesteine. Hier dominieren die Foliation und/oder das Linear das Gesteinsgefüge, definiert meist durch eine Kombination von stark bis sehr stark ausgeprägter Kornformregelung und stark bis sehr stark ausgeprägtem Materialwechsel. In dieser Fraktion (insgesamt neun Proben) befinden sich ausschließlich phyllosilikathaltige Gneise (Gra1c, Be1, VA, Cal, Be2) und Mylonite (MrMy5, Iv1b, Iv2, Gra3a) mit granitischer bis granodioritischer Zusammensetzung (Abb.5-9). Die Foliation ist im Fall der Proben MrMy5 und Be1 unruhig/flaserig ausgeprägt und wird über die Proben VA, Cal bis Be2, Gra3a zunehmend straffer. Ein Sonderfall ist die Probe Gra1c: durch einen sehr straffen Lagenbau entsteht ein stark anisotroper Eindruck, ähnlich dem der Ultramylonite Gra3a und Iv2, der aber einer eingehenden Betrachtung nicht standhält. So zeigen lediglich die Biotite eine ausgeprägte Kornformregelung, deren Volumenanteil mit 5\% niedrig ist. Daher sind, wie schon bei der Probe Grala aus Gruppe I, die Glimmer zwar in bestimmten Bereichen angereichert, aber als einzelne Körner isoliert und bilden keine penetrativen Lagen. Wahrscheinlich wird diese Probe bezüglich ihrer Anisotropie überschätzt. Eine endgültige Klärung kann allerdings erst aufgrund des mikroskopischen Befundes stattfinden. 


\section{$\underline{\text { 5. Gesteinsmaterial }}$}

Die Gefüge der Gruppe III sind entweder flächendominierte S-Typen oder S-L-Typen; reine L-Typen kommen nicht vor.

Die Ergebnisse der Gruppeneinteilung aufgrund des makroskopischen Erscheinungsbildes sind nebst Gruppendefinitionen und beispielhaften Gefügeschemata in Tab.5-5 zusammengefasst.

\subsection{Mikrogefüge}

Analog zu den Makrogefügen weisen auch die Mikrogefüge der Gesteine eine in ihrer unterschiedlichen geologischen Geschichte begründete breite Variation auf. Im folgenden Abschnitt werden die in Kap.5.5 definierten Gefügegruppen bezüglich ihres mikrostrukturellen Inventars beschrieben. Es geht hierbei mehr um die Darstellung von repräsentativen Gruppen-Charakteristika und nicht um eine eingehende mikrotektonische Analyse jeder einzelnen Probe. Detaillierte Mikrogefügeanalysen werden im Rahmen von Fallstudien sowie bei der Betrachtung von Spezialfällen (Kap.7) vorgenommen und dort zu einer weiterführenden Interpretation der Gefügeabhängigkeit gesteinstechnischer Eigenschaften herangezogen. Eine Charakterisierung der Riss-Systeme wird in Kap.5.9/Anhang II vorgenommen.

\subsubsection{Gruppe I- isotrope Gesteine}

Die Gefüge der Gruppe I erweisen sich auch im Dünnschliff als richtungslos körnig bis leicht foliiert. Ersteres zeigen der porphyrische, grobkörnige Granit Koess (Abb.5-10) und der annähernd equigranulare, mittelkörnige Granodiorit PDD. Letzteres trifft auf den fast equigranularen, mittelkörnigen NI (Gabbro-Norit) und den porphyrisch feinkörnigen MrGr3 (Granit) zu, wobei MrGr3 (Abb.5-10) lagige, wenn auch nicht penetrative Anreicherungen von Muskovit und Biotit aufweist. 

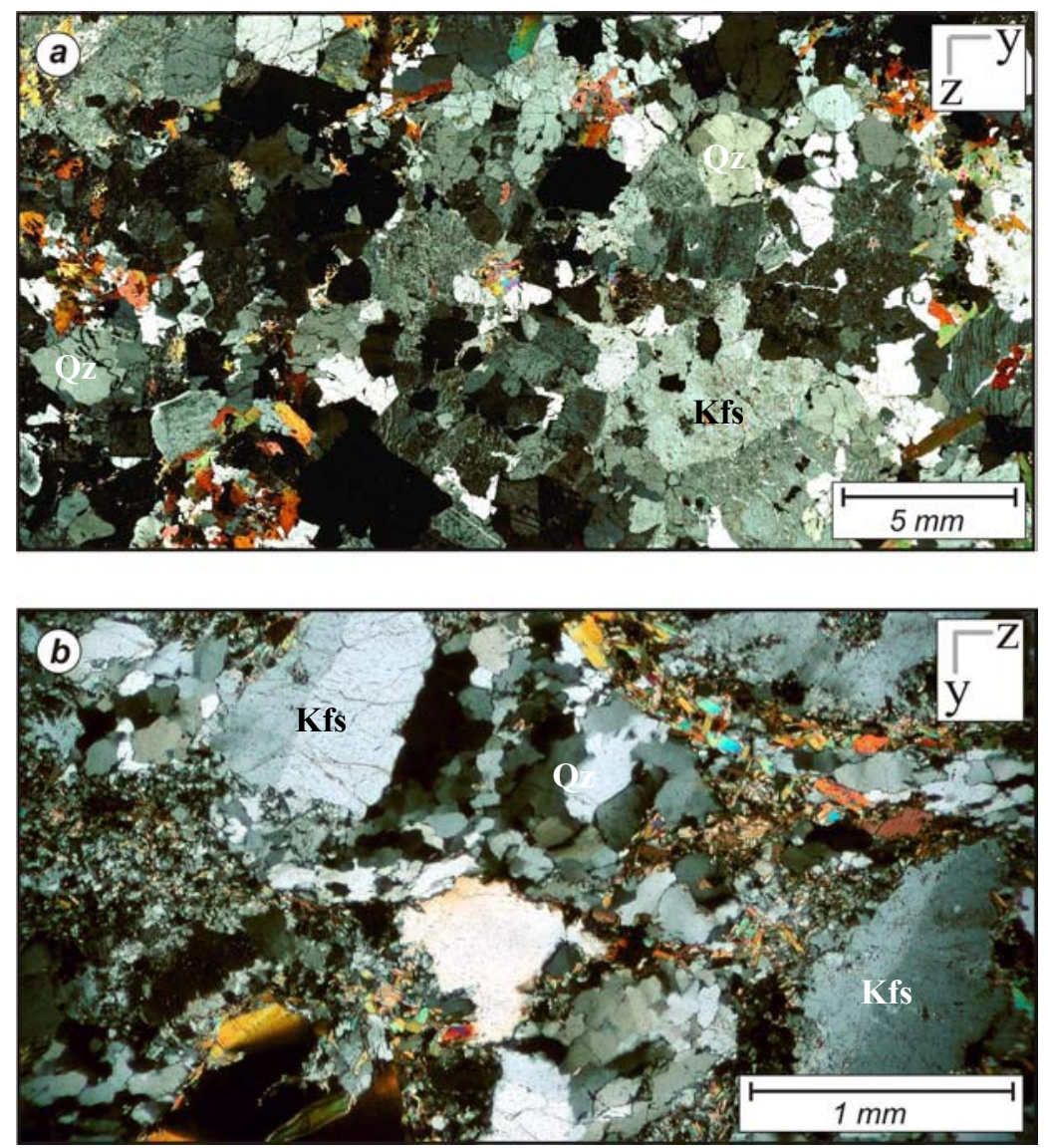

Abb.5-10: Mikrogefügebilder aus der Gruppe I: Isotrope Gesteine. Kfs - Kalifeldspat. Qz - Quarz. Ms - Muskovit. Bt Biotit. Details im Text und in Anhang II.

(a) Kösseine Granit (Koess); Dünnschliffscan, gekreuzte Polarisatoren. (b) Granit (MrGr3); Mikroskopbild, gekreuzte Polarisatoren.

Diese Minerale sind im Fall des Granits und Granodiorits ausschließlich in Nestern angereichert. Die schwach ausgeprägte Foliation des Gabbro-Norits wird in der Hauptsache durch die bevorzugte Orientierung xenomorph bis hypideomorph ausgebildeter Plagioklase definiert. Die Quarze innerhalb dieser Gruppe löschen gerade (Koess) bis leicht undulös (MrGr3) aus, weisen also nur geringe Merkmale einer Kaltdeformation auf. Die Korngrenzen sind polygonal bis gebogen, überwiegend geschlossen und gelegentlich belegt.

\subsubsection{Gruppe II - anisotrope Gesteine}

Die Gesteine der Gruppe II sind im Hinblick auf ihre Mineralogie und Entstehungsgeschichte deutlich heterogener, als die der Gruppen I und III und damit schwerer zu klassifizieren. Daher wird im Folgenden neben den gemeinsamen Merkmalen auch auf einige spezielle Gefügeausprägungen eingegangen. Die Gesteine sind durchweg fein- bis mittelkörnig und equigranular bis inequigranular ausgebildet. Bei allen Proben weist mindestens eine Mineralkomponente des meist xenomorphen, gelegentlich hypideomorphen Mineralbestands eine Kornformregelung auf. Bei den Meta-Gabbros ist das der Fall für Plagioklas, Hornblende und Klinopyroxen (Abb.5-11). Aber auch Phyllosilikate, Quarz, Kalzit, Olivin und Orthopyroxen zeigen bei unterschiedlichen Proben eine bevorzugte Kornformorientierung. 


\section{$\underline{\text { 5. Gesteinsmaterial }}$}

Die vom Habitus anisotropen und innerhalb dieser Gruppe immer mikrokristallinen bis feinkörnigen Phyllosilikate weisen die stärkste Tendenz zu einer Einregelung auf und sind im Fall der Probe Iv1 (granodioritischer Protomylonit) in länglichen Aggregaten und bei der Probe Grala (granitischer Gneis) in lagigen Bereichen konzentriert, ohne allerdings penetrative Lagen zu bilden. Die Probe AI, ein hydrothermal überprägter Quarzit, weist eine Serizitisierung entlang der kornformanisotropen und schwach eingeregelten Quarze auf (Abb.5-11). Bezüglich der Probe Grala bestätigt sich der makroskopische Eindruck, nach dem die Biotite als isolierte Kristalle in die umgebenden Quarze und Feldspäte eingebettet sind. Quarz sowie die Feldspäte zeigen hier ein granoblastisches Gefüge ohne eine mikroskopisch beobachtbare Vorzugsorientierung.

Mit Ausnahme der eben geschilderten Anreicherungen der Phyllosilikate lassen sich Materialwechsel im Dünnschliffbefund nicht belegen. Dafür mag der kleinere Maßstab verantwortlich sein, durch den z.B. die makroskopisch sichtbare, angedeutete Segregation im Meta-Gabbro Mg2 sowie die lagige Variation beim Quarzit AI nicht abgebildet werden kann. Quarz, als eine Hauptkomponente in drei der Proben vorhanden, löscht gerade (Quarzit) bis undulös aus und zeigt bei der Probe Iv1 randliche Subkornbildung als ein erstes Zeichen dynamischer Rekristallisation, was zur Einordnung als Protomylonit führt. Die großen Kalifeldspäte sind hier bruchhaft deformiert. Der fein bis mittelkörnige Kalzit des Marmors (Rek) zeigt eine Einregelung der Kornlangachsen parallel zur Foliation und weist eine große Anzahl von Druckzwillingen auf (Abb.5-11). In der gesamten Gruppe sind die Korngrenzen polygonal bis leicht gebogen, zumeist geschlossen und gelegentlich belegt. 

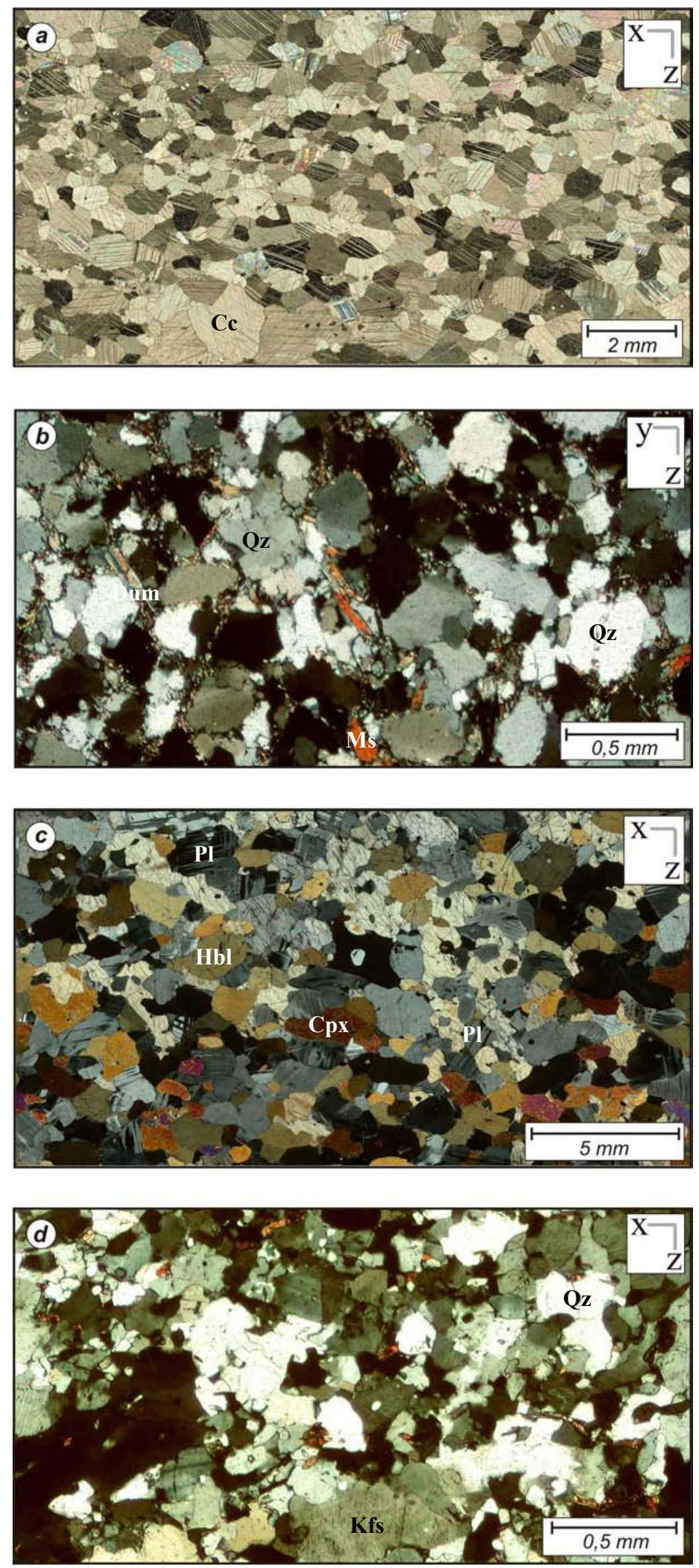

Abb.5-11: Mikrogefügebilder aus der Gruppe II: Anisotrope Gesteine. Cc - Kalzit. Dum Dumortierit. Qz - Quarz. Ms Muskovit. Pl-Plagioklas. $\mathrm{Hbl}-$ Hornblende. CpxKlinopyroxen. Kfs Kalifeldspat. Details im Text und Anhang II.

(a) Kalzit-Marmor (Rek); Dünnschliffscan gekreuzte Polarisatoren; (b) Quarzit (Al); Mikroskopbild gekreuzte Polarisatoren; (c) Meta-Gabbro (Mg1b). Dünnschliffscan gekreuzte Polarisatoren. (d) granitischer Gneis (Gra1a); Mikroskopbild gekreuzte Polarisatoren. 


\section{$\underline{\text { 5. Gesteinsmaterial }}$}

\subsubsection{Gruppe III - ausgeprägt anisotrope Gesteine}

Die Gruppe III beinhaltet ausschließlich phyllosilikathaltige Gneise und Mylonite von granitischer bis granodioritischer Komposition. Eine starke bis sehr starke Kornformregelung von mindestens einer, meist aber mehrerer Mineralkomponenten und die durchgreifende Segregation in Lagen unterschiedlicher Zusammensetzung sind die Hauptmerkmale dieser Gruppe auch im mikroskopischen Erscheinungsbild. Die Minerale sind mit Ausnahme von Granat und Phyllosilikaten xenomorph entwickelt. Die mittel- bis feinkörnigen Korngefüge reichen von stark inequigranular beim „Augen“-Gneis (Be1 - $\boldsymbol{A b} \boldsymbol{b} .5-12)$ und Protomylonit (MrMy5) über weniger inequigranular beim Paragneis (Cal) und Orthogneis (VA) bis zu equigranular bei den straff foliierten Ultramyloniten (Gra3a, Iv2) und dem Gneis (Be2). Damit einher geht ein in dieser Abfolge zunehmender Grad der Gefügeanisotropie sowie eine Abnahme der mittleren Korngröße. Bei ersterem bilden große Kalifeldspäte Klasten in einer feinkörnigen Matrix aus Quarz, Plagioklas und Phyllosilikaten. Quarz löscht stets undulös aus und ist in Bezug auf die Mylonite teilweise (Protomylonit) bis durchgreifend (Ultramylonit) dynamisch rekristallisiert. Beim Hochtemperatur-Ultramylonit Gra3a (Duyster 1991; Ullemeyer 1992-Abb.5-12) trifft das auch auf die Feldspäte zu, welche hingegen in den Proben Iv1b/Iv2 eine bruchhafte Deformation aufweisen. Die Glimmer als ein Hauptmerkmal dieser Gruppe sind durchweg stark bis sehr stark mit ihrer (001)-Fläche parallel zur Foliation eingeregelt und in Lagen angereichert. Der granitische Gneis Be2 zeigt eine ansatzweise rotationale Anordnung der Glimmer um das von polykristallinen Aggregaten gebildete Linear. Eine weitere Besonderheit bildet die schon in Kap.5.5.1 diskutierte Probe Gralc. Im Gegensatz zu ihrem makroskopisch durch den Materialwechsel bedingten stark anisotropen Eindruck, ist mikroskopisch kaum eine Gefügeanisotropie zu erkennen. Der feinkörnige Biotit liegt in Form isolierter Körner vor, deren Texturierung auch wesentlich weniger deutlich ausgeprägt ist als vom makroskopischen Bild her zu erwarten gewesen wäre. Der restliche Mineralbestand zeigt keinerlei Vorzugsorientierung und bildet ein granoblastisches Gefüge. 

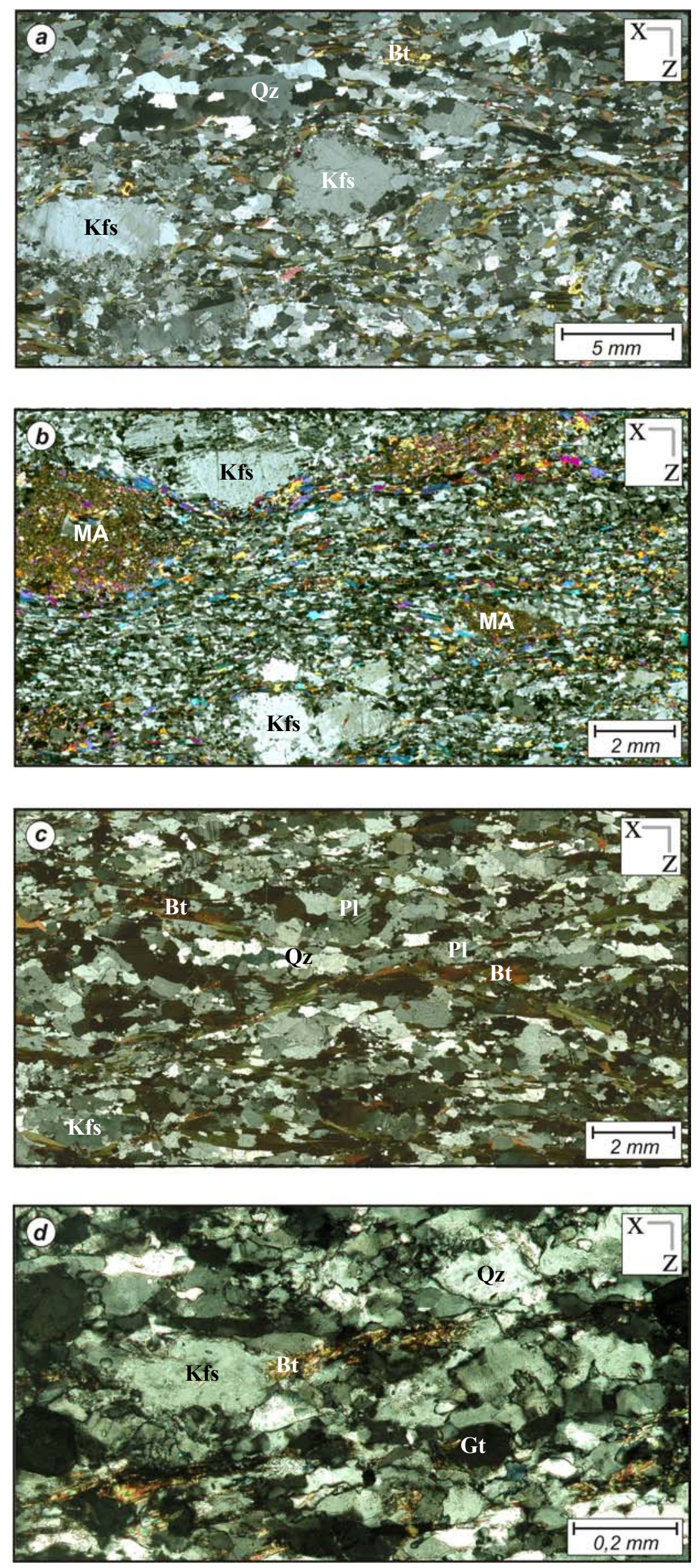

Abb.5-12: Mikrogefügebilder aus der Gruppe III: Stark bis sehr stark anisotrope Gesteine. Kfs - Kalifeldspat. Qz - Quarz. Bt - Biotit. MA -

mikrokristallines Aggregat. PIPlagioklas. Gt - Granat. Details im Text und in Anhang II.

(a) granodioritischer Gneis (Be1); Dünnschliffscan gekreuzte Polarisatoren; (b) granodioritischer Mylonit (IV1b); Dünnschliffscan gekreuzte Polarisatoren; (c) granodioritischer Gneis (Cal). Dünnschliffscan gekreuzte Polarisatoren. (d) granitischer Gneis (Gra3a); Mikroskopbild gekreuzte Polarisatoren. 


\section{$\underline{\text { 5. Gesteinsmaterial }}$}

Die geschlossenen Korngrenzen haben hier eine polygonale Form, wohingegen in den restlichen Proben der Gruppe interlobate bis stark interlobate (verzahnte) Korngrenzen verbreitet sind.

\subsection{Texturen}

Wie aus Studien von z.B. Siegesmund \& Kern (1990), Siegesmund et al. (1995), Strohmeyer \& Siegesmund (2002) und Zeisig et al. (2002) hervorgeht, kann die Textur der Minerale einen großen Einfluss auf die physikalisch-technischen Eigenschaften eines Gesteins haben. Wichtige Faktoren sind diesbezüglich der prozentuale Volumenanteil des jeweiligen Minerals im Gestein, ihre Einkristalleigenschaften (Kap.4) und die Textur.

Bisher wurden die untersuchten Gesteine nach dem makroskopischen Erscheinungsbild in Gruppen unterschiedlicher Gefügeanisotropie unterteilt und das Ergebnis mit mikrostrukturellen Untersuchungen untermauert. Es stellt sich nun die Frage, ob sich die beobachtete Anisotropisierung innerhalb des Probensatzes auch in den Texturen widerspiegelt. Da Deformationsmechanismen, wie z.B. die Rotation und Einregelung stark formanisotroper Minerale sowie kristallplastische Prozesse mit der Ausbildung von Foliation und Linear eine Gefügeanisotropie (Passchier \& Trouw 1998; Blenkinsop 2000) verursachen können, ist ebenfalls eine kristallographische Vorzugsorientierung der gesteinsbildenden Minerale (Textur) zu erwarten. Die verschiedenen Minerale weisen ein deutlich unterschiedliches Deformationsverhalten auf. So konkurrieren beispielsweise beim Quarz drei unterschiedliche Gleitsysteme miteinander, die je nach Druck-, Temperatur-, und Deformationsbedingungen bevorzugt aktiviert werden, was $\mathrm{zu}$ unterschiedlichen Texturen führen kann (z.B. Tullis 1977; Mainprice et al. 1986; Passchier \& Trouw 1998). Auch bei Olivin, Pyroxenen, Hornblende, Kalzit und Feldspäten, die als Hauptgemengteile auftreten, sind die texturbildenden Deformationsprozesse verhältnismäßig komplex (z.B. Kruhl 1985; Dietrich 1986; Ji \& Mainprice 1988; Skrotzki et al. 1992; Ersekine et al. 1993; Dornbusch 1995; Passchier \& Trouw 1998; Leiss et al. 2002), so dass eine einfache Korrelation von Makrogefügen und Textur häufig nicht möglich ist. Allein die Glimmer weisen neben einer starken Formanisotropie auch ein einfaches Gleitsystem (Basisgleiten parallel zur (001)Fläche) auf und sind daher mit ihren einfachen und zumeist senkrecht zur Foliation ausgerichteten c-Achsen-Maxima sowohl gut geeignet die Textur eines Gesteins als auch die Gefügesymmetrie zu beschreiben (Wenk \& Pannetier 1990; Ullemeyer 1992; Ullemeyer 1999). Das Regelungsverhalten ist über einen weiten Druck- und Temperaturbereich konstant 


\section{$\underline{\text { 5. Gesteinsmaterial }}$}

und wird durch Biegung und Knickung des Kristallgitters und einem Basisgleiten parallel zur (001)-Fläche dominiert. Rekristallisation konnte im Experiment für Phlogopit erst $\mathrm{ab} 1050^{\circ} \mathrm{C}$ und $1000 \mathrm{MPa}$ nachgewiesen werden (Etheridge \& Hobbs 1974). Zudem weisen die Glimmer aufgrund ihres ausgeprägt anisotropen Habitus und der damit verbundenen dominanten Kornrotation ((001)-Flächen rotieren in Richtung der Langachsen des finiten Strainellipsoids) während der Deformation stets die stärkste Textur (c-Achse) auf. Das, in Verbindung mit der ausgeprägten Anisotropie gegenüber vielen physikalischen Eigenschaften, lässt einen großen Einfluss der Glimmer auf die Anisotropie der technischen Gesteinseigenschaften vermuten (z.B. Gottschalk et al. 1990, Shea \& Kronenberg 1993). Daher scheinen die Glimmer also gut geeignet, mit ihrer Textur eine Aussage sowohl über die Gefügeanisotropie als auch die Gefügesymmetrie zu treffen.

In 15 der untersuchten Gesteine kommen Biotit und/oder Muskovit vor. Bei 14 von ihnen wurde die Glimmertextur ((001)-Pole) bestimmt. Chlorit-Texturen wurden nicht ermittelt, aber aufgrund des ähnlichen Regelverhaltens von Glimmern und Chlorit ist eine Analogie der Texturen beider Minerale zu erwarten. Sekundäre, postdeformative Bildungen von Chlorit bleiben dabei unberücksichtigt, sind aber innerhalb des Probensatzes auch eher selten zu beobachten. Beim Quarzit (AI) konnte die Muskovit-Textur aufgrund der feinkörnigen Ausbildung als Serizit nicht quantifiziert werden. Hier wurde die Quarztextur (c-Achse) bestimmt. Die Charakterisierung der sonstigen, glimmerfreien Proben erfolgte auf der Basis des am stärksten texturierten Minerals im Gestein (z.B. Kalzit c-Achse beim Marmor; Hornblende (001)-Pole bei den Meta-Gabbros).

Im Folgenden werden die jeweiligen Gruppen aus der makroskopischen Bewertung der Gesteine bezüglich ihrer Texturvariationen charakterisiert. Da es zunächst um eine generelle Gefügeklassifizierung der Gesteine geht, wird auf eine umfassende Darstellung der Texturen verzichtet. Detailiert aufgeschlüsselte Texturen werden im Rahmen von Fallstudien (Kap.7) erörtert und zur Betrachtung von Spezialfällen in den jeweiligen Kapiteln (z.B. Kap.6.5 thermische Dehnung) herangezogen.

\subsubsection{Gruppe I - isotrope Gesteine}

Die Gruppe I beinhaltet mit den Proben Koess (Granit), PDD (Granodiorit) und MrGr3 (Granit) drei glimmerhaltige Gesteine, die durch ihre Glimmertexturen charakterisiert werden. (Für die Probe NI (Gabbro-Norit) liegen keine Texturuntersuchungen vor). Die maximalen Intensitäten der (001)-Pole bewegen sich zwischen 2,89 mrd (multiples of random 
distribution) und 4,1 mrd und sind sowohl im Verhältnis zu anderen Proben dieser Studie als auch im Vergleich mit Literaturdaten (z.B. Shea \& Kronenberg 1993) als niedrig einzustufen.

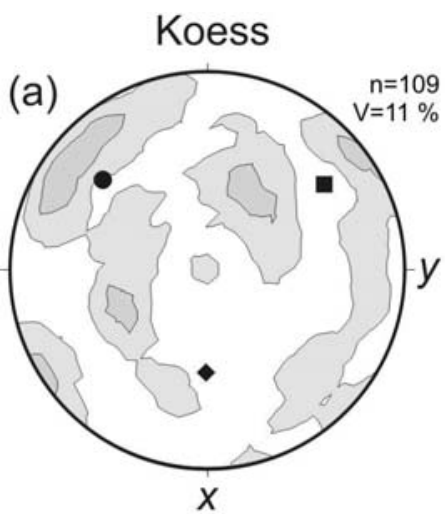

max. dens. $=2.89($ at $309 / 12)$

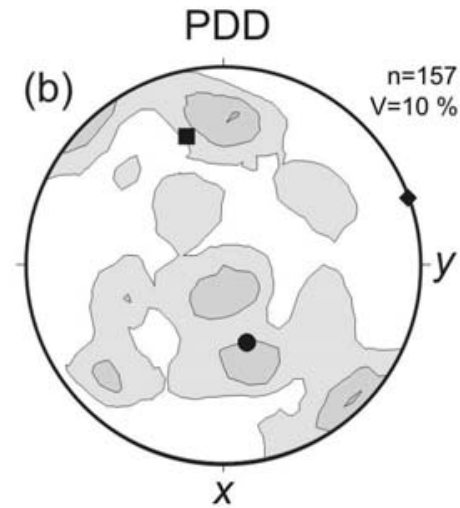

max. dens. $=3.11($ at $137 / 6)$

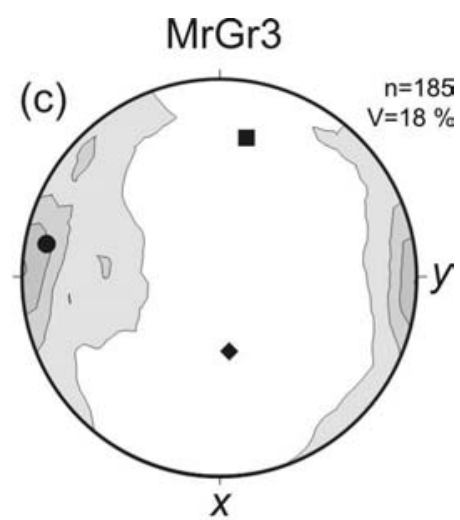

max. dens. $=4.10($ at $92 / 0)$

Abb.5-13: Glimmertexturen der Gruppe I. Richtungen x,y des Referenzsystems sind bezeichnet, z steht senkrecht auf der Papierebene. Azimuth $0^{\circ}$ ist oben (Nord) und $90^{\circ}$ ist in y-Richtung. Isolinienlevel bei 1,2,3,..,n [mrd]. $n$ - Anzahl der gemessenen Körner. V - Volumenanteil der Phyllosilikate im Gestein. Lage der Eigenvektoren: Kreis - großer Eigenvektor; Quadrat - mittlerer Eigenvektor; Raute - kleiner Eigenvektor. (a) Granit (Biotit). (b) Granodiorit (Biotit). (c) Granit (Muskovit, Biotit).

Die annähernd richtungslosen Koess und PDD weisen keine eindeutige Textursymmetrie auf, wohingegen der Granit (MrGr3) ein klar definiertes, wenn auch schwaches Maximum der (001)-Pole parallel zur y-Achse des Referenzsystems und eine oblate Textursymmetrie zeigt (Abb.5-13).

\subsubsection{Gruppe II - anisotrope Gesteine}

In der Gruppe II befinden sich zwei durch Glimmertexturen definierte (Iv1, Gra1a) und fünf durch die Texturen weniger anisotroper Minerale gekennzeichnete Gesteine. Die maximalen Intensitäten der Glimmer-(001)-Flächenpole liegen zwischen 5,54 mrd (granodioritischer Protomylonit - Iv1) und 5,71 mrd (granitischer Gneis - Gra1a). Iv1 offenbart eine Gürtelverteilung der (001)-Pole um das Linear und damit eine prolate Symmetrie, die auf ein rotationales Gefüge schließen lässt. Das Maximum der (001)-Pole ist etwa $60^{\circ}$ zur x-y-Ebene geneigt und steht senkrecht auf x; die Foliation des Gesteins ist damit um $30^{\circ}$ gegen die x-yEbene geneigt (Rotation um das Linear-Abb.5-14). Die Probe Grala hingegen weist ein Punktmaximum senkrecht zur Foliation und eine stark oblate Symmetrie auf (Abb.5-14). Die Olivin (100)-Pole des Peridotit (Pila) zeigen ein Punktmaximum (5,33 mrd), das mit der x-yEbene sowie mit der x-Richtung einen Winkel von ca. $30^{\circ}$ einschließt. Die Untersuchungen von Skrotzki et al. (1992) am gleichen Material zeigen ein (100)-Polmaximum parallel zum Linear, was zu dem Schluss führt, dass die Probe Pila in dieser Studie durchgehend 


\section{Gesteinsmaterial}

schiefwinklig zur Foliation und zur Lineation präpariert wurde. Der Marmor (Rek) zeigt ein leicht in Richtung des Linears gelängtes Punktmaximum der Kalzit c-Achsenverteilung mit einer maximalen Intensität von 2,5 mrd annähernd senkrecht zur Foliation (Abb.5-14). Die Quarz c-Achsen des Quarzits (AI) sind in Form eines Gürtels um die z- Achse des Referenzsystems orientiert, liegen also tendenziell in der Foliationsebene. Die Regelung ist mit einer maximalen Intensität von 1,38 mrd schwach und bestätigt den makroskopisch und mikroskopisch gewonnenen Eindruck eines schwach deformierten Gesteins. Die MetaGabbros, welche vom Rand (Mg1b) in Richtung auf das Zentrum einer Scherzone (Mg2) beprobt wurden, werden durch die Verteilung der Hornblende (001)-Pole der Probe Mg1b beschrieben.

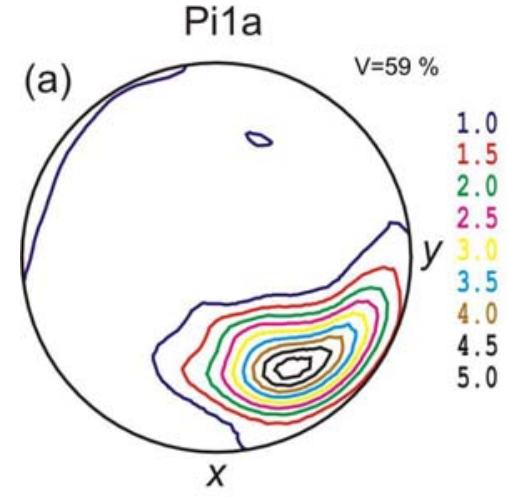

max. dens. $=5.33($ at $145 / 30)$ Olivin (100)-Pole

$\mathrm{Mg} 1 \mathrm{~b} / \mathrm{Mg} 2$

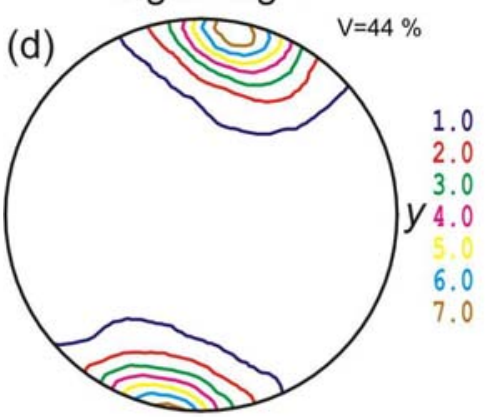

$X$

max. dens. $=7.79($ at $010 / 05)$

Hornblende (001)-Pole

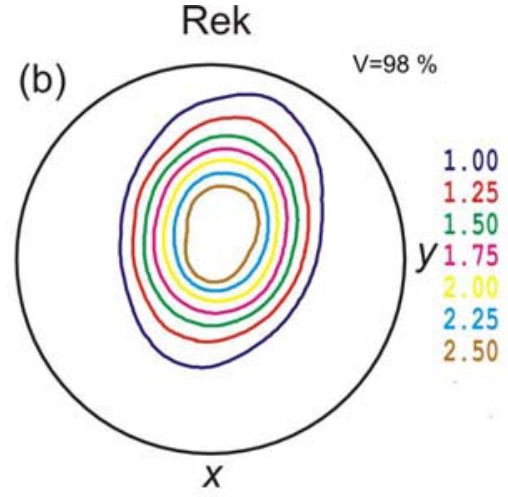

max. dens. $=2.88($ at $020 / 70)$ Kalzit c-Achse

Iv1

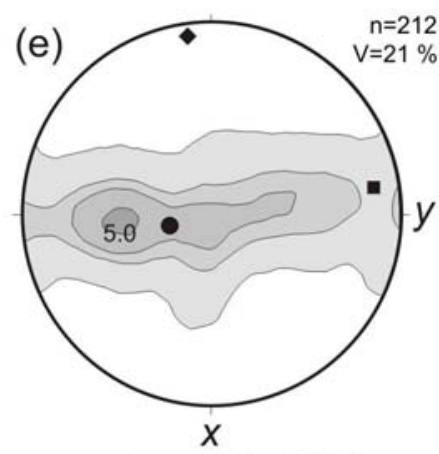

max. dens. $=5.54($ at $270 / 54)$

Muskovit (001)-Pole

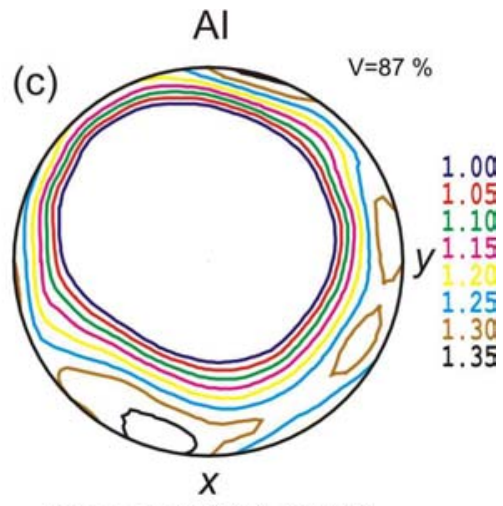

$\max$. dens. $=1.38($ at $195 / 05)$

Quarz c-Achse

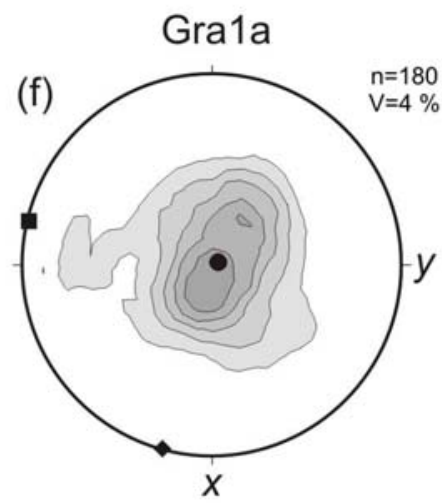

max. dens. $=5.71$ (at 210/78)

Biotit (001)-Pole

Abb.5-14: Texturen der Gruppe II. Richtungen x,y des Referenzsystems sind bezeichnet, z steht senkrecht auf der Papierebene. Azimuth $0^{\circ}$ ist oben (Nord) und $90^{\circ}$ ist in y-Richtung. Isolinienlevel bei $1,2,3, \ldots, n$ [mrd] bzw. bezeichnet. $n$ - Anzahl der gemessenen Körner. V - Volumenanteil des texturierten Minerals (a-d) bzw. Volumenanteil der Phyllosilikate im Gestein (e,f). Lage der Eigenvektoren: Kreis - großer Eigenvektor; Quadrat - mittlerer Eigenvektor; Raute - kleiner Eigenvektor. (a) Peridotit (Olivin). (b) Kalzit-Marmor (Kalzit). (c) Quarzit (Quarz).(d)Meta-Gabbros (Hornblende). (e) Granodioritischer Protomylonit (Muskovit). (f) Granitischer Gneis (Biotit).

Deren Punktmaximum, annähernd parallel zum Linear, hat eine maximale Intensität von 7,79 mrd (Abb.5-14). Für die Probe Mg2 wurden keine Texturuntersuchungen durchgeführt, doch implizieren die Ergebnisse von Dornbusch (1995) lediglich eine leichte Intensivierung der 


\section{Gesteinsmaterial}

Hornblende (001)-Textur mit zunehmender Deformation innerhalb der Scherzone, so dass eine gemeinsame Charakterisierung beider Proben an dieser Stelle legitim erscheint. Im Detail werden die Texturen der Meta-Gabbros im Rahmen einer Fallstudie in Kap.7.2 beleuchtet. 


\subsubsection{Gruppe III - ausgeprägt anisotrope Gesteine}

In der Gruppe III mit ihren neun ausschließlich phyllosilikathaltigen, granitischen bis granodioritischen Myloniten und Gneisen kommen maximale Intensitäten der Glimmer (001)Pole von 8,61 mrd (Gra1a) bis 23,3 mrd (Gra3a) vor.

\section{MrMy5}

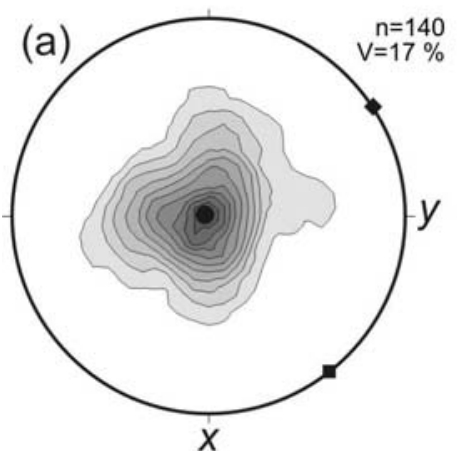

max. dens. $=11.07$ (at $0 / 90$ )

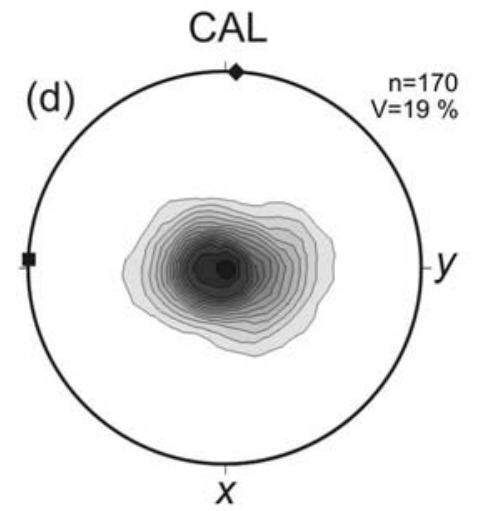

max. dens. $=16.95($ at $0 / 90)$

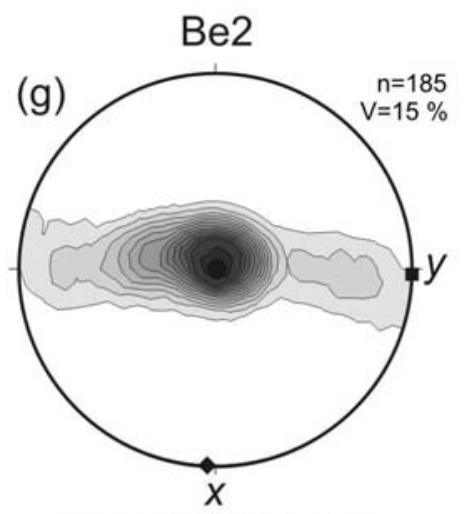

max. dens. $=16.12($ at $0 / 90)$
Be1

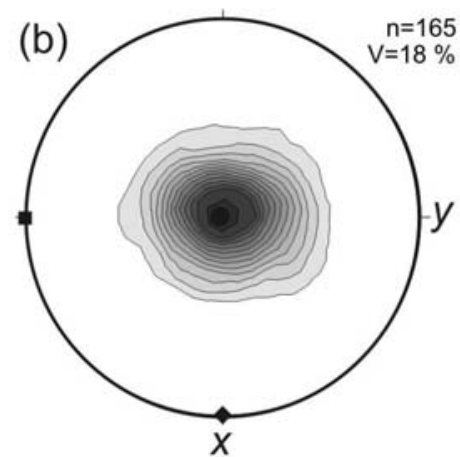

max. dens. $=15.48$ (at $0 / 90)$

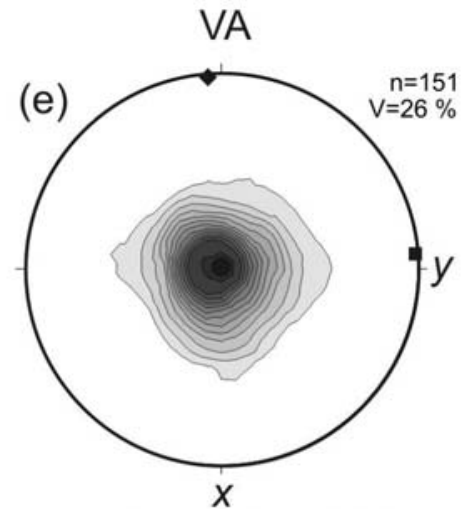

max. dens. $=15.89($ at $0 / 90)$

Iv2

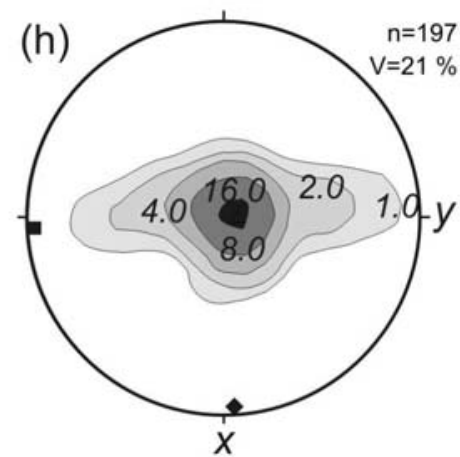

max. dens. $=17.56($ at $60 / 87)$

\section{Iv1b}

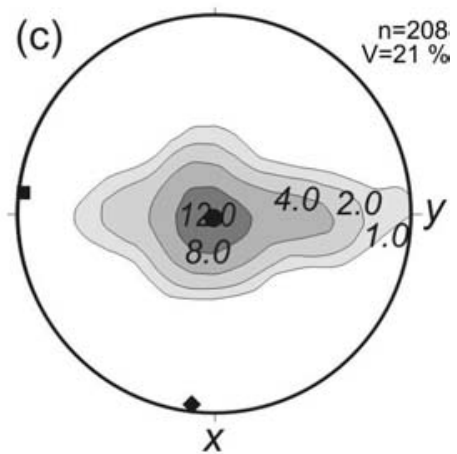

max. dens. $=12.25($ at $0 / 90)$

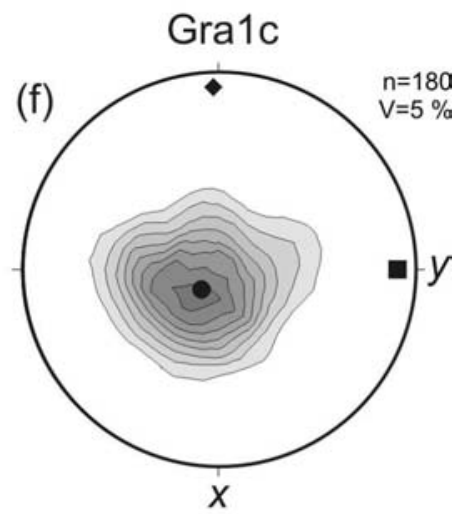

- $\max$. dens. $=8.61$ (at 210/78)

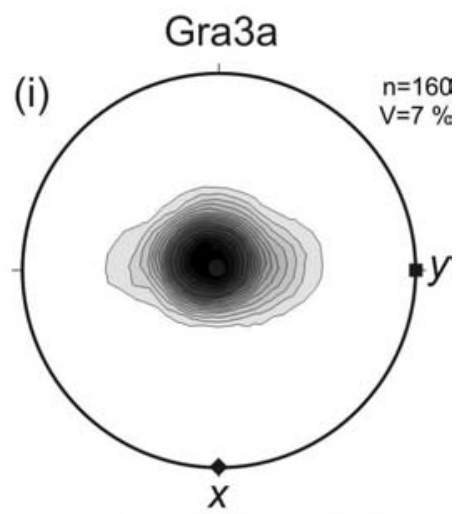

max. dens. $=23.30($ at $0 / 90)$

Abb.5-15: Glimmertexturen der Gruppe III. Richtungen x,y des Referenzsystems sind bezeichnet, z steht senkrecht auf der Papierebene. Azimuth $0^{\circ}$ ist oben (Nord) und $90^{\circ}$ ist in y-Richtung. Isolinienlevel bei 1,2,3,..,n [mrd] bzw. bezeichnet. $n$ - Anzahl der gemessenen Körner. V Volumenanteil der Phyllosilikate im Gestein. Lage der Eigenvektoren: Kreis - großer Eigenvektor; Quadrat - mittlerer Eigenvektor; Raute - kleiner Eigenvektor. (a) Granitischer Protomylonit (Biotit, Muskovit). (b) Granosioritischer Gneis (Biotit). (c) Granodioritischer Mylonit (Muskovit). (d) Granodioritischer Gneis (Biotit). (e) Granitischer Gneis (Muskovit). (f) Granitischer Gneis (Biotit). (g) Granitischer Gneis (Biotit, Muskovit). (h) Granodioritischer Ultramylonit (Muskovit). (i) Granitischer Ultramylonit (Gra3a). 


\section{$\underline{\text { 5. Gesteinsmaterial }}$}

Alle Proben zeigen ein Maximum der (001)-Pole senkrecht zur Foliation, wobei die Proben MrMy5, Be1, Cal, VA und Gra1c eine oblate Glimmertextur und die Proben Gra3a, Iv2, Iv1b und $\mathrm{Be} 2$ ein in y-Richtung gelängtes Maximum bzw. unvollkommene Gürtelverteilungen um das Linear aufweisen (Abb.5-15). Auffällig innerhalb dieser Gruppe ist der granitische Gneis Gra1c, dessen Regelungsgrad der (001)-Pole deutlich niedriger ausfällt (Abb.5-15).

\subsection{Korrelation von makroskopischem Erscheinungsbild und Textur}

Zunächst einmal fällt auf, dass sich sämtliche glimmerfreien Proben in den Gruppen I (NI) und II (Pila, Rek, Mg1b, Mg2) und keine in der Gruppe III der stark bis sehr stark anisotropen Gesteine befinden. Dabei ist der Regelungsgrad der Olivin (100)-Pole (max. 5,33 mrd) im Peridotit und der Hornblende (001)-Pole (max. 7,79 mrd) in den Meta-Gabbros im Vergleich mit Literaturdaten durchaus als hoch anzusehen (publizierte Vergleichsdaten z.B. in: Skrotzki et al. 1992; Leiss et al. 2002; Zucali et. al. 2002). Eine mögliche Erklärung für die trotzdem niedrige, makroskopisch beobachtbare Gefügeanisotropie liefert die gegenüber den Phylloslikaten geringere Formanisotropie der Minerale. Neben dem weniger anisotropen Habitus ist auch die Anisotropie der mechanischen und elastischen Eigenschaften weniger deutlich ausgeprägt (Kap.4), so dass selbst eine starke Textur von Olivin und Hornblende vermutlich einen kleineren Einfluss auf die Gesamtgesteinseigenschaften hat, als das bei Phyllosilikaten der Fall ist. Aus diesem Grunde und in Verbindung mit einer kaum vorhandenen Segregation kann die Einteilung der Proben Pila und Mg1b/Mg2 in die Gruppe II argumentativ untermauert werden. Die Textur des Kalzits im Marmor (Rek) ist im

Vergleich zu natürlich vorkommenden Regelungsgraden durchschnittlich stark (cAchsenmax. 2,5 mrd) und die des Quarz im Quarzit (AI) schwach (c-Achsenmax. 1,38 mrd) ausgeprägt (publizierte Vergleichsdaten z.B. in Mancktelow 1987; Siegesmund et al. 2000; Zeisig et al. 2002). Die Einordnung des Marmors in die Gruppe der anisotropen Gesteine ist daher gerechtfertigt, wobei der Quarzit (AI) aufgrund der Textur eher der Gruppe I zugerechnet werden könnte.

Von 14 der 20 untersuchten Gesteine liegen Glimmertexturen vor, die schwache bis sehr starke maximale Intensitäten aufweisen (publizierte Vergleichsdaten z.B. in: Means et al. 1984; Ullemeyer 1992; Shea \& Kronenberg 1993; Siegesmund et al. 1995).

Das erlaubt sowohl den direkten Vergleich des Regelungsgrads von (001)-Glimmerpolen der Proben untereinander als auch eine Korrelation der Texturintensitäten und -muster mit den makroskopisch beobachteten Gefügeanisotropien. Zu diesem Zweck wurden die Daten unter 


\section{$\underline{\text { 5. Gesteinsmaterial }}$}

Verwendung der Textureigenvektoren und -eigenwerte im Woodcock-Diagramm und in einem modifizierten Jelinek-Diagramm dargestellt. Im Woodcock-Diagramm steht ein ansteigender Wert des Parameters $\mathrm{c}_{\mathrm{W}}$ für eine stärkere Regelungsschärfe (stärkere Anisotropie) und der Faktor $\mathrm{k}_{\mathrm{W}}$ für die Symmetrie der Textur; $\mathrm{k}_{\mathrm{W}}=\infty$ für ideal oblat, $\mathrm{k}_{\mathrm{W}}=0$ für ideal prolat. Im modifizierten Jelinek-Diagramm zeigt die $\mathrm{x}$-Achse mit den maximalen Intensitäten [mrd] ein Maß für den Regelungsgrad und der Faktor T die Symmetrie der Textur von $\mathrm{T}=+1$ (ideal oblat) bis $\mathrm{T}=-1$ (ideal prolat) $(\boldsymbol{A b b . 5 - 1 6})$.

Die Glimmertexturen untermauern mit ihren Intensitäten und Symmetrien eindrucksvoll die Einteilung nach dem makroskopischen Erscheinungsbild. Die Eigenvektoren der Glimmertexturen sind annähernd parallel zu den Achsen der makroskopischen Gefüge orientiert. Im Grundsatz korrelieren die Regelungsschärfe $\left(\mathrm{c}_{\mathrm{W}}\right)$ und Intensität der Glimmerregelung positiv mit der beobachteten Gefügeanisotropie. Die S-Typ-Gesteine weisen tendenziell oblate, die L-Typ-Gesteine tendenziell prolate Texturmuster auf. Dazwischen liegen die S-L-Typen mit $\mathrm{k}_{\mathrm{W}}$-Werten zwischen 0,37 und 3 bzw. Form-Faktoren (T) von 0,5 bis -0,5 (Abb.5-16). Folglich lassen sich die Gruppen I, II und III der makroskopischen Gefüge bezüglich der Glimmertexturen wie folgt definieren:

\section{Anisotropie nach makroskopischem Gefüge}

Gruppe I: Isotrope Gesteine

Gruppe II: Anisotrope Gesteine

Gruppe III: Ausgeprägt anisotrope Gesteine

Symmetrie für alle Gruppen: $\quad$ S-Typ

$$
\begin{aligned}
& \text { S-L-Typ } \\
& \text { L-Typ }
\end{aligned}
$$

$$
\begin{aligned}
& \text { Anisotropie nach Glimmertextur } \\
& \mathrm{c}_{\mathrm{W}}<1 \text { und } \mathrm{I}_{\max }<5 \mathrm{mrd} \\
& 1 \leq \mathrm{c}_{\mathrm{W}} \leq 2 \text { und } 5 \mathrm{mrd} \leq \mathrm{I}_{\max } \leq 10 \mathrm{mrd} \\
& \mathrm{c}_{\mathrm{W}}>2 \text { und } \mathrm{I}_{\max }>10 \mathrm{mrd}
\end{aligned}
$$

$$
\begin{aligned}
& \mathrm{k}_{\mathrm{W}}>3 \text { und } \mathrm{T}>0,5 \\
& 0,375 \leq \mathrm{k}_{\mathrm{W}} \leq 3 \text { und }-0,5 \leq \mathrm{T} \leq 0,5 \\
& \mathrm{k}_{\mathrm{W}}<0,375 \text { und } \mathrm{T}<-0,5
\end{aligned}
$$

$\mathrm{Zu}$ beachten bleibt die Probe Gra1c (granitischer Gneis), die aufgrund der Anisotropie ihres makroskopischen Erscheinungsbilds zwischen den Proben VA und Be2 eingeordnet wurde, vom Regelungsgrad der Glimmer her aber wesentlich niedriger anisotrop einzustufen ist (Abb.5-15). Dieser Eindruck bestätigt die Ergebnisse der mikroskopischen Untersuchungen (Kap.5.6.3) und führt zu der Einsicht, dass die makroskopische Einordnung, bedingt durch den ausgeprägten Farbkontrast des Lagenbaus, zu einer Überschätzung der Gefügeanisotropie geführt hat. Die Einteilung wird dennoch beibehalten und die Konsequenzen dieser Tatsache 


\section{$\underline{\text { 5. Gesteinsmaterial }}$}

im Hinblick auf die Korrelation von Gefüge und gesteinstechnischen Eigenschaften in den jeweiligen Kapiteln diskutiert.

(a)

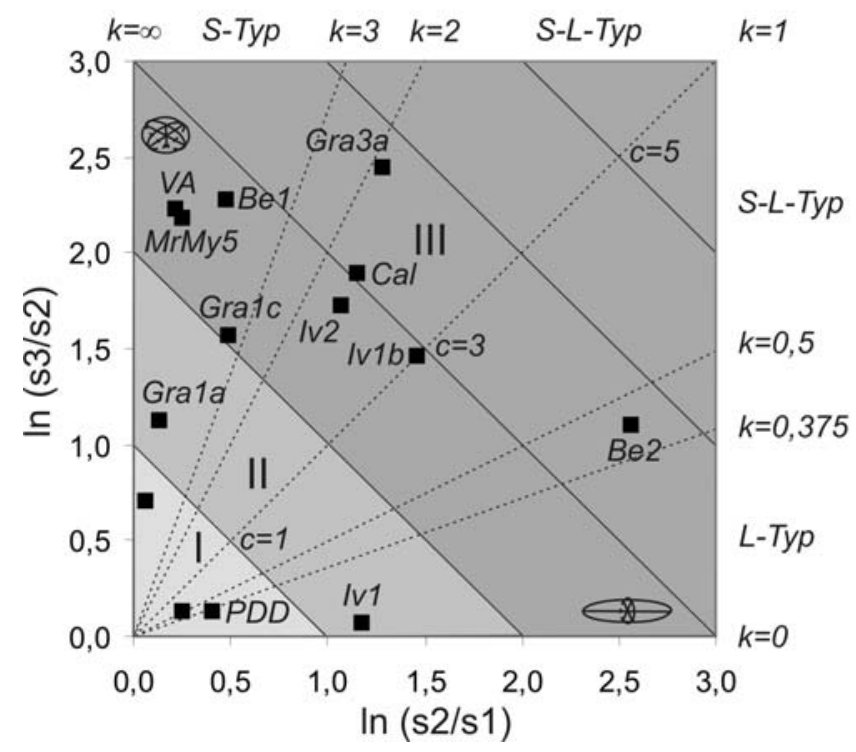

(b)

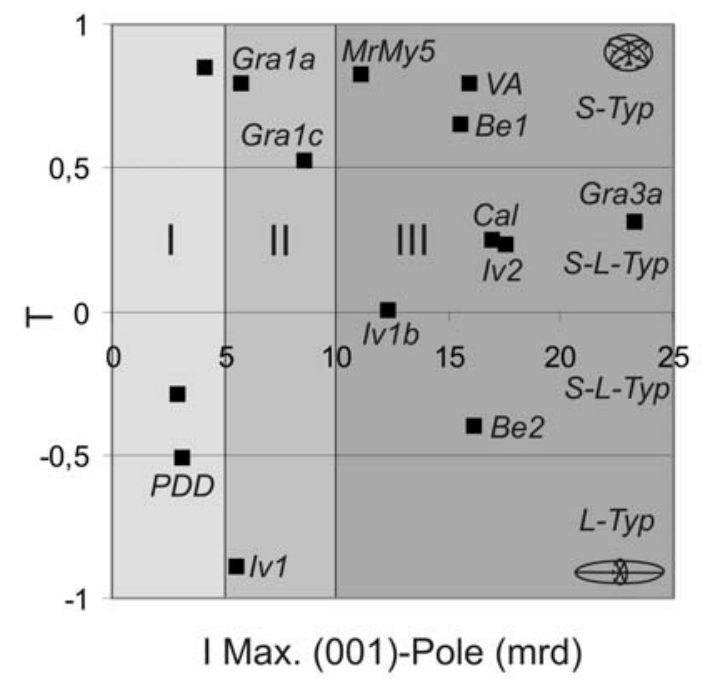

Abb.5-16: Gesteine mit Glimmertexturen im Woodcock-Diagramm (a) und im modifizierten JelinekDiagramm (b). Makrogefüge-Gruppen: I - hellgrau; II - mittelgrau; III - dunkelgrau. s1 - kleiner Eigenwert; s2 - mittlerer Eigenwert; s3 - großer Eigenwert. Symmetrie innerhalb der MakrogefügeGruppen ist mit S-Typ, S-L-Typ und L-Typ bezeichnet. Gra1a/1c/Be2/VA - granitischer Gneis. Iv1 granodioritischer Protomylonit. Iv1b - granodioritischer Mylonit. Iv2 - granodioritischer Ultramylonit. Cal/Be1 - granodioritischer Gneis. MrMy5 - granitischer Protomylonit. PDD - Granodiorit. Gra3a granitischer Ultramylonit. Weitere Details im Text.

\subsection{Mikrorisse}

Alle Gesteine enthalten Mikrorisse, deren Entstehung in der teilweise komplexen geologischen Vergangenheit begründet liegt. Ihre Geometrie (z.B. aspect ratio), Lage (z.B. intra- oder transgranular) und die Art (z.B. offen oder verheilt) hängen ebenso von der Genese des jeweiligen Gesteins ab, wie eine mögliche bevorzugte Orientierung und die Rissdichte. Wichtige Prozesse der Mikrorissbildung sind tektonische Hebungen, regionale Spannungsfelder in Verbindung mit Deformation und thermisch induzierte Rissbildung. Neben den geologischen Prozessen haben auch der Mineralbestand und seine Textur einen Einfluss auf die Anzahl und die bevorzugte Orientierung von Mikrorissen (Willaime et al. 1979; Wong \& Biegel 1985; Vollbrecht et al. 1991). Sekundäre Alteration und Verwitterung greifen insbesondere an offenen Riss-Systemen an und führen zur Aufweitung der präexistierenden Mikrorisse sowie zur Bildung neuer Riss-Systeme (z.B. Winkler 1994; Siegesmund et al. 2000) bis hin zum vollständigen Kohäsionsverlust.

In dem umfassenden Probensatz mit seiner großen Bandbreite an mineralogischen und genetischen Variationen kommen nahezu sämtliche in der Literatur beschriebenen Typen von 


\section{$\underline{\text { 5. Gesteinsmaterial }}$}

Mikrorissen (z.B. Kranz 1983; Passchier \& Trouw 1998; Blenkinsop 2000) vor. Zur Nomenklatur siehe Kap.2.3.2. Intra- und transgranulare Risse sind ebenso wie Korngrenzenrisse als offene, verheilte und mineralisierte Risse verbreitet. Prinzipiell überwiegen bei allen Risslagen die offenen Risse, wobei im Einzelfall auch verheilte oder mineralisierte Risse als dominantes Riss-System auftreten können. In Anhang II sind sämtliche Riss-Systeme der unterschiedlichen Proben nach dem Mineralbestand aufgeschlüsselt. Innerhalb dieses Kapitels werden die Gesteine bezüglich ihrer offenen Risse charakterisiert, klassifiziert und mit dem Makrogefüge verglichen. Den offenen Rissen, ihrer Geometrie, Quantität und bevorzugten Orientierung im Gestein kommt eine große Bedeutung im Hinblick auf die technischen Gesteinseigenschaften zu (z.B. Peck et al. 1985; Sheorey 1997; Prikryl 1998; Brosch et al. 2000; Stravogin \& Tarasov 2001). Betrachtet man zunächst die Zugspannungen, so ist der offene Riss mit einer Kohäsion von Null das mechanisch schwächste Element im Gestein. Bei Scherspannungen spielt auch die Beschaffenheit der Rissoberfläche eine Rolle (z.B. Barton \& Choubey 1977; Haberfield \& Johnston 1994; Grasselli \& Egger 2003). In beiden Fällen kommt es zu einer Spannungskonzentration an der Riss-Spitze in Ausbreitungsrichtung; daher sind offene Mikrorisse potenzielle „Keime“ der Rissausbreitung und des Gesteinsversagens gegenüber mechanischer Belastung. Sowohl die Rissgeometrie als auch die Orientierung eines Risses zu den einwirkenden Spannungen sowie das elastische Verhalten des Gesteins sind Einflussgrößen (Griffith-Kriterium: z.B. Wittke 1984; Eisbacher 1996; Twiss \& Moores 1997). Weitere Parameter, die wesentlich durch die Anzahl und die räumlichen Ausmaße offener Risse bestimmt werden, sind die Wasseraufnahme und die elastischen Gesteinseigenschaften (z.B. Fitzner 1988; Siegesmund et al. 1991; Meglis et al. 1996), aber auch der Einfluss auf die thermische Dilatation eines Gesteins wird diskutiert (Siegesmund et al. 2000).

Im Rahmen dieser Arbeit werden die offenen Mikrorisse mittels Kompressionswellengeschwindigkeiten (Vp) quantifiziert. Dabei wird die Differenz (Vp $\left.p_{Q R i s s}\right)$ aus dem Voigt-Reuss-Hill-Mittel ( $\left(\mathrm{Vp}_{\mathrm{GVHR}}\right)$ des Gesamtgesteins und dem Mittelwert der trockenen Kugelmessung ( $\left.\mathrm{Vp}_{\text {trockm}}\right)$ als $\mathrm{Maß}$ für die Quantität (Rissdichte/Rissfrequenz) aller offenen Risse im Gestein verwendet. Die Anisotropie $\left(\mathrm{A}_{\Delta \mathrm{Vp}}\right)$ wird aus den maximalen und minimalen $\Delta \mathrm{Vp}$ des Differenzisolinienplots der Kugelmessung abgeschätzt (vgl. Kap.2.4.5.1). Weitere Informationen bezüglich der offenen Mikrorisse geben die Porenradienverteilung und die zur Charakterisierung des Porenraums verwendete effektive Porosität, die eine gute lineare Korrelation zu den $\mathrm{Vp}_{\mathrm{QRiss}}-$ Werten zeigt (Abb.5-17). 


\section{$\underline{\text { 5. Gesteinsmaterial }}$}

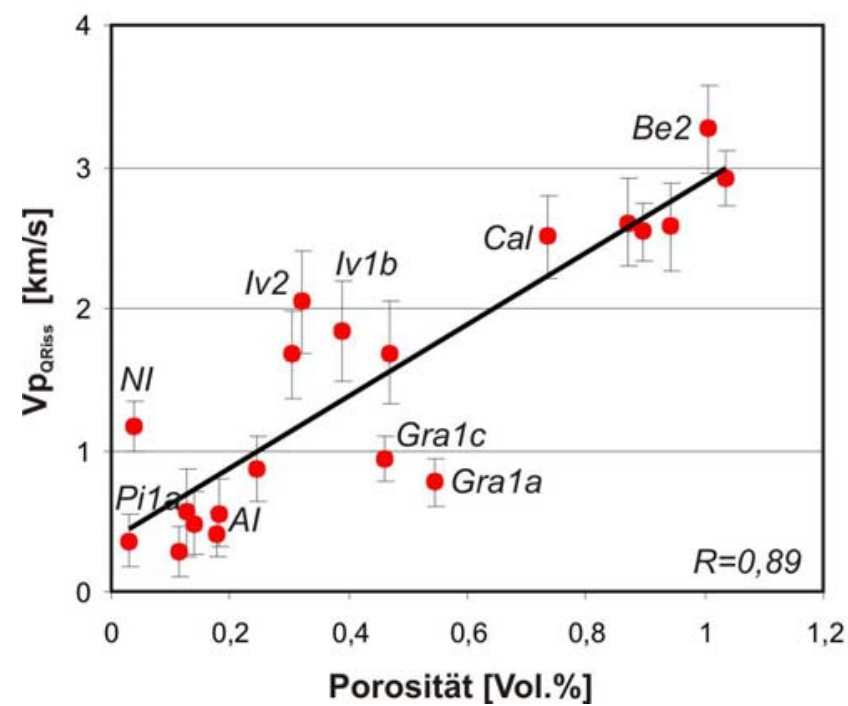

Abb.5-17: Zusammenhang zwischen dem Rissquantifizierungsparameter ( $\left.V p_{Q R i s s}\right)$ und der effektiven Porosität. NI - Gabbro-Norit. Pi1a - Peridotit. Al - Quarzit. Gra1a/1c - granitischer Gneis. Iv1b granodioritischer Mylonit. Iv2 - granodioritischer Ultramylonit. Cal - granodioritischer Gneis. Be2 granitischer Gneis. Fehlerbalken markieren 1-s-Fehler. 


\section{Gesteinsmaterial}

\subsubsection{Gruppe I - isotrope Gesteine}

Die $V p_{\mathrm{QRiss}}-$ Werte der Gruppe I rangieren zwischen 0,28 km/s beim Granit (Koess) und 2,91 $\mathrm{km} / \mathrm{s}$ beim Granit (MrGr3). Dazwischen liegen der Granodiorit (PDD) mit 0,86 km/s und der Gabbro-Norit (NI) mit 1,17 km/s. Der Mittelwert der Gruppe I von 1,31 km/s hat aufgrund der großen Variation wenig Aussagekraft. Die Anisotropie liegt zwischen 30\% und 87\%; der Mittelwert beträgt 47\% (Abb.5-18).
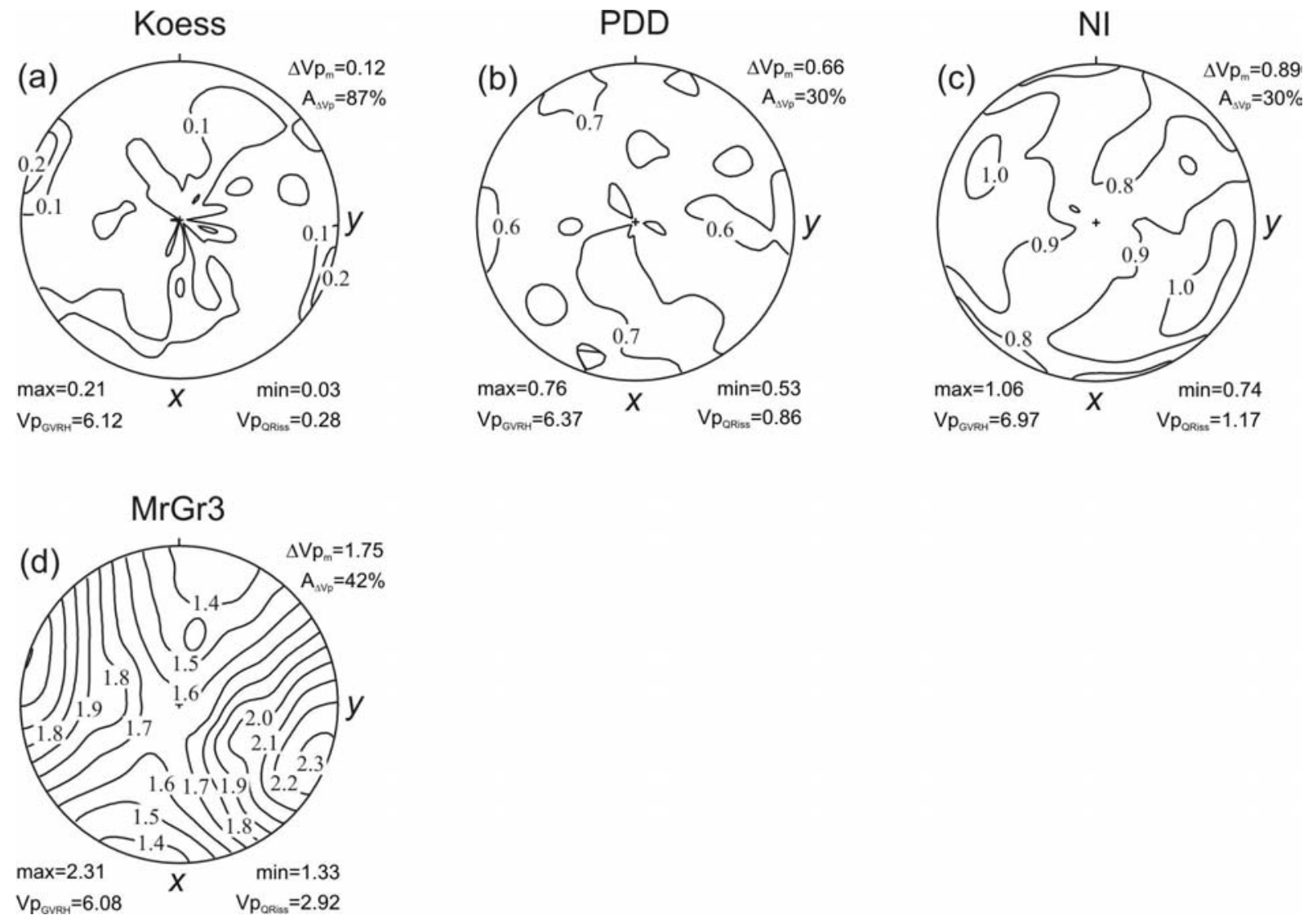

Abb.5-18: Isolinienplot der $\Delta V p$-Werte aus der Kugelmessung. Richtung $x, y$ des Referenzsystems sind bezeichnet; z senkrecht zur Papierebene. $p$-Wellengeschwindigkeiten in [km/s]. Der Fehler der $\Delta V p$-Werte beträgt +/-0,1 km/s. $\Delta V p_{m}$ - Mittelwert der richtungsabhängigen $\Delta V p-W e r t e . A_{\Delta V p}-$ Anisotropie der $\Delta V p$-Werte [\%]. $V p_{G V H R}-$ aus Modalbestand und Voigt-Reuss-Hill-Vp der Minerale berechnete Gesamtgesteins-Vp. V $p_{Q R i s s}$ - zur Rissquantifizierung verwendete Differenz $V p_{G V H R^{-}}$ $V p_{\text {trockm. }}$ (a) Granit. (b) Granodiorit. (c) Gabbro-Norit. (d) Granit.

\subsubsection{Gruppe II - anisotrope Gesteine}

Die $V p_{\mathrm{QRiss}}$-Werte liegen zwischen 0,36 km/s für den Peridotit (Pila) und 1,69 km/s für den granodioritischen Mylonit (Iv1). Der Mittelwert beträgt $0,87 \mathrm{~km} / \mathrm{s}$. Die Anisotropie ist beim Quarzit (AI) mit 100\% am höchsten und beim Meta-Gabbro (Mg1b) mit 21\% am niedrigsten. Der Mittelwert liegt bei 56\%, wobei die Probe Pila nicht berücksichtigt wurde. Hier liegt die Anisotropie im Bereich des Messfehlers und birgt daher keine verwertbare Aussage (Abb.519). 


\section{$\underline{\text { 5. Gesteinsmaterial }}$}

Pi1a

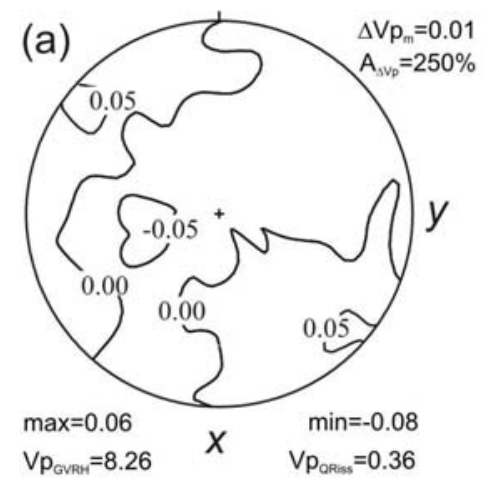

$\mathrm{Mg} 1 \mathrm{~b}$

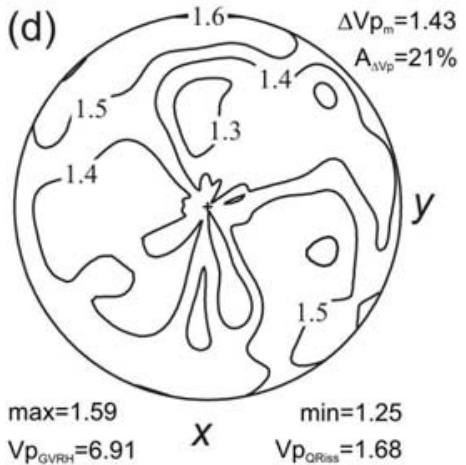

Rek

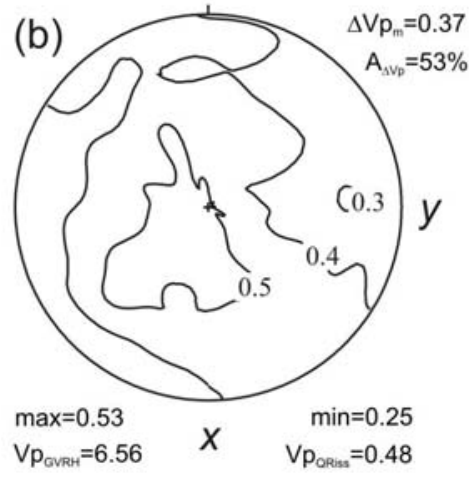

Mg2

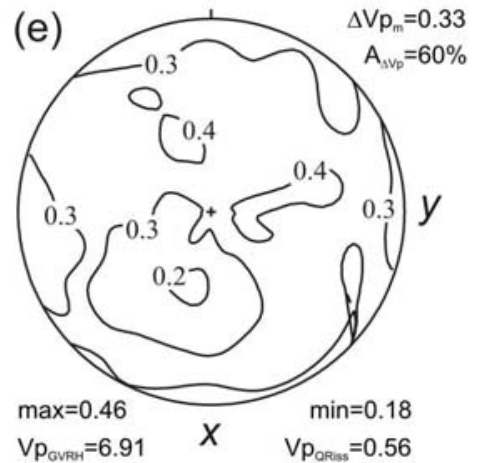

Al

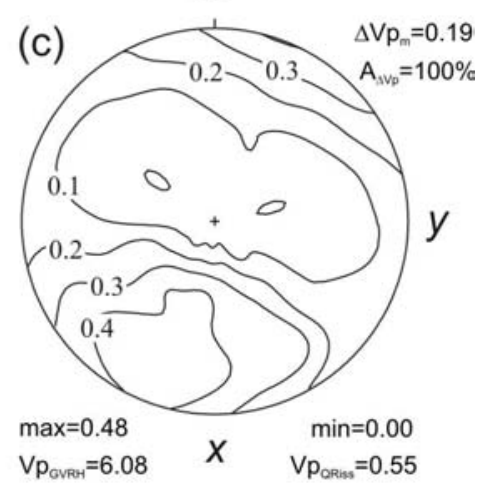

$\operatorname{lv} 1$
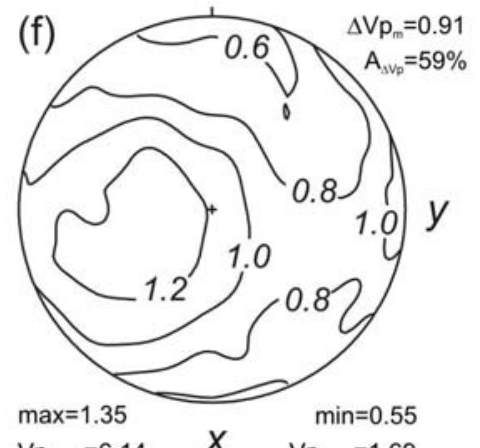

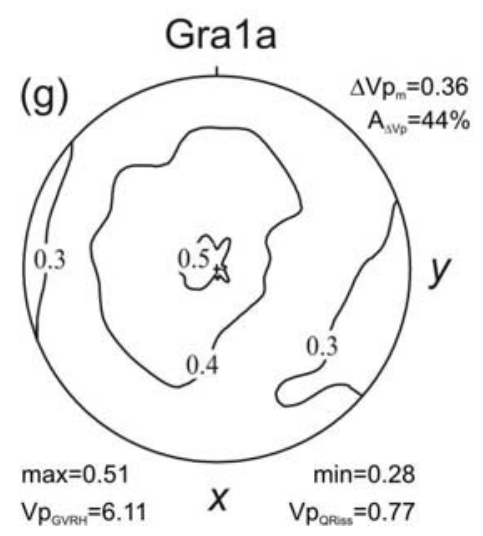

Abb.5-19: Isolinienplot der $\Delta V p$-Werte aus der Kugelmessung. Richtung $x$, y des Referenzsystems sind bezeichnet; z senkrecht zur Papierebene. p-Wellengeschwindigkeiten in [km/s] Der Fehler der $\Delta V p$-Werte beträgt $+/-0,1 \mathrm{~km} / \mathrm{s} . \quad \Delta V p_{m}$ - Mittelwert der richtungsabhängigen $\Delta V p$-Werte. $A_{\Delta V p}-$ Anisotropie der $\Delta V p$-Werte [\%]. V $p_{G V H R}$ - aus Modalbestand und Voigt-Reuss-Hill-Vp der Minerale berechnete Gesamtgesteins-Vp. V $p_{Q R i s s}$ - zur Rissquantifizierung verwendete Differenz $V p_{G V H R^{-}}$ $V p_{\text {trockm. }}$ (a) Peridotit. (b) Kalzit-Marmor. (c) Quarzit. (d, e) Meta-Gabbro. (f) Granodioritischer Protomylonit. (g) Granitischer Gneis. 


\subsubsection{Gruppe III - ausgeprägt anisotrope Gesteine}

In der Gruppe III weist der granitische Gneis (Be2) die höchste Dichte der offenen Risse $\left(\mathrm{Vp}_{\mathrm{QRiss}}=3,27 \mathrm{~km} / \mathrm{s}\right.$ ) und der granitische Ultramylonit (Gra3a) die geringste Rissdichte $\left(\mathrm{Vp}_{\mathrm{QRiss}}{ }^{=} 0,41 \mathrm{~km} / \mathrm{s}\right)$ auf $(\boldsymbol{A b b} .5-20)$.
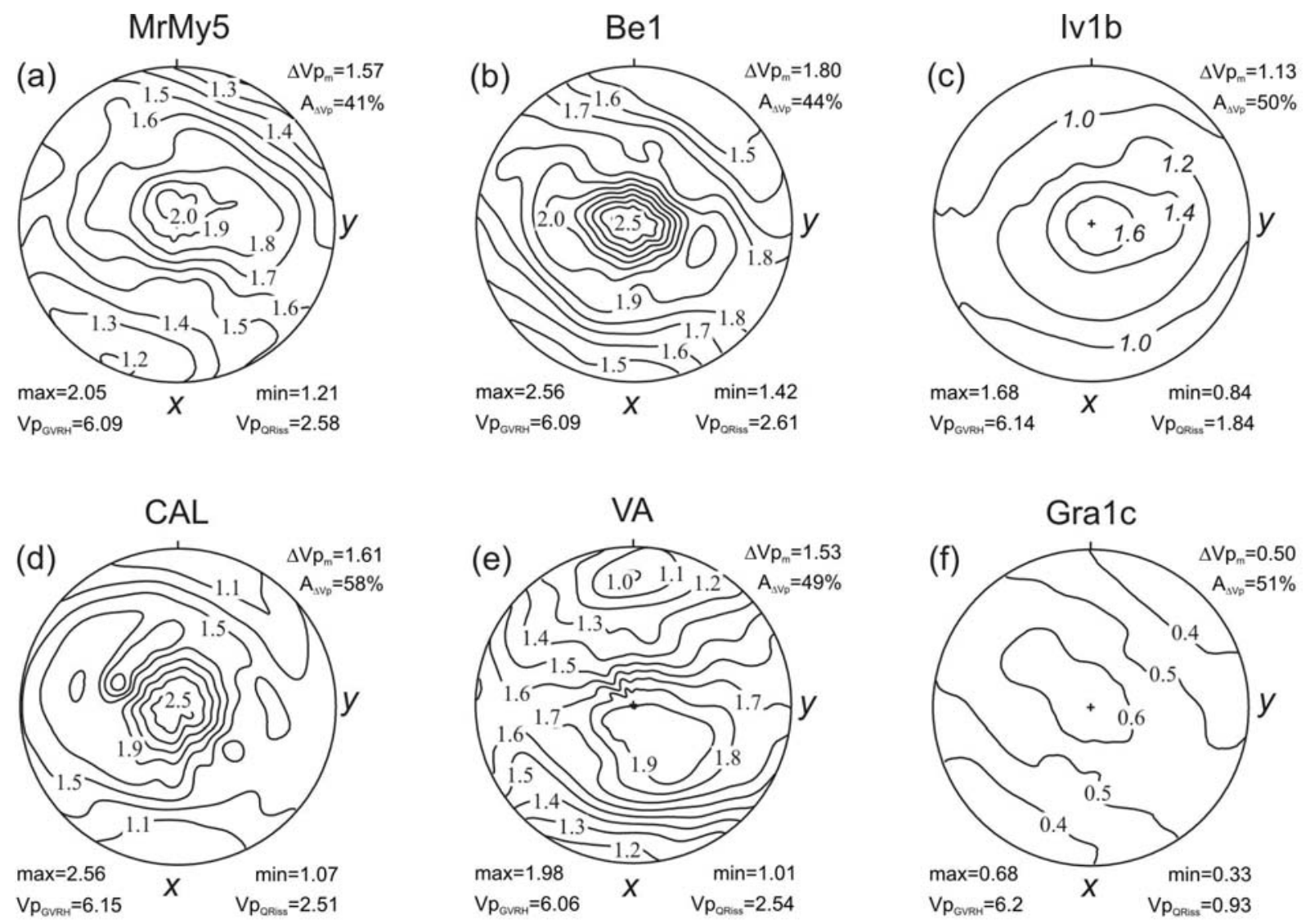

\section{$\mathrm{Be} 2$}
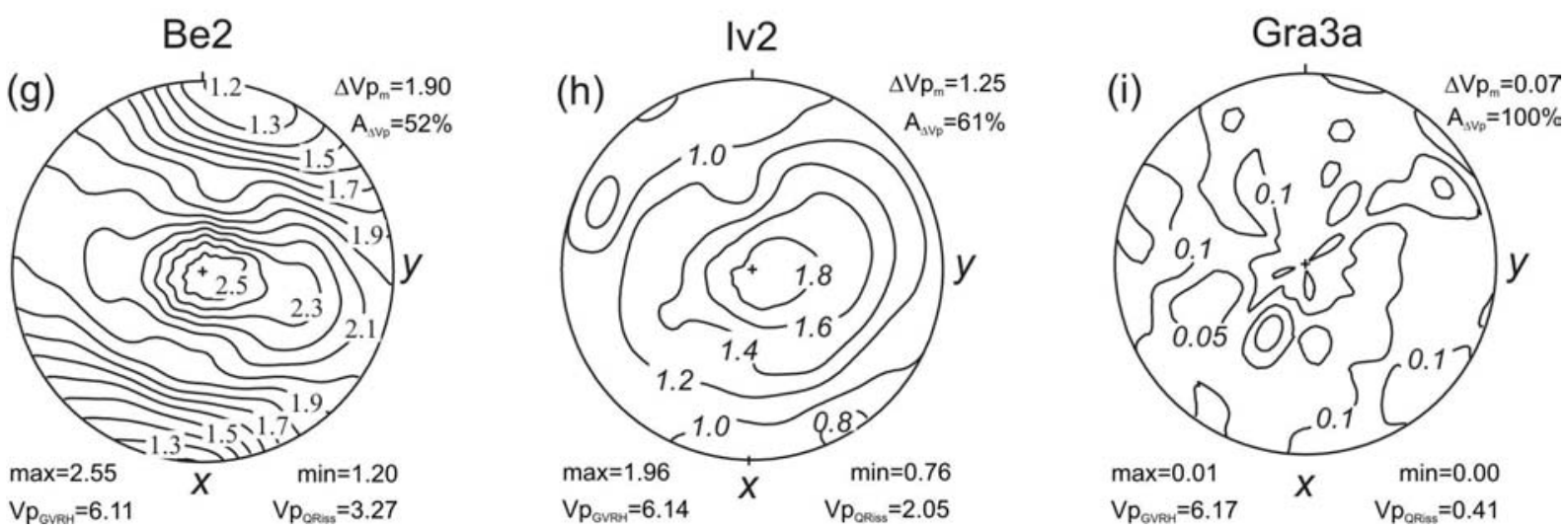

Abb.5-20: Isolinienplot der $\Delta V p$-Werte aus der Kugelmessung. Richtung $x$, y des Referenzsystems sind bezeichnet; z senkrecht zur Papierebene. p-Wellengeschwindigkeiten in [km/s]. Der Fehler der $\Delta V p$-Werte beträgt $+/-0,1 \mathrm{~km} / \mathrm{s} . \Delta V p_{m}-$ Mittelwert der richtungsabhängigen $\Delta V p$-Werte. $A_{\Delta v_{p}}-$ Anisotropie der $\Delta V p$-Werte [\%]. V $p_{G V H R}$ - aus Modalbestand und Voigt-Reuss-Hill-Vp der Minerale berechnete Gesamtgesteins-Vp. V $p_{\text {QRiss }}$ - zur Rissquantifizierung verwendete Differenz $V p_{G V H R^{-}}$ $V p_{\text {trockm. }}$ (a) Granitischer Protomylonit. (b) Granodioritischer Gneis. (c) Granodioritischer Mylonit. (d) Granodioritischer Gneis. (e) Granitischer Gneis. (f) Granitischer Gneis. (g) Granitischer Gneis. (h) Granodioritischer Ultramylonit. (i) Granitischer Ultramylonit. 


\section{$\underline{\text { 5. Gesteinsmaterial }}$}

Hier, wie auch schon in der Gruppe I, ist der Mittelwert aller Proben von $\mathrm{Vp}_{\mathrm{QRiss}}=2,08 \mathrm{~km} / \mathrm{s}$ aufgrund der großen Streuung der Daten nicht aussagekräftig. Die Werte der Anisotropie schwanken zwischen $61 \%$ für den granodioritischen Ultramylonit (Iv2) und $41 \%$ für den granitischen Protomylonit (MrMy5). Der 100\%-Wert der Probe Gra3a wurde nicht verwendet, da die rissbedingte Anisotropie im Bereich des Messfehlers liegt. Im arithmetischen Mittel beträgt die Anisotropie 51\%.

\subsubsection{Gruppierung nach der Rissdichte offener Mikrorisse}

Im Gegensatz zu den Texturen zeigt sich keine eindeutige Übereinstimmung von mikrorissinduzierter Anisotropie und der beobachteten makroskopischen Gefügeanisotropie. Die Anisotropie-Mittelwerte der Gruppen I bis III sind sich mit 47\%, 56\% bzw. 51\% sehr ähnlich und lassen nicht auf eine Zunahme der rissbedingten Gefügeanisotropie von der Gruppe I zur Gruppe III schließen. Dieser Effekt ist zum einen durch die Art der Berechnung erklärbar (der $\Delta \mathrm{Vp}_{\max }-$ Wert, auf den die Anisotropien bezogen werden, schwankt innerhalb des Probensatzes zwischen $0,06 \mathrm{~km} / \mathrm{s}$ und $2,56 \mathrm{~km} / \mathrm{s}$ ), zum anderen finden sich in jeder Gruppe Proben mit Anisotropie-Werten, die weit außerhalb des Mittelwertes liegen. Mit Ausnahme der Probe Gra3a und den annähernd regellosen Gesteinen Koess und PDD sowie der Probe AI (keine Glimmertexturen bestimmt) zeigen alle phyllosilikathaltigen Proben eine bevorzugte Orientierung der offenen Risse parallel zur Verteilung der Glimmerspaltflächen (001-Flächen), deren maximale Rissdichte/Rissfrequenz (maximale $\Delta \mathrm{Vp}$-Werte) an den Regelungsgrad und den Volumenanteil der jeweiligen Phyllosilikate gekoppelt ist. Tendenziell weisen Gesteine mit einem hohen Gehalt an Phyllosilikaten und einer starken Textur auch eine hohe rissbedingte Anisotropie und eine hohe maximale Rissdichte/Rissintensität auf. Dieser beobachtbare Zusammenhang lässt sich jedoch nicht verallgemeinern und mit Sicherheit nicht quantifizieren. Zudem haben andere, nicht an die Glimmerspaltflächen gebundene Rissscharen einen Einfluss auf die $\Delta \mathrm{Vp}$-Verteilung und verursachen z.T. eine Rotation oder Verzerrung des $\Delta \mathrm{Vp}$-Musters gegenüber den Eigenvektoren/Hauptachsen der Glimmertexturen. Daher stimmen die Achsen der Mikrorissverteilungen häufig auch nicht mit den Achsen der makroskopischen Gefüge überein. Alles in allem existiert zwischen den Mustern der bevorzugten Mikrorissorientierung und den Makrogefügen keine ausreichende Übereinstimmung, die eine Einteilung nach den Mikrorissen analog zu den Makro-Gefüge-Gruppen erlaubt. Aus diesem Grund werden für eine spätere Bewertung des Mikrorisseinflusses auf die technischen Gesteinseigenschaften 


\section{Gesteinsmaterial}

Rissklassen definiert. Das Kriterium für die Gruppierung ist die Rissdichte. Die Rissklassen $A_{R}$ (geringe Rissdichte), $B_{R}$ (mittlere Rissdichte) und $C_{R}$ (hohe Rissdichte) sind in Tab.5-3 definiert.

Tab.5-3: Rissklassen

\begin{tabular}{|c|c|c|c|}
\hline & $\begin{array}{c}\text { Rissklasse } A_{R}-\text { geringe } \\
\text { Rissdichte }\end{array}$ & $\begin{array}{c}\text { Rissklasse } B_{R}-\text { mittlere } \\
\text { Rissdichte }\end{array}$ & $\begin{array}{c}\text { Rissklasse } C_{R}-\text { hohe } \\
\text { Rissdichte }\end{array}$ \\
\hline $\begin{array}{c}\text { Definition der } \\
\text { Klassen- } \\
\text { merkmale }\end{array}$ & $V p_{Q R i s s}<0,8 \mathrm{~km} / \mathrm{s}$ & $\begin{array}{c}0,8 \mathrm{~km} / \mathrm{s} \leq \bigvee p_{\text {QRiss }} \leq 1,8 \\
\mathrm{~km} / \mathrm{s}\end{array}$ & $V p_{Q R i s s}>1,8 \mathrm{~km} / \mathrm{s}$ \\
\hline $\begin{array}{c}\text { zugehörige } \\
\text { Proben }\end{array}$ & $\begin{array}{c}\text { Rissklasse } A_{R}-\text { geringe } \\
\text { Rissdichte }\end{array}$ & $\begin{array}{c}\text { Rissklasse } B_{R}-\text { mittlere } \\
\text { Rissdichte }\end{array}$ & $\begin{array}{c}\text { Rissklasse } C_{R}-\text { hohe } \\
\text { Rissdichte }\end{array}$ \\
\hline & $\begin{array}{c}\text { Granit (Koess) } \\
\text { Peridotit (Pi1a) } \\
\text { granit. Ultramylonit (Gra3a) } \\
\text { Kalzit-Marmor (Rek) } \\
\text { Quarzit (Al) } \\
\text { Meta-Gabbro (Mg2) } \\
\text { granit. Gneis (Gra1a) }\end{array}$ & $\begin{array}{c}\text { Granodiorit (PDD) } \\
\text { granit. Gneis (Gra1c) } \\
\text { Gabbro-Norit (NI) } \\
\text { Meta-Gabbro (Mg1b) } \\
\text { granod. Protomylonit (IV1) }\end{array}$ & $\begin{array}{c}\text { granod. Mylonit (Iv1b) } \\
\text { granod. Ultramylonit (Iv2) } \\
\text { granod. Paragneis (Cal) } \\
\text { granit. Orthogneis (VA) } \\
\text { granit. Protomylonit (MrMy5) } \\
\text { granod. Gneis (Be1) } \\
\text { Granit (MrGr3) } \\
\text { granit. Gneis (Be2) }\end{array}$ \\
\hline
\end{tabular}

$\mathrm{Ob}$ eine ausgeprägte bevorzugte Orientierung offener Riss-Systeme eine Auswirkung auf die Anisotropie der technischen Gesteinseigenschaften hat, hängt maßgeblich von der Rissdichte ab. So kann beispielsweise ein einzelner Mikroriss (sehr klein im Verhältnis zu den Abmessungen, der beim Ermitteln der technischen Eigenschaft benutzten Probe) mit einer Anisotropie von 100\% nur einen sehr geringen Einfluss auf die Gesamtgesteinsanisotropie haben.

\subsubsection{Beispiele für offene Mikrorisse in den untersuchten Gesteinen}

Das folgende Kapitel beinhaltet eine repräsentative Auswahl offener Mikrorisse und deren Lokalisation. Die erste Folge von mikroskopischen Dünnschliffbildern fokussiert auf die bevorzugte Orientierung offener Mikrorisse entlang der Spaltbarkeit der gesteinsbildenden Minerale (Abb.5-21). Es handelt sich dabei zumeist um intrakristalline Risse. Die zweite Serie zeigt intra-, inter- und transgranulare Risse, die keiner bestimmten kristallographischen Fläche folgen (Abb.5-22). Die Richtungen des Probenreferenzsystems sind in den Abbildungen gekennzeichnet und die dargestellten Mikrorisse und Minerale hervorgehoben. 


\section{Gesteinsmaterial}
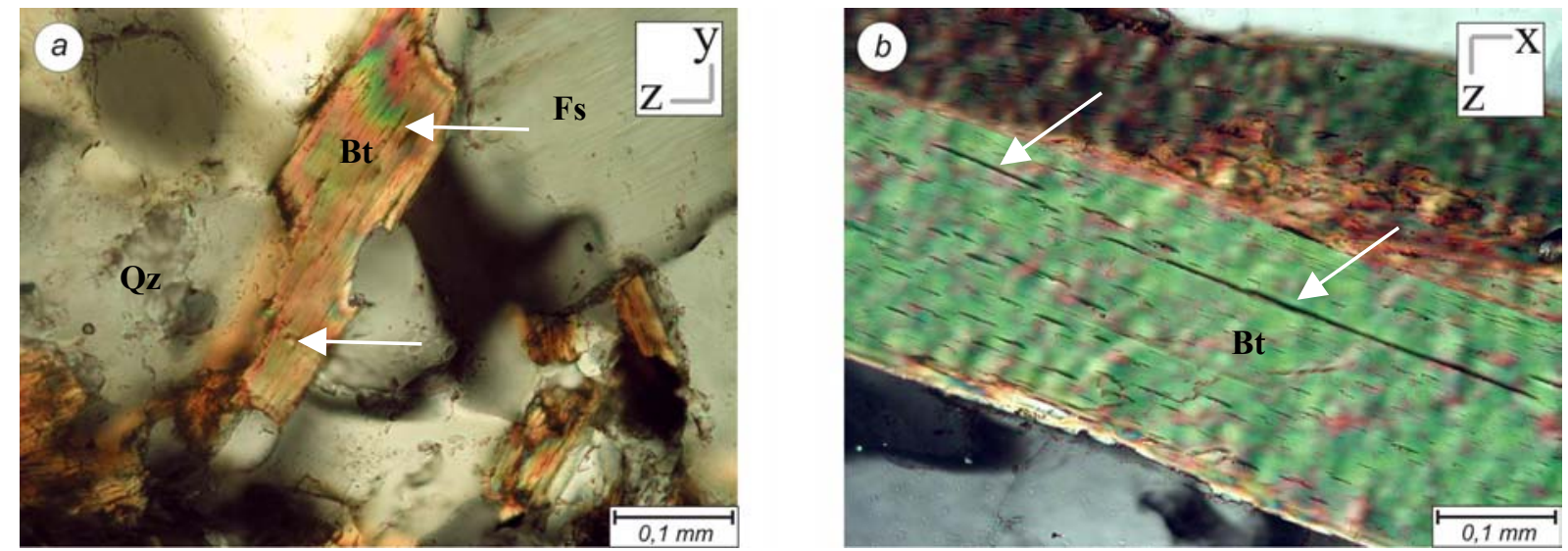

Abb.5-21ab: Mikrogefüge (Durchlicht, gekreuzte Polarisatoren). ( $a, b)$ Offene Mikrorisse in Biotit, die der (001)-Fläche folgen. (a) Probe Gra1a (granitischer Gneis). (b) Probe Cal (granodioritischer Gneis). Qz - Quarz. Bt - Biotit. Fs - Feldspat.
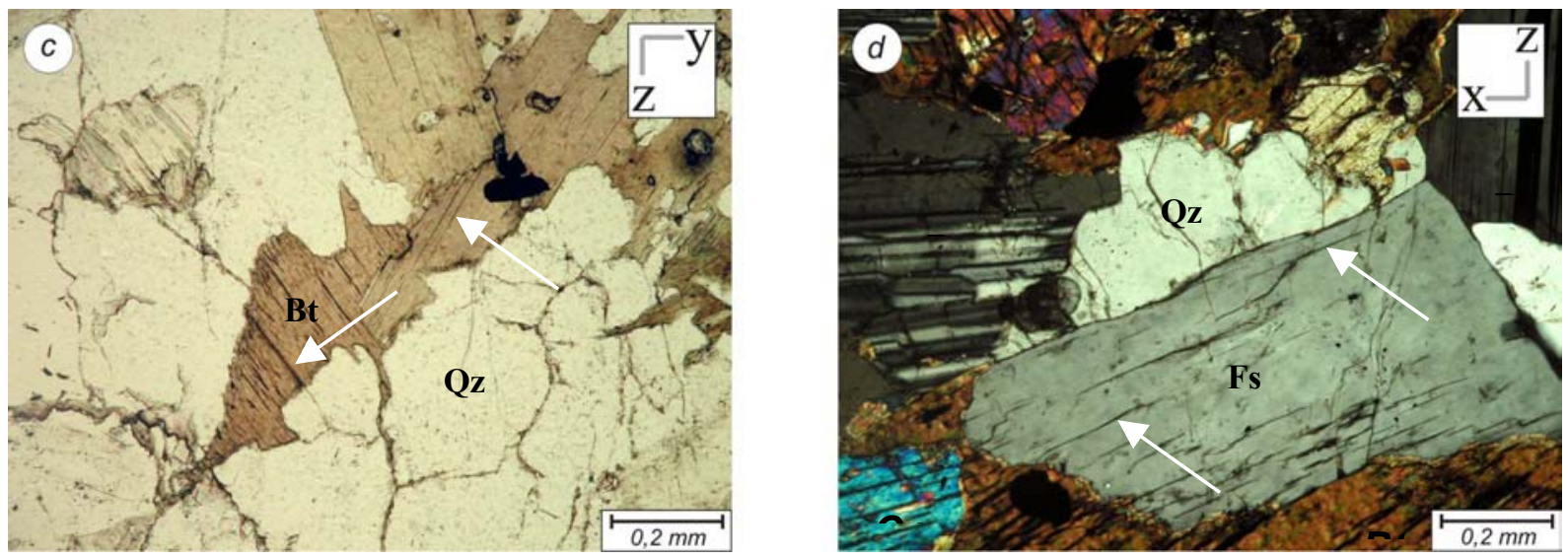

Abb.5-21cd: Mikrogefüge (Durchlicht) (c); Mikrogefüge (Durchlicht, gekreuzte Polarisatoren) (d). (c) Offene Mikrorisse in Biotit (parallel zur (001)-Fläche) der Probe Koess (Granit). (d) Offene Spaltflächen in Feldspat der Probe PDD (Granodiorit). Bt - Biotit. Qz - Quarz. PI - Plagioklas. Fs Feldspat. Cpx - Klinopyroxen.
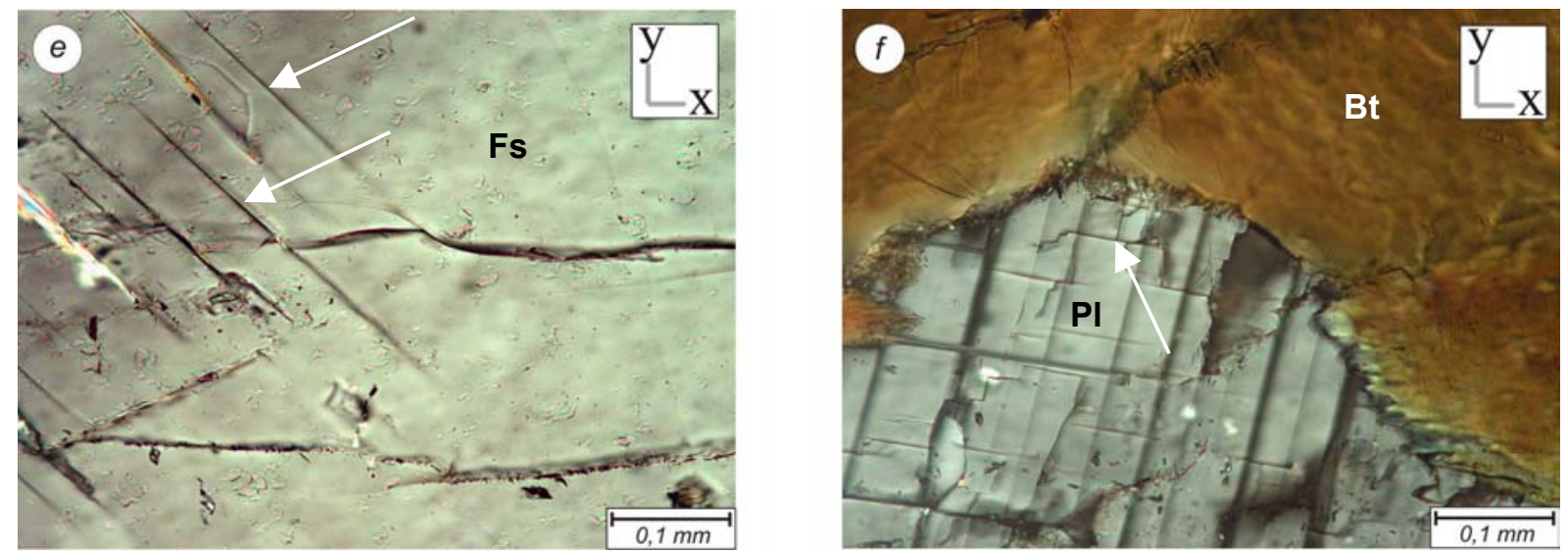

Abb.5-21ef: Mikrogefüge (Durchlicht, gekreuzte Polarisatoren). (e, f) Offene Spaltflächen in Feldspat der Probe NI (Gabbro-Norit) bzw. PDD (Granodiorit). Fs - Feldspat. Bt - Biotit. PI - Plagioklas. 


\section{Gesteinsmaterial}
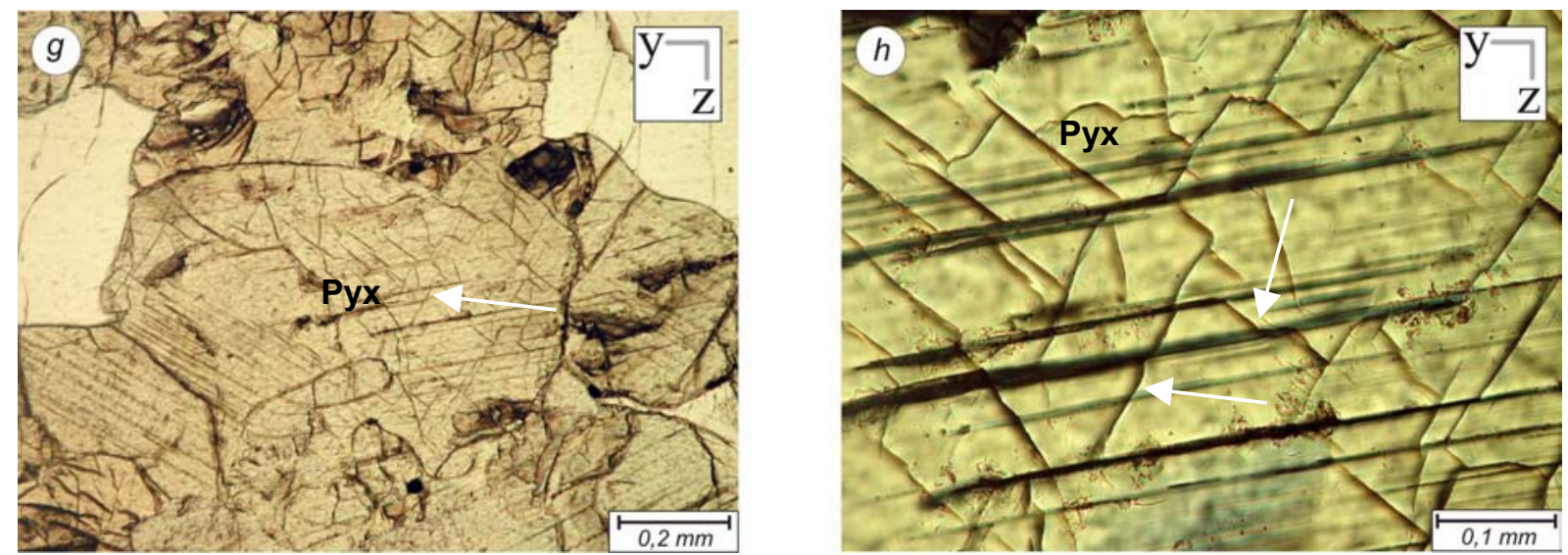

Abb.5-21gh: Mikrogefüge (Durchlicht). linear polarisiertes Licht. $(g, h)$ Offene Spaltflächen in Pyroxen der Probe NI (Gabbro-Norit). Pyx - Pyroxen.
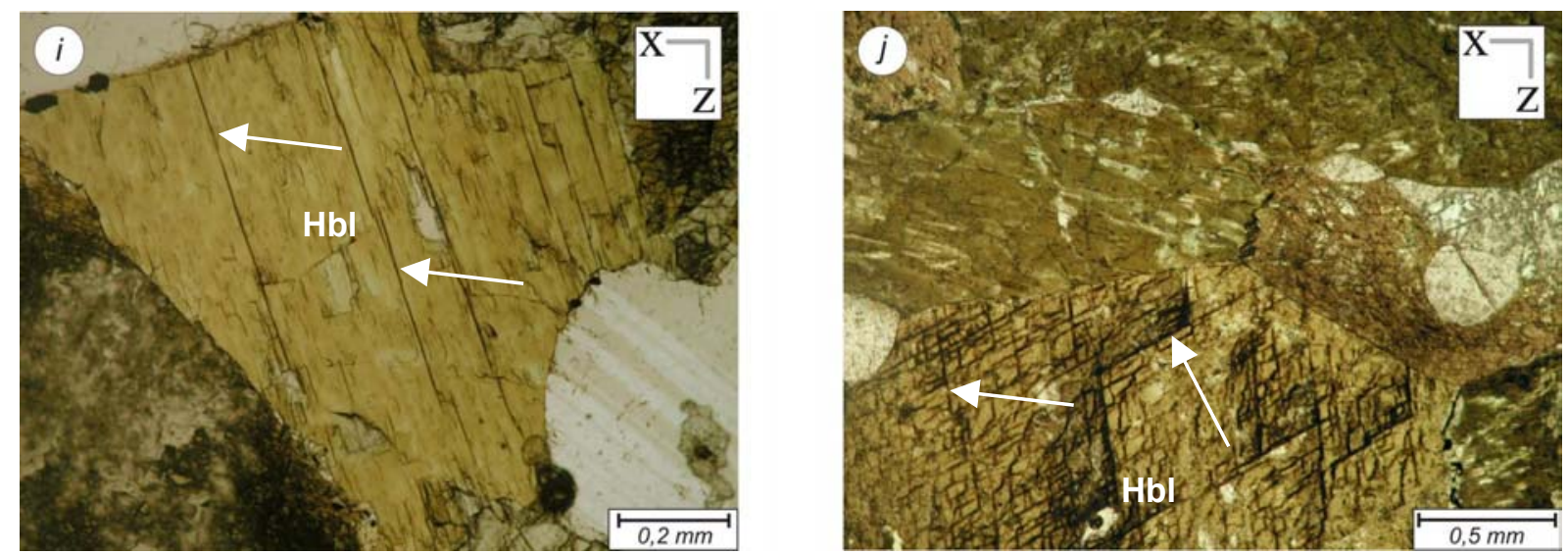

Abb.5-21ij: Mikrogefüge (Durchlicht). (i, j) Offene Spaltflächen in Hornblende in unterschiedlichen Schnittlagen. Proben Mg1b (i) und Mg2 (j); Meta-Gabbros. Hbl - Hornblende.
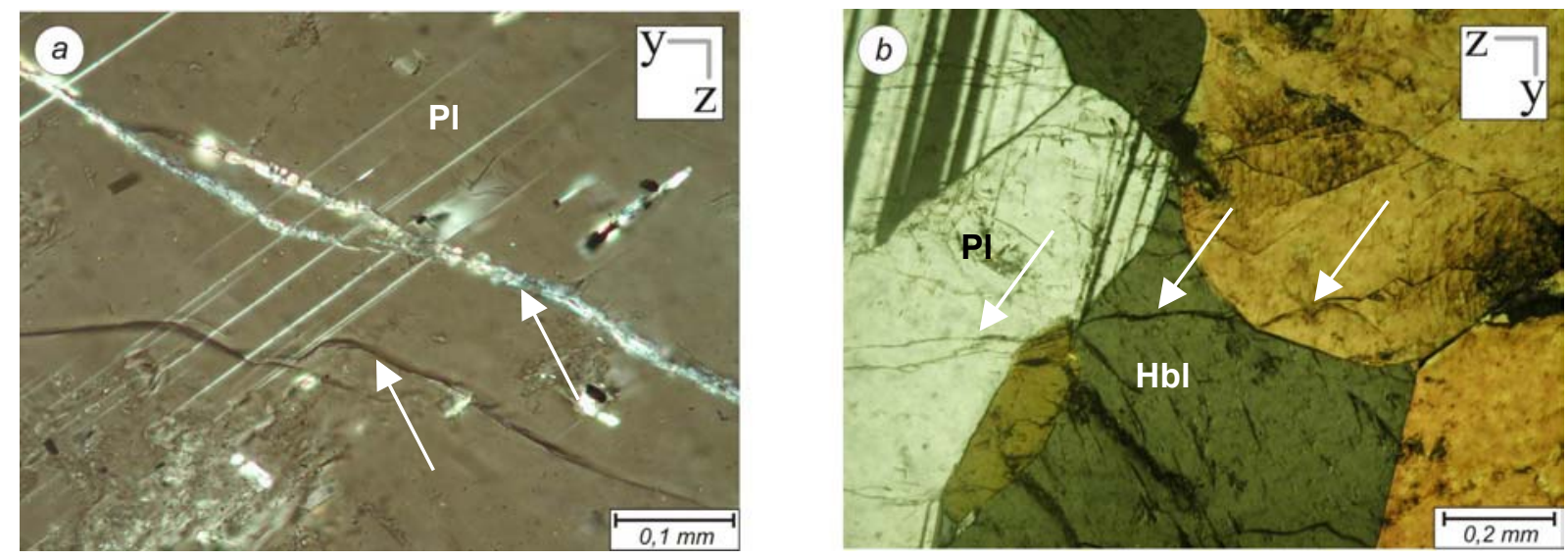

Abb.5-22ab: Mikrogefüge (Durchlicht, gekreuzte Polarisatoren). (a) Intrakristalliner Riss in Plagioklas (Probe NI - Gabbro-Norit). (b) Intra- und transgranulare Risse in Plagioklas und Hornblende (Probe Mg2 - Meta-Gabbro). Pl- Plagioklas. Hbl - Hornblende. 


\section{Gesteinsmaterial}
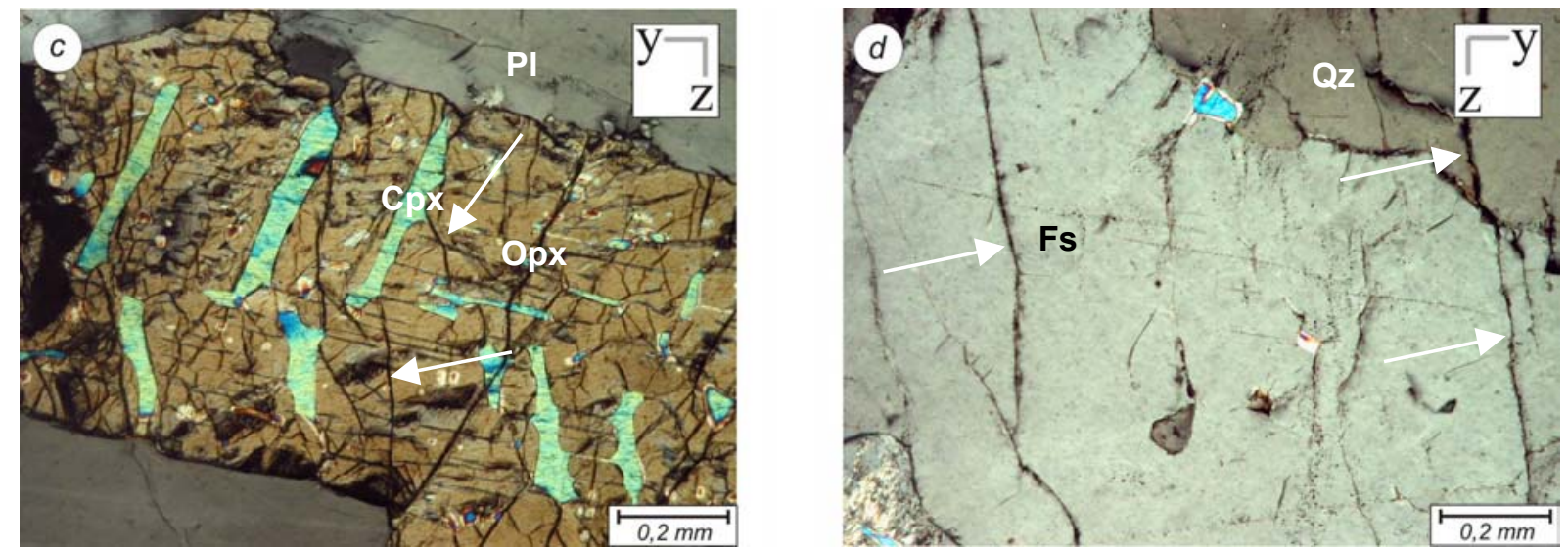

Abb.5-22cd: Mikrogefüge (Durchlicht, gekreuzte Polarisatoren). (c) Intrakristalline Risse in Orthopyroxen (mit Entmischungslamellen nach Klinopyroxen) der Probe NI (Gabbro-Norit). (d) Intraund transgranulare Risse in Feldspat und Quarz der Probe Koess (Granit). Opx - Orthopyroxen. Cpx - Klinopyroxen. Fs - Feldspat. Qz - Quarz.
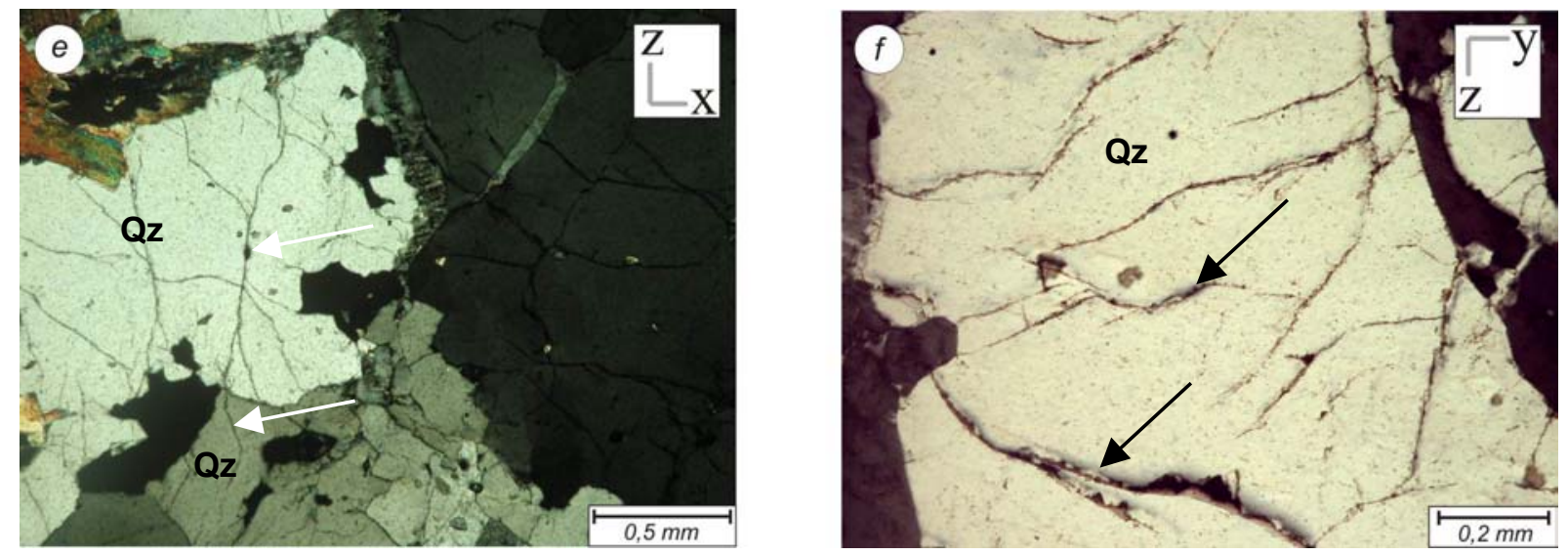

Abb.5-22ef: Mikrogefüge (Durchlicht, gekreuzte Polarisatoren). (e) Intra- und transgranulare Risse in Quarz (Probe Koess - Granit). (f) Intragranulare Risse in Quarz (Probe Koess - Granit). Qz - Quarz.
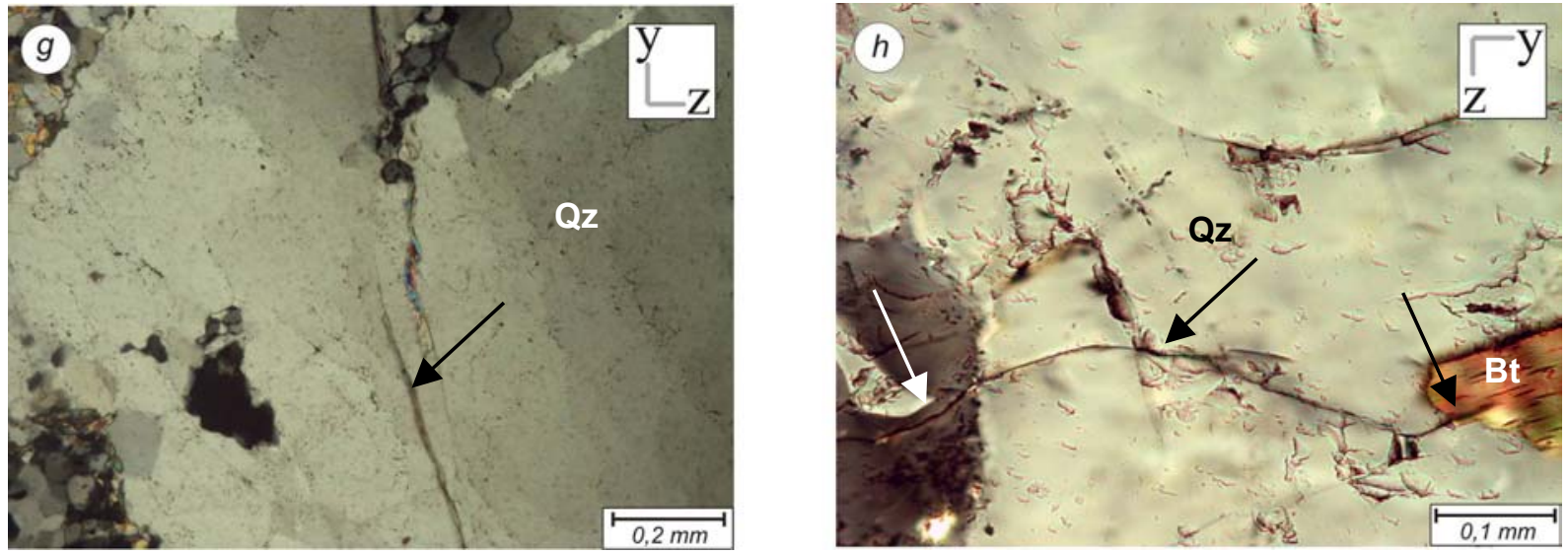

Abb.5-22gh: Mikrogefüge (Durchlicht, gekreuzte Polarisatoren). (g) Intrakristalliner Riss in Quarz der Probe Iv1 (granodioritischer Protomylonit). (h) Intra- und transgranulare Risse in Quarz und Biotit der Probe Cal (granodioritischer Gneis). Qz - Quarz. Bt - Biotit. 


\section{Gesteinsmaterial}
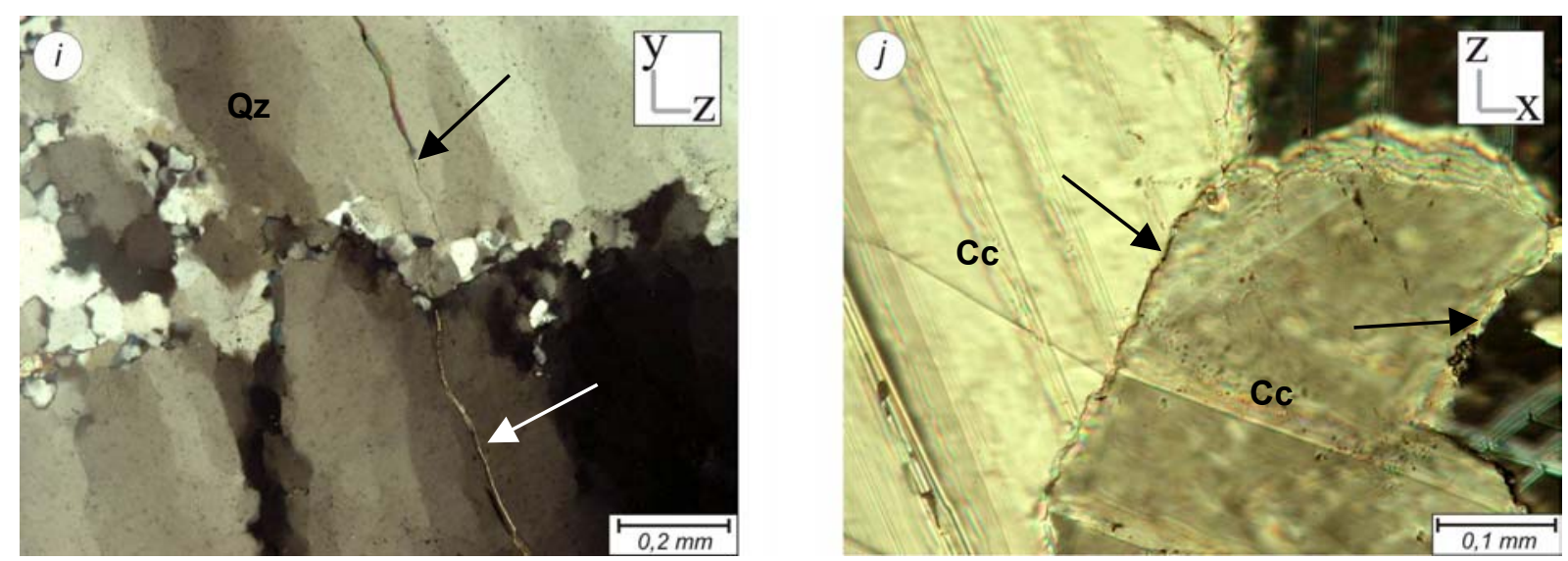

Abb.5-22ij: Dünnschliffphotos; gekreuzte Polarisatoren. (i) Transgranularer Riss in Quarz (Probe Iv1 granodioritischer Protomylonit). (j) Mikroverzahnte und teilweise offene Korngrenze am Übergang von Kalzit zu Kalzit (Probe Rek - Kalzit-Marmor).

\subsection{Korngröße}

Die Korngröße hat einen Einfluss auf die mechanischen Gesteinseigenschaften. So beobachtete Prikryl (1998) eine Antikorrelation von der mittleren Korngröße und der Druckfestigkeit von Graniten. Zu vergleichbaren Ergebnissen kommen Olsson (1974) und Hugman \& Friedmann (1979) für Marmore bzw. Karbonate sowie Onodera \& Kumara (1980) für kristalline Gesteine. Ansonsten und sind die Aussagen vage. Peschel (1983) spricht allgemein von einer Beeinflussung der physikalisch-technischen Eigenschaften durch die Korngröße, und Hirschwald (1912) bemerkte eine Änderung der Kornbindungseigenschaften mit variierender Korngöße. Beide Aussagen werden nicht näher spezifiziert.

Die Quantifizierung der Korngrößen birgt eine Reihe von Schwierigkeiten. Zum einen liegen verschiedene Mineralbestandteile eines Gesteins in zum Teil völlig unterschiedlichen Korngrößen vor, zum anderen weisen viele der untersuchten Gesteine eine deutliche Kornformanisotropie auf, die eine dreidimensionale Betrachtung zur Erfassung der Korngeometrie erfordert. Eine mittlere Korngröße ist daher nicht für alle Schnittlagen repräsentativ und damit für stark deformierte Gesteine eine wenig aussagekräftige Größe. Eine vollquantitative und nach dem Mineral aufgeschlüsselte Analyse erscheint aus diesem Grund wenig sinnvoll und wurde nicht durchgeführt.

Im Rahmen dieser Arbeit werden die Gesteine semiquantitativ nach ihrer Korngröße eingeteilt. Dabei wird das in Tab.5-4 dargestellte Schema von de Quervain (1967) verwendet, wobei die Einordnung nach der am Mikroskop abgeschätzten, vorwiegenden mittleren Korngröße aller Minerale aus den drei Schnittlagen senkrecht zu den Achsen des Referenzsystems vorgenommen wurde. 


\section{Gesteinsmaterial}

Tab.5-4: Korngrößen der Gesteine

\begin{tabular}{|c|c|c|}
\hline nach de Quervain (1967) & $\begin{array}{c}\text { Korngröße } \\
{[\mathrm{mm}]}\end{array}$ & zugehörige Proben \\
\hline extrem feinkörnig (ef) & $<0,2$ & granit. Ultramylonit (Gra3a) \\
\hline sehr feinkörnig (sf) & $0,2-0,5$ & $\begin{array}{c}\text { granit. Gneis (Gra1a) } \\
\text { granit. Gneis (Gra1c) } \\
\text { Quarzit (AI) }\end{array}$ \\
\hline feinkörnig (f) & $0,5-2$ & $\begin{array}{c}\text { granod. Ultramylonit (Iv2) } \\
\text { granit. Gneis (Be2) } \\
\text { Granit (MrGr3) } \\
\text { Kalzit-Marmor (Rek) } \\
\text { granod. Mylonit (Iv1b) } \\
\text { granod. Gneis (Cal) } \\
\text { granit. Gneis (VA) }\end{array}$ \\
\hline mittelkörnig (m) & $2-5$ & $\begin{array}{c}\text { Meta-Gabbro (Mg1b) } \\
\text { Meta-Gabbro (Mg2) } \\
\text { Granodiorit (PDD) } \\
\text { Gabbro-Norit (NI) } \\
\text { Peridotit (Pi1a) } \\
\text { granod. Protomylonit (Iv1) } \\
\text { granit. Protomylonit (Mrmy5) } \\
\text { granod. Gneis (Be1) }\end{array}$ \\
\hline grobkörnig (g) & $5-10$ & Granit (Koess) \\
\hline sehr grobkörnig (sg) & $>10$ & \\
\hline
\end{tabular}




\section{$\underline{\text { 5. Gesteinsmaterial }}$}

\subsection{Zusammenfassung}

Die Untersuchungen der mineralogischen und chemischen Zusammensetzung sowie der Gesteinsgefüge haben folgende Einteilungen der Gesteine ergeben (Tab.5-5):

Tab.5-5: Gruppierungen nach Mineralogie, Makrogefüge, Glimmertextur und offenen Mikrorissen

\begin{tabular}{|c|c|c|c|}
\hline Phyllosilikat & Gruppe $A<10$ Vol. $\%$ & Gruppe B 10-20 Vol.\% & Gruppe C >20 Vol. $\%$ \\
\hline $\begin{array}{l}\text { zugehörige } \\
\text { Proben }\end{array}$ & $\begin{array}{c}\text { granit. Gneis (Gra1a) } \\
\text { granit. Gneis (Gra1c) } \\
\text { granit. Ultramylonit } \\
\text { (Gra3a) } \\
\text { Quarzit (Al) }\end{array}$ & $\begin{array}{c}\text { Granodiorit (PDD) } \\
\text { Granit (Koess) } \\
\text { granit. Gneis (Be2) } \\
\text { granit. Protomyl. (MrMy5) } \\
\text { granod. Gneis (Be1) } \\
\text { Granit (MrGr3) } \\
\text { granod. Paragneis (Cal) }\end{array}$ & $\begin{array}{c}\text { granod. Protomylonit (Iv1) } \\
\text { granod. Mylonit (Iv1b) } \\
\text { granod. Ultramylonit (Iv2) } \\
\text { granit. Orthogneis (VA) }\end{array}$ \\
\hline $\begin{array}{l}\text { Makrogefüge } \\
\text { Textur }\end{array}$ & $\begin{array}{c}\text { Gruppe } I\left(c_{w}<1 \text { und } I_{\max }\right. \\
<5 \mathrm{mrd})\end{array}$ & $\begin{array}{c}\text { Gruppe II (1 } \leq c_{w} \leq 2 \text { und } 5 \\
\left.m r d \leq I_{\max } \leq 10 \mathrm{mrd}\right)\end{array}$ & $\begin{array}{c}\text { Gruppe III }\left(c_{w}>2 \text { und }\right. \\
\left.I_{\max }>10 \mathrm{mrd}\right)\end{array}$ \\
\hline $\begin{array}{l}\text { makrosk. } \\
\text { Gefüge- } \\
\text { merkmale der } \\
\text { Gruppen } \\
\text { (schematische } \\
\text { Beispiele) }\end{array}$ & 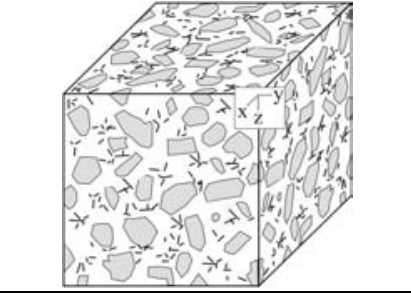 & 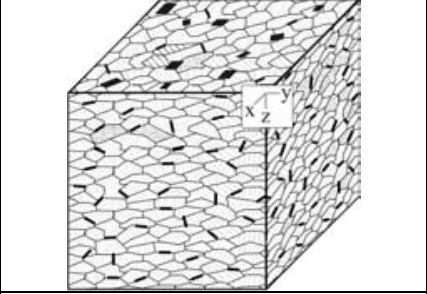 & 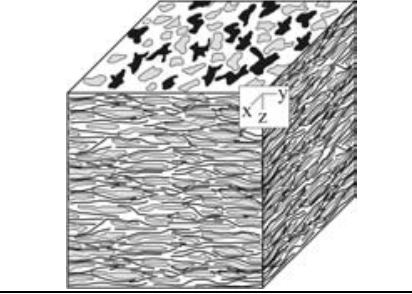 \\
\hline $\begin{array}{c}\text { S-Typ } \\
\left(k_{w}>3,00 \text { und }\right. \\
T>0,5)\end{array}$ & \multirow{3}{*}{$\begin{array}{c}\text { Granit (Koess) } \\
\text { Granodiorit (PDD) } \\
\text { Gabbro-Norit (NI) } \\
\text { Granit (MrGr3) }\end{array}$} & $\begin{array}{c}\text { Kalzit-Marmor (Rek) } \\
\text { granit. Gneis (Gra1a) } \\
\text { Quarzit (Al) }\end{array}$ & $\begin{array}{c}\text { granit. Protomyl. (MrMy5) } \\
\text { granit. Gneis (Gra1c) } \\
\text { granod. Gneis (Be1) } \\
\text { granit. Orthogneis (VA) }\end{array}$ \\
\hline $\begin{array}{c}\text { S-L-Typ } \\
\left(0,375<k_{w}<3 \text { und }\right. \\
-0,5<T<0,5)\end{array}$ & & $\begin{array}{l}\text { Meta-Gabbro (Mg2) } \\
\text { Peridotit (Pi1a) }\end{array}$ & $\begin{array}{c}\text { granod. Mylonit (Iv1b) } \\
\text { granod. Paragneis (Cal) } \\
\text { granod. Ultramylonit (Iv2) } \\
\text { granit. Ultramylonit } \\
\text { (Gra3a) granit. Gneis } \\
\text { (Be2) }\end{array}$ \\
\hline $\begin{array}{c}\text { L-Typ } \\
\left(\mathrm{k}_{\mathrm{w}}<0,375 \text { und }\right. \\
\mathrm{T}<-0,5)\end{array}$ & & $\begin{array}{c}\text { Meta-Gabbro (Mg1b) } \\
\text { granod. Protomylonit (Iv1) }\end{array}$ & \\
\hline $\begin{array}{c}\text { Offene } \\
\text { Mikrorisse }\end{array}$ & $\begin{array}{c}\text { Rissklasse } A_{R}-\text { geringe } \\
\text { Rissdichte }\left(V p_{Q R i s s}<0,8\right. \\
k m / s)\end{array}$ & $\begin{array}{c}\text { Rissklasse } B_{R}-\text { mittlere } \\
\text { Rissdichte }(, 8 \\
\left.\text { km/s } \leq V p_{Q R i s s} \leq 1,8 \mathrm{~km} / \mathrm{s}\right) \\
\end{array}$ & $\begin{array}{c}\text { Rissklasse } C_{R}-\text { hohe } \\
\text { Rissdichte }\left(p_{\text {QRiss }}>1,8\right. \\
\mathrm{km} / \mathrm{s})\end{array}$ \\
\hline $\begin{array}{l}\text { zugehörige } \\
\text { Proben }\end{array}$ & $\begin{array}{c}\text { Granit (Koess) } \\
\text { Peridotit (Pi1a) } \\
\text { granit. Ultramylonit } \\
\text { (Gra3a) } \\
\text { Kalzit-Marmor (Rek) } \\
\text { Quarzit (Al) } \\
\text { Meta-Gabbro (Mg2) } \\
\text { granit. Gneis (Gra1a) }\end{array}$ & $\begin{array}{c}\text { Granodiorit (PDD) } \\
\text { granit. Gneis (Gra1c) } \\
\text { Gabbro-Norit (NI) } \\
\text { Meta-Gabbro (Mg1b) } \\
\text { granod. Protomylonit (IV1) }\end{array}$ & $\begin{array}{c}\text { granod. Mylonit (Iv1b) } \\
\text { granod. Ultramylonit (Iv2) } \\
\text { granod. Paragneis (Cal) } \\
\text { granit. Orthogneis (VA) } \\
\text { granit. Protomyl. (MrMy5) } \\
\text { granod. Gneis (Be1) } \\
\text { Granit (MrGr3) } \\
\text { granit. Gneis (Be2) }\end{array}$ \\
\hline
\end{tabular}

Die mineralogischen Eigenarten und die Gefügeelemente, die zur Gruppierung der Gesteine geführt haben, wurden aufgrund ihrer potenziellen Relevanz hinsichtlich der technischen Gesteinseigenschaften und deren Anisotropie ausgewählt. Die Phyllosilikate sind in Bezug auf viele physikalische Eigenschaften stark anisotrop (vgl. Kap.4), daher kann ihr 


\section{$\underline{\text { 5. Gesteinsmaterial }}$}

Volumenanteil ebenso wie ihre Textur einen entscheidenden Einfluss auf die Gesamtgesteinseigenschaften haben. Das makroskopische Erscheinungsbild bietet einen umfassenden Eindruck des Gesteinsgefüges und seiner Anisotropie, der sowohl die Formanisotropie der Minerale und deren bevorzugte Orientierung als auch farbliche, durch Materialwechsel bedingte Variationen beinhaltet. Inwiefern die makroskopisch sichtbare Anisotropie eine Korrelation mit den gesteinstechnischen Eigenschaften zeigt, wird im Rahmen dieser Studie ebenso erläutert wie die Auswirkung von offenen Mikroriss-Systemen, die als potenzielle Quelle für Rissausbreitung gelten und damit entscheidend für den Versagensprozess bei mechanischer Belastung sind. 


\section{Technische Gesteinseigenschaften}

Im Rahmen dieses Kapitels werden die Ergebnisse der gesteinstechnischen Untersuchungen dargestellt und zu dem makroskopischen Erscheinungsbild und speziellen Gefügeelementen in Beziehung gesetzt. Das Hauptziel ist es, die Abhängigkeit der technischen Parameter und ihrer Anisotropie bestimmten Gefügemerkmalen gegenüberzustellen. Als skalare Größen werden die Dichte und die Porosität und als vektorielle Größen die kapillare Wasseraufnahme sowie die mechanischen und die elastischen Eigenschaften in die Analyse einbezogen.

\subsection{Indexeigenschaften}

\subsubsection{Dichte}

Die Dichte ist ein Standardparameter zur Charakterisierung von Gesteinen aus gesteinstechnischer Sicht und findet sowohl in Bereichen der Ingenieurgeologie als auch im Bereich der Naturwerksteine und der Bautechnik eine breite Anwendung (z.B. Koroneos 1980; Peschel 1983; Reinsch 1991; Prinz 1997). Zum einen ist bei Berechnungen zur Statik von Gebäuden oder Gebäudeteilen das Eigengewicht der Naturwerksteine zu berücksichtigen und damit eine Kenntnis der Dichte nötig, zum anderen werden Dichteangaben häufig mit anderen gesteinstechnischen Kennwerten (z.B. Druckfestigkeit) in Verbindung gebracht. Letzteres wird in Bezug auf die untersuchten Kristallingesteine in Kap.8.1 diskutiert. Nach DIN EN 1936 wird zwischen der Reindichte (in dieser Arbeit: Matrixdichte) und der Rohdichte unterschieden. Deren Bestimmung wird in unterschiedlichen Produktnormen zu Naturwerksteinen ausdrücklich gefordert (z.B. DIN EN 771-6 - Festlegungen für Mauersteine). Die Matrixdichte $\left(\rho_{M}\right)$ ist allein durch die mineralogische Zusammensetzung und die Dichte der gesteinsbildenden Minerale bestimmt, wohingegen die Rohdichte ( $\left.\rho_{\text {Roh }}\right)$ die Porosität mit einbezieht und damit die mittlere Dichte von Mineralbestand und Porenraum beschreibt. Rohdichte und Matrixdichte sind nach G.6-1 miteinander verknüpft.

(G.6-1) $\quad \rho_{\text {Roh }}=\rho_{M}(1-\phi) \quad\left[\mathrm{g} / \mathrm{cm}^{3}\right]$ $\rho_{\text {Roh }}-$ Rohdichte $\left[\mathrm{g} / \mathrm{cm}^{3}\right]$. $\rho_{M}-$ Matrixdichte $\left[\mathrm{g} / \mathrm{cm}^{3}\right]$. $\phi$ - Porosität [\%]. 


\section{Technische Gesteinseigenschaften}

\subsubsection{Rohdichte}

Im weiteren Verlauf dieser Arbeit wird die Rohdichte verwendet. Aufgrund der niedrigen Porositäten von $\leq 1$ Vol.\% liegen Matrixdichte und Rohdichte maximal $0,03 \mathrm{~g} / \mathrm{cm}^{3}$ auseinander; folglich wird die Rohdichte der Proben fast ausschließlich durch die Dichte des Mineralbestands und die mineralogische Komposition des Gesteins bestimmt.

Die Rohdichten liegen zwischen 2,64 g/ $\mathrm{cm}^{3}$ (z.B. granitischer Gneis - Be2) und 3,34 g/ $\mathrm{cm}^{3}$ (Peridotit - Pila) und decken damit einen großen Bereich der niedrigporösen, silikatischen und karbonatischen Kristallingesteine ab (Abb.6-1). Die Proben Be2 und Pila können als typische Vertreter oberkrusten-assoziierter saurer Gesteine, bzw. oberer-Mantel-assoziierter ultrabasischer Gesteine angesehen werden. Im Vergleich dazu weisen hochporöse Sedimentgesteine wesentlich niedrigere Rohdichten auf. Werte von $<2 \mathrm{~g} / \mathrm{cm}^{3}$ sind keine Seltenheit.

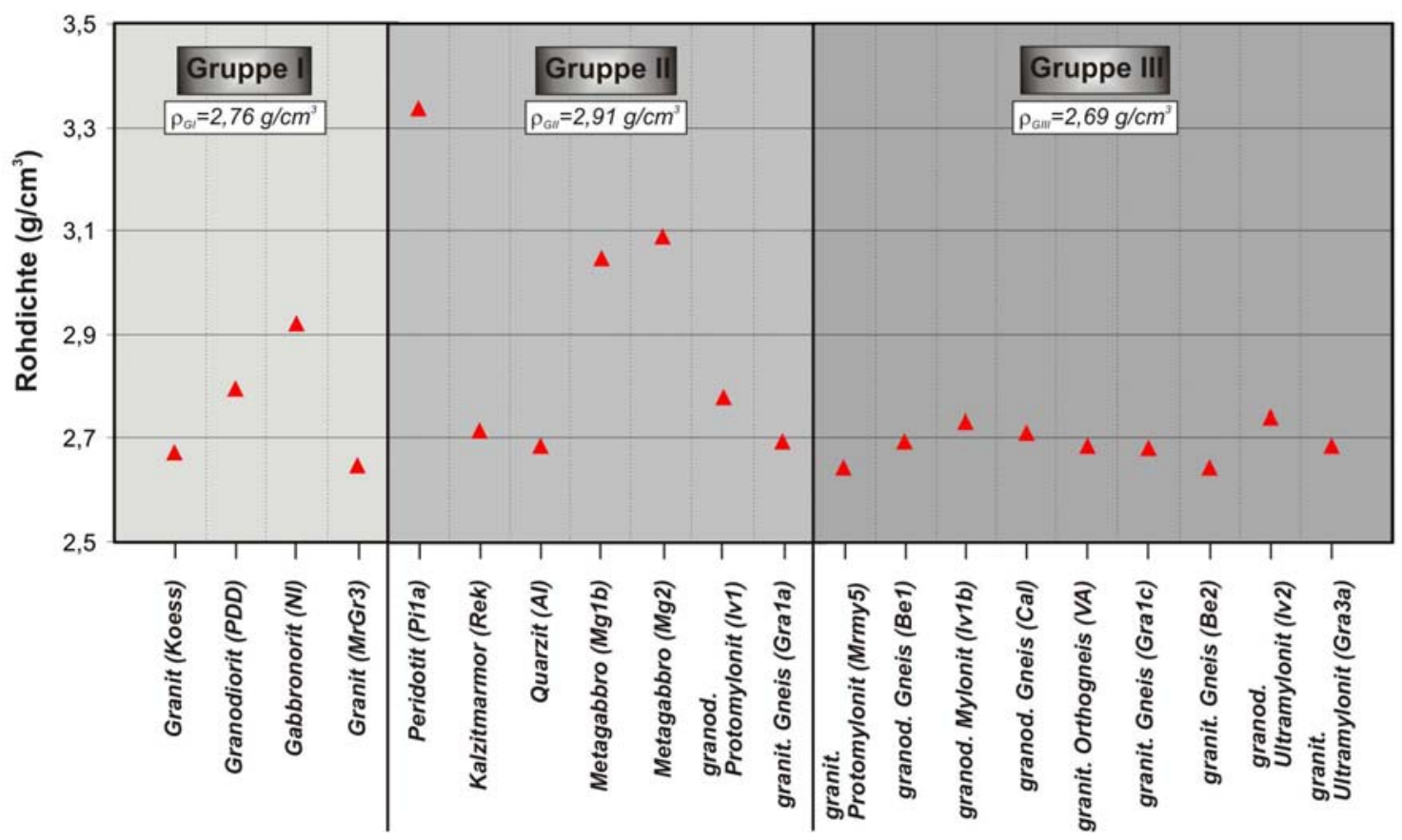

Abb.6-1: Rohdichte ( $\rho_{\text {roh }}$ ) der Makrogefüge-Gruppen I/IIIII. Mittelwerte der Rohdichte für die jeweilige Gruppe sind angegeben. 1- $\sigma$-Fehler innerhalb der Symbolgröße.

Die mittlere Dichte der Makrogefüge-Gruppen I/II/III bewegt sich zwischen 2,69 $\mathrm{g} / \mathrm{cm}^{3}$ (Gruppe III) und $2,91 \mathrm{~g} / \mathrm{cm}^{3}$ (Gruppe II) und zeigt keinerlei signifikante Trends in Abhängigkeit von der makroskopischen Gefügeanisotropie. 


\section{Technische Gesteinseigenschaften}

\subsubsection{Porenraum}

Dem Porenraum kommt aus ganz unterschiedlichen Sichtweisen eine Bedeutung für die Bewertung von Naturwerksteinen zu. Zum ersten haben sein Volumenanteil im Gestein (Porosität), seine Geometrie und seine räumliche Anordnung einen entscheidenden Einfluss auf viele gesteinstechnische Eigenschaften (z.B. mechanische Festigkeiten - vgl. Kap.6.3.4) und deren Anisotropie (z.B. Smorodinov et al. 1970; Kelsall et al. 1986). Zum zweiten bildet der Porenraum Wegsamkeiten für fluide Phasen und chemische Reaktanten, die, katalytisch beschleunigt durch Temperaturschwankungen und das spezielle thermische Dehnungsverhalten der Naturwerksteine (vgl. Kap.6.5), zur chemischen und physikalischen Verwitterung des Gesteinsmaterials beitragen (z.B. Fitzner \& Snethlage 1982; Alm et al. 1985; Klopfer 1985; Fitzner 1988; Poschlod 1989; Weiss 1992; Kownatzki 1997; Grimm 1999). Wichtige Eigenschaften sind die effektive Porosität, der mittlere Porenradius und der Anteil der Kapillarporen, weil durch sie die Fähigkeit eines Gesteins, Wasser aufzunehmen und abzugeben, maßgeblich bestimmt wird.

\subsubsection{Porosität}

Im Rahmen dieser Arbeit wird die effektive Porosität verwendet (offene Porosität nach DIN EN 1936). Die Werte liegen zwischen 0,03 Vol.\% (Peridotit - Pila) und 1,03 Vol.\% (Granit Mrgr3). Damit sind alle untersuchten Gesteine als niedrig porös einzustufen und befinden sich tendenziell am unteren Ende der für die jeweiligen Gesteinstypen angegebenen Porositätsbereiche. So können nach Peschel (1983) für Granite und Granodiorite 6,9 Vol.\% und für Peridotite 5 Vol.\% Porosität erreicht werden. Noch deutlich höhere Werte können Sedimentgesteine wie z.B. Sandsteine (ca. 35 Vol.\%) und Travertine (bis 60 Vol.\%) aufweisen. 


\section{Technische Gesteinseigenschaften}

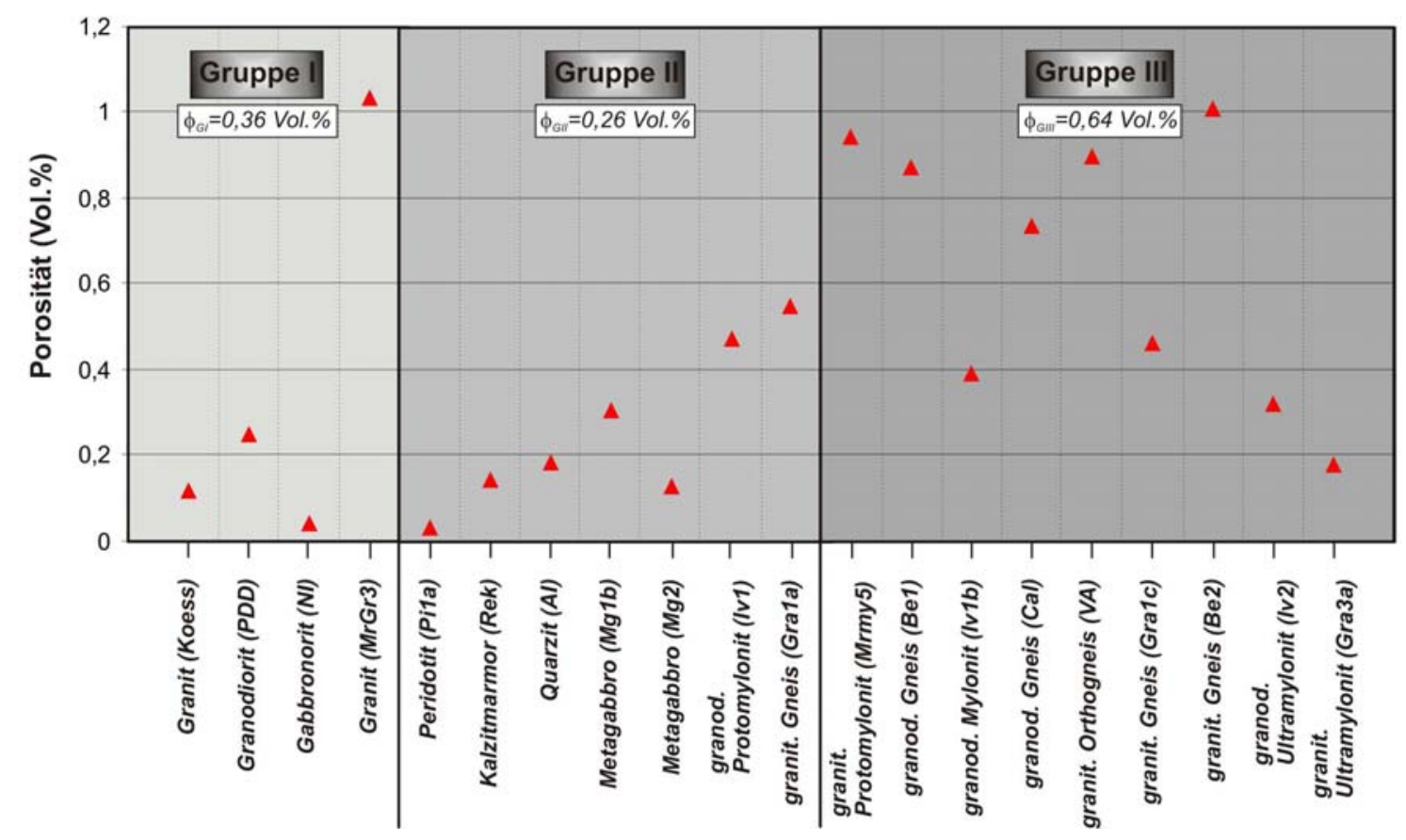

Abb.6-2: Porosität ( $\phi)$ der Makrogefüge-Gruppen I/IIIII. Mittelwerte der Porosität für die jeweilige Gruppe sind angegeben. 1- $\sigma$-Fehler innerhalb der Symbolgröße.

Innerhalb der Makrogefüge-Gruppen mit ihren Mittelwerten von 0,36 Vol.\% (Gruppe I) 0,26 Vol.\% (Gruppe II) und 0,64 Vol.\% (Gruppe III) ist keine Korrelation der makroskopischen Gefügeanisotropie mit den effektiven Porositäten zu beobachten (Abb.6-2).

\subsubsection{Mittlerer Porenradius}

Der mittlere Porenradius ( $\mathrm{r}_{\mathrm{m}}$ ) wurde aus den Messergebnissen der Hg-Porosimetrie berechnet. Für die Proben NI, Pila und Mg2 konnten keine Werte ermittelt werden. Die Daten bewegen sich zwischen 0,01 $\mu \mathrm{m}$ (z.B. Quarzit - AI) und 0,16 $\mu \mathrm{m}$ (granitischer Gneis - Be2). Vergleichswerte liefert Rüdrich (2003), der für 6 frische Marmore mit Porositäten zwischen 0,2-0,33 Vol.\% die dominante Porenklasse mit 0,04-0,237 $\mu \mathrm{m}$ und für deren verwitterte Derivate mit 0,237-2,371 $\mu \mathrm{m}$ angibt. Für bestimmte Sandsteine (Porosität 11,6-25,2 Vol.\%) wurden von Koch \& Siegesmund (2001) deutlich höhere mittlere Porenradien von 2,7-17,7 $\mu \mathrm{m}$ ermittelt. Die untersuchten Gesteine weisen also eine Rissporosität mit sehr niedrigen mittleren Porenradien auf, die typisch für unverwitterte, niedrigporöse Kristallingesteine ist. 


\section{Technische Gesteinseigenschaften}

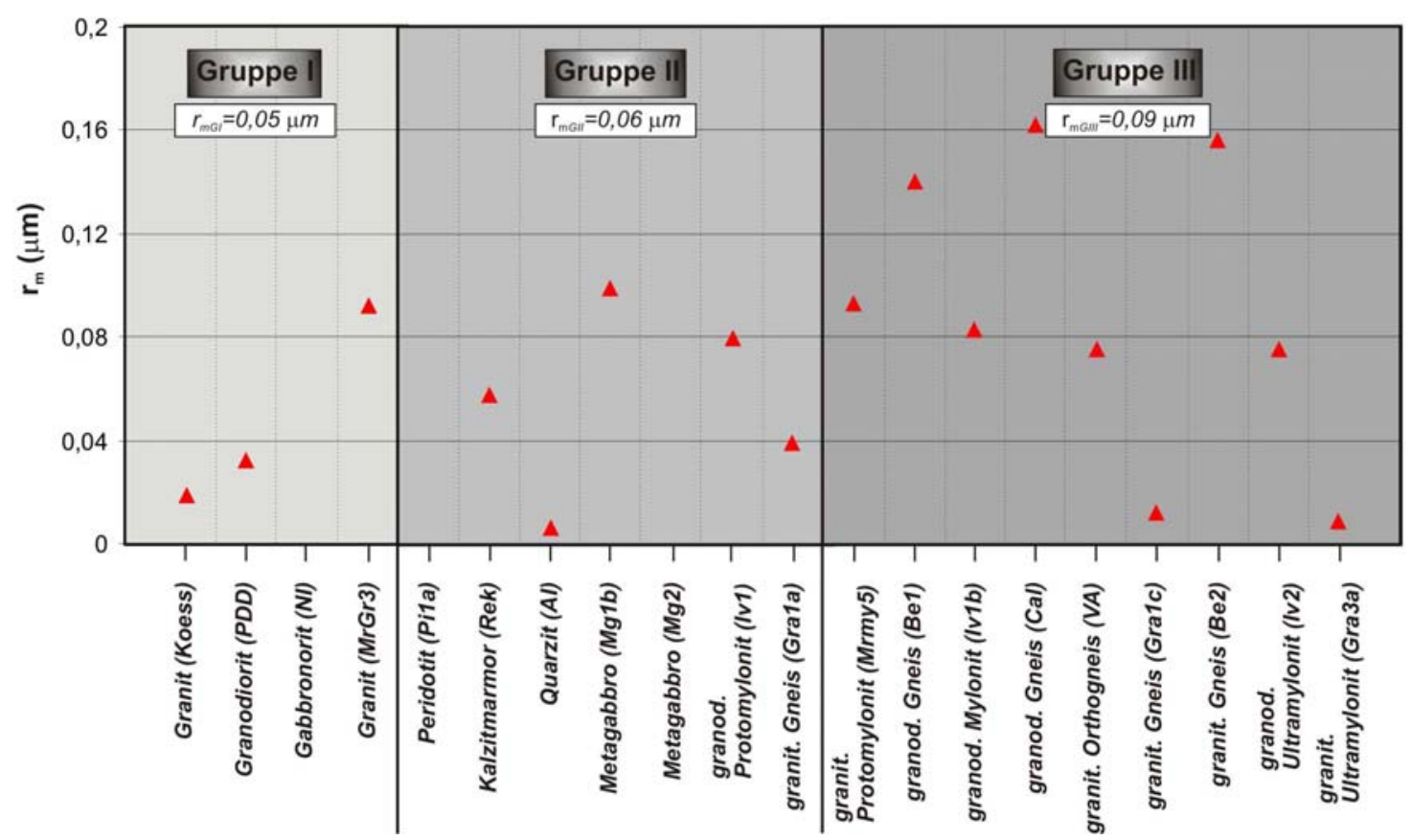

Abb.6-3: Mittlerer Porenradius $\left(r_{m}\right)$ der Makrogefüge-Gruppen I/II/III. Mittelwerte des mittleren Porenradius für die jeweilige Gruppe sind angegeben. 1- $\sigma$-Fehler beträgt 0,03 $\mu$ m (vgl. Anhang I.4).

Die Mittelwerte der Makrogefüge-Gruppen I/II/III (Abb.6-3) und auch die Gesteine innerhalb der Gruppen zeigen keinen Trend des mittleren Porenradius in Abhängigkeit von der makroskopischen Gefügeanisotropie.

\subsubsection{Volumenanteil der Kapillarporen}

Der Volumenanteil der Kapillarporen $\left(\phi_{\text {rkap }}\right)$ am Gesamtgesteinsvolumen (Porenhalsradien $0,05 \mu \mathrm{m}$ bis $500 \mu \mathrm{m}$ - Klopfer 1985; Poschlod 1989) wurde aus den Ergebnissen der HgPorosimetrie ermittelt. Für die Proben NI, Pila und Mg2 liegen keine Daten vor. Die Werte variieren zwischen 0,00 Vol.\% (z.B. granitischer Ultramylonit - Gra3a) und 0,87 Vol.\% Kapillarporenanteil (granitischer Gneis - Be2). Damit erreicht der Anteil der Kapillarporen an der Porosität maximal 87\% (Probe Be2). Die Makrogefüge-Gruppen haben Mittelwerte von 0,25 Vol.\% (Gruppe I), 0,14 Vol.\% (Gruppe II) und 0,39 Vol.\% (Gruppe III), ohne dass eine Abhängigkeit des Kapillarporenanteils vom makroskopischen Erscheinungsbild zu erkennen ist $(\boldsymbol{A b b} .6-4)$. 


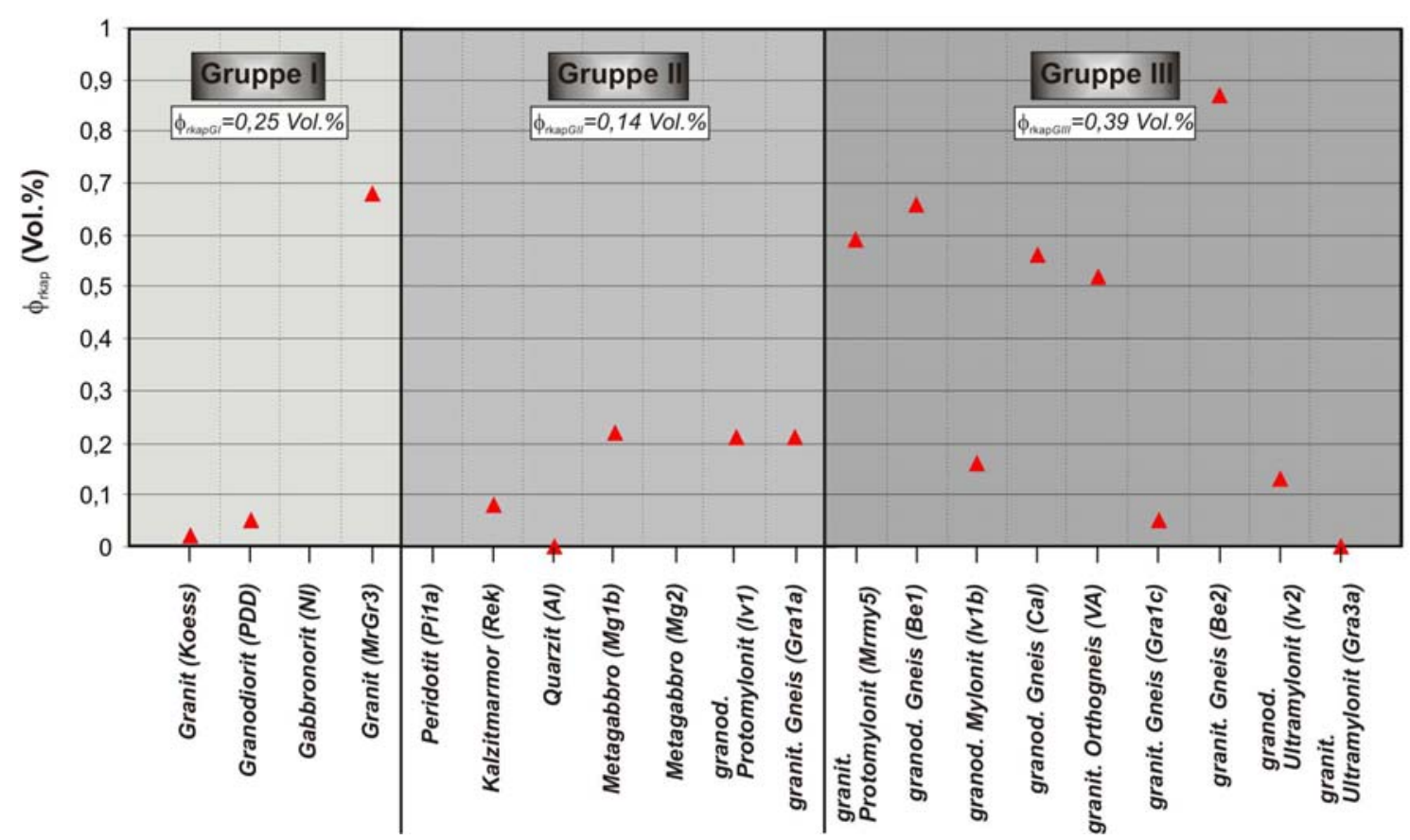

Abb.6-4: Volumenanteil der Kapillarporen ( $\phi_{\text {rkap }}$ ) am Gesamtgesteinsvolumen der MakrogefügeGruppen I/IIIIII. Mittelwerte von $\phi_{\text {rkap }}$ für die jeweilige Gruppe sind angegeben. 1- $\sigma$-Fehler beträgt 0,09 Vol.\% (vgl. Anhang I.4).

\subsection{Kapillare Wasseraufnahme}

Feuchtigkeit in Gesteinen verändert deren technische Eigenschaften und beeinflusst das Verwitterungsverhalten. Wassergesättigte Proben weisen stets niedrigere mechanische Festigkeiten auf als trockene (z.B. Stavrogin \& Tarasov 2001). In Bezug auf die Gesteinsverwitterung wird der Feuchte eine katalytische Wirkung nachgesagt. Sowohl im Zusammenhang mit dem thermischen Dehnungsverhalten und der damit verbundenen Gefügeauflockerung als auch bei der Frost- und Salzverwitterung (Weiss 1992; Ondrasina et al. 2002) spielt der Wasserhaushalt des Gesteins eine große Rolle. Daher ist die Fähigkeit eines Gesteins, Wasser aufzunehmen, ein bedeutender technischer Kennwert, der Rückschlüsse auf die Verwitterungscharakteristik der Gesteine zulässt. Unter mitteleuropäischen Klimaten sind Naturwerksteine in ihrer Verwendung im Außenbereich wechselfeuchten Bedingungen ausgesetzt. Die Feuchtigkeit wird dabei abhängig von der Porengeometrie durch Kapillardiffusion und -kondensation sowie durch kapillares Saugen oder Strömung aufgenommen (Klopfer 1985; Poschlod 1989; Reinsch 1991).

Im Rahmen der vorliegenden Studie wurden 20 Kristallingesteine auf ihre kapillare Saugfähigkeit als den vermutlich dominanten Mechanismus des Wassertransportes untersucht. Die Wasseraufnahme ist eng an die Eigenschaften des Porenraums geknüpft und wird daher 


\section{Technische Gesteinseigenschaften}

schon innerhalb dieses Kapitels mit dessen Volumenanteilen und den Porendimensionen in Verbindung gebracht.

\subsection{1 w-Wert}

Als Maß für die kapillare Saugfähigkeit der Gesteine wurde aus den Daten des Saugversuchs der richtungsabhängige w-Wert in mindestens zwei Orientierungen bestimmt. Den maximalen mittleren w-Wert (arithmetisches Mittel aus x-, y-, z-Richtung) weist die Probe Be2 (granitischer Gneis) mit $\mathrm{w}=0,22 \mathrm{~kg} / \mathrm{m}^{2} * \sqrt{ } \mathrm{h}$, den niedrigsten mit $\mathrm{w}=0,003 \mathrm{~kg} / \mathrm{m}^{2} * \sqrt{\mathrm{h}}$ die Probe Koess (Granit) auf. Elf der 20 Proben zeigen w-Werte, die kleiner als $0,06 \mathrm{~kg} / \mathrm{m}^{2} * \sqrt{\mathrm{h}}$ sind (Abb.6-5).

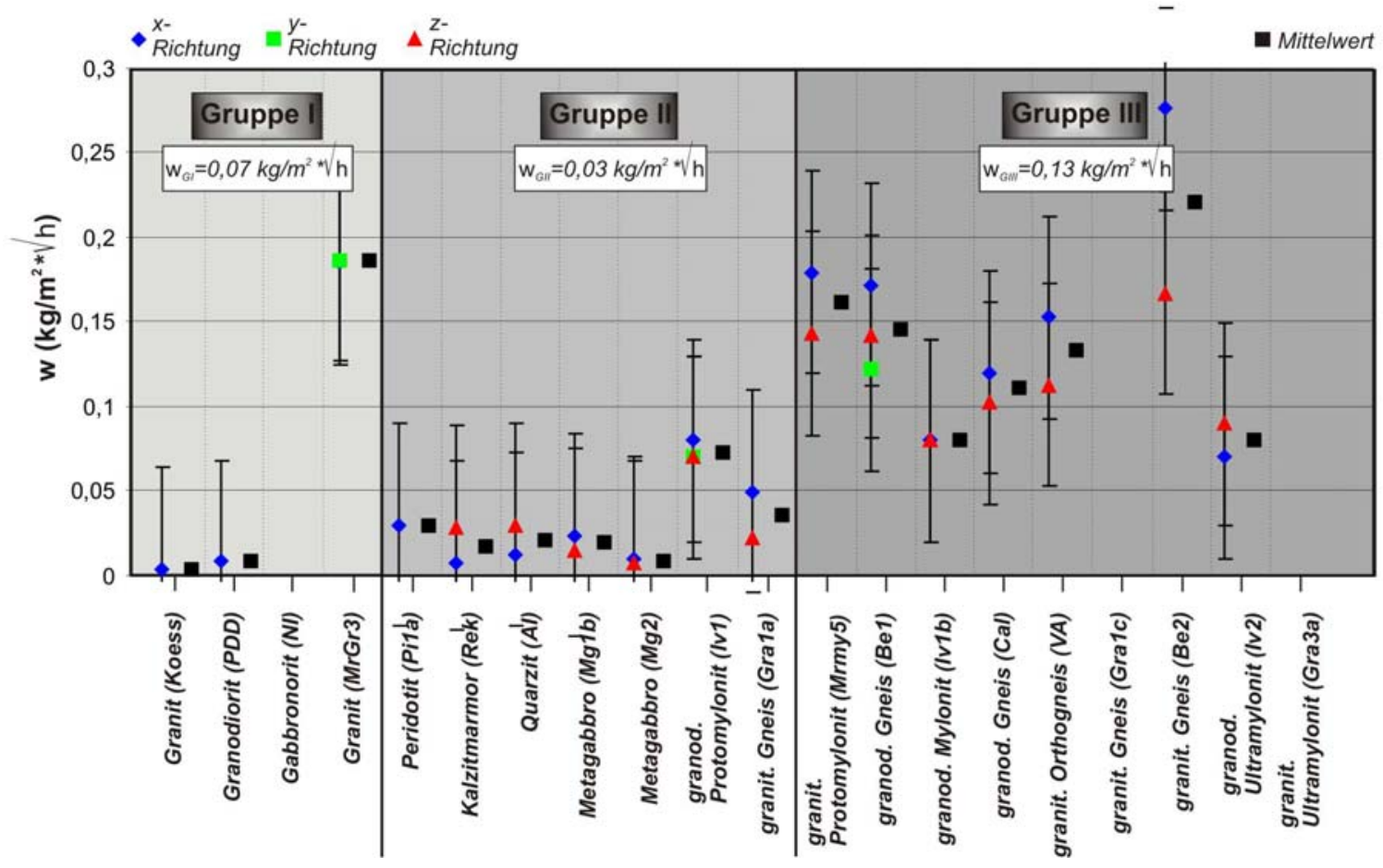

Abb.6-5: w-Wert (w) der Makrogefüge-Gruppen I/II/III. Mittelwerte des w-Wertes für die jeweilige Gruppe sind angegeben. Fehlerbalken markieren 1- $\sigma$-Fehler.

Diese Daten liegen innerhalb des versuchsbedingten Messfehlers und sind nur im Sinne einer nicht vorhandenen kapillaren Wasseraufnahme $\mathrm{zu}$ interpretieren. Den maximalen Sättigungsgrad $\left(\mathrm{S}_{\text {Kap }}\right)$ der Kapillarporen erreicht die Probe Be2. 0,7 Vol.\% des offenen Porenraums werden hier durch kapillares Saugen gefüllt, was einem Sättigungsgrad von ca. $80 \%$ entspricht. Die ermittelten sehr niedrigen $\mathrm{w}$-Werte sind typisch für niedrig poröse Kristallingesteine. Vergleichswerte für Marmor (Porosität 0,4-0,74 Vol.\%) liefert Rüdrich (2003) mit w-Werten zwischen $0,08 \mathrm{~kg} / \mathrm{m}^{2} * \sqrt{\mathrm{h}}$ und $0,19 \mathrm{~kg} / \mathrm{m}^{2} * \sqrt{\mathrm{h}}$. Stärker poröse Gesteine mit einer entsprechenden Porenradienverteilung können wesentlich höhere w-Werte 
erreichen. Beispiele hierfür finden sich bei Koch \& Siegesmund (2001) (Sandstein mit $\mathrm{w}=44,7 \mathrm{~kg} / \mathrm{m}^{2} * \sqrt{\mathrm{h}}$ ), bei Weiss (1992) (Sandsteine mit $\mathrm{w}=0,71-2,21 \mathrm{~kg} / \mathrm{m}^{2} * \sqrt{\mathrm{h}}$ ) und Metz (1992) (Sandsteine und Karbonate mit $\mathrm{w}=1,2-27,7 \mathrm{~kg} / \mathrm{m}^{2} * \sqrt{\mathrm{h}}$ ).

Die w-Mittelwerte für die Makrogefüge-Gruppen I/II/III liegen zwischen $0,03 \mathrm{~kg} / \mathrm{m}^{2} * \sqrt{\mathrm{h}}$ und $0,13 \mathrm{~kg} / \mathrm{m}^{2} * \sqrt{\mathrm{h}}(\boldsymbol{A} \boldsymbol{b} \boldsymbol{b} .6-5)$. Die Anisotropie der w-Werte ist stark fehlerbehaftet. Daher werden für die weitere Betrachtung der Wasseraufnahme nur Proben herangezogen, welche signifikante w-Werte oberhalb des versuchsbedingten Fehlers aufweisen (Proben: Mrgr3, Mrmy5, Iv1, Iv2, Cal, Be1, Be2, VA).
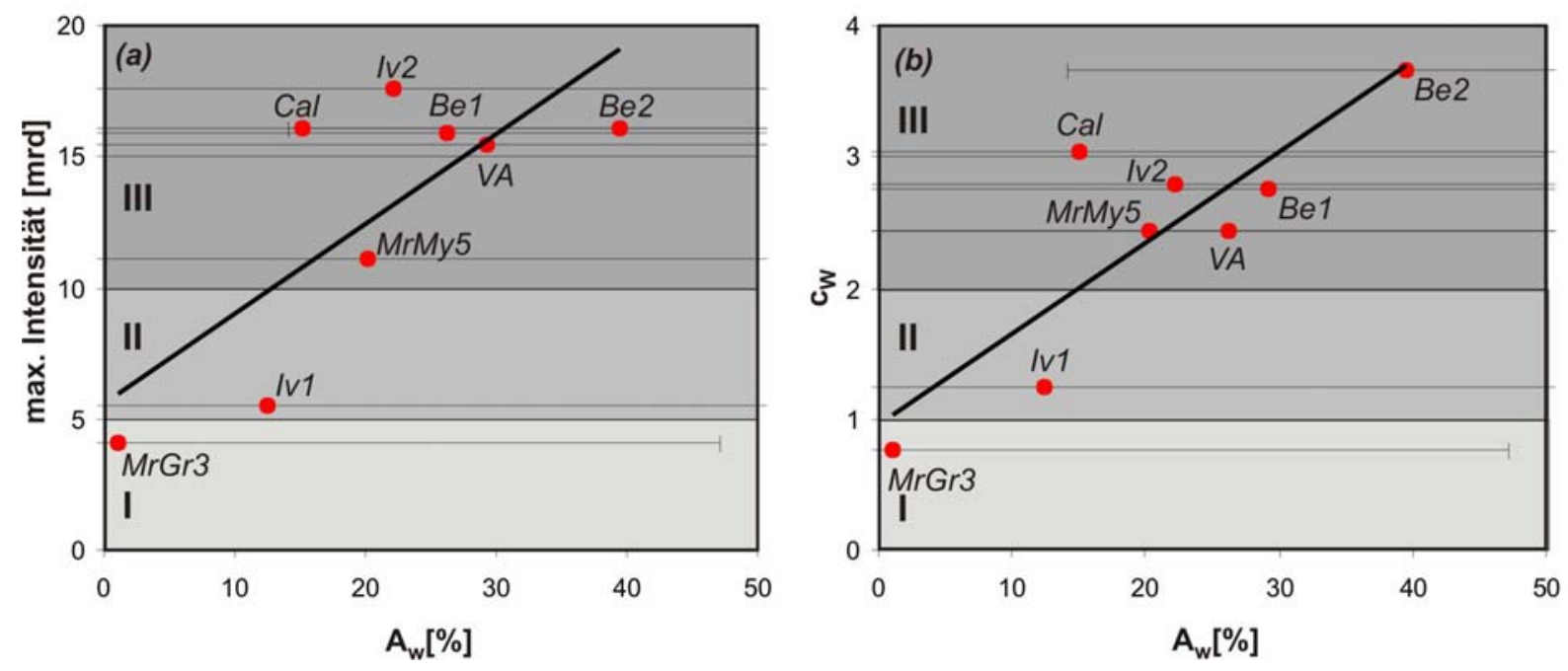

Abb.6-6: Glimmertextur und Anisotropie der w-Werte. (a) Max. Intensität der (001)-Pole vs. Anisotropie der w-Werte. (b) Regelungsschärfe $\left(c_{w}\right)$ vs. Anisotropie der w-Werte. Die Bereiche der Makrogefüge-Gruppen I/IIIIII sind gekennzeichnet. Mrgr3 - Granit. IV1 - granodioritischer Protomylonit. IV2 - granodioritischer Ultramylonit. MrMy5 - granitischer Protomylonit. VA/Be2 granitischer Gneis. Be1/Cal - granodioritischer Gneis. Fehlerbalken markieren den 1- $\sigma$-Fehler.

Sämtliche der genannten Proben enthalten Glimmer, so dass gemäss der Einteilung aus Kap.5.7 die max. Intensität der Glimmer (001)-Pole bzw. deren Regelungsschärfe $\left(\mathrm{c}_{\mathrm{W}}\right)$ stellvertretend für die makroskopische Gefügeanisotropie gewählt wurde. Es zeigt sich eine gute Korrelation von max. Intensität sowie der Regelungsschärfe der (001)-Pole mit der Anisotropie der w-Werte (Abb.6-6). Zu bemerken bleibt der bereits erwähnte große Fehler der Anisotropie-Werte, aufgrund dessen die Ergebnisse kritisch betrachtet werden müssen. Auf die Angabe von Korrelationskoeffizienten wurde daher verzichtet.

\subsection{2 w-Werte und Porenraumeigenschaften}

Der mittlere w-Wert der Gesteine steht in einem engen Verhältnis zum Volumen und der Geometrie des Porenraums. In Abb.6-7 werden die mittleren w-Werte mit der Rissdichte 


\section{Technische Gesteinseigenschaften}

$\left(\mathrm{Vp}_{\mathrm{QRiss}}\right)$, der Porosität $(\phi)$, dem mittleren Porenhalsradius $\left(\mathrm{r}_{\mathrm{m}}\right)$ und dem Volumenanteil der Kapillarporen $\left(\phi_{\text {rkap }}\right)$ verglichen.
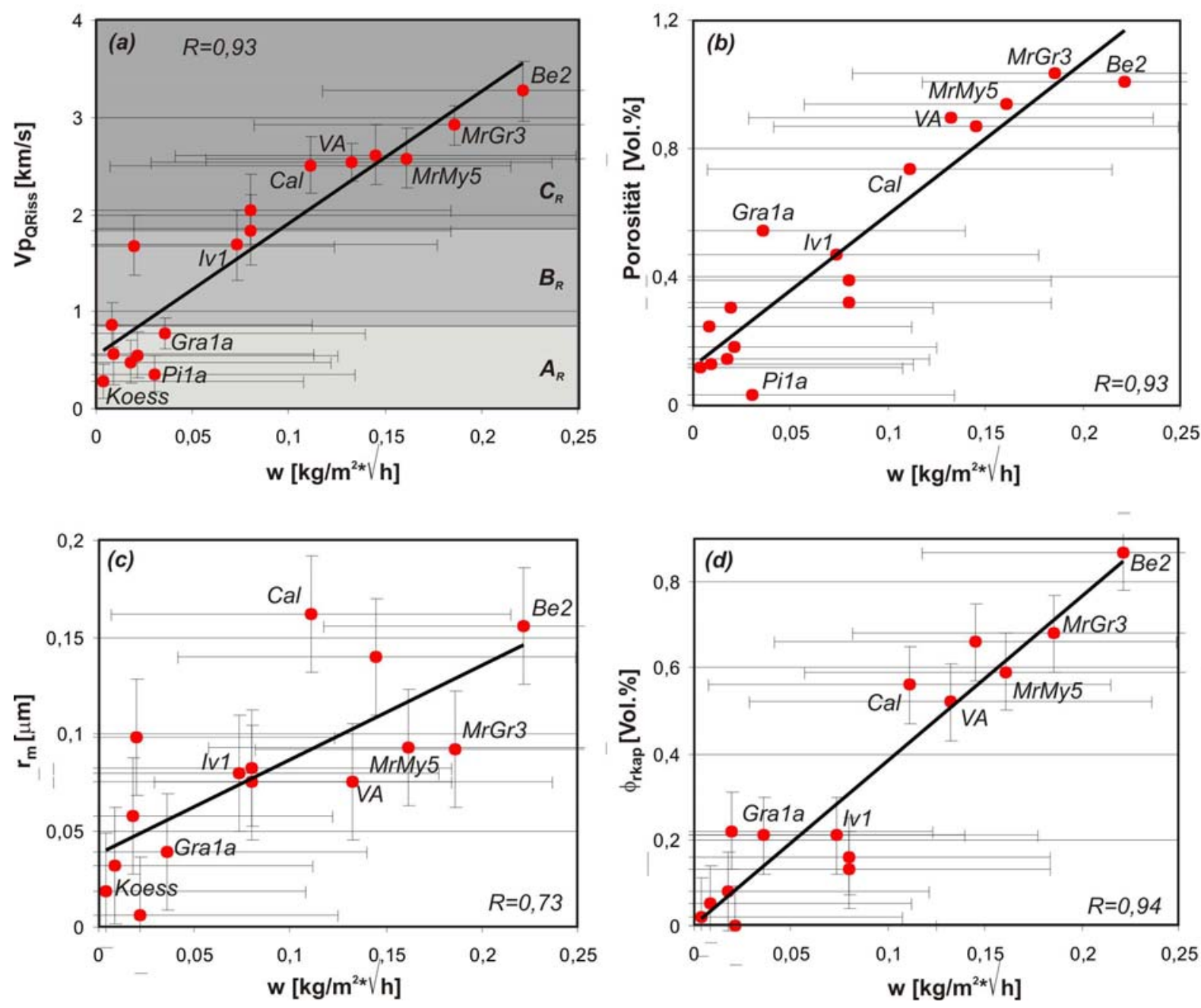

Abb.6-7: w-Wert (arithmetisches Mittel aus w-Werten der x-, y-, z-Richtung) in Abhängigkeit von Porenraumeigenschaften. (a) w-Werte in Abhängigkeit von der Rissdichte (V $\left.p_{Q R i s s}\right)$; die Rissklassen $A_{R}$ (geringe Rissdichte), $B_{R}$ (mittlere Rissdichte) und $C_{R}$ (hohe Rissdichte) sind indiziert. (b) w-Werte in Abhängigkeit von der Porosität ( $\phi)$. (c) $w$-Werte in Abhängigkeit vom mittleren Porenradius $\left(r_{m}\right)$. (d) $w$ Werte in Abhängigkeit vom Volumenanteil der Kapillarporen ( $\left.\phi_{\text {rkap }}\right)$. Es werden alle Proben betrachtet, für die das jeweilige Datenpaar vorliegt (vgl. Anhang l.4). Be2/VA/Gra1a granitischer Gneis. Mrgr3/Koess - Granit. Mrmy5 - granitischer Protomylonit. Cal - granodioritscher Gneis. Iv1 granodioritischer Protomylonit. Pi1a - Peridotit. Fehlerbalken markieren den 1- $\sigma$-Fehler.

In allen Fällen zeigt sich eine gute bis sehr gute positive lineare Korrelation von w-Werten und den jeweiligen Porenraumeigenschaften. Den statistisch signifikantesten Trend $(R=0,94)$ weist der w-Wert in Abhängigkeit vom Volumenanteil der Kapillarporen auf.

Die gefundenen Zusammenhänge lassen sich für Kapillaren mit einem kreisförmigen Querschnitt theoretisch untermauern. Nach G.6-2 ist die Masse des kapillar aufgenommenen Wassers $\left(\mathrm{m}_{\mathrm{auf}}\right)$ proportional zum Kapillarporenradius $(\mathrm{r})$. 


$$
m_{\text {auf }}=\pi * r * \frac{2 * \sigma * \cos \phi}{g} \quad[\mathrm{~kg}] \quad \text { mit } 0,05 \mu \mathrm{m} \leq \mathrm{r} \leq 500 \mu \mathrm{m}
$$

$m_{\text {auf }}$ - Masse des aufgenommenen Wassers [kg]. $r$ - Kapillarradius [m]. $\sigma$-Oberflächenspannung (für Wasser bei $20^{\circ} \mathrm{C} \mathrm{s}=0,07 \mathrm{~J}^{2} \mathrm{~m}^{2}$; Bergmann 1995)[ $\left[\mathrm{J}^{2}\right]$. $\phi$ - Randwinkel [ ${ }^{\circ}$ ]. $\mathrm{g}$ - Erdbeschleunigung $\left[9,81 \mathrm{~m} / \mathrm{s}^{2}\right]$.

Mit der Definition für den w-Wert

(G.6-3) $\quad w=\frac{m_{\text {auf }}}{A^{*} \sqrt{t}} \quad\left[\mathrm{~kg} / \mathrm{m}^{2} * \sqrt{\mathrm{h}}\right]$

$w$ - Wasseraufnahmekoeffizient $\left[\mathrm{kg} / \mathrm{m}^{2 *} \sqrt{\mathrm{h}}\right] . m_{\text {auf }}$ - Masse des aufgenommenen Wassers [kg]. A Fläche $\left[\mathrm{m}^{2}\right]$. $t-$ Zeit [Std.].

und konstantem $\mathrm{A}$ und $\mathrm{t}$ folgt daraus eine lineare Abhängigkeit des $\mathrm{w}$-Wertes vom Kapillarporenradius und der Dichte der zur Verfügung stehenden Kapillarporen (G.6-4).

$$
w=\frac{\pi * r * n * \frac{2 * \sigma * \cos \phi}{g}}{A^{*} \sqrt{t}}
$$

$\left[\mathrm{kg} / \mathrm{m}^{2} * \sqrt{\mathrm{h}}\right] \quad$ mit $0,05 \mu \mathrm{m} \leq \mathrm{r} \leq 500 \mu \mathrm{m}$

w - Wasseraufnahmekoeffizient $\left[\mathrm{kg} / \mathrm{m}^{2 *} \sqrt{\mathrm{h}}\right] . \quad r$ - Kapillarradius [m]. $n$ - Kapillarporendichte/ Kapillarporenfrequenz. $\sigma$-Oberflächenspannung (für Wasser bei $20^{\circ} \mathrm{C} s=0,07 \mathrm{~J} / \mathrm{m}^{2}$; Bergmann 1995) $\left[\mathrm{J} / \mathrm{m}^{2}\right] . \phi$-Randwinkel $\left[{ }^{\circ}\right] . g-$ Erdbeschleunigung $\left[9,81 \mathrm{~m} / \mathrm{s}^{2}\right]$. ]. A - Fläche $\left[\mathrm{m}^{2}\right]$. t - Zeit [Std.].

Eine lineare Abhängigkeit zwischen dem w-Wert und dem mittleren Porenradius $\left(\mathrm{r}_{\mathrm{m}}\right)$ liegt also insbesondere dann nahe, wenn ein möglichst großer Anteil der Gesamtporosität durch Kapillarporen gebildet wird und damit die Bedingungen von G.6-4 erfüllt sind. Im Fall der untersuchten Proben schwankt der Anteil der Kapillarporen an der Gesamtporosität zwischen $0 \%$ und $87 \%$, so dass die Bedingungen nur teilweise gegeben sind und bezüglich einiger Proben ein zu hoher Anteil von Nicht-Kapillarporen in die Berechnung von $r_{m}$ eingeht. Eine separate Betrachtung des mittleren Radius der Kapillarporen $\left(r_{\text {kapm }}\right)$ verspricht eine noch deutlich bessere Korrelation mit den w-Werten. Die sehr gute Korrelation des Volumenanteils der Kapillarporen mit den w-Werten geht ebenfalls aus G.6-4 hervor. Der zunehmende Volumenanteil ist entweder durch eine Zunahme des Kapillarporenradius r oder durch eine Erhöhung der Kapillarporendichte zu erklären, wobei beide Größen linear in die Berechnung des w-Wertes eingehen. Die gute Übereinstimmung von höheren Porositäten mit höheren wWerten ist nicht direkt ableitbar und muss im Zusammenhang mit der Entwicklung des Volumenanteils der Kapillarporen mit zunehmender Porosität gesehen werden. Bei den untersuchten Proben bedingt ein Anstieg der effektiven Porosität auch immer einen Anstieg 


\section{Technische Gesteinseigenschaften}

des Kapillarporenanteils, was zu der beobachteten guten Korrelationen von Porositäten und w-Werten führt. Untersuchungen von Koch \& Siegesmund (2001) an Sandsteinen zeigen einen nur mäßig guten Trend von hohen Porositäten zu hohen w-Werten. Hier wird der jeweilige Anteil der Kapillarporen am Gesamtgesteinsvolumen nicht berücksichtigt. Eine höhere Porosität muss also nicht zwingend mit einem Anstieg des Kapillarporenanteils übereinstimmen, sondern kann auch durch einen erhöhten Anteil von Poren außerhalb des Bereichs kapillarer Saugfähigkeit bedingt sein.

Der in dieser Arbeit entwickelte Rissquantifizierungsparameter $\mathrm{V} p_{\mathrm{QRiss}}$ steht stellvertretend für die Dichte der offenen Risse des gesamten Porenraums. Ein Anstieg der V $p_{Q R i s s}-W e r t e$ kann also mit einer Zunahme der Rissdichte und bei ähnlicher Rissgeometrie (ist bei den untersuchten Proben gegeben) mit einer Zunahme der Porosität gleichgesetzt werden. Dieses wiederum birgt einen Anstieg des Kapillarporenanteils und führt zu höheren w-Werten. In dieser Argumentationskette ist die sehr gute lineare Korrelation von $\mathrm{Vp}_{\mathrm{QRiss}}$ mit w-Werten zu verstehen.

Zusammenfassend: Die guten bis sehr guten Korrelationen von Porenraumeigenschaften mit den $\mathrm{w}$-Werten lassen sich theoretisch durch die physikalischen Vorgänge in idealen Kapillaren erklären. Entscheidend für die Größe der w-Werte ist immer der Anteil des kapillaren Porenvolumens bzw. dessen mittlerer Porenradius. Der Porenraum mit Porenausmaßen und -geometrien, die außerhalb des Bereichs kapillaren Saugens (für Wasser) liegen, muss bei der Untersuchung der Wasseraufnahmefähigkeit von Gesteinen separat betrachtet werden. Die gefundenen Korrelationen (Abb.6-7) sind auf niedrig poröse Kristallingesteine mit einer dominanten Rissporosität anwendbar und lassen eine gute Abschätzung der w-Werte zu. In Sedimentgesteinen mit deutlich anderen Porengeometrien und evt. auch anderen Benetzungswinkeln/Randwinkeln (G.6-2; Vogel 1999) können sich abweichende Korrelationen ergeben.

\subsection{Druck-, Zug-, Biege- und Abriebfestigkeit}

Die Eignung eines Naturwerksteins für eine bestimmte Anwendung wird maßgeblich durch seine mechanischen Eigenschaften bestimmt. Druck-, Biege- und Zugfestigkeit sind Größen, welche in die Statik-Berechnungen von Gebäuden und Gebäudefassaden eingehen (z.B. Wanetschek \& Wanetschek 2000; Purtak 2001) und damit von Architekten und Bauingenieuren bei der Planung und Konstruktion von Gebäuden berücksichtigt werden müssen. Einen Ansatz, welche Untersuchungen für welchen Verwendungszweck erforderlich 


\section{Technische Gesteinseigenschaften}

sind, liefern die deutschen und europäischen Produktnormen (z.B. DIN EN 1341 - Platte aus Naturstein; DIN EN 1342 - Pflastersteine; DIN 18516-3 - Außenwandbekleidung). Hier sind die empfohlenen Prüfungen mit Verweisen auf die jeweilige Prüf-Norm festgelegt. Beispielsweise erfordert eine Platte aus Naturstein nach DIN EN 1341 die Feststellung der Biegefestigkeit nach DIN EN 12372 und der Abriebfestigkeit nach DIN 52108. Der Tatsache, dass Naturwerksteine inhomogene und anisotrope Körper sind, wird durch eine vorgeschriebene Mindestanzahl von Prüfkörpern und der standardmäßigen Prüfung parallel und senkrecht zum Lager (Foliation, Lagenbau, u.s.w.) Rechnung getragen. Dass diese Maßnahmen nicht in jedem Fall ausreichend sind, um die mechanische Gesteinsanisotropie zu erfassen, wird in diesem Kapitel dargelegt.

Aus dem Bereich der Naturwerksteine existieren einige Lehrbücher und Datensammlungen, die sich sowohl an Steinmetze und Architekten als auch an Bauingenieure und Geologen richten (Hirschwald 1912, Peschel 1983; Reinsch 1991; Winkler 1994; Müller 2001). Diese Werke enthalten unter anderem Hinweise zur Untersuchung der Gesteinsfestigkeiten sowie Kompilationen von Festigkeitsdaten marktgängiger Naturwerksteine mit Bereichsangaben zu bestimmten Gesteinsgruppen. Die Aussagen zur Anisotropie der mechanischen Gesteinseigenschaften bleiben qualitativ und weisen keinen direkten Bezug zu den ursächlichen Gefügemerkmalen auf. In Bezug auf die Gefügeabhängigkeit der mechanischen Gesteinseigenschaften wurden detaillierte Untersuchungen von Siegesmund et al. (1999) für Marmore, Koch \& Siegesmund (2001) für Sandsteine und Strohmeyer \& Siegesmund (2002) für granitoide Mylonite durchgeführt. Hier wird auf die mineralogischen, petrographischen und mikrostrukturellen Besonderheiten der Gesteine und deren Auswirkungen auf die Gesteinsanisotropie eingegangen.

Eine große Anzahl von Studien zum mechanischen Verhalten von Gesteinen liegt aus dem Bereich der Rheologie und der Ingenieurgeologie vor. Die Arbeiten an einer Vielzahl von magmatischen, metamorphen und sedimentären Gesteinen umfassen sowohl Untersuchungen zur Druckfestigkeit unter einaxialen (z.B. Howarth \& Rowlands 1987; Azzoni et al. 1996; Li et al. 1998; Prikryl 1998/2001; Brosch et al. 2000) und triaxialen Bedingungen (z.B. Gottschalk et al. 1990; Shea \& Kronenberg 1993; Stavrogin \& Tarasov 2001) als auch Studien zur Biege- und Zugfestigkeit (Peck et al. 1985; Al-Lahyani et al. 1995). Die Abriebfestigkeit bzw. Bohrbarkeit von Gesteinen wird bei Howarth \& Rowlands (1987) und Ersoy \& Waller (1995) behandelt. Je nach Zielstellung der Arbeit stehen die mineralogische Zusammensetzung und/oder unterschiedliche Gefügeelemente, wie präexistierende Mikrorisse, die Kornform und die Textur der gesteinsbildenden Minerale sowie deren 


\section{Technische Gesteinseigenschaften}

Auswirkungen auf die Gesteinsfestigkeit im Vordergrund. Studien mit dem Schwerpunkt auf der mechanischen Anisotropie von Gesteinen und deren Ursachen beinhalten die Publikationen von Ramamurthy (1993), Shea \& Kronenberg (1993), Prikryl (1998) und Brosch et al. (2000).

Im Rahmen der vorliegenden Arbeit werden die mechanischen Gesteinseigenschaften und deren Anisotropie in Abhängigkeit vom makroskopischen Erscheinungsbild des Gesteins und der Textur der gesteinsbildenden Minerale diskutiert. Zudem wird der Einfluss des Mineralbestands und der Rissdichten offener Risse auf die Gesteinsfestigkeit dargelegt.

Die 20 untersuchten kristallinen Gesteine weisen eine starke Kornbindung und niedrige Porositäten $(<1$ Vol.\%) auf. Folglich ist zu vermuten, dass neben den offenen MikrorissSystemen die mechanischen Eigenschaften des Mineralbestands einen großen Einfluss auf die Gesamtgesteinseigenschaften haben. Daher wurden unter Berücksichtigung der mineralogischen Zusammensetzung sowie der Härte und Spaltbarkeit der Minerale, die mittlere Härte des Gesteins $\left(\mathrm{H}_{\mathrm{G}}\right)$ und der Spaltbarkeitsindex $\left(\mathrm{I}_{\mathrm{SpG}}\right)$ als ein Maß für die mechanische Stabilität der Gesteine berechnet (vgl. Kap.2.4.4.1) und zu den Festigkeitseigenschaften in Beziehung gesetzt.

\subsubsection{Ergebnisse in Relation zum makroskopischen Erscheinungsbild}

\subsubsection{Druckfestigkeit}

Die Mittelwerte der einaxialen Druckfestigkeit liegen zwischen $81 \mathrm{MPa}$ für den KalzitMarmor (Rek) und $347 \mathrm{MPa}$ für den granitischen Ultramylonit (Gra3a) und decken damit beinahe den gesamten Bereich der für Naturwerksteine verwirklichten Druckfestigkeiten ab (Winkler 1994; Müller 2001). Lediglich hochporöse Sedimentgesteine weisen noch niedrigere Druckfestigkeiten als Rek auf, und die 347 MPa der Probe Gra3a werden in einigen Fällen von hypabyssalen Basalten erreicht, zeigen mit diesen aber die absolute Spitze der vorkommenden Druckfestigkeiten. Die Makrogefüge-Gruppen I/II/III weisen mittlere Druckfestigkeiten von $210 \mathrm{MPa}$ (III) bis $219 \mathrm{MPa}$ (II) auf und zeigen damit keine signifikante Variation. Korrelierend mit der Anisotropie des makroskopischen Gefüges steigt die mittlere Anisotropie der Druckfestigkeiten von 7,6\% in der Gruppe I über 11,0\% in der Gruppe II auf 21,3\% in der Gruppe III an (Abb.6-8). 


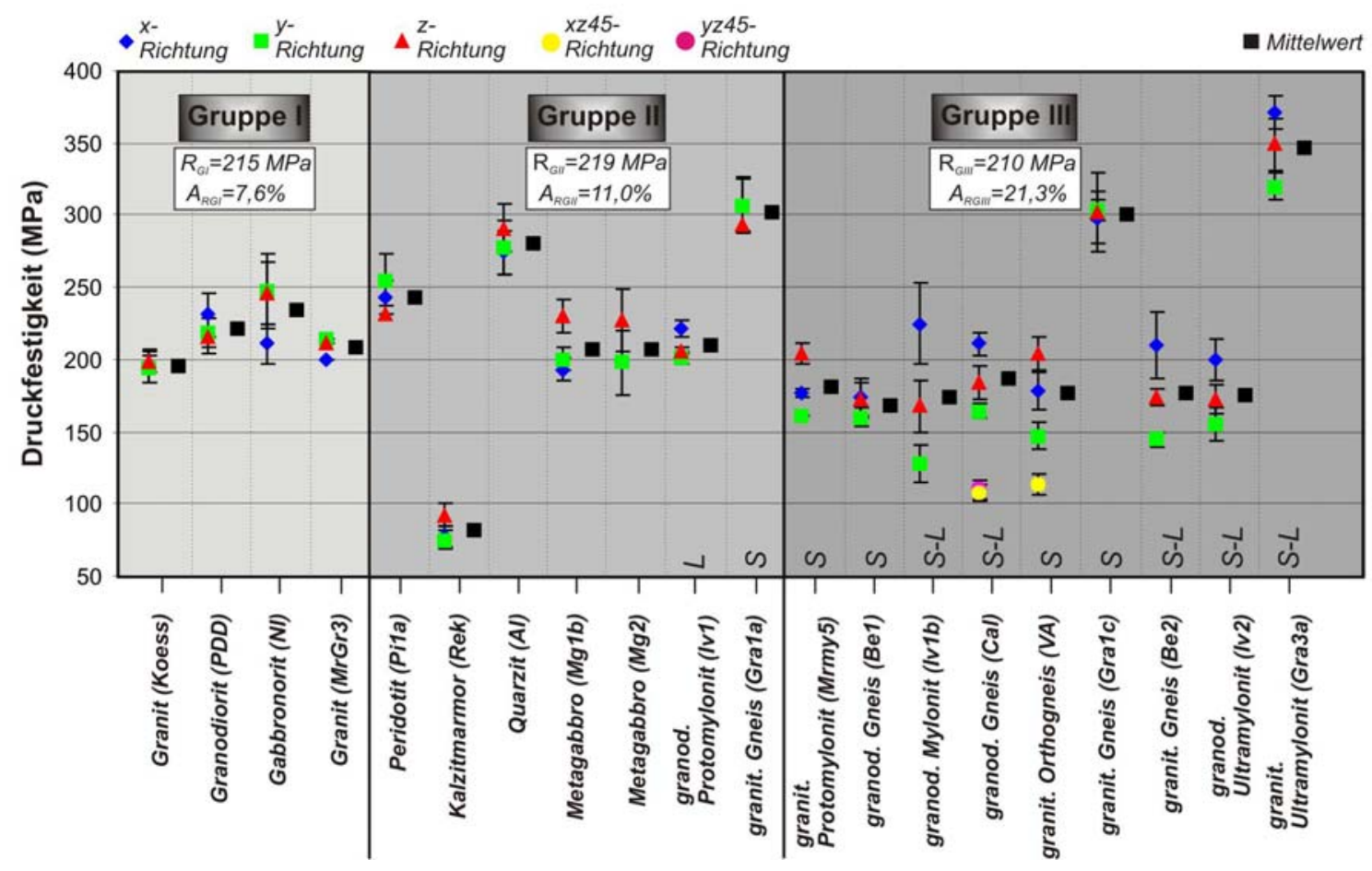

Abb.6-8: Einaxiale Druckfestigkeiten (R) der Makrogefüge-Gruppen I/IIIII. Mittelwerte der Druckfestigkeiten und mittlere Anisotropie für die jeweilige Gruppe sind angegeben. xz-45 Richtung und yz-45 Richtung: Druckfestigkeitsprüfkörper sind mit ihrer Kernachse $45^{\circ}$ zwischen der $x$ - und zRichtung bzw. der y- und z-Richtung des Probenreferenzsystems orientiert, die Richtungen wurden für die Berechungen der Mittelwerte und der Anisotropien nicht berücksichtigt. S - Flächengefüge dominant. S-L - sowohl Flächengefüge als auch Linear deutlich ausgebildet. L - Linear dominant. Fehlerbalken zeigen den 1- $\sigma$-Fehler.

Es zeigt sich also eine gute Übereinstimmung zwischen der makroskopischen Gefügeanisotropie und der Anisotropie der Druckfestigkeiten. Allerdings ist dieser Anstieg der Festigkeitsanisotropie in noch deutlicherer Form zu erwarten, da bei der Messung der Druckfestigkeiten mit den hier verwendeten Probenorientierungen wohl annähernd die Maxima, nicht aber die Minima der Druckfestigkeiten innerhalb einer Probe erfasst werden (vgl. Shea \& Kronenberg 1993; Brosch et al. 2000; Strohmeyer \& Siegesmund 2002). Die an den Proben Cal (granodioritischer Gneis) und VA (granitischer Gneis) durchgeführten Druckfestigkeitsprüfungen an $45^{\circ}$ zur Foliation (xy-Ebene) geneigten Kernen bestätigen diese Aussage. Die 45-Richtungen weisen mit 108/110 MPa (Cal xz45- bzw. yz45-Richtung) und 113 MPa (VA xz45-Richtung) die niedrigsten Druckfestigkeiten für die jeweilige Probe auf (Abb.6-8). Der Mittelwert sinkt dadurch für Cal von $186 \mathrm{MPa}$ auf $148 \mathrm{MPa}$ und für VA von 176MPa auf $145 \mathrm{MPa}$. Im Gegensatz dazu steigen die Anisotropien der Druckfestigkeit von $22 \%$ auf $48 \%$ bei Cal und von $28 \%$ auf $45 \%$ bei der Probe VA an. Dieses Phänomen wirkt insbesondere auf die anisotropen und ausgeprägt anisotropen Gesteine (Gruppen II/III), wohingegen die isotropen Gesteine (Gruppe I) davon nur geringfügig oder gar nicht berührt werden (vgl. Kap.8.2). Folglich sind die tatsächlichen Druckfestigkeits-Anisotropien der 


\section{Technische Gesteinseigenschaften}

Gruppen II und III deutlich höher und deren Festigkeits-Mittelwerte deutlich niedriger einzuschätzen, als nach dem angewendeten Verfahren ermittelt wurde.

Des Weiteren weisen die Druckfestigkeiten eine Abhängigkeit von der Gefügesymmetrie auf. Bei Gesteinen mit einem dominanten S-Gefüge (S-Typ - vgl. Kap. 8.2.1) zeigt die z-Richtung stets die höchste Druckfestigkeit, wohingegen bei den S-L-Typen die x-Richtung am stärksten ausgeprägt ist (Abb.6-8). Die mikrostrukturellen Ursachen für dieses Phänomen werden in Kap.8.2.1 vorgestellt.

Auffällig innerhalb des gesamten Probensatzes sind die Proben Grala und Gralc (granitische Gneise), die trotz ihres anisotropen bzw. ausgeprägt anisotropen Gefüges nur eine geringe, im Bereich des Fehlers liegende Anisotropie der Druckfestigkeiten aufweisen. Als Ursache dafür ist der geringe Vernetzungsgrad der Biotite $\mathrm{zu}$ nennen, der bereits im Zuge der Mikrogefügeuntersuchung erkannt wurde (vgl. Kap.5.6).

\subsubsection{Spaltzugfestigkeit}

Die Mittelwerte der Spaltzugfestigkeiten liegen zwischen 6,8 MPa für den Kalzit-Marmor (Rek) und 18,3 MPa für den Quarzit (AI). Die mittleren Spaltzugfestigkeiten der Makrogefüge-Gruppen sind mit 11,9 (Gruppe I) und 12,0 (Gruppe II/III) nahezu identisch. Mit zunehmender makroskopischer Gefügeanisotropie nimmt auch die Anisotropie der Spaltzugfestigkeiten deutlich zu. So weist die Gruppe I eine mittlere Anisotropie von 12,5\%, die Gruppe II von 24,2\% und die Gruppe III von 50,4\% auf (Abb.6-9). Mit Ausnahme der bezüglich ihrer Gefügeanisotropie nach dem makroskopischen Erscheinungsbild überschätzten Proben Grala und Gra1c, zeigt sich auch innerhalb der Gruppe eine Tendenz zur Übereinstimmung von höherer Gefügeanisotropie mit einer höheren Anisotropie der Spaltzugfestigkeiten. Bei allen Gesteinen mit einer ausgeprägten Foliation und einem Maximum der Glimmer (001)-Pole senkrecht zum Flächengefüge weist die z-Richtung die niedrigsten Spaltzugfestigkeiten auf. Häufig hat gleichzeitig die x-Richtung die höchsten Festigkeiten. Eine Analyse dieser Gegebenheiten wird in Kap.8.2.1 vorgenommen. 


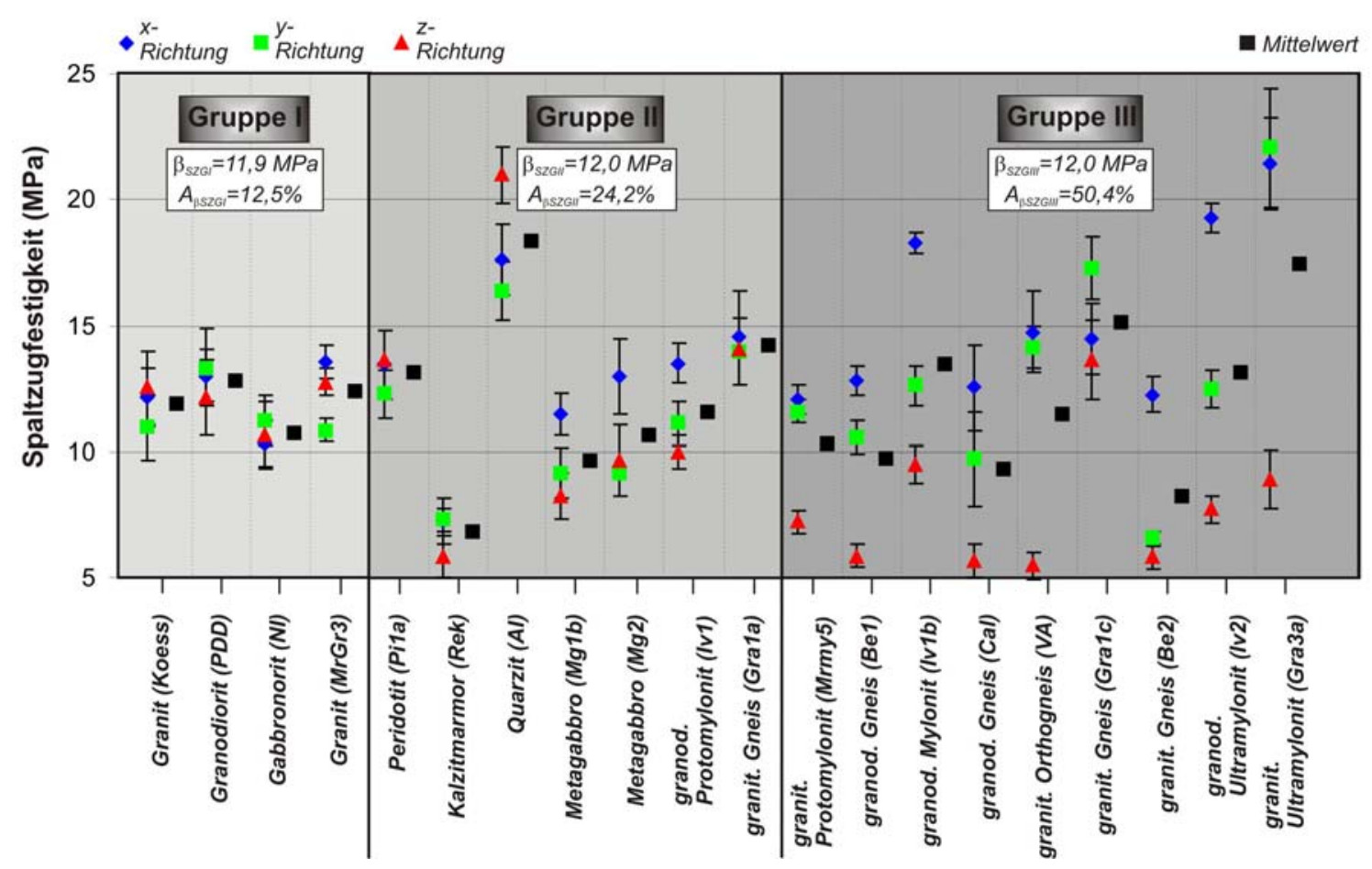

Abb.6-9: Spaltzugfestigkeit ( $\beta_{\text {Sz }}$ ) der Makrogefüge-Gruppen I/II/III. Mitte/werte der Spaltzugfestigkeiten und mittlere Anisotropie für die jeweilige Gruppe sind angegeben. Fehlerbalken zeigen den 1- $\sigma$ Fehler.

\subsubsection{Biegefestigkeit}

Aufgrund des hohen Materialaufwands konnten lediglich zwölf der 20 Gesteine auf ihre Biegefestigkeit hin untersucht werden. Die Mittelwerte rangieren zwischen 14,9 MPa beim Meta-Gabbro (Mg1b) und 32,8 MPa beim granitischen Ultramylonit (Gra3a). Abgesehen von den Proben Grala und Gra3a, die mit ihren Biegefestigkeiten von >30 MPa im Bereich der kristallinen Schiefer (Müller 2001) liegen, befinden sich alle Proben in dem als typisch für die jeweilige Gesteinsgruppen angegebenen Bereich.

Die mittleren Biegefestigkeiten der Makrogefüge-Gruppen bewegen sich zwischen 19,4 MPa (Gruppe I) und 22,3 MPa (Gruppe II), ohne eine signifikante Tendenz in Abhängigkeit von der makroskopischen Gefügeanisotropie zu zeigen. Prinzipiell steigt die Anisotropie der Biegefestigkeit parallel zur Zunahme der makroskopischen Gefügeanisotropie. Die Gruppe I weist eine Anisotropie von 12,7\%, die Gruppe II von 40,8\% und die Gruppe III von 77,5\% auf (Abb.6-10). Im Vergleich zur Druck- und Spaltzugfestigkeit scheint die Biegefestigkeit am deutlichsten mit einer Anisotropiezunahme auf die progressive bevorzugte Orientierung von Gefügeelementen zu reagieren. Allerdings muss beachtet werden, dass hier nicht sämtliche Proben untersucht wurden. Wie auch schon bei den Spaltzugfestigkeiten ist die zRichtung der anisotropen und ausgeprägt anisotropen Gesteine mit einem Maximum der 


\section{Technische Gesteinseigenschaften}

Glimmer (001)-Pole senkrecht zur Foliation am schwächsten ausgebildet (Gründe: vgl. Kap.8.2.1).

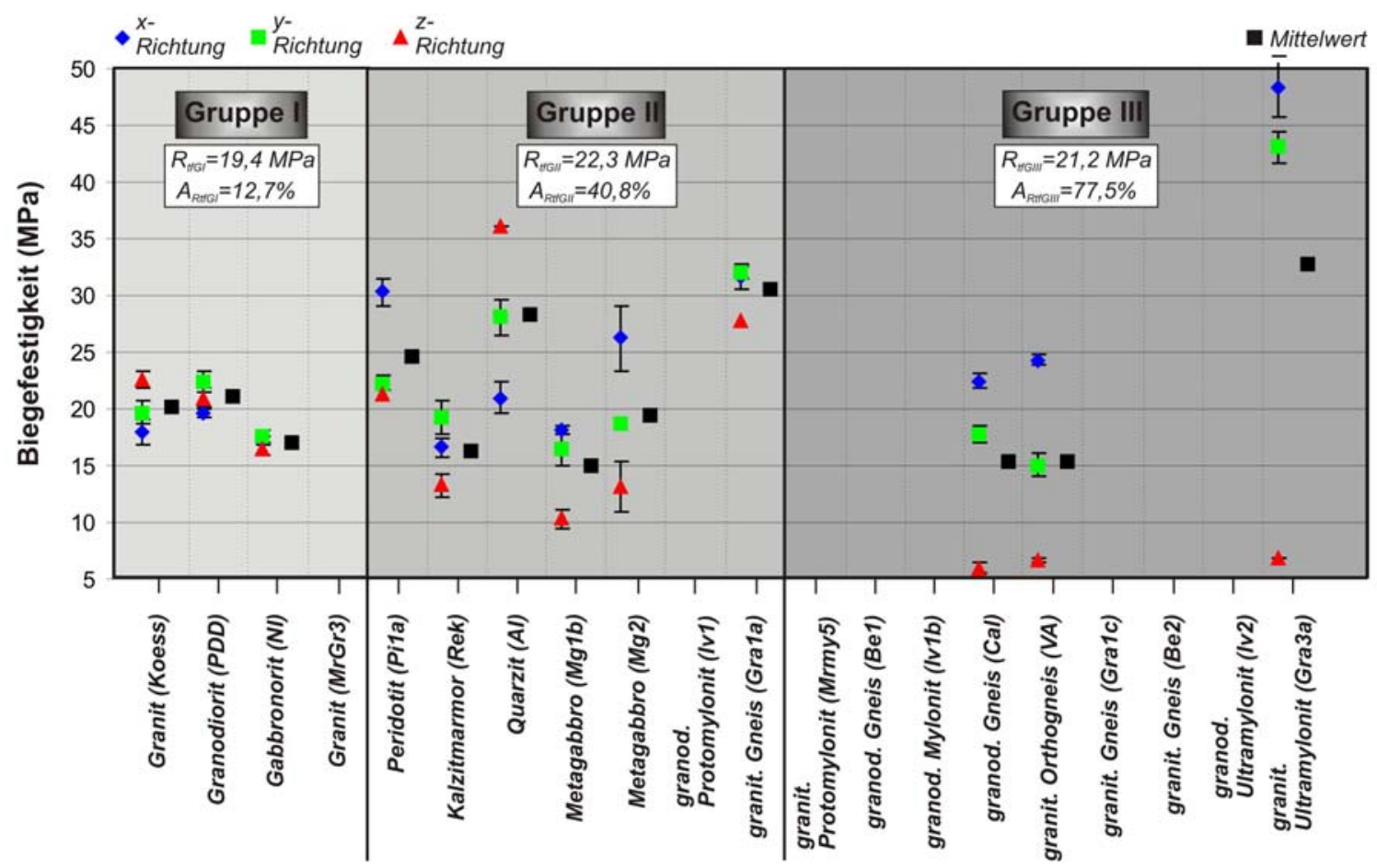

Abb.6-10: Biegefestigkeit $\left(R_{t t}\right)$ der Makrogefüge-Gruppen I/IIIIII. Mitte/werte der Biegefestigkeiten und mittlere Anisotropie für die jeweilige Gruppe sind angegeben. Fehlerbalken zeigen den 1- $\sigma$-Fehler.

Wiederum auffällig ist die Probe Grala, deren Festigkeitsanisotropie deutlich geringer ausfällt als aufgrund der Einschätzung nach dem makroskopischen Erscheinungsbild zu erwarten gewesen wäre.

\subsubsection{Abriebfestigkeit}

Die mittlere Abriebfestigkeit ist am niedrigsten für den Kalzit-Marmor (Rek) mit 24,9 $\mathrm{cm}^{3}$ und am höchsten für den Quarzit (AI) mit 4,3 $\mathrm{cm}^{3}$. Im Vergleich zu anderen Gesteinen markiert der Quarzit die höchstmöglichen der bei den Naturwerksteinen verwirklichten Abriebfestigkeiten, wohingegen der typische Kalzit-Marmor von hochporösen Sedimentgesteinen mit schwacher Kornbindung noch um das drei- bis vierfache des Volumenverlusts übertroffen werden kann (Müller 2001).

Die mittlere Abriebfestigkeit der Makrogefüge-Gruppe II fällt mit $9,03 \mathrm{~cm}^{3}$ schwächer aus als die der Gruppen I/III $\left(6,74 \mathrm{~cm}^{3}\right.$ bzw. 7,35 $\left.\mathrm{cm}^{3}\right)$. Das liegt jedoch ausschließlich an den gegenüber den anderen Gesteinen extrem niedrigen Abriebfestigkeiten des Marmors (Abb.611). 


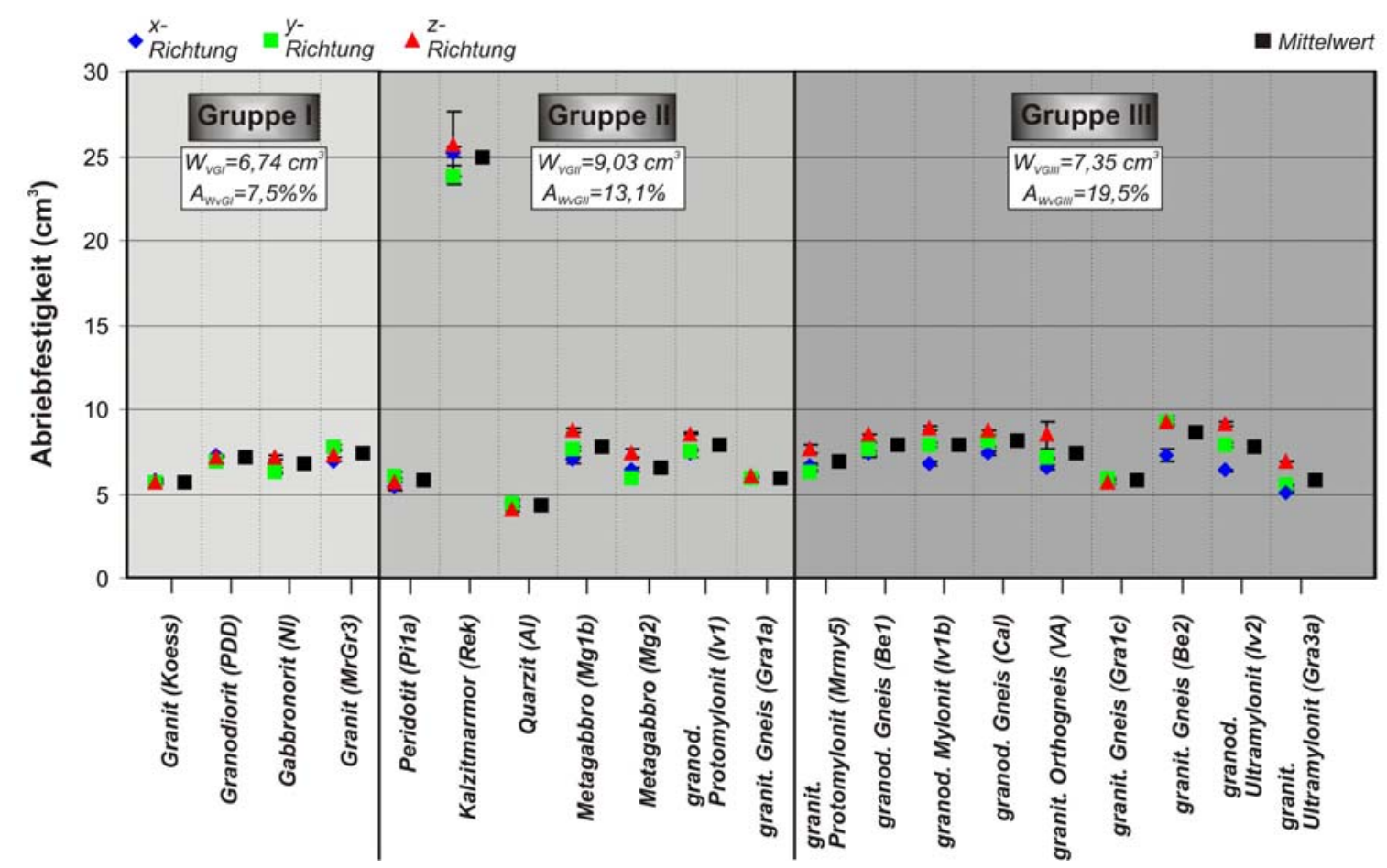

Abb.6-11: Abriebfestigkeit $\left(W_{v}\right)$ der Makrogefüge-Gruppen I/II/III. Mittelwerte der Abriebfestigkeiten und mittlere Anisotropie für die jeweilige Gruppe sind angegeben. Fehlerbalken zeigen den 1- $\sigma$ Fehler.

Die Anisotropie der Abriebfestigkeit steigt mit zunehmender makroskopischer Gefügeanisotropie von Gruppe I (7,5\%) über Gruppe II (13,1\%) zur Gruppe III (19,5\%) zu. Hier, wenn auch nicht so offensichtlich, sind die Proben Grala und Gra1c niedriger anisotrop als vom makroskopischen Erscheinungsbild zu erwarten ist.

Von den mechanischen Eigenschaften reagieren die Abriebfestigkeiten am geringsten mit einem Anstieg der Anisotropie auf die Zunahme der Gefügeanisotropie. Sowohl bei der Druck- als auch bei den Spaltzug- und Biegefestigkeiten ist dieser Effekt deutlich stärker ausgeprägt. Eine Begründung liefern die unterschiedlichen Versagensmuster bei der Bestimmung der Festigkeitseigenschaften. Ähnlich der Spaltzug- und Biegefestigkeit weist die z-Richtung bei den Gesteinen mit einem deutlichen Planar-Gefüge die niedrigsten Abriebfestigkeiten auf (Begründung in Kap.8.2).

\subsubsection{Gesteinsfestigkeiten und mineralogische Zusammensetzung}

Nachdem, wie in Kap.6.3.1 dargelegt, die Anisotropie der mechanischen Eigenschaften hinreichend aus dem makroskopischen Erscheinungsbild abgeschätzt werden kann, gilt es nun zu überprüfen, welche Einflussfaktoren die mittleren Gesteinsfestigkeiten bestimmen. 

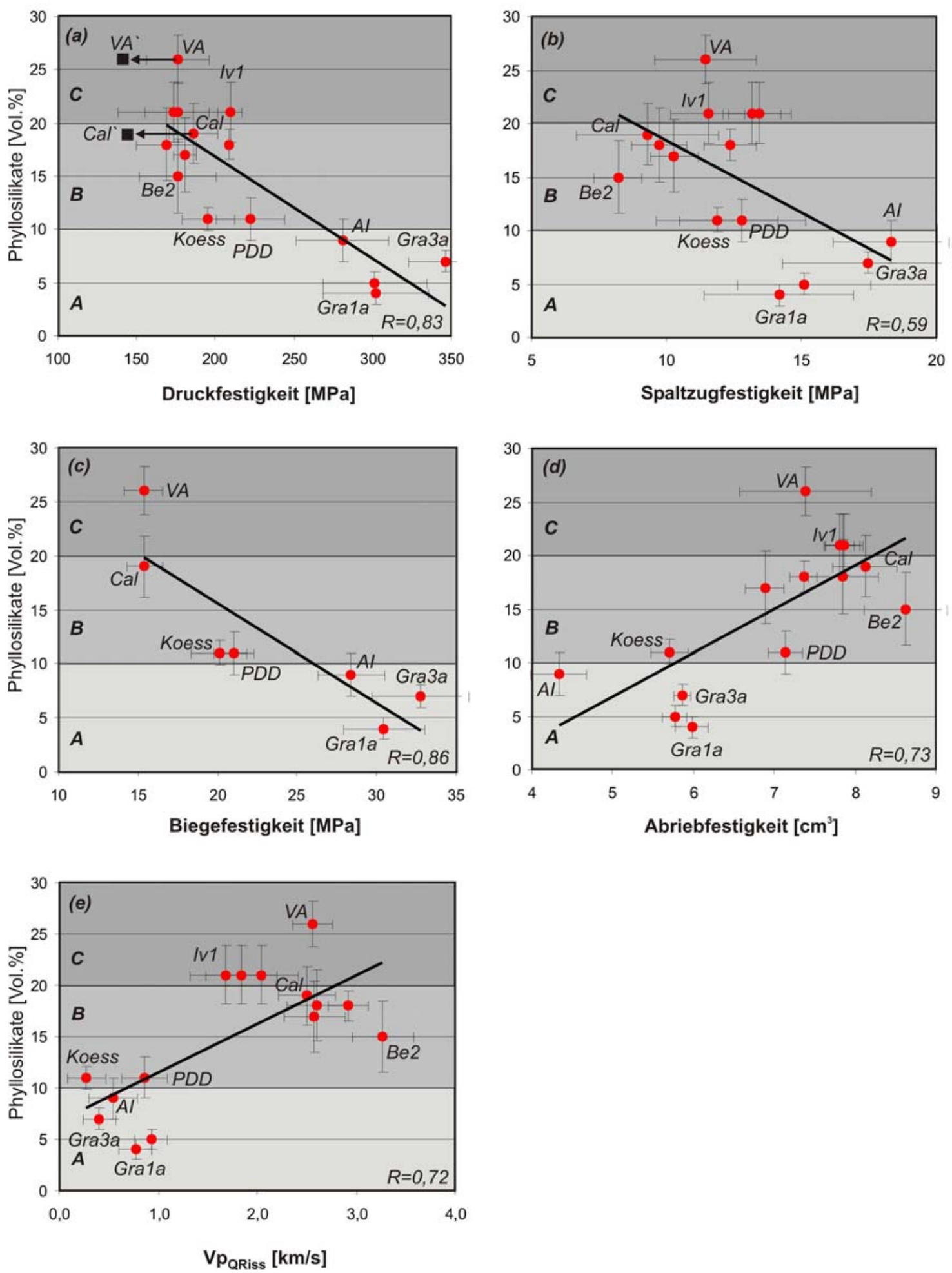

Abb.6-12: Gesteinsfestigkeit und Volumenanteil der Phyllosilikate. (a) Einaxiale Druckfestigkeit; Cal'

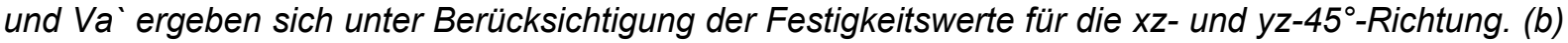
Spaltzugfestigkeit. (c) Biegefestigkeit. (d) Abriebfestigkeit. (e) Verhältnis von Phyllosilikatanteil zum Rissquantifizierungsparameter (V $p_{\text {QRiss }}$ ). Die Phyllosilikat-Gruppen A (<10 Vol.\%), B (10-20 Vol.\%) und C (>20 Vol.\%) sind gekennzeichnet. Gra1a - granitischer Gneis. Gra3a - granitischer Ultramylonit. Cal - granodioritischer Gneis. VA - granitischer Gneis. Koess - Granit. PDD - Granodiorit. IV1 granodioritischer Protomylonit. Al - Quarzit. Be2 - granitischer Gneis. Festigkeitsmittelwerte berechnet als arithmetisches Mittel aus den Festigkeiten von $x$-, $y$ - und z-Richtung. Fehlerbalken zeigen den absoluten Fehler (Phyllosilikatanteil) bzw. für die Festigkeiten und für die $V p_{Q R i s s}-$ Werte den 1- $\sigma$-Fehler. 


\section{Technische Gesteinseigenschaften}

Gelingt es, sowohl die Anisotropie als auch den Mittelwert der Festigkeitseigenschaften von der Mineralogie und den Gefügemerkmalen abzuleiten, so ist in Verbindung mit den in Kap.8.2.1 aufgezeigten Versagensmodellen eine vollständige Beschreibung der Höhe und des richtungsabhängigen Verhaltens der Gesteinsfestigkeiten möglich.

Da den Glimmermineralen neben ihrer ausgeprägten Anisotropie auch eine Schwächung der mechanischen Gesteinseigenschaften nachgesagt wird (z.B. Tapponnier \& Brace 1976; Gottschalk et al. 1990), wird zunächst der Einfluss des Volumenanteils der Phyllosilikate auf die mittlere Gesteinsfestigkeit behandelt.

Tatsächlich zeigen die Druck-, Spaltzug-, Biege- und Abriebfestigkeiten eine Antikorrelation zu den Volumenanteilen der Phyllosilikate (Abb.6-12). Allerdings suggeriert die starke Streuung der Daten mit gegenläufigen Trends (Beispiel: Probengruppe mit Proben Be2, Iv1, Cal und VA zeigt eine positive Korrelation) innerhalb bestimmter Gesteinsgruppen einen Einfluss weiterer Faktoren. Abb.6-12 belegt, dass die Gesteine auch bei ähnlichen Phyllosilikatgehalten unterschiedliche Rissdichten aufweisen können und dieser Umstand eine mögliche Ursache für die Abweichung bestimmter Proben vom generellen Trend ist. Als Beispiele können die Proben Iv1, Cal und Be2 herangezogen werden. Die Probe Cal (granodioritischer Gneis) liegt stets in der Nähe der generellen Entwicklungslinie von Gesteinsfestigkeiten und den Phyllosilikatgehalten. Ihre Rissdichte $\left(\mathrm{Vp}_{\mathrm{QRiss}}=2,51 \mathrm{~km} / \mathrm{s}\right)$ wird im Folgenden als Vergleichswert verwendet. Die Probe Be2 (granitischer Gneis) zeigt stets niedrigere Festigkeiten als Cal und eine deutlich höhere Rissdichte ( $\mathrm{Vp}_{\text {QRiss }}=3,27 \mathrm{~km} / \mathrm{s}$ ) (Abb.6-12). Da die Phyllosilkatanteile bei Be2 mit 15 Vol.\% niedriger sind als bei Cal mit 19 Vol.\%, kann nur die größere Rissdichte für die mechanische Schwächung von Be2 verantwortlich sein. Umgekehrt verhält es sich bei der Probe Iv1, deren Phyllosilikatanteil bei $21 \mathrm{Vol} \%$ liegt und $\mathrm{zu}$ tendenziell niedrigeren Festigkeitswerten gegenüber der Probe Cal führen sollte. Wie aus $\boldsymbol{A b b . 6 - 1 2}$ hervorgeht, ist das jedoch nicht der Fall und kann mit der niedrigeren Rissdichte $\left(\mathrm{Vp}_{\mathrm{QRiss}}=1,69 \mathrm{~km} / \mathrm{s}\right)$ erklärt werden. Der zunehmende Phyllosilikatanteil und die zunehmende Rissdichte sind somit zwei Faktoren, die beide eine mechanische Schwächung des Gesteins hervorrufen, die bezüglich der Quantität ihres Einflusses aber nur schwer zu unterscheiden sind.

Um ebenso Gesteine ohne Phyllosilikate betrachten $\mathrm{zu}$ können und auch andere Mineralbestandteile und deren mechanische Eigenschaften in die Betrachtungen einzubeziehen, werden die Festigkeitseigenschaften in Abb.6-13 und Abb.6-14 in Beziehung zum Spaltbarkeitsindex $\left(\mathrm{I}_{\mathrm{SpG}}\right)$ bzw. der mittleren Härte $\left(\mathrm{H}_{\mathrm{G}}\right)$ des Gesteins gesetzt. Es wird 
somit die mittlere mechanische Stabilität des Mineralbestands mit den Mittelwerten der Gesteinsfestigkeiten verglichen.
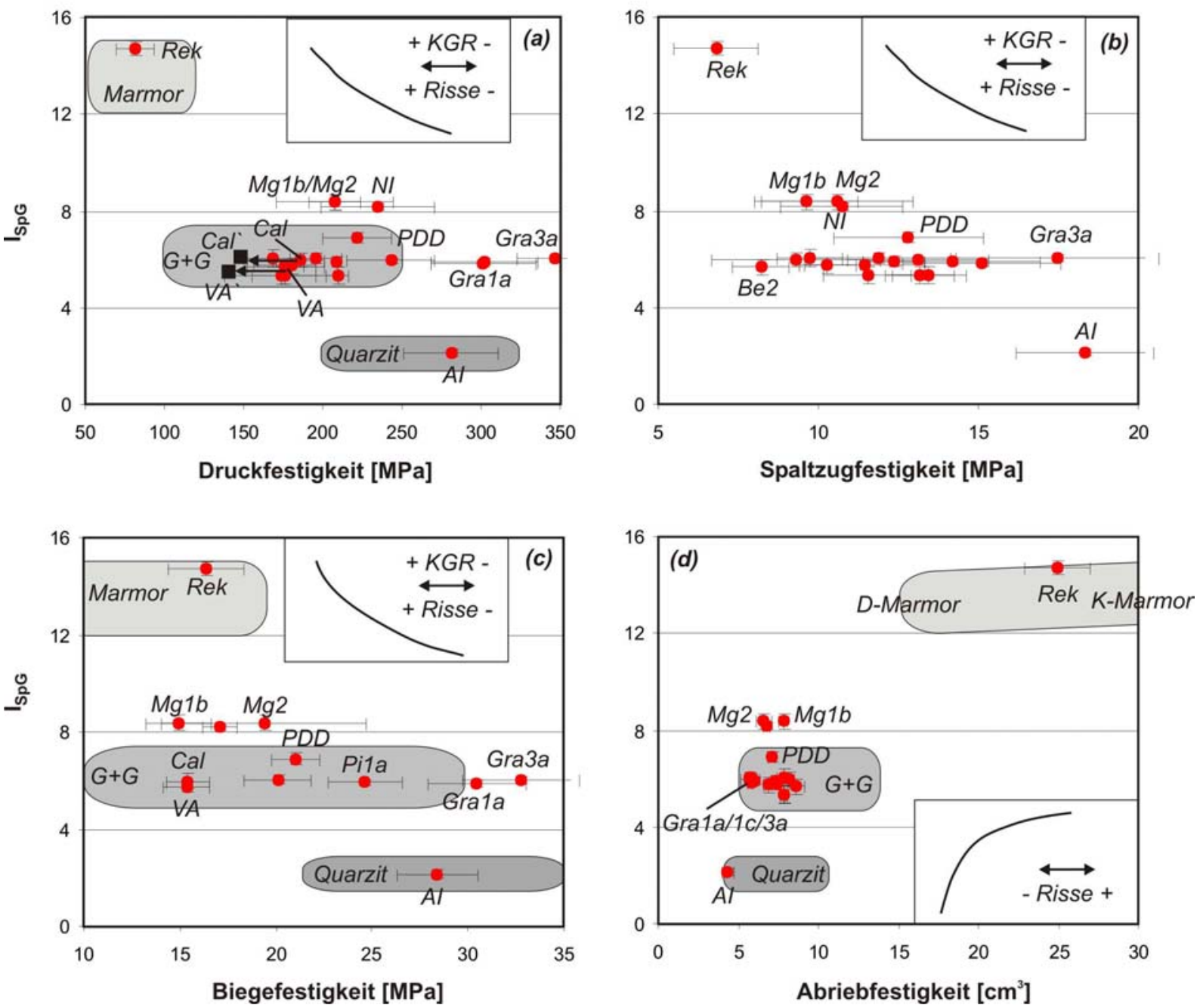

Abb.6-13: Gesteinsfestigkeiten und Spaltbarkeitsindex (I $\left.I_{S G G}\right)$. (a) Einaxiale Druckfestigkeit; Cal' und

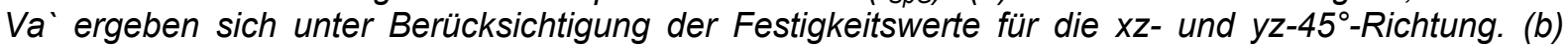
Spaltzugfestigkeit. (c) Biegefestigkeit. (d) Abriebfestigkeit. Die Bereiche für Marmore (D-Marmor Dolomit-Marmor; K-Marmor - Kalzit-Marmor); Gneise und Granitoide (G+G) sowie Quarzite sind gekennzeichnet. Die $I_{S p G^{-}}$Werte für die Bereiche ergeben sich aus der theoretisch möglichen Variationsbreite der mineralogischen Zusammensetzung. Die zugehörigen Festigkeitswerte für die Gesteinsgruppen entstammen der Datensammlung von Börner \& Hill (2002); Marmor (34 Einzelgesteine); Gneis und Granitoide (68 Einzelgesteine); Quarzit (6 Einzelgesteine). Gra1a granitischer Gneis. Gra3a - granitischer Ultramylonit. Cal - granodioritischer Gneis. VA - granitischer Gneis. Rek - Kalzit-Marmor. PDD - Granodiorit. Pi1a - Peridotit. NI - Gabbro-Norit. Mg1b/Mg2 Meta-Gabbro. Al - Quarzit. Be2 - granitischer Gneis. Auswirkungen der Variation von Rissdichten und Korngrößen (KGR) sowie Datentrends sind in Kästen bezeichnet. Festigkeitsmitte/werte berechnet als arithmetisches Mittel aus den Festigkeiten von $x$-, $y$ - und z-Richtung. Fehlerbalken zeigen den 1- $\sigma$ Fehler.

Für alle vier Festigkeitseigenschaften zeigt sich ein Trend zur Übereinstimmung niedriger $\mathrm{I}_{\mathrm{SpG}}-$ Werte (und damit hoher mittlerer mechanischer Festigkeiten der gesteinsbildenden Minerale eines Gesteins - vgl. Kap.2.4.4.1/4.2) mit hohen Gesteinsfestigkeiten. Das bedeutet, ein Gestein mit einer durchschnittlich schlechten Spaltbarkeit seiner Minerale zeigt eine Tendenz zu hohen Festigkeiten (Abb.6-13). Umgekehrt verhält es sich bei der Betrachtung 


\section{Technische Gesteinseigenschaften}

von mittlerer Härte des Gesteins und den mittleren Festigkeitseigenschaften (Abb.6-14). Gesteine mit einer hohen mittleren Härte ihres Mineralbestands weisen eine Tendenz zu hohen Festigkeiten auf. Trotz der beobachtbaren Trends wird deutlich, dass neben den Eigenschaften des Mineralbestands noch andere Parameter einen Einfluss auf die mittleren Gesteinsfestigkeiten haben müssen. Deutlich wird das bei der Betrachtung von Gesteinen mit ähnlichen $\mathrm{I}_{\mathrm{SpG}}-$ und $\mathrm{H}_{\mathrm{G}}-$ Werten.
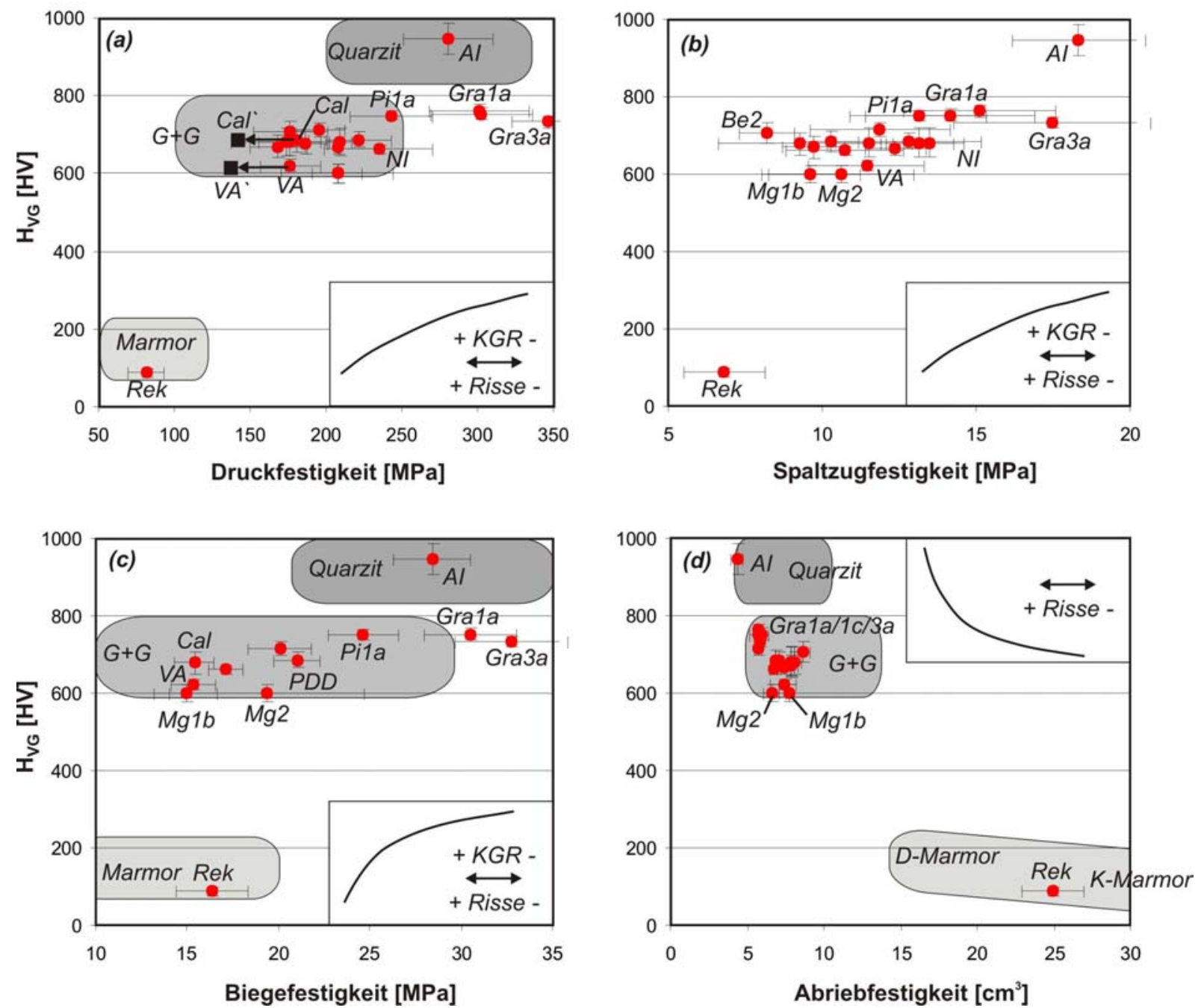

Abb.6-14: Gesteinsfestigkeiten und mittlere Härte der Gesteine. (a) Einaxiale Druckfestigkeit; Cal'

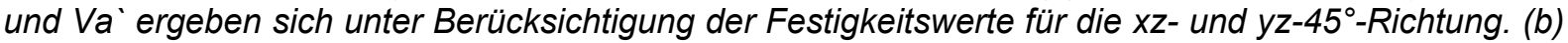
Spaltzugfestigkeit. (c) Biegefestigkeit. (d) Abriebfestigkeit. Die Bereiche für Marmore (D-Marmor Dolomit-Marmor; K-Marmor - Kalzit-Marmor); Gneise und Granitoide $(G+G)$ sowie Quarzite sind gekennzeichnet. Die $I_{S p G^{-}}$Werte für die Bereiche ergeben sich aus der theoretisch möglichen Variationsbreite der mineralogischen Zusammensetzung. Die zugehörigen Festigkeitswerte für die Gesteinsgruppen entstammen der Datensammlung von Börner \& Hill (2002); Marmor (34 Einzelgesteine); Gneis und Granitoide (68 Einzelgesteine); Quarzit (6 Einzelgesteine). Gra1a/1c granitischer Gneis. Gra3a - granitischer Ultramylonit. Cal - granodioritischer Gneis. VA - granitischer Gneis. Rek - Kalzit-Marmor. PDD - Granodiorit. Pi1a - Peridotit. NI - Gabbro-Norit. Mg1b/Mg2 Meta-Gabbro. Al - Quarzit. Be2 - granitischer Gneis. Auswirkungen der Variation von Rissdichten und Korngrößen (KGR) sowie Datentrends sind in Kästen bezeichnet. Festigkeitsmitte/werte berechnet als arithmetisches Mittel aus den Festigkeiten von $x$-, $y$ - und z-Richtung. Fehlerbalken zeigen den 1- $\sigma$ Fehler. 
Die in $\boldsymbol{A b b . 6 - 1 3 / A b b . 6 - 1 4}$ als grau unterlegte Kästchen ausgehaltenen Gesteinstypen weisen deutliche Unterschiede der Festigkeiten (Festigkeitsdaten aus Börner \& Hill 2002) auf, obwohl ihr Spaltbarkeitsindex und ihre mittlere Härte aufgrund der Variationsmöglichkeit der mineralogischen Zusammensetzung nur gering ausfallen. Für eine eingehende Betrachtung wurden der Bereich $\mathrm{I}_{\mathrm{SpG}}=5,5-7$, der die granitischen und granodioritischen Gneise und Mylonite sowie den Peridotit umfasst, bzw. der Bereich $\mathrm{H}_{\mathrm{G}}=600-800 \mathrm{HV}$, der zusätzlich die Meta-Gabbros und den Gabbro-Norit beinhaltet (Abb.6-13/Abb.6-14), ausgewählt. Die Gesteine beider Gruppen zeigen bei relativ ähnlicher mechanischer Stabilität ihres Mineralbestands deutlich variierende Festigkeitseigenschaften. Dieser Effekt ist bezüglich Druck-, Spaltzug- und Biegefestigkeiten stark und für die Abriebfestigkeiten gering ausgeprägt, was die maximale Abweichung vom Mittelwert der Gruppe beweist. Für die Druckfestigkeiten beträgt der Mittelwert der Gruppe mit $\mathrm{I}_{\mathrm{SpG}}=5,5-7218 \mathrm{MPa}$, und die maximale Abweichung vom Mittelwert zeigt mit 59\% die Probe Gra3a (Spaltzugfestigkeitsmittelwert: 12,3 MPa, Abweichung: 42\%, Probe: Gra3a; Biegefestigkeitsm:: 22,8 MPa, Abw.: 44\%, P.: Gra3a; Abriebfestigkeitsm.: 7,1 cm ${ }^{3}$, Abw.: 21\%, P.: Be2). Die Ursachen für die Festigkeitsunterschiede bei ähnlicher mechanischer Stabilität des Mineralbestands werden anhand der Beispielgruppen im folgenden Kapitel erläutert.

\subsubsection{Beziehung zwischen der Anisotropie der Gesteinsfestigkeiten und der Glimmertextur}

Die maximale Intensität der Glimmertextur ((001)-Pole) und deren Regelungsschärfe $\left(\mathrm{c}_{\mathrm{W}}\right)$ sind geeignet, die Gefügeanisotropie der glimmerhaltigen Gesteine stellvertretend zu beschreiben. Es stellt sich also die Frage, ob zwischen der bevorzugten Orientierung der Glimmer und der Anisotropie der Gesteinsfestigkeiten nicht nur eine qualitative Übereinstimmung (was die in Kap.6.3.1 beschriebenen Relationen von Makrogefüge-Gruppen und Festigkeitsanisotropie suggerieren), sondern auch ein quantifizierbarer Zusammenhang besteht. Bezüglich der Druckfestigkeiten (Abb.6-15) ergibt sich eine schlechte Korrelation von maximaler Intensität der Glimmer (001)-Pole und der Festigkeitsanisotropie. 

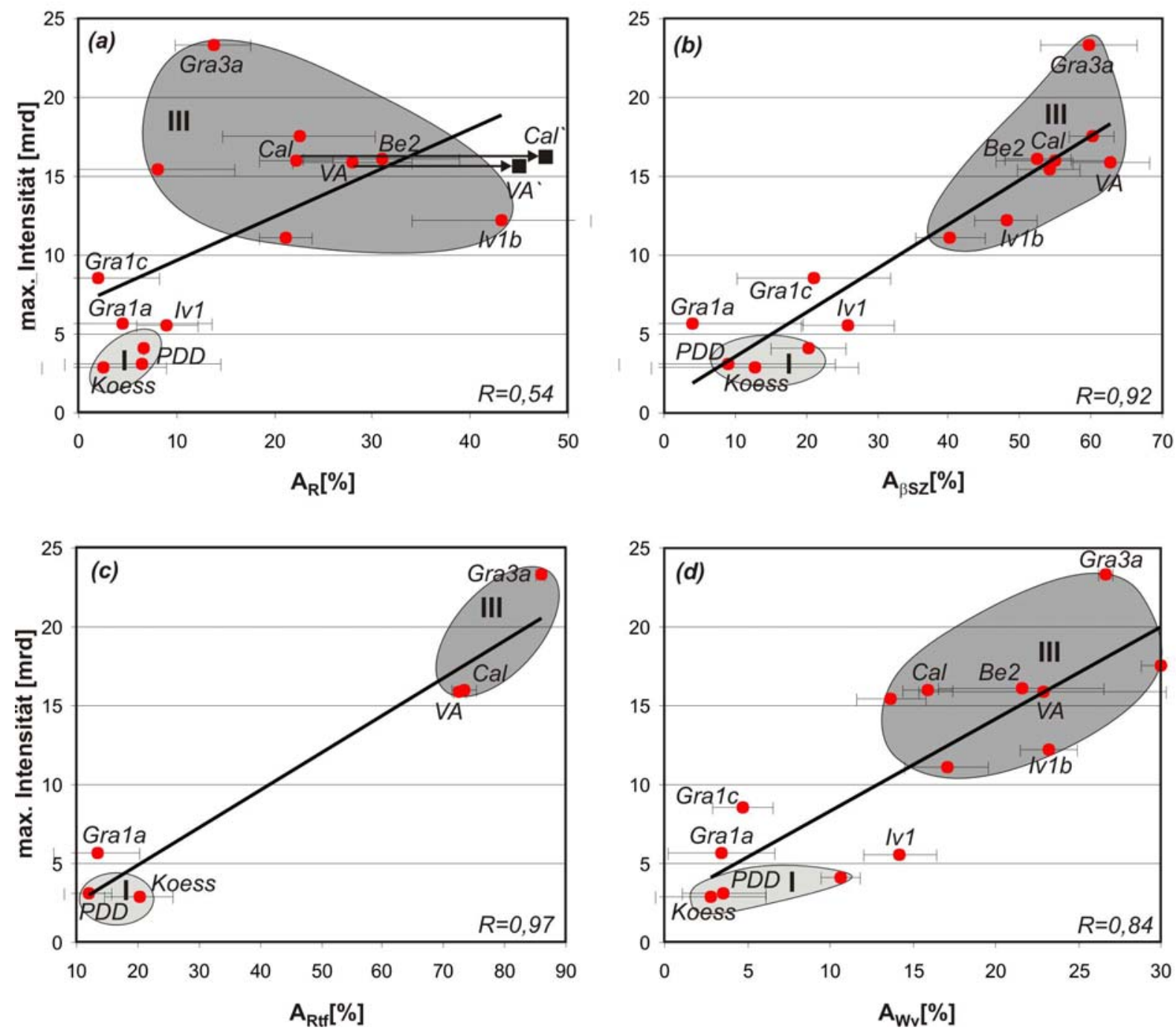

Abb.6-15: Anisotropie der Gesteinsfestigkeiten in Abhängigkeit von der maximalen Intensität der Glimmertextur ((001)-Pole). (a) Einaxiale Druckfestigkeit; Cal und Va' ergeben sich unter Berücksichtigung der Festigkeitswerte für die xz- und yz-45-Richtung. (b) Spaltzugfestigkeit. (c) Biegefestigkeit. (d) Abriebfestigkeit. Die Makrogefüge-Gruppen I (hellgrau) und III (dunkelgrau) sind gekennzeichnet. Gra1a - granitischer Gneis. Gra1c - granitischer Gneis. Gra3a - granitischer Ultramylonit. Cal - granodioritischer Gneis. VA - granitischer Gneis. Koess - Granit. PDD Granodiorit. Iv1 - granodioritischer Protomylonit. Iv1b - granodioritischer Mylonit. Be2 - granitischer Gneis. Fehlerbalken zeigen den 1- $\sigma$-Fehler.

Zwar zeigt sich die Tendenz, dass die Proben der Gruppe III mit ihren stärkeren Texturen auch eine höhere Anisotropie der Druckfestigkeiten aufweisen, doch ist die Streuung der Daten sehr hoch. Proben wie z.B. der granitische Ultramylonit (Gra3a) zeigen deutlich niedrigere Druckfestigkeitsanisotropien als nach dem Trend $\mathrm{zu}$ erwarten ist. Ursächlich verantwortlich ist der Umstand, dass unter Berücksichtung der drei Hauptrichtungen (x-, y-, zRichtung) das Minimum der Druckfestigkeiten nicht erfasst wird und damit die Anisotropien bei bestimmten Gesteinen zu niedrig ausfallen. Deutlich wird das an den Proben Cal (granodioritischer Gneis) und VA (granitischer Gneis), deren tatsächliche Anisotropie unter Berücksichtigung der xz/yz45-Richtungen (Datenpunkte Cal’ und VA') mit 48\% bzw. 45\% 


\section{Technische Gesteinseigenschaften}

wesentlich höher sind als die vorher ermittelten $22 \%$ und 28\%. Für die Probe Gra3a, stellvertretend für die Proben der Gruppe III, ist ein ähnlicher Effekt zu erwarten. Die isotropen Gesteine der Gruppe I würden nur einen geringen oder gar keinen Anstieg der Anisotropie aufweisen, da die Vorzugsorientierungen hier nur eine untergeordnete Rolle spielen.

Für die Spaltzug-, Biege- und Abriebfestigkeiten (Abb.6-15) ergibt sich eine statistisch signifikante Korrelation von Festigkeitsanisotropie und maximaler Intensität der Glimmertextur. $\mathrm{Zu}$ beachten ist, dass nur sechs der glimmerhaltigen Proben auf ihre Biegefestigkeit untersucht wurden. Da die Datenpunkte der Gruppe I und der Gruppe III weit auseinander liegen, kann es sich um eine Scheinkorrelation handeln.

Analog zu den beschriebenen Zusammenhängen zwischen der Anisotropie der Gesteinsfestigkeiten und der maximalen Intensität der Glimmertextur stellen sich auch die Verhältnisse zwischen der Regelungsschärfe $\left(\mathrm{c}_{\mathrm{W}}\right)$ und der Anisotropie der Gesteinsfestigkeiten dar (Abb.6-16). Beide Parameter sind also geeignet, eine zumindest semiquantitative Abschätzung der Anisotropie der Spalt-, Biege- und Abriebfestigkeiten zu liefern. Auch für die Druckfestigkeit ist ein solcher Zusammenhang zu erwarten, wenn bei der Messung die tatsächliche Festigkeitsanisotropie ermittelt wird. Eine endgültige Verifizierung dieser Aussage und eine anschließende Quantifizierung kann aufgrund der bestehenden Daten allerdings nicht erfolgen. 

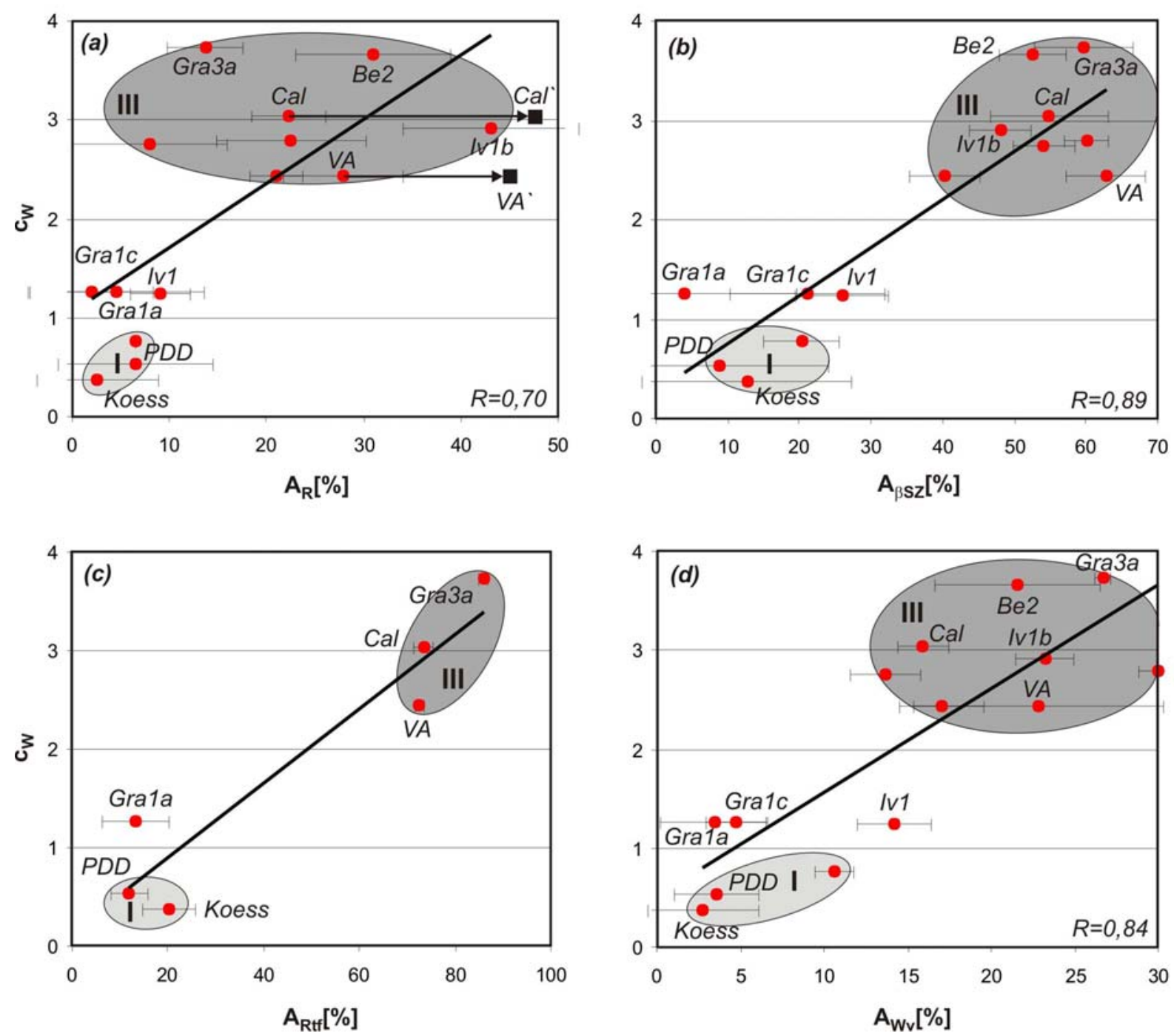

Abb.6-16: Anisotropie der Gesteinsfestigkeiten in Abhängigkeit von der Regelungsschärfe ( $\left.c_{w}\right)$ der Glimmer (001)-Pole. (a) Einaxiale Druckfestigkeit; Cal' und Va' ergeben sich unter Berücksichtigung

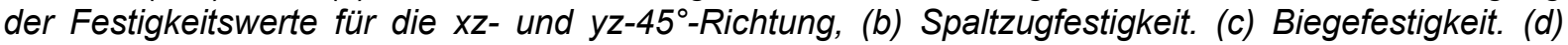
Abriebfestigkeit. Die Makrogefüge-Gruppen I (hellgrau) und III (dunkelgrau) sind im Diagramm gekennzeichnet. Gra1a - granitischer Gneis. Gra1c - granitischer Gneis. Gra3a - granitischer Ultramylonit. Cal - granodioritischer Gneis. VA - granitischer Gneis. Koess - Granit. PDD Granodiorit. Iv1 - granodioritischer Protomylonit. Iv1b - granodioritischer Mylonit. Be2 - granitischer Gneis. Fehlerbalken zeigen den 1- $\sigma$-Fehler.

\subsubsection{Gesteinsfestigkeiten, offene Mikroriss-Systeme und Korngröße}

Um den Einfluss der offenen Mikroriss-Systeme und der Korngröße auf die mittleren Gesteinsfestigkeiten festzustellen, werden für die Druck-, Spaltzug- und Biegefestigkeiten die Gesteinsgruppe mit $\mathrm{I}_{\mathrm{SpG}}=5,5-7$ und für die Abriebfestigkeiten die Gesteinsgruppe mit $\mathrm{H}_{\mathrm{VG}}=600-800 \mathrm{HV}$ (vgl. Kap.6.3.2) untersucht. In allen vier Fällen werden die Festigkeiten gegen die Rissdichten, quantifiziert durch $\mathrm{Vp}_{\mathrm{QRiss}}$, aufgetragen (Abb.6-17). Bei der Druck-, Spalt- und Biegefestigkeit sind zwei unterschiedliche Trends zu beobachten. Zum einen korrelieren hohe Rissdichten (hohe $\mathrm{Vp}_{\mathrm{QRiss}}-\mathrm{Werte)}$ mit niedrigen Festigkeiten, zum anderen 
existiert eine Überlagerung durch Korngrößeneffekte, die besonders an Proben der Rissklasse $A_{R}$ aufgezeigt werden kann. Trotz ähnlicher Eigenschaften des Mineralbestands und zudem ähnlicher Rissdichten weisen die Gesteine Gra3a (granitischer Ultramylonit), Pila (Peridotit) und Koess (Granit) unterschiedliche Gesteinsfestigkeiten auf. Ursachen hierfür können die unterschiedlichen Korngrößen sein. Die Probe Gra3a ist extrem feinkörnig und zeigt stets die höchsten Festigkeiten $\left(\mathrm{R}=347 \mathrm{MPa} ; \beta_{\mathrm{SZ}}=17,5 \mathrm{MPa} ; \mathrm{R}_{\mathrm{tf}}=32,8 \mathrm{MPa}\right)$, wohingegen die Probe Koess grobkörnig ist und die niedrigsten mechanischen Festigkeiten $\left(\mathrm{R}=195 \mathrm{MPa} ; \beta_{\mathrm{SZ}}=11,9\right.$ $\left.\mathrm{MPa} ; \mathrm{R}_{\mathrm{tf}}=20,1 \mathrm{MPa}\right)$ aufweist. Dazwischen liegt der mittelkörnige Peridotit $(\mathrm{R}=243 \mathrm{MPa}$; $\beta_{\mathrm{SZ}}=13,1 \mathrm{MPa} ; \mathrm{R}_{\mathrm{tf}}=24,6 \mathrm{MPa}(\boldsymbol{A b b . 6 - 1 7})$.
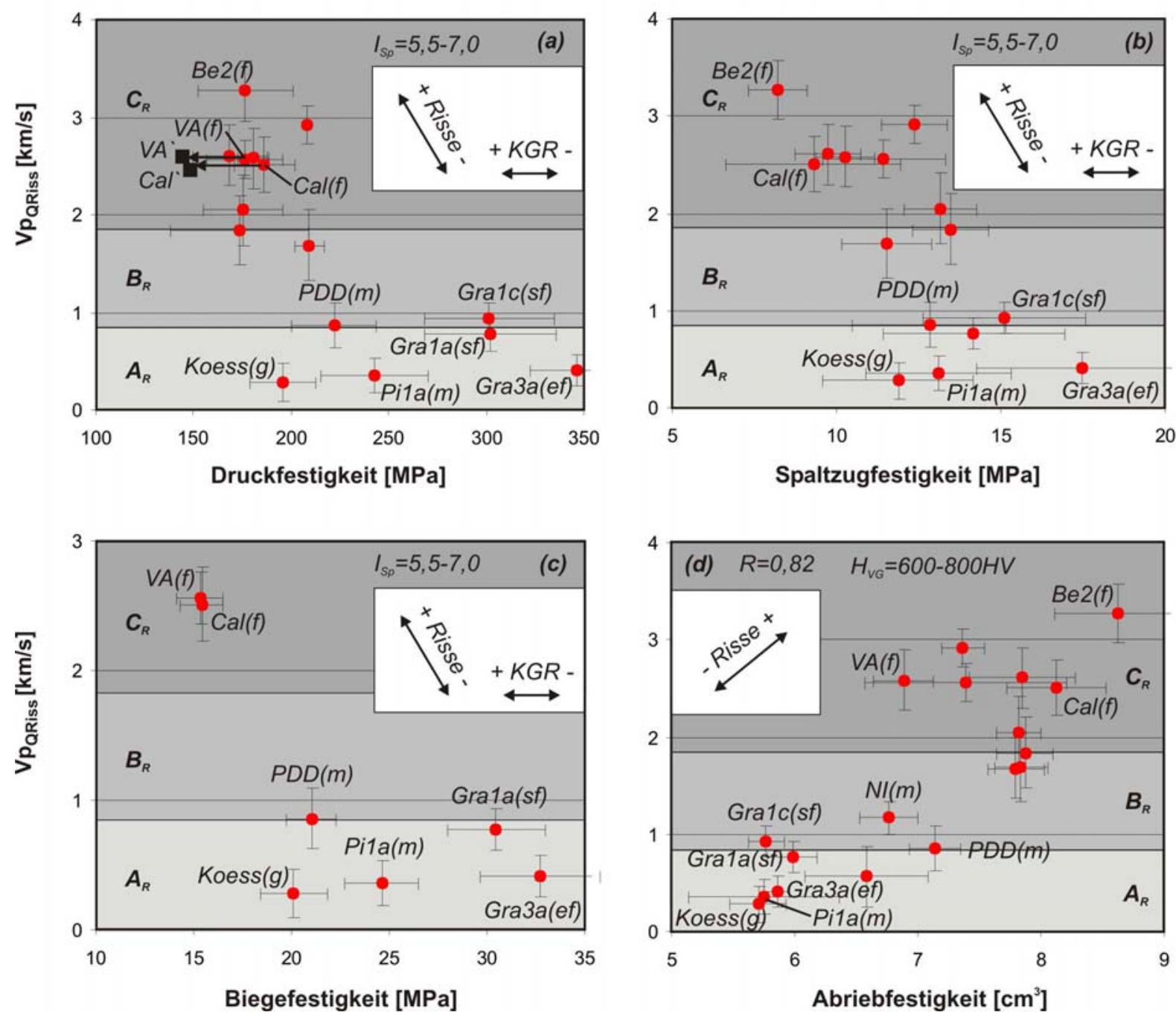

Abb.6-17: Gesteinsfestigkeiten und Rissquantifizierungs-Parameter ( $\left.V p_{Q R i s s}\right)$. (a) Einaxiale Druckfestigkeit; Cal' und Va' ergeben sich unter Berücksichtigung der Festigkeitswerte für die xz- und yz-45-Richtung. (b) Spaltzugfestigkeit. (c) Biegefestigkeit. (d) Abriebfestigkeit. Die Rissklassen $A_{R}$ (geringe Rissdichte), $B_{R}$ (mittlere Rissdichte) und $C_{R}$ (hohe Rissdichte) sind gekennzeichnet. Gra1a/1C - granitischer Gneis. Gra3a - granitischer Ultramylonit. Cal - granodioritischer Gneis. VA granitischer Gneis. PDD - Granodiorit. Pi1a - Peridotit. NI - Gabbro-Norit. Mg1b/Mg2 - Meta-Gabbro. Koess - Granit.Be2 - granitischer Gneis. Korngrößen: ef - extrem feinkörnig; sf - sehr feinkörnig; $f$ feinkörnig; $m$ - mittelkörnig; $g$ - grobkörnig. Auswirkungen der Variation von Rissdichten und Korngrößen (KGR) sind in Kästen bezeichnet. Mittelwerte berechnet als arithmetisches Mittel aus den Festigkeiten von $x-, y$ - und z-Richtung. Fehlerbalken zeigen den 1- $\sigma$-Fehler. 


\section{Technische Gesteinseigenschaften}

Es besteht also eine Antikorrelation von der Korngröße zur Druck-, Spaltzug- und Biegefestigkeit. Betrachtet man die Abriebfestigkeiten, so ist auch hier die Übereinstimmung von hohen Rissdichten mit niedrigen Festigkeiten festzustellen; ein Korngrößeneffekt auf die Abriebfestigkeit ist jedoch nicht ausgeprägt. Der variierende Einfluss der Korngröße auf die verschiedenen Gesteinsfestigkeiten liegt in den unterschiedlichen Versagensmechanismen bei der experimentellen Bestimmung der Druck-, Spaltzug-, Biege- und Abriebfestigkeit begründet (vgl. Kap.8.2.1).

Zusammenfassend: Bei ähnlicher durchschnittlicher mechanischer Stabilität des Mineralbestands der Gesteine haben die Frequenz der offenen Risse und die Korngröße einen entscheidenden Einfluss auf die mechanischen Gesteinsfestigkeiten. Vergleichsweise größere Korngrößen und höhere Rissdichten führen dabei zu niedrigeren Festigkeiten.

Diese Thesen stehen im Einklang mit der Griffith-Theorie (Griffith 1924), nach der bei gegebener äußerer Spannung an Risspitzen offener Risse Spannungskonzentrationen auftreten (G.6-5).

$$
\text { (G.6-5) } \quad \sigma_{z \max }=2 \sigma_{z} * \sqrt{\frac{C}{r}} \quad[\mathrm{MPa}]
$$

$\sigma_{\text {zmax }}$ - maximale Zugspannung an der Riss-Spitze [MPa]. $\sigma_{z}$ - äußere Zugspannung [MPa]. C - halbe Risslänge [mm]. $r$ - kleinster Radius an der Riss-Spitze [mm].

Diese führen bei der Überschreitung der Gesteinsfestigkeit zur Ausbreitung von Rissen und letztendlich zum Bruch. Folglich beinhaltet eine größere Rissdichte auch eine stärkere Schwächung des Gesteins eben durch eine volumenbezogen höhere Anzahl möglicher Herde der Spannungskonzentration und Rissausbreitung. Die G.6-5 bezieht sich auf Zugspannungen und kann in dieser Form nicht auf Bedingungen des einaxialen Druckversuchs angewendet werden, da der Bruch in der Regel durch ein Zug-Scher-Risspaar eintritt (z.B. Stavrogin \& Tarasov 2001). Damit nimmt die Scherfestigkeit des Gesteins und der Rissoberflächen neben den Winkeln zwischen der äußeren Spannung und den präexistierenden Rissen Einfluss auf die Spannungskonzentration und Lokalisation (z.B. Wittke 1984; Twiss \& Moores 1997). Die qualitative Aussage, dass eine Spannungskonzentration an der Riss-Spitze auftritt, ist jedoch ohne Einschränkungen möglich.

Die Korngrößenabhängigkeit der Druckfestigkeiten wurde von Prikryl (1998) an Graniten, von Olsson (1974) und Hugman \& Friedman (1979) an Karbonaten sowie von Fahy \& Guccione (1979) und Haney \& Shakoor (1994) an Sandsteinen untersucht. In allen Studien wurde eine Antikorrelation der mittleren Korngröße zur Druckfestigkeit gefunden, die von 


\section{Technische Gesteinseigenschaften}

Prikryl (1998) mit der Griffith-Risslänge in Verbindung gebracht wird. Demnach haben grobkörnige Gesteine gegenüber feinkörnigen Gesteinen größere Griffith-Risslängen, was zu einer Erhöhung der Spannungskonzentration an der Riss-Spitze bei konstanter äußerer Spannung führt. Dadurch wird die Gesteinsfestigkeit schon bei niedrigeren äußeren Spannungen überschritten und herabgesetzt. Die Ergebnisse der vorliegenden Studie erweitern diese Aussagen auch auf die Spaltzug- und Biegefestigkeiten. Da in beiden Fällen ausschließlich Zugspannungen zum Gesteinsversagen führen, kann ein Einfluss der GriffithRisslänge nach G.6-5 angenommen werden.

\subsubsection{Bevorzugt aktivierte Gefügeelemente beim Spaltzug- und Abriebversuch}

Die beim Spaltzug- und Abriebversuch entstandenen Bruch- und Abriebflächen wurden mittels rasterelektronenmikroskopischer Bildverfahren (Sekundärelektronen) analysiert und in zwei Serien von Bildern aufgeteilt. Die erste hat ihren Schwerpunkt auf dem Bruch- und Abriebverhalten der Minerale. Hier werden die unterschiedlichen Bruchmuster innerhalb verschiedenen Minerale, also die Ausbreitung der während der Experimente neu entstehenden Risse, beschrieben (Abb.6-18). Die zweite Serie beinhaltet aktivierte präexistierende Risse und Korngrenzen (Abb.6-19). Aufgrund der besseren Bildqualität sind hauptsächlich Aufnahmen der Spaltzugproben dargestellt. Die bevorzugt aktivierten Gefügeelemente sind für den Spaltzug- und den Abriebversuch identisch, so dass die Ergebnisse für beide Verfahren Gültigkeit besitzen. Die mikroskopischen Betrachtungen der beim Druckversuch entstandenen Bruchflächen suggerieren eine Übertragbarkeit auch hinsichtlich der Druckfestigkeiten.

Die Richtungen des Referenzsystems sind in den Abbildungen gekennzeichnet und die dargestellten Minerale und Versagensphänomene hervorgehoben. Informationen zur Vergrößerung (Maßstab) und Beschleunigungsspannung befinden sich in der Informationszeile am unteren Rand der Bilder. 

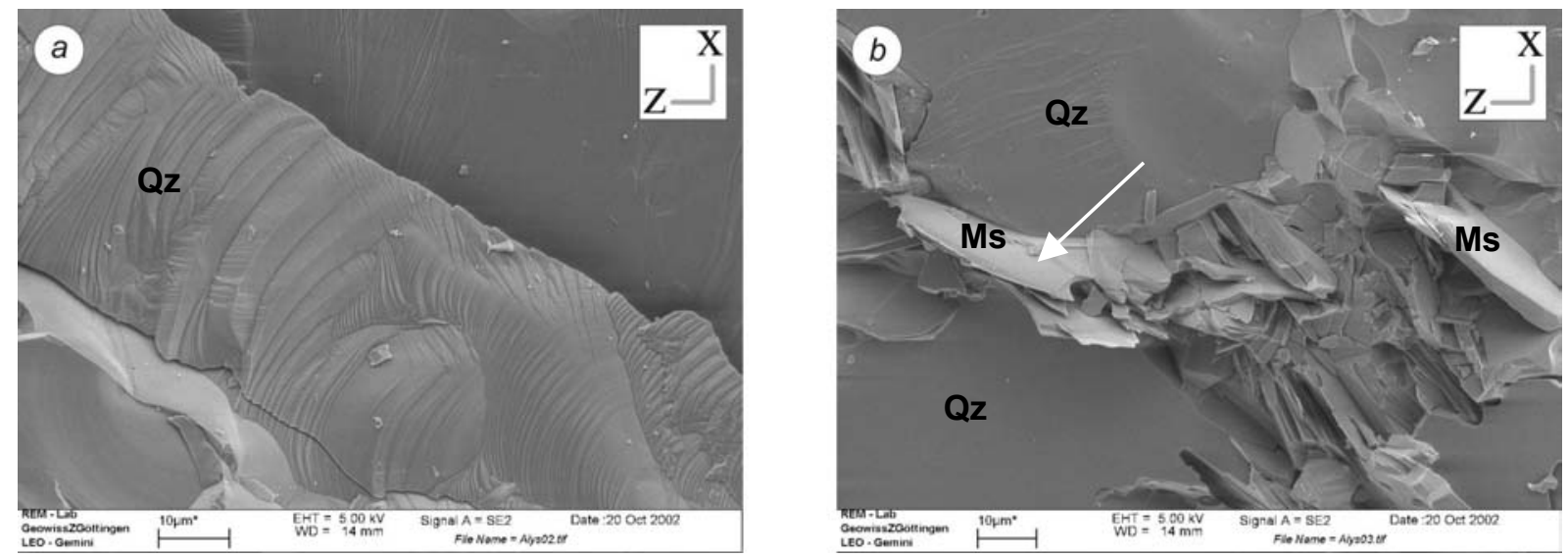

Abb.6-18ab: (a) Spaltzugprobe (Al - Quarzit); muscheliger Bruch in Quarz. (b) Spaltzugprobe (Al Quarzit). Zwickel zwischen Quarzkörnern; innerhalb des Zwickels befinden sich Muskovit (Serizit) und Dumortierit als hydrothermale Bildungen; die (001)-Spaltflächen des Muskovits (Pfeil) wurden während des Spaltzugversuchs aktiviert. Qz - Quarz. Ms - Muskovit.
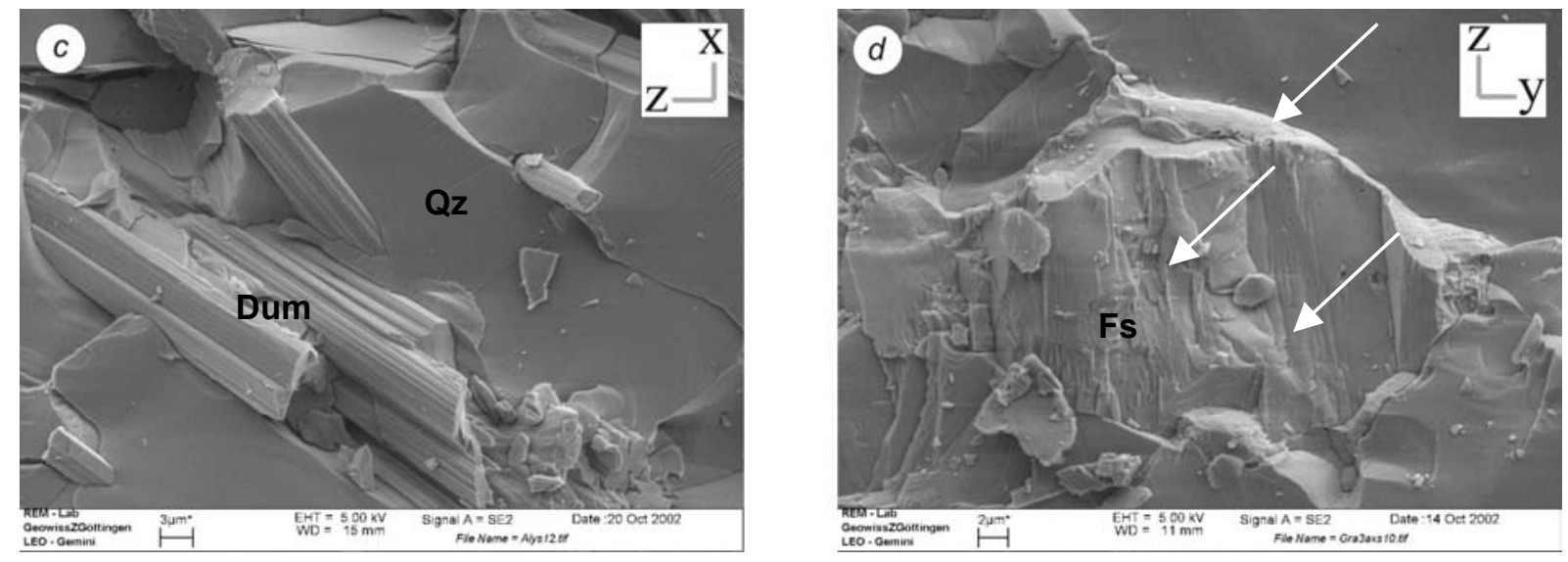

Abb.6-18cd: (c) Spaltzugprobe Al (Quarzit); Bruchmuster in Quarz und Dumortierit. (d) Spaltzugprobe Gra3a (granitischer Ultramylonit); zentral im Bild ein Feldspat mit beim Versuch aktivierten Spaltflächen nach (001) und (010) und teilweise aktivierter Korngrenze. Dum - Dumortierit. Qz Quarz. Fs - Feldspat.
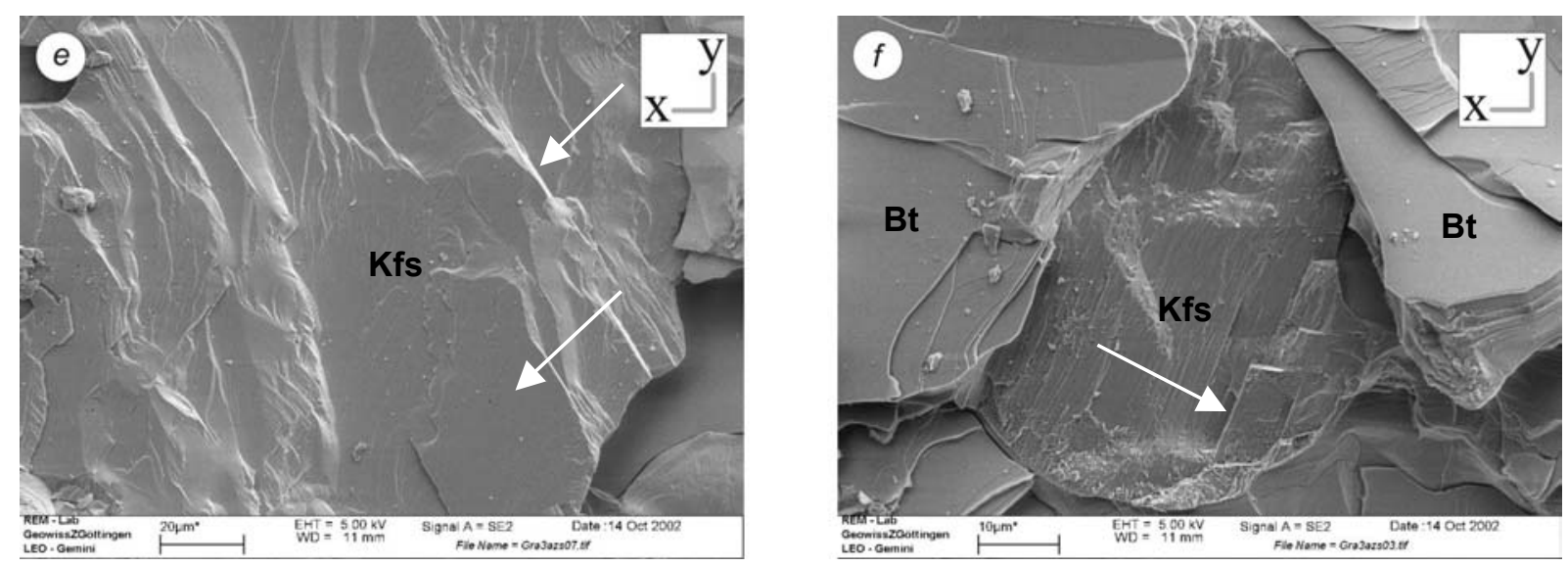

Abb.6-18ef: Spaltzugprobe Gra3a (granitischer Ultramylonit); (e) Bruch in Kalifeldspat mit aktivierten Spaltflächen. (f) Zentral im Bild ein Kalifeldspat mit aktivierten Spaltflächen; links und rechts davon Biotit. Kfs - Kalifeldspat. Bt - Biotit. 

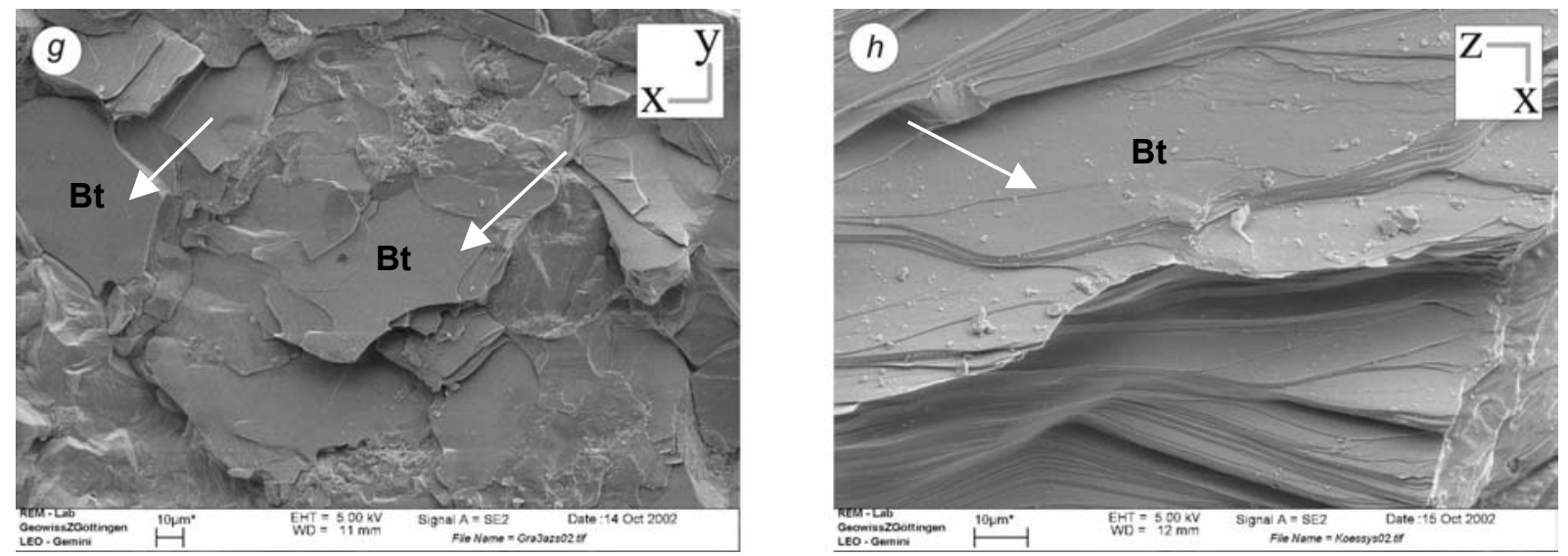

Abb.6-18gh: (g) Spaltzugprobe Gra3a (granitischer Ultramylonit); Biotit mit beim Versuch aktivierten (001)-Flächen. (h) Spaltzugprobe Koess (Granit); Biotitkristall: Deutlich der Unterschied im Bruchverhalten zwischen der (001)-Basisfläche und den kristallographischen Elementen senkrecht dazu. Bt-Biotit.
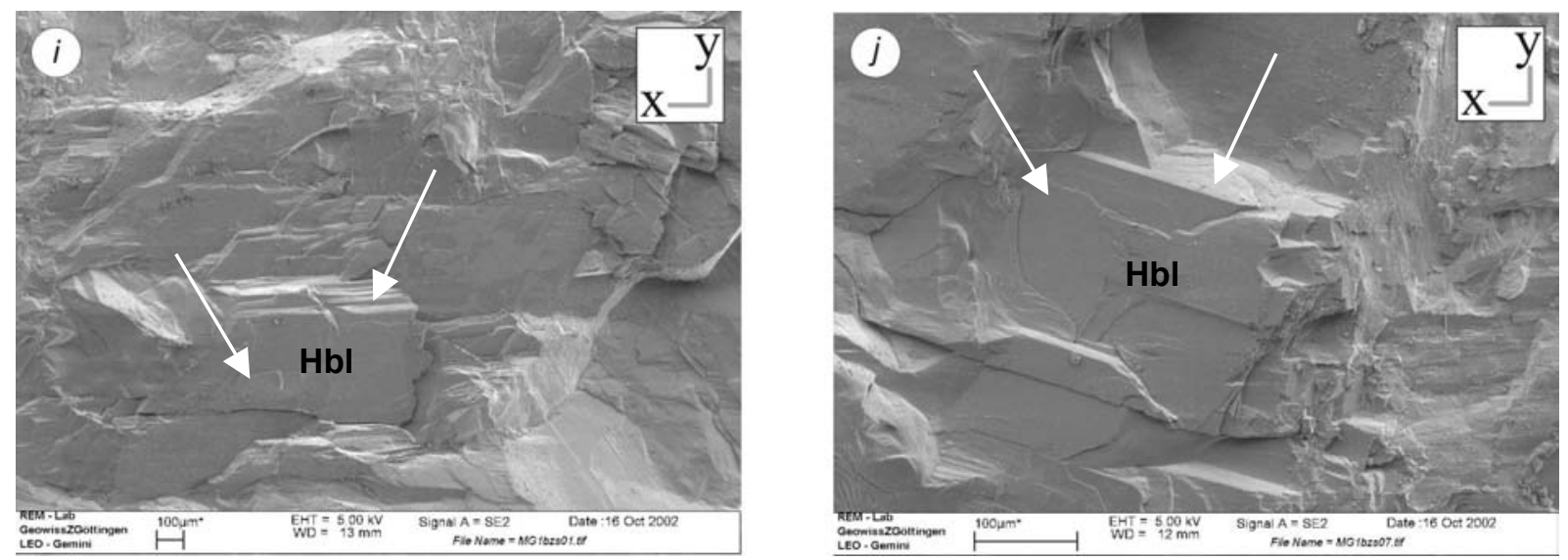

Abb.6-18ij: Spaltzugprobe Mg1b (Meta-Gabbro). Hornblende mit aktivierten Spaltflächen (110) und (1-10). Hbl-Hornblende.
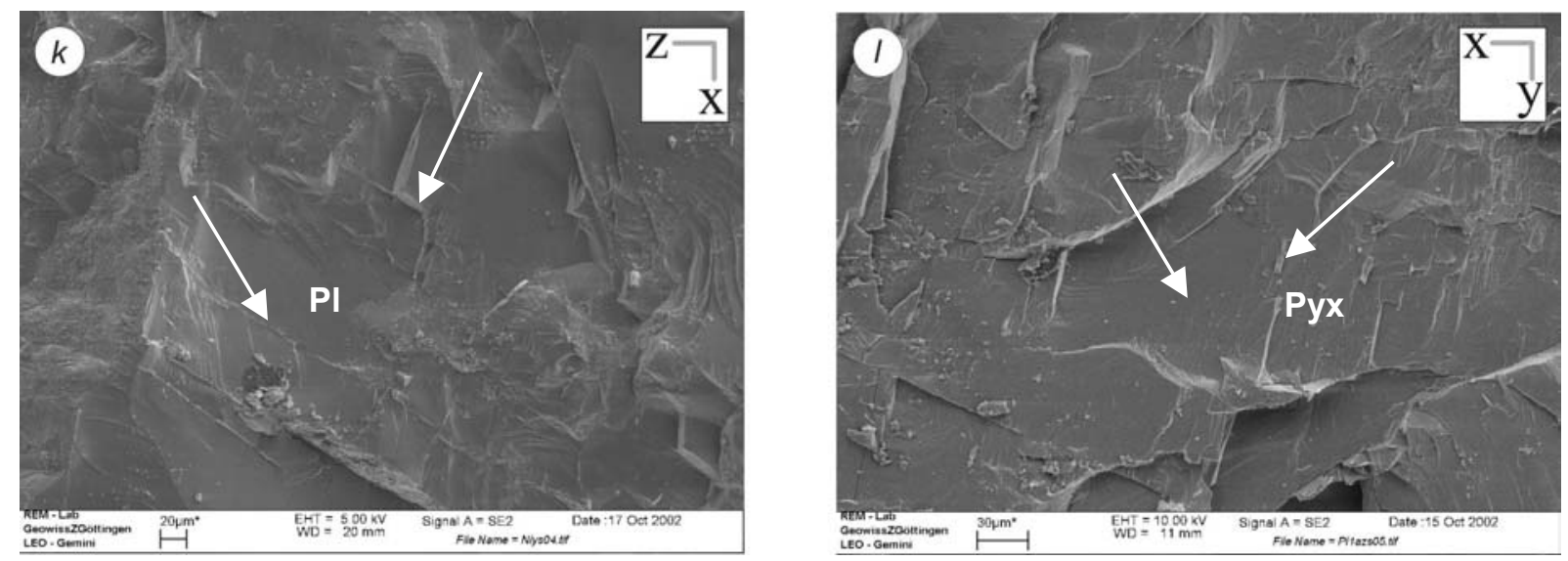

Abb.6-18kl: (k) Spaltzugprobe NI (Gabbro-Norit); Spaltflächen und aktivierte präexistierende Mikrorisse in Plagioklas. (I) Spaltzugprobe Pi1a (Peridotit); Spaltflächen Pyroxenkristall. PI Plagioklas. Pyx - Pyroxen. 

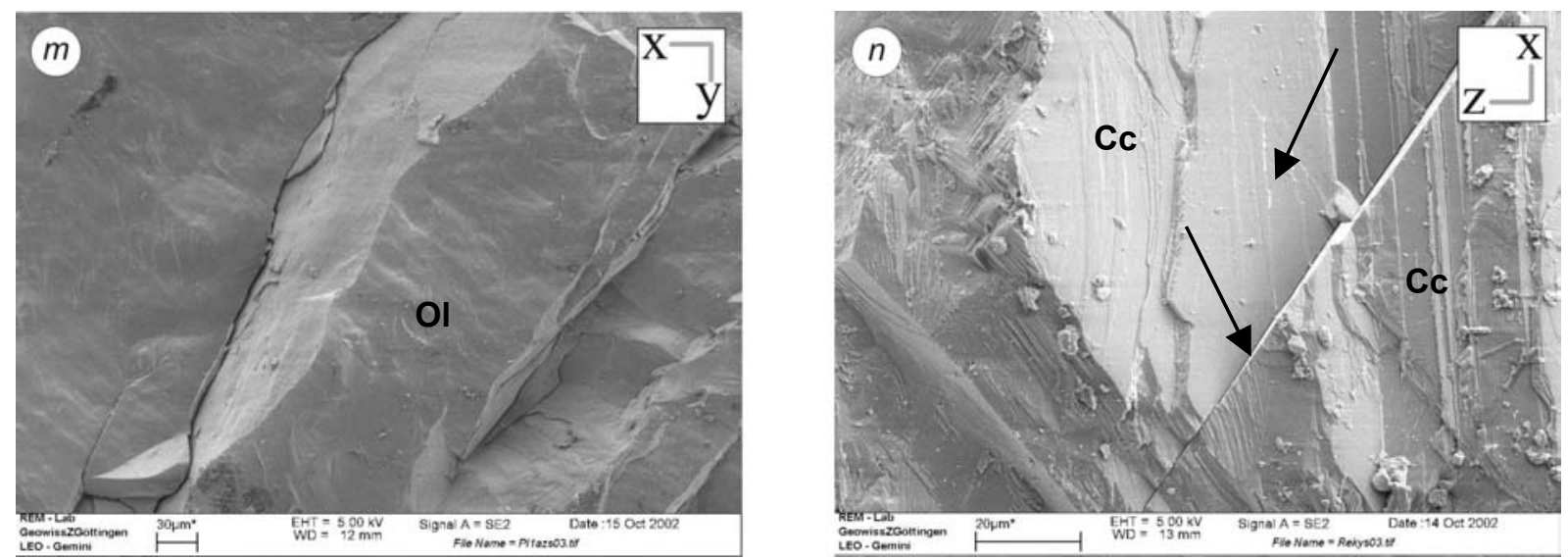

Abb.6-18mn: $(m)$ Spaltzugprobe Pi1a (Peridotit); Bruchmuster in Olivin mit aktivierten präexistierenden Mikrorissen. (n) Spaltzugprobe Rek (Kalzit-Marmor); aktivierte Spaltflächen (Rhomboeder-Flächen) und aktivierter präexistierender Mikroriss in Kalzit. Ol - Olivin. Cc-Kalzit.
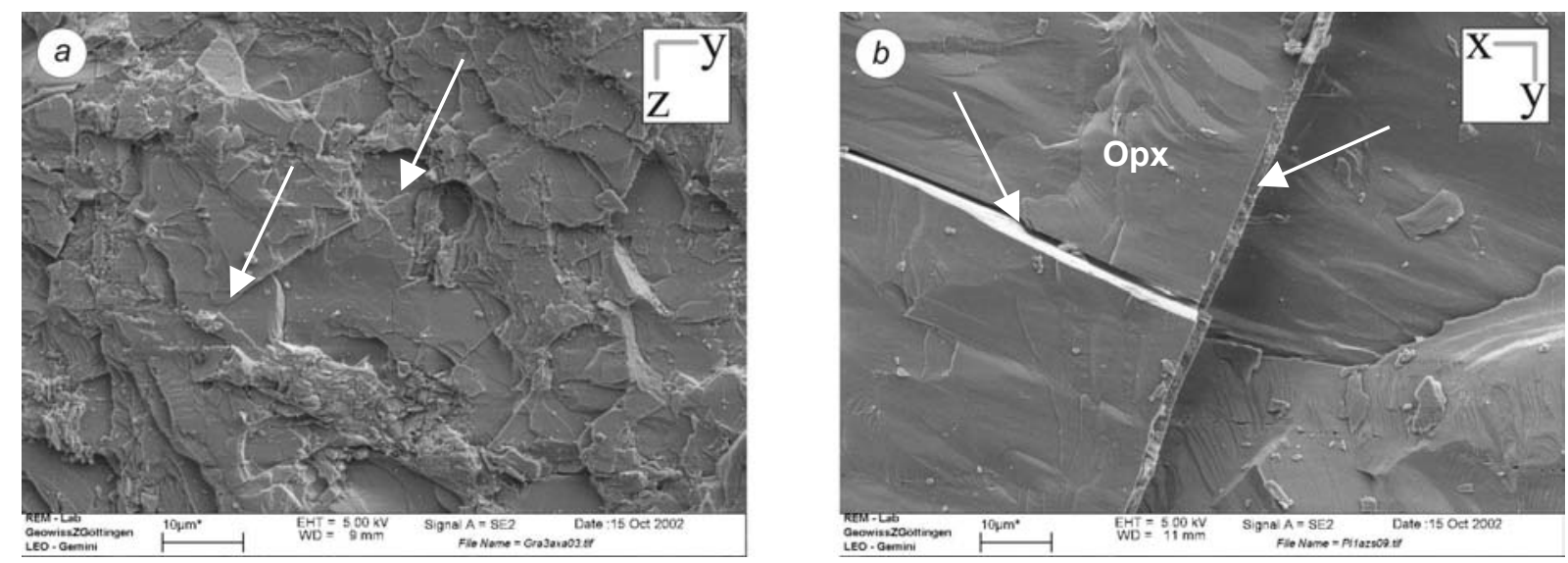

Abb.6-19ab: (a) Abriebprobe Gra3a (granitischer Ultramylonit); von links unten nach rechts oben verlaufen präexistierende offene Mikrorisse, welche während des Versuchs teilweise aktiviert werden. (b) Spaltzugprobe Pi1a (Peridotit); Bild zeigt einen mineralisierten und einen offenen Mikroriss in Orthopyroxen. Opx - Orthopyroxen.
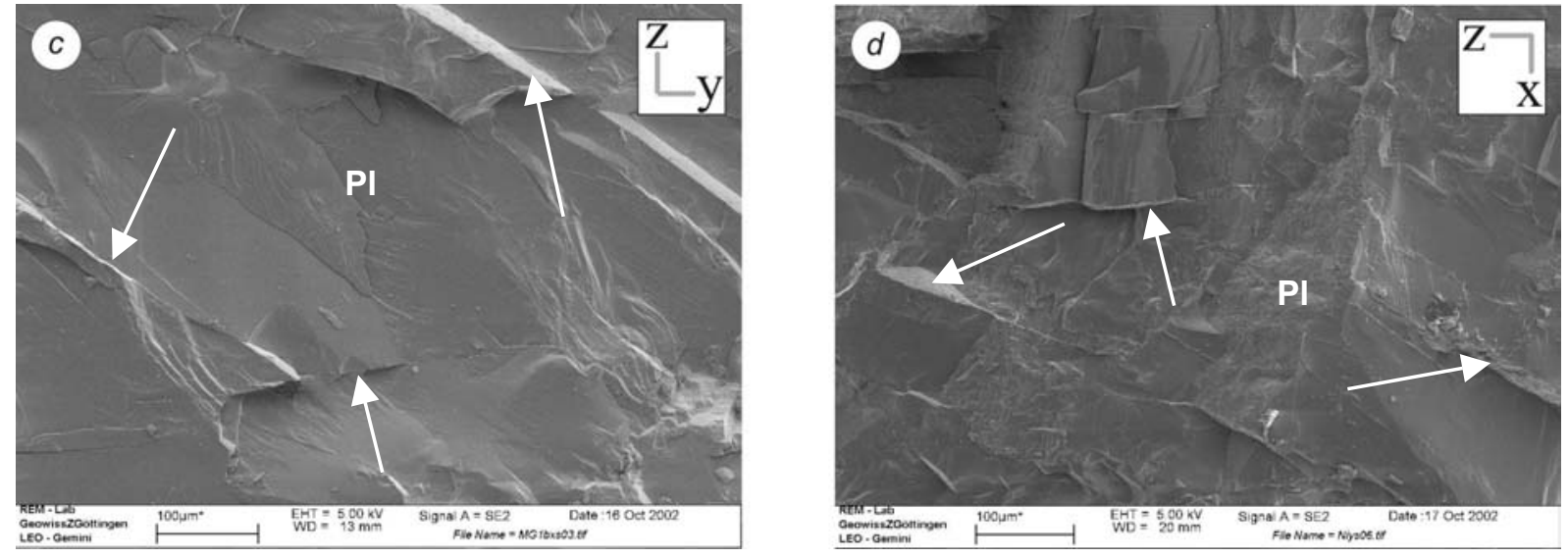

Abb.6-19cd: (c) Spaltzugprobe Mg1b (Meta-Gabbro); Bruchfläche in Plagioklas mit aktivierten Spaltflächen und präexistierenden offenen Mikrorissen. (d) Spaltzugprobe NI (Gabbro-Norit); aktivierte präexistierende offene Mikrorisse in Plagioklas. PI - Plagioklas. 

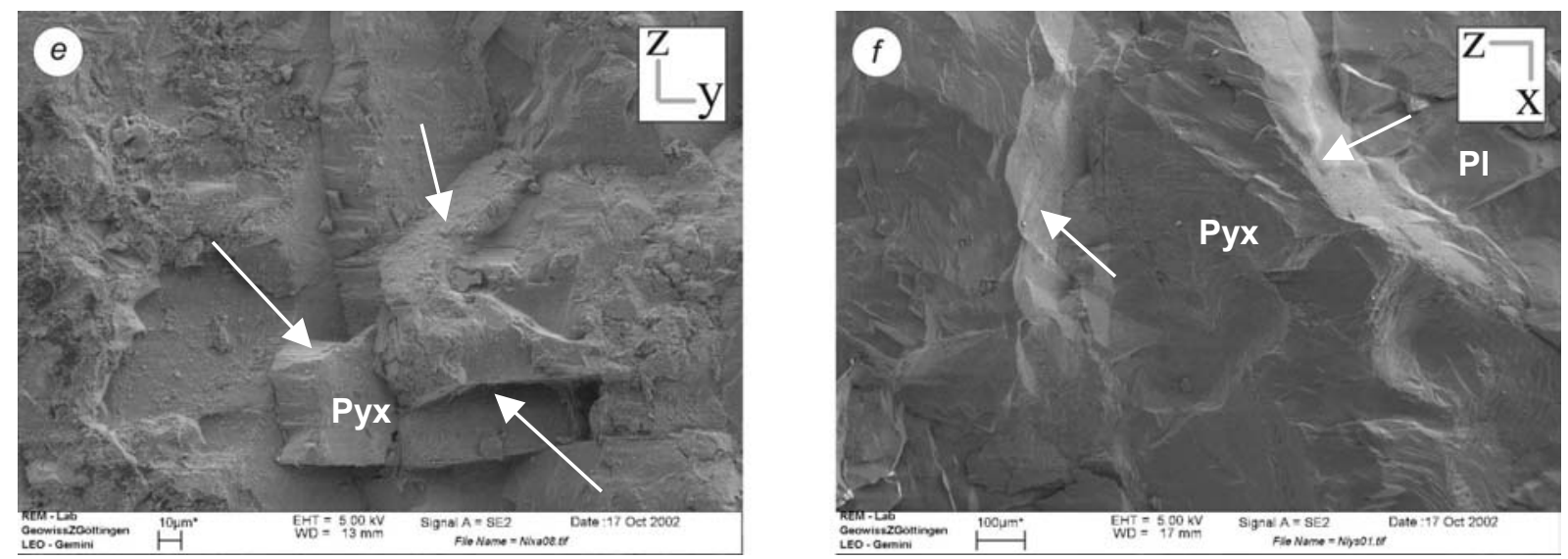

Abb.6-19ef: (e) Abriebprobe NI (Gabbro-Norit); aktivierte präexistierende offene Mikrorisse in Pyroxen. (f) Spaltzugprobe NI (Gabbro-Norit); aktivierte präexistierende offene Mikrorisse in Pyroxen und aktivierte Korngrenze am Übergang Pyroxen zu Plagioklas. Pyx - Pyroxen. PI-Plagioklas.
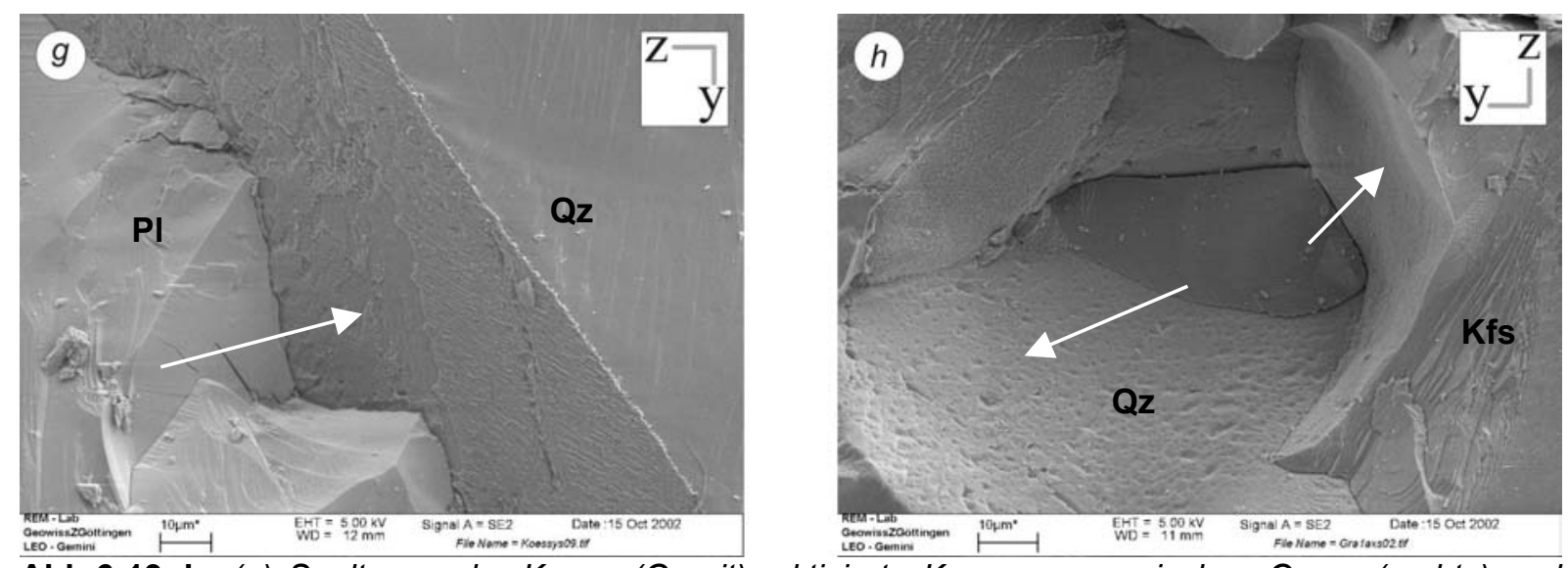

Abb.6-19gh: (g) Spaltzugprobe Koess (Granit); aktivierte Korngrenze zwischen Quarz (rechts) und Plagioklas (links). (h) Spaltzugprobe Gra1a (granitischer Gneis); Korngrenze eines Quarzkristalls; rechts Übergang zu Kalifeldspat. PI - Plagioklas. Qz - Quarz. Kfs - Kalifeldspat.
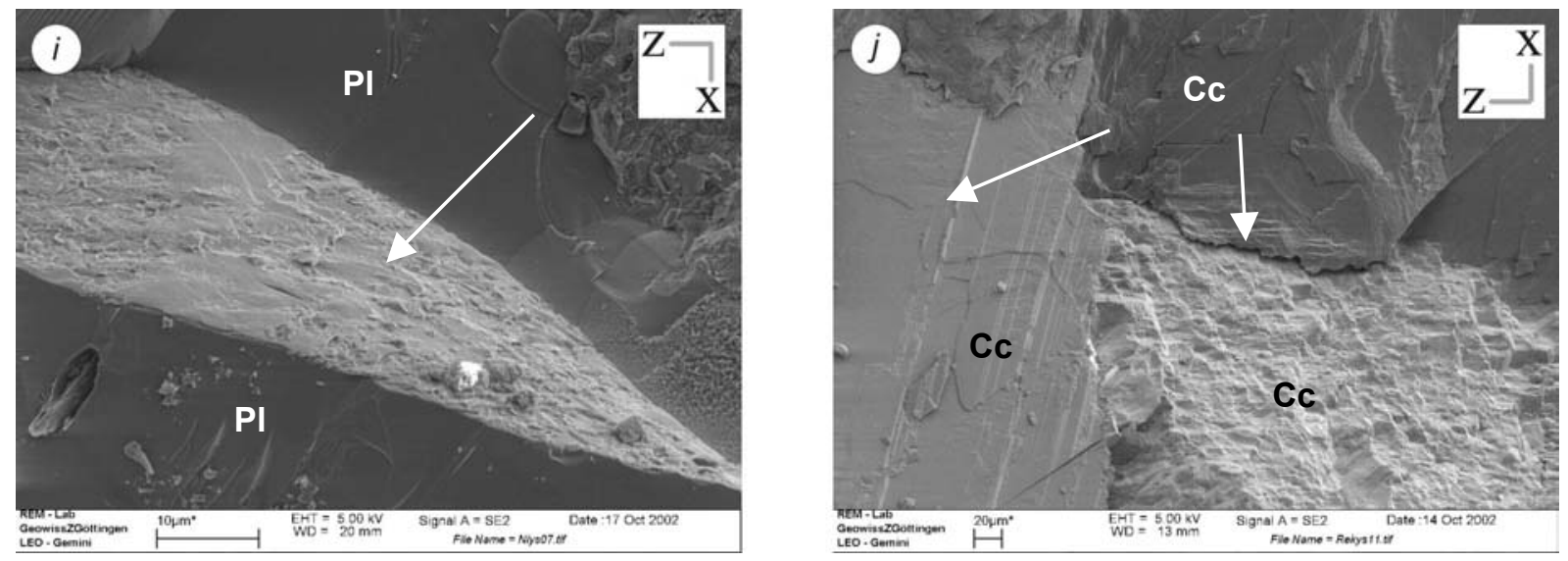

Abb.6-19ij: (i) Spaltzugprobe NI (Gabbro-Norit); aktivierter präexistierender offener Mikroriss in Plagioklas. (j) Spaltzugprobe Rek (Kalzit-Marmor); Korngrenze mit Mikroverzahnung in Kalzit unten rechts und aktivierte Spaltflächen in Kalzit (links und oben). PI - Plagioklas. Cc - Kalzit. 


\section{Technische Gesteinseigenschaften}

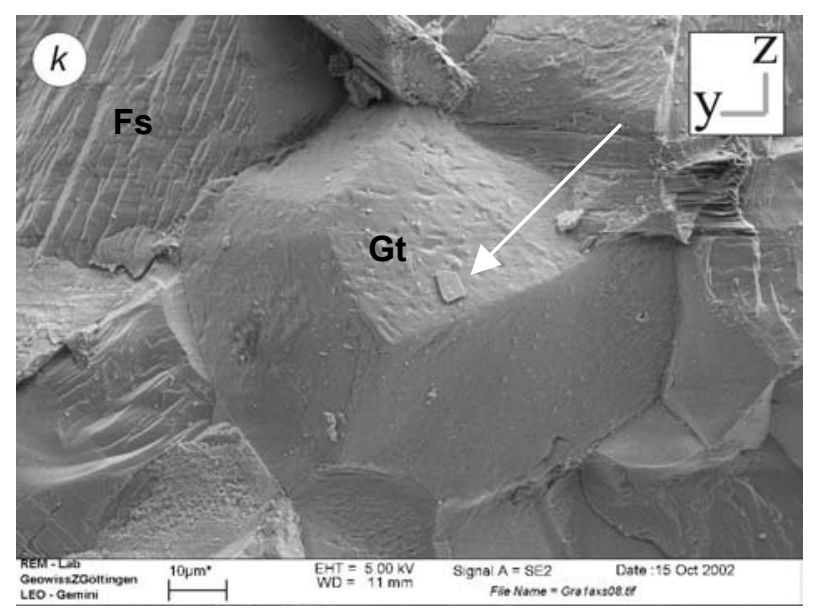

Abb.6-19k: Spaltzugprobe Gra1a (granitischer Gneis); Korngrenze von Granatkristall. Fs - Feldspat. Gt - Granat.

Offene Mikrorisse sowie die Spaltflächen der Minerale und in Ansätzen auch Korngrenzen sind Gefügeelemente, die bei den mechanischen Tests bevorzugt aktiviert werden. Qualitativ kann festgestellt werden, dass die offenen Mikrorisse das mechanisch schwächste Glied darstellen, gefolgt von den Spaltflächen, sofern eine zumindest gute Spaltbarkeit vorhanden ist. Die Korngrenzen sind bei den untersuchten Gesteinen relativ stabil und werden tendenziell seltener aktiviert. Die genannten Gefügeelemente sind im Vergleich zu sonstigen kristallographischen Flächen mechanische Schwachstellen im Gestein und tragen über ihre Frequenz zu den mittleren mechanischen Eigenschaften und im Falle einer bevorzugten Orientierung auch zu deren Anisotropie bei.

Bei den Abriebversuchen treten entgegen den Erwartungen keinerlei Kratz- und Schleifspuren auf den Mineralen auf. Selbst die gegenüber dem Schleifpulver (SIC) weicheren Minerale, wie z.B. Kalzit, werden nicht spanend abgetragen, sondern erleiden eine Kristallzertrümmerung, die schließlich über eine Gefügeauflockerung zur Zerstörung des gesamten Mineralverbandes führt. 


\subsection{Elastische Eigenschaften}

Der Elastizitätsmodul (E) und die Poisson-Zahl ( $v$ ) sind wichtige gesteinstechnische Kennwerte, die das Spannungs-Dehnungs-Verhalten der Gesteine beschreiben. Dabei wird von einem linear elastischen Verhalten ausgegangen und der E-Modul nach dem Zusammenhang

(G.6-6) $\quad E=\frac{\delta \sigma}{\delta \varepsilon} \quad[\mathrm{GPa}]$

E-Elastizitätsmodul [GPa]; $\sigma-$ Spannung [MPa]; $\varepsilon-D e h n u n g$ [\%].

definiert. Die Poisson-Zahl beschreibt das Verhältnis von Querdehnung zur Längsdehnung bei einer volumenkonstanten Verformung (z.B. Vogel 1999; Schön 1983).

(G.6-7) $\quad v=\frac{\Delta \varepsilon_{\text {quer }}}{\Delta \varepsilon_{\text {längs }}}$

v- Poisson-Zahl; $\Delta \varepsilon_{q u e r}-$ Querdehnung der Probe; $\Delta \varepsilon_{\text {längs }}$ - Längsdehnung der Probe.

Beide Kennwerte sind u.a. Eingangsgrößen für gesteinsmechanische Modelle, beispielsweise bei der Berechnung von Spannungsverteilungen während der hydraulischen Rissbildung (z.B. Gudmundsson 2000) oder bei der Analyse von Gebirgsspannungen im Tunnel- und Bergbau (z.B. Wittke 1984). Aber auch die Modellierung von Spannungsverteilungen in Bauwerken, wie Brücken und Häusern, erfordert eine Kenntnis des Spannungs-Dehnungs-Verhaltens der verwendeten Werkstoffe (z.B. Purtak 2001). Der E-Modul und die Poisson-Zahl können prinzipiell nach zwei unterschiedlichen Verfahren ermittelt werden: nach der statischen und der dynamischen Methode. Im Bereich der Naturwerksteine ist eine Untersuchung des elastischen Gesteinsverhaltens nach prEN14580 (statische Methode) und prEN14146 (dynamische Methode) vorgesehen. Im ersten Fall wird der E-Modul aus den SpannungsDehnungs-Daten des einaxialen Druckversuchs bestimmt; im zweiten Fall erfolgt die Bestimmung mittels Ultraschall nach dem Resonanzverfahren. Beide Untersuchungsmethoden finden unter Laborbedingungen statt, also ohne Umschließungsdruck, was am ehesten den Verwendungsbedingungen der Naturwerksteine entspricht. Die prEN14146 schließt die Untersuchung unter verschiedenen Wassersättigungsgraden mit ein. Abweichend zur EN können der dynamische E-Modul und die Poisson-Zahl auch nach dem Durchschallungsverfahren ermittelt werden. Hier werden die 
Laufzeiten von Kompressionswelle (p) und Scherwelle (s) in einer Probe bekannter Länge und derer entsprechenden Geschwindigkeiten (Vp; Vs) verwendet, um nach StandardGleichungen unter Annahme eines linear-elastischen, isotropen Festkörpers (vgl. Kap.2.4.5.2; Gebrande 1982; Schön 1983) den E-Modul und die Poisson-Zahl zu berechnen. Dabei ist die Frequenzabhängigkeit der Ergebnisse zu beachten (Tatham 1985).

Analog zu den beiden Hauptuntersuchungsmethoden gliedern sich die bisherigen Ergebnisse zur Gefügeabhängigkeit der elastischen Gesteinseigenschaften in Betrachtungen der statischen bzw. der dynamischen elastischen Parameter. Prikryl (1998) beobachtete eine Anisotropie des statischen E-Moduls von Graniten in Abhängigkeit von bevorzugt orientierten Mikroriss-Systemen. Studien von Stavrogin \& Tarasov (2001) zeigen flachere Spannungs-Dehnungs-Kurven und damit niedrigere E-Moduln bei einer Orientierung von Mikroriss-Systemen parallel zum Maximum der während des Versuchs auftretenden Scherspannungen. Shea \& Kronenberg (1993) untersuchten glimmerhaltige Gesteine unter ein- und triaxialen Bedingungen und fanden neben einer ausgeprägten Anisotropie eine Tendenz zu einem mehr duktilen rheologischen Verhalten (niedrigerer E-Modul) bei einer Orientierung der maximalen Scherspannungen parallel zum Maximum der Glimmer-(001)Flächen. Sowohl mineralogische Komponenten und deren bevorzugte Orientierung als auch Gefügeelemente wie Mikrorisse stehen also im Verdacht das statische elastische Verhalten zu beeinflussen.

Zur gefügebedingten Anisotropie der dynamischen elastischen Eigenschaften wurde eine Vielzahl von Studien mit dem Ziel durchgeführt, Rückschlüsse auf das elastischen Verhalten der Erdkruste und des Erdmantels zu erhalten. Diese generellen Erkenntnisse werden wiederum angewandt, um aus relativ einfach $\mathrm{zu}$ gewinnenden seismischen Daten Rückschlüsse auf tektonische Strukturen, aber auch auf andere physikalische Gesteinseigenschaften, wie z.B. den Wärmefluss oder die Wärmeproduktion (z.B. Rybach \& Buntebarth 1982) zu ermöglichen. Diese Untersuchungen beschäftigen sich fast ausschließlich mit der mineralogisch und gefügebedingten Geschwindigkeit von p-Wellen und s-Wellen sowie deren Anisotropie, ohne direkt auf den E-Modul und die Poisson-Zahl einzugehen. Insbesondere zwei Einflussfaktoren, die mineralogische Zusammensetzung und der Porenraum, beeinflussen das elastische Gesteinsverhalten (z.B. Siegesmund 1989; Siegesmund \& Kern 1990; Siegesmund \& Vollbrecht 1991; Siegesmund et al. 1993; Popp 1994; Jahns 1995; Siegesmund 1996; Weiß 1998; Zimmer 2001; Dürrast et al. 2002). Als Kernaussagen gelten: 


\section{Technische Gesteinseigenschaften}

1. Die p-Wellen- und die s-Wellen-Geschwindigkeiten und damit verknüpft auch der EModul und die Poisson-Zahl werden durch den Mineralbestand beeinflusst. Gesteine mit einem hohen Volumenanteil von Mineralen mit z.B. hohen Vp führen auch zu einer hohen Vp im Gesamtgestein. Die bevorzugte Orientierung besonders von Mineralen mit einer hohen elastischen Anisotropie (z.B. Glimmer) verursacht eine Anisotropie der elastischen Gesteinseigenschaften.

2. Die Geschwindigkeiten der $\mathrm{p}$ - und s-Welle werden durch offene Mikrorisse herabgesetzt. Dabei ist sowohl die Rissdichte als auch die Geometrie der Risse von Bedeutung. So führt eine Erhöhung der Rissdichte ebenso wie eine Erniedrigung des aspect-ratios (Verhältnis von Risskurzachse zur Risslangachse) zur Verringerung der p- und s-Wellen-Geschwindigkeiten (z.B. Wilkens et al. 1991). Das bedeutet fast immer auch einen niedrigeren resultierenden E-Modul bzw. eine niedrigere PoissonZahl (vgl. Gleichungen in Kap.2.4.5.2). Die bevorzugte Orientierung von Mikrorissen hat eine große Bedeutung für die Anisotropie des Gesamtgesteins.

Den starken Einfluss offener Mikrorisse auf die dynamischen elastischen Eigenschaften macht man sich zunutze, um mittels Vp und Vs Aussagen über Mikrorissdichten und den damit in Verbindung stehenden Porositäten und Permeabilitäten vorzunehmen. Es ist also möglich, aufgrund von Vp und Vs eine Information bezüglich der Qualität eines Gesteins im Hinblick auf einen bestimmten Verwendungszweck zu bekommen. Schild (2001) und Zimmer (2001) charakterisierten z.B. die Mikrorissgefüge von Graniten und Salzgesteinen, um die Eignung als potenzielle atomare Endlager $\mathrm{zu}$ untersuchen. Auch im Bereich der Naturwerksteine werden elastische Eigenschaften als Qualitätskriterium verwendet. Eine niedrige Vp, Vs und ein niedrigerer E-Modul in einem Gestein und damit verbunden relativ höhere Rissdichten und eine höhere Porosität werden hier als niedrigere Qualität gewertet. Dahinter steht die Annahme, dass hohe Mikrorissdichten zu einer Schwächung der mechanischen Eigenschaften des Gesteins führen, zusätzlich die Wasseraufnahme verbessern und folglich die Verwitterungsgeschwindigkeit beschleunigen. Die Ergebnisse beziehen sich auf Sandsteine (z.B. Weiss 1992; Fitzner 1988) und Marmore (z.B. Köhler 1991; Rüdrich et al. 2001; Ondrasina et al. 2002; Weiss et al. 2002b; Rüdrich 2003). Häufig wird auch das Verwitterungsverhalten im Sinne einer progressiven Gefügeauflockerung (und damit die Bildung neuen Porenraums) indirekt über die Vp- und E-Modul-Änderungen charakterisiert. 


\section{Technische Gesteinseigenschaften}

\subsubsection{Dynamische elastische Eigenschaften}

Im Rahmen dieser Arbeit wurden die Ultraschallgeschwindigkeiten einer p-Welle und zweier senkrecht zueinander polarisierten s-Wellen parallel zu den drei Gefügehauptrichtungen $(\mathrm{x}, \mathrm{y}$, z) bestimmt und daraus der E-Modul (E) und die Poisson-Zahl (v) berechnet. Die Messungen wurden mit einer Frequenz von $0,5 \mathrm{MHz}$ an trockenen Würfelproben und unter Laborbedingungen $\left(20^{\circ} \mathrm{C} ; 1013 \mathrm{hPa}\right)$ sowie mit $1 \mathrm{MHz}$ an Zylindern unter hydrostatischem Druck von $2 \mathrm{MPa}$ bis $400 \mathrm{MPa}$ durchgeführt. Aus den druckabhängigen Messungen wurden die Vp und Vs für $0 \mathrm{MPa}$ Umschließungsdruck interpoliert.
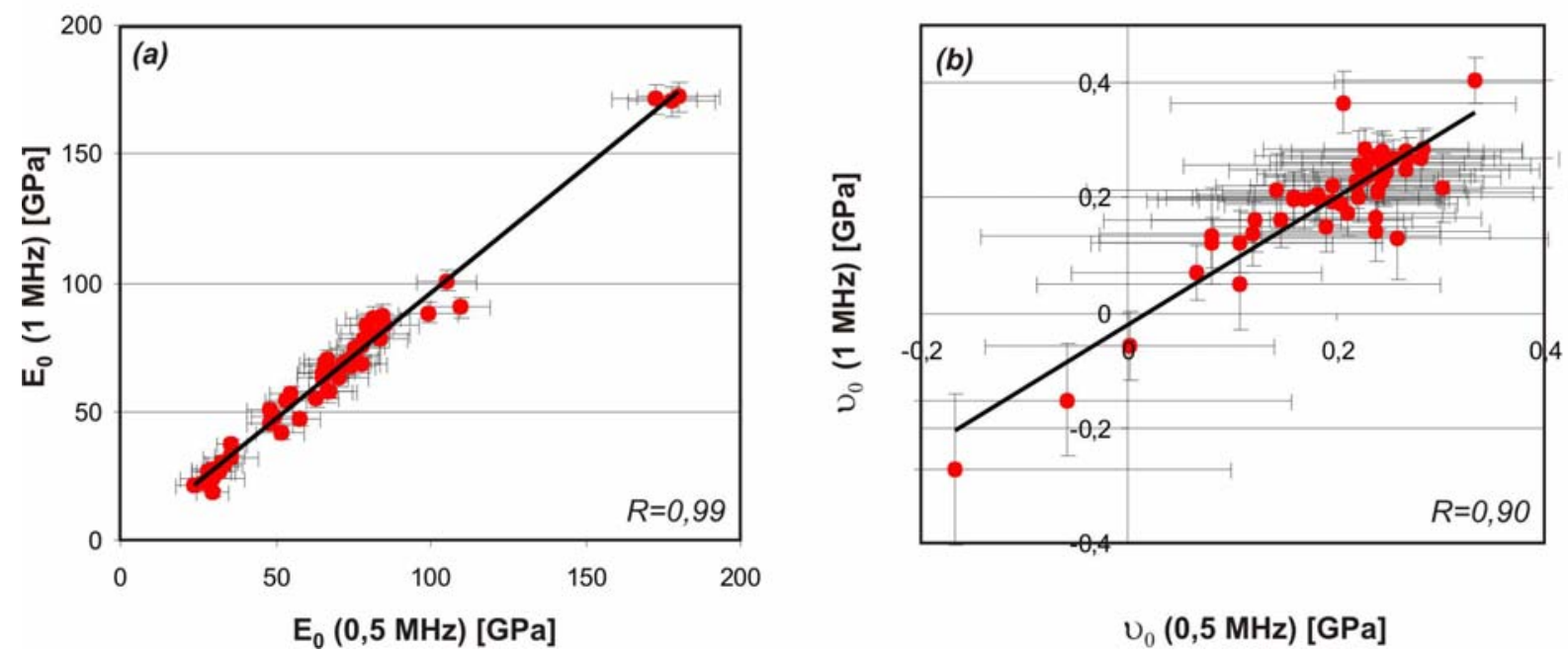

Abb.6-20: E-Modul und Poisson-Zahl (dynamisch). Vergleich der Daten von E-Modul und PoissonZahl. Vp und Vs ermittelt mit 0,5 MHz bzw. 1,0 MHz Anregungsfrequenz und bei $0 \mathrm{MPa}$ Umschließungsdruck. Es sind die drei Hauptrichtungen $(x, y, z)$ aller Proben berücksichtigt. Fehlerbalken zeigen den 1- $\sigma$-Fehler.

Bei der Berechnung des E-Modul und der Poisson-Zahl wurde der Mittelwert aus der schnelleren Scherwelle $\left(\mathrm{Vs}_{1}\right)$ und der langsameren Scherwelle $\left(\mathrm{Vs}_{2}\right)$ verwendet. Die Auswirkungen des Scherwellen-Splittings auf den E-Modul werden im weiteren Verlauf separat diskutiert. $\boldsymbol{A} \boldsymbol{b} \boldsymbol{b} .6-20$ zeigt die E-Moduln und Poisson-Zahlen der Würfelmessungen $(0,5 \mathrm{MHz})$ und der Zylindermessungen $(1 \mathrm{MHz})$ unter $0 \mathrm{MPa}$ Umschließungsdruck im Vergleich.

Die Werte für E beider Untersuchungsmethoden plotten mit einer sehr guten Korrelation $(\mathrm{R}=0,99)$ entlang der Linie $\mathrm{E}_{0}(0,5 \mathrm{MHz})=\mathrm{E}_{0}(1 \mathrm{MHz})$. Für die Poisson-Zahlen ist die Korrelation schlechter, doch ergibt sich auch hier ein durchschnittliches Verhältnis von annähernd $v_{0}(0,5 \mathrm{MHz}): v_{0}(1 \mathrm{MHz}) \approx 1: 1$. Allerdings ist die Poisson-Zahl stark fehlerbehaftet, so dass die Daten kritisch $\mathrm{zu}$ betrachten sind. Aufgrund der gerätebedingt größeren Messgenauigkeit bei der Zylindermessung (1 MHz) und um einen in sich konsistenten Datensatz im Druckbereich von $0 \mathrm{MPa}$ bis $400 \mathrm{MPa}$ zu erhalten, werden für die weitere 


\section{Technische Gesteinseigenschaften}

Interpretation die Daten aus der Zylindermessung verwendet. Einen Überblick über die Qualität der Daten und die Variationsbreite der Vp- und Vs-Werte aus den unterschiedlichen Verfahren gibt Anhang I.6.

\subsubsection{Dynamischer E-Modul}

Der mittlere dynamisch bestimmte E-Modul bei einem Umschließungsdruck von $0 \mathrm{MPa}$ (arithmetischer Mittelwert aus x-, y-, z-Richtung) bewegt sich zwischen 20,1 GPa (granitischer Gneis Be2) und 171,4 GPa (Peridotit - Pi1a). Der granitische Orthogneis (VA) weist zwar einen noch niedrigeren mittleren E-Modul auf, jedoch ist hier die z-Richtung negativ, was darauf hinweist, dass die Voraussetzungen eines linear elastischen Körpers nicht gegeben sind. Vergleichswerte liefern Weiss (1992) für Sandsteine und Ondrasina et al. (2002) für frische und verwitterte, kalzitische und dolomitische Marmore mit Werten zwischen $7 \mathrm{GPa}$ und $24 \mathrm{GPa}$ bzw. zwischen $10 \mathrm{GPa}$ und $80 \mathrm{GPa}$.

Die Mittelwerte für die Makro-Gefügegruppen I/II und III betragen $63 \mathrm{GPa}, 83 \mathrm{GPa}$ bzw. 37 GPa (Abb.6-21).

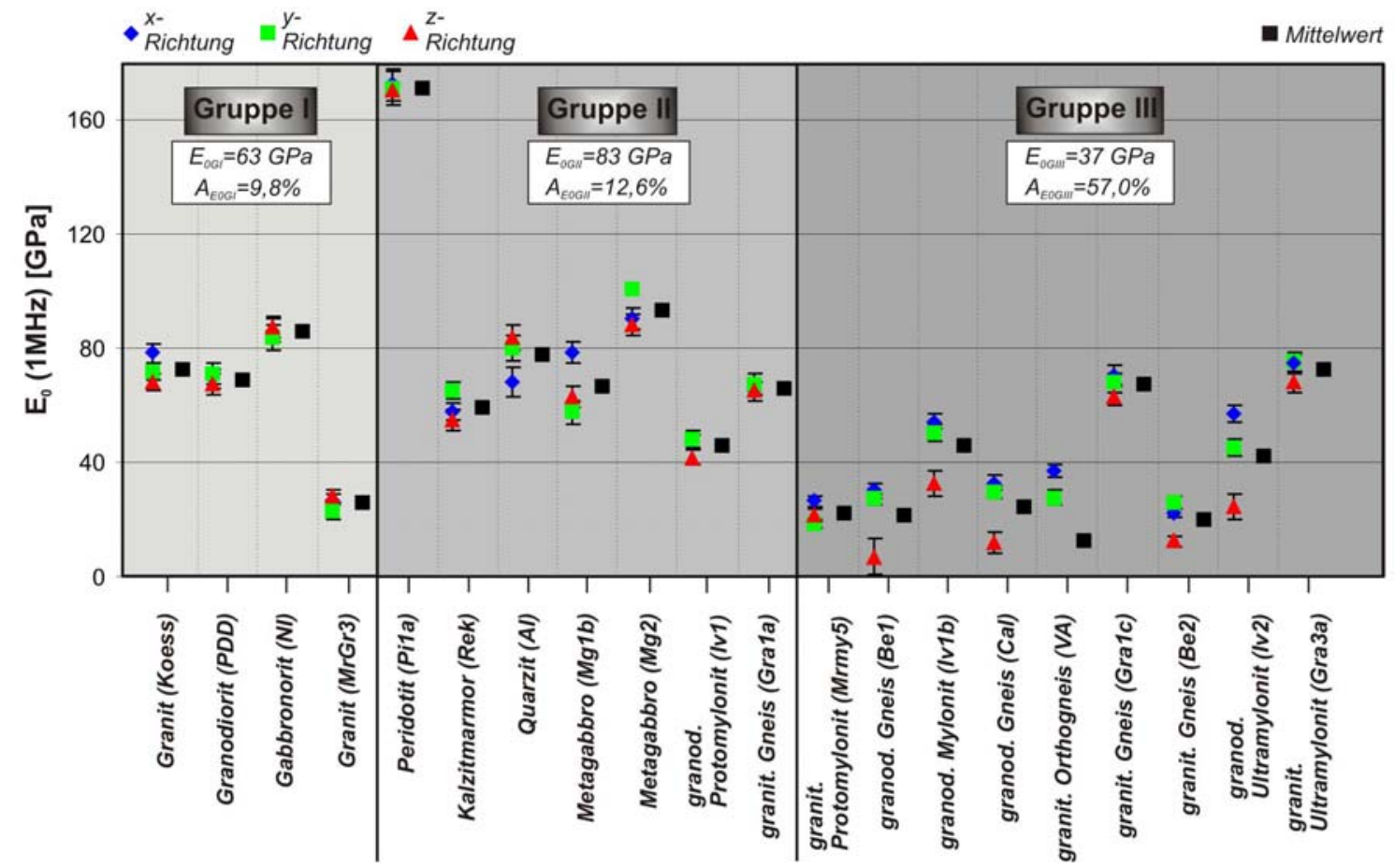

Abb.6-21: E-Modul (dynamisch) bei $0 \mathrm{MPa}$ Umschließungsdruck ( $\left.E_{0}(1 \mathrm{MHz})\right)$ [GPa] der MakrogefügeGruppen I/IIIII. Mittelwerte des E-Modul und mittlere Anisotropie für die jeweilige Gruppe sind angegeben. Fehlerbalken zeigen den 1- $\sigma$-Fehler.

Die mittlere Anisotropie steigt mit zunehmender makroskopischer Gefügeanisotropie von 9,8\% (Gruppe I) über 12,6\% (Gruppe II) auf 57\% (Gruppe III). Die Probe VA wurde aus oben genannten Gründen nicht in die Berechnungen einbezogen. Im Vergleich fallen die 
Elastizitätsmoduln der untersuchten Proben unter einem Umschließungsdruck von $400 \mathrm{MPa}$ wesentlich höher und die Anisotropien wesentlich niedriger aus. Die untere und obere Grenze der $\mathrm{E}_{400}$-Werte markieren die Proben Be2 (granitischer Gneis) mit 80,0 GPa und Pila (Peridotit) mit 190,2 GPa. Die Makrogefüge-Gruppen weisen Mittelwerte zwischen 90 GPa (Gruppe I) und $120 \mathrm{GPa}$ (Gruppe II) auf (Abb.6-22). Die Anisotropie liegt zwischen 4,6\% (Gruppe II) und 7,6\% (Gruppe III), ohne dass eine signifikante Veränderung parallel zur Entwicklung des Makro-Gefüges sichtbar ist. Da die Ultraschallgeschwindigkeit unter 400 MPa Umschließungsdruck die intrinsischen Gesteinseigenschaften widerspiegelt (vgl. Siegesmund 1989/1996, Meglis et al. 1996; Rasolofosoan et al. 2000), handelt es sich bei der Differenz zwischen elastischen Eigenschaften unter hohem Druck und denen unter Normalbedingungen um eine rissbedingte Änderung von Vp und Vs und damit auch des EModuls. Das Phänomen der rissinduzierten Erniedrigung des E-Moduls und der entsprechenden Erhöhung der Anisotropie wird in Kap.6.4.1.5 näher diskutiert.

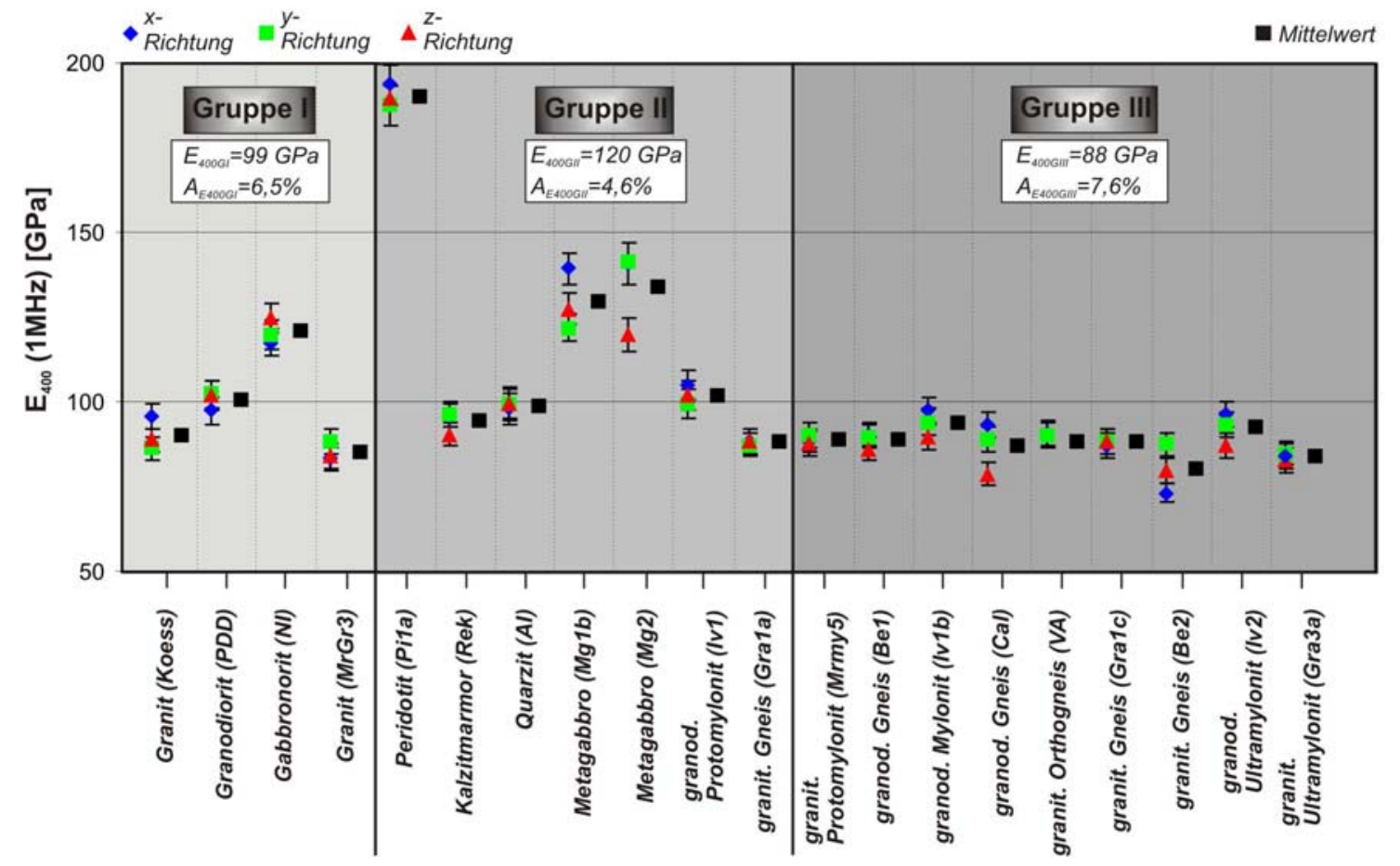

Abb.6-22: E-Modul (dynamisch) bei $400 \mathrm{MPa}$ Umschließungsdruck $\left(E_{400}(1 \mathrm{MHz})\right)$ [GPa] der Makrogefüge-Gruppen I/IIIIII. Mitte/werte des E-Moduls und mittlere Anisotropie für die jeweilige Gruppe sind angegeben. Fehlerbalken zeigen den 1- $\sigma$-Fehler.

\subsubsection{Dynamisch ermittelte Poisson-Zahl}

Ähnlich den Gegebenheiten bezüglich des E-Moduls verhält es sich bei der Poisson-Zahl. Die Mittelwerte unter Normaldruck $\left(v_{0}\right)$ bewegen sich zwischen $-0,10$ (granodioritischer Gneis Be1) und 0,28 (Granit Koess). Die negativen Poisson-Zahlen, die bei den glimmerhaltigen Gesteinen in z-Richtung auftreten, zeigen deutlich, dass die Bedingungen eines linear 
elastischen Körpers hier nicht erfüllt sind. Die Mittelwerte der Makrogefüge-Gruppen liegen bei 0,23 (Gruppe I), 0,19 (Gruppe II) bzw. 0,00 (Gruppe III). Die Anisotropie der PoissonZahlen steigt mit zunehmender makroskopischer Gefügeanisotropie von 27\% (Gruppe I) über 44,6\% (Gruppe II) auf 232,7\% (Gruppe III) (Abb.6-23). Im Vergleich sind die Poisson-Zahlen unter einem Umschließungsdruck von $400 \mathrm{MPa}\left(v_{400}\right)$ im Mittel höher (0,26/0,23/0,22 für Gruppe I/II/III) und die Anisotropien mit 12,0\% für die Gruppe I, 10,1\% für die Gruppe II und 10,6\% für die Gruppe III wesentlich niedriger (Abb.6-24).

Auch hier zeigt der Vergleich der intrinsischen Poisson-Zahlen und denen unter Normaldruck ermittelten die starke Rissabhängigkeit. Bei Betrachtung der $v_{400}$-Werte fällt zudem das niedrige Quer-zu-Längsdehnungs-Verhältnis der Probe AI (Quarzit) von 0,11 auf, welches den Einfluss des Quarzes wiederspiegelt. Dieser hat eine mittlere Poisson-Zahl von 0,08 (Birch 1960/1961). Damit ist die Poisson-Zahl eher als der E-Modul geeignet, zwischen quarzreichen und quarzarmen Gesteinen zu unterscheiden (Christensen \& Fountain 1975; Kern 1982; Holbrook et al. 1987), solange der Risseinfluss durch hohen Umschließungsdruck oder aber auch durch eine Fluidsättigung des Porenraums unterdrückt ist.

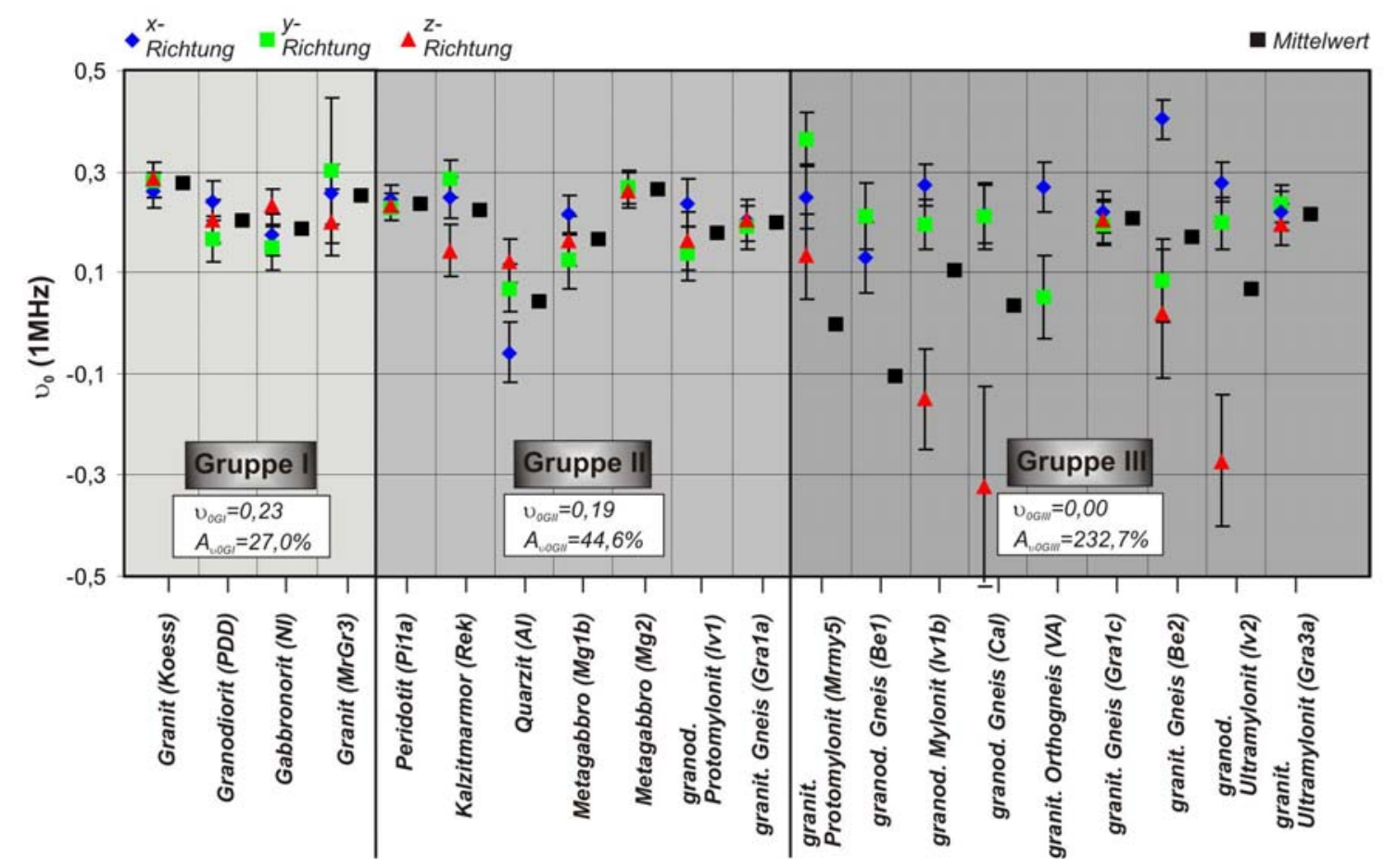

Abb.6-23: Poisson-Zahl (dynamisch) bei 0 MPa Umschließungsdruck (voll MHz)) der Makrogefügegruppen I/IIIII. Mittelwerte der Poisson-Zahl und mittlere Anisotropie für die jeweilige Gruppe sind angegeben. Fehlerbalken zeigen den 1- $\sigma$-Fehler. 


\section{Technische Gesteinseigenschaften}

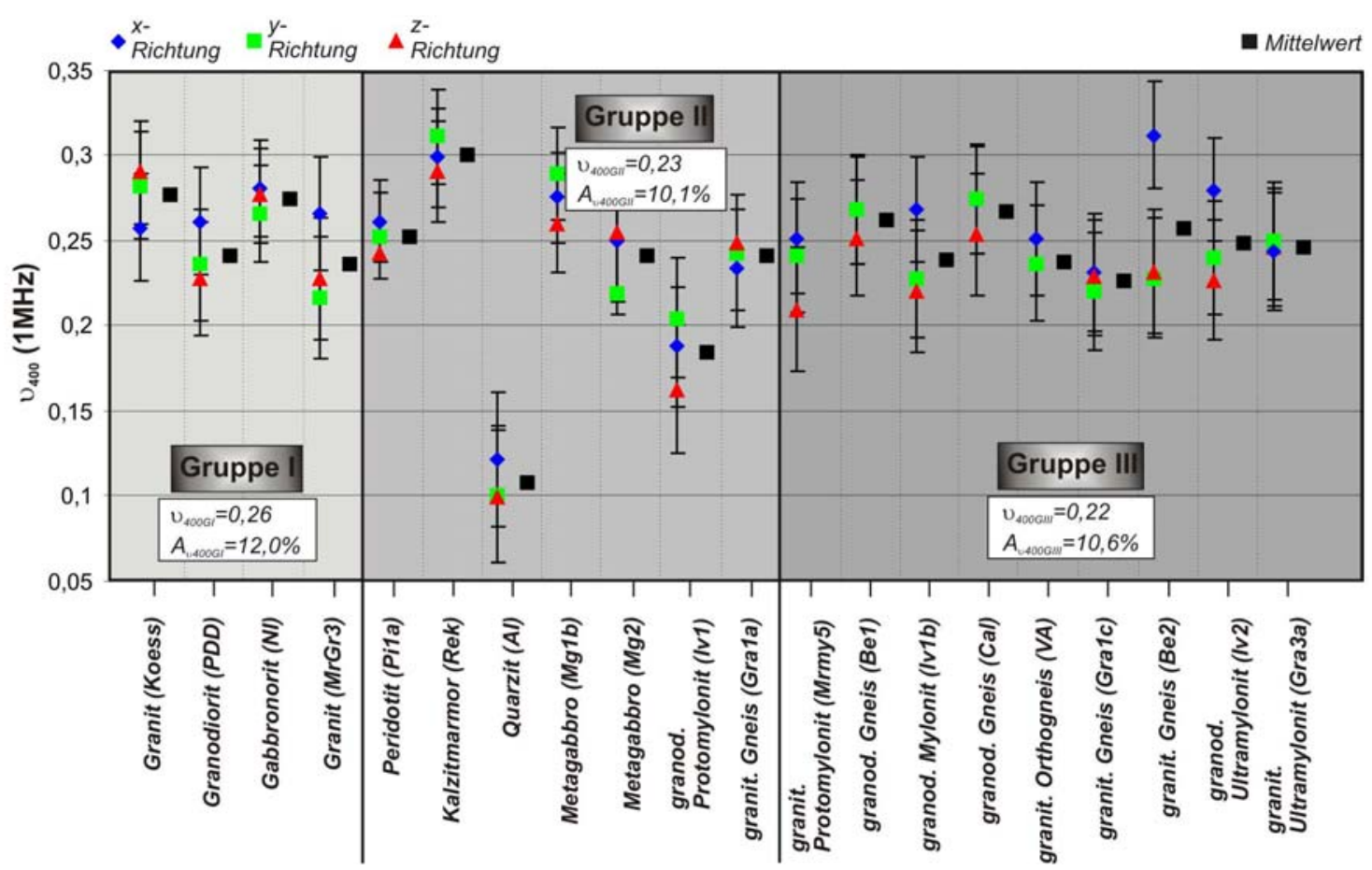

Abb.6-24: Poisson-Zahl (dynamisch) bei $400 \mathrm{MPa}$ Umschließungsdruck (v400(1 MHz)) der Makrogefüge-Gruppen I/IIIIII. Mittelwerte der Poisson-Zahl und mittlere Anisotropie für die jeweilige Gruppe sind angegeben. Fehlerbalken zeigen den 1- $\sigma$-Fehler.

\subsubsection{Scherwellen-Splitting}

Die bisher verwendeten E-Moduln wurden mit dem Mittelwert der Geschwindigkeit für $\mathrm{s}_{1}$ (schnellere Scherwelle) und $s_{2}$ (langsamere Scherwelle) ermittelt. In isotropen Körpern kann $\mathrm{Vs}_{1} \approx \mathrm{Vs}_{2}$ angenommen werden, so dass die Auswirkungen des Scherwellen-Splittings $\left(\mathrm{Vs}_{1} \neq \mathrm{Vs}_{2}\right)$ auf die Proben der Gruppe I als gering eingeschätzt werden können. In anisotropen Körpern gehört das Scherwellen-Splitting zu den häufig auftretenden Phänomenen und kann sowohl durch den Mineralbestand und seine Textur, als auch durch die Anwesenheit bevorzugt orientierter, offener Mikroriss-Systeme bedingt sein (z.B. Christensen 1984; Crampin 1987; Siegesmund \& Kern 1990; Siegesmund 1996). Um einen Ansatz über die zu erwartende Größenordnung der Auswirkungen auf den E-Modul unter Normaldruck zu bekommen, wurde der prozentuale Anteil der durch Scherwellen-Splitting bedingten Differenz $\mathrm{E}_{0 \mathrm{~s} 1}-\mathrm{E}_{0 \mathrm{~s} 2}\left(\mathrm{E}-\mathrm{Modul}\right.$ berechnet mit Vs1 bzw. Vs2) am E-Modul ( $\mathrm{E}_{0}-$ mit Mittelwert aus $\mathrm{Vs}_{1}$ und $\mathrm{Vs}_{2}$ berechneter E-Modul) nach G.6-8 ermittelt.

$$
\Delta E_{0 s 1 / 2}=\frac{\left(E_{0 s 1}-E_{0 s 2}\right)}{E_{0}} * 100
$$

$\Delta E_{0 s 1 / 2}$ - prozentualer Anteil der durch Scherwellen-Splitting bedingten Differenz $E_{0 s 1}-E_{0 s 2}$ an $E_{0}[\%]$; $E_{0 s 1}-E-M o d u l ~ b e r e c h n e t ~ m i t ~ V s_{1}[G P a]$. $E_{0 s 2}-E$-Modul berechnet mit $V s_{2}$ [GPa]. $E_{0}-E-M o d u l$ berechnet mit Mittelwert aus $V s_{1}$ und $V s_{2}[G P a]$; alle Werte unter Normaldruck ermittelt. 
Die relativen Abweichungen $\left(\Delta \mathrm{E}_{0 \mathrm{~s} 1 / 2}\right)$ von den bisher verwendeten $\mathrm{E}_{0}$-Werten liegen für die Mittelwerte der Proben (arithmetisches Mittel aus x, y, z-Richtung) zwischen 1,6\% (granitischer Gneis - Gralc) und 26,2\% (granodioritischer Gneis - Cal). Die mittleren Abweichungen für die Makrogefüge-Gruppen liegen zwischen 5,1\% (Gruppe I) und 12,1\% (Gruppe III). Ein Trend parallel zur zunehmenden Gefügeanisotropie kann aufgrund der Datenqualität nicht eindeutig nachgewiesen werden. Dennoch entsteht der Eindruck, dass mit Ausnahme der Probe Gralc (granitischer Gneis) und der Probe Gra3a (granitischer Ultramylonit) mit einem gering ausgeprägten Scherwellen-Splitting, die Proben der Gruppe III deutlich höhere relative Abweichungen von $\mathrm{E}_{0}$ aufweisen als die der Gruppe II und Gruppe I zumal bei letzterer die Probe MrGr3 (Granit) mit einem hohen mittleren $\Delta \mathrm{E}_{0 \mathrm{~s} 1 / 2}$-Wert von 15,4\% den Mittelwert der Gruppe maßgeblich beeinflusst (Abb.6-25). Zusätzlich lässt sich feststellen, dass eine Richtungsabhängigkeit des Scherwellen-Splittings besteht. Tendenziell sind die $\mathrm{x}$ - und die $\mathrm{y}$-Richtung am stärksten betroffen, weisen also die höchsten $\Delta \mathrm{E}_{0 \mathrm{~s} 1 / 2}$-Werte auf, wohingegen die z-Richtung das geringste Scherwellen-Splitting zeigt.

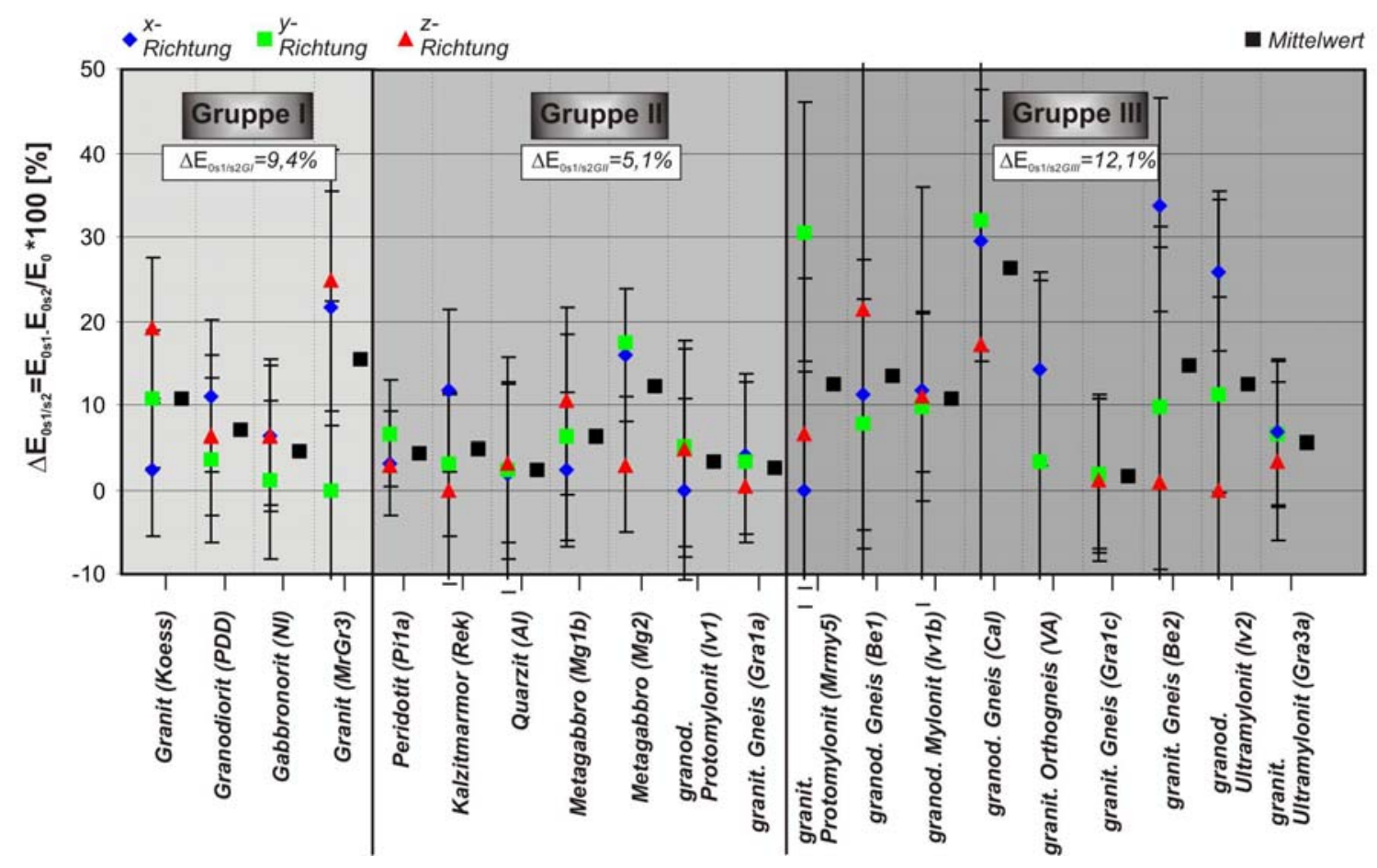

Abb.6-25: Scherwellen-Splitting. Prozentualer Anteil der durch Scherwellen-Splitting bedingten EModul-Unterschiede am Mittelwert des E-Modul [\%]. E-Moduln bei $1 \mathrm{MHz}$ Anregungsfrequenz und einem Umschließungsdruck von $0 \mathrm{MPa}$. $E_{0 \mathrm{~s} 1}-E$-Modul bestimmt mit der schnelleren Scherwelle $\left(\mathrm{s}_{1}\right)$. $E_{0 \mathrm{~s} 2}-E-M o d u l$ bestimmt mit der langsameren Scherwelle $\left(s_{2}\right)$. Fehlerbalken zeigen den 1- $\sigma$-Fehler.

Besonders deutlich ist dieser Effekt bei glimmerhaltigen Gesteinen ausgeprägt (z.B. granitischer Gneis - Be2; granodioritischer Gneis - Cal). Folglich sind die Richtungen am stärksten betroffen, bei denen sich die Scherwellen parallel zur Foliation ausbreiten und eine der Scherwellen senkrecht zur makroskopischen Foliation und damit häufig auch senkrecht 


\section{Technische Gesteinseigenschaften}

zum Maximum der offenen Mikrorissflächen polarisiert ist. Diese hat stets die höhere Laufzeit und damit die niedrigere Geschwindigkeit der beiden Scherwellen. Diese Erkenntnisse stimmen mit Ergebnissen von Nur \& Simmons (1969), Christensen (1984) und Siegesmund \& Kern (1990) überein, die ein ausgeprägtes Scherwellen-Splitting bei einer sWellenausbreitungsrichtung parallel zur Foliation, mit einer s-Welle parallel und einer senkrecht zur Foliation polarisiert, beobachteten.

Bei Ultraschallmessungen an Gebäuden sollte zumindest für stark foliierte Gneise die Polarisationsrichtung der Scherwelle berücksichtigt werden. Ist das messtechnisch nicht möglich, so ist $\mathrm{zu}$ empfehlen, die $\mathrm{zu}$ erwartenden Unterschiede in den Schwerwellengeschwindigkeiten und die damit verbundenen Unterschiede in den E-Moduln in die Interpretation einzubeziehen.

\subsubsection{Anisotropie des dynamischen E-Moduls}

Im Folgenden wird die Anisotropie des E-Moduls in Abhängigkeit vom Mineralbestand und den Gefügemerkmalen diskutiert. In $\boldsymbol{A b b . 6 - 2 6}$ ist die aufgrund des Mineralbestands mögliche maximale Anisotropie der Gesteine $\left(\mathrm{A}_{\mathrm{E} 0 \mathrm{Gmax}}\right)$ gegen die unter Normaldruck experimentell ermittelte Anisotropie $\left(\mathrm{A}_{\mathrm{E} 0}\right)$ aufgetragen. Einige der Proben, wie z.B der granitische Gneis $(\mathrm{Be} 2)$ und der granodioritische Gneis (Cal) zeigen deutlich höhere Anisotropien als aufgrund der mineralogischen Komposition und den Einkristallanisotropien (vgl. Kap.4.3) möglich ist. Es muss daher ein nicht berücksichtigtes Gefügeelement zumindest für einen Teil der Anisotropie verantwortlich sein. In $\boldsymbol{A} \boldsymbol{b} \boldsymbol{b}$.6-26 wird die Anisotropie des E-Moduls unter einem Umschließungsdruck von $400 \mathrm{MPa}\left(\mathrm{A}_{\mathrm{E} 400}\right)$ betrachtet (intrinsischer Anteil). Alle Proben befinden sich oberhalb der 1:1-Linie, was bedeutet, dass die experimentell beobachteten Anisotropien durchweg niedriger ausfallen als die theoretisch möglichen. Zudem ist eine leichte Tendenz von hohen Anisotropiepotentialen mit höheren Anisotropien zu beobachten. Analog zu den Ergebnissen bei der thermischen Dehnung (vgl. Kap.6.5) hängt die Anisotropie des E-Moduls nicht direkt von dem Anisotropiepotential $\left(\mathrm{A}_{\mathrm{E} 0 \mathrm{Gmax}}\right) \mathrm{ab}$, sondern vielmehr zeigen hohe $A_{E 0 G m a x}-W e r t e$ die Gefährdung eines Gesteins für eine hohe Anisotropie an. Diese muss jedoch erst durch die starke Textur eines möglichst anisotropen Minerals mit einem hohen Volumenanteil im Gestein aktiviert werden. Insgesamt geht aus der deutlichen Verringerung der Anisotropie der Gesteine durch einen hohen Umschließungsdruck hervor (Abb.6-26), dass die Anisotropie neben den intrinsischen Eigenschaften (Mineralbestand und Textur) massiv von der Anwesenheit offener Mikrorisse und deren bevorzugter Orientierung bestimmt wird. Weiter untermauert wird diese Aussage durch $\boldsymbol{A b b . 6 - 2 6}$, die einen Vergleich 


\section{Technische Gesteinseigenschaften}

des Produkts Anisotropiepotential $\left(\mathrm{A}_{\mathrm{E} 0 \mathrm{Gmax}}\right) *$ Rissanisotropiefaktor $\left(\mathrm{A}_{\text {Riss }}\right)$ mit der Anisotropie des E-Moduls unter Normaldruck $\left(\mathrm{A}_{\mathrm{E} 0}\right)$ beinhaltet. Es zeigt sich eine gute Korrelation, obwohl die Texturen und damit die intrinsische Anisotropien noch nicht berücksichtigt sind. Damit ist klar, dass die Anisotropie der offenen Mikroriss-Systeme und deren Rissdichte (quantifiziert durch den Rissanisotropiefaktor $\mathrm{A}_{\mathrm{Riss}}=\mathrm{A}_{\Delta \mathrm{Vp}} * \mathrm{~V}_{\mathrm{QRiss}}$ - vgl. Kap.2.4.5.1) einen großen Einfluss auf die Anisotropie des E-Moduls unter Laborbedingungen haben. Bei zunehmendem Umschließungsdruck geht der Risseinfluss zurück, bis nur noch die intrinsischen Eigenschaften die Anisotropie des E-Moduls dominieren.
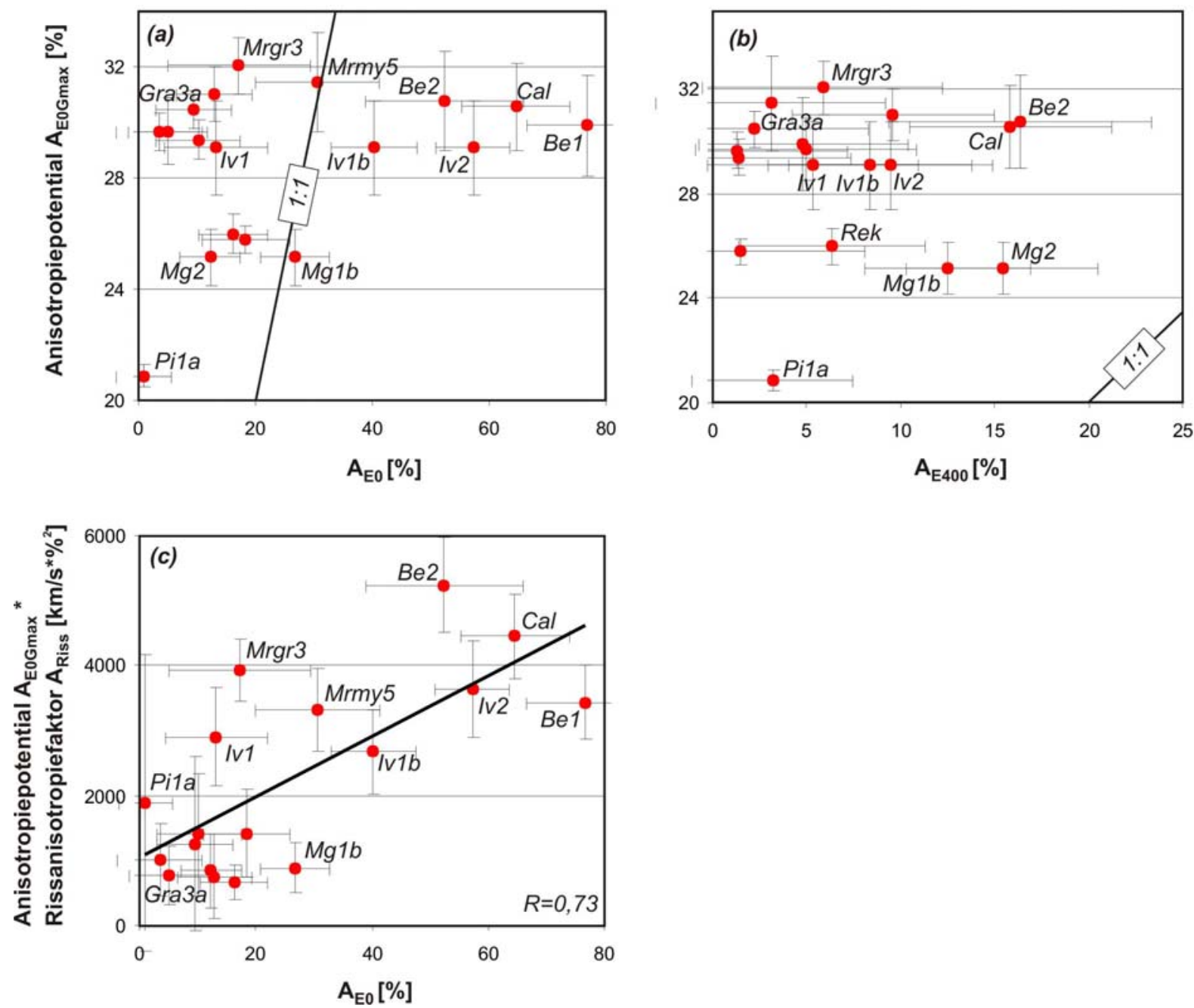

Abb.6-26: Anisotropie des E-Moduls bei $0 \mathrm{MPa}$ bzw. $400 \mathrm{MPa}$ Umschließungsdruck. (a) Anisotropiepotential $\left(A_{E O G \max }\right)$ berechnet aus der mineralogischen Zusammensetzung und den Einkristalleigenschaften und Anisotropie des E-Moduls (O MPa) aus den experimentellen Daten. (b) Anisotropiepotential $\left(A_{E O G \max }\right)$ und Anisotropie des E-Moduls (400 MPa) aus den experimentellen Daten. (c) Anisotropiepotential $\left(A_{E 0 G \max }\right)[\%]{ }^{*}$ Rissanisotropiefaktor $\left(A_{\text {Riss }}=\right.$ Anisotropie aus dem Differenzisolinienplot der Kugelmessung [\%] (vgl. Kap.2.4.5.1)* Rissquantifizierungsparameter $V p_{Q R i s s}$ $[\mathrm{km} / \mathrm{s}]$ ) und Anisotropie des E-Moduls (0 MPa) aus experimentellen Daten. Be2 - granitischer Gneis. $\mathrm{Cal}$ - granodioritischer Gneis. Be1 - granodioritischer Gneis. Iv1 - granodioritischer Protomylonit. Iv1b - granodioritischer Mylonit. Iv2 - granodioritischer Ultramylonit. Mrgr3 - Granit. Mrmy5 - granitischer Protomylonit. Mg1b/Mg2 - Meta-Gabbro. Rek - Kalzit-Marmor. Pi1a - Peridotit. Gra3a - granitischer Ultramylonit. Fehlerbalken markieren den 1- $\sigma$-Fehler. 


\section{Technische Gesteinseigenschaften}

Weiterführend stellt sich die Frage, ob die Glimmer mit ihren z.T. sehr starken Texturen und ihrer hohen Anisotropie der elastischen Eigenschaften als Stellvertreter für die makroskopische Gefügeanisotropie geeignet sind, einen Hinweis auf die zu erwartende Anisotropie des E-Moduls unter Normaldruck $\left(\mathrm{A}_{\mathrm{E} 0}\right)$ zu liefern. $\boldsymbol{A} \boldsymbol{b} \boldsymbol{b} .6-27$ zeigt auf den ersten Blick eine gute lineare Korrelation zwischen der Intensität der Glimmertexturen und der Anisotropie des E-Moduls. Auf den zweiten Blick fällt die Probe Gra3a (granitischer Ultramylonit) auf, die weit außerhalb des Trends liegt und zu dem verhältnismäßig niedrigen Korrelationskoeffizienten von $\mathrm{R}=0,58$ führt.
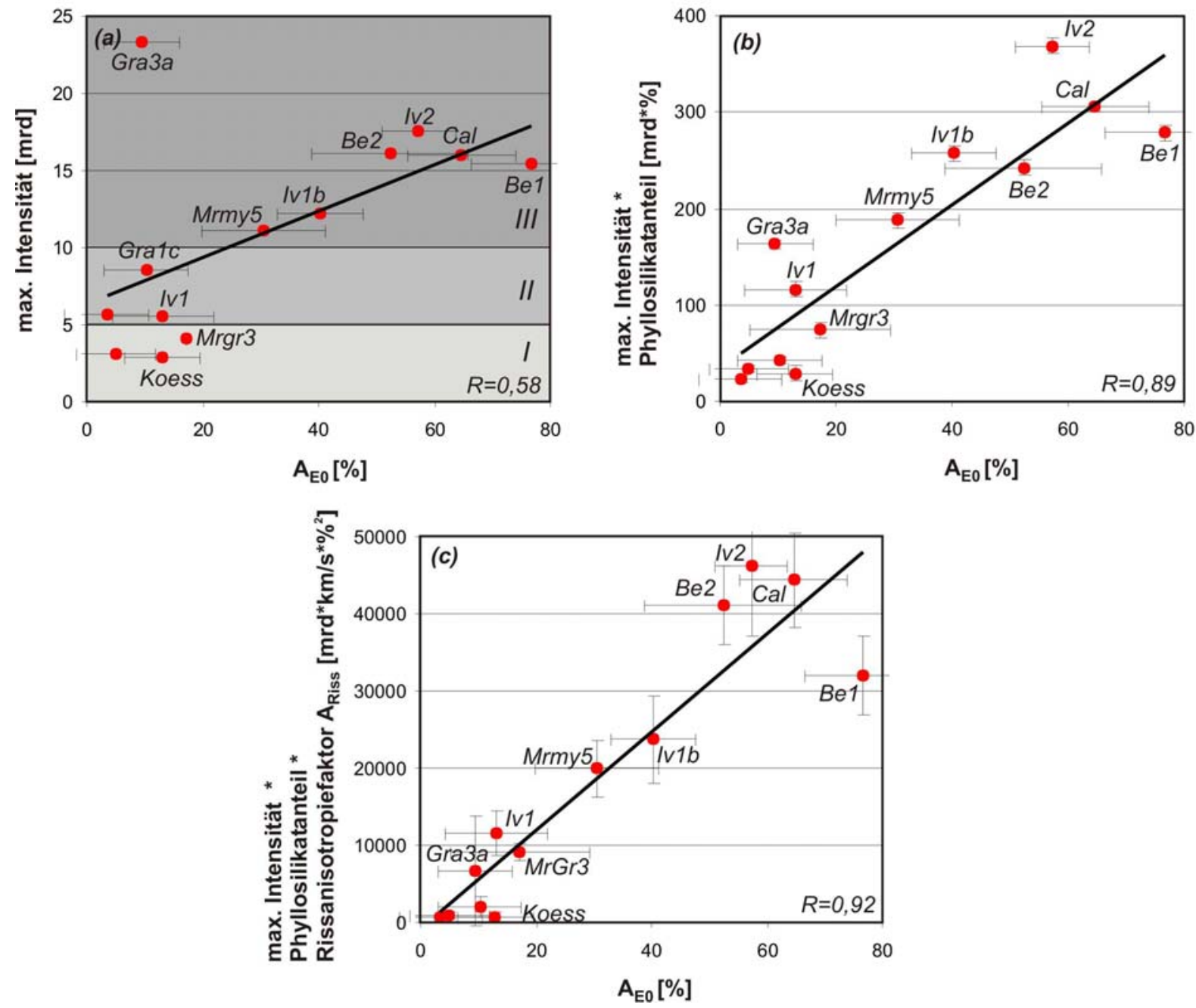

Abb.6-27: Anisotropie des E-Moduls aus experimentellen Daten bei 0 MPa Umschließungsdruck. (a) Maximale Intensität der Glimmer-(001)-Pole [mrd]; Makrogefüge-Gruppen I/II/III sind indiziert. (b) Maximale Intensität der Glimmer-(001)-Pole [mrd] * Volumenanteil der Phyllosilikate [\%]. (c) Maximale Intensität der Glimmer-(001)-Pole [mrd] * Volumenanteil der Phyllosilikate [\%] * Rissanisotropiefaktor $\left(A_{\text {Riss }}=A_{\Delta v_{p}}[\%]^{*} V p_{Q R i s s}[\mathrm{~km} / \mathrm{s}]\right)$. Be2 - granitischer Gneis. Cal - granodioritischer Gneis. Be1 granodioritischer Gneis. Iv1 - granodioritischer Protomylonit. Iv1b - granodioritischer Mylonit. Iv2 granodioritischer Ultramylonit. Mrgr3 - Granit. Mrmy5 - granitischer Protomylonit. Koess - Granit. Gra1c-granitischer Gneis. Gra3a - granitischer Ultramylonit. Fehlerbalken markieren den 1- $\sigma$-Fehler.

Die Intensität der Glimmertextur alleine kann die Anisotropie des E-Moduls also nicht vorhersagen. Bezieht man die Volumenanteile der Phyllosilikate mit ein, so verbessert sich 


\section{Technische Gesteinseigenschaften}

die Korrelation deutlich. Eine weitere Erhöhung des Korrelationskoeffizienten bringt die Einbindung des Rissanisotropiefaktors (Abb.6-27).

Es darf an dieser Stelle nicht der Eindruck entstehen, die Anisotropie des dynamischen EModuls unter Normaldruck hinge hauptsächlich von der Glimmertextur und dem Volumenanteil der Glimmer ab. Vielmehr soll herausgestellt werden, dass die Glimmertexturen als Stellvertreter für die Gesamtgesteinsanisotropie geeignet sind. Dies schließt gerade bezüglich der elastischen Eigenschaften auch die offenen Mikroriss-Systeme ein, welche häufig die bevorzugte Orientierung der Glimmer nachzeichnen.

Ursächlich entscheidend für die Anisotropie des E-Moduls unter Normaldruck sind daher die offenen Mikrorisse, wohingegen die intrinsische Anisotropie in Abhängigkeit von der mineralogischen Zusammensetzung und der Textur der gesteinsbildenden Minerale meist nur gering dazu beiträgt. Ein anderes Bild ergibt sich unter hohem Umschließungsdruck von 400 MPa. Hier sind die Mikrorisse größtenteils geschlossen und ihr Einfluss auf die Anisotropie des E-Moduls wird unterdrückt. Aber auch bei sehr rissarmen Proben (niedrige Rissdichte) sind die intrinsischen Gesteinseigenschaften dominant (z.B. Proben Pila - Peridotit; Gra3a granitischer Ultramylonit).

\subsubsection{Dynamischer E-Modul, offene Mikrorisse und Mineralbestand}

Nicht nur die Anisotropie des E-Moduls, sondern auch die Mittelwerte (arithmetisches Mittel aus x-, y-, z-Richtung) der einzelnen Proben sind stark von der Anwesenheit offener Mikrorisse beeinflusst. Der aufgrund des Mineralbestands und dem Voigt-Reuss-Hill-Mittel des E-Moduls der gesteinsbildenden Minerale ermittelte Gesamtgesteins-E-Modul ( $\left.\mathrm{E}_{\mathrm{GVRH}}\right)$ liegt durchweg höher als der Mittelwert der experimentellen Daten (Abb.6-28). Dagegen ergibt sich beim Vergleich von $\mathrm{E}_{\mathrm{GVRH}}$ mit den experimentell unter $400 \mathrm{MPa}$ ermittelten Werten ein Verhältnis von annähernd 1:1. Die Differenz zwischen den $\mathrm{E}_{\mathrm{VRH}}-$ Werten und den $\mathrm{E}_{0}$-Werten von maximal $68 \mathrm{GPa}$ (Probe $\mathrm{Be} 2$ - granitischer Gneis) ist daher rissbedingt, was durch die gute Korrelation von $\mathrm{E}_{\mathrm{GVRH}}-\mathrm{E}_{0}$ mit dem Rissquantifizierungsparameter ( $\mathrm{V} \mathrm{p}_{\mathrm{QRiss}}$ ) bestätigt wird (Abb.6-28). Im Mittel liegt der rissbedingte Abfall des E-Moduls bei 41,3 GPa, was etwa 42\% vom $\mathrm{E}_{\mathrm{GVRH}}-$ Mittelwert aller Proben (99,4 GPa) entspricht. Wird ein Gestein am Gebäude untersucht und aufgrund der p- und s-Wellengeschwindigkeiten der E-Modul ermittelt, so muss neben der Anisotropie auch die rissbedingte Reduktion der Mittelwerte berücksichtigt werden. Bei alleiniger Betrachtung der mittleren Verringerung des E-Moduls von 41,3 GPa kann sich der am Gebäude bestimmte E-Modul der untersuchten Gesteine zwischen dem $\mathrm{E}_{\mathrm{GVRH}}-\mathrm{Mittelwert}$ von 99,4 GPa und 58,1 GPa bewegen. Das ist der Fall, weil 
die Wassersättigung des Porenraumes eine ähnliche Auswirkung auf die elastischen Eigenschaften hat wie ein hoher Umschließungsdruck (z.B. Schild et al. 2001). Mit zunehmendem Sättigungsgrad steigen die Vp-Werte und die Vs-Werte an, wodurch sich der errechnete E-Modul erhöht. Da der Sättigungsgrad des Gesteins am Gebäude nur schwer zu ermitteln ist und außerdem kaum Referenzdaten für die Ultraschallgeschwindigkeiten in wassergesättigten Gesteinen vorliegen, bleibt die Interpretation solcher Daten ohne detaillierte Kenntnisse des Gesteinszustandes schwierig.
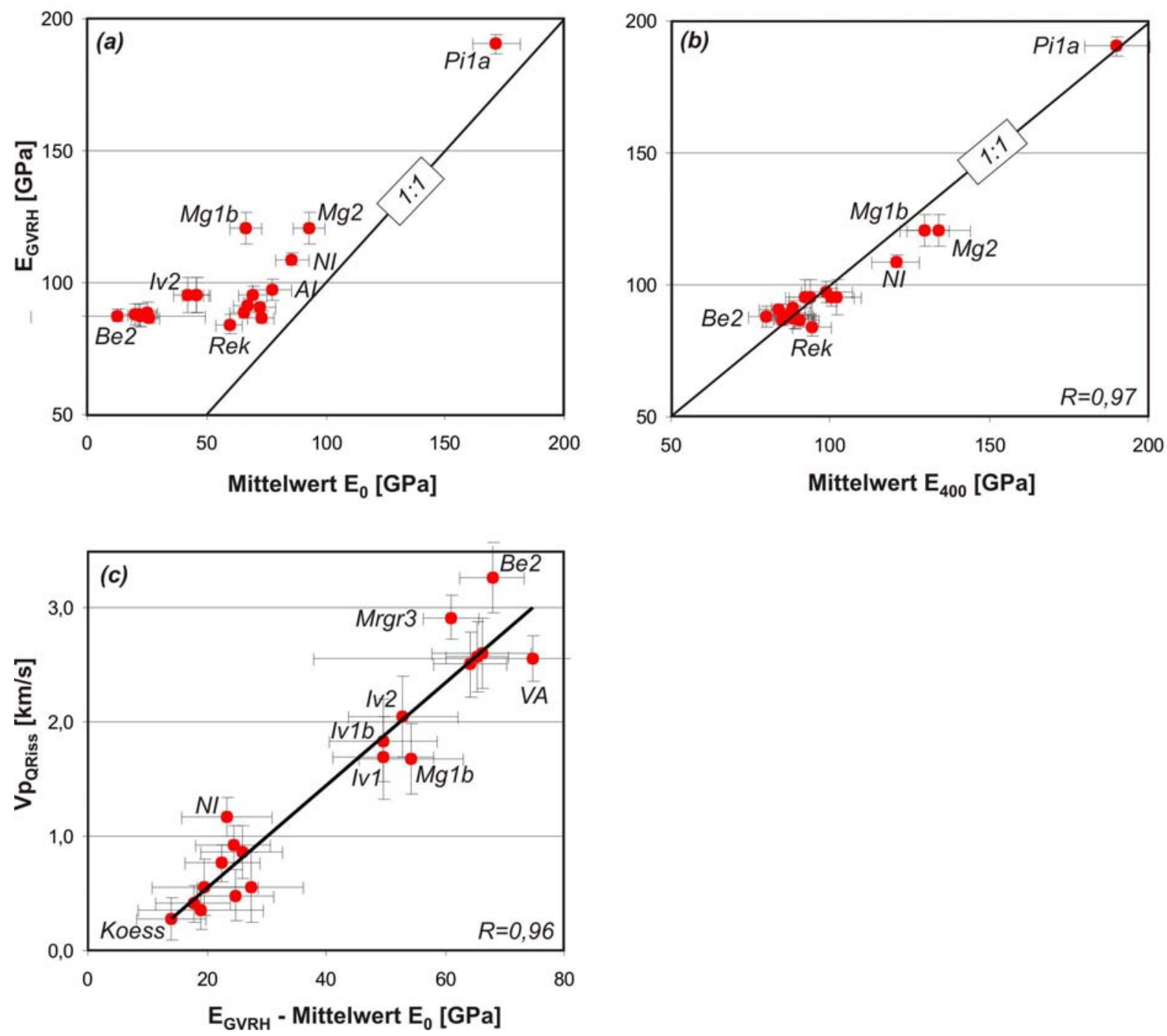

Abb.6-28: Einfluss der mineralogische Zusammensetzung und der offenen Mikroriss-Systeme auf die Mittelwerte (arithmetisches Mittel aus $x$-, $y$ - und z-Richtung) des E-Moduls (aus experimentellen Daten bei $0 \mathrm{MPa}$ bzw. $400 \mathrm{MPa}$ ). (a) Aus dem Mineralbestand und den Einkristalldaten berechneter E-Modul $E_{G V R H}$ (Voigt-Reuss-Hill-Mittel) [GPa] vs. Mittelwert des E-Moduls $E_{0}(0 \mathrm{MPa}$ ) [GPa]. (b) Aus dem Mineralbestand und den Einkristalldaten berechneter E-Modul $E_{G V R H}$ (Voigt-Reuss-Hill-Mittel) [GPa] vs. Mittelwert des E-Moduls $E_{400}\left(400 \mathrm{MPa}\right.$ ) [GPa]. (c) Rissquantifizierungsparameter $V p_{\text {QRiss }}[\mathrm{km} / \mathrm{s}]$ vs. $E_{G V R H}-E_{0}[G P a]$. Be2 - granitischer Gneis. Cal - granodioritischer Gneis. Iv1 - granodioritischer Protomylonit. Iv1b - granodioritischer Mylonit. IV2 - granodioritischer Ultramylonit. Mrgr3 - Granit. VA - granitischer Gneis. Mg1b/Mg2 - Meta-Gabbro. Rek - Kalzit-Marmor. Pi1a - Peridotit. NI - GabbroNorit. Koess - Granit. Al - Quarzit. Fehlerbalken markieren den 1- $\sigma$-Fehler. 


\section{Technische Gesteinseigenschaften}

In $\boldsymbol{A b b . 6 - 2 9}$ ist die Abhängigkeit des mittleren E-Moduls bei $0 \mathrm{MPa}\left(\mathrm{E}_{0}\right)$ und $400 \mathrm{MPa}\left(\mathrm{E}_{400}\right)$ Umschließungsdruck vom Kationenpackungsindex (Buntebarth 1982) dargestellt. Der Kationenpackungsindex $\left(\mathrm{k}_{\mathrm{M}}\right)$ beschreibt die Anzahl der Kationen je Volumeneinheit für Minerale. Aus dem Mineralbestand und den Einkristalldaten kann der Kationenpackungsindex des Gesamtgesteins $\left(\mathrm{k}_{\mathrm{G}}\right)$ berechnet werden (vgl. Kap.2.2.4), der für niedrig poröse Kristallingesteine eine gute Korrelation mit der Rohdichte und den Vp zeigt (Rybach \& Buntebarth 1982). Ein hoher Kationenpackungsindex stimmt dabei mit hohen Dichten und hohen Vp überein. Bezüglich des E-Moduls der untersuchten Gesteine ergibt sich eine gute Korrelation zwischen mittlerem $\mathrm{E}_{0}$ und dem Kationenpackungsindex und eine noch bessere Korrelation zwischen $\mathrm{E}_{400}$ und dem $\mathrm{k}_{\mathrm{G}}$. Daraus wird deutlich, dass der Kationenpackungsindex sehr gut geeignet ist, die intrinsischen elastischen Eigenschaften widerzuspiegeln. Im Hinblick auf die mittleren E-Moduln unter Normaldruck, deren Höhe sowohl von den Mikrorissen als auch von den Mineraleigenschaften bestimmt werden, ist die gute Korrelation (Abb.6-29) mit Vorsicht zu betrachten, zumal die Daten eine gewisse Streuung aufweisen. Außerdem ist die von Rybach und Buntebarth (1982) gefundene lineare Korrelation von $\mathrm{k}_{\mathrm{G}}$ und $\mathrm{Vp}$ nur für einen gewissen Umschließungsdruck gültig (50 MPa), bei dem bereits ein Teil des Mikrorisseinflusses unterdrückt ist.
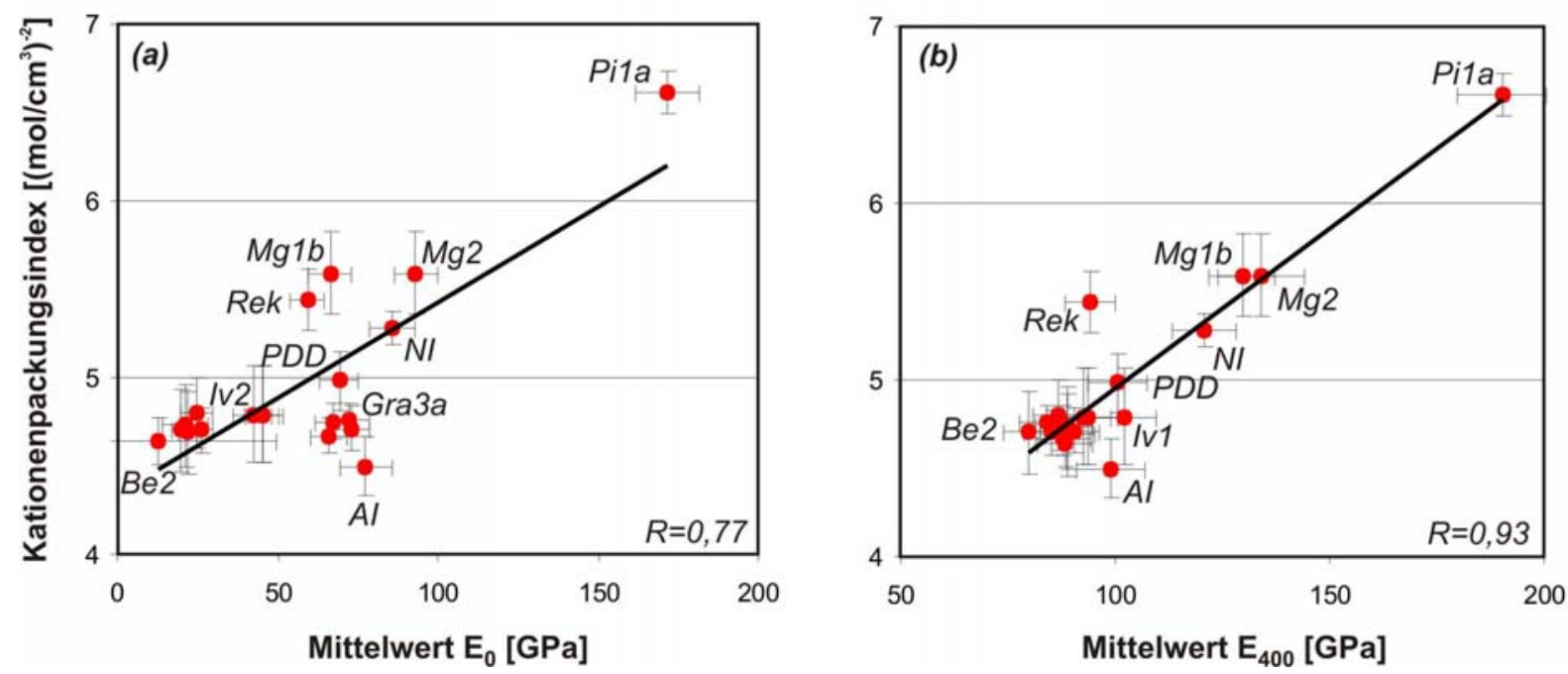

Abb.6-29: Zusammenhang zwischen E-Modul bei $0 \mathrm{MPa}$ (a) bzw. $400 \mathrm{MPa}$ (b) Umschließungsdruck und dem Kationenpackungsindex nach Buntebarth (1982). Be2 - granitischer Gneis. Cal granodioritischer Gneis. Iv1 - granodioritischer Protomylonit. IV2 - granodioritischer Ultramylonit. Mg1b/Mg2 - Meta-Gabbro. Rek - Kalzit-Marmor. Pi1a - Peridotit. Gra3a - granitischer Ultramylonit. $\mathrm{NI}$ - Gabbro-Norit. PDD - Granodiorit. AI - Quarzit. Fehlerbalken markieren den 1- $\sigma$-Fehler.

Diese Tatsache in Kombination mit dem starken Einfluss von offenen Mikrorissen auf die Vp, Vs und den E-Modul (die Größenordnung wird im nächsten Abschnitt noch näher beleuchtet) führt $\mathrm{zu}$ dem Schluss, dass die Verbindung von $\mathrm{k}_{\mathrm{G}}$-Werten mit den $\mathrm{E}_{0}$-Werten schwierig ist, da die offenen Mikrorisse nicht in die Berechnung des Kationenpackungsindex eingehen. 


\section{Technische Gesteinseigenschaften}

Im Verlauf dieses Kapitels wurde immer wieder auf den starken Einfluss der Mikrorisse auf die Höhe (Mittelwerte) und die Anisotropie des dynamischen E-Moduls hingewiesen. Die Modelle von O'Connel \& Budiansky (1974) sowie Hudson (1981) bieten Möglichkeiten, den Einfluss offener Risse unter trockenen und wassergesättigten Bedingungen zu quantifizieren. Nach Rathore et al. (1991), Assad et al. (1992) und Zimmer (2001) ist das Modell von Hudson am besten geeignet, die Zustände in trockenen, niedrig porösen Gesteinen mit vorwiegender Rissporosität (niedrige aspect-ratios) zu erfassen. In diesem Modell werden die Verschiebungsdiskontinuitäten an einem Riss mit den Achsenverhältnissen $a_{1}=a_{2}>>b_{3}$ (pennyshaped) verwendet, um die Veränderung des elastischen Tensors zu berechnen (vgl. Zimmer 2001). Für den isotropen Fall, der im Folgenden zusammen mit den experimentellen Mittelwerten der Gesteine betrachtet wird, ergibt sich die resultierende Geschwindigkeit der p-Welle nach

$$
V p^{2}=V p_{0}{ }^{2}\left[1-v a^{3}\left\{\frac{4\left(3 V p_{0}{ }^{2}-4 V s_{0}{ }^{2}\right)^{2}}{27 V s_{0}{ }^{2}\left(V p_{0}{ }^{2}-V s_{0}{ }^{2}\right)}+\frac{14\left(9 V p_{0}{ }^{2}-8 V s_{0}{ }^{2}\right) V s_{0}{ }^{2}}{15\left(3 V p_{0}{ }^{2}+2 V s_{0}{ }^{2}\right)\left(V p_{0}{ }^{2}-V s_{0}{ }^{2}\right)}\right\}\right]
$$

$V p$ - resultierende $V p[\mathrm{~km} / \mathrm{s}] ; V p_{0}-V p$ im rissfreien Gestein $[\mathrm{km} / \mathrm{s}] ; V_{0}-V s$ im rissfreien Gestein $[\mathrm{km} / \mathrm{s}] ; v-$ Risskonzentration/Rissdichte $\left[1 / \mathrm{cm}^{3}\right] ;$ a - Rissradius [cm]; es gilt $v^{*} a^{3}<<1$.

und die Geschwindigkeit der s-Welle nach

$$
V s^{2}=V s_{0}{ }^{2}\left[1-v a^{3}\left\{\frac{16 V p_{0}{ }^{2}\left(9 V p_{0}{ }^{2}-8 V s_{0}{ }^{2}\right)}{45\left(3 V p_{0}{ }^{2}-2 V s_{0}{ }^{2}\right)\left(V p_{0}{ }^{2}-V s_{0}{ }^{2}\right)}\right\}\right]
$$

$V s$ - resultierende Vs $[\mathrm{km} / \mathrm{s}] ; V p_{0}-V p$ im rissfreien Gestein $[\mathrm{km} / \mathrm{s}] ; V_{s_{0}}-V s$ im rissfreien Gestein $[\mathrm{km} / \mathrm{s}] ; v-$ Risskonzentration/Rissdichte $\left[1 / \mathrm{cm}^{3}\right]$; a - Rissradius [cm]; es gilt $v^{*} a^{3}<<1$.

Beide Zusammenhänge gelten für kleine Rissdichten mit $\mathrm{v}^{*} \mathrm{a}^{3}<<1$. Im Fall größerer Rissdichten muss die Wechselwirkung der elastischen Felder an den einzelnen Rissen berücksichtigt werden (Zimmer 2001). Sowohl die resultierende Vp, als auch die resultierende Vs gehen in die Berechnung des E-Moduls und der Poisson-Zahl ein (vgl. Kap.2.4.5.2) und führen zu einer Modellvorhersage in Abhängigkeit von der Rissdichte*a ${ }^{3}$ (Abb.6-30). Aus dem untersuchten Probensatz wurden acht Proben mit ähnlichen Vp und Vs unter einem Umschließungsdruck von $400 \mathrm{MPa}$ (rissfreier Zustand) ausgewählt. Aus deren Mittelwerten $(\mathrm{Vp}=6,27 \mathrm{~km} / \mathrm{s} ; \mathrm{Vs}=3,63 \mathrm{~km} / \mathrm{s})$ und der mittleren Rohdichte der Proben von $\rho=2,710 \mathrm{~g} / \mathrm{cm}^{3}$ ergibt sich ein mittlerer E-Modul von 89,1 GPa und eine mittlere Poisson-Zahl von 0,25, welche als Ausgangspunkt, also als rissfreier Zustand, sowohl für die Modellvorhersage als 
auch für die Entwicklung innerhalb des Probensatzes angenommen wird. Als Maß für die Rissdichte der Proben wird der Rissquantifizierungsparameter $V \mathrm{p}_{\mathrm{QRiss}}$ verwendet. Es zeigt sich eine gute qualitative Übereinstimmung der Kurvenverläufe von den in Abhängigkeit von $\mathrm{V}^{*} \mathrm{a}^{3}$ modellierten und den experimentellen in Abhängigkeit von $\mathrm{V} \mathrm{p}_{\mathrm{QRiss}}$ dargestellten $\mathrm{E}$ Moduln und Poisson-Zahlen. Zum ersten scheint das Modell von Hudson gut geeignet zu sein, eine Vorhersage des mittleren E-Moduls und der mittleren Poisson-Zahl eines Gesteins bei bekannter Rissdichte und -geometrie zu treffen. Zum zweiten drängt sich eine Analogie zwischen dem Rissquantifizierungsparameter ( $\mathrm{Vp}_{\mathrm{QRiss}}$ ) und dem im Hudson-Modell verwendeten Parameter $\mathrm{v}^{*} \mathrm{a}^{3}$ auf. Die beinahe deckungsgleichen Kurven aus Abb.6-30 suggerieren ein Verhältnis von $\mathrm{Vp}_{\mathrm{QRiss}}[\mathrm{km} / \mathrm{s}] / \mathrm{v}^{*} \mathrm{a}^{3}$ von ca. 10:1, welches wahrscheinlich nicht exakt ist, aber eventuell die Möglichkeit bietet, den $\mathrm{Vp}_{\mathrm{QRiss}}-\mathrm{Wert}$ in $[\mathrm{km} / \mathrm{s}] / 10$ stellvertretend als dimensionslosen Parameter für $\mathrm{v}^{*} \mathrm{a}^{3}$ in die Modellgleichungen einzusetzen.
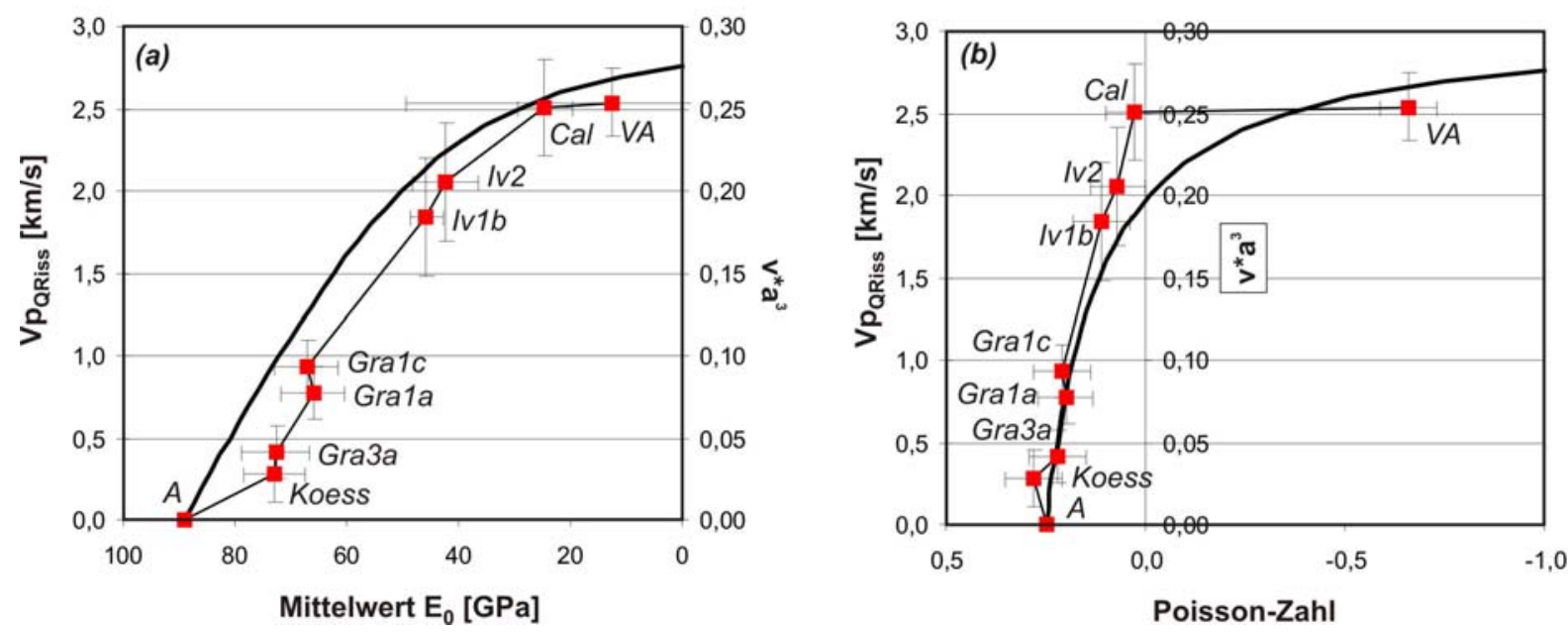

Abb.6-30: Abhängigkeit des E-Moduls (a) und der Poisson-Zahl (b) unter O MPa Umschließungsdruck von offenen Mikroriss-Systemen. Schwarze Kurven beschreiben die theoretische Entwicklung von EModul und Poisson-Zahl in Abhängigkeit vom Parameter $v^{*} a^{3}$ (mit $v$ - Rissdichte $\left[1 / \mathrm{cm}^{3}\right]$; a Risslänge/Rissbreite [mm]) folgend dem Modell von Hudson (1981). Die Probendaten bestehen aus Wertepaaren $E_{0} /$ Poisson-Zahl zu V V QRiss. $_{\text {A }}$-mittlerer E-Modul/mittlere Poisson-Zahl der acht Proben unter einem Umschließungsdruck von $400 \mathrm{MPa}$ (rissfreier Zustand) Vp $p_{Q R i s s}-$ Rissquantifizierungsparameter [km/s]. Koess - Granit. Gra3a - granitischer Ultramylonit. Gra1a/c granitischer Gneis. Iv1b - granodioritischer Mylonit. Iv2 - granodioritischer Ultramylonit. Cal granodioritischer Gneis. VA-granitischer Gneis. Fehlerbalken markieren den 1- $\sigma$-Fehler. 


\section{Technische Gesteinseigenschaften}

Damit könnte man die aufwendige Bestimmung der Rissdichten und der Rissachsenverhältnisse umgehen. Den theoretischen Hintergrund liefert G.6-10, die den Einfluss von $\mathrm{v}^{*} \mathrm{a}^{3}$ auf die Vp beschreibt. Damit gilt:

$$
V p^{2}=V p_{0}{ }^{2}\left[1-v a^{3}\left\{\frac{4\left(3 V p_{0}{ }^{2}-4 V s_{0}{ }^{2}\right)^{2}}{27 V s_{0}{ }^{2}\left(V p_{0}{ }^{2}-V s_{0}{ }^{2}\right)}+\frac{14\left(9 V p_{0}{ }^{2}-8 V s_{0}{ }^{2}\right) V s_{0}{ }^{2}}{15\left(3 V p_{0}{ }^{2}+2 V{s_{0}}^{2}\right)\left(V p_{0}{ }^{2}-V s_{0}{ }^{2}\right)}\right\}\right]
$$

$V p$ - resultierende Vp $[\mathrm{km} / \mathrm{s}] ; V p_{0}-V p$ im rissfreien Gestein $[\mathrm{km} / \mathrm{s}] ; V s_{0}-V s$ im rissfreien Gestein [km/s]; v - Risskonzentration/Rissdichte [1/ $\left.\mathrm{cm}^{3}\right]$; a - Rissradius [cm]; es gilt $v^{*} a^{3}<<1$.

Vereinfacht ergibt sich daraus:

$$
V p^{2}=V p_{0}^{2}\left[1-v a^{3}\{D\}\right]
$$

$V p$ - resultierende $V p[\mathrm{~km} / \mathrm{s}] ; V p_{0}-V p$ im rissfreien Gestein $[\mathrm{km} / \mathrm{s}] ; v$ - Risskonzentration/Rissdichte $\left[1 / \mathrm{cm}^{3}\right]$; a - Rissradius [cm]; es gilt $v^{*} a^{3}<<1$. D - Konstante bei gleichbleibender $V p_{0}$ und $V s_{0}$.

$\mathrm{Vp}_{0}$ ist die Kompressionswellengeschwindigkeit im rissfreien Zustand und kann daher mit dem $\mathrm{Vp}_{\mathrm{GVRH}}$-Wert gleichgesetzt werden, der die theoretische Vp eines Gesteins aufgrund des Mineralbestands und der Einkristalldaten und damit ebenfalls den rissfreien Zustand beschreibt. Daraus ergibt sich mit

$$
\begin{array}{llr}
\mathrm{Vp}_{0}=\mathrm{Vp}_{\mathrm{GVRH}} & \text { und } \\
\mathrm{Vp}_{\mathrm{QRiss}}=\mathrm{Vp}_{\mathrm{GVRH}}-\mathrm{Vp}_{\text {trockm }} & (\text { vgl. } \text { Kap. 2.4.5.1) } & \text { und } \\
\mathrm{Vp}_{\text {trockm }}=\mathrm{Vp} & &
\end{array}
$$

$V p_{0}-V p$ im rissfreien Gestein nach Hudson (1981)[km/s]. V $p_{G V R H}$ - theoretische Vp im Gestein, basierend auf der mineralogischen Komposition und den Einkristalldaten [km/s]. $V p_{Q R i s s}-$ Rissquantifizierungsparameter [km/s]. V $p_{\text {trockm }}-$ Mittelwert der trockenen Kugelmessung $[\mathrm{km} / \mathrm{s}]$. Vp resultierende Vp bei bekannter Rissdichte und-geometrie nach Hudson (1981) [km/s].

der Zusammenhang:

$$
V p_{Q R i s s}=V p_{G V R H}-V p_{G V R H} \sqrt{1-v a^{3} * D}
$$

$V p_{V R H}$ - theoretische $V p$ im Gestein, basierend auf der mineralogischen Komposition und den Einkristalldaten [km/s]. $V p_{Q R i s s}$ - Rissquantifizierungsparameter [km/s]. v-Risskonzentration/ Rissdichte [1/ $\left.\mathrm{cm}^{3}\right]$; a - Rissradius [cm]; es gilt $v^{*} a^{3}<<1$.

Dieser nichtlineare Zusammenhang zwischen $V p_{\mathrm{QRiss}}$ und $\mathrm{v}^{*} \mathrm{a}^{3}$ ist für $\mathrm{Vp}_{\mathrm{GVRH}}=6,27 \mathrm{~km} / \mathrm{s}$ und $\mathrm{Vs}=3,63 \mathrm{~km} / \mathrm{s}$ (Vs geht in D ein - G.6-11/G.6-12) in Abb.6-31 dargestellt. 


\section{Technische Gesteinseigenschaften}

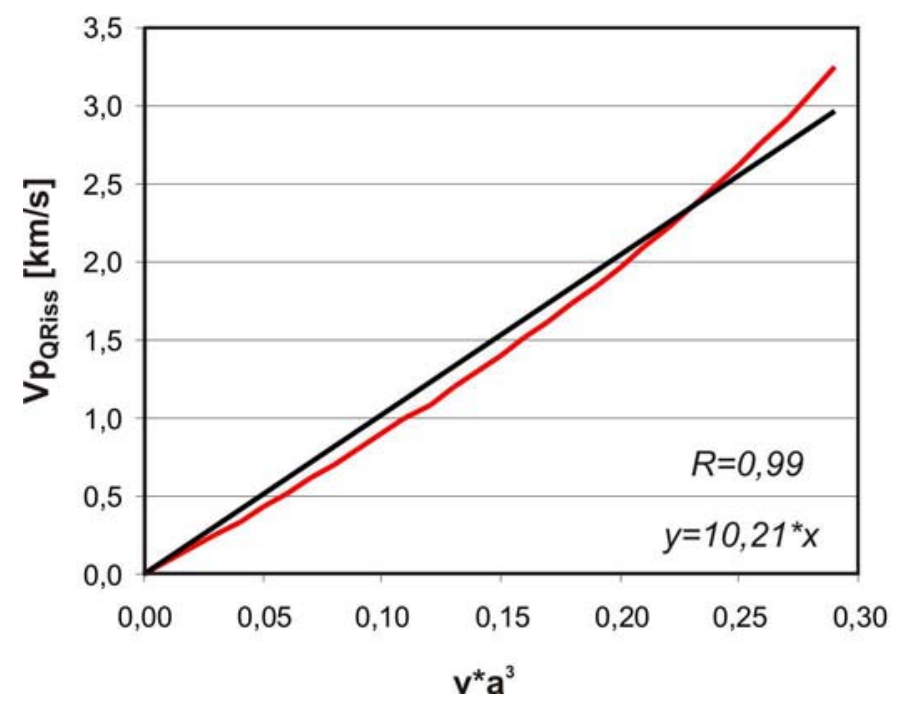

Abb.6-31: Theoretischer Zusammenhang zwischen dem Rissquantifizierungsparameter $V p_{Q R i s s}[\mathrm{~km} / \mathrm{s}]$ und dem Parameter $v^{*} a^{3}$ zur Quantifizierung der Rissdichte; mit der Rissdichte (v) $\left[1 / \mathrm{cm}^{3}\right]$ und der Risslänge (a) [mm]. Die rote Kurve beschreibt die theoretische Entwicklung von $V p_{Q R i s s}$ in Abhängigkeit von $v^{*} a^{3}$ nach dem Modell von Hudson (1981). Die schwarze Gerade ist die Regressionsgerade mit Schnittpunkt bei 0 für die theoretische Entwicklung; Geradengleichung und Korrelationskoeffizient sind angegeben.

Es zeigt sich, dass die theoretische Ableitung eine Näherung durch eine lineare Regression zulässt und damit im verwirklichten Rissdichte-Bereich ein Ratio $V p_{\mathrm{QRiss}}[\mathrm{km} / \mathrm{s}] / \mathrm{v}^{*} \mathrm{a}^{3}$ von 10:1 als mittleres Verhältnis angenommen werden kann. Daher bietet der Rissquantifizierungsparameter $\mathrm{Vp}_{\mathrm{QRiss}}$ sowohl eine gute Variante zur Abschätzung der Rissdichten bei bekanntem a (Rissachsenlänge) als auch die Möglichkeit einer Vorhersage der zu erwartenden mittleren rissbedingten E-Modul- und Poisson-Zahl-Reduktion.

\subsubsection{Statische elastische Eigenschaften}

Der statische E-Modul wurde in mindestens drei Richtungen (x-, y-, z-Richtung) aus den Spannungs-Dehnungs-Daten des einaxialen Druckversuchs nach der Sekanten- und der Tangenten-Methode bestimmt. Im Weiteren werden die Werte $\mathrm{E}_{\text {stat10, }} \mathrm{E}_{\text {stat50, (statischer E- }}$ Modul bei 10\% bzw. 50\% Bruchlast) und $\mathrm{E}_{\text {stats }}$ (Bruchmodul) und ihre Abgängigkeit von der mineralogischen Zusammensetzung und dem Gesteinsgefüge untersucht. Die Bezeichnung statischer E-Modul wird vereinfachend für den nach der statischen Methode bestimmten EModul verwendet, obwohl der Ausdruck aufgrund der immer auftretenden plastischen Verformungsanteile nicht korrekt ist. 


\subsubsection{Statischer E-Modul}

Der mittlere (arithmetisches Mittel aus x-, y- und z-Richtung) statische E-Modul bei 50\% der Bruchlast $\left(\mathrm{E}_{\text {stat50) }}\right.$ ) liegt zwischen 25,4 GPa (Kalzit-Marmor Rek) und 57,5 GPa (Peridotit Pila). Vergleichswerte liefert Prikryl (1998), der Granite untersuchte und für diese statische E-Moduln zwischen $23 \mathrm{GPa}$ und $72 \mathrm{GPa}$ fand. Winkler (1994) gibt für Granite einen Bereich von 20-60 GPa und für Gabbros von 70-110 GPa an. Für Sandsteine existieren Daten von Weiss (1992) die zwischen 1-24 GPa liegen.

Der mittlere E-Modul der Gruppe I beträgt 41 GPa, der Gruppe II 42 GPa und der Gruppe III $37 \mathrm{GPa}$. Die Anisotropie steigt mit zunehmender makroskopischer Gefügeanisotropie von $6,5 \%$ (Gruppe I) auf 20,2\% (Gruppe III).

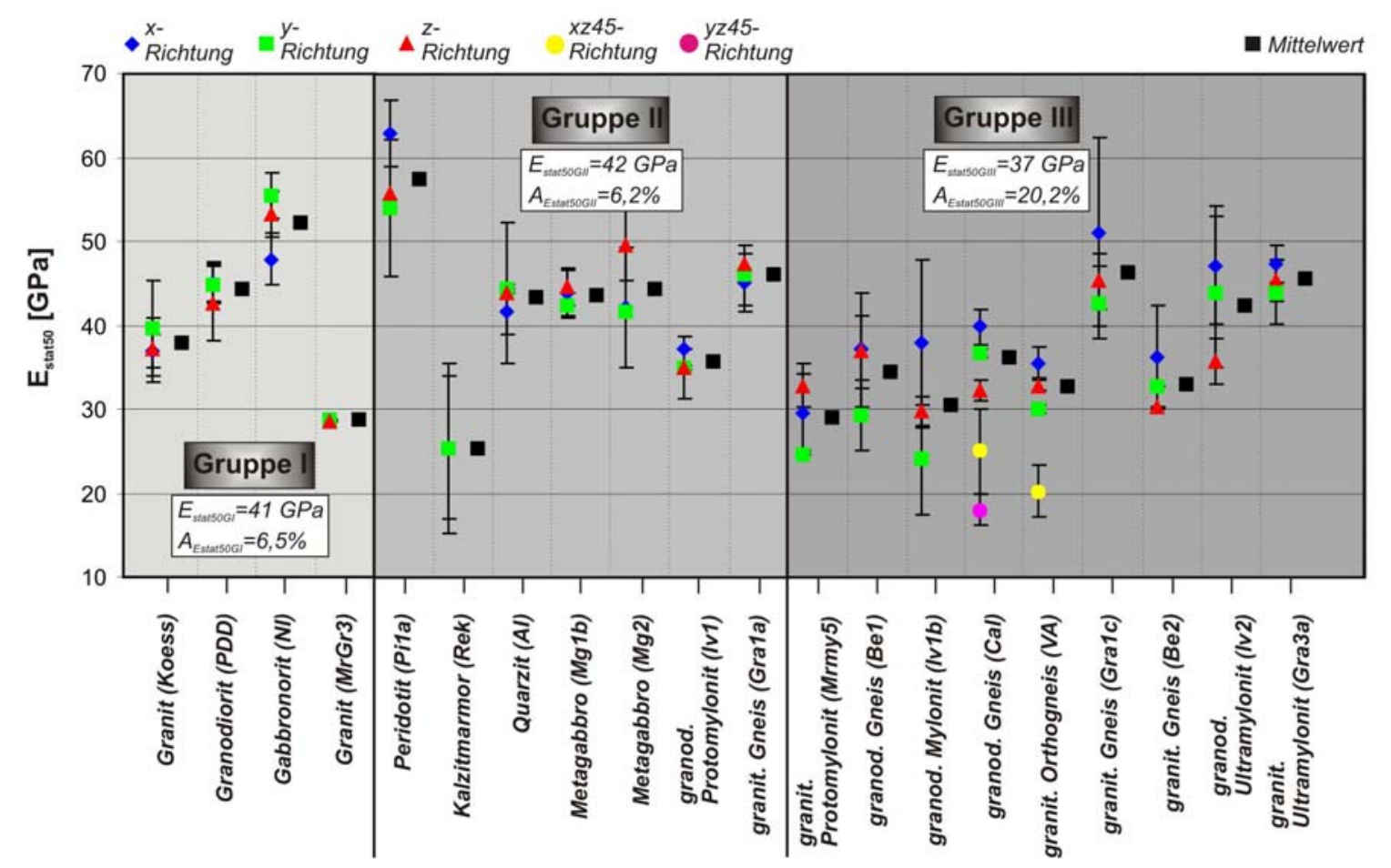

Abb.6-32: E-Modul (statisch) bei 50\% der Bruchlast ( $E_{\text {stat50) }}$ [GPa] der Makrogefüge-Gruppen I/IIIII. Mittelwerte des E-Moduls und mittlere Anisotropie für die jeweilige Gruppe sind angegeben. Fehlerbalken zeigen den 1- $\sigma$-Fehler.

Analog zur Vorgehensweise bei den Druckfestigkeiten wurden auch bei der Ermittlung des EModuls die $45^{\circ}$-Richtungen der Proben Cal (granodioritischer Gneis) und VA (granitischer Gneis) in die Untersuchungen einbezogen. Die $45^{\circ}$-Richtungen weisen mit 25,0 GPa/18,3 GPa (Cal) und 20,2 GPa (VA) jeweils die niedrigsten E-Moduln innerhalb des Gesteins auf (Abb.6-32). Die Mittelwerte der Proben Cal und VA sinken bei Berücksichtigung dieser Richtungen von 36,2 GPa und 32,8 GPa auf 28,9 GPa bzw. 26,5 GPa. Dahingegen steigt die Anisotropie von 19,1\% (Cal) und 15,5\% (VA) auf 54,0\% bzw. 43,1\% an. Es kann daher geschlossen werden, dass bei der standardmäßigen Untersuchung der drei Hauptgefüge- 
Richtungen die Minima der statischen E-Moduln nicht erfasst werden. Somit ist gerade bei den anisotropen und ausgeprägt anisotropen Gesteinen der Gruppen II/III eine deutlich höhere Anisotropie zu erwarten. Die niedrig anisotropen Gesteine sind von diesem Effekt nur gering oder gar nicht betroffen, weil im isotropen Körper keine Richtungsabhängigkeit der physikalisch-technischen Eigenschaften besteht.

Der statische E-Modul nach der Sekanten-Methode ( $\mathrm{E}_{\text {stats }}$ ), auch Bruchmodul genannt, bewegt sich zwischen 15,9 GPa (Granit Mrgr3) und 39,7 GPa (granitischer Ultramylonit Gra3a). Die Mittelwerte der Makrogefüge-Gruppen betragen $27 \mathrm{GPa}, 30 \mathrm{GPa}$ und $26 \mathrm{GPa}$ für die Gruppen I/II/III. Die Anisotropie liegt bei 21,2\% für die Gruppe I, 12,2\% für die Gruppe II und 18,5\% für die Gruppe III, so dass keine Abhängigkeit von der makroskopischen Gefügeanisotropie nachgewiesen werden kann (Abb.6-33). Zum einen sind die Daten stark fehlerbehaftet, zum anderen zeigen die $45^{\circ}$-Richtungen der Proben Cal und VA (siehe oben) die niedrigsten $\mathrm{E}_{\text {stats }}{ }^{-}$

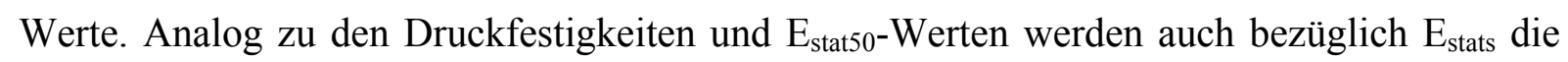
Minima nicht erfasst.

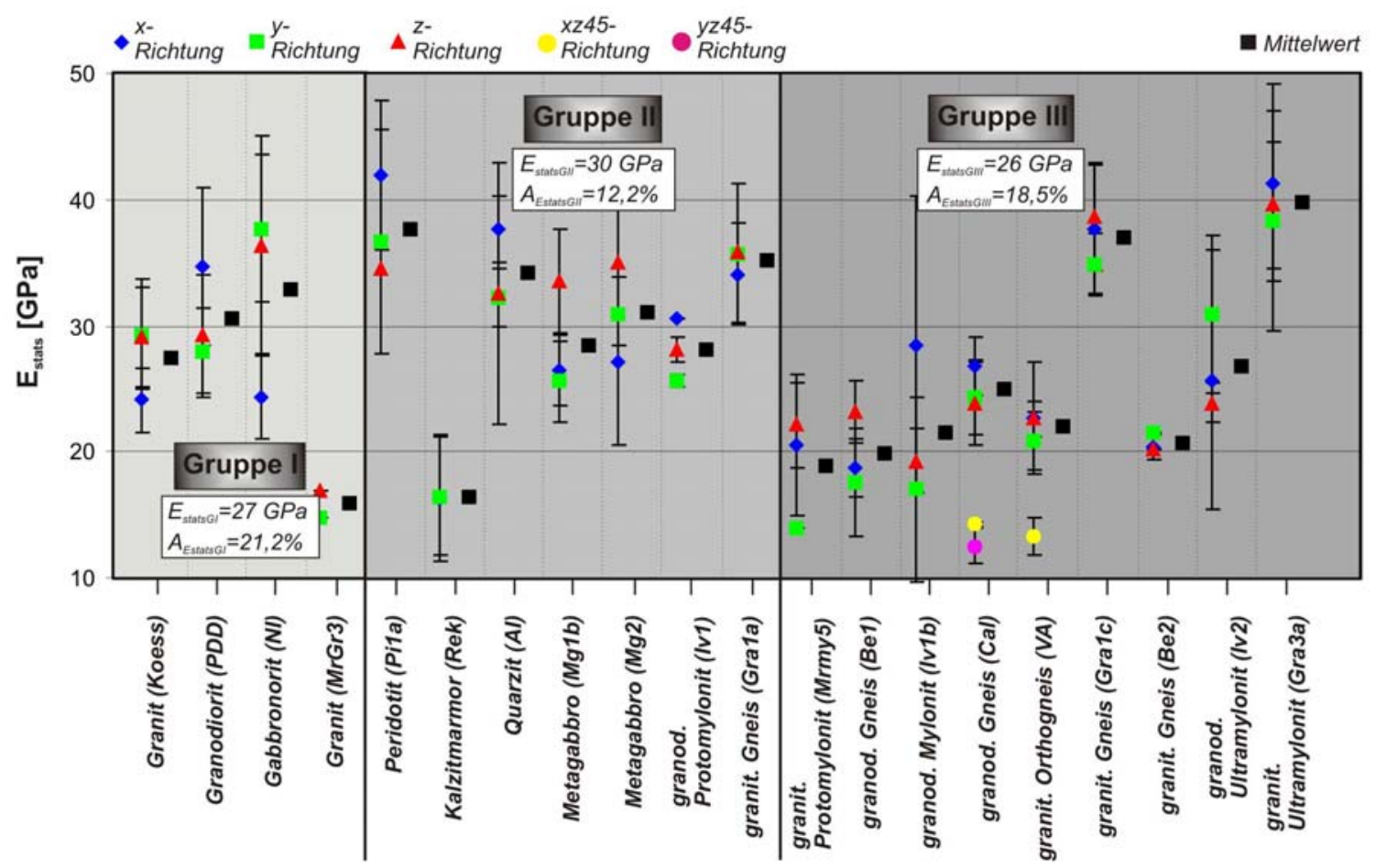

Abb.6-33: E-Modul ( $\left.E_{\text {stats }}\right)$ nach der Sekanten-Methode (Bruchmodul) ermittelt [GPa] der Makrogefüge-Gruppen I/II/III. Mittelwerte des E-Moduls und mittlere Anisotropie für die jeweilige Gruppe sind angegeben. Fehlerbalken zeigen den 1- $\sigma$-Fehler.

Bei den Proben der Gruppen II/III ist daher die tatsächliche Anisotropie des Bruchmoduls höher einzuschätzen, als bei der alleinigen Betrachtung der drei Hauptrichtungen. Folglich ist auch bezüglich der Bruchmodul-Anisotropie eine deutliche Abhängigkeit von der makroskopischen Gefügeanisotropie zu erwarten (siehe oben). 


\section{Technische Gesteinseigenschaften}

\subsubsection{Statischer E-Modul, Mineralbestand und offene Mikrorisse}

Alle untersuchten Proben zeigten beim einaxialen Druckversuch ein Spannungs-DehnungsVerhalten, welches sich wie folgt charakterisieren lässt. Zu Beginn des Versuchs kommt es zur Konsolidierung der Probe. In dieser Phase werden offene präexistierende Mikrorisse geschlossen. Ab etwa 30\% bis $70 \%$ der Bruchlast verhält sich das Gestein annähernd linear elastisch (wobei an dieser Stelle keine Aussage über eine mögliche Hysterese gemacht werden kann). Danach setzt vermehrt Rissbildung ein, die schließlich zum Gesteinsversagen führt (Abb.6-34).

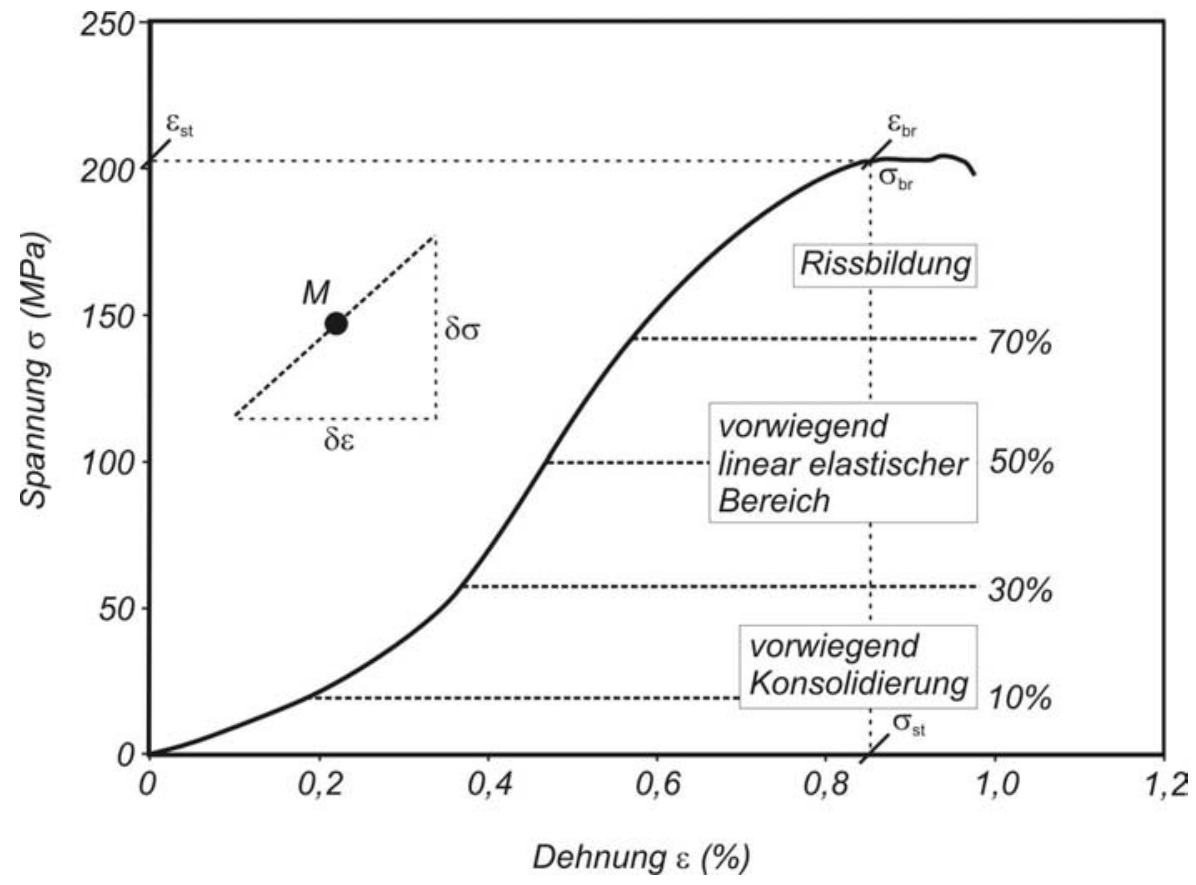

Abb.6-34: Generelles Spannungs-Dehnungs-Verhalten der Proben beim einaxialen Druckversuch. \%Zahlen zeigen die Spannung bei 10\%.....70\% der Bruchlast. $\varepsilon_{s t}$ - Dehnung zu Beginn des Druckversuchs. $\varepsilon_{b r}-$ Bruchdehnung. $\sigma_{s t}$ - Spannung zu Beginn des Druckversuchs [MPa]. $\sigma_{b r}-$ Bruchspannung [MPa].

Grundsätzlich treten alle drei Prozesse, die Konsolidierung, das linear elastische Verhalten und die Rissbildung parallel, jedoch mit unterschiedlicher Gewichtung auf (Abb.6-35). Innerhalb des Probensatzes variiert die Steigung der Spannungs-Dehnungs-Kurve in den verschiedenen Segmenten, ohne dass das grundsätzliche Schema verlassen wird. So hängt der Grad der Konsolidierung stark von der Rissdichte der offenen Mikrorisse ab, wohingegen das linear elastische Verhalten hauptsächlich durch den Mineralbestand und dessen elastischen Eigenschaften bestimmt wird. Wann und wo sich in einer Probe neue Risse bilden, ist wiederum durch die präexistierenden Mikrorissen und deren Geometrie und Dichte, aber auch durch die mechanische Stabilität der Minerale und die gefügebedingten Interaktionen zwischen mechanisch schwachen und starken Mineralen bedingt (Segall \& Pollard 1980). 


\section{Technische Gesteinseigenschaften}

\section{Konsolidierung}

(Mikrorisse werden geschlossen)

linear elastische Dehnung der Probe

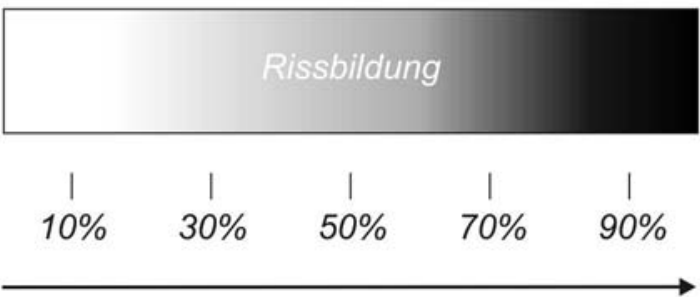

Beginn des

Druckversuchs

Bruch der

Probe

Abb.6-35: Prozesse während des einaxialen Druckversuchs. Schwarz - Prozess dominant. Weiß Prozess nur von untergeordneter Bedeutung. \%-Zahlen zeigen Anteil der Bruchlast.

Geht man davon aus, dass der E-Modul bei $50 \%$ der Bruchlast $\left(\mathrm{E}_{\mathrm{stat} 50}\right)$ vorwiegend durch das elastische Verhalten des Mineralbestands geprägt ist und der E-Modul bei 10\% der Bruchlast ( $\left.\mathrm{E}_{\text {stat10 }}\right)$ durch den Mechanismus der Konsolidierung dominiert wird, so liefert der Quotient $\mathrm{E}_{\text {stat50 }} / \mathrm{E}_{\text {stat10 }}$ eine Möglichkeit, den Risseinfluss auf das Spannungs-Dehnungs-Verhalten zu charakterisieren.

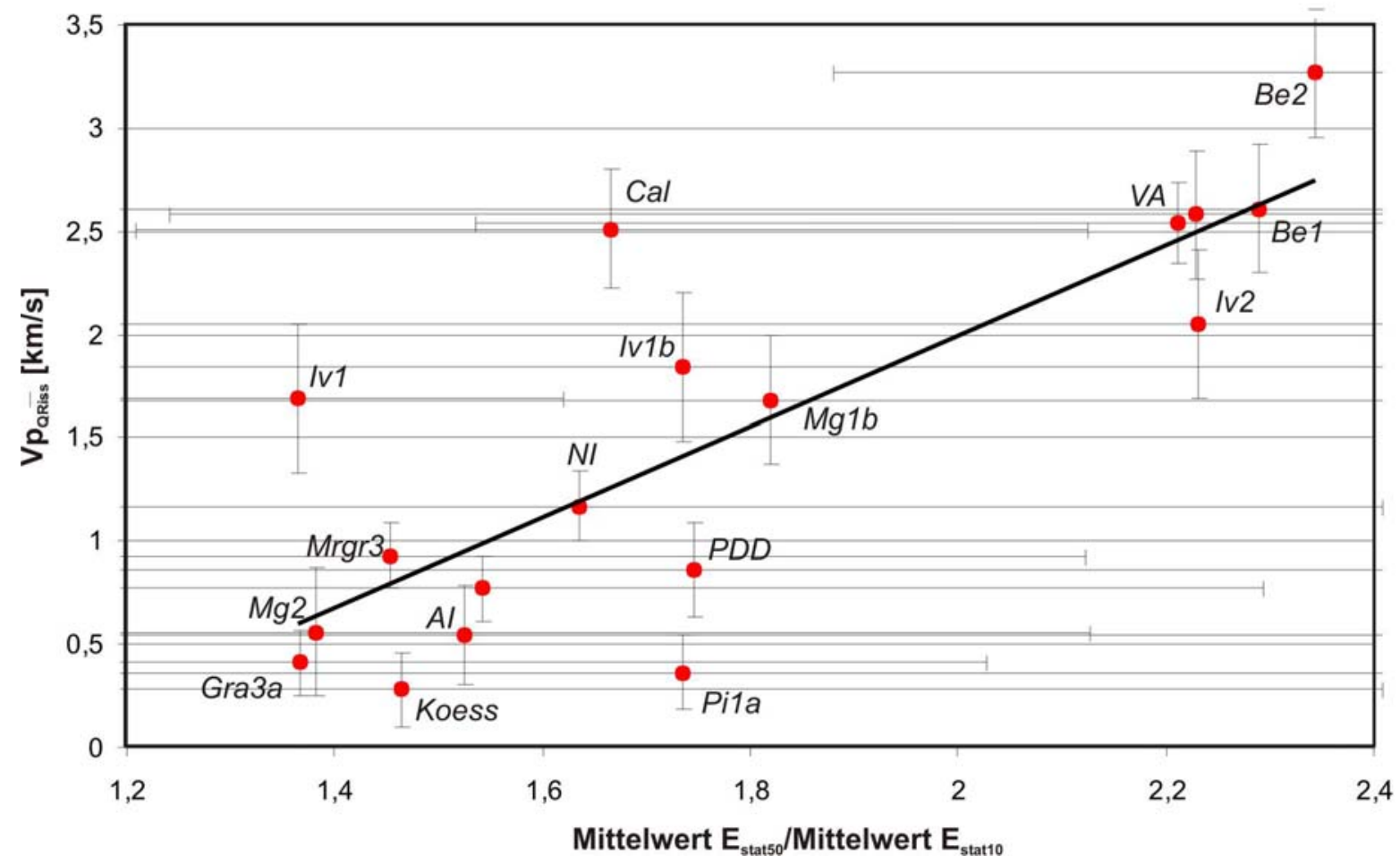

Abb.6-36: Rissquantifizierungsparameter $V p_{\text {QRiss }}[\mathrm{km} / \mathrm{s}]$ und Mittelwert $E_{\text {stat5 } 0} /$ Mitte/wert $E_{\text {stat10. }} E_{\text {stat50 }}-$

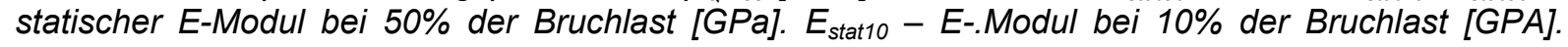
Weitere Details im Text. Fehlerbalken zeigen den 1- $\sigma$-Fehler. 


\section{Technische Gesteinseigenschaften}

Relativ höhere $\mathrm{E}_{\mathrm{stat} 50} / \mathrm{E}_{\mathrm{stat} 10}$-Werte bedeuten bei Proben ähnlicher elastischer Eigenschaften des Mineralbestands eine hohe Rissdichte und korrelieren gut mit hohen $\mathrm{Vp}_{\mathrm{QRiss}}$-Werten (Abb.6-36).

Des Weiteren wird der Einfluss des Mineralbestands näher beleuchtet. Betrachtet man den mittleren statischen E-Modul bei 50\% der Bruchlast, also den vorwiegend linear elastischen Bereich, und vergleicht die Daten mit den theoretisch zu erwartenden E-Moduln $\left(\mathrm{E}_{\mathrm{GVRH}}\right)$, so fällt folgendes auf (Abb.6-37):

1. Die $\mathrm{E}_{\mathrm{sta} 50}-\mathrm{Werte}$ sind alle deutlich niedriger als die $\mathrm{E}_{\mathrm{GVRH}}-\mathrm{Werte}$.

2. Die Korrelation zwischen $E_{\text {stat50 }}$ und $E_{G V R H}$ ist schlecht, auch wenn eine Tendenz von höheren $\mathrm{E}_{\mathrm{GVRH}}$ und damit höheren durchschnittlichen E-Moduln des Mineralbestands $\mathrm{zu}$ höheren $\mathrm{E}_{\mathrm{stat} 50}$-Werten sichtbar ist.

3. Die Proben zwischen dem Kalzit-Marmor (Rek) und dem granitischen Ultramylonit (Gra3a) mit ähnlichen elastischen Eigenschaften des Mineralbestands (annähernd gleiche $\left.\mathrm{E}_{\mathrm{GVRH}}\right)$ weisen eine große Streuung der mittleren statischen E-Moduln auf.
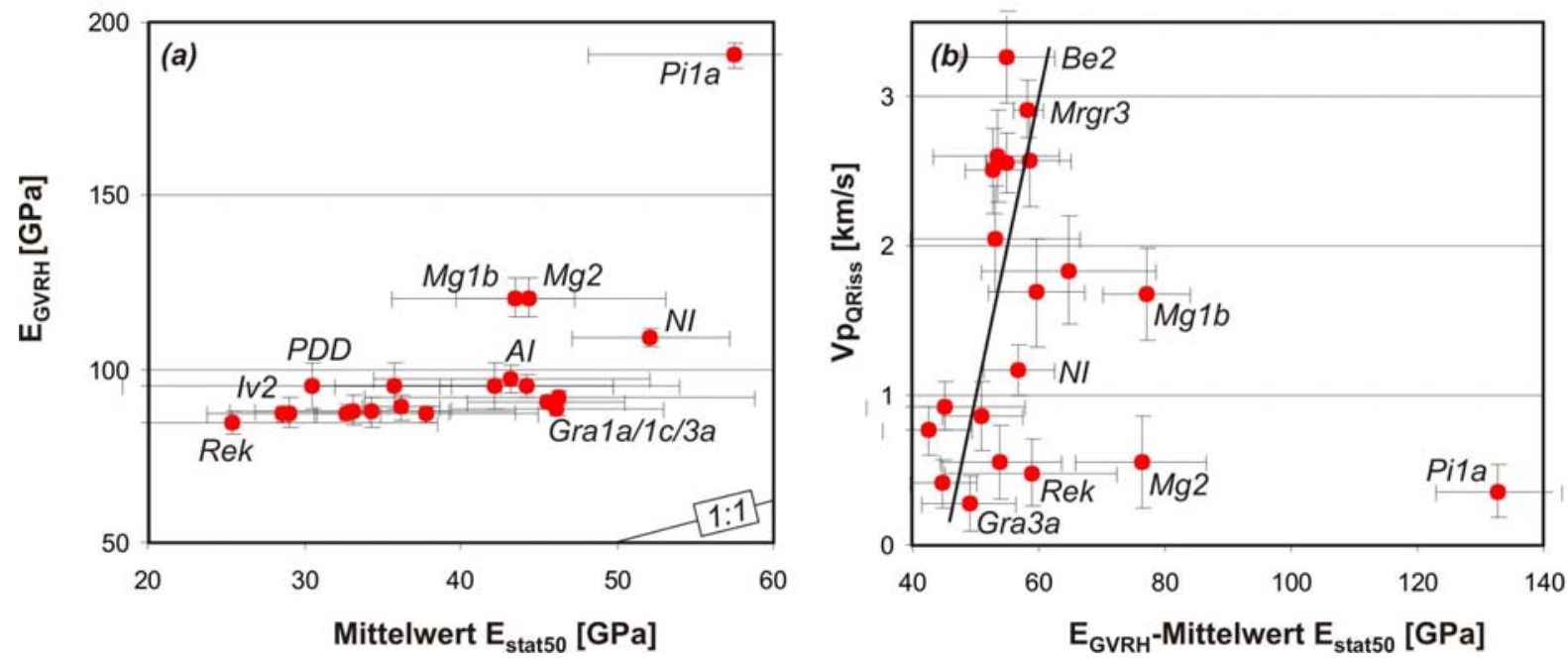

Abb.6-37: (a) Abhängigkeit der Mittelwerte des statischen E-Moduls bei 50\% Bruchlast $E_{\text {stat50 }}$ [GPa] von den mittleren elastischen Eigenschaften des Mineralbestands $E_{G V R H}$ [GPa]. Rissquantifizierungsparameter $V P_{\text {Qriss }} v$ v. $E_{G V R H}-E_{\text {stat50 }}[\mathrm{GPa}$ ]. Fehlerbalken zeigen 1- $\sigma$-Fehler.

Die beobachteten Phänomene zeigen, dass der statische E-Modul $\left(\mathrm{E}_{\text {stat50 }}\right)$ durch eine komplexe Kombination unterschiedlicher Parameter beeinflusst wird. Proben mit einer hohen Rissdichte und ähnlichen elastischen Eigenschaften des Mineralbestands weisen höhere Differenzen $\mathrm{E}_{\mathrm{GVRH}}-\mathrm{E}_{\mathrm{stat} 50}$ auf, liegen also weiter von den intrinsischen dynamischen elastischen Eigenschaften entfernt. $\boldsymbol{A b b . 6 - 3 7}$ zeigt in Ansätzen einen Zusammenhang von hohen $\mathrm{E}_{\mathrm{GVRH}}-\mathrm{E}_{\text {stat50 }}$ mit hohen Rissdichten $\left(\mathrm{Vp}_{\mathrm{QRiss}}\right)$; der Trend für eine Gruppe mit ähnlichen theoretischen elastischen Eigenschaften $\left(\mathrm{E}_{\mathrm{GVRH}}<100 \mathrm{GPa}\right)$ ist angedeutet. Das bedeutet auch, 


\section{Technische Gesteinseigenschaften}

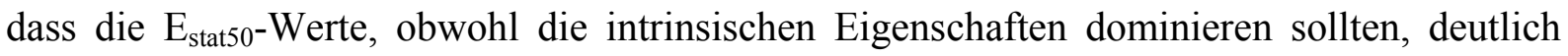
durch eine hohe Rissdichte beeinflusst, nämlich erniedrigt werden. Insgesamt scheint der statische E-Modul wesentlich weniger von den elastischen Eigenschaften des Mineralbestands bestimmt zu werden als das beim dynamischen E-Modul der Fall ist. Einen Hinweis darauf gibt der Peridotit (Pila). Mit einer geringen Rissdichte $\left(\mathrm{V}_{\mathrm{QRiss}}=0,36 \mathrm{~km} / \mathrm{s}\right)$ und einem sehr hohen $\mathrm{E}_{\mathrm{GVRH}}-$ Wert $(190,4 \mathrm{GPa})$ liegt die Probe mit ihrem $\mathrm{E}_{\text {stat50-Wert }}$ (57,5 GPa) am weitesten vom $\mathrm{E}_{\mathrm{GVRH}}-$ Wert entfernt $\left(\mathrm{E}_{\mathrm{GVRH}}-\mathrm{E}_{\mathrm{stat} 50}=132,9 \mathrm{GPa}\right)$. Das bedeutet, dass der Einfluss der dynamischen elastischen Eigenschaften des Mineralbestands hier deutlich geringer ausfällt als beim dynamischen E-Modul, der mit 171,4 GPa relativ nahe an den $\mathrm{E}_{\mathrm{GVRH}}-$ Werten liegt. In Analogie zu den Druckfestigkeiten scheinen auch beim statischen EModul die mechanischen Eigenschaften der Minerale und die Korngröße von Bedeutung zu sein, die nicht in jedem Falle mit den elastischen Eigenschaften korrelieren (Kap.4.5). Zusätzlich ist der Einfluss der offenen Mikrorisse unbestreitbar eine wichtige Einflussgröße.

\subsubsection{Vergleich von $E_{\text {stat5o }}$ mit dem dynamischen E-Modul $E_{0}$}

Wie am Ende des letzten Abschnitts erwähnt, wird der statische E-Modul $\left(\mathrm{E}_{\text {stat } 50}\right)$ durch eine komplexe Wechselwirkung der elastischen und mechanischen Eigenschaften des Mineralbestands, der offenen Mikrorisse und der Korngröße bestimmt. Der dynamische EModul unter Normaldruck $\left(\mathrm{E}_{0}\right)$ hingegen ist fast ausschließlich von den offenen MikrorissSystemen und den elastischen Eigenschaften des Mineralbestands dominiert. Auch wenn sich zwei der Einflussgrößen, nämlich die Rissdichte und die intrinsischen elastischen Eigenschaften, gleichen, so ist deren Wirkungsintensität auf den dynamischen und den statischen E-Modul deutlich unterschiedlich und führt zu unkonstanten Verhältnissen $\mathrm{E}_{0} / \mathrm{E}_{\text {stat50 }}$ (Abb.6-38). Es zeigt sich, dass eine gute lineare Korrelation zwischen $\mathrm{E}_{0}$ und $\mathrm{E}_{\text {stat50 }}$ besteht, wobei die Verhältnisse $\mathrm{E}_{0} / \mathrm{E}_{\text {stat50 }}$ zwischen 0,39 (granitischer Gneis - VA) und 3,0 (Peridotit Pila) schwanken. Bei Betrachtung der Gruppe von Proben ähnlicher intrinsischer elastischer Eigenschaften (Abb.6-38 - grau hinterlegt) wird deutlich, dass die offenen Mikrorisse einen größeren Einfluss auf den dynamischen und einen im Verhältnis geringeren Einfluss auf den statischen E-Modul haben. Proben mit einer hohen Rissdichte, wie z.B (VA) mit $\mathrm{Vp}_{\mathrm{QRiss}}=2,56$ $\mathrm{km} / \mathrm{s}$ oder der granitische Gneis $(\mathrm{Be} 2)$ mit $\mathrm{Vp}_{\mathrm{QRiss}}=3,27 \mathrm{~km} / \mathrm{s}$ liegen unterhalb der 1:1-Linie, zeigen also höhere statische als dynamische E-Moduln. Andersherum verhält es sich bei Proben mit einer niedrigen Rissdichte (z.B. granitischer Ultramylonit - Gra3a mit $\mathrm{Vp}_{\mathrm{QRiss}}=0,41 \mathrm{~km} / \mathrm{s}$ ), die alle oberhalb der 1:1-Linie gruppieren und gegenüber den dynamischen, niedrigere statische E-Moduln aufweisen. Die statischen E-Moduln steigen 


\section{Technische Gesteinseigenschaften}

zwar mit abnehmender Rissdichte an, jedoch deutlich weniger stark im Vergleich zu den $\mathrm{E}_{0^{-}}$ Werten.

Beim einaxialen Druckversuch kommt es neben der elastischen immer zu einer plastischen Verformung des Gesteinsmaterials. Selbiges ist aufgrund wesentlich geringerer Zeiten der Spannungseinwirkung bei der dynamischen Methode nicht zu erwarten (Schön 1983). Folglich sollte der dynamische E-Modul immer höher ausfallen als der statische.

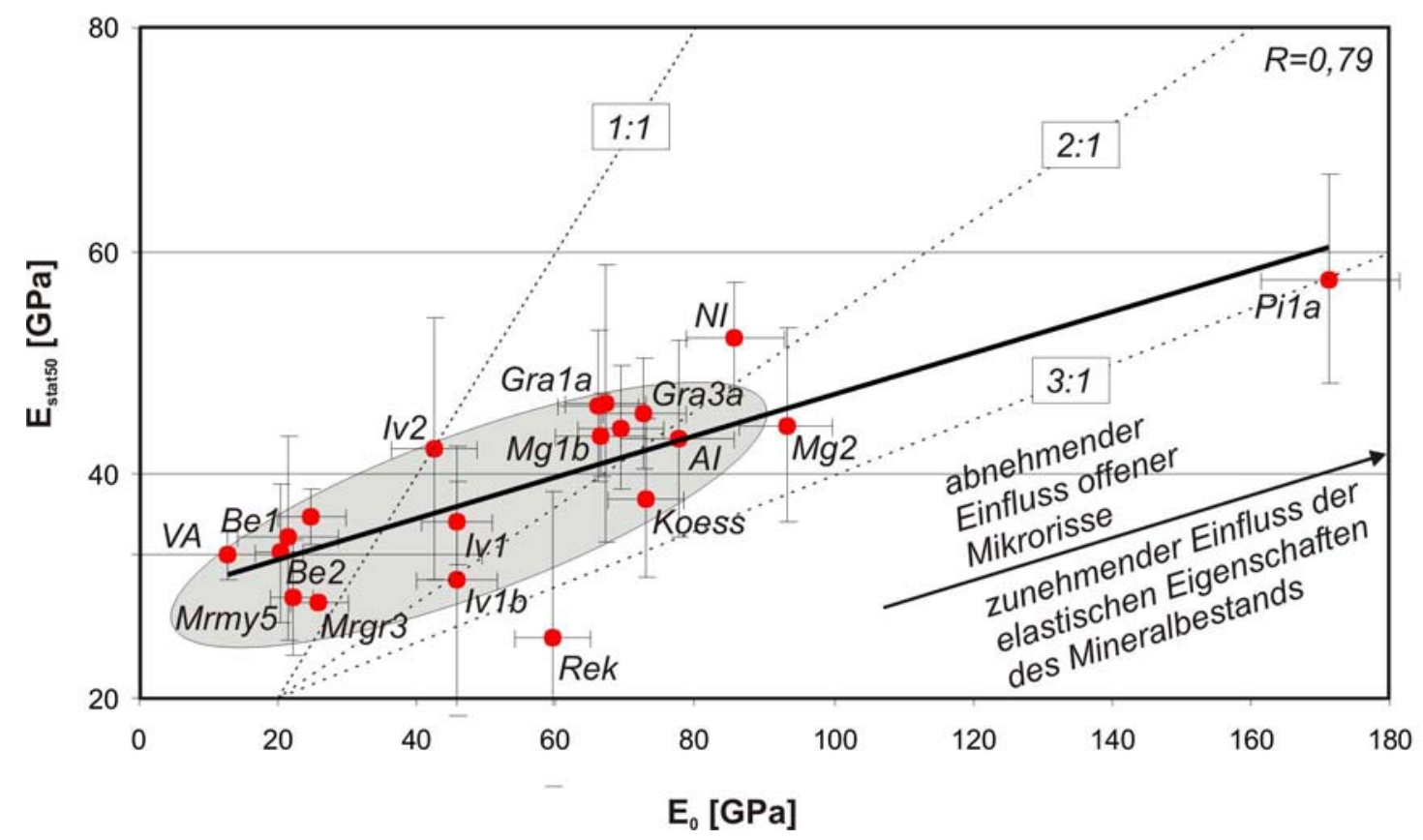

Abb.6-38: Verhältnis von dynamischem E-Modul unter $0 \mathrm{MPa}$ Umschließungsdruck ( $\left.E_{0}\right)$ [GPa] zu statischem E-Modul bei $50 \%$ Bruchlast $\left(E_{\text {stat5o }}\right)$ [GPa]. Werte sind arithmetische Mittel aus $x$-, $y$ - und zRichtung. Isolinien gleicher Verhältnisse von $E_{0} / E_{\text {stat5o }}$ sind gekennzeichnet. Gruppe ähnlicher intrinsischer elastischer Eigenschaften ( $E_{V R H}<100$ GPa mit Ausnahme des Kalzit-Marmors Rek) ist grau hinterlegt. Fehlerbalken markieren den 1- $\sigma$-Fehler.

Nach Aussagen von Schön (1983) soll dieser Effekt mit zunehmender Porosität noch größer werden $\left(\mathrm{E}_{0}>>\mathrm{E}_{\text {stat50 }}\right)$, was er auf den parallel zur Porosität zunehmenden Anteil der plastischen Verformung beim Druckversuch zurückführt. Ergebnisse von Weiss (1992) für Sandsteine bestätigen diesen Zusammenhang in Ansätzen. Einiger der im Rahmen dieser Arbeit untersuchten Gesteine zeigen abweichend zu diesen Darstellungen deutlich höhere statische als dynamische E-Moduln (z.B. Proben Be1, Va). Zudem weisen die Daten einen genau gegenläufigen Trend von Gesteinen höherer Rissdichte und damit auch höherer Porosität (die Rissgeometrien sind innerhalb des Probensatzes sehr ähnlich - vgl. Kap.6.1.2.2) zu niedrigeren $\mathrm{E}_{0} / \mathrm{E}_{\mathrm{stat} 50}$-Verhältnissen auf (Abb.6-38). Der Grund für diese unterschiedlichen Ergebnisse sind die verschiedenen Gesteine, die den Beobachtungen als Basis dienen. Sowohl die Ergebnisse von Weiss (1992) als auch die Aussagen von Schön (1983) fußen auf Untersuchungen an deutlich poröseren Gesteinen (z.B. 4,4-19,1 Vol.\% bei Sandsteinen von 
Weiss gegenüber $\leq 1$ Vol.\% für die Gesteine dieser Studie) mit wesentlich differierenden Porengeometrien (z.B. Maxima der Porenradienverteilung tendenziell zwischen 0,1-10 $\mu \mathrm{m}$ bei Sandsteinen von Weiss gegenüber 0,01-1 $\mu \mathrm{m}$ für die Gesteine dieser Studie). Diese Faktoren beinhalten völlig unterschiedliche Wirkungsweisen der Porenraumeigenschaften auf den statischen und den dynamischen E-Modul. Daher sind die Aussagen nicht auf die hier untersuchten dichten kristallinen Gesteine übertragbar.

Insgesamt scheint der dynamische E-Modul aufgrund des bedeutenden Einflusses der offenen Mikrorisse, welcher bei der Probe VA in z-Richtung sogar einen negativen dynamischen EModul bewirkt (vgl. Kap.6.4.1.5 - Model nach Hudson (1981)), nicht geeignet, das wirkliche Spannungs-Dehnungs-Verhalten eines Gesteins unter Normaldruck zu beschreiben. Allerdings macht eine Qualitätseinschätzung oder die Bewertung des Verwitterungsverhaltens von niedrigporösen Gesteinen mit dominanter Rissporosität aufgrund des besonders risssensitiven dynamischen E-Moduls durchaus Sinn und wurde z.B. von Ondrasina et al. (2002) bereits angewendet.

Um eine Idee über das tatsächliche Verformungsverhalten zu erlangen, sind Druckversuche unter entsprechenden Rahmenbedingungen aber unumgänglich.

\subsection{Thermische Dehnung}

Das thermische Dehnungsverhalten ist eine wichtige Eigenschaft zur Charakterisierung von Naturwerksteinen. Bautechnische Einsatzgebiete, wie z.B. der Hochbau (Fassadenplatte) und die Verwendung als Bodenbelag erfordern eine standardmäßige Einschätzung des Dehnungsverhaltens bei thermischer Beanspruchung (z.B. Peschel 1983). Das schließt sowohl die Volumenausdehnung des Gesteins als auch die Anisotropie der thermischen Dehnung und die Restdehnung (und damit thermische Rissbildung) mit ein. Anhand der thermischen Dehnung sind, gepaart mit anderen petrophysikalischen Eigenschaften, Rückschlüsse auf die Eignung eines Gesteins für eine bestimmte Verwendung möglich. Die Höhe der Volumenausdehnung liefert in Verbindung mit den zu erwartenden Temperaturschwankungen die Grundlage für die Berechnung einer minimalen Fugenbreite; die Anisotropie der thermischen Dehnung sowie die Restdehnung und deren richtungsabhängiges Verhalten erlauben Aussagen bezüglich der Anfälligkeit eines Gesteins gegenüber thermischer Verwitterung. Zudem sind einige Gesteine aufgrund ihres besonderen Dehnungsverhaltens für spezielle Zwecke geeignet. So werden z.B. gabbroische Gesteine mit einem besonders hohen Plagioklasanteil, einer schwachen Textur und einem resultierenden niedrigen 


\section{Technische Gesteinseigenschaften}

Volumenausdehnungskoeffizienten und einer niedrigen Anisotropie der thermischen Dehnung in Metallwerkstätten als Anreißplatten mit einer auf eine Ebenheit von 0,001 mm geschliffenen Oberfläche verwendet. Für Naturwerksteine liegen vorwiegend Untersuchungen für das Dehnungsverhalten von Sandsteinen (Weiss 1992) und Marmoren vor, die hauptsächlich auf das Verwitterungsverhalten abzielen. Es wird deutlich, das insbesondere Kalzit-Marmore ein stark anisotropes Dehnungsverhalten und eine starke und ebenfalls anisotrope Restdehnung, also eine bleibende Schädigung des Gesteins durch thermische Rissbildung aufweisen können. Erklärt wird dieses Phänomen mit der stark anisotropen thermischen Dehnung des Kalzit-Einkristall in Verbindung mit einer Texturierung des Mineralbestands (z.B. Weiss et al. 2002). In diesem Zusammenhang werden gesteinstypische Verwitterungsmerkmale von Marmoren, wie z.B. die Verbiegung von Fassadenplatten (Widhalm et al. 1996; Tschegg et al. 1999; Koch \& Siegesmund 2002) und teilweise extreme Gefügeauflockerung durch Mikrorissbildung (Siegesmund et al. 1999; Siegesmund et al. 2000; Rüdrich et al. 2001; Strohmeyer et al. 2001; Zeisig et al. 2002), aber auch die Konsequenzen für die Restauration und Konservierung bereits geschädigter Bauwerksteile und Kulturgüter (Rüdrich et. al. 2002) in Abhängigkeit vom Gefüge und Mineralbestand der Marmore diskutiert. Die thermisch induzierte Rissbildung findet bei Marmoren schon unter mitteleuropäischen Klimabedingungen statt und führt in der Regel zu einer deutlichen Verringerung der Gesteinsfestigkeit und damit $\mathrm{zu}$ einer Verschlechterung der Gebrauchseigenschaften (Ondrasina et al. 2002; Prinzipien in Ferrero \& Marini 2001; für Sandsteine Weiss 1992).

Studien von Ide (1937), Simmons \& Cooper (1978), Richter \& Simmons (1974), Bauer \& Johnson (1979) und Glover et al. (1995), die sich mit dem thermischen Dehnungsverhalten von Magmatiten (z.B. Granit, Norit) bzw. Quarziten (Lu \& Jackson 1998) befassen, zeigen, dass auch hier eine Bildung von thermischen Rissen zu erwarten ist. Allerdings zielen diese Untersuchungen größtenteils auf das Dehnungsverhalten der Gesteine unter den Druck- und Temperaturbedingungen der Erdkruste ab und umfassen daher Temperaturen bis $\mathrm{zu} 700^{\circ} \mathrm{C}$ und entsprechend hohe Drücke (bis zu 250 MPa bei Lu \& Jackson 1998). Aussagen auf das Dehnungsverhalten unter mitteleuropäischen Temperaturen und Normaldruck können aus diesem Grund kaum abgeleitet werden, zumal Anisotropieeffekte komplett unberücksichtigt bleiben. Letztere werden in Peschel (1983) kurz angeschnitten, jedoch weder genauer definiert noch quantifiziert. Der Einfluss von Quarz auf die thermische Dehnung wird von Peschel (1983) und Weiss (1992) diskutiert. Nach der G.6-14 von Correns (1968) ist der Quarz infolge seiner Volumenausdehnung und seiner Kompressibilität in der Lage, schon bei 


\section{Technische Gesteinseigenschaften}

niedrigen Temperaturdifferenzen $\left(40^{\circ} \mathrm{C}\right)$ einen Druck auf die umgebenden Minerale von 54,5 MPa aufzubauen, was zwar die Druckfestigkeiten der meisten Kristallingesteine nicht überschreiten würde, jedoch infolge von Spannungskonzentrationen und assoziierten Zugspannungen eine Bildung von thermisch induzierten Mikrorissen erwarten lässt.

$$
p=\frac{\alpha^{*} \Delta t}{\chi\left(1+\alpha^{*} \Delta t\right)} \quad\left[\mathrm{N} / \mathrm{mm}^{2}\right][\mathrm{MPa}]
$$

Gleichung nach Correns (1968). $p$ - Druck [MPa]. $\Delta t$ - Temperaturdifferenz. $\alpha-$ Volumenausdehnungskoeffizient von Quarz $\left(36,2^{*} 10^{-6} 1 / K\right) \cdot \chi-$ Kompressibilität von Quarz $\left(2,67^{*} 10^{-11}\right.$ $\left.m^{2} / N\right)$.

Im Rahmen der vorliegenden Arbeit wurden die Gesteine in einem Temperaturbereich von $20^{\circ} \mathrm{C}$ bis $90^{\circ} \mathrm{C}$ in den drei Richtungen parallel zu den Achsen des Probenreferenzsystems auf ihr thermisches Dehnungsverhalten untersucht und sowohl die richtungsabhängigen Ausdehnungskoeffizienten und sRestdehnungen als auch die Volumenausdehnungskoeffizienten bestimmt. Diese Parameter werden in ihrer Relation zu Gefügeparametern und der mineralogischen Komposition der Gesteine diskutiert und mögliche Konsequenzen für das thermische Verwitterungsverhalten aufgezeigt.

\subsubsection{Thermischer Ausdehnungskoeffizient}

Die Mittelwerte des thermischen Ausdehnungskoeffizienten (arithmetisches Mittel aus X-, y-, z-Richtung) liegen zwischen $\alpha_{20-90}=5,02 * 10^{-6} 1 / \mathrm{K}$ (Gabbro-Norit $\left.-\mathrm{NI}\right)$ und $\alpha_{20-90}=11,3 * 10^{-6}$ 1/K (Quarzit - AI) und damit in der Größenordnung der in Peschel (1983) für verschiedene Gesteinsgruppen angegebenen Bereiche (z.B. $\alpha=9,0-13,4 * 10^{-6} 1 / \mathrm{K}$ für Quarzit). Der in diesem Kapitel zusätzlich untersuchte quarzitische Ultramylonit (QM) hat einen mittleren thermischen Ausdehnungskoeffizienten von $\alpha_{20-90}=12,5 * 10^{-6} 1 / \mathrm{K}$ und damit den höchsten aller betrachteten Gesteine. Entgegen anderer technischer Eigenschaften zeigt das thermische Dehnungsverhalten keine signifikante Erhöhung der Anisotropie in Korrelation mit der Anisotropie des makroskopischen Gefüges. Mit einer mittleren Anisotropie von 18\% weist die Gruppe II den höchsten Wert auf; die Gruppe I und die Gruppe III haben eine niedrigere mittlere Anisotropie von 14\% bzw. 12\%. Die vermeintlich höhere Richtungsabhängigkeit der thermischen Dehnung in der Gruppe II ist jedoch nur auf den Einfluss einer einzigen Probe, nämlich den des Kalzit-Marmors (Rek), zurückzuführen, der mit einer Anisotropie von 74,2\% weit über dem Durchschnitt liegt. 


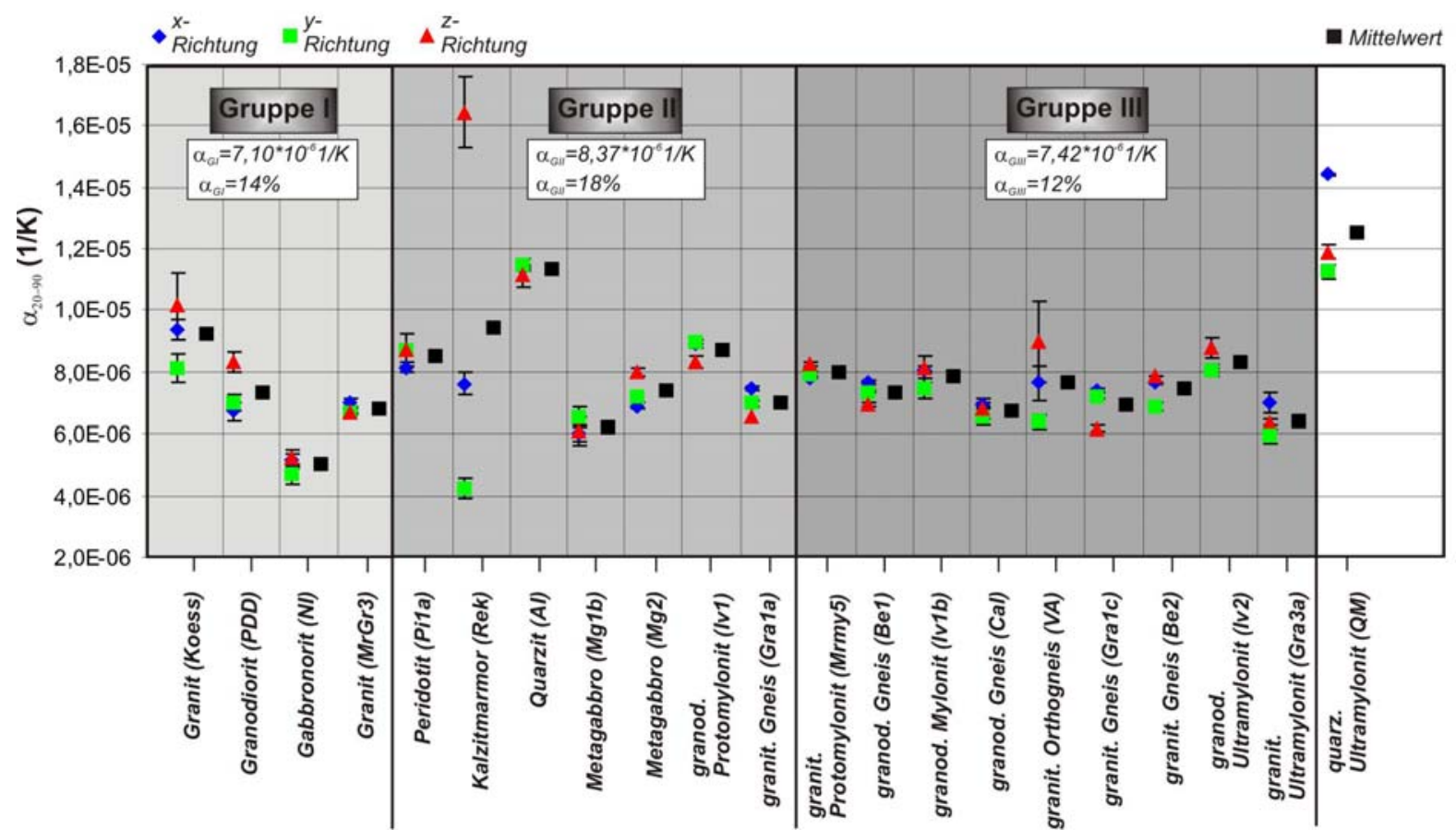

Abb.6-39: Thermische Ausdehnungskoeffizienten ( $\left.\alpha_{20-90}\right)$ der Makrogefüge-Gruppen I/II/III im Temperaturbereich von $20^{\circ} \mathrm{C}$ bis $90^{\circ} \mathrm{C}$. Mittelwerte der Ausdehnungskoeffizienten und mittlere Anisotropie für die jeweilige Gruppe sind angegeben.

Damit ist der Mittelwert der Anisotropie hier genausowenig aussagekräftig wie der in der Gruppe II gegenüber den beiden anderen Gruppen leicht erhöhte mittlere Ausdehnungskoeffizient, der durch die höheren Ausdehnungskoeffizienten des Quarzit (AI) und des Kalzit-Marmors (Rek) bedingt ist (Abb.6-39).

Von den analysierten Gesteinen zeigt lediglich Rek eine nachweisbare und richtungsabhängige Restdehnung; $\varepsilon_{\text {Restx }}=0,202 \mathrm{~mm} / \mathrm{m}$ für die X-Richtung, $\varepsilon_{\text {Resty }}=0,131 \mathrm{~mm} / \mathrm{m}$ für die y-Richtung und $\varepsilon_{\text {Restz }}=0,223 \mathrm{~mm} / \mathrm{m}$ für die z-Richtung (Abb.6-40). Die Restdehnung sämtlicher anderer Proben liegt bei $+-0,05 \mathrm{~mm} / \mathrm{m}$ und damit im Bereich des Gerätefehlers. Die Daten sind in Anhang I.8/Tab.6-6 zusammengefasst. 

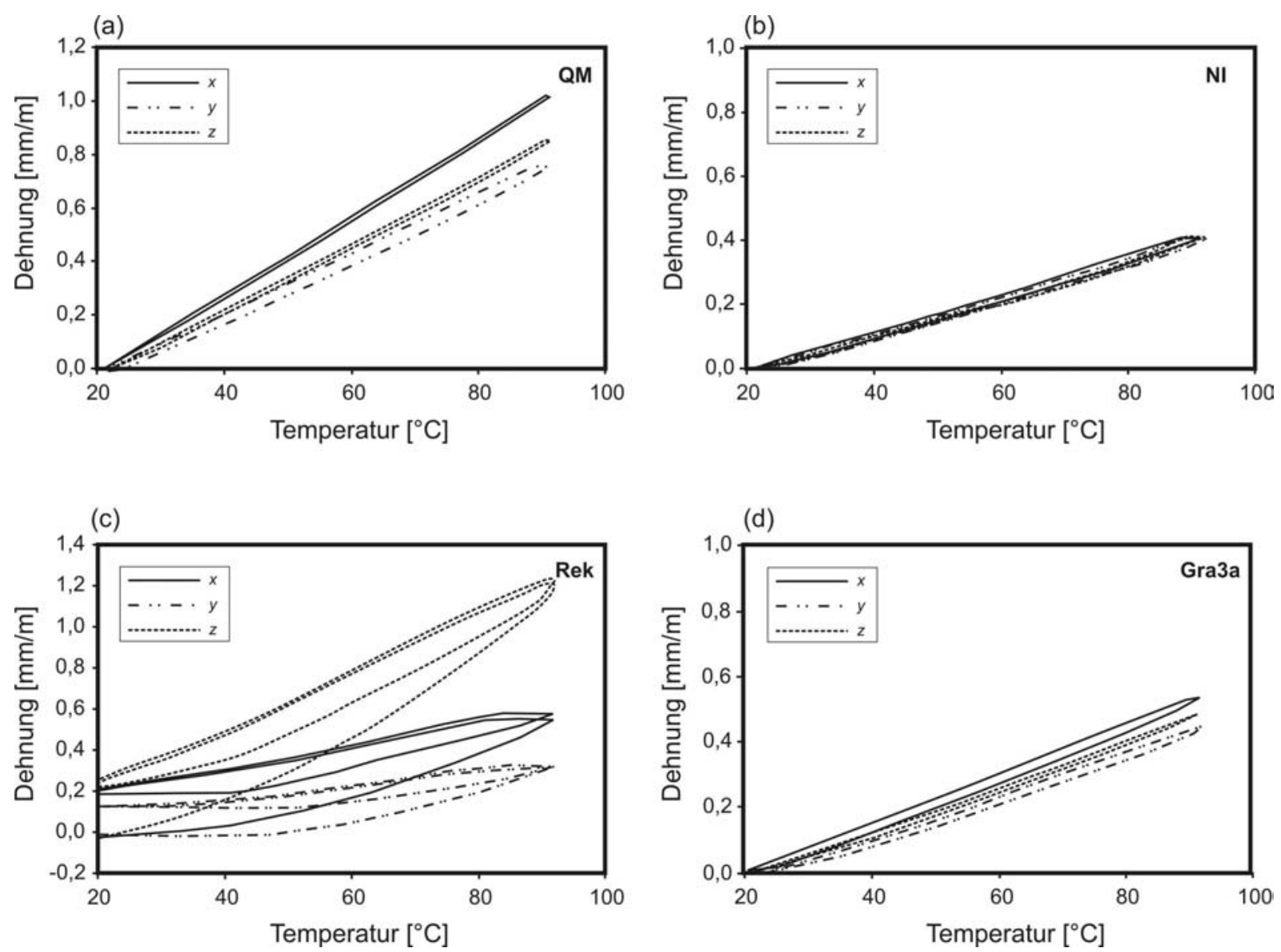

Abb.6-40: Dehnungs-Temperatur-Kurven. (a) QM - Quarzit. (b) NI - Gabbro-Norit (c) Rek - KalzitMarmor. (d) Gra3a Granitischer Ultramylonit.

\subsubsection{Thermische Dehnung, Mineralbestand und Textur}

Ist die an einigen Proben (z.B. Rek, QM) beobachtbare Anisotropie der thermischen Dehnung nicht auf die zunehmende Anisotropie der Makrogefüge und der Texturen entsprechend der getroffenen Einordnung in die Makrogefüge-Gruppen I/II/III zurückzuführen, so müssen andere, bei der Gruppeneinteilung nicht berücksichtigte Elemente einen Einfluss haben. Aufschluss darüber liefert $\boldsymbol{A b b . 6 - 4 1}$. Hier ist die maximal mögliche Anisotropie des Gesteins, berechnet aus den Volumenanteilen der Minerale und den Einkristallanisotropien, gegen die experimentell bestimmte Anisotropie der thermischen Dehnung aufgetragen. Die Daten liegen vollständig oberhalb der $\mathrm{A}_{\alpha \mathrm{Gmax}}=\mathrm{A}_{\alpha \mathrm{G}}-$ Linie, welche die maximale Anisotropie markiert. Diese kann nur verwirklicht werden, wenn eine 100\%ige Texturierung (alle Kristalle eines Minerals haben eine identische Raumlage) in Verbindung mit einer aufgrund der Orientierung additiven Verknüpfung des Anisotropiepotentials der verschiedenen Minerale vorliegt. Eine solche Konstellation kommt in der Natur nicht vor, so dass alle Daten mehr oder minder weit von der $A_{\alpha \mathrm{Gmax}}=\mathrm{A}_{\alpha \mathrm{G}}$-Linie entfernt plotten. 


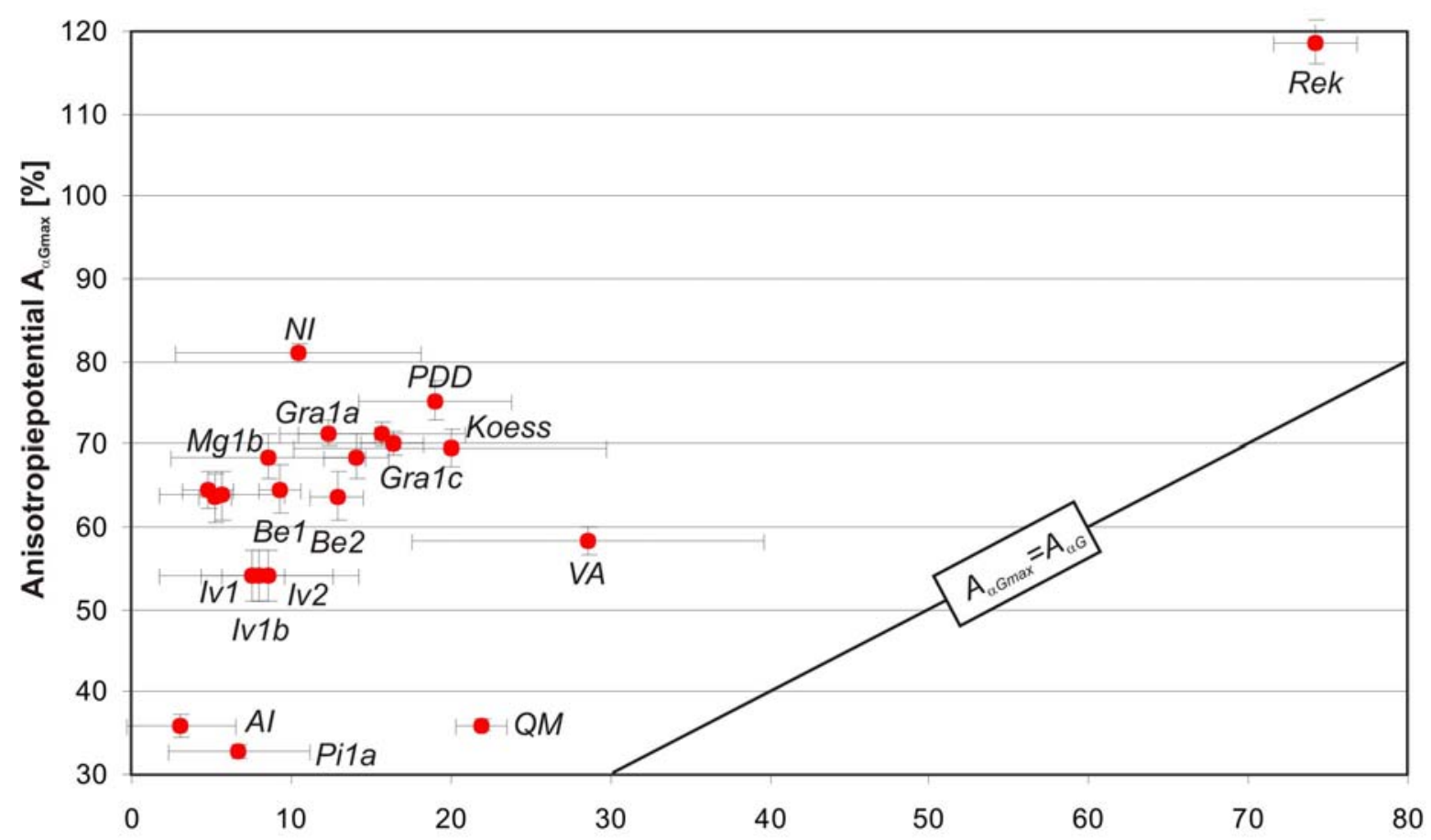

Anisotropie der thermischen Dehnung $\mathbf{A}_{\alpha \mathrm{G}}$ (experimentell) [\%]

Abb.6-41: Anisotropiepotential $\left(A_{\alpha G \max }\right)$ und experimentell ermittelte Anisotropie der thermischen Ausdehnungskoeffizienten $\left(A_{\alpha G}\right)$. Linie $A_{\alpha G \max }=A_{\alpha G}$ markiert die höchstmögliche Anisotropie, die aufgrund der mineralogischen Zusammensetzung im Gestein vorkommen kann. Rek - Kalzit-Marmor. $\mathrm{NI}$ - Gabbro-Norit. PDD - Granodiorit. Gra1a - granitischer Gneis. Koess - Granit. Mg1b - MetaGabbro. Gra1c - granitischer Gneis. Be1 - granodioritischer Gneis. Be2 - granitischer Gneis. Iv1 granodioritischer Protomylonit. Iv1b - granodioritischer Mylonit. Iv2 - granodioritischer Ultramylonit. VA - granitischer Orthogneis. Al - Quarzit. Pi1a - Peridotit. QM - quarzitischer Ultramylonit.

Im Diagramm ist ein schwacher Trend zu erkennen, der eine positive Korrelation von $\mathrm{A}_{\alpha \mathrm{Gmax}}$ und $\mathrm{A}_{\alpha \mathrm{G}}$ vermuten lässt und im Zusammenhang mit den Makrogefügen und den Texturen zu dem Schluss führt, dass bei einem Gestein mit einem hohen Anisotropiepotential schon eine verhältnismäßig niedrige Gefügeanisotropie bzw. Textur eine hohe Anisotropie der thermischen Dehnung verursacht. Ein gutes Beispiel ist der Marmor (Rek), der mit einem gegenüber den Glimmertexturen der Gruppe ÍII niedrigen Regelungsgrad der Kalzit-c-Achsen (max. 2,88 mrd) und der hohen Einkristallanisotropie des Kalzit von 120\% ein sehr stark anisotropes Dehnungsverhalten $\left(\mathrm{A}_{\alpha \mathrm{G}}=74,2 \%\right)$ offenbart. Ein anderes Beispiel zeigt deutlich den Einfluss der Textur auf das Dehnungsverhalten. Die Proben QM (100 Vol.\% Quarz) und AI (89 Vol.\% Quarz) haben aufgrund ihrer ähnlichen Komposition ein identisches Anisotropiepotential $\mathrm{A}_{\alpha \mathrm{Gmax}}=36 \%$. Die Probe QM mit der stärkeren Quarz-c-Achsenregelung (max. $>14$ mrd) zeigt im Vergleich mit AI (c-Achsen max. 1,38) die deutlich höhere Anisotropie und liegt näher an der maximal möglichen Anisotropie der thermischen Dehnung (Abb.6-41). An den Proben QM und Rek lässt sich auch die Abhängigkeit der thermischen Ausdehnungskoeffizienten von der bevorzugten Orientierung kristallographischer Elemente 
belegen. Beim Quarzit (QM) zeigt die y-Richtung in Übereinstimmung mit dem Maximum der Quarz-c-Achsen und deren im Vergleich zu den a-Achsen niedrigeren Ausdehnungskoeffizienten einen ebenfalls niedrigeren Gesamtgesteinsausdehnungskoeffizienten.

(a)

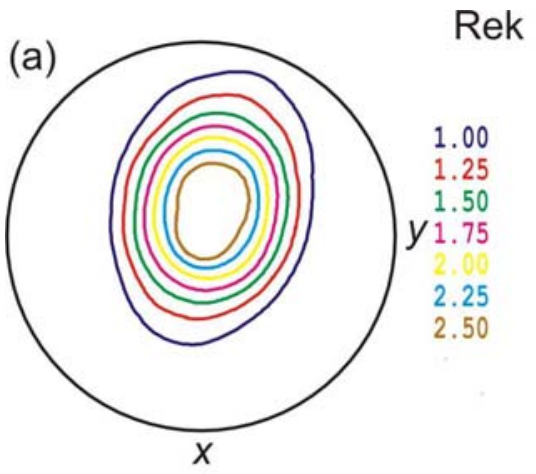

max. dens. $=2.88($ at $020 / 70)$ Kalzit c-Achse
(1)

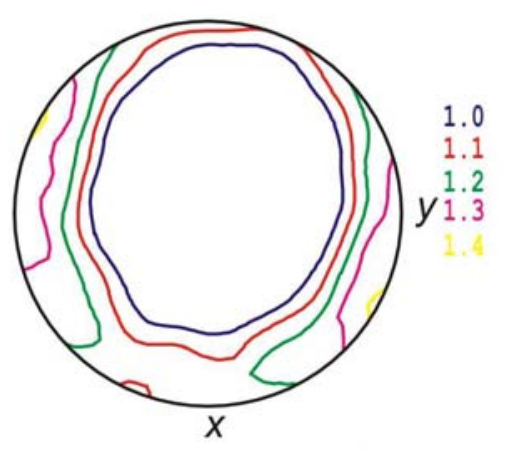

max. dens. $=1.42($ at $120 / 00)$ Kalzit a-Achse

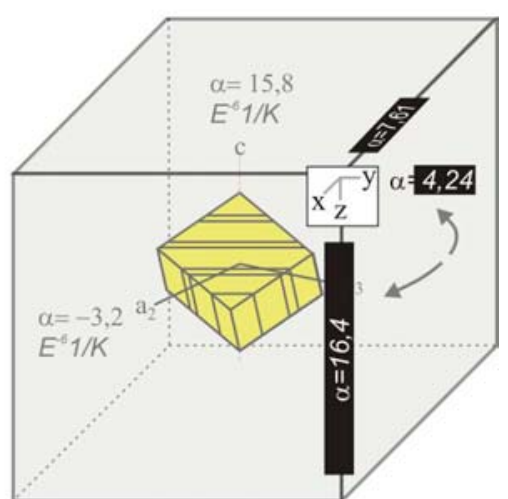

(b)

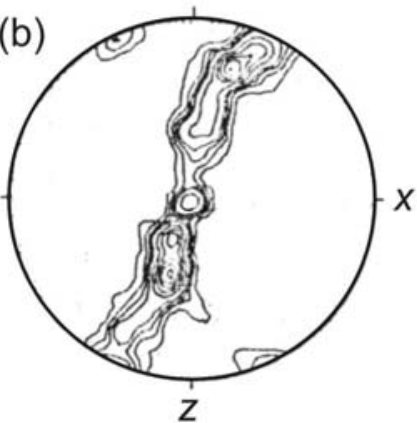

Konturen bei $1,2,3, \ldots .14 \mathrm{mrd}$

Quarz c-Achse
QM

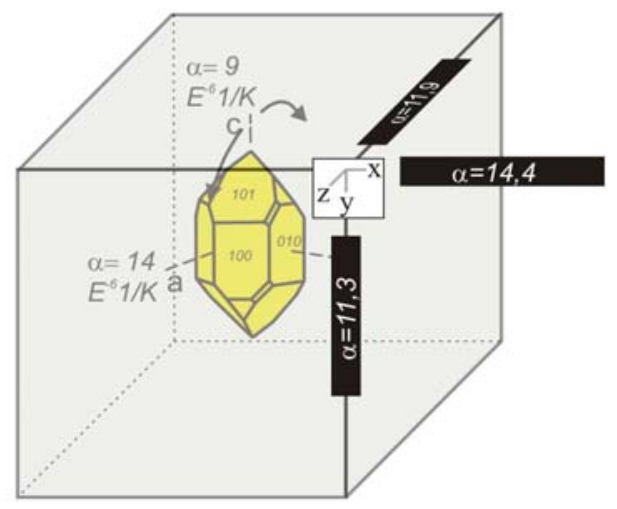

Abb.6-42: Abhängigkeit der thermischen Dehnung von der Textur am Beispiel des Kalzit-Marmor (Rek; a) und des quarz. Ultramylonit (QM; b). Richtungsabhängige Ausdehnungskoeffizienten $(\alpha)$ sind in $\left[{ }^{*} 10^{-6} 1 / \mathrm{K}\right]$ angegeben. Die Kristalle (mit thermischen Ausdehnungskoeffizienten für den Temperaturbereich von $20^{\circ} \mathrm{C}$ bis $90^{\circ} \mathrm{C}$ ) in den Würfeln sind nach den c-Achsen-Maxima der Texturen orientiert, die Tendenz der Verteilungen ist mit Pfeilen gekennzeichnet. $x, y, z$ sind die Richtungen des Probenreferenzsystems. Quarz-c-Achsen-Verteilung für QM aus Mancktelow (1987).

Für den Kalzit-Marmor gilt das selbe Prinzip, nur dass hier die Richtung der c-Achsen im Kalzit gegenüber den a-Achsen einen höheren Ausdehnungskoeffizienten aufweist. Dadurch ist der Ausdehnungskoeffizient des Gesteins parallel zur bevorzugten Orientierung der cAchsen am höchsten (vgl. Siegesmund et al. 1999; Rüdrich et al. 2001; Zeisig et al. 2002; Abb.6-42). Dass die richtungsabhängigen thermischen Ausdehnungskoeffizienten des Gesamtgesteins zum Teil höher liegen als die höchsten Werte der entsprechenden Einkristalle, kann mit dem Phänomen der thermisch induzierten Rissbildung erklärt werden, welches im weiteren Verlauf diskutiert wird. Die Glimmer haben im Gegensatz zu ihren stark anisotropen mechanischen und elastischen Eigenschaften nur eine niedrige Anisotropie der thermischen Dehnung $\left(\mathrm{A}_{\alpha \mathrm{M}}=28 \%\right.$ für Muskovit $)$ und tragen mit ihren niedrigen Volumenanteilen von 
kleiner als 22 Vol.\% (die Volumenanteile der Minerale gehen in die Berechnung von $\mathrm{A}_{\alpha \mathrm{Gmax}}$ ein), trotz z.T. sehr starker Texturierung (max. 23,3 mrd für Gra3a), nur gering zur Anisotropie des Gesamtgesteins bei. Insgesamt ist die Anisotropie der thermischen Dehnung eines Gesteins also durch das Anisotropiepotential des Gesteins und die Texturierung des Mineralbestands, insbesondere der stark anisotropen Mineralbestandteile und deren Volumenanteile bestimmt. Die Höhe der mittleren thermischen Dehnung hängt von der mineralogischen Zusammensetzung und den Einkristalldaten der gesteinsbildenden Minerale ab. Sowohl die thermische Dehnungs-Anisotropie der Einkristalle als auch die Volumenanteile der Minerale gehen nicht in die Bewertungskriterien zur Bildung der Makrogefüge-Gruppen ein, was die schlechte Übereinstimmung von der durch Materialwechsel und Formanisotropie sowie der Textur des Mineralbestands bestimmten makroskopischen Gefügeanisotropie und der Anisotropie der thermischen Dehnung erklärt.

\subsubsection{Risspufferung und thermisch induzierte Rissbildung}

Dehnt sich eine Probe aufgrund von Temperaturänderungen (hier Aufheizen) aus, so entstehen aus der thermischen Dehnung der Minerale in der Hauptsache zwei konkurrierende Prozesse. Zum einen dehnen sich Minerale in den bereits bestehenden Porenraum hinein und schließen diesen teilweise, so dass die thermische Ausdehnung des Mineralbestands kompensiert wird (Risspufferung).

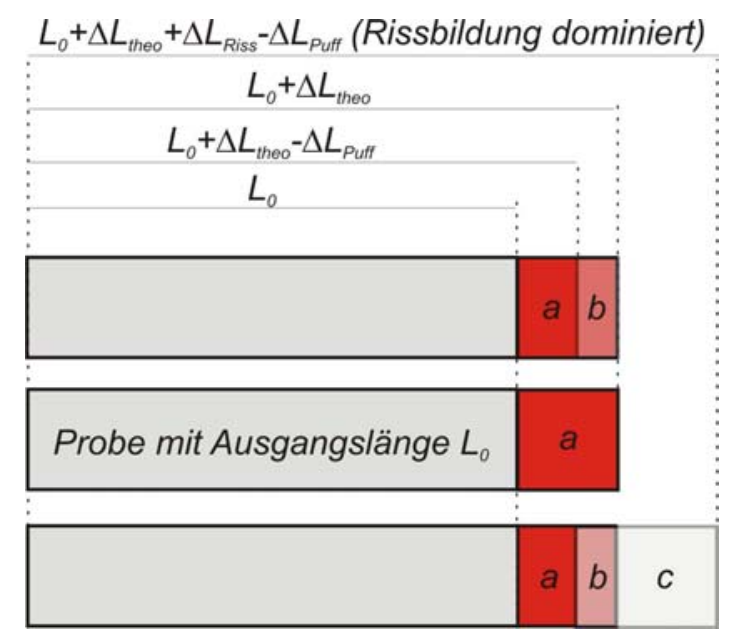

Abb.6-43: Schema zu Prozessen bei der thermischen Dehnung von Gesteinen. Auf die Dehnung bezogen eindimensionale Darstellung. $L_{0}$ - Ausgangslänge der Probe $[\mathrm{mm}]$. $\Delta L_{\text {theo }}$ (a) - theoretische Längenänderung der Probe auf der Basis der mineralogischen Zusammensetzung und der mittleren Ausdehnungskoeffizienten der Minerale. $\Delta L_{\text {Puff }}$ (b) - Längenänderung (negativ) durch Risspufferung. $\Delta L_{\text {Riss. }}(c)$ - Längenänderung der Probe aufgrund von thermisch induzierter Rissbildung.

Zum anderen dehnen sich die Minerale gegeneinander aus, was zu einer Längenänderung und dem Aufbau von Spannungen führt, die beim Überschreiten der Gesteinskohäsion eine 


\section{Technische Gesteinseigenschaften}

thermisch induzierten Rissbildung verursachen (Abb.6-43). Dieser Prozess bedingt eine höhere thermische Dehnung der Probe als aufgrund der Mineralbestandteile und deren Textur zu erwarten wäre (Studien an Marmoren - z.B. Battaglia et al. 1993; Franzini 1995; Rüdrich et al. 2001). Eine Abschätzung, welcher der beiden Prozesse bezüglich der untersuchten Gesteine überwiegt und damit das Dehnungsverhalten bestimmt, liefert der Vergleich der experimentell und theoretisch (vgl. Kap.2.4.6) bestimmten Volumenausdehnungskoeffizienten (Abb.6-44). Für die Betrachtungen wird modellhaft eine orthorhombische Symmetrie der thermischen Dehnungseigenschaften der Gesteine angenommen.

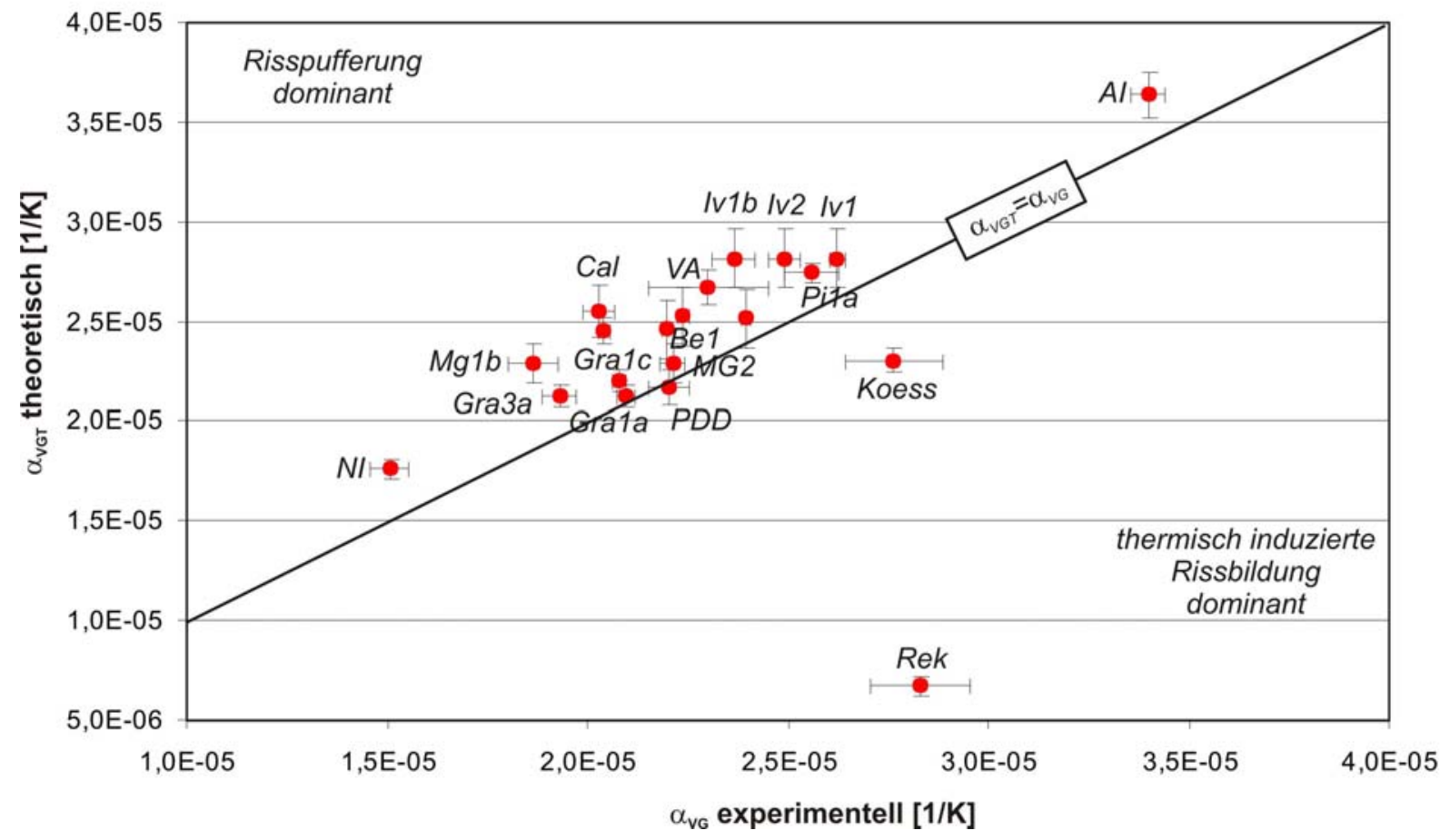

Abb.6-44: Theoretischer Volumenausdehnungskoeffizient ( $\left.\alpha_{V G T}\right)$ und experimentell ermittelter Volumenausdehnungskoeffizient ( $\alpha_{V G}$ ) für den Temperaturbereich von $20^{\circ} \mathrm{C}$ bis $90^{\circ} \mathrm{C}$. Rek $-K a l z i t-$ Marmor. NI - Gabbro-Norit. PDD - Granodiorit. Gra1a - granitischer Gneis. Koess - Granit. Mg1b Meta-Gabbro. Gra1c - granitischer Gneis. Gra3a - granitischer Ultramylonit. Be1 - granodioritischer Gneis. Cal - granodioritischer Gneis. Iv1 - granodioritischer Protomylonit. Iv1b - granodioritischer Mylonit. Iv2 - granodioritischer Ultramylonit. VA - granitischer Orthogneis. AI - Quarzit. Pi1a Peridotit. Mg2 - Meta-Gabbro. Fehlerbalken markieren den 1- $\sigma$-Fehler.

Mit Ausnahme der Proben Rek (Kalzit-Marmor) und Koess (Granit) liegen alle Daten oberhalb der $\alpha_{V G T}=\alpha_{V G}$-Linie. Daraus folgt, dass in dem Probensatz für den betrachteten Temperaturbereich der Prozess der Risspufferung dominant und die thermische Rissbildung nur bei Rek und Koess deutlich ausgeprägt ist. Naheliegend ist daher die Frage, ob zwischen der Rissdichte der Gesteine, bestimmt durch den Rissquantifizierungsparameter $\mathrm{Vp}_{\mathrm{QRiss}}$ und der Risspufferung eine Wechselbeziehung besteht. Dieser Zusammenhang ist in Abb.6-45 dargestellt. Trotz der recht hohen Streuung der Daten zeigt sich eine positive Korrelation von 
Rissdichte $\left(\mathrm{Vp}_{\mathrm{QRiss}}\right)$ und $\Delta \alpha_{\mathrm{V}}=\alpha_{\mathrm{VGT}}$ (theoretisch)- $\alpha_{\mathrm{VG}}$ (experimentell) als ein Maß für die Risspufferung in der von Risspufferung dominierten Gesteinsgruppe.

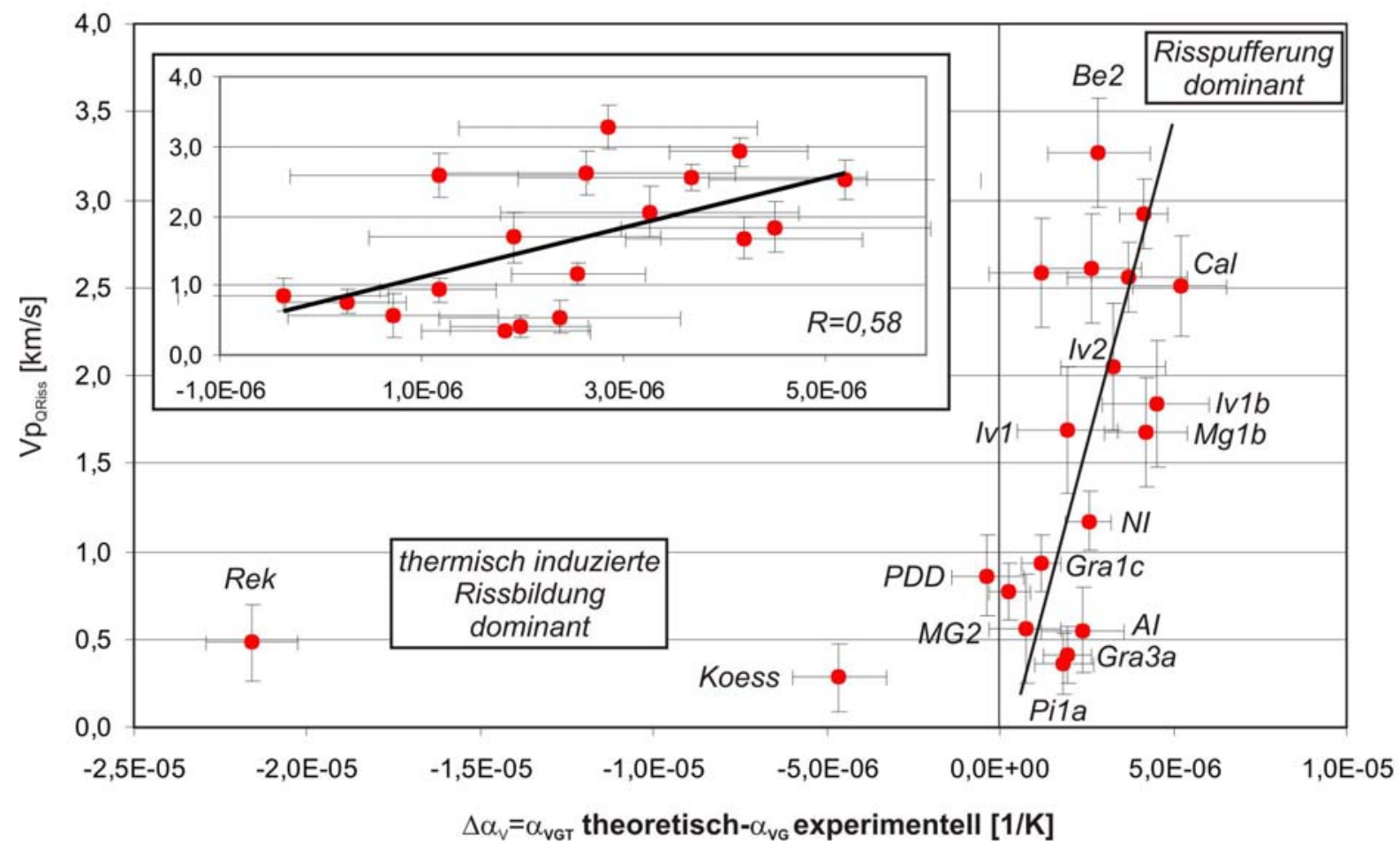

Abb.6-45: Rissquantifizierungsparameter $V p_{Q R i s s}[\mathrm{~km} / \mathrm{s}]$ und $\Delta \alpha_{V}=\alpha_{V G T}-\alpha_{V G}[1 / \mathrm{K}]$. Diagramm oben links zeigt die Proben ohne (Rek) (Koess), also den durch Risspufferung dominierten Bereich. Rek - KalzitMarmor. NI - Gabbro-Norit. PDD - Granodiorit. Koess - Granit. Mg1b - Meta-Gabbro. Gra1c granitischer Gneis. Gra3a - granitischer Ultramylonit. Be2 - granitischer Gneis. Cal - granodioritischer Gneis. Iv1 - granodioritischer Protomylonit. Iv1b - granodioritischer Mylonit. IV2 - granodioritischer Ultramylonit. VA - granitischer Orthogneis. Al - Quarzit. Pi1a - Peridotit. Mg2 - Meta-Gabbro. Fehlerbalken markieren den $1-\sigma-F e h l e r$.

Es bleibt darauf hinzuweisen, dass Risspufferung und thermische Rissbildung bezüglich der thermischen Ausdehnung gegenläufig wirken (Abb.6-43), sich theoretisch gegenseitig aufheben und zu einer Nullausdehnung der Probe führen können, ohne dass die Quantifizierung der Beträge der einzelnen Effekte möglich ist.

Beim Kalzit-Marmor (Rek) mit seiner niedrigen Porosität von 0,14 Vol.\% und seinen hohen mittleren Ultraschallgeschwindigkeiten von $\mathrm{Vp}=5,99 \mathrm{~km} / \mathrm{s}$ (trockene Kugel - Kap.5.9.2; Anhang I.6) kann in Übereinstimmung mit Untersuchungen von Rüdrich et al. (2001) der Einfluss der Risspufferung als niedrig gegenüber der thermisch bedingten Rissbildung eingeschätzt werden. Mit dieser Annahme lässt sich aus dem durch thermische Rissbildung induzierten Betrag von $\Delta \alpha_{V}$ das bei einer Aufheizung des Marmors von $20^{\circ} \mathrm{C}$ auf $90^{\circ} \mathrm{C}$ neu gebildete Porenvolumen ( $\Delta \mathrm{V}_{\text {Riss }}$ ) [\%] berechnen (G.6-15/G.6-16). Voraussetzung ist, dass die Achsen des Dehnungstensors in etwa mit den Achsen des Probenreferenzsystems 
übereinstimmen, was aufgrund der Textursymmetrie des Kalzit (Abb.6-42) angenommen werden kann.

(G.6-15)

(G.6-16)

$$
\Delta V_{\text {Riss }}=\Delta \alpha_{V} * \Delta T * 100
$$

mit

$\Delta V_{\text {Riss }}$ - neu gebildetes Rissvolumen im Temperaturintervall $(\Delta T)$ [\%]. $\Delta \alpha_{V}$ - Differenz aus theoretisch und experimentell ermittelten Volumenausdehnungskoeffizienten [1/K]. $\alpha_{V G T}-$ theoretisch ermittelter Volumenausdehungskoeffizient. $\alpha_{V G T}-$ theoretischer Volumenausdehnungskoeffizient (aus Mineralbestand und Einkristalldaten) $[1 / K] \quad \alpha_{V G}-e_{\text {experimentell ermittelter }}$ Volumenausdehnungskoeffizient [1/K]. $\Delta T$ - Temperaturintervall [K].

Unter Berücksichtigung der richtungsabhängigen Beträge der thermischen Dehnung bei $90^{\circ} \mathrm{C}$ und der Restdehnung nach der ersten $90^{\circ} \mathrm{C}$-Rampe (vgl. Kap.2.4.6) lässt sich ein vollständiges Bild des thermischen Dehnungsverhalten des Kalzit-Marmors (Rek) zeichnen (Tab.6-6).

Tab.6-6: Änderungen des Proben- und Porenvolumens für den Kalzit-Marmor Rek im Temperaturbereich von $20^{\circ} \mathrm{C}$ bis $90^{\circ} \mathrm{C}$.

\begin{tabular}{|c|c|}
\hline \multicolumn{2}{|l|}{ Eingangsgrössen } \\
\hline$a_{x}=7,61^{*} 10^{-6} 1 / K$ & $\varepsilon_{90 x}=0,53+-0,02 \mathrm{~mm} / \mathrm{m}$ \\
\hline$a_{y}=4,24 * 10^{-6} 1 / K$ & $\varepsilon_{90 \mathrm{y}}=0,32+-0,02 \mathrm{~mm} / \mathrm{m}$ \\
\hline$a_{z}=16,4^{*} 10^{-6} 1 / K$ & $\varepsilon_{90 \mathrm{z}}=1,18+-0,07 \mathrm{~mm} / \mathrm{m}$ \\
\hline$\alpha_{V G}=28,25^{*} 10^{-6} 1 / \mathrm{K}$ & $\varepsilon_{\text {Restx }}=0,202+-0,017 \mathrm{~mm} / \mathrm{m}$ \\
\hline$\alpha_{V G T}=6,70 * 10^{-6} 1 / \mathrm{K}$ & $\varepsilon_{\text {Resty }}=0,131+-0,021 \mathrm{~mm} / \mathrm{m}$ \\
\hline$\Delta \alpha_{V}=21,6^{*} 10^{-6} 1 / \mathrm{K}$ & $\varepsilon_{\text {Restz }}=0,223+-0,014 \mathrm{~mm} / \mathrm{m}$ \\
\hline \multicolumn{2}{|l|}{ Berechnungsgrundlagen } \\
\hline$\Delta \mathrm{V}_{90}=\left(\mathrm{V}_{90}-\mathrm{V}_{0}\right) / \mathrm{V}_{0} * 100[\%]$ mit & ${ }_{90}=\mathrm{L}_{0 \mathrm{x}}{ }^{*}\left(1+\varepsilon_{90 \mathrm{x}} / 1000\right)^{*} \mathrm{~L}_{0 \mathrm{y}}\left(1+\varepsilon_{90 \mathrm{y}} / 1000\right)^{*} \mathrm{~L}_{0 \mathrm{z}}\left(1+\varepsilon_{90 \mathrm{z}} / 1000\right)\left[\mathrm{mm}^{3}\right]$ \\
\hline \multicolumn{2}{|c|}{ analog dazu kann $\Delta \mathrm{V}_{\text {Rest }}[\%]$ mit $\varepsilon_{\text {Restx }, y, z}$ ermittelt werden } \\
\hline \multirow{2}{*}{\multicolumn{2}{|c|}{$\begin{array}{l}\Delta \mathrm{V}_{\text {Ming0 }}=\alpha_{V G T}{ }^{*} \Delta T^{*} 100[\%] \\
\text { Ergebnisse für die Änderung des Proben- und Porenvolumens der Probe Rek bezogen auf } \\
\text { ein Aufheizen auf } 90^{\circ} \mathrm{C} \text { und anschließendes Abkühlen auf } 20^{\circ} \mathrm{C}\end{array}$}} \\
\hline & \\
\hline \multirow{4}{*}{$\begin{array}{l}\text { Start bei } 20^{\circ} \mathrm{C} \\
\text { Probentemperatur } 90^{\circ} \mathrm{C}\end{array}$} & Ausgangsvolumen $\mathrm{V}_{0}$ \\
\hline & Vergrößerung der Probenvolumens $\left(\Delta \mathrm{V}_{90}\right)$ : \\
\hline & davon durch den Mineralbestand bedingt $\left(\Delta \mathrm{V}_{\text {Min90 }}\right): 0,05 \%$ \\
\hline & davon durch die therm. Rissb. bedingt $\left(\Delta \mathrm{V}_{\text {Riss }}\right)$ : \\
\hline Probentemperatur $20^{\circ} \mathrm{C}$ & volumenbezogene Restdehnung $\left(\Delta \mathrm{V}_{\text {Rest }}\right)$ beträgt: \\
\hline
\end{tabular}

$\alpha_{x}, \quad \alpha_{y}, \quad \alpha_{z}-$ Ausdehnungskoeffizienten des Gesteins in $x-, \quad y$-, z-Richtung $[1 / K] . \alpha_{V G}-$ Volumenausdehnungskoeffizient des Gesteins aus experimentellen Daten [1/K]. $\alpha_{V G T}-$ Volumenausdehnungskoeffizient des Gesteins theoretisch ermittelt [1/K]. $\Delta \alpha_{V}-/ \alpha_{V G T} \alpha_{V G} /[1 / K]$. $\varepsilon_{90 x, y, z}-$ Dehnung der Probe bei $90^{\circ} \mathrm{C}$ in $x$-, $y$-, z-Richtung $[\mathrm{mm} / \mathrm{m}]$. $\varepsilon_{\text {Rest } x, y, z}-$ Restdehnung der Probe nach der ersten $90^{\circ} \mathrm{C}$-Rampe bei $20^{\circ} \mathrm{C}$ in $x$-, y-, z-Richtung $[\mathrm{mm} / \mathrm{m}] . \Delta V_{90}$ - relative Volumenänderung der Probe bei $90^{\circ} \mathrm{C}[\%] . \quad V_{90}-$ Volumenänderung der Probe bei $90^{\circ} \mathrm{C} \quad\left[\mathrm{mm}^{3}\right] . \quad \Delta V_{\text {Min9o }}-$ Volumenänderung der Probe bei $90^{\circ} \mathrm{C}$ aufgrund der Volumenausdehnung der gesteinsbildenden Minerale [\%]. $\Delta V_{\text {Rest }}$ - relative volumenbezogene Restdehnung nach der ersten $90^{\circ} \mathrm{C}$-Rampe [\%]. $\Delta V_{\text {Riss }}$ - relative Volumenänderung der Probe bei $90^{\circ} \mathrm{C}$ aufgrund von thermischer Rissbildung [\%].

Bei einer Aufheizung von $20^{\circ} \mathrm{C}$ auf $90^{\circ} \mathrm{C}$ vergrößert sich das Probenvolumen um $0,20 \%$, davon entfallen lediglich 0,05\% auf die Volumenänderung des Mineralbestands und 0,15\% 
auf neu gebildeten Porenraum durch thermisch induzierte Risse. Diese Werte stimmen größenordnungsmäßig mit Ergebnissen von Weiss et al. (2001) überein, die den Anteil des neugebildeten Porenraums mit Hilfe von Rissdichten und aspect-ratios abgeschätzt haben $\left(0,12 \%\right.$ für einen Kalzit-Marmor). Nach der Abkühlung auf $20^{\circ} \mathrm{C}$ bleibt eine volumenbezogene Restdehnung von $0,05 \%$ bestehen. Daraus folgt, dass im Fall des betrachteten Kalzit-Marmors 0,10\% des gebildeten Rissvolumens wieder geschlossen werden. Es besteht daher die Möglichkeit, dass eine deutlich stärkere Entfestigung eines Gesteins stattfindet als aufgrund von Restdehnung und Ultraschalluntersuchungen (z.B. Weiss et al. 2002b) zu erwarten ist. Daher ist der Parameter $\Delta \mathrm{V}_{\text {Riss, }}$, also die relative Volumenänderung durch Mikrorisse, als Ergänzung zu den richtungsabhängigen Dehnungsmessungen und Restdehnungen von Bedeutung, weil er den tatsächlich gebildeten Porenraum beschreibt und damit ein Maß für die Gefügeauflockerung und mechanische Schwächung des Gesteins beinhaltet.

\subsection{Zusammenfassung}

Die Abhängigkeit der technischen Gesteinseigenschaften von der mineralogischen Zusammensetzung und dem Gesteinsgefüge ist ein komplexes Thema. Viele verschiedene Faktoren wirken in unterschiedlicher Weise auf die jeweilige Eigenschaft ein und erschweren damit die Diskriminierung zwischen den unterschiedlichen Parametern und deren Einflussintensitäten. Trotzdem konnten im Rahmen der vorliegenden Studie einige Größen herausgearbeitet werden, die für die technischen Eigenschaften von Naturwerksteinen von Bedeutung sind. Nicht immer handelt es sich bei den beobachteten Korrelationen um direkte kausale Zusammenhänge. So sind die makroskopische Gefügeanisotropie und die Glimmertextur beispielsweise als Stellvertreter für die Anisotropie aller Gefügeelemente zu verstehen, ohne dass zwischen ihnen selbst und der jeweiligen technischen Gesteinseigenschaft eine physikalisch begründbare Abhängigkeit existieren muss. Die Eignung dieser Faktoren zur Prognose der Anisotropie der unterschiedlichen Eigenschaften wurde in Kap.6 diskutiert und die Ergebnisse sind im Folgenden zusammengefasst. An dieser Stelle wird keine Aussage über die Art der Korrelation im Sinne von linear, exponentiell u.s.w. getroffen. Für jede Eigenschaft sind Indexminerale angegeben, die gemäß ihrer Einkristalleigenschaften für die jeweilige Eigenschaft und deren Anisotropie obere und untere Grenzen darstellen. 


\section{Technische Gesteinseigenschaften}

\section{Rohdichte}

Alle untersuchten Gesteine weisen eine Porosität von kleiner gleich 1 Vol.\% auf. Daher hängt die Dichte zu mindestens $99 \%$ von der Dichte des Mineralbestands ab. Die Rissdichten spielen aufgrund der sehr niedrigen aspect-ratios der vorkommenden, offenen Mikrorissen eine untergeordnete Rolle.

Indexminerale: Olivin, Granat (hohe Dichte); Quarz, Feldspat (niedrige Dichte)

\section{Porosität}

Der Porenraum wird ausschließlich durch offene Mikrorisse (auch offene Korngrenzen) gebildet, die damit über die Rissdichte und die Geometrie (aspect-ratio) zu $100 \%$ die Porosität bestimmen.

\section{Kapillare Wasseraufnahme}

a) Mittelwerte: Die Mittelwerte der kapillaren Wasseraufnahme (charakterisiert durch den w-Wert) hängen von der Rissdichte der offenen Mikrorisse und dem Volumenanteil der Kapillarporen ab. Weitere gute lineare Korrelationen ergeben sich mit der Porosität und dem mittleren Porenradius, sofern diese Werte sich proportional zur Rissdichte und dem Volumenanteil der Kapillarporen ändern.

b) Anisotropie: Sowohl die makroskopische Gefügeanisotropie, als auch die Intensität der Glimmertextur sind geeignet die Anisotropie der kapillaren Wasseraufnahme abzuschätzen, ohne dass ein direkter kausaler Zusammenhang zwischen diesen beiden Parametern und der kapillaren Wasseraufnahme besteht. Vielmehr sind die makroskopische Gefügeanisotropie und die Glimmertextur hier Stellvertreter für die bevorzugte Orientierung der offenen Mikrorisse.

\section{Druck-, Spaltzug- und Biegefestigkeit}

a) Mittelwerte: Die Mittelwerte der Druck-, Spaltzug-, und Biegefestigkeit werden durch eine Kombination der mechanischen Eigenschaften des Mineralbestands und der Rissdichte der offenen Mikrorisse bestimmt. Der Mineralbestand gibt dabei einen Grundwert (Höchstwert) der mechanischen Festigkeit vor, der durch die Anwesenheit von Mikrorissen herabgesetzt wird. Der Einfluss der Korngöße ist indirekt. Größere Korngrößen werden mit 


\section{Technische Gesteinseigenschaften}

höheren Risslängen und resultierend niedrigeren Festigkeiten assoziiert, aber unterschiedliche Spannungsverteilungen im Gesteinsgefüge aufgrund unterschiedlicher Korngrößen spielen ebenso eine Rolle.

b) Anisotropie: Die Anisotropie der Druckfestigkeit kann indirekt über die makroskopische Gefügeanisotropie und die max. Intensität der Glimmertextur abgeschätzt werden. Grundsätzlich hat ein Mineral mit einer hohen Anisotropie der mechanischen Eigenschaften, einer starken Textur und einem hohen Volumenanteil einen entsprechenden Einfluss auf die Anisotropie der Festigkeiten (Beispiel Glimmer in Gneisen; Hornblende in Metagabbros). Ein weiterer wichtiger Punkt ist die Verteilung besonders anisotroper Minerale im Gesteinsgefüge. Sind z.B. Glimmer in Lagen konzentriert, so kommt es beim Druckversuch zur Lokalisation von Bruchzonen in eben diesen Lagen. Die Eigenschaften des restlichen Mineralbestands sind dann nicht mehr von Bedeutung. Das mechanische schwächste Glied im Gesteinsgefüge bestimmt die Druckfestigkeit in Abhängigkeit von der relativen Orientierung der angreifenden Spannungen. In diesem Fall genügen auch schon geringe Volumenanteile eines entsprechenden Minerals, um im Zusammenhang mit einer starken Textur eine deutliche Anisotropie der Festigkeitseigenschaften hervorzurufen. Die bevorzugte Orientierung der Mikrorisse folgt in der Regel der makroskopischen Gefügeanisotropie und der bevorzugten Orientierung der Glimmerspaltflächen (oder Spaltflächen anderere Minerale), intensiviert deren Einfluss auf die mechanischen Eigenschaften und liefert in Verbindung mit der Rissdichte ebenfalls eine brauchbare Aussage über die zu erwartende Anisotropie.

Indexminerale:

Mittelwerte: Quarz (hohe Festigkeit); Kalzit (niedrige Festigkeit)

Anisotropie: Glimmer (hohe Anisotropie); Granat (niedrige Anisotropie)

5. Abriebfestigkeit

a) Mittelwerte: Die Mittelwerte der Abriebfestigkeit sind sehr stark von den mechanischen Eigenschaften des Mineralbestands, insbesondere von der durchschnittlichen Härte, abhängig. Bei glimmerhaltigen Granitoiden oder Metagranitoiden bietet der Glimmergehalt einen guten Anhaltspunkt in Bezug auf die zu erwartenden Abriebfestigkeiten. Die Rissdichte und auch die 


\section{Technische Gesteinseigenschaften}

Korngröße haben einen im Vergleich zu den Druck-, Spaltzug- und Biegefestigkeiten wesentlich geringeren Einfluss.

b) Anisotropie: Die Anisotropie der Abriebfestigkeit zeigt eine gute Übereinstimmung mit der makroskopischen Gefügeanisotropie und der max. Intensität der Glimmertextur, jedoch sind die Richtungsabhängikeiten der Abriebfestigkeiten in Relation zum bei Naturwerksteinen verwirklichten Bereich sehr gering. Aus diesem Grund ist sowohl der Einfluss der Intensität der Glimmertextur als auch der bevorzugten Orientierung der Mikrorissysteme, trotz der guten Korrelation, als verhältnismäßig niedrig einzustufen. Bei einer entsprechend starken Textur und einem hohen Volumenanteil kann auch ein weniger anisotropes Mineral eine Gesamtgesteinsanisotropie hervorrufen, wie z.B. der Kalzit beim Kalzit-Marmor (Rek) und die Hornblende bei den MetaGabbros $(\mathrm{Mg} 1 \mathrm{~b} / 2)$.

Indexminerale:

Mittelwerte: Quarz (hohe Abriebfestigkeit); Kalzit (niedrige Abriebfestigkeit) Anisotropie: Glimmer (hohe Anisotropie); Granat (niedrige Anisotropie)

6. Dynamischer E-Modul

a) Mittelwerte: Die Mittelwerte des dynamischen E-Moduls hängen zum einen von den elastischen Eigenschaften des Mineralbestands und zum anderen von der Rissdichte und der Rissgeometrie der offenen Mikrorisse ab. Die mineralogische Zusammensetzung bestimmt dabei den höchstmöglichen EModul, wohingegen die offenen Mikrorisse zu einer starken Reduktion des EModuls führen. Der Volumenanteil der Phyllosilikate geht gemäß der Einkristalleigenschaften in die Gesamtgesteinseigenschaften ein, ohne jedoch eine besondere Stellung bezüglich der mittleren elastischen Eigenschaften einzunehmen.

b) Anisotropie: Betrachtet man die Anisotropie des dynamischen E-Moduls, so zeigt sich eine gute Übereinstimmung mit der makroskopischen Gefügeanisotropie. Entscheidend ist jedoch die rissinduzierte Anisotropie, die dazu führen kann, dass die Gesteinsanisotropie, die aufgrund des Mineralbestands höchstmögliche Anisotropie überschreitet. Die max. Intensität der Glimmertextur ist in Verbindung mit dem Volumenanteil der Glimmer ein guter Indikator für die zu erwartende Anisotropie des E-Moduls. Hier spielen 


\section{Technische Gesteinseigenschaften}

zwei Effekte eine Rolle: Erstens weisen die Phyllosilikate sehr anisotrope elastische Eigenschaften auf die in Verbindung mit der Textur und dem Volumenanteil als intrinsische Eigenschaften auf das Gesamtgestein vererbt werden. Zweitens treten häufig offene Mikrorisse entlang der (001)-Flächen auf und intensivieren den Effekt der Phyllosilikate auf die Anisotropie der dynamischen elastischen Eigenschaften. Auch der Einfluss der NichtPhyllosilikat-Minerale auf die elastischen Eigenschaften ist nicht $\mathrm{zu}$ unterschätzen. Hohe Volumenanteile eines Minerals mit einer deutlichen Anisotropie können schon bei verhältnismäßig schwachen Texturen eine hohe Anisotropie hervorrufen (Beispiel Kalzit im Kalzit-Marmor Rek).

Indexminerale:

Mittelwerte: Granat, Olivin (hoher dynamischer E-Modul); Kalzit, Albit (niedriger dynamischer E-Modul)

Anisotropie: Glimmer (hohe Anisotropie); Granat (niedrige Anisotropie)

\section{Statischer E-Modul}

a) Mittelwerte: Der mittlere statisch bestimmte E-Modul bei $50 \%$ der Bruchlast hängt von den elastischen und von den mechanischen Eigenschaften des Mineralbestands sowie der Rissdichte der offenen Mikrorisse ab. Auch der Volumenanteil der Glimmer hat einen Einfluss auf den statischen E-Modul. Im Gegensatz zu den dynamischen elastischen Eigenschaften ist nicht die Elastizität der gesteinsbildenden Minerale ausschlaggebend für das SpannungsDehnungs-Verhalten des Gesteins, sondern vielmehr deren mechanisches Verhalten. Es bestehen gewisse Analogien zur Gefügeabhängigkeit der Druckfestigkeiten. Daher ist zu vermuten, dass auch die Korngröße in den statischen E-Modul mit eingeht.

b) Anisotropie: Die Anisotropie des statischen E-Moduls zeigt eine gute Übereinstimmung mit der makroskopischen Gefügeanisotropie und der max. Intensität der Glimmertextur. Der Einfluss der letzteren wird durch die häufig parallel zu den Phyllosilikat-(001)-Flächen orientierten offenen Mikrorisse verstärkt. Die meist deutlich schwächeren Texturen der sonstigen gesteinsbildenden Minerale haben aufgrund der gegenüber den Glimmern deutlich niedrigeren mechanischen Einkristallanisotropien einen geringen Einfluss auf die Richtungsabhängigkeit des statischen E-Moduls. 


\section{Technische Gesteinseigenschaften}

Indexminerale:

Mittelwerte: Olivin, Quarz (hoher statischer E-Modul); Glimmer (niedriger statischer E-Modul)

Anisotropie: Glimmer (hohe Anisotropie); Granat (niedrige Anisotropie)

8. Thermische Dehnung

a) Mittelwerte: Der thermische Volumenausdehnungskoeffizient wird hauptsächlich durch die thermischen Dehnunggseigenschaften des Mineralbestands bestimmt. Präexistierende offene Mikrorisse führen über eine Risspufferung zu einer leichten Verringerung der thermischen Dehnung. Neben der Risspufferung kann auch thermisch bedingte Rissbildung auftreten, die einen gegenläufigen Effekt hat und zu höheren Volumenausdehnungskoeffizienten führt.

b) Anisotropie: Zwischen der makroskopischen Gefügeanisotropie, der max. Intensität der Glimmertextur und der Anisotropie der thermischen Dehnung besteht kein Zusammenhang. Als Ursache hiefür gelten die geringe Einkristallanisotropie der Glimmer und die Tatsache, dass Texturen von hochanisotropen Mineralen, wie z.B. des Kalzit, nicht in die Definition des makroskopischen Gefügebildes eingehen. Gerade diese Minerale dominieren über ihre Textur die Gesamtgesteinsanisotropie. Gegebenenfalls (z.B. beim Kalzit) kommt der richtungsabhängige Prozess der thermischen Rissbildung hinzu und verstärkt den Anisotropieeffekt, ohne dass die aufgrund des Mineralbestands maximal mögliche Anisotropie überschritten wird.

Indexminerale:

Mittelwerte: Quarz (hoher thermischer Volumenausdehnungskoeffizient); Kalzit, Feldspat (niedriger thermischer Volumenausdehnungskoeffizient Anisotropie: Kalzit (hohe Anisotropie); Granat (niedrige Anisotropie) 


\section{Richtungsabhängigkeit technischer Gesteinseigenschaften an Fallbeispielen}

Die technischen Gesteinseigenschaften zeigen in Abhängigkeit von der makroskopischen Gefügeanisotropie eine z.T starke Richtungsabhängigkeit. Verantwortlich dafür sind z.B. die Textur der gesteinsbildenden Minerale und die bevorzugte Orientierung von offenen Mikrorissen (Kap.6). Um das Verhalten der einzelnen Eigenschaften in Bezug auf eine bestimmte Gefügerichtung zu charakterisieren und die Abhängigkeit von der räumlichen Anordnung der Gefügeelemente darzustellen, wurden zwei Gesteinsserien mit jeweils identischer mineralogischer Komposition ausgewählt. Zum einen handelt es sich um drei Proben granodioritischer Zusammensetzung mit einem zunehmenden Grad an mylonitischer Deformation (Proben Iv1, Iv1b, Iv2), zum anderen um zwei Meta-Gabbros (Mg1b, Mg2) mit einem vergleichbaren Deformationsgrad und ähnlicher Textur des Mineralbestands, jedoch unterschiedlichen Rissdichten. In beiden Fällen werden ausgewählte Gesteinseigenschaften und ihre Richtungsabhängigkeit zur bevorzugten Orientierung der Gefügeelemente in Beziehung gesetzt.

\subsection{Sesia-Lanzo-Mylonite}

Die aus drei Proben bestehende Serie der Sesia-Lanzo-Mylonite stammt aus einer mylonitischen Scherzone, die der Sesia-Lanzo-Einheit in Norditalien zugehörig ist. Die Gesteine zeichnen sich durch einen unterschiedlichen Grad der mylonitischen Deformation aus (Abb.7-1). Iv1 ist der nahezu undeformierte Protomylonit, Iv1b ein Mylonit und Iv2 ein Ultramylonit. Die Proben gehören zu den Makrogefüge-Gruppen II (Iv1) und III (Iv1b/Iv2). Die progressive Mylonitisierung bei identischer mineralogischer Zusammensetzung bietet die Möglichkeit, die resultierende Entwicklung der technischen Eigenschaften Druck-, Spaltzug-, Abriebfestigkeit, dynamischer E-Modul, statischer E-Modul und thermische Dehnung parallel zur Gefügeentwicklung zu erfassen. Sowohl die Gefügeelemente und ihre räumliche Anordnung als auch die in den drei Hauptgefügerichtungen (x-, y-, z-Richtung) ermittelten technischen Eigenschaften werden zum Probenreferenzsystem (vgl. Kap.2.1.1) in Bezug gesetzt. 


\subsubsection{Mineralogische Zusammensetzung und Gefüge}

Die Sesia-Lanzo-Mylonite bestehen aus 35 Vol.\% Quarz, 27 Vol.\% Plagioklas (Albit), 15 Vol.\% Muskovit, 6 Vol.\% Chlorit, 6 Vol.\% Klinozoisit, 5 Vol.\% Kalifeldspat und 4 Vol.\% Granat. Akzessorien sind Apatit und Zirkon. Makroskopisch grün gefärbte Aggregate bestehen aus Chlorit, Albit, Granat und Serizit. Nach Techmer (1996) handelt es sich dabei um alterierte Kalifeldspäte.

Der Protolith Iv1 zeigt ein dominantes L-Gefüge, dessen deutliches Linear von gelängten Quarzkristallen und mikrokristallinen Aggregaten definiert wird. Die Foliation ist schwach ausgeprägt und nur anhand von leicht geplätteten Quarzkristallen zu erkennen. Der Mylonit Iv1b und der Ultramylonit Iv2 zeigen makroskopisch ein S-L-Gefüge. Die mit zunehmender Mylonitisierung engständigere Foliation wird sowohl durch die bevorzugte Orientierung von Mineralen und Korngrenzen als auch durch einen deutlichen (Material)-Wechsel von Quarz/Albit- und Chlorit/Klinozoisit-reichen Lagen gebildet (Abb.7-1).

IV1

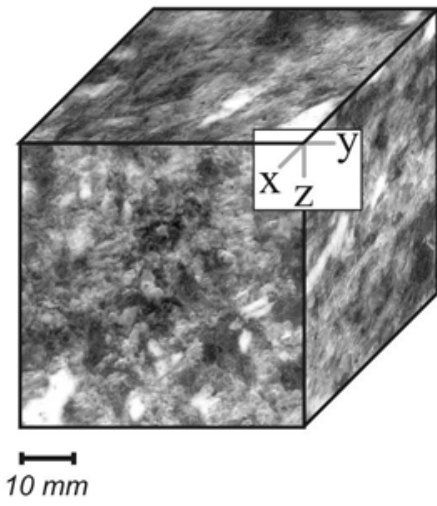

zunehmende mylonitische Deformation

Protomylonit
IV1b
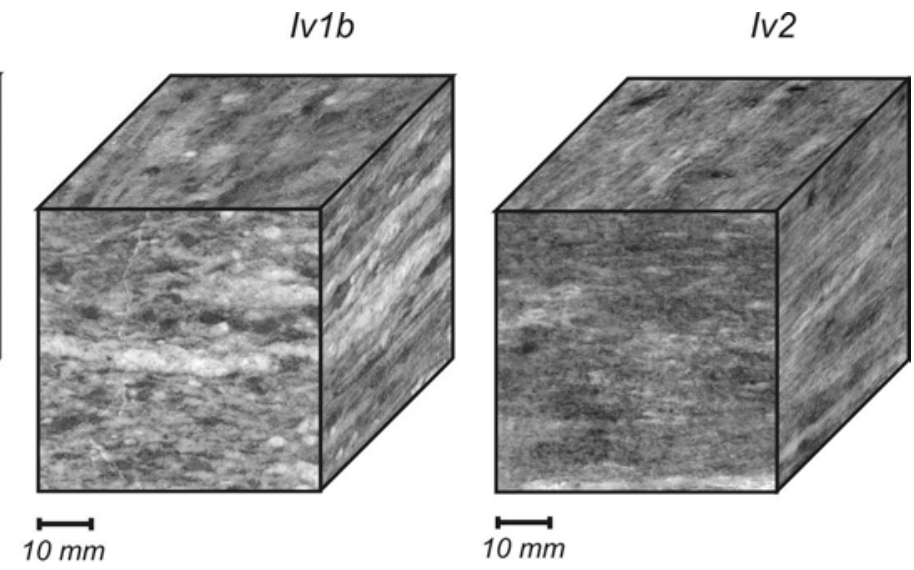

Ultramylonit

Abb.7-1: Sesia-Lanzo-Mylonite (granodioritische Zusammensetzung). Würfelscans. Iv1 Protomylonit. Iv1b - Mylonit. Iv2 - Ultramylonit.

Die Gefügeentwicklung ist begleitet von einer Korngrößenreduktion. In der Probe Iv1 kommen Quarz und Feldspat in Kristallen mit einer durchschnittlichen Korngröße von 1 bis 3 mm vor. Dabei zeigen die Quarze mit Subkornbildung und Korngrenzenmigration typische Erscheinungsformen der dynamischen Rekristallisation. Die Kalifeldspäte sind in der Regel bruchhaft verformt. Die grünen Aggregate haben einen Durchmesser von 3-4 mm, wobei Albit, Chlorit und Sericit als Teil dieser Aggregate sehr feinkörnig (1-2 $\mu \mathrm{m})$ ausgebildet sind. Außerhalb der Aggregate liegen diese Minerale in einer Korngröße von 100-150 $\mu \mathrm{m}$ vor. Die myonitische Deformation führt bei Iv2 zu einem annähernd equigranularen Gefüge mit Korngrößen zwischen 100-300 $\mu \mathrm{m}$ (Abb.7-2). Das Vorkommen von großen Quarz- und 
Feldspatkörnern nimmt von Iv1 bis Iv2 ab, die mikrokristallinen Aggregate werden stark geplättet und sind in Lagen konzentriert (Iv1b, Iv2).

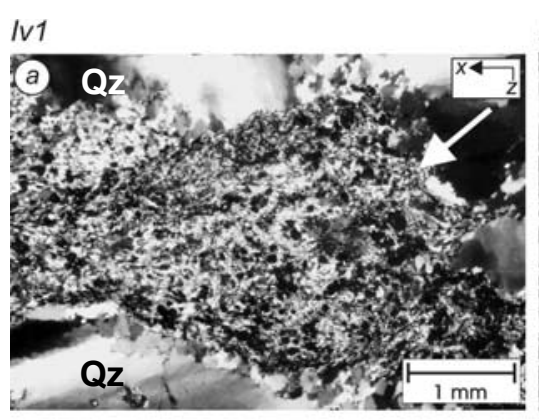

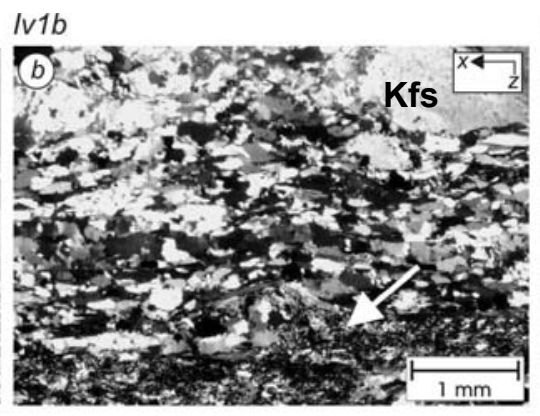

zunehmende mylonitische Deformation

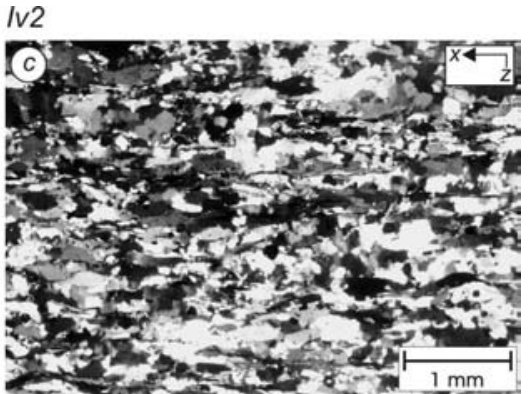

$1 \mathrm{~mm}$

Abb.7-2: Dünnschliffphotos der Sesia-Lanzo-Mylonite im xz-Schnitt; gekreuzte Polarisatoren. Mikrokristalline Aggregate sind mit weißen Pfeilen markiert. (a) Iv1. (b) Iv1b. (c) Iv2. Qz - Quarz. Kfs Kalifeldspat.

Die Dichte der Proben liegt zwischen $2,74 \mathrm{~g} / \mathrm{cm}^{3}$ und 2,79 g/ $\mathrm{cm}^{3}$ und die Porosität zwischen 0,32 Vol.\% und 0,47 Vol.\%. Das Maximum der Porenradien ist zwischen 0,01 $\mu \mathrm{m}$ und 0,1 $\mu \mathrm{m}$ zu finden.

\subsubsection{Textur und Mikrorisse}

Für die Minerale Muskovit, Quarz und Albit wurden Texturuntersuchungen (vgl. Kap.2.3.1) durchgeführt. Für die Verteilung der (001)-Pole des Albit ergibt sich unabhängig vom Mylonitisierungsgrad ein undeutliches Texturmuster mit einer niedrigen maximalen Intensität von 1,08 mrd. Die Quarztextur (c-Achse) zeigt deutlich ausgeprägte Verteilungsmuster. Für die Probe Iv1 bilden die c-Achsen einen Kreuzgürtel, wohingegen Iv1b und Iv2 Gürteltexturen aufweisen (Strohmeyer \& Siegesmund 2002). Die maximalen Intensitäten sind auch hier verhältnismäßig gering (1,82 mrd für die Probe Iv2), so dass ein Einfluss der Quarzund der Albit-Textur auf die technischen Gesteinseigenschaften nicht zu erwarten ist. Eine wesentlich größere Bedeutung kommt vermutlich der bevorzugten Orientierung der Muskovite zu. Die maximale Intensität der (001)-Pole steigt parallel zur zunehmenden Mylonitisierung von 5,54 mrd (Iv1) über 12,25 mrd (Iv1b) auf 17,56 mrd (Iv2). Die Probe Iv1 weist eine Gürteltextur mit einem Maximum auf, dass mit $\mathrm{x}$ als Rotationsachse einen Winkel von $30^{\circ}$ mit z einschließt (Abb.7-3). Iv1b und Iv2 zeigen schwach entwickelte Gürtel mit starken Maxima der (001)-Pole parallel zur z-Richtung der Probenreferenzsystems.

Die Proben Iv1b und Iv2 weisen ein fast orthogonales Mikroriss-System auf, welches annähernd senkrecht zu den Hauptgefügeachsen (x-, y-, z-Richtung) und damit etwa parallel 
zu den xy-, xz- und yz-Flächen ausgerichtet ist. Die Rissscharen der Probe Iv1 sind mit denen von Iv1b und Iv2 vergleichbar, nur dass sie mit $30^{\circ}$ um die x-Achse rotiert sind (Abb. $\left.7-3\right)$. Es kommen verheilte, mineralisierte und offene Mikrorisse vor (Anhang II). Die Entwicklung der offenen Mikroriss-Systeme in Abhängigkeit von der Gefügeentwicklung spiegelt sich in den $\Delta \mathrm{Vp}$-Verteilungen wider.
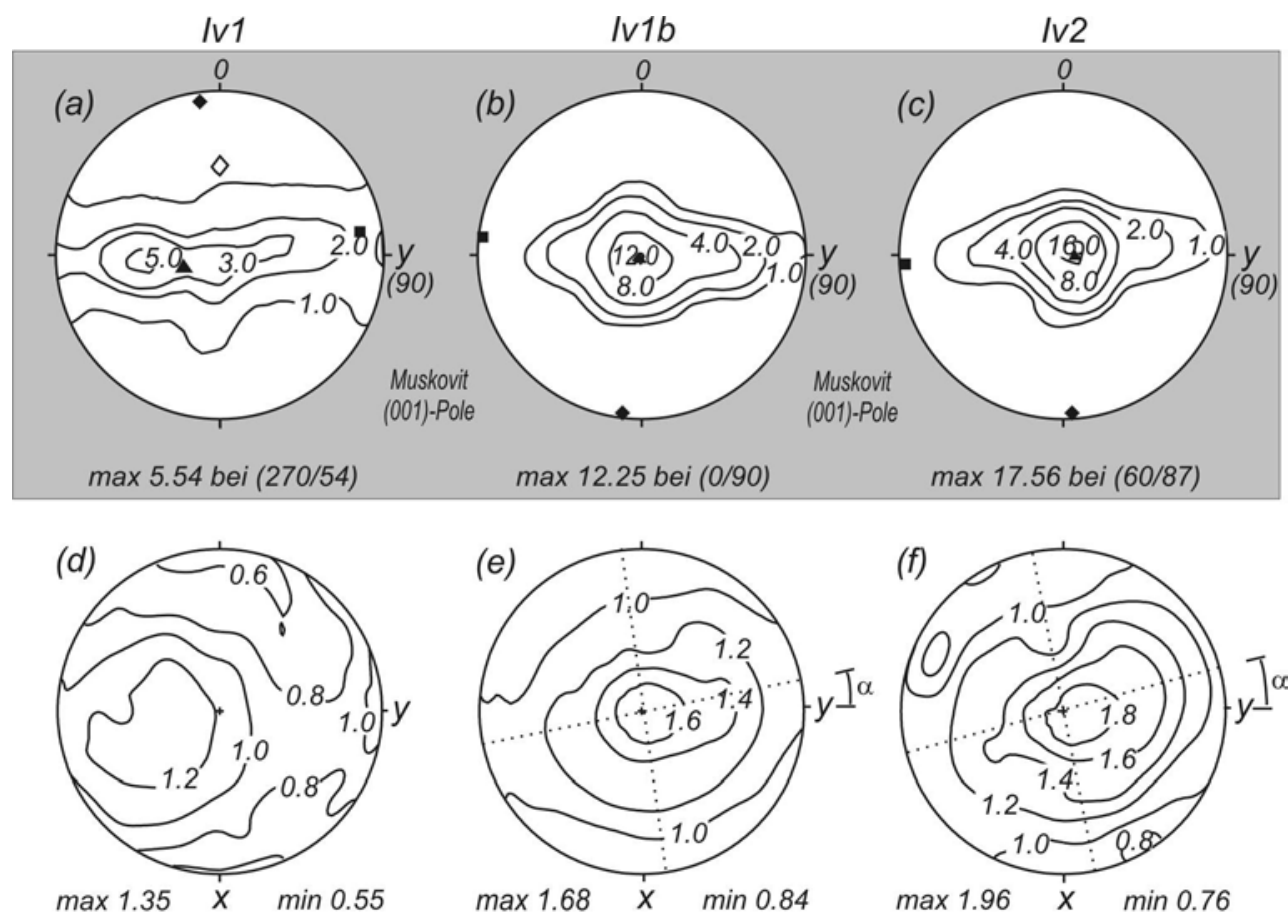

(g)

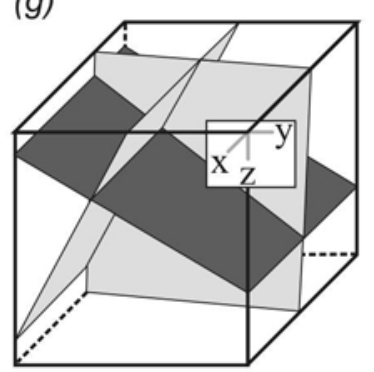

(h)

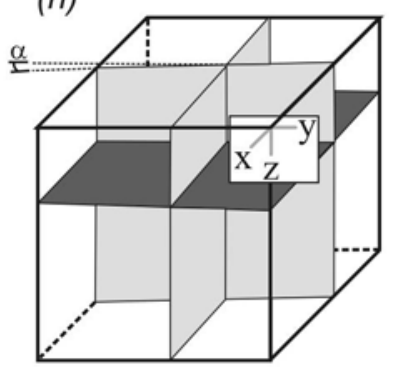

(i)

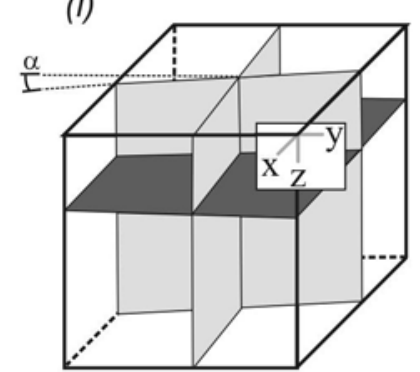

zunehmende mylonitische Deformation

Abb.7-3: Muskovit-Textur, $\Delta V p$ und offene Mikrorisse. (a-c) Verteilung der Muskovit (001)-Pole [mrd]; Eigenvektoren der Textur: Rauten - min; Quadrate - mittel; Dreiecke - max. (d-f) Aus der VpMessung der wassergesättigten und trockenen Kugel berechnete $\Delta$ Vp-Verteilung (vgl. Kap.2.4.5.1). (g-h) Bevorzugte Orientierung der präexistierenden Mikroriss-Systeme; dunkelgraue Flächen zeigen das Maximum der Muskovit (001)-Spaltflächen an.

Die Lage der höchsten $\Delta \mathrm{Vp}$-Werte kennzeichnet dabei die höchste Rissdichte offener Mikrorisse mit einer Orientierung senkrecht dazu (vgl. Kap.2.4.5.1). Diese Werte treten stets parallel zum Maximum der Muskovit (001)-Pole auf. Das dominante Mikroriss-System bilden daher die offenen Spaltflächen ((001)-Flächen) des Muskovit. Folglich nimmt die bevorzugte 
Orientierung der offenen Mikrorisse analog zur Intensivierung der Muskovit-Textur und analog zum steigenden Grad der Mylonitisierung zu. Die im Vergleich zu den Glimmertexturen-Hauptachsen leicht verschobenen Muster der offenen Riss-Systeme (siehe Muster der $\Delta \mathrm{Vp}$-Verteilungen) zeigen den, wenn auch untergeordneten, Einfluss anderer offener Mikrorisse auf die Gesamtverteilungsmuster (Abb.7-3). Hier sind insbesondere die offenen Mikrorisse in Quarz und in Kalifeldspat zu nennen, deren Rissdichte mit zunehmender mylonitischer Deformation abnimmt.

\subsubsection{Technische Gesteinseigenschaften}

Für die verschiedenen technischen Gesteinseigenschaften erfolgt die Richtungsindizierung (x, y-, z-Richtung) gemäß der Definitionen aus Kap.2.

Die einaxiale Druckfestigkeit liegt zwischen $128 \mathrm{MPa}$ (Probe Iv1b - y-Richtung) und 225 MPa (Probe Ivlb - x-Richtung). Mit zunehmender Mylonitisierung geht keine eindeutige Entwicklung der Druckfestigkeiten einher. Die Anisotropie steigt von 9\% (Iv1) auf 43\% (Iv1b), nimmt dann jedoch wieder ab (Iv2 - 23\%). Die x-Richtung zeigt durchweg die höchsten und die y-Richtung die niedrigsten Druckfestigkeitswerte (Abb.7-4; vgl. auch Druckfestigkeiten für S-L-Gefügetypen - Kap.8.2.1).
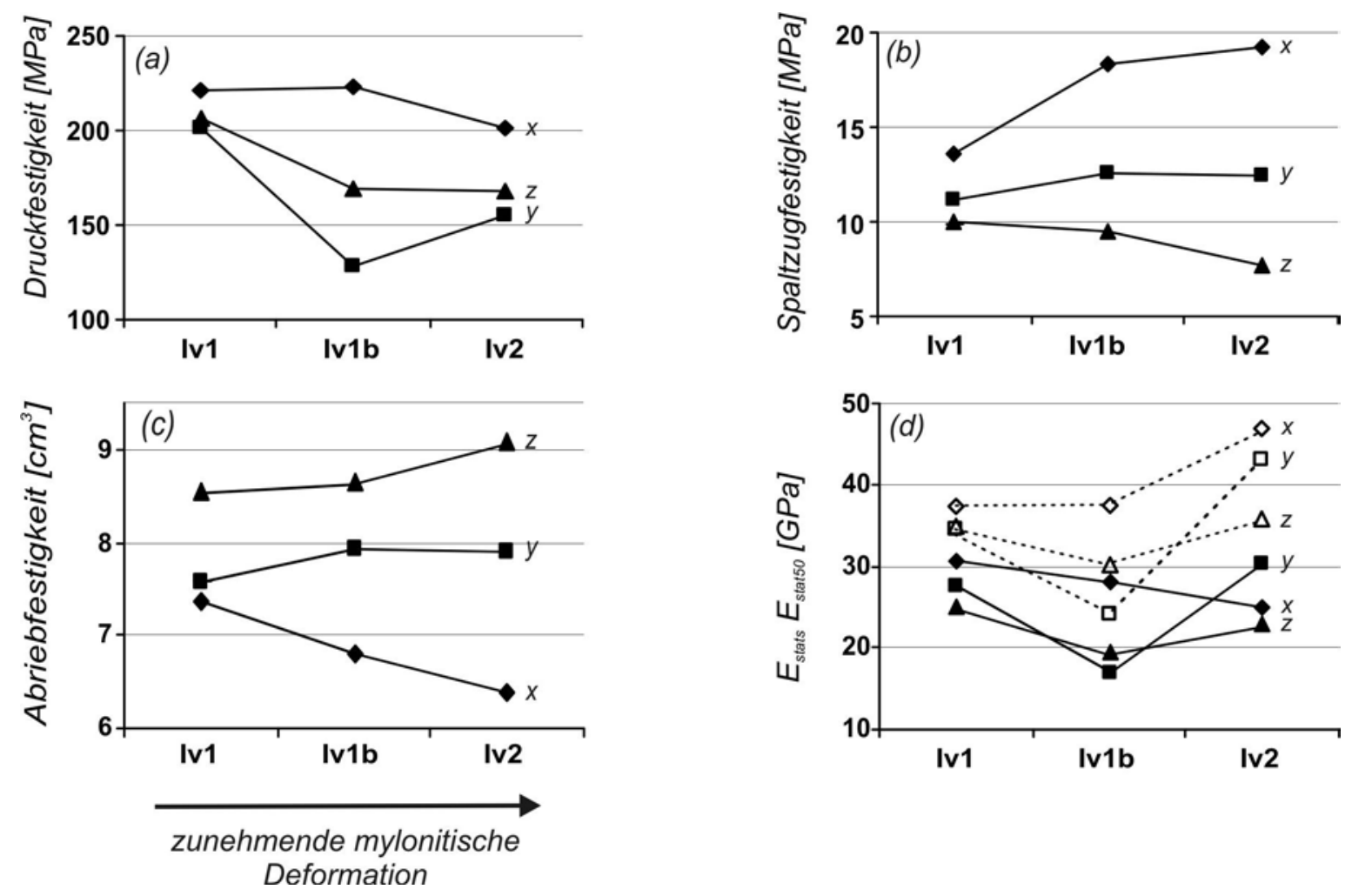

Abb.7-4: Mechanische Parameter und statischer E-Modul in Abhängigkeit von der Gefügeentwicklung. Die Richtungen sind angegeben. (a) Druckfestigkeit. (b) Spaltzugfestigkeit. (c)

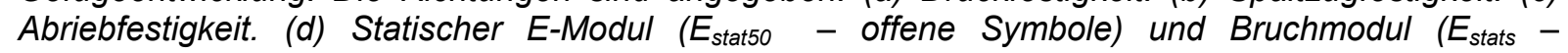
geschlossene Symbole). Fehlerangaben in Anhang I. 
Das Bruchmuster für die x-Richtung verändert sich parallel zur Gefügeentwicklung von einem konischen $\mathrm{zu}$ einem dachförmigen (Abb.7-5; vgl. auch Bruchmuster für S-LGefügetypen - Kap.8.2.1). Dabei ist das Muster bezüglich der Probe Iv2 nicht deutlich ausgeprägt und beinhaltet eine Vielzahl von makroskopischen Zugrissen und Knickungen, die bei den Proben Iv1 und Ivlb nicht zu beobachten sind.

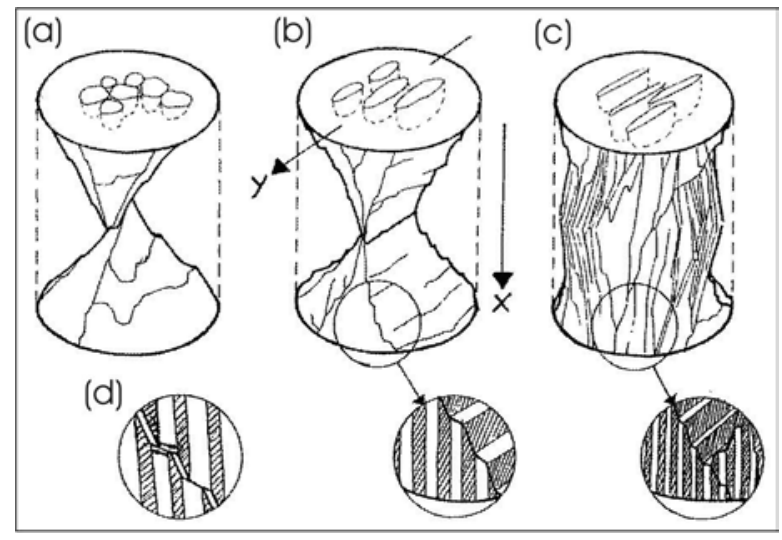

Abb.7-5: Bruchmuster der x-Richtung beim Druckversuch in Abhängigkeit von der Gefügeentwicklung. (a) Protomylonit Iv1. (b) Mylonit Iv1b. (c) Ultramylonit Iv2. (d) Rissausbreitung beim Druckversuch; Pfeile zeigen Verschiebungsrichtung (vgl. Kap.8.2.1).

Die Spaltzugfestigkeiten decken den Bereich zwischen 7,7 MPa (Iv2 - z-Richtung) und 19,3 MPa (Iv2 - x-Richtung) ab. Die Anisotropie der Spaltzugfestigkeiten nimmt mit steigender Gefügeanisotropie von 26\% (Iv1) über 48\% (Iv1b) auf 60\% (Iv2) zu. Die x-Richtung zeigt stets die höchsten Spaltzugfestigkeiten mit einer Festigkeitserhöhung bei zunehmendem Mylonitisierungsgrad (Abb.7-4). Dahingegen ist die z-Richtung gegenüber den Zugspannungen am mechanisch schwächsten mit einer Abnahme der Festigkeit parallel zur Gefügeentwicklung. Die y-Richtung weist mittlere Werte und für alle drei Proben annähernd konstante Werte auf.

Die Abriebfestigkeiten variieren zwischen $6,4 \mathrm{~cm}^{3}$ (Iv2 - x-Richtung) und 9,14 $\mathrm{cm}^{3}$ (Iv2 - zRichtung). Die Anisotropie nimmt mit zunehmender Mylonitisierung von 14\% (Iv1) auf 30\% (Iv2) zu. Die x-Richtung zeigt die höchste und die z-Richtung die niedrigste Abriebfestigkeit, wobei parallel zur Gefügeentwicklung die x-Richtung mechanisch stärker und die z-Richtung mechanisch schwächer wird $(\boldsymbol{A b b} .7-4)$.

Der dynamische E-Modul ( $\mathrm{E}_{0}$ ) liegt zwischen 24,4 GPa (Iv2 - z-Richtung) und 57,2 GPa (Iv2 - x-Richtung). Die Anisotropie des E-Moduls steigt mit zunehmender Gefügeanisotropie von 13\% (Iv1) über 40\% (Iv1b) auf 57\% (Iv2). Die x-Richtung mit den stets höchsten E-Moduln zeigt einen Anstieg der $\mathrm{E}_{0}$-Werte und die z-Richtung mit den durchweg niedrigsten E-Moduln eine Verringerung der $\mathrm{E}_{0}$-Werte in Abhängigkeit von der Gefügeentwicklung (Abb.7-6). 
Die Werte für den statischen E-Modul $\left(\mathrm{E}_{\text {stat50 }}\right)$ bei $50 \%$ der Bruchlast und dem Bruchmodul ( $\left.E_{\text {stats }}\right)$ liegen zwischen $E_{\text {stat } 50}=24,0 \mathrm{GPa} / \mathrm{E}_{\text {stats }}=19,3 \mathrm{GPa}\left(\mathrm{Iv} 1 \mathrm{~b}-\mathrm{y}\right.$-Richtung) und $\mathrm{E}_{\text {stat } 50}=47,1$ GPa (Iv2 - x-Richtung)/ $E_{\text {stats }}=30,9$ GPa (Iv2 - y-Richtung) und zeigen keine einheitlichen Entwicklungstrends in Abhängigkeit vom Mylonitisierungsgrad. Die Anisotropie für $\mathrm{E}_{\text {stat50 }}$ und $E_{\text {stats }}$ ist für die Probe Iv1 am geringsten (6\%/16\%) und für die Probe Iv1b am höchsten (37\%/40\%). Iv2 liegt mit Werten von 24\% und 23\% dazwischen. Auffällig sind die analog zu den Druckfestigkeiten niedrigsten $\mathrm{E}_{\text {stat50 }}$ bzw. $\mathrm{E}_{\text {stats }}$-Werte (24,0 GPa und 19,3 GPa) für die yRichtung der Probe Iv1b (Abb. 7-4).
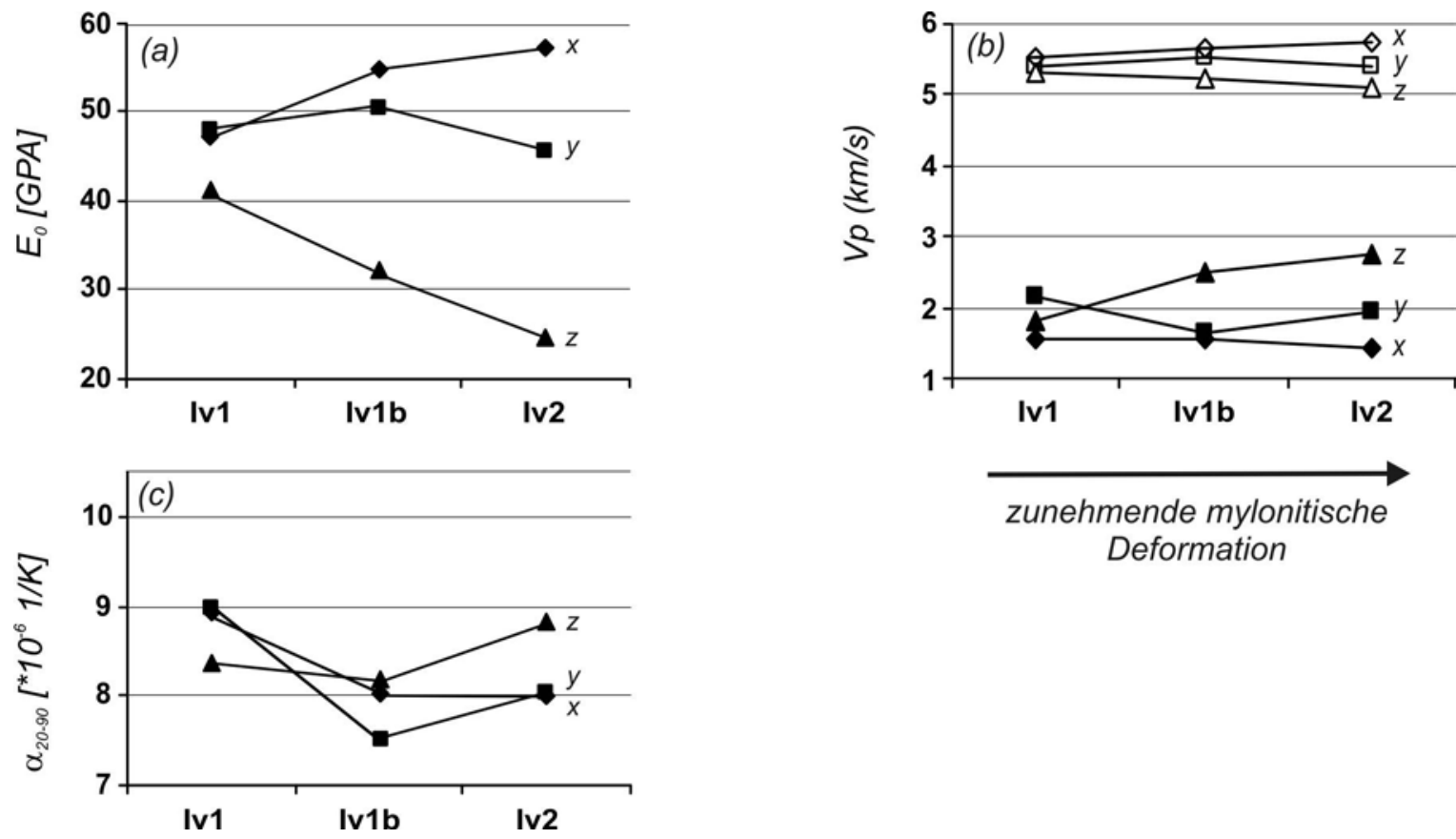

Deformation

Abb.7-6: Dynamische elastische Eigenschaften und thermische Dehnung in Abhängigkeit von der Gefügeentwicklung. Die Richtungen sind angegeben. (a) Dynamischer E-Modul. (b) Kompressionswellengeschwindigkeiten (Vp). Offene Symbole - aus Messung der wassergesättigten Kugel; geschlossene Symbole - aus der Messung der wassergesättigten und der trockenen Kugel berechnete $\triangle \mathrm{Vp}$. (c) Thermischer Ausdehnungskoeffizient ( $\left.\alpha_{20-90}\right)$. Fehlerangaben in Anhang I.

Der thermische Ausdehnungskoeffizient $\left(\alpha_{20-90}\right)$ rangiert zwischen 9,00*10 $0^{-6} 1 / \mathrm{K}(\mathrm{Iv} 1-\mathrm{X}$ Richtung) und $7,46 * 10^{-6} 1 / \mathrm{K}$ (Ivlb - y-Richtung). Die Gefügeentwicklung innerhalb des Probensatzes scheint keinen signifikanten Einfluss auf die Richtungsabhängigkeit der thermischen Dehnung zu haben. Die Anisotropie ist mit etwa 8\% für alle Proben annähernd gleich. Außerdem zeigt keine der drei Hauptgefügerichtungen einen Trend zu höheren oder niedrigeren Ausdehnungskoeffizienten parallel zur Gefügeentwicklung. Zusätzlich weist keine der betrachteten Richtungen für alle drei Proben den Maximal- oder Minimalwert auf (Abb.7-6). 


\subsubsection{Diskussion}

\subsubsection{Mechanische und statische elastische Eigenschaften}

Die mechanischen Eigenschaften und deren Richtungsabhängigkeit sind maßgeblich von der max. Intensität der Glimmer (001)-Pole und der bevorzugten Orientierung der offenen Mikroriss-Systeme abhängig (vgl. Kap.6.3). Die Glimmer sind bezüglich ihres mechanischen Verhaltens die anisotropesten Minerale (Anisotropie der Spaltbarkeit $\mathrm{A}_{\mathrm{ISPM}}=4$ - vgl. Kap.4.2) und bilden mit ihrer höchst vollkommenen (Spaltbarkeit 5 nach Rösler 1991) spaltenden (001)-Basisfläche das wohl mechanisch schwächste Glied nach den offenen Mikrorissen. Dieses kristallographische Element zeigt Aktivierungsenergien für Versetzungsgleiten von $87 \mathrm{~kJ} / \mathrm{mol}$ (Biotit -Kronenberg et al. 1990) und 47kJ/mol (Muskovit - Mares \& Kronenberg 1993), die sehr niedrig sind gegenüber denen stärkerer Silikate wie z.B. Plagioklas (237kJ/mol - Shelton 1981; Tullis et al. 1991). Auch die spezifische Bruchflächenenergie der Muskovit (001)-Fläche ist mit 0,38 J/m² gegenüber z.B. der Orthoklas (001)-fläche mit 7,77 $\mathrm{J} / \mathrm{m}^{2}$ (Atkinson \& Meredith 1987) sehr gering. Die Glimmer (001)-Flächen werden aus diesem Grunde bei den mechanischen Tests bevorzugt aktiviert (vgl. Kap.6.3.5). Folglich lässt sich die Entwicklung der mechanischen Eigenschaften mit der parallel zur Mylonitisierung beobachtbaren Intensivierung der Muskovit-Textur erklären. Deren Einfluss wird durch die bevorzugte Orientierung der offenen Mikrorisse parallel zu den (001)-Flächen noch verstärkt. Immer mehr (001)-Flächen und damit auch offene Glimmerspaltflächen konzentrieren sich in einer Raumlage parallel zur Foliation und verursachen einen Trend zu niedrigeren Spaltzugund Abriebfestigkeiten in z-Richtung. Die x-Richtung wird hingegen mechanisch stärker, was sich nicht vollständig mit der Entwicklung der Muskovit-Textur, wohl aber mit der Reduktion der offenen Mikrorisse in Quarz und Feldspat mit zunehmendem Mylonitisierungsgrad begründen lässt. Die Druckfestigkeiten mit ihrer gefügeabhängig uneinheitlichen Entwicklung stehen unter dem Einfluss einer Vielzahl sich überlagernder Faktoren. Wahrscheinlich ist dieses Verhalten durch eine Kombination von Korngrößenreduktion und zunehmend engständigerer Foliation sowie der Muskovit-Textur und der bevorzugten Orientierung der offenen Mikrorisse bedingt. Grundsätzlich gilt auch hier: Das Angebot an potenziell mechanisch schwachen Flächen parallel zu den Flächen der maximalen Scherspannungen bestimmt die Druckfestigkeit. Daraus ergibt sich die beobachtbare niedrigere Druckfestigkeit der y-Richtung von Probe Iv1b und Iv2 (128 MPa/155 MPa) gegenüber den stärkeren x- (225 $\mathrm{MPa} / 200 \mathrm{MPa})$ und $z$-Richtungen $(168 \mathrm{MPa} / 172 \mathrm{MPa})$, die typisch ist für glimmerhaltige Gesteine mit einem S-L-Gefüge (vgl. Kap.8.2.1). Ergebnisse von Gottschalk et al. (1990), die ein Maximum der Druckfestigkeiten parallel zur Lineation feststellten, gehen in die gleiche 
Richtung. Brosch et al. 2000 fanden das Maximum der Druckfestigkeiten parallel zur zRichtung, also senkrecht zur Foliation, untersuchten jedoch einen Gneis mit einem Punktmaximum der Glimmer(001)-Pole. Das lässt auf einen S-Gefüge-Typ schließen, bei dem die z-Richtung der Druckfestigkeit, dem Modell des „Angebots schwacher Flächen“ folgend, die höchsten Druckfestigkeitswerte aufweist (vgl. Kap.8.2.1).

Die Entwicklung der statischen elastischen Eigenschaften verhält sich beinahe analog zu der der Druckfestigkeiten. Die Parameter Druckfestigkeit und $\mathrm{E}_{\text {stat50}} / \mathrm{E}_{\text {stats }}$ werden aus dem Druckversuch ermittelt, so dass sowohl die beim Versuch auftretenden Spannungen und Prozesse, als auch die Abhängigkeit vom Gefüge sehr ähnlich sind. Ein „weicheres“ Spannungs-Dehnungs-Verhalten und damit ein niedrigerer E-Modul tritt bei einem höheren Angebot von mechanisch schwachen Flächen parallel zur Fläche der maximalen Scherspannungen auf, was auch zu niedrigeren Druckfestigkeiten führt und daraus folgend eine deutliche Korrelation zwischen den statischen elastischen Eigenschaften und den Druckfestigkeiten bedingt (vgl. Kap.8.3.2). Zu ähnlichen Ergebnissen kommen Christoffersen \& Kronenberg (1993) und Shea \& Kronenberg (1993), die für eine Orientierung des Maximums der Glimmer (001)-Flächen parallel zum Maximum der Scherspannungen ein mehr „duktiles“ Spannungs-Dehnungs-Verhalten feststellten.

\subsubsection{Dynamischer E-Modul und thermische Dehnung}

Die Richtungsabhängigkeit des dynamischen E-Moduls unter Normaldruck $\left(\mathrm{E}_{0}\right)$ ist stark von der Textur anisotroper Minerale und der bevorzugten Orientierung von offenen Mikrorissen abhängig. Der Muskovit ist mit einer Anisotropie des dynamischen E-Moduls von 69\% das Mineral mit der deutlichsten Richtungsabhängigkeit der elastischen Eigenschaften (Abb.7-7). Senkrecht zur (001)-Fläche und damit parallel zu den (001)-Polen beträgt der E-Modul 54,1 GPa und senkrecht dazu 178 GPa (Gebrande 1982). Ein zunehmender Grad der Einregelung der Glimmer (001)-Flächen parallel zur Foliation bedingt folglich eine Entwicklung hin zu niedrigeren E-Moduln senkrecht zur Foliation (z-Richtung) und eine Erhöhung der $\mathrm{E}_{0}$-Werte in der Foliationsebene (aufgespannt durch die x- und y-Richtung). Dieser Effekt ist umso deutlicher ausgeprägt, je höher der Volumenanteil von Glimmern im Gestein ist. Die innerhalb des Probensatzes für die x- und z-Richtung deutlich erkennbare Entwicklung kann durch die bevorzugte Orientierung von offenen Mikrorissen intensiviert, aber auch destruktiv überlagert werden. Offene Mikrorisse, die senkrecht zur Durchschallungsrichtung orientiert, den E-Modul am stärksten herabsetzen (Hudson 1981 - vgl. Kap.6.4.1.5), wirken in die gleiche Richtung auf den E-Modul wie die Glimmer mit einer identischen Raumlage der 
Basisfläche. Da das dominante offene Riss-System den Glimmerspaltflächen folgt, ist die Erniedrigung des E-Moduls in z-Richtung durch einen kombinierten Einfluss von Intensivierung der Glimmertextur und damit einhergehend stärkerer bevorzugter Orientierung der offenen Mikrorisse begründbar. Die uneinheitliche Entwicklung der y-Richtung kann nicht direkt an die Glimmertexturen gekoppelt werden. Eine mögliche Erklärung liefern Überlagerungsphänomene von der Veränderung der Glimmertextur mit der Reduktion der offenen Mikrorisse in Quarz und Feldspat aufgrund der fortschreitenden Mylonitisierung. Eine endgültige Aussage diesbezüglich ist jedoch nicht möglich.
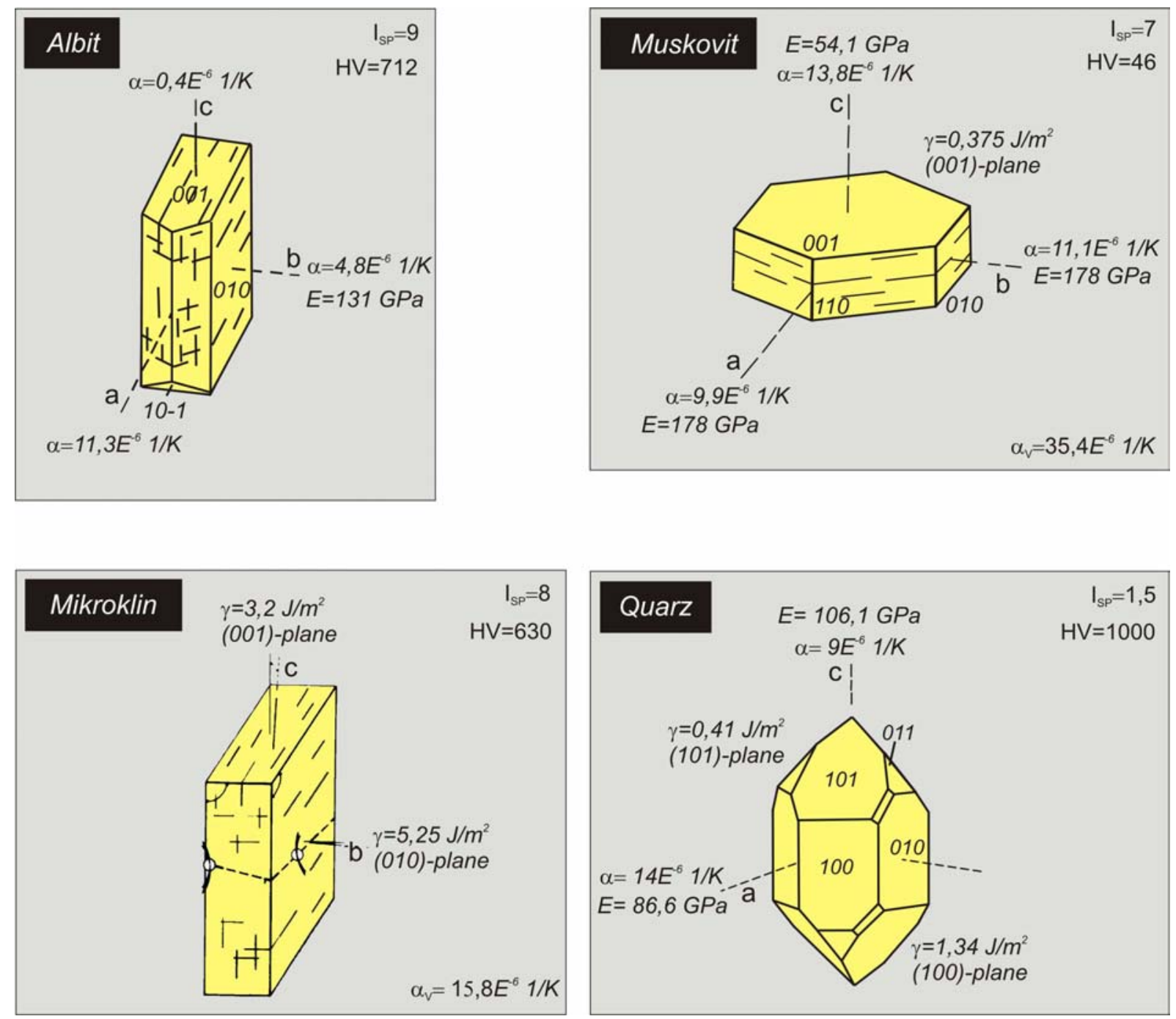

Abb.7-7: Kristallhabitus und die Richtungsabhängigkeit physikalischer Eigenschaften für (a) Albit, (b) Muskovit, (c) Mikroklin, (d) Quarz. ISP - Spaltbarkeitsindex (vgl. Kap.4.2); HV - Härte nach Vickers. $\gamma$ - spezifische Bruchflächenenergie [J/m²] (Daten aus Atkinson \& Meredith 1987); $\alpha$ - thermischer Ausdehnungskoeffizient im Temperaturbereich von 20 bis $90^{\circ} \mathrm{C}[1 / \mathrm{K}]$ (Daten aus Fei 1995, für Quarz aus Kleber 1990); $\alpha_{V}$ - thermischer Volumenausdehnungskoeffizient 20 bis $90^{\circ} \mathrm{C}$ [1/K] (Daten aus Fei 1995); E - dynamischer E-Modul unter Normalbedingungen [GPa] (Daten aus Gebrande 1982).

Völlig von der Gefügeentwicklung der Sesia-Lanzo-Mylonite abgekoppelt sind augenscheinlich die thermischen Ausdehnungskoeffizienten. Nach den Ergebnissen aus 
Kap.6.5 ist die Anisotropie der thermischen Dehnung hauptsächlich von der Textur der bezüglich ihrer Dehnungseigenschaften sehr anisotropen Minerale abhängig. Im Zuge der progressiven Mylonitisierung verändert sich in größerem Umfang nur die Textur der Muskovite; die Albit- und auch die Quarz-Textur bleiben in ihrer Intensität nahezu konstant. Zusammen mit Chlorit ist Muskovit zu 21 Vol.\% an der Gesteinszusammensetzung beteiligt und weist eine nur schwache Anisotropie der thermischen Dehnung von 28\% aus (vgl. Kap.4.4). Der höchste Ausdehnungskoeffizient ist dabei parallel zur c-Achse $\left(13,8^{*} 10^{-6} 1 / \mathrm{K}\right)$ und der niedrigste parallel zur a-Achse $\left(9,9 * 10^{-6} 1 / \mathrm{K}\right) \mathrm{zu}$ finden. Trotz der relativ niedrigen Anisotropie sollte die extreme Steigerung des Einregelungsgrads der Muskovit-(001)-Flächen zu einer messtechnisch erfassbaren Erhöhung der $\alpha_{20-90}$-Werte parallel zur z-Richtung und einer entsprechenden Tendenz zu niedrigeren $\alpha_{20-90}$-Werten in der Foliationsebene (xy-Ebene) führen. Im betrachteten Temperaturbereich wirkt jedoch der Prozess der Risspufferung (Kap.6.5.3) dieser Entwicklung entgegen. Mit zunehmender Mylonitisierung konzentrieren sich die offenen Risse in einer Raumlage nahe der Foliationsebene, was zu einer verstärkten Risspufferung in z-Richtung führt und damit niedrigere thermische Ausdehnungskoeffizienten verursacht. Somit können sich beide Effekte ausgleichen (was in Bezug auf die Sesia-LanzoMylonite vermutlich der Fall ist), und der zunehmende Grad der Deformation und der Gefügeanisotropie bringt daher keine signifikante richtungsabhängige Entwicklung der thermischen Ausdehnungskoeffizienten mit sich.

\subsubsection{Zusammenfassung}

Im Grundsatz ist die Anisotropie einer technischen Gesteinseigenschaft und deren Entwicklung in Abhängigkeit von einer progressiven Deformation aus der Entwicklung der Gefügemerkmale ableitbar, sofern folgende Fragen eindeutig beantwortet werden können:

1. Von welchen Gefügeelementen hängt die untersuchte Eigenschaft ab? Welche dieser Gefügeelemente sind im Gestein vertreten?

2. Welche Minerale des Mineralbestands sind bezüglich der technischen Eigenschaft anisotrop und welches kristallographische Element zeigt welchen Wert? Mit welchem Volumenanteil sind diese Minerale vertreten?

3. Welche Gefügeelemente verändern ihre bevorzugte Orientierung aufgrund der zunehmenden Deformation? (z.B. offene Mikrorisse; Textur von Mineralen) 
Stark anisotrope Minerale und Gefügeelemente mit einem starken Einfluss auf die betrachtete Eigenschaft verursachen, in Kombination mit einer starken Veränderung der bevorzugten Orientierung, eine deutliche richtungsabhängige Entwicklung der technischen Gesteinseigenschaften in Abhängigkeit von der Gefügeentwicklung.

\subsection{Meta-Gabbros}

Im Rahmen dieser Fallstudie werden zwei Meta-Gabbros (Mg1b, Mg2) hinsichtlich der Gefügeabhängigkeit der Spaltzugfestigkeiten untersucht. Die Spaltzugfestigkeiten wurden in 23 verschiedenen Richtungen bestimmt und die Ergebnisse zur Textur von Hornblende und Diopsid, der Kornformorientierung sowie der Rissdichte und bevorzugten Orientierung der offenen Mikrorisse ins Verhältnis gesetzt.

\subsubsection{Mineralogische Zusammensetzung und Gefüge}

Die ausgewählten Proben stammen aus einer Hochtemperatur-Scherzone bei Anzola/Norditalien und entsprechen der Probe DT46 von Dornbusch (1995). Sie weisen eine nahezu identische mineralogischen Zusammensetzung mit 44 Vol.\% Hornblende, 35 Vol.\% Plagioklas, 16 Vol.\% Klinopyroxen und 5 Vol.\% Orthopyroxen auf. Als Akzessorien kommen Granat, Ilmenit, Biotit und Titanit vor.
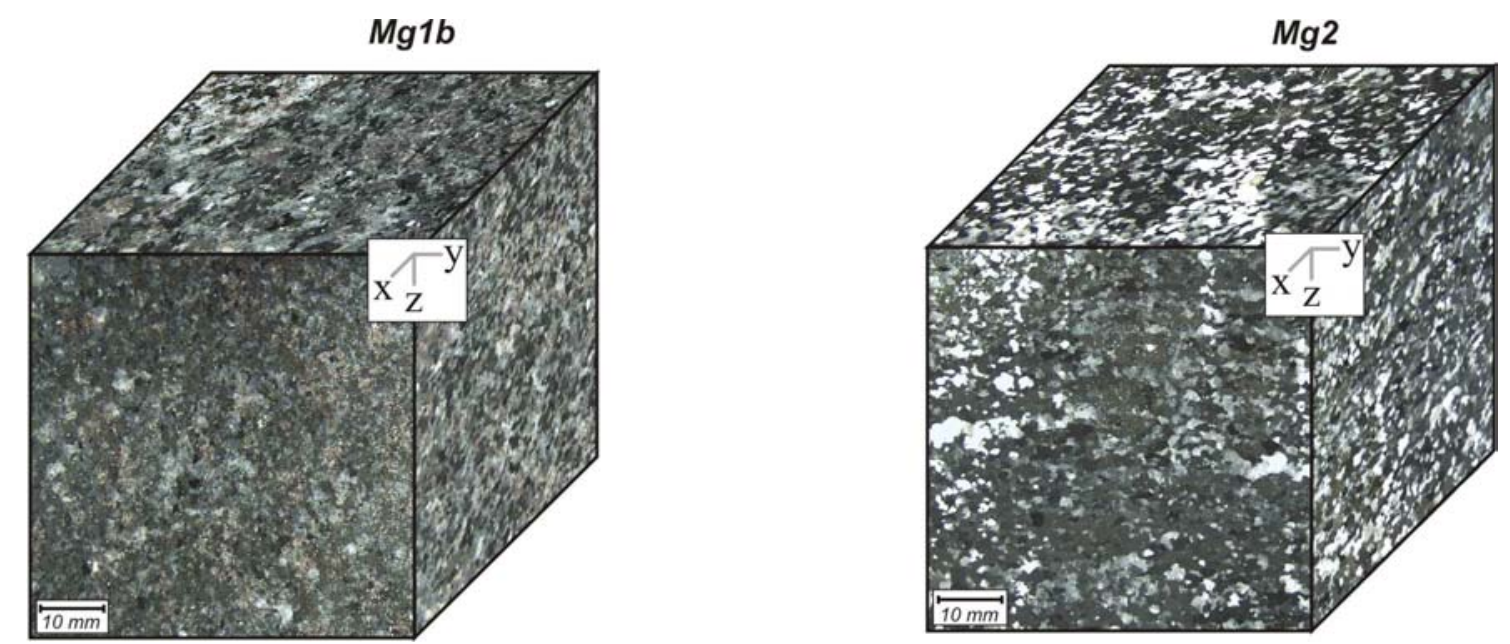

Abb.7-8: Meta-Gabbros von Anzola; Würfelscans. (a) Probe Mg1b. (b) Probe Mg2.

Das makroskopische Gefüge der Probe MG1b wird durch ein Linear dominiert, welches durch die Kornformregelung der hypideomorph ausgebildeten Minerale Hornblende, Plagioklas und Klinopyroxen gebildet wird. Im Unterschied dazu ist bei der Probe Mg2 die Foliation etwas 
deutlicher ausgeprägt und durch einen angedeuteten Lagenbau von hellen (plagioklasreicheren) und dunklen (hornblendereicheren) Bereichen bestimmt (Abb. 7-8).

(a)

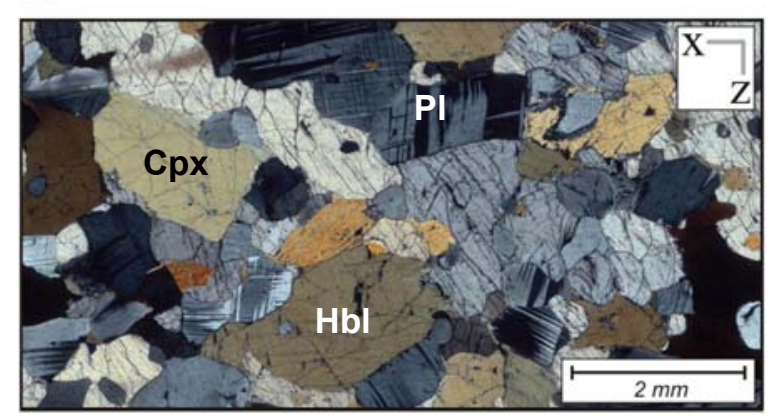

(b)

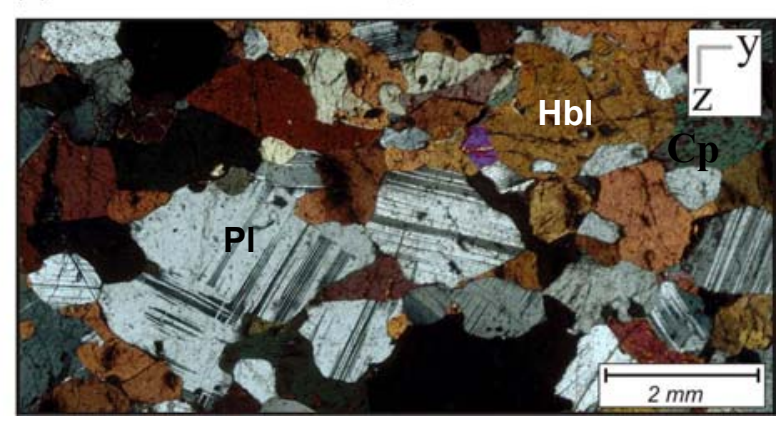

Abb.7-9: Dünnschliffscans der Meta-Gabbros von Anzola; gekreuzte Polarisatoren. (a) Probe Mg1b im xz-Schnitt. (b) Probe Mg2 im y-z-Schnitt. PI - Plagioklas. Hbl-Hornblende. Cpx - Klinopyroxen.

Beide Proben sind mit Korngrößen von 2-5 mm mittelkörnig, wobei Mg2 innerhalb dieses Bereiches zu etwas größeren Korngrößen tendiert. Die Korngrenzen sind generell gerade und zumeist geschlossen (Abb.7-9). Die Dichte liegt zwischen 3,06 g/ $\mathrm{cm}^{3}$ und 3,09 g/ $\mathrm{cm}^{3}$. Die Porosität beträgt 0,31 Vol.\% (Mg1b) bzw. 0,13 Vol.\% (Mg2), und der mittlere Porenradius rangiert für die Probe $\mathrm{Mg} 1 \mathrm{~b}$ bei $0,10 \mu \mathrm{m}$. Für die Probe $\mathrm{Mg} 2$ konnte dieser Wert nicht bestimmt werden.

\subsubsection{Textur}

Für die Probe Mglb wurden die Texturen für Hornblende, Klinopyroxen und Plagioklas per Neutron-Beugungs-Messungen und anschließender quantitativer Texturanalyse bestimmt. Zur Charakterisierung werden für Hornblende und Klinopyroxen die Polverteilungen der (001)Flächen und der (110)-Spaltflächen verwendet. Die Textur von Plagioklas ((001)-Pole) ist mit einer maximalen Intensität von 1,10 schwach und undeutlich (vgl. auch Dornbusch 1995) und wird nicht in die Analyse einbezogen. Das gleiche gilt für Orthopyroxen, dessen Volumenanteil mit 5\% gering und daher ein entscheidender Einfluss auf die Spaltzugfestigkeiten unwahrscheinlich ist, zumal die Orthopyroxene keine durchgreifenden Lagen im Gesteinsgefüge bilden. Da beide Proben aus einem strukturell ähnlichen Bereich der Probenlokation stammen und auch in ihrem Gefügebild sehr ähnlich sind, wird eine vergleichbare Texturierung des Mineralbestands angenommen und die Ergebnisse der Texturuntersuchungen von $\mathrm{Mg} 1 \mathrm{~b}$ auf Mg2 übertragen. Die Hornblende zeigt die mit Abstand stärkste Textur mit einer bevorzugten Orientierung der (001)-Pole annähernd parallel zum Linear (parallel x-Richtung). Die (110)-Pole zeigen eine Gürtelverteilung um das Linear, liegen also bevorzugt in der y-z-Ebene $(\boldsymbol{A b} \boldsymbol{b} .7-10)$. 

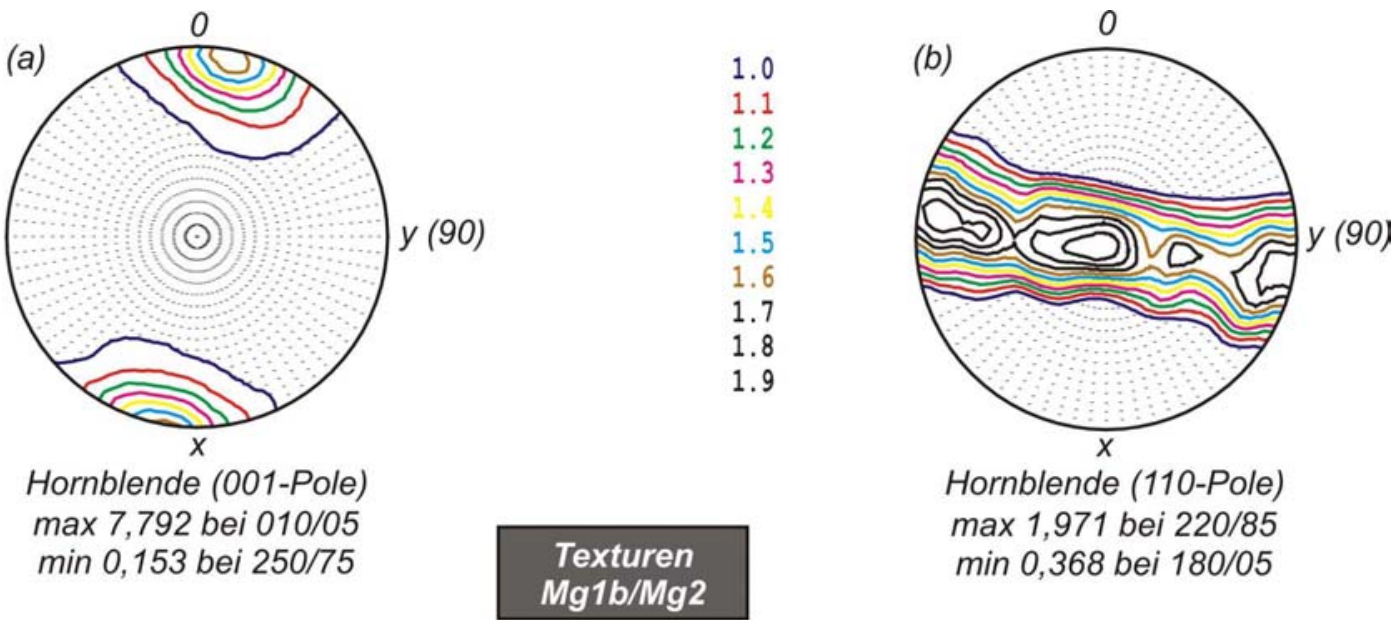

Hornblende (001-Pole) $\max 7,792$ bei $010 / 05$ $\min 0,153$ bei $250 / 75$

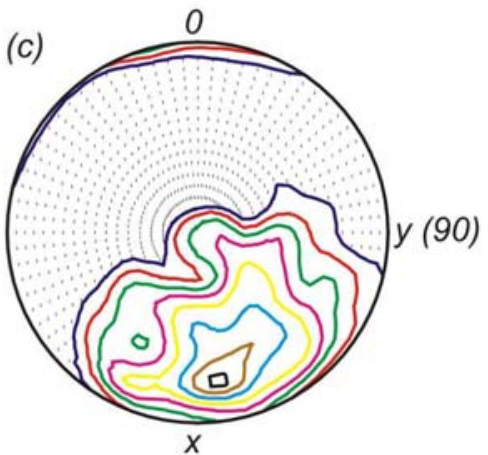

Diopsid (001-Pole)

$\max 1,356$ bei $080 / 20$ $\min 0,738$ bei $280 / 40$

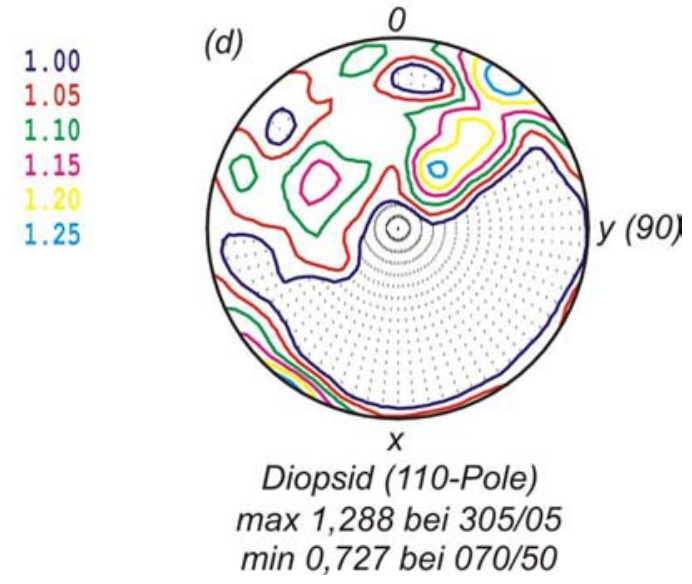

Abb.7-10: Hornblende- und Diopsid-Textur der Meta-Gabbros. Intensitäten in mrd. (a) Hornblende (001)-Pole. (b) Hornblende (110)-Pole. (c) Diopsid (001-Pole). (d) Diopsid (110)-Pole.

Die maximale Intensität der Hornblende-Textur beträgt 7,79 mrd für die (001)-Pole und 1,97 mrd für die (110)-Pole. Die Textur des Diopsid ist mit einer maximalen Intensität der (001)Pole von 1,36 mrd und der (110)-Pole von 1,29 mrd deutlich schwächer ausgeprägt (Abb.710). Hier liegt das Maximum der (001-Pole) ca. $20^{\circ}$ von der $x$-Achse entfernt und damit in der Nähe des Linears. Die (110)-Pole zeigen eine undeutliche Gürtelverteilung. Die Ergebnisse der Texturuntersuchungen stimmen qualitativ mit denen von Dornbusch (1995) überein. Die wenn auch nicht bedeutetenden Abweichungen der Intenstitätswerte liegen in den unterschiedlichen Untersuchungsmethoden (U-Tisch bei Dornbusch) begründet.

\subsubsection{Kornformregelung}

Die Ergebnisse der Kornformanalyse basieren auf Daten von Dornbusch (1995) für die Probe DT46 und werden auf die Proben $\mathrm{Mg} 1 \mathrm{~b} / \mathrm{Mg} 2$ angewandt. Sowohl Hornblende als auch Plagioklas zeigen eine deutliche Kornformanisotropie und Kornformregelung, die für 
Orthopyroxen und Diopsid weniger deutlich ausgeprägt ist. $\boldsymbol{A} \boldsymbol{b} \boldsymbol{b} \cdot \mathbf{7 - 1 1}$ zeigt die bevorzugte Korngrenzenorientierung aller Mineralbestandteile.

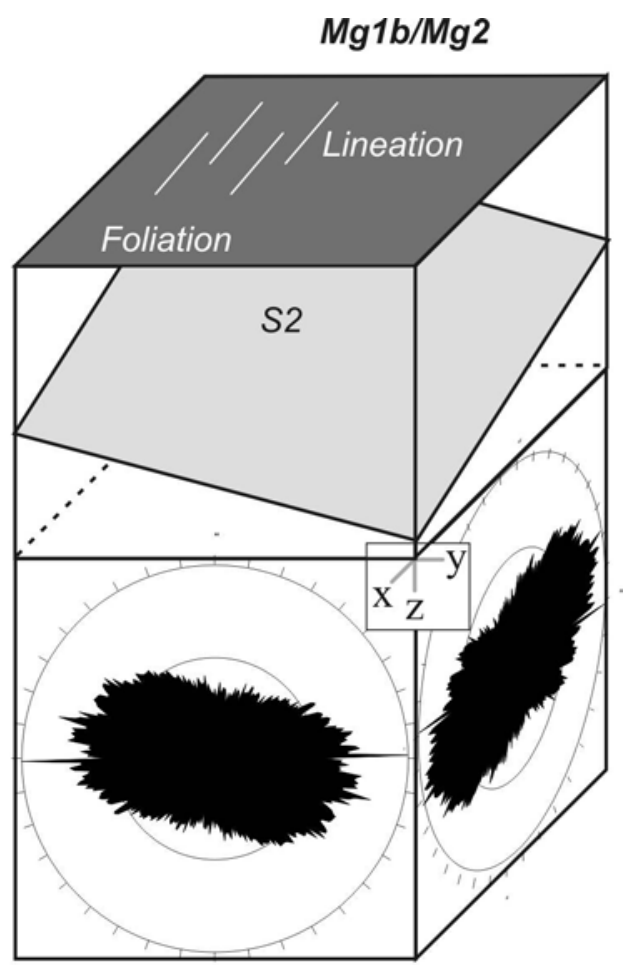

Abb.7-11: Bevorzugte Orientierung der Korngrenzen von Hornblende, Plagioklas, Diopsid und Orthopyroxen der Meta-Gabbros von Anzola. Richtungen des Probenreferenzsystems sind gekennzeichnet. Graue Fläche - Schematische Lage der Korngrenzen-Foliation S2.

Die Verteilung der Korngrenzen wurde in den Schnittlagen xz und yz ermittelt und sind gemäss der Streichrichtungen in Form von Rosendiagrammen dargestellt. Es zeigt sich, dass die Korngrenzen bevorzugt schräg zur makroskopischen und durch den stofflichen Lagenbau definierten Foliation orientiert sind und im Winkel von ca. $15^{\circ}$ eine zweite Foliation (S2Abb.7-11) bilden. Aufgrund dieser Lage ist das Angebot von Korngrenzen etwa senkrecht zur z-Richtung deutlich größer als selbiges senkrecht zur x- und y-Richtung.

\subsubsection{Offene Mikrorisse}

Es existieren sowohl intra- wie auch transgranulare offene und verheilte Risse (Anhang II.8/9). Insbesondere in Hornblende, aber auch in Klinopyroxen und Plagioklas folgen die offenen Risse bevorzugt der Spaltbarkeit der Minerale. Das Hauptaugenmerk liegt auf der Rissdichte und der bevorzugten Orientierung der offenen Mikrorisse, die das dominante RissSystem darstellen und außerdem mechanisch schwach sind. Der Differenz-Isolinien-Plot aus der Vp-Messung der wassergesättigten und der trockenen Kugel birgt eine Information bezüglich der Rissdichte und der bevorzugten Orientierung der offenen Mikrorisse (vgl. 
Kap.2.4.5.1). Zusätzlich wird der Rissquantifizierungsparameter (Vp $\left.p_{\mathrm{QRiss}}\right)$ zur richtungsunabhängigen Quantifizierung der Rissdichte verwendet.

Die Probe $\mathrm{Mg} 1 \mathrm{~b}$ liegt mit einem $\mathrm{Vp}_{\mathrm{QRiss}}-$ Wert von $1,68 \mathrm{~km} / \mathrm{s}$ in der Rissklasse $\mathrm{B}_{\mathrm{R}}$ (mittlere Rissdichte), wohingegen die Probe $\mathrm{Mg} 2$ mit $\mathrm{Vp}_{\mathrm{QRiss}}=0,56 \mathrm{~km} / \mathrm{s}$ eine wesentlich geringere Rissdichte aufweist und zur Rissklasse $A_{R}$ (geringe Rissdichte) gezählt wird. Beide Proben sind bezüglich ihrer Rissverteilung nur schwach anisotrop.
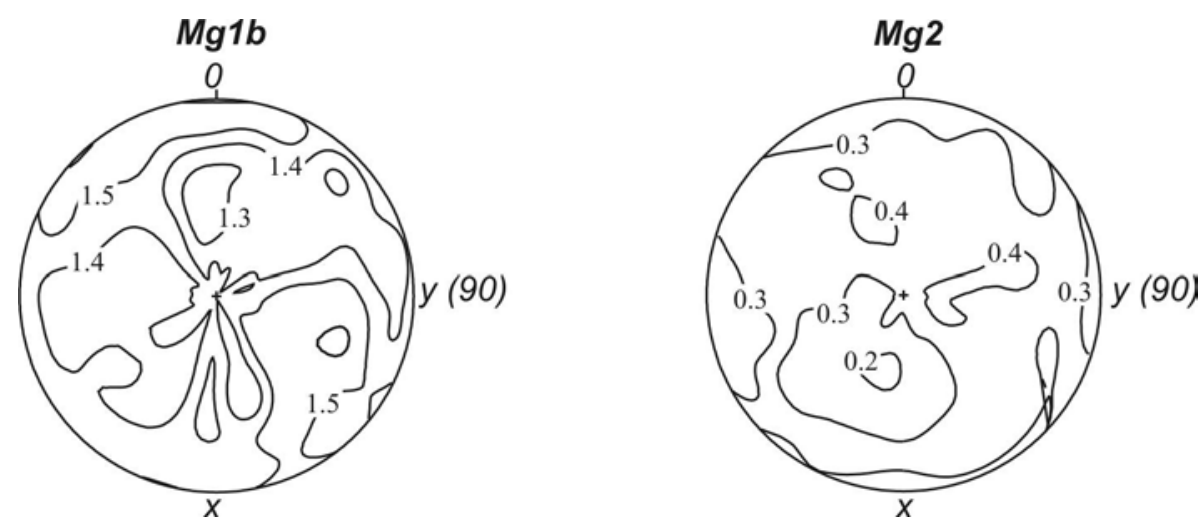

Abb.7-12: $\quad$ VVp-Isolinien-Plots der Meta-Gabbros, berechnet aus der Vp-Messung der wassergesättigten und der trockenen Kugel [km/s]. Der Fehler beträgt $0,1 \mathrm{~km} / \mathrm{s}$.

Die Unterschiede innerhalb des Differenzisolinien-Plots liegen für $\mathrm{Mg} 1 \mathrm{~b}$ bei $0,24 \mathrm{~km} / \mathrm{s}$ und für $\mathrm{Mg} 2$ bei $0,26 \mathrm{~km} / \mathrm{s}(\boldsymbol{A} \boldsymbol{b} \boldsymbol{b} .7-12)$. Bei einem $1-\sigma-F e h l e r$ von $0,1 \mathrm{~km} / \mathrm{s}$ ist keine eindeutige Aussage über eine bevorzugte Orientierung der offenen Mikrorisse möglich, was auch bedeutet, dass diese, wenn vorhanden, nur sehr gering ausgeprägt ist im Vergleich zu höher anisotropen Proben wie Iv1 und Be1 (vgl. Kap.5.9). Auch die häufig beobachtbare bevorzugte Orientierung der offenen Mikrorisse parallel zur Foliation (z.B. Kalzit-Marmor - Rek) ist hier nicht einmal in Ansätzen zu erkennen. Obwohl die offenen Mikrorisse nach qualitativen Beobachtungen bevorzugt der Spaltbarkeit, insbesondere der Hornblende und des Diopsid folgen (vgl. Kap.5.9.5), ist keine Übereinstimmung zwischen der Verteilung der (110)Spaltflächen mit dem Muster der Mikrorissverteilung festzustellen. Aus diesem Grund kann der Einfluss der Textur von Diopsid und Hornblende auf die Verteilung der offenen Mikrorisse als gering eingeschätzt werden.

\subsubsection{Spaltzugfestigkeit}

Die Spaltzugfestigkeit wurde gemäß $\boldsymbol{A} \boldsymbol{b} b .7-13$ in der yz- und der xz-Ebene bestimmt. Als Kriterium für die Orientierung dienen die Richtungen der zum Bruch führenden Zugspannungen. Die einzelnen Proben sind von $0^{\circ}$ bis $180^{\circ}$ gemäß ihres Drehwinkels 
gekennzeichnet. Die Abfolge in allen Diagrammen orientiert sich am Messschema (Abb.713).

Die Spaltzugfestigkeiten der Probe Mg1b liegen zwischen 7,0 MPa und 11,4 MPa, wobei die niedrigeren Spaltzugfestigkeiten tendenziell senkrecht zur Foliation auftreten (z-Richtung) und die höchsten bei einer Zugbelastung parallel zum Linear (x-Richtung). Die mittlere Spaltzugfestigkeit der Probe Mg1b, berechnet als arithmetisches Mittel der drei HauptgefügeRichtungen (x-, y-,z-Richtung), beträgt 9,6 MPa.

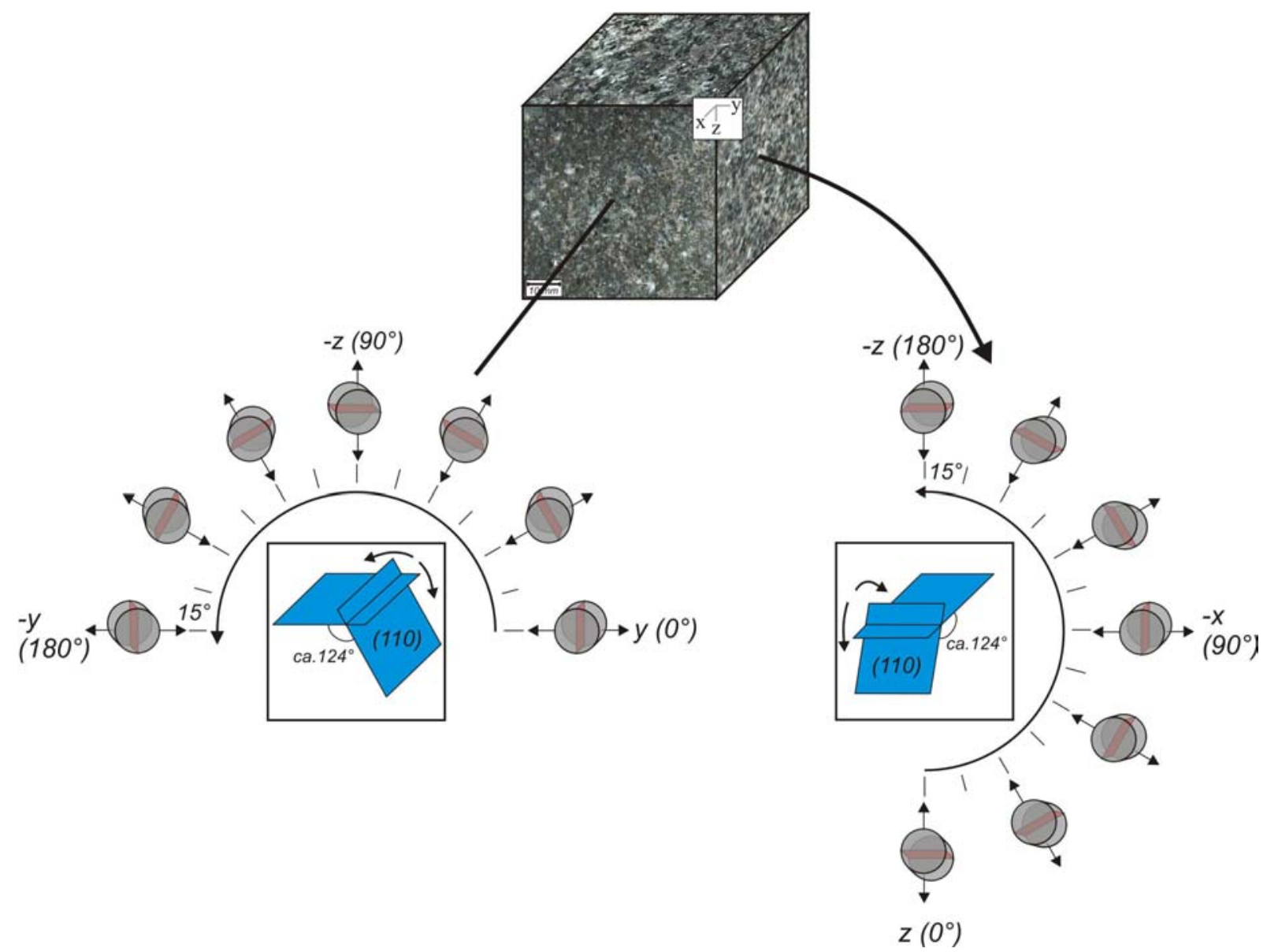

Abb.7-13: Schema zur räumlichen Orientierung der Spaltzugkörper. Rote Flächen - Bruchflächen beim Spaltzugversuch. Blaue Flächen - Spaltflächen von Hornblende mit schematischer Raumlage gemäß der Hornblende-(110-Textur. Die Richtungen des Probenreferenzsystems und die korrespondierenden Gradzahlen sind indiziert.

Die Probe Mg2 weist eine minimale Spaltzugfestigkeit von 7,1 MPa und eine maximale von 13,5 MPa auf. Wie bei der Probe Mglb sind die Richtungen annähernd senkrecht zur Foliation bzw. mechanisch am schwächsten und die parallel zum Linear am stärksten. Der Mittelwert der Spaltzugfestigkeit (Berechnung siehe oben) liegt bei 10,8 MPa und ist damit etwas höher als der von Mglb. Bezüglich beider Proben werden beim Spaltzugversuch häufig präexistierende Mikrorisse und die dominanten Spaltflächen der gesteinsbildenden Minerale Hornblende, Plagioklas und Diopsid aktiviert. Die Häufigkeit aktivierter Korngrenzen ist im 
Vergleich deutlich geringer (qualitative Beobachtung aus den rasterelektronenmikroskopischen Untersuchungen - vgl. Kap.6.3.5).

\subsubsection{Gefügeabhängigkeit der Spaltzugfestigkeit}

Die Anisotropie der Spaltzugfestigkeiten wird im Falle der Meta-Gabbros hauptsächlich von der Textur der gesteinsbildenden Minerale und der bevorzugten Orientierung der offenen Mikrorisse bestimmt (vgl. Kap.6.3). Mechanische Schwachstellen sind dabei die Spaltbarkeit der Minerale, soweit eine zumindest gute Spaltbarkeit ausgebildet ist, und natürlich die offenen Mikrorisse. Doch auch die Korngrenzen können ggf. beim Bruch aktiviert werden und mit ihrer bevorzugten Orientierung einen Einfluss auf die Anisotropie haben. Um einen Vergleich zwischen dem Gesteinsgefüge und dem richtungsabhängigen Verhalten der Spaltzugfestigkeiten zu ermöglichen, wurden die relativen Häufigkeiten der Gefügeelemente in den gleichen Orientierungen wie die Spaltzugfestigkeiten ermittelt. Dabei wurden sämtliche Gefügeelemente berücksichtigt, die einen Einfluss auf die Anisotropie der Spaltzugfestigkeiten haben können. Die Hornblende weist mit ihrer vollkommenen Spaltbarkeit (4) nach $(110 ; 1-10)$ potenziell mechanisch schwache Flächen und zudem eine hohe mechanische Anisotropie von $\mathrm{A}_{\mathrm{ISpM}}=3$ (vgl. Kap.4.2) auf. Der Diopsid zeigt eine deutlich geringere Spaltbarkeit der (110/1-10)-Flächen von 2 bis 3 und eine niedrigere Anisotropie von $\mathrm{A}_{\mathrm{ISpM}}=1,5$. Die Intensitäten der (110)-Pole wurden für beide Minerale ermittelt und bieten eine Aussage über die relative Häufigkeit der Spaltflächen senkrecht zur betrachteten Richtung. Als weiteres Element werden die Korngrenzen als Gesamtheit aller Korngrenzen der vorkommenden Minerale in die Analyse einbezogen. Eine hohe relative Häufigkeit der Streichrichtungen bedeutet dabei ein niedriges Angebot an Korngrenzen in der Richtung senkrecht dazu. Die Rissdichte der offenen Mikrorisse, quantifiziert über die $\Delta \mathrm{Vp}$ Werte, wurde ebenfalls richtungsabhängig bestimmt. Hohe $\Delta \mathrm{Vp}$-Werte sind dabei gleichzusetzen mit einem hohen Angebot an offenen Mikrorissen mit einer Orientierung senkrecht zur entsprechenden Richtung. Insgesamt liegt der Schwerpunkt des Interesses auf dem Angebot mechanisch schwacher Flächen mit einer bevorzugten Orientierung senkrecht zu den beim Spaltzugversuch auftretenden Zugspannungen, bzw. parallel zur entstandenen Bruchfläche. Hier sind die niedrigsten Spaltzugfestigkeiten zu erwarten. Die Ergebnisse sind für die xz-Ebene in $\boldsymbol{A b b . 7 - 1 4}$ und für die yz-Ebene in $\boldsymbol{A b b . 7 - 1 5}$ dargestellt. 


\section{Mg1b}
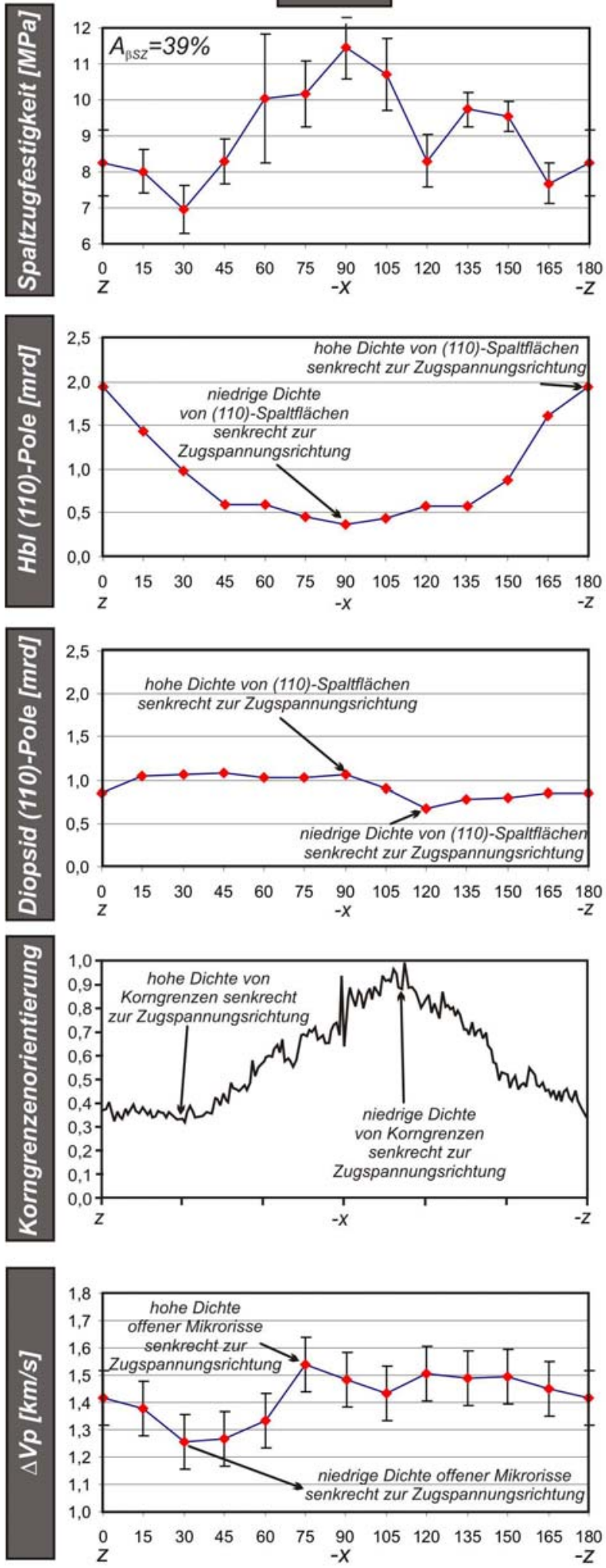

\section{Mg2}
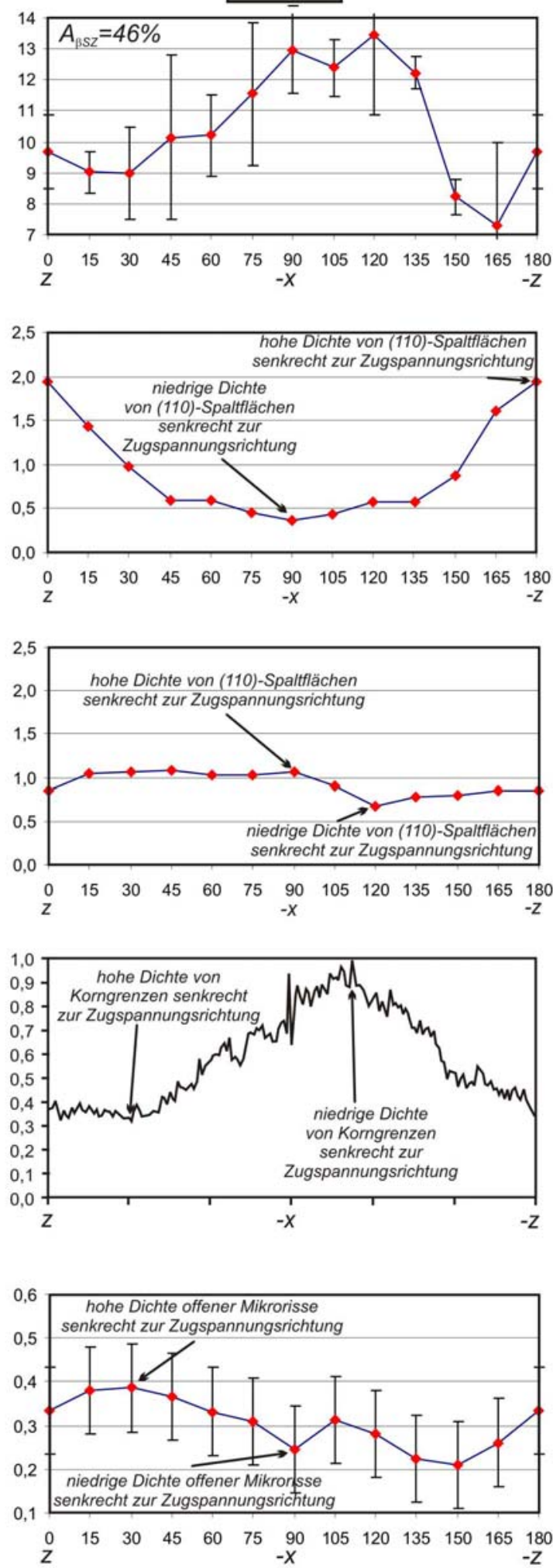

Abb.7-14: Richtungsabhängige Korrelation der Spaltzugfestigkeit mit Gefügeelementen in der xzEbene. Die Häufigkeiten der Korngrenzenorientierung sind auf das Maximum normiert. Die Minima und Maxima der relativen Häufigkeit der Gefügeelemente mit einer Ausrichtung senkrecht zu den Zugspannungsrichtungen sind mit Pfeilen gekennzeichnet. $A_{\beta S z}-$ Anisotropie der Spaltzugfestigkeit. Fehlerbalken markieren den 1- $\sigma$-Fehler. 
Die xz-Ebene zeigt eine deutliche Anisotropie der Spaltzugfestigkeiten (39\% - Mg1b/46\% Mg2), die in der Hauptsache an die bevorzugte Orientierung der Hornblende (110)-Flächen geknüpft ist. Ein niedriges (niedrig und hoch werden immer zur Darstellung der Relationen in Bezug auf ein Gefügeelement verwendet) Angebot an Spaltflächen senkrecht zur Zugspannungsrichtung bedingt hohe Spaltzugfestigkeiten und umgekehrt. Das Muster der Spaltzugfestigkeiten der Probe $\mathrm{Mg} 2$ ist asymmetrisch und das Maximum der Spaltzugfestigkeit um ca. $30^{\circ}$ Richtung $120^{\circ}$ verschoben (Abb.7-14), was mit der HornblendeTextur nicht zu erklären ist. Möglicherweise ist dieser Effekt auf eine Überlagerung des HblTextureinflusses durch die Korngrenzenorientierung und die Textur der Diopsid-(001)Flächen zurückzuführen. Letzteres erscheint dabei unwahrscheinlich, da die Diopsid-Textur schwach, die mechanische Anisotropie des Diopsid niedrig und dessen Volumenanteil am Gestein mit 16 Vol.\% verhältnismäßig gering ist, gegenüber dem der Hornblende (44 Vol.\%). Mit der Verteilung der Korngrenzen hingegen ließe sich das Verhalten der Spaltzugfestigkeiten in der xz-Ebene sogar vollständig erklären, ohne den Einfluss der Hornblende-Textur für die Argumentation $\mathrm{zu}$ verwenden. Die beiden Kurven (Spaltzugfestigkeit und relative Häufigkeit der Korngrenzenstreichrichtungen) verlaufen annähernd parallel, und ein hohes Angebot von Korngrenzen senkrecht zur Zugspannungsrichtung korreliert mit niedrigen Festigkeiten und umgekehrt. Zwei Argumente sprechen jedoch gegen eine starke Bedeutung der Korngrenzenorientierung und für eine lediglich schwach den Einfluss der Hornblende-Textur überlagernde Funktion der Korngrenzen. Zum einen ergeben die rasterelektronenmikroskopischen Untersuchungen, dass die Hbl-(110)-Flächen deutlich häufiger als die Korngrenzen beim Spaltzugversuch aktiviert werden. Zum zweiten ist der Einfluss der Kornformorientierung in der yz-Ebene nicht nachweisbar, obwohl auch dort vorhanden (Abb.7-15). In dieser Ebene zeigt die Spaltzugfestigkeit mit 19\% (Mg1b) und 33\% (Mg2) eine niedrigere Anisotropie und kein bestimmtes Verteilungsmuster, sondern unstete Kurvenverläufe. Betrachtet man die Gefügemerkmale, so besteht der Hauptunterschied zwischen der xy-Ebene und der yz-Ebene im Verteilungsmuster der Hornblende-(110)-Flächen. Die yz-Ebene liegt in Bezug darauf innerhalb des Gürtels und zeigt gegenüber der xz-Ebene, die etwa senkrecht dazu orientiert ist, nur eine geringe Varianz der (001)-Pol-Intensitäten.

Zusammenfassend kann geschlossen werden, dass das richtungsabhängige Verhalten der Spaltzugfestigkeiten in der Hauptsache von der bevorzugten Orientierung der Spaltflächen der Hornblende abhängt. Möglicherweise existiert eine schwache Überlagerung durch die Korngrenzenorientierung, die aber keinesfalls das dominante Gefügeelement ist. 


\section{$M g 1 b$}
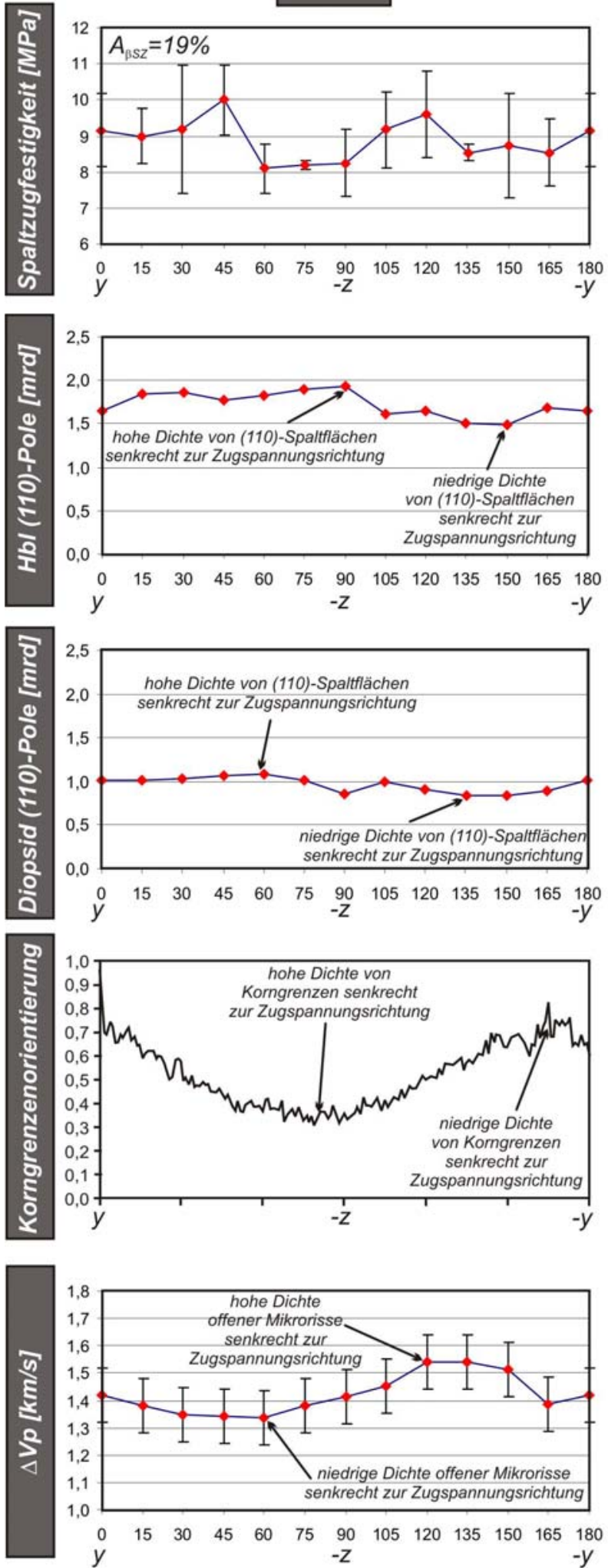

Mg2
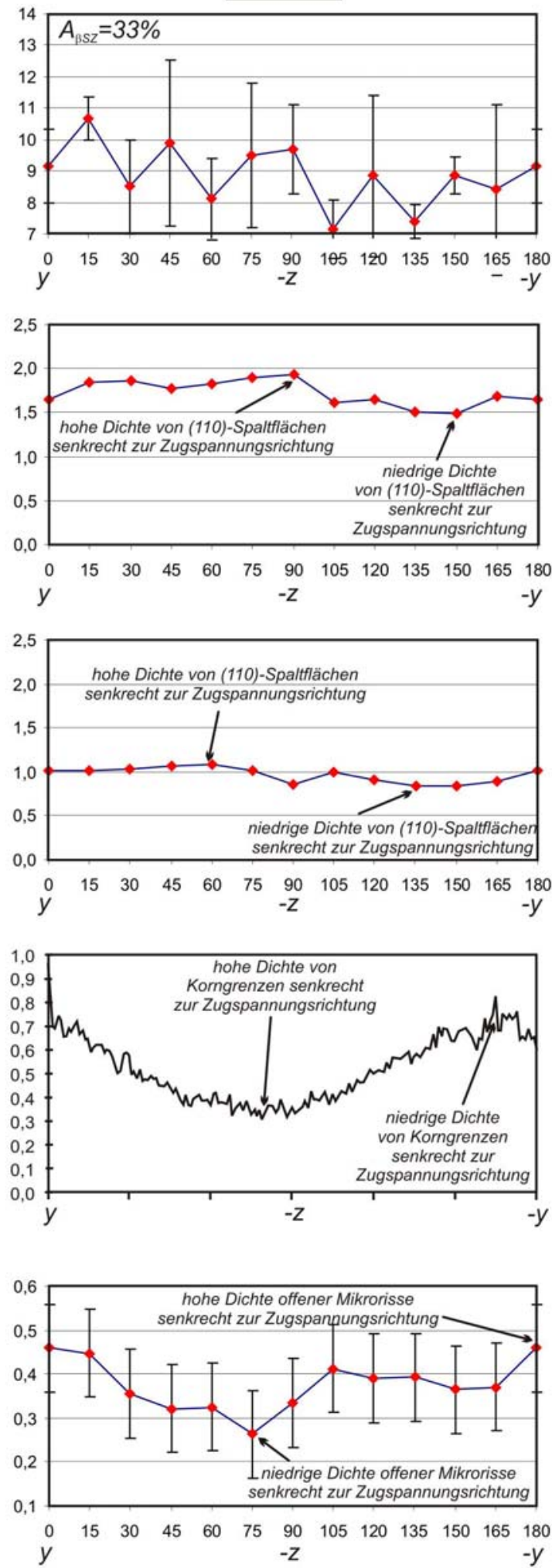

Abb.7-15: Richtungsabhängige Korrelation der Spaltzugfestigkeit mit Gefügeelementen in der yzEbene. Die Häufigkeiten der Korngrenzenorientierung sind auf das Maximum normiert. Die Minima und Maxima der relativen Häufigkeit der Gefügeelemente mit einer Ausrichtung senkrecht zu den Zugspannungsrichtungen sind mit Pfeilen gekennzeichnet. $A_{\beta S Z}-$ Anisotropie der Spaltzugfestigkeit. Fehlerbalken markieren den 1- $\sigma$-Fehler. 
Der Einfluss der Diopsid-Textur und der offenen Mikrorisse auf die Anisotropie der Spaltzugfestigkeiten ist als gering einzuschätzen. Eine andere Bedeutung der offenen Mikrorisse erschließt sich jedoch beim Vergleich der mittleren Spaltzugfestigkeiten. Die Probe Mg1b liegt mit einem Mittelwert von 9,6 MPa niedriger, als die Probe Mg2 (10,8 $\mathrm{MPa})$, was negativ korreliert mit der höheren Rissdichte von $\operatorname{Mg} 1 \mathrm{~b}\left(\mathrm{Vp}_{\mathrm{QRiss}}=1,68 \mathrm{~km} / \mathrm{s}\right)$, gegenüber der von $\mathrm{Mg} 2\left(\mathrm{Vp}_{\mathrm{QRiss}}=0,56 \mathrm{~km} / \mathrm{s}\right)$. Die offenen Mikrorisse haben also einen signifikanten Einfluss auf die Festigkeiten (vgl. auch Kap.6.3.4), tragen jedoch aufgrund ihrer homogenen Verteilung nicht nachweisbar zur Anisotropie der Spaltzugfestigkeiten bei. 


\section{Korrelation der technischen Gesteinseigenschaften}

Bisherige Untersuchungen und Datenkompilationen zur Korrelation technischer Gesteinseigenschaften verfolgen in der Regel zwei unterschiedliche Zielrichtungen. Übergeordnet geht es darum, die Gesamtheit der technischen Gesteinseigenschaften aus möglichst einfach zu ermittelnden Kennwerten, wie z.B. den Indexeigenschaften Dichte und Porosität, extrapolieren zu können. In diesem Zusammenhang sind eine Vielzahl von Korrelationen der verschiedenen technischen Kennwerte in der Literatur vorhanden. Aus der Gesteinsphysik (z.B. Schön 1983), aber auch im Sektor der Naturwerksteine (z.B. Peschel 1983; Winkler 1994) sind Korrelationen der Dichte und Porosität mit anderen technischen Gesteinseigenschaften, wie der Druckfestigkeit, Biegefestigkeit und auch der kapillaren Wasseraufnahme zu finden. Besonders wichtig sind Vergleiche der mechanischen Eigenschaften untereinander (Peschel 1983) (Abb.8-1).
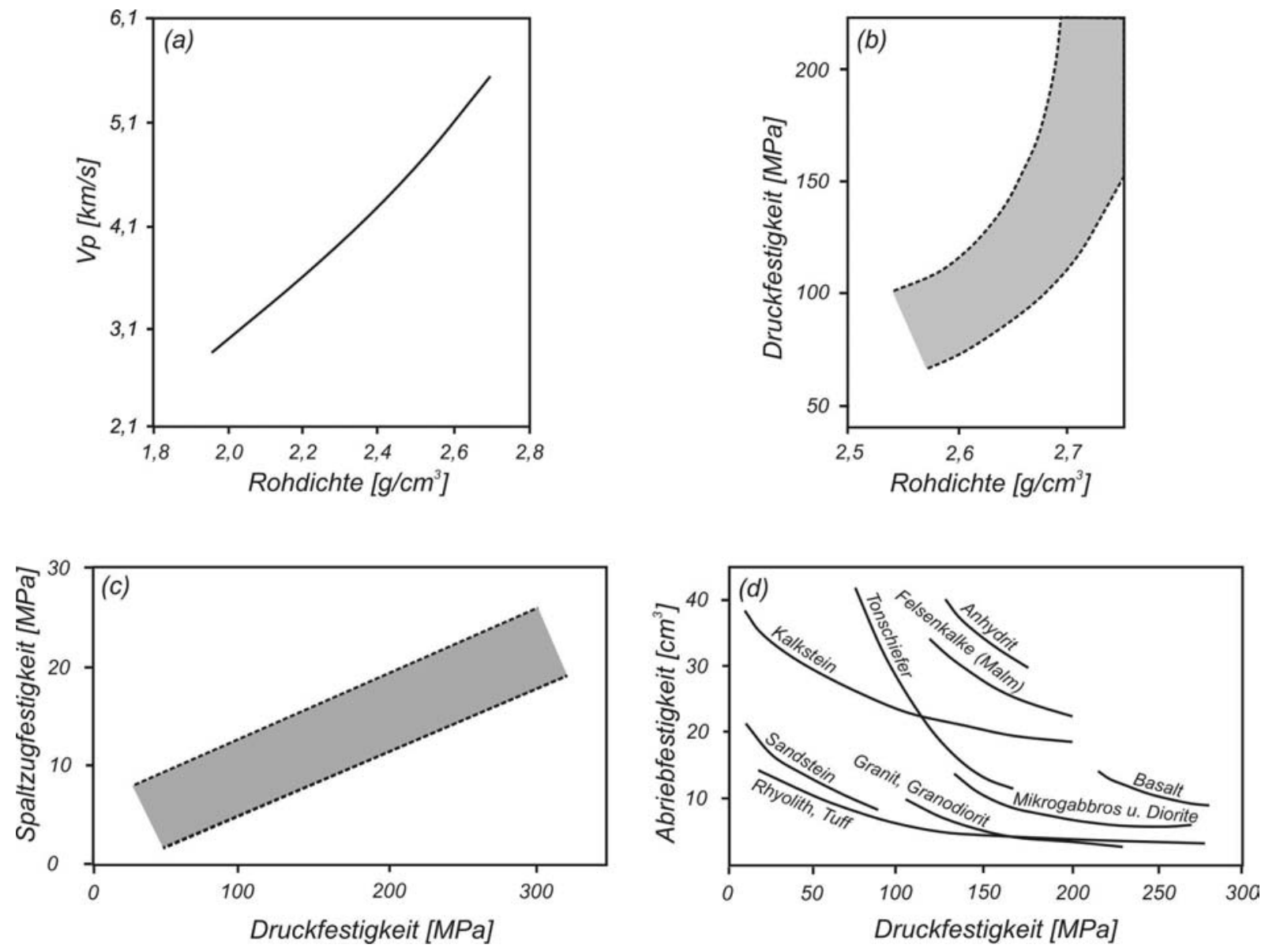

Abb.8-1: Schemata zu Kreuzkorrelationen gesteinstechnischer Kennwerte. (a) Rohdichte vs Kompressionswellen-geschwindigkeit (Vp) verändert aus Winkler (1994). (b) Druckfestigkeit vs. Rohdichte. (c) Spaltzugfestigkeit vs. Druckfestigkeit. (d) Abriebfestigkeit vs. Druckfestigkeit. (b, c, d) verändert aus Peschel (1983). 


\section{$\underline{\text { 8. Korrelation der technischen Gesteinseigenschaften }}$}

Im letzten Fall wird versucht, mit zerstörungsfreien Methoden, wie z.B. der Ultraschallmessung (Vp, dyn. E-Modul), eine Aussage über den Zustand eines Gesteins und damit eine indirekte Einschätzung der Festigkeiten abzuleiten. Diese Art der Herangehensweise hat gerade bei der Schadensanalyse von Naturwerksteinen eine große Bedeutung, weil hier nur selten Probenmaterial zur direkten Bestimmung der mechanischen Gesteinseigenschaften vorhanden ist. Für Marmore wurde die Ultraschallanalyse bereits mehrfach angewendet und beschrieben (z.B. Köhler 1991; Dürrast et al. 1999; Siegesmund et al. 2000; Ondrasina et al. 2002). Auch für Sandsteine (Weiss 1992) und Granit (Falls et al. 1992) sind derartige Versuche unternommen worden. Mit Ausnahme von Weiss (1992), der eine positive lineare Korrelation des dynamischen E-Moduls mit der Biegefestigkeit feststellte, und Köhler (1991) mit einer positiven aber unlinearen Korrelation zwischen VpWerten und Druck-/Biegefestigkeiten wurden klare Aussagen bezüglich der aus den Ultraschallmessungen hervorgehenden Festigkeiten vermieden und lediglich Schädigungsgrade herausgearbeitet. Ein Gestein mit niedrigen Vp ist dabei stärker geschädigt als eines mit hohen Ultraschallgeschwindigkeiten. Die bekannteste Skala der Schädigungsgrade von Marmoren mit einer qualitativen Beschreibung der jeweiligen Gefügezustände liefert Köhler (1991). Die Interpretationen der Ultraschallgeschwindigkeiten und des dynamisch bestimmten E-Moduls basieren auf dem Einfluss offener Mikrorisse. Je höher die Rissporosität, desto niedriger die Ultraschallgeschwindigkeiten und desto niedriger auch die zu erwartende Gesteinsfestigkeit (vgl. Kap.6.3). An vielen historischen Gebäuden und Statuen ist diese Art der Untersuchung häufig die einzig mögliche Methode und erfordert eine gute Kenntnis der Zusammenhänge zwischen den Festigkeiten und den elastischen Gesteinseigenschaften.

Gesteine sind in Bezug auf ihren Mineralbestand und ihre Gefügeeigenschaften sehr variabel, so dass bei der Etablierung von Korrelationen immer die Gefahr einer nicht repräsentativen Probenauswahl besteht. Folglich können verschiedene empirische Studien, je nach untersuchtem Probenmaterial, zu unterschiedlichen Ergebnissen kommen. Nur die detaillierten Kenntnisse über die Gefügeabhängigkeit der technischen Eigenschaften führen zu einer sicheren Interpretation der Korrelationsdiagramme und damit $\mathrm{zu}$ einer vertrauenswürdigen Einschätzung der Zusammenhänge. Auch die Richtungsabhängigkeit der gesteinstechnischen Kennwerte hat bisher wenig Beachtung gefunden und wird im Rahmen der vorliegenden Arbeit genauer untersucht.

Im Folgenden werden die skalaren (richtungsunabhängigen) Kennwerte Porosität und Dichte mit den Mittelwerten (arithmetisches Mittel aus x-, y-,z-Richtung) anderer technischer 


\section{$\underline{\text { 8. Korrelation der technischen Gesteinseigenschaften }}$}

Eigenschaften verglichen. Die nichtskalaren (richtungsabhängigen) Eigenschaften werden mittels zweier unterschiedlicher Ansätze näher analysiert (Abb.8-2).
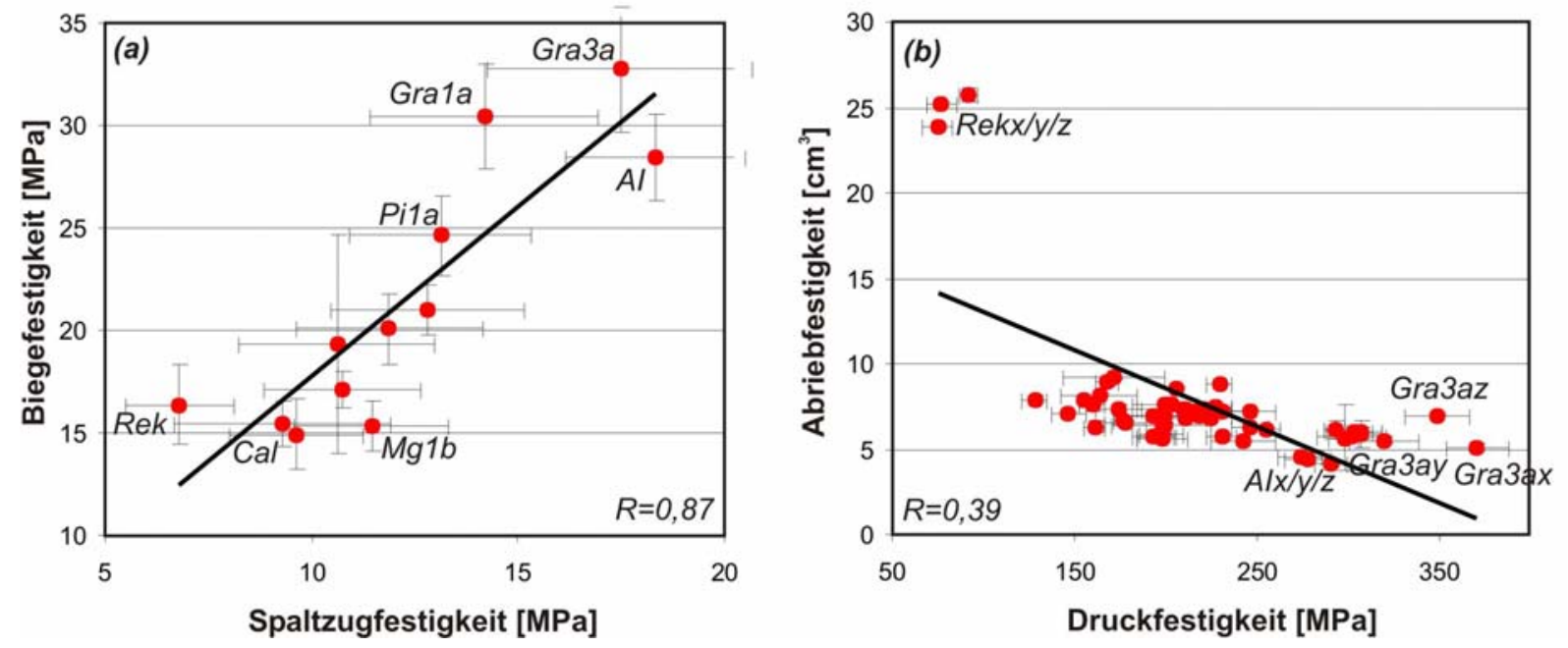

Abb.8-2: (a) Beispiel für die Kreuzkorrelation von Mittelwerten (arithmetisches Mittel aus x-, y-, zRichtung). (b) Richtungsabhängige Betrachtung der Zusammenhänge zwischen zwei technischen Parametern. Proben sind mit Richtungsangabe indiziert.

Zum einen werden hier die Mittelwerte, andererseits aber auch die richtungsabhängigen Kennwerte miteinander in Verbindung gebracht. Dabei besteht ein Wertepaar immer aus den Größen zweier technischer Gesteinseigenschaften, die in derselben Richtung (x-, y- oder zRichtung einer bestimmten Probe- Definition siehe Kap.2) ermittelt wurden (Abb.8-2). Wenn möglich, wird für jede Korrelation ein Korrelationskoeffizient (R) angegeben. Nichtlineare Zusammenhänge werden durch lineare Regressionen angenähert, sofern kein plausibles Modell zur Quantifizierung besteht. Korrelationen mit $\mathrm{R} \geq 0,70$ sind statistisch signifikant (gute Korrelation) und solche mit $0,6<\mathrm{R}<0,7$ zumindest noch als deutlich zu bezeichnen. $\mathrm{R}$ wird immer positiv angegeben und die Art der Korrelation separat spezifiziert. Die Allgemeingültigkeit der Zusammenhänge für niedrigporöse kristalline Gesteine ist auch bei hohen Korrelationskoeffizienten nicht in jedem Fall gegeben und wird an entsprechender Stelle diskutiert. Werden im Folgenden Mikrorisse ohne nähere Spezifizierung erwähnt, so handelt es sich stets um offene Mikrorisse.

Um die bestehenden Zusammenhänge zwischen den technischen Gesteinseigenschaften zu analysieren und die Ursachen für die Art und Qualität der Korrelationen zu erklären, liegt der Schwerpunkt auf der Beantwortung folgender Kernfragen:

1. Wie ist die Abhängigkeit der Eigenschaften vom Mineralbestand? Von welchen Gefügeelementen hängen die betrachteten Eigenschaften ab? 
2. Welche Minerale und Gefügeelemente bestimmen die Anisotropie der betrachteten Eigenschaften?

3. Besteht eine Korrelation der betrachteten technischen Gesteinseigenschaften für die gesteinsbildenden Minerale?

4. Wie ist die Orientierung der bei den Experimenten zur Bestimmung der technischen Kennwerte auftretenden Kräfte und Spannungen? Welche Prozesse kommen bei der Bestimmung der Parameter zum Tragen? (z.B. Orientierung der zum Bruch führenden Spannungen beim Druck- und Spaltzugversuch; Gesteinsversagen durch unterschiedliche Arten der Rissbildung, wie z.B. Zugrisse und Scherrisse)

Haben zwei unterschiedliche technische Parameter eine möglichst große Anzahl an Gemeinsamkeiten in Bezug auf ihre Abhängigkeit vom Mineralbestand und dem Gesteinsgefüge, als auch auf die Orientierung der Spannungen und der Prozesse während der Experimente, so ist eine gute Korrelation zu erwarten.

Zunächst werden die Vergleiche zwischen den Indexeigenschaften und der Druck-, Spaltzug-, Biege-, Abriebfestigkeit und den dynamischen und statischen elastischen Gesteinseigenschaften $\left(\mathrm{E}_{0} ; \mathrm{E}_{\text {stat50}} ; \mathrm{E}_{\text {stats }}\right)$ sowie der thermischen Dehnung $\left(\alpha_{20 \mathrm{bis} 90}\right)$ gezogen. Es folgt eine Analyse der Zusammenhänge bezüglich der mechanischen Festigkeiten untereinander, und abschließend werden die elastischen Gesteinseigenschaften mit den mechanischen und der thermischen Dehnung in Verbindung gebracht.

\subsection{Technische Gesteinseigenschaften und Indexeigenschaften}

Die bisherigen Korrelationen der Porosität mit anderen gesteinstechnischen Kennwerten basieren in der Hauptsache auf der Annahme, dass eine zunehmende Porosität einhergeht mit dem Verlust an Mineralvolumen, welches zu den technischen Eigenschaften beiträgt. Ein Teil des Mineralbestands mit seinen spezifischen physikalischen Kennwerten wird also durch luftgefüllten Porenraum mit deutlich abweichenden Eigenschaften ersetzt. Nimmt man das Beispiel der mechanischen Gesteinseigenschaften, so hat der Porenraum eine Festigkeit von Null und reduziert mit steigendem Volumen die Festigkeit, wobei die Porengeometrie zunächst unberücksichtigt bleibt. Analog zur Porosität wird auch der Einfluss der Dichte (Rohdichte) auf die sonstigen technischen Gesteinseigenschaften bewertet. Niedrige Dichten werden häufig mit hohen Porositäten assoziiert und eine dementsprechende Veränderung der Eigenschaften angenommen. Für die untersuchten niedrigporösen ( $\phi \leq 1$ Vol.\%) Gesteine sind 


\section{$\underline{\text { 8. Korrelation der technischen Gesteinseigenschaften }}$}

diese Zusammenhänge nicht gegeben. Die Porosität ist eine Rissporosität und wirkt weniger über das reine Volumen als vielmehr über die Geometrie der Mikrorisse (Porengeometrie) auf die Gesteinseigenschaften. Zwar bedingen die unterschiedlichen Rissdichten auch verschiedene Beträge der Porosität, doch führen diese Zusammenhänge aufgrund der niedrigen aspect-ratios nur $\mathrm{zu}$ einem maximalen Porenvolumen von $\leq 1$ Vol.\%. Die Auswirkungen der Rissdichten auf die gesteinstechnischen Parameter ist dahingegen wesentlich höher einzuschätzen (vgl. Kap.6.3.4). Die Dichte hängt in erster Linie nicht von der Porosität, sondern von der Dichte der gesteinsbildenden Minerale ab. Überhaupt sind die Dichte und die Porosität nur dann direkt miteinander verknüpft, wenn die untersuchten Gesteine eine identische Matrixdichte aufweisen.

Die Porosität und die mechanischen Eigenschaften Druck-, Spaltzug-, Biegefestigkeit sowie der thermische Ausdehnungskoeffizient $\left(\alpha_{20-90}\right)$ korrelieren schlecht miteinander $(\boldsymbol{A b b} .8-3)$.

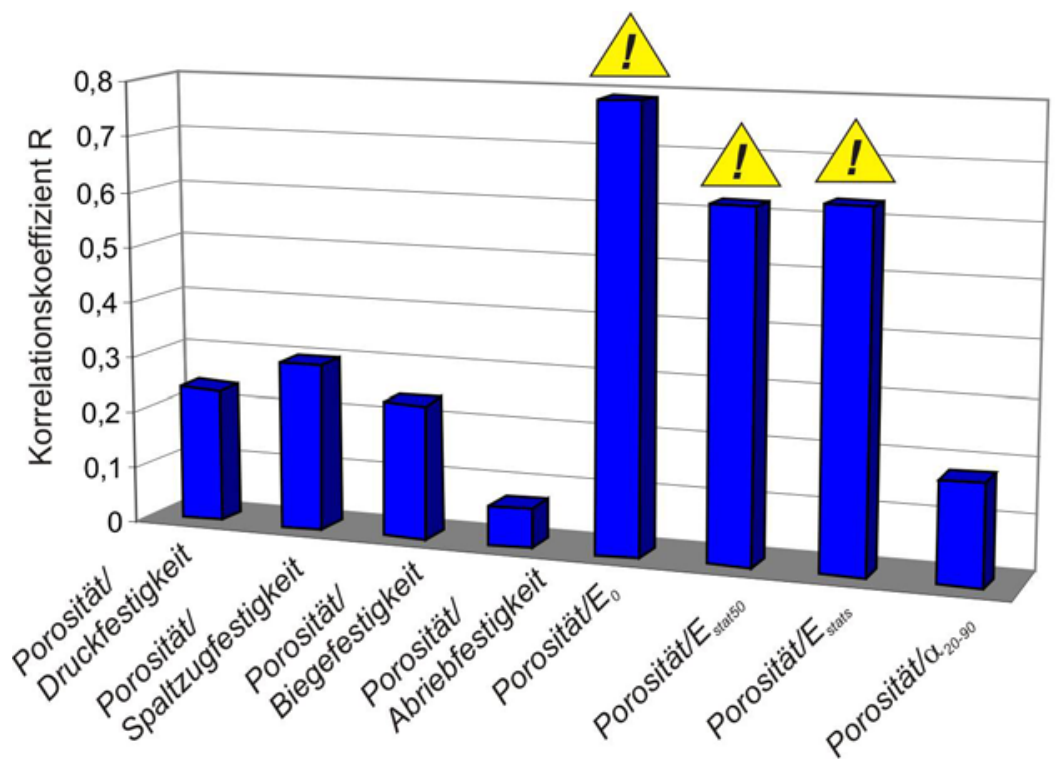

Abb.8-3: Korrelationskoeffizienten $R$ für die Abhängigkeiten der technischen Gesteinseigenschaften von der Porosität. Gelbe Dreiecke - die Korrelationen sind nicht allgemeingültig für niedrigporöse kristalline Gesteine.

Diese Eigenschaften werden zwar zu einem gewissen Anteil auch über die Rissdichte der offenen Mikrorisse gesteuert, aber maßgeblich durch die mechanischen und thermischen (Dehnungs-)Eigenschaften der Minerale dominiert, die keinerlei Bezug zur Porosität eines Gesteins aufweisen.

Die $\boldsymbol{A b b . 8 - 4 ~ z e i g t ~ d i e ~ A b h a ̈ n g i g k e i t e n ~ e i n i g e r ~ m e c h a n i s c h e r ~ F e s t i g k e i t e n ~ v o n ~ d e r ~ P o r o s i t a ̈ t ~ f u ̈ r ~}$ die Kristallingesteine ergänzt durch Daten sedimentärer Gesteine. Bei Betrachtung aller Gesteine besteht ein deutlicher Trend von hohen Porositäten $\mathrm{zu}$ niedrigeren Gesteinsfestigkeiten. Der lässt sich dadurch erklären, dass der Porenraum nicht zur 
Gesteinskohäsion beiträgt. Im Bereich der niedrigporösen Gesteine existieren große Unterschiede in den Festigkeiten ohne eine nennenswerte Änderung der Porositätswerte.
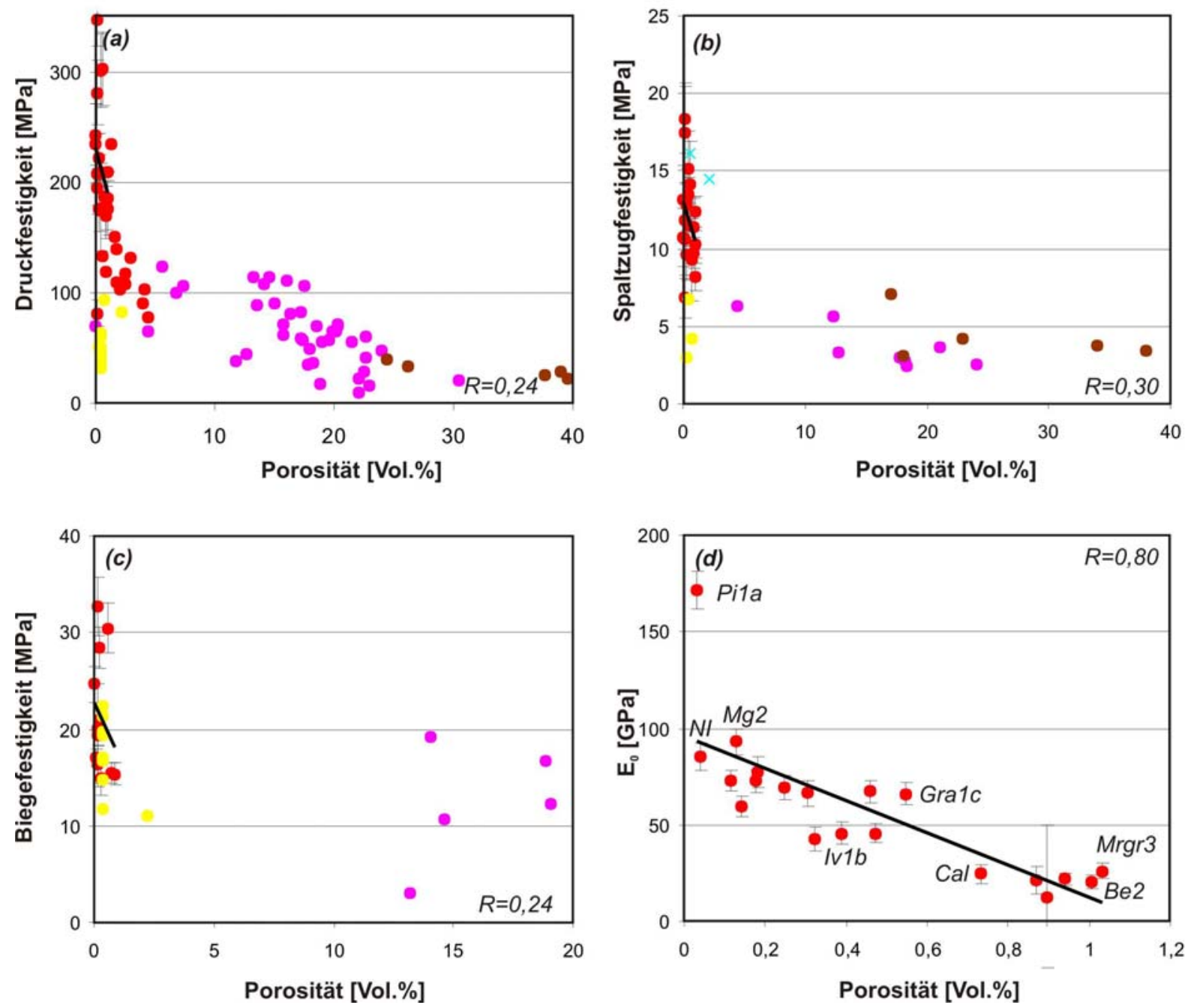

Abb.8-4: Korrelationsdiagramme. Mittelwerte technischer Eigenschaften und ihre Abhängigkeiten von der Porosität. Gelbe Datenpunkte - Marmore. Rote Datenpunkte - Granitoide/Gneise/Gabbros/ Peridotit/Quarzit. Pinke Datenpunkte - Sandsteine. Braune Datenpunkte - Tuff/Ignimbrit. Cyanfarbende Datenkreuze - Granite. Die Korrelationskoeffizienten und Trendlinien gelten für die eigenen Daten. (a) Porosität vs. Druckfestigkeit. Daten für Sandsteine aus Howarth \& Rowlands (1987); Fitzner (1988); Metz (1992); Weiss (1992); Daten für Granitoide/Gneise/ Gabbros/Peridotit/Quarzit aus Prikryl (1998); Gupta \& Rao (2000); eigene Daten. Daten für Marmore aus Koroneos (1980); Howarth \& Rowlands (1987); eigene Daten (b) Porosität vs. Spaltzugfestigkeit. Daten für Sandsteine aus Howarth \& Rowlands (1987); Daten für Granitoide/Gneise/ Gabbros/Peridotit/Quarzit aus Gupta \& Rao (2000); eigene Daten. Daten für Ignimbrit aus Moon (1993); Daten für Marmore aus Howarth \& Rowlands (1987); Siegesmund et al. (1999); eigene Daten. Eigene Daten für Granitoide/Gneise/ Gabbros/Peridotit//Quarzit und aus Gupta \& Rao (2000) (c) Porosität vs. Biegefestigkeit. Eigene Daten für Granitoide/Gneise/Gabbros/Peridotit//Quarzit. Daten für Marmor aus Koroneos (1980); eigene Daten. Daten für Sandsteine aus Weiss (1992). (d) Porosität vs. dyn. E-Modul. Pi1a - Peridotit. NI Gabbro-Norit. Mg2 - Meta-Gabbro. Iv1b - granodioritischer Mylonit. Gra1c-granitischer Gneis. Cal granodioritischer Gneis. Mrgr3 - Granit. Be2 - granitischer Gneis. Fehlerbalken markieren den 1- $\sigma$ Fehler.

Besonders die Festigkeitsunterschiede zwischen den Marmoren und den Graniten/Gneisen belegen, dass hier die mechanische Stabilität der Minerale entscheidend ist und die Porosität über ihr Volumen keinen signifikanten Einfluss hat. Zwischen der Porosität und den 
elastischen Eigenschaften besteht auf den ersten Blick ein guter oder zumindest deutlicher Zusammenhang. Bei genauerer Analyse zeigt sich jedoch, dass eine Verallgemeinerung der Beziehung auf alle kristallinen Gesteine nicht möglich ist. Die Daten des Peridotit (Pi1a) zeigen deutlich, dass die elastischen Mineraleigenschaften $\left(\mathrm{E}_{\mathrm{GVRH}}=190,4 \mathrm{GPa}\right)$ eine bedeutende Rolle für die Höhe des E-Moduls spielen (vgl. Kap.6.4.1/Abb.8-4). Lediglich für Gesteine mit ähnlichen elastischen Eigenschaften des Mineralbestands ( $\mathrm{E}_{\mathrm{GVRH}}-$ Werte zwischen $85 \mathrm{GPa}$ und $120 \mathrm{GPa}$ für die Gesteine dieser Studie) und Rissgeometrien ergibt sich eine gute und auch modellhaft begründbare Übereinstimmung von höheren Porositäten mit niedrigeren dynamischen E-Moduln. Im Fall der statischen E-Moduln ( $\mathrm{E}_{\text {stat50}}$; $\left.\mathrm{E}_{\text {stats }}\right)$ wirkt eine Kombination von Rissdichte der Mikrorisse und mechanischen und elastischen Mineraleigenschaften sowie der Korngröße auf die Höhe des Kennwerts. Folglich liegen Gesteine mit extremen mechanischen (Kalzit-Marmor - Rek mit $\mathrm{I}_{\mathrm{SPG}}=15$ ) und elastischen Eigenschaften (Peridotit - Pila mit $\mathrm{E}_{\mathrm{GVRH}}=190,4 \mathrm{GPa}$ ) weit außerhalb des generellen Trends. Daher ist auch hier der aufgrund der Korrelationskoeffizienten von $\mathrm{R}=0,62$ bzw. $\mathrm{R}=0,63$ (Abb.8-3) zu vermutende lineare Zusammenhang zwischen den statischen elastischen Eigenschaften und der Porosität nicht auf alle niedrigporösen Kristallingesteine übertragbar.

Die Dichte und die mechanischen Gesteinseigenschaften zeigen eine schlechte Korrelation (Abb.8-5) Die Rohdichte ist zu annähernd 100\% durch die Dichte des Mineralbestands bestimmt, wohingegen die Gesteinsfestigkeiten durch eine Kombination aus der mechanischen Stabilität der Minerale, die eine Art Maximalwert der Festigkeit indiziert, und der Schwächung durch offene Mikrorisse dominiert wird.

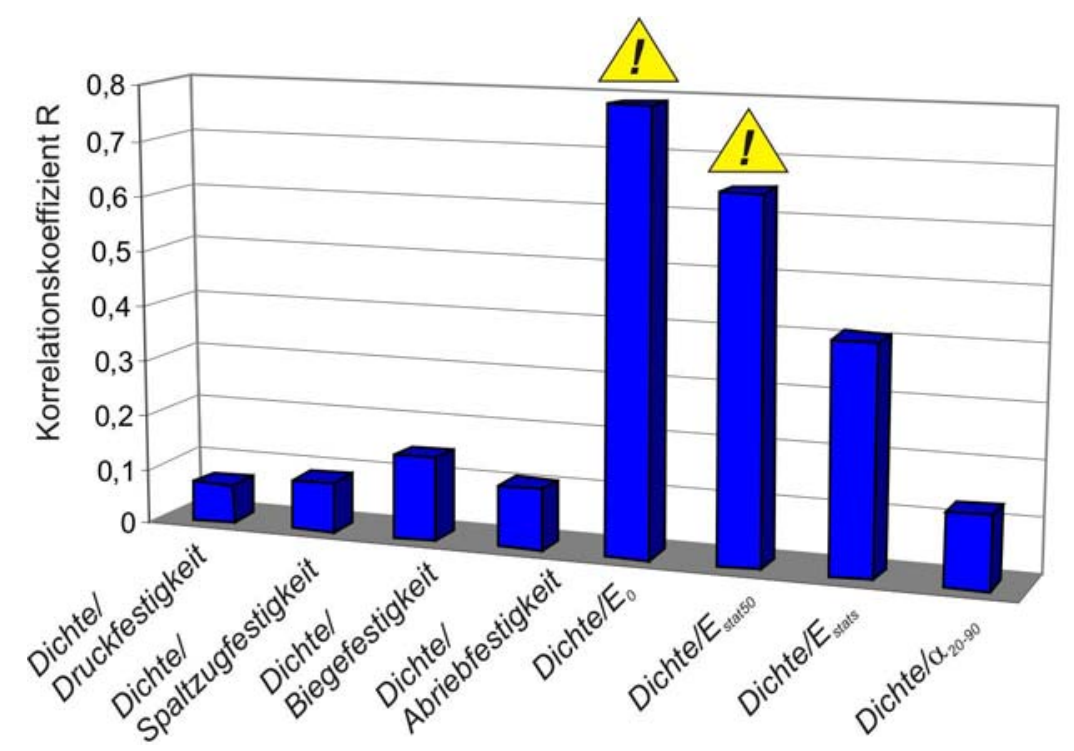

Abb.8-5: Korrelationskoeffizienten $R$ für die Abhängigkeiten der technischen Gesteinseigenschaften von der Dichte. Gelbe Dreiecke - die Korrelationen sind nicht allgemeingültig für niedrigporöse kristalline Gesteine. 
Zwar besteht bezüglich des Mineralbestands eine Korrelation zwischen der Dichte und der Härte/Spaltbarkeitsindex, doch wirkt sich das nicht im Sinne einer ebenfalls guten Übereinstimmung bezüglich der betrachteten Gesteinseigenschaften aus. Ein Grund ist die bereits erwähnte Abhängigkeit der Festigkeiten von der Rissdichte, zum zweiten weisen gerade wichtige gesteinsbildende Minerale, wie Quarz, aber auch die Feldspäte Besonderheiten bezüglich der Korrelation der Eigenschaften auf und liegen außerhalb des Trends (vgl. Kap.4.5). Als dritter Punkt ist ein Teil des Gesteinsgefüges anzuführen, nämlich die räumliche Verteilung der Glimmer im Kornverband, die nicht in die Dichte eingeht, sehr wohl aber eine große Bedeutung für die mechanischen Gesteinseigenschaften hat (vgl. Kap.6.3). Zwischen der Dichte und der thermischen Dehnung besteht kein Zusammenhang, obwohl beide Parameter hauptsächlich durch die Mineraleigenschaften bestimmt werden. Hier ist die schlechte Korrelation der beiden Eigenschaften für die gesteinsbildenden Minerale ausschlaggebend (vgl. Kap.4.5).

Wie bei der Porosität zeigt sich auch bei der Dichte eine gute $\left(E_{0}\right)$ bis deutliche $\left(E_{\text {stat50 }}\right)$ Korrelation mit den elastischen Gesteinseigenschaften (Abb.8-6). $\mathrm{E}_{0}$ ist neben der Mikrorissdichte stark von den elastischen Eigenschaften des Mineralbestands abhängig.
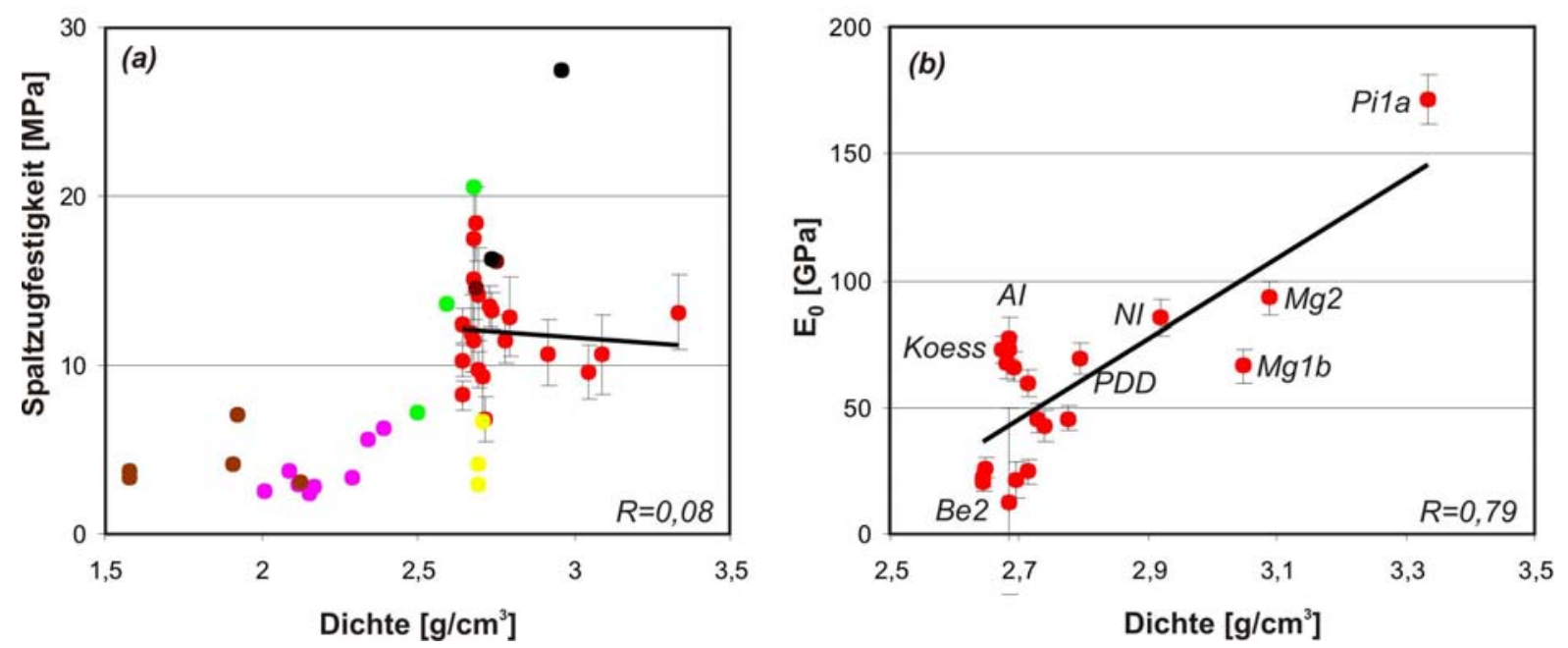

Abb.8-6: Korrelationsdiagramme. Mittelwerte technischer Eigenschaften und ihre Abhängigkeiten von der Dichte. Gelbe Datenpunkte - Marmore. Rote Datenpunkte - Granitoide/ Gneise/ Gabbros/ Peridotit/ Quarzit. Pinke Datenpunkte - Sandsteine. Braune Datenpunkte - Ignimbrit. Grüne Datenpunkte - Quarzite. Schwarze Datenpunkte - Basalt. Die Korrelationskoeffizienten und Trendlinien gelten für die eigenen Daten. (a) Dichte vs. Spaltzugfestigkeit. Daten für Granitoide/ Gneise/ Gabbros/ Peridotit/ Quarzite sind eigene Daten und aus Gupta \& Rao (2000). Daten für Basalt aus Gupta \& Rao (2000). Daten für Sandsteine aus Howarth \& Rowlands (1987); Koch \& Siegesmund (2001). Daten für Marmore aus Howarth \& Rowlands (1987); Siegesmund et al. (1999). Daten für Ignimbrit aus Moon (1993). (b) Dichte vs. dyn. E-Modul (Eo). Pi1a - Peridotit. Mg1b/2 - Meta-Gabbro. $\mathrm{NI}$ - Gabbro-Norit. PDD - Granodiorit. Al - Quarzit. Koess - Granit. Be2 - granitischer Gneis. Fehlerbalken markieren den 1- $\sigma$-Fehler.

Die gute Korrelation von Dichte und dynamischen elastischen Eigenschaften in Bezug auf den Mineralbestand überträgt sich hier in Ansätzen auf das Gestein. Dabei ist der Einfluss der 
offenen Mikrorisse auf $E_{0}$ nicht berücksichtigt. Dieser führt jedoch zu einer Streuung der Daten innerhalb der Granitoide und Gneise mit ähnlichen elastischen Eigenschaften des Mineralbestands und unterschiedlichen Rissdichten (Abb.8-6; granitischer Gneis Be2 - hohe Rissdichte; Granit Koess - niedrige Rissdichte). Die Zusammenhänge zwischen der Rohdichte eines Gesteins und dem statisch ermittelten Spannungs-Dehnungs-Verhalten sind sehr kompliziert, und die deutliche Korrelation zwischen $\mathrm{E}_{\text {stat50 }}$ und der Dichte kann nur als zufällig interpretiert werden. Der statische E-Modul hängt sowohl von den mechanischen und elastischen Eigenschaften des Mineralbestands als auch von der Rissdichte und der Korngröße ab. Dadurch, dass die letztgenannten Parameter kaum einen bzw. gar keinen Einfluss auf die Dichte haben und zusätzlich nur schwer kategorisierbare Zusammenhänge zwischen der Dichte und den mechanischen/elastischen Eigenschaften der Minerale bestehen, ist die deutliche Korrelation von $E_{\text {stat50 }}$ und der Dichte mit Sicherheit nicht auf andere Kristallingesteine übertragbar. Ein weiteres Argument für diese Sichtweise liefert die wesentlich schlechtere Übereinstimmung zwischen $E_{\text {stats }}$ (Bruchmodul) und der Dichte, obwohl für $\mathrm{E}_{\text {stats }}$ gegenüber $\mathrm{E}_{\text {stat50 }}$ lediglich ein etwas höherer Einfluss der Mikrorisse gilt.

Basierend auf 261 Datensätzen, bestehend aus Granitoiden, Gneisen, Gabbros, Marmoren, Kalksteinen, Sandsteinen, Quarziten und Schiefern wurden für die Eigenschaften Dichte, Druck-, Biege- und Abriebfestigkeit Korrelationsdiagramme mit drei Komponenten erstellt mit dem Ziel, die Dichteabhängigkeit der mechanischen Eigenschaften nach Gesteinstypen aufzuschlüsseln. Für die Druck- und Biegefestigkeiten besteht ein dichte-sensitiver Bereich mit Gesteinsdichten $\leq 2,6 \mathrm{~g} / \mathrm{cm}^{3}$ und ein dichte-insensitiver Bereich mit Dichten $>2,6 \mathrm{~g} / \mathrm{cm}^{3}$ (Abb.8-7). Im ersten Fall korrelieren niedrige Dichten mit beiderseitig niedrigen Festigkeiten. Im zweiten Fall besteht kein derartiger Zusammenhang. Die Druckfestigkeiten der Gesteine können sich hier um ca. $200 \mathrm{MPa}$ und die Biegefestigkeiten um bis zu $20 \mathrm{MPa}$ unterscheiden, bei lediglich geringen Änderungen der Dichte $\left(0,2 \mathrm{~g} / \mathrm{cm}^{3}\right)$. Es zeigt sich hier deutlich, dass die gängigen positiven Korrelationen zwischen der Dichte und den Druck- und Biegefestigkeiten, die auf einer Verringerung der Dichte (höheren Porosität) beruhen, nicht auf niedrigporöse Gesteine (z.B. Gneise, Granite, Quarzite, Marmore, niedrigporöse Kalk- und Sandsteine) zutreffen. Hier sind allgemein die Gesteinsgefüge und dabei im speziellen die offenen Mikrorisse und ihre Geometrie, aber auch die mechanischen Eigenschaften der Minerale entscheidend für die Gesteinsfestigkeiten.

Für die Abriebfestigkeiten ist die Abhängigkeit von der Dichte weniger klar als für die Druckund Biegefestigkeiten (Abb.8-7). Insgesamt zeigt sich die Abriebfestigkeit verhältnismäßig dichte-insensitiv auch im Bereich der Dichten $\leq 2,6 \mathrm{~g} / \mathrm{cm}^{3}$. 

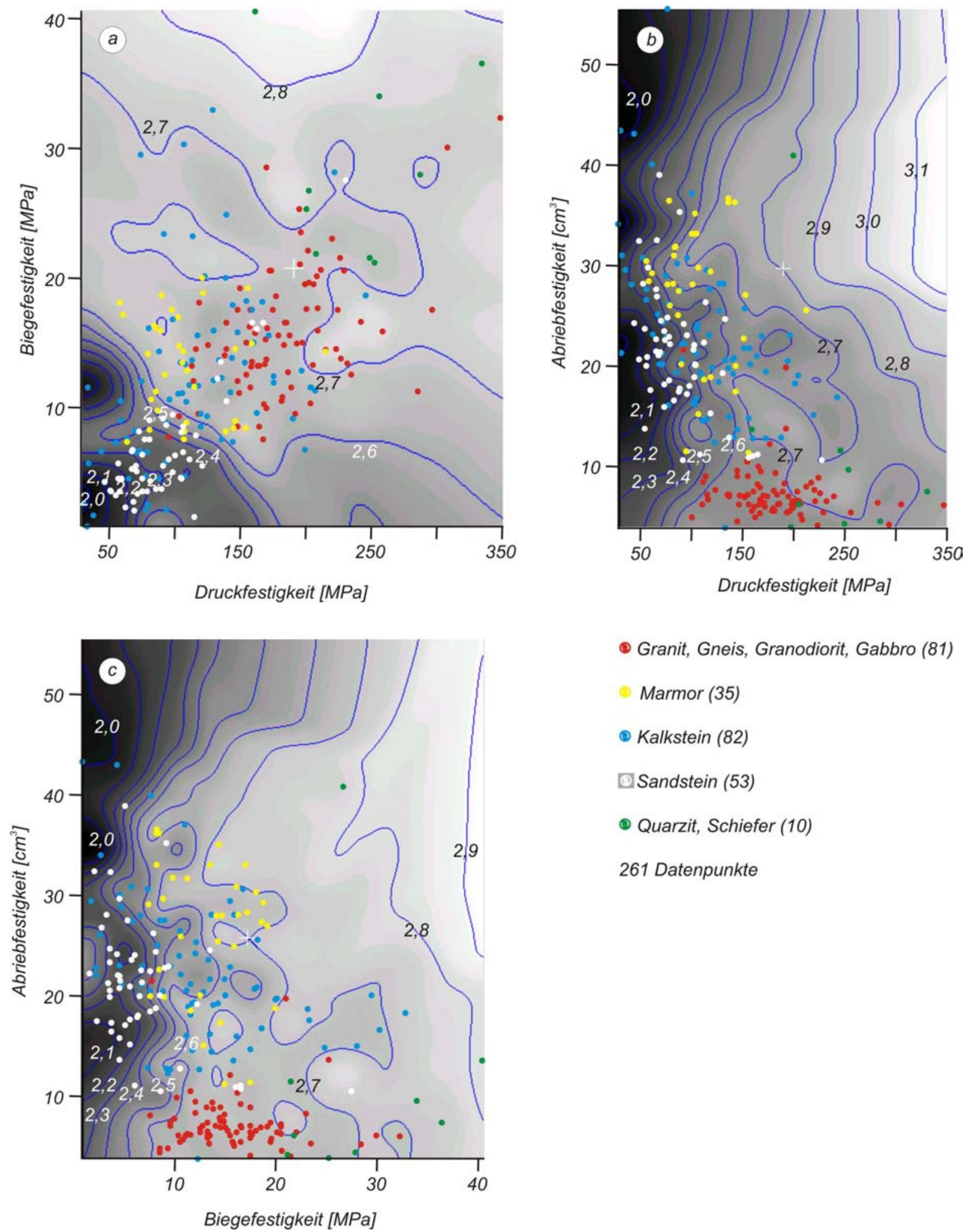

- Granit, Gneis, Granodiorit, Gabbro (81)

Marmor (35)

๑ Kalkstein (82)

QSandstein (53)

(1) Quarzit, Schiefer (10)

261 Datenpunkte

Abb.8-7: Ternäre Korrelationsdiagramme. Zusammenhänge zwischen mechanischen Eigenschaften und der Dichte für unterschiedliche Gesteinsgruppen. Die Dichte $\left[\mathrm{g} / \mathrm{cm}^{3}\right]$ ist in der dritten Dimension in der Form von Isolinien (Isolinienabstände $0,1 \mathrm{~g} / \mathrm{cm}^{3}$ ) indiziert. Daten aus Börner \& Hill (2002). Die Oberfläche wurde auf der Basis aller 261 Datenpunkte berechnet (interpolation distance transform); smoothing mit overrelaxation technique. (a) Druckfestigkeit, Biegefestigkeit, Dichte. (b) Druckfestigkeit, Abriebfestigkeit, Dichte. (c) Biegefestigkeit, Abriebfestigkeit, Dichte.

So können Gesteine mit einer niedrigen Dichte von beispielsweise ca. 2,1 g/ $\mathrm{cm}^{3}$ durchaus im Bereich von $15 \mathrm{~cm}^{3}$ bis $40 \mathrm{~cm}^{3}$ in ihren Abriebfestigkeiten variieren. Hier bestimmt die 
mineralogische Zusammensetzung die Festigkeit (vgl. Kap.6.3.2). Gesteine mit einer hohen durchschnittlichen Härte $(\mathrm{HV}>600)$ wie z.B. Gneise und Granit weisen durchweg hohe Abriebfestigkeiten von $<10 \mathrm{~cm}^{3}$ auf, wohingegen die Marmore bei einer vergleichbaren Dichte, aber einer geringeren Härte $(\mathrm{HV}>250)$ im Bereich zwischen $15 \mathrm{~cm}^{3}$ und $40 \mathrm{~cm}^{3}$ liegen.

\subsection{Korrelation der mechanischen Eigenschaften}

Bislang wurden die Mittelwerte der mechanischen Eigenschaften, ihre Abhängigkeit von der Porosität und der Dichte und in Ansätzen auch die Verhältnisse zueinander dargelegt. Im Folgenden wird im Detail auf die Korrelation der mechanischen Kennwerte untereinander eingegangen mit einem Schwerpunkt auf der Richtungsabhängigkeit. Auf der Basis des Angebots mechanisch schwacher Flächen wird ein Erklärungsmodell für die Richtungsabhängigkeit der mechanischen Gesteinseigenschaften und für die sich daraus ergebenden wechselseitigen Beziehungen zwischen den einzelnen Parametern entwickelt. Abb.8-8 zeigt die sechs möglichen Kreuzkorrelationen der mechanischen Kennwerte Druck-, Spaltzug-, Biege- und Abriebfestigkeit für die Mittelwerte (arithmetisches Mittel aus x-, y-, zRichtung) und die einzelnen Richtungen (x-, y-, z-Richtung).

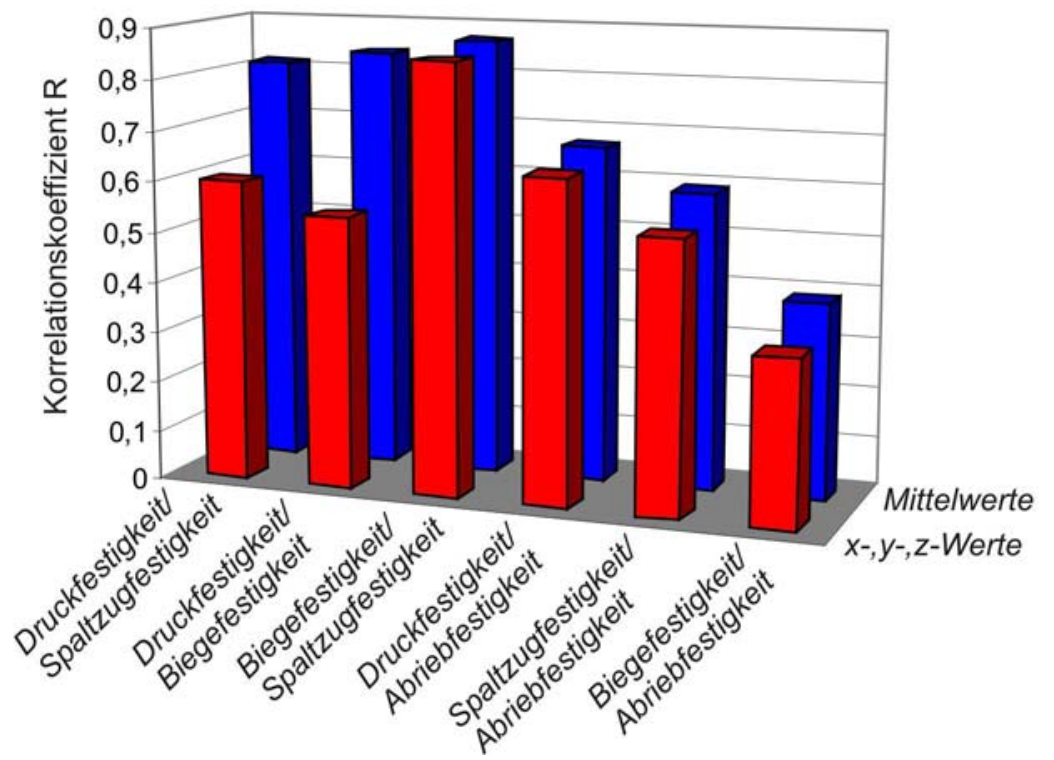

Abb.8-8: Korrelationskoeffizienten für die Kreuzkorrelationen der mechanischen Eigenschaften.

Für die Mittelwerte besteht eine gute Korrelation zwischen der Druckfestigkeit und der Spaltzug- und Biegefestigkeit sowie zwischen der Biegefestigkeit und der Spaltzugfestigkeit. Der Zusammenhang zwischen der Druck- und der Abriebfestigkeit ist noch deutlich, wobei zu bemerken ist, dass alle Korrelationen mit der Abriebfestigkeit nichtlinearer Natur sind. Dieses 
Phänomen wird im Weiteren noch diskutiert. Grundsätzlich fällt die Korrelation der Einzelrichtungen schlechter aus als die der Mittelwerte. Dieser Effekt ist besonders deutlich bei der Korrelation Druck-/Spaltzugfestigkeit und Druck-/Biegefestigkeit und geringer für die Verhältnisse Biege-/Spaltzugfestigkeit und Druck-/Abriebfestigkeit ausgeprägt und suggeriert eine Gefügeabhängigkeit der Korrelationen. Um diese Vermutungen zu bestätigen, werden beispielhaft die Mittelwerte und die x-, y-, z-Werte für die Korrelationen Biege/Spaltzugfestigkeit und Druck-/Spaltzugfestigkeit nach dem makroskopischen Erscheinungsbild der Gesteine (Makrogefüge-Gruppen I/II/III) aufgeschlüsselt ( $\boldsymbol{A} \boldsymbol{b} \boldsymbol{b} . \boldsymbol{8}$ 9/Abb.8-10). Es sind jeweils die mittleren sowie die maximalen und minimalen Verhältnisse angegeben. Eine geringe Abweichung letzterer vom Durchschnittswert bedeutet ein konstantes Verhältnis der Kennwerte (Quotient aus den betrachteten Kenngrößen) und damit eine gute Korrelation.

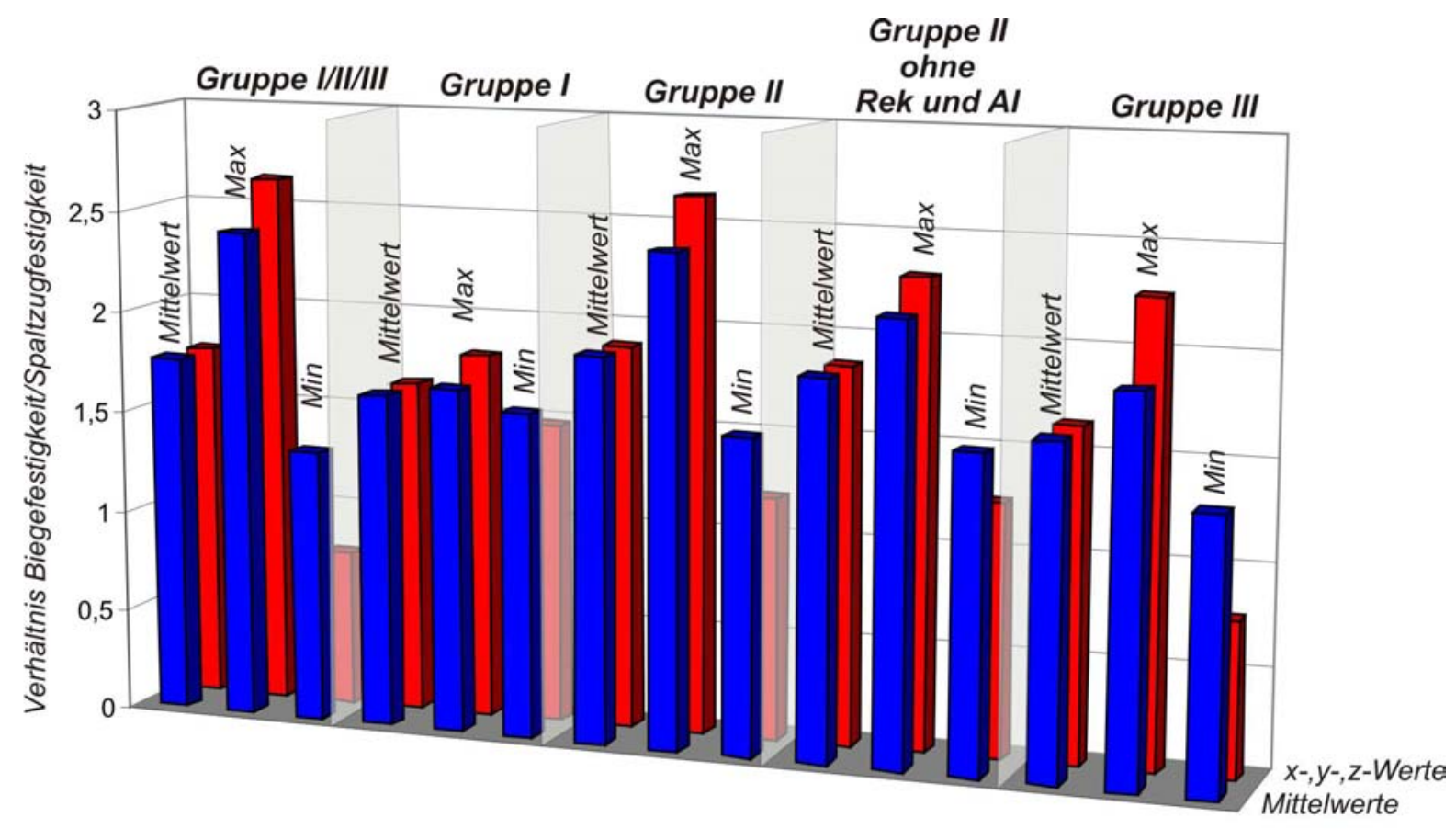

Abb.8-9: Quotient Biege-/Spaltzugfestigkeit unterteilt nach den Makrogefüge-Gruppen I/IIIII.

Das Verhältnis der Biege- zur Spaltzugfestigkeit bewegt sich zwischen 1,06 (granitischer Gneis VAz) und 2,63 (Kalzit-Marmor - Reky) bei einem Mittelwert von 1,76. Dabei zeigt die Makrogefüge-Gruppe I (isotrope Gesteine) die geringste Variationsbreite von 1,48 bis 1,8 (Granit - Koessx bzw. Koessy). Die der Gruppe II (anisotrope Gesteine) mit 1,19 (Quarzit AIx) bis 2,63 (Reky) und der Gruppe III (ausgeprägt anisotrope Gesteine) von 0,76 (granitischer Ultramylonit - Gra3az) bis 2,25 (Gra3ax) sind wesentlich höher. Die Unterschiede in den Verhältnissen der Gruppe II verringern sich, wenn die, von der mineralogischen Zusammensetzung her, extremen Proben AI (Quarzit) und Rek (Kalzit- 
Marmor) nicht in die Betrachtungen einbezogen werden (Abb.8-9). Grundsätzlich variieren die Verhältnisse für die Mittelwerte weniger stark als für die x-,y-,z-Werte.

Das Verhältnis von Druck- zu Spaltzugfestigkeit beträgt im Mittel 17,83. Der Minimalwert liegt bei 10,15 (granodioritischer Mylonit - Iv1b), der Maximalwert bei 39,25 (granitischer Ultramylonit - Gra3az). Das Verhalten in Abhängigkeit von der makroskopischen Gefügeanisotropie ähnelt qualitativ dem der Verhältnisse von Biege- zu Spaltzugfestigkeit. Als deutlicher Unterschied sticht die wesentlich größere Diskrepanz zwischen den Quotienten (Druck-/Spaltzugfestigkeit) für die Mittelwerte und für die richtungsabhängigen x-, y-, zWerte heraus. Die Varianz ist insbesondere bei den Makrogefüge-Gruppen mit höherer Gefügeanisotropie wesentlich stärker ausgebildet (Abb.8-10).

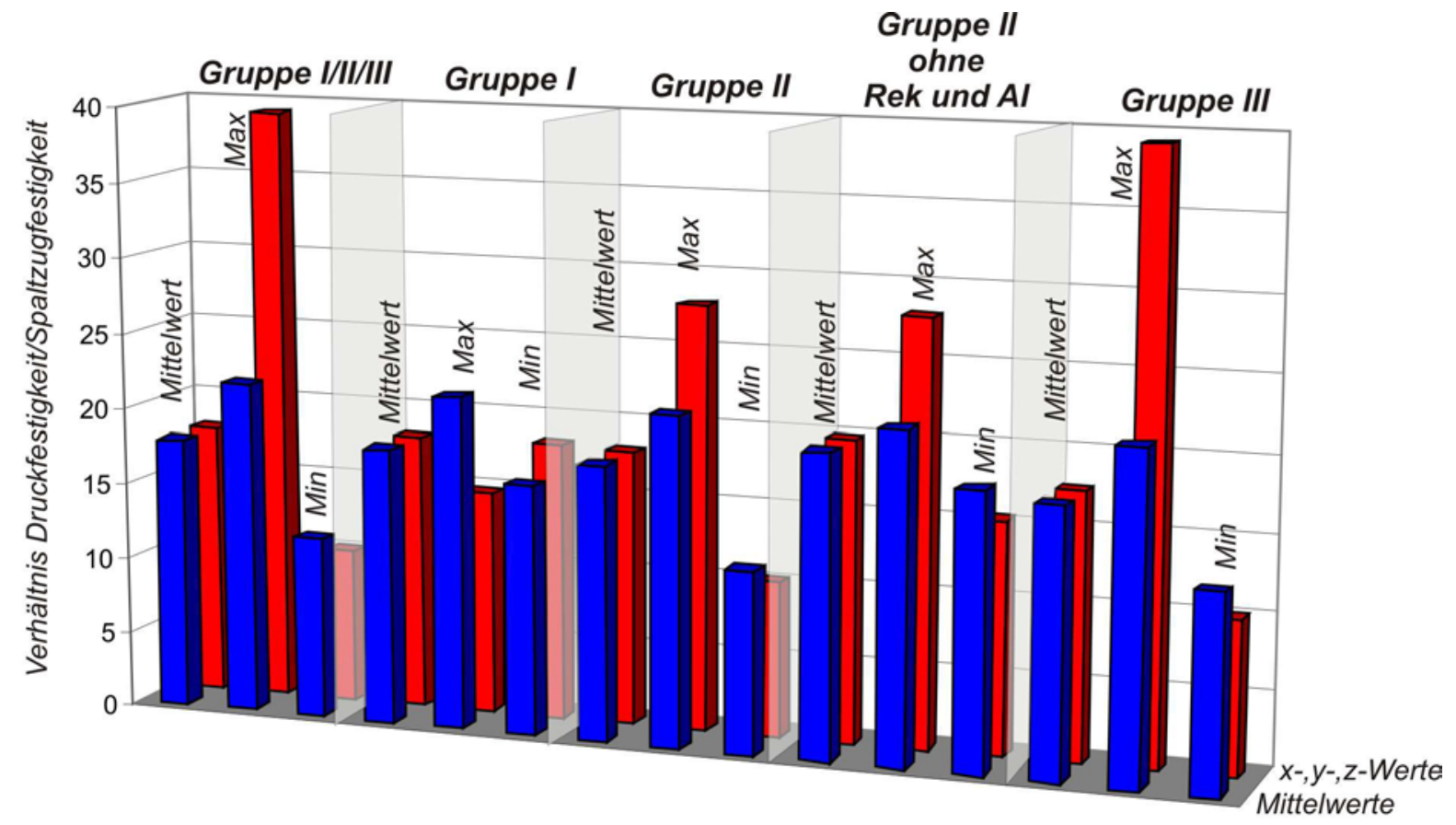

Abb.8-10: Quotient Druck-/Spaltzugfestigkeit unterteilt nach den Makrogefüge-Gruppen I/IIIIII.

Zusammengefasst ergeben sich die folgenden Aussagen:

1. Die Verhältnisse der Mittelwerte zeigen eine geringere Variationsbreite als die der X-, y- und z-Werte. Dieser Effekt ist beim Verhältnis Druck-/Spaltzugfestigkeit besonders stark ausgeprägt.

2. Die Makrogefüge-Gruppen II/III mit einer höheren Gefügeanisotropie weisen im Vergleich zur Gruppe I (isotrope Gesteine) eine größere Variationsbreite der Verhältnisse auf. Das gilt besonders für die Korrelation Druck- zu Spaltzugfestigkeit.

3. Innerhalb der Gruppe II sinkt die Variationsbreite der Verhältnisse, wenn die Proben AI (Quarzit) und Rek (Kalzit-Marmor) nicht einbezogen werden. 
Die Relationen der mechanischen Kennwerte zueinander sind also auch von der mineralogischen Zusammensetzung abhängig. Diese Aussage wird durch die extremen Verhältnisse für die Proben AI und Rek, und zusätzlich durch die unterschiedliche Gruppierung von z.B. Marmoren und Granitoiden in Abb.8-7 bestätigt. Zudem sind die Verhältnisse der Parameter richtungsabhängig und werden daher durch die Gefügeanisotropie beeinflusst.

\subsubsection{Versagensmodelle und Festigkeitsprognosen}

Warum also besteht diese Gefügeabhängigkeit für die wechselseitigen Beziehungen der mechanischen Parameter? Und warum ist dieses Phänomen für den Quotienten Druck/Spaltzugfestigkeit stärker ausgeprägt als für den Quotienten Biege-/Spaltzugfestigkeit? Aufschluss liefern die Versagensmechanismen bei der Durchführung der mechanischen Tests und die räumliche Orientierung der Prozesszonen in Relation zur Orientierung der Gefügeelemente (Abb.8-11). Beim Druckversuch bildet sich aus Scherriss-Zugriss-Paaren eine makroskopische Bruchfläche, entlang der das Gesteinsversagen eintritt (Prikryl 1998; Stavrogin \& Tarasov 2001). Der Bruch entsteht etwa in der Ebene der maximalen Scherspannungen, die mit ca. $35-40^{\circ}$ zur Achse der angreifenden Kräfte geneigt ist. Die während des Spaltzugversuchs wirkenden Spannungen führen zu einer von Zugrissen dominierten Prozesszone, die senkrecht $\mathrm{zu}$ den auftretenden Kräften orientiert ist. Beim Biegeversuch entstehen sowohl Druckspannungen als auch Zugspannungen. Das Gestein versagt aufgrund der gegenüber den Druckfestigkeiten deutlich niedrigeren Zugfestigkeiten durch die Bildung von Zugrissen mit einer bevorzugten Orientierung senkrecht zu den Zugspannungen. Ein sehr komplexes Bild ergibt sich für den Abriebversuch. Der Mineralverband wird durch die Bildung von Scherrissen und Zugrissen zerstört, die jedoch kaum bestimmten Richtungen zugeordnet werden können, zumal die Probe während des Versuchs mehrfach um $90^{\circ}$ gedreht wird. Lediglich eine Tendenz der bevorzugten Orientierung von Scherrissen parallel zur Basis(Abrieb)-Fläche des Abriebkörpers ist zu vermuten (Abb.8-11). Die räumliche Lage der verschiedenen Prozesszonen bzw. die Orientierung der zum Bruch führenden Spannungen können für jede betrachtete Richtung (x-, y- ,z-Richtung - Hauptgefüge-Richtungen) mit der Anordnung der Gefügeelemente in Verbindung gebracht werden. 


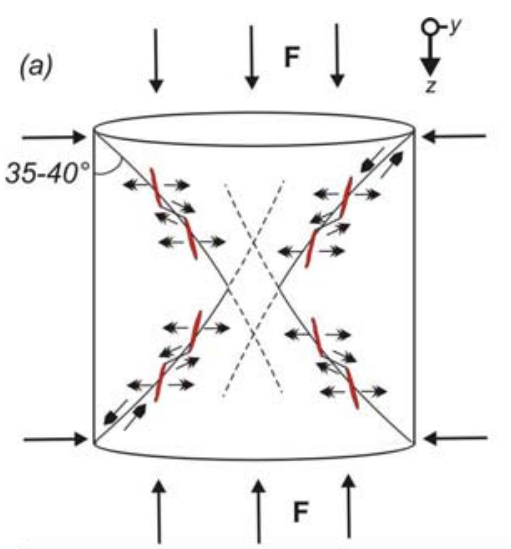

Gesteinsversagen durch Scherrisse und Zugrisse Es kommt zu Lokalisation von Prozesszonen; die Minerale und Gefügeelemente mit der geringsten mechanischen Festigkeit sind entscheidend

(c)
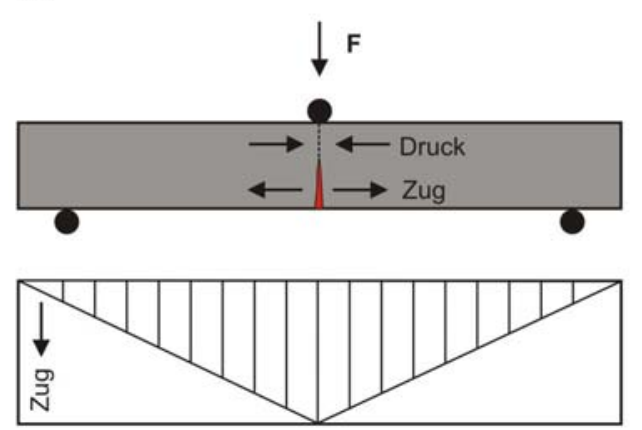

Spannungsverteilung an der Unterseite der Probe

\section{Gesteinsversagen durch Zugrisse}

Es kommt zu Lokalisation von Prozesszonen; die Minerale und Gefügeelemente mit der geringsten mechanischen Festigkeit sind entscheidend

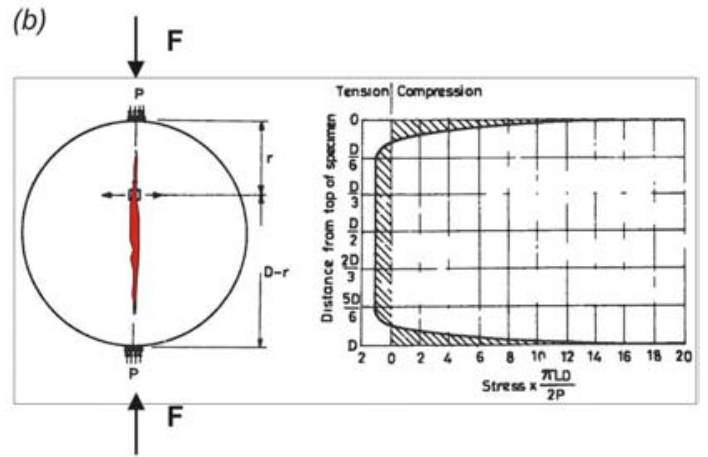

Gesteinsversagen durch Zugrisse

Es kommt zu Lokalisation von Prozesszonen; die Minerale und Gefügeelemente mit der geringsten mechanischen Festigkeit sind entscheidend

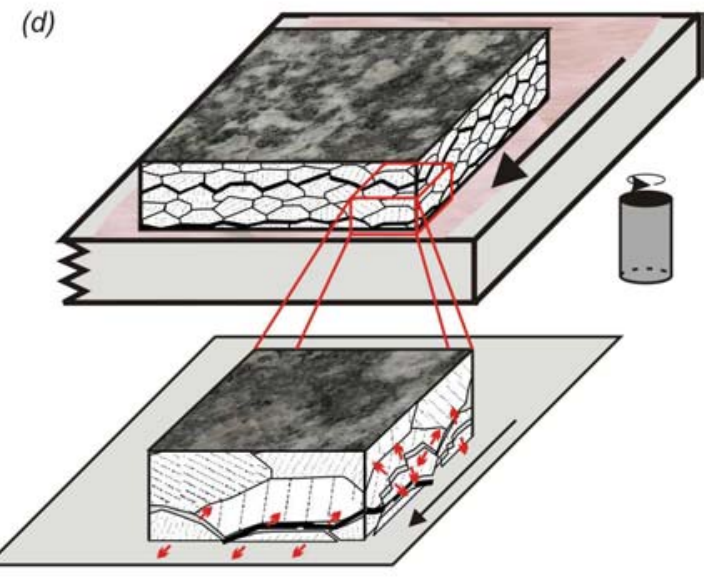

Gesteinsversagen durch Scherrisse und Zugrisse Das Gesteinsgefüge wird durchgreifend entfestigt; alle Minerale und Gefügelelemente (nach Kap.6.3) tragen zur Abriebfestigkeit bei

Abb.8-11: Versagensmechanismen und räumliche Orientierung der Spannungen und Prozesszonen während der mechanischen Tests. (a) Einaxialer Druckversuch. (b) Spaltzugversuch. (c) Biegeversuch. (d) Abriebversuch nach Böhme.

Beispielhaft sind diese räumlichen Zusammenhänge in $\boldsymbol{A b b . 8 - 1 2}$ für einen idealisierten SGefüge-Typ (Flächengefüge dominant) und in $\boldsymbol{A b b . 8 - 1 3}$ für einen S-L-Gefüge-Typ (Foliation und Linear gleichermaßen ausgeprägt) dargestellt. Die schwarzen Elemente deuten dabei nicht zwingend eine Foliation, sondern vielmehr die Raumlage potenziell mechanisch schwacher Flächen an. Das können sowohl die (001)-Flächen der Phyllosilikate als auch offene Mikrorisse oder durch den mineralogischen Lagenbau (Materialwechsel) bedingte Schwächezonen sein. Aus den Abb.8-12/Abb.8-13 lassen sich zum einen die bereits beschriebenen Korrelationen der Festigkeitskennwerte erklären, und zum anderen kann die Richtungsabhängigkeit der mechanischen Eigenschaften begründet und eine Vorhersage 
abgegeben werden, welche Richtung die relativ höheren und welche die niedrigeren Festigkeiten zeigt.
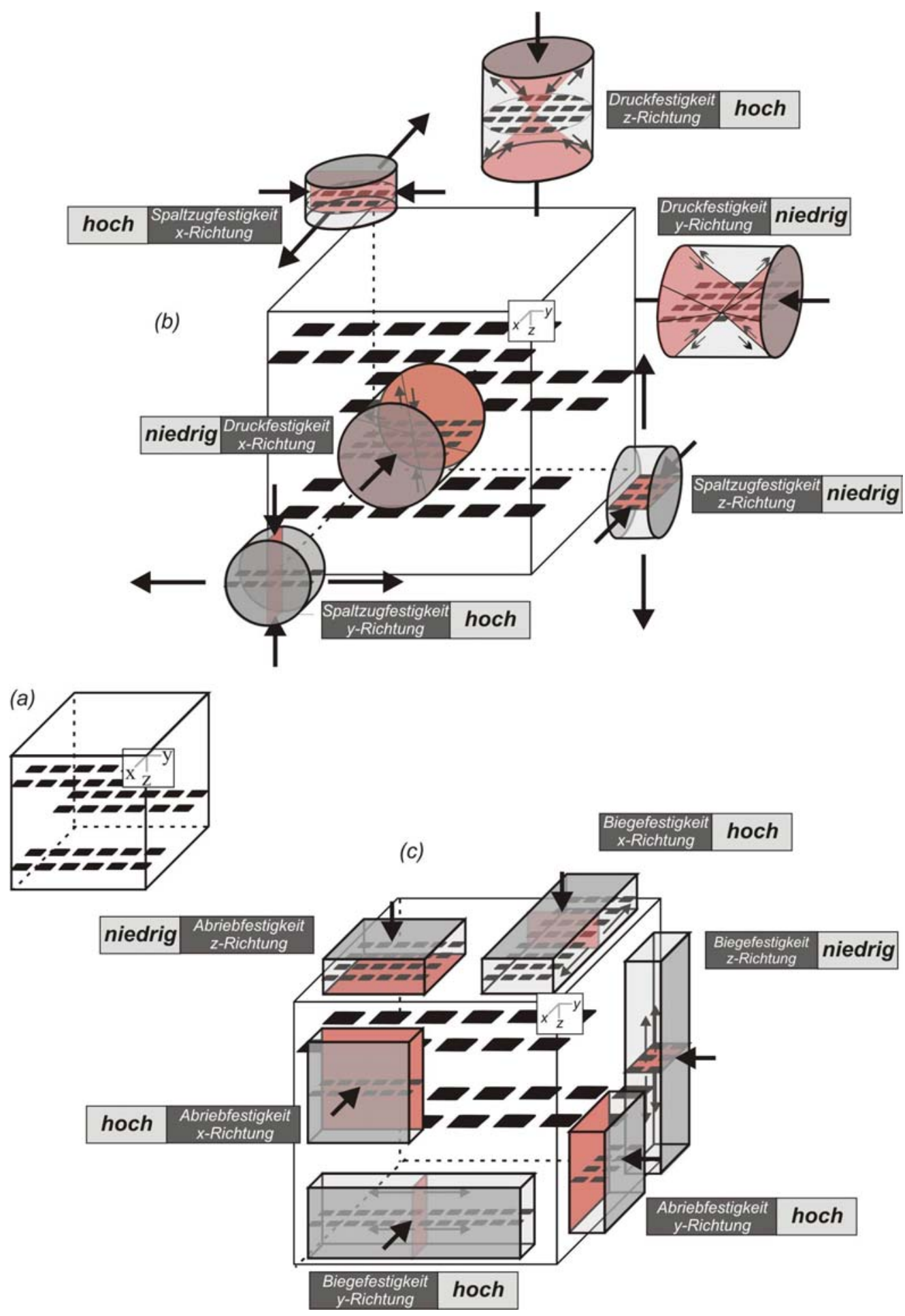

Abb.8-12: S-Gefüge-Typ. Räumliche Anordnung der mechanisch schwachen Flächen und der Probenkörper für die mechanischen Tests in Bezug auf das Probenreferenzsystem. Die Richtungen sind indiziert und die relative Höhe der Festigkeiten (hoch, niedrig, mittel) nach dem Modell des Schwächezonen-Angebots angegeben. Rote Flächen - Prozesszonen. Dicke Pfeile - Orientierung der beim Versuch auf die Probe gebrachten Kräfte. Dünne Pfeile - Orientierung der zum Bruch führenden Spannungen. (a) Schema. (b) Druck- und Spaltzugversuch. (c) Biege- und Abriebversuch. 
1a) Korrelation Spaltzug- und Biegefestigkeit

Lediglich beim Spaltzug- und beim Biegeversuch findet eine Zerstörung der Probe durch den gleichen Prozess (Bildung von Zugrissen) entlang von Prozesszonen identischer Raumlage (senkrecht zu den Hauptgefüge-Achsen) statt. Dadurch werden die gleichen Gefügeelemente in der gleichen Orientierung auf nahezu identische Weise aktiviert, was zu der beobachteten guten Korrelation sowohl der Mittelwerte, als auch der x-, y-, z-Werte (richtungsabhängige Betrachtung) der Spaltzug- und Biegefestigkeitswerte führt.

1b) Korrelation Druck- und Spaltzug-/Biegefestigkeit

Bei der Ermittlung der Druckfestigkeiten sind die Prozesszonen zu den HauptgefügeAchsen geneigt, und im Gegensatz zum Spaltzug- und Biegeversuch findet der Bruch durch eine Kombination von Scher- und Spaltrissen statt. Folglich werden die Gefügeelemente beim Druckversuch in einer anderen Richtung und auch auf eine andere Art aktiviert als das bei der Bestimmung der Spaltzug- und Biegefestigkeiten der Fall ist. Dieser Umstand wirkt sich insbesondere auf die Korrelation der x-, y-, zWerte von Druck- zur Spaltzug-/Biegefestigkeit aus, wobei die anisotropen und ausgeprägt anisotropen Gesteine der Gruppen II/III am stärksten betroffen sind und stark schwankende Verhältnisse (hier: schlechte Korrelationen) zeigen (Abb.8-10). Für die isotropen Gesteine mit einer ohnehin nur geringen Vorzugsorientierung der Gefügemerkmale bestehen nahezu keine Richtungsabhängigkeiten, so dass die Korrelationen (Druck- zu Spaltzug-/Biegefestigkeit) gut und die Verhältnisse weitestgehend konstant sind. Für die Mittelwerte aller Gruppen spielt die Richtungsabhängigkeit nahezu keine Rolle. Folglich kommt der Einfluss der Gefügeanisotropie nicht zum Tragen und die Korrelationen der Mittelwerte sind stets besser und damit die Verhältnisse konstanter, als die der x-, y-, z-Werte. Die Varianzen bezüglich der Korrelationen der Mittelwerte sind daher eher im Bereich der mineralogischen Zusammensetzung zu suchen.

1c) Korrelation Abrieb- und Druck-/Spaltzug-/Biegefestigkeit

Eine einheitliche Aussage für die Korrelation der Abriebfestigkeiten mit den Druck/Spalt-/Biegefestigkeiten ist nur schwer möglich. Die Orientierung der beim Abriebversuch auftretenden Scher-, Druck- und Zugspannungen, die zu Scher- und Zugrissen führen, ist schlecht definiert und kaum zu erfassen. Zudem kommt es bei der Bestimmung der Abriebfestigkeit nicht zu einer geometrisch gut bestimmten Bruchzone, sondern der Abrieb findet in Form einer durchgreifenden 
Gefügeentfestigung statt. Mehr als bei den anderen mechanischen Kennwerten, die durch die Verteilung und Quantität der schwächsten Gefügeelemente dominiert werden, tragen hier alle Minerale und Gefügeelemente gleichermaßen zur Festigkeit bei. Mit Sicherheit ist damit der Einfluss der mechanischen Eigenschaften des Mineralbestands (z.B. der Härte) auf die Abriebfestigkeit deutlich höher als der Beitrag der Rissdichte offener Mikrorisse (vgl. Kap.6.3). Die Zusammenhänge zwischen der Abriebfestigkeit und der Druck-/Spaltzug-/Biegefestigkeit sind unlinear und nur durch empirische Ansätze (vgl. Abb.8-7) mit einem möglichst großen Datensatz sinnvoll zu erfassen (z.B. Peschel 1983). Modellhafte Begründungen auf der Basis von Versagensprozessen und Spannungsorientierungen führen hier aufgrund der multiplen Überlagerungen von Einflussgrößen nicht zum Erfolg.

2) Richtungsabhängigkeit der mechanischen Eigenschaften

Die relative Abfolge der mechanische Stärke in den drei Hauptgefüge-Richtungen lässt sich über das jeweilige Angebot an mechanischen Schwächezonen in Bezug zu den angreifenden Spannungen prognostizieren. Bei den vier mechanischen Tests treten sowohl Scherspannungen, als auch Zugspannungen auf, die bei Überschreitung der Kohäsion des Kornverbandes zum Gesteinsversagen führen. Mechanisch schwache Gefügeelemente (z.B. Glimmer-(001)-Flächen, offene Mikrorisse) mit einer Orientierung parallel/subparallel zu den auftretenden Scherspannungen bzw. senkrecht zu den Zugspannungen bewirken die deutlichste Reduktion der Festigkeiten. Daraus ergeben sich für den S-Gefüge-Typ (Abb.8-12) und den S-LGefüge-Typ (Abb.8-13) folgende Zusammenhänge, wenn man für die Abriebfestigkeit von einer Tendenz $\mathrm{zu}$ einer bevorzugten Orientierung der Scherspannungen und neu gebildeten Risse parallel zur Abriebfläche ausgeht.

2a) S-Gefüge-Typ - Druckfestigkeit

Die z-Richtung zeigt die höchsten Festigkeiten und ein symmetrisches konisches Bruchbild. Die $x-$ und die $y$-Richtung weisen identische, aber niedrigere Druckfestigkeiten auf. Da die in der xy-Ebene orientierten Schwächezonen bevorzugt aktiviert werden, ist das Bruchbild dachförmig (Abb.8-12).

2b) S-Gefüge-Typ - Spaltzug-/Biegefestigkeit

Die z-Richtung weist die niedrigsten Festigkeitswerte, und die $\mathrm{x}$ - und y-Richtung zeigen identische, aber höhere Werte. 

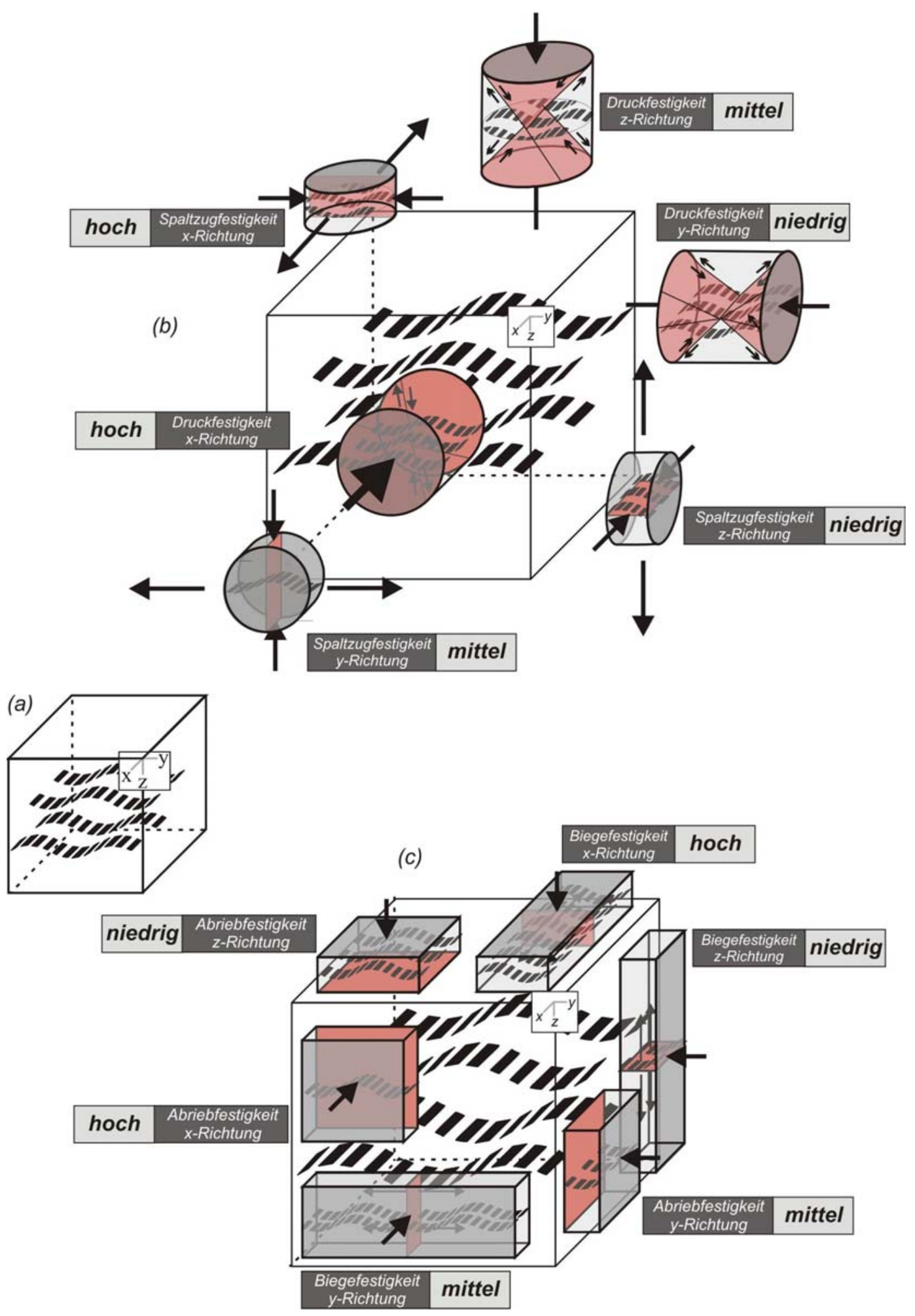

Abb.8-13: S-L-Gefüge-Typ. Räumliche Anordnung der mechanisch schwachen Flächen und der Probenkörper für die mechanischen Tests in Bezug auf das Probenreferenzsystem. Die Richtungen sind indiziert und die relative Höhe der Festigkeiten (hoch, niedrig, mittel) nach dem Modell des Schwächezonen-Angebots angegeben. Rote Flächen - Prozesszonen. Dicke Pfeile - Orientierung der beim Versuch auf die Probe gebrachten Kräfte. Dünne Pfeile - Orientierung der zum Bruch führenden Spannungen. (a) Schema. (b) Druck- und Spaltzugversuch. (c) Biege- und Abriebversuch. 
2c) S-Gefüge-Typ - Abriebfestigkeit

Die z-Richtung zeigt die niedrigsten Abriebfestigkeiten, wohingegen die x- und yRichtung höhere und identische Werte aufweisen.

2d) S-L-Gefüge-Typ - Druckfestigkeit

Die x-Richtung ist am stärksten und die y-Richtung am schwächsten; die z-Richtung liegt dazwischen. Das Bruchmuster ist für alle Richtungen dachförmig (Abb.8-13).

2e) S-L-Gefüge-Typ - Spaltzug-/Biegefestigkeit

Die x-Richtung zeigt die höchsten Festigkeitswerte, wohingegen die y-Richtung intermediäre und die z-Richtung die niedrigsten Werte aufweist.

2f) S-L-Gefüge-Typ - Abriebfestigkeit

Die Abriebfestigkeit ist am niedrigsten für die z-Richtung und am höchsten für die XRichtung. Mittlere Werte weist die y-Richtung auf.

Zwischen den beiden idealisierten Gefüge-Typen kommen in der Natur alle Variationen vor. Die aufgrund des Modells vorhergesagten Relationen werden durch die experimentellen Ergebnisse bestätigt. Allerdings sind die S-Gefüge-Typen innerhalb des Probensatzes nicht so ideal, wie das in $\boldsymbol{A b b . 8 - 1 2}$ dargestellte Modellgestein, und beinhalten immer eine, wenn auch schwach ausgeprägte lineare Komponente, bewegen sich also bereits in Richtung S-LGefüge-Typ. Das beinhaltet eine gegenüber der y-Richtung geringfügig mechanisch stärkere $\mathrm{x}$-Richtung. Beispiele für S-Gefügetypen mit der vorausgesagten relativen Festigkeitsabfolge in Bezug auf die Hauptgefüge-Richtungen sind die Proben Bel (granodioritischer Gneis), VA (granitischer Gneis) und Gra1a/1c (granitische Gneise). Typische S-L-Typen sind die Proben Iv1b (granodioritischer Mylonit) und Be2 (granitischer Gneis) (vgl. Kap.5.5.1/6.3.1.1).

\subsection{Elastische, mechanische Eigenschaften, thermische Dehnung}

Welcher der elastischen Parameter $\left(\mathrm{E}_{0}, \mathrm{E}_{\mathrm{stat50}}, \mathrm{E}_{\text {stats }}\right)$ geeignet ist, über eine gute Korrelation mechanische Gesteinsfestigkeiten und das thermische Dehnungsverhalten vorherzusagen, ist die zentrale Frage, welche im Zuge dieses Kapitels beantwortet werden soll.

Gerade in Bezug auf die Schadensanalyse von Naturwerksteinen ist immer wieder der Zusammenhang zwischen den elastischen Eigenschaften und den mechanischen Kennwerten gefragt, um z.B. aus den weitestgehend zerstörungsfreien Ultraschallmessungen (Vp, dynamischer E-Modul) den Erhaltungszustand eines Naturwerksteins einschätzen zu können. Der Korrelation des dynamischen E-Moduls $\left(\mathrm{E}_{0}\right)$ mit den Gesteinsfestigkeiten gilt also ein 
besonderes Interesse. Wie bei der Bewertung der schon beschriebenen Korrelationen ist ein grundlegendes Verständnis der Zusammenhänge nur über die Analyse der Gefügeabhängigkeit und der Abhängigkeit der einzelnen Kennwerte von der mineralogischen Zusammensetzung sowie der detaillierten Betrachtung der beim Experiment wirkenden Prozesse möglich.

Im Folgenden werden die Korrelationen der elastischen mit den mechanischen und thermischen Kennwerten für die Mittelwerte und für die x-, y- und z-Werte dargestellt und ihre Verallgemeinerbarkeit auf kristalline Gesteine eingeschätzt.

\subsubsection{Dynamischer E-Modul, mechanische Kennwerte, thermische}

\section{Dehnung}

Zwischen dem dynamischen E-Modul $\left(\mathrm{E}_{0}\right)$ und den mechanischen Eigenschaften Druck-, Spaltzug-, Biege- und Abriebfestigkeit besteht kein statistisch signifikanter Zusammenhang.

Das gilt für die Mittelwerte und für die x-, y-, z-Werte (Abb.8-14/Abb.8-15).

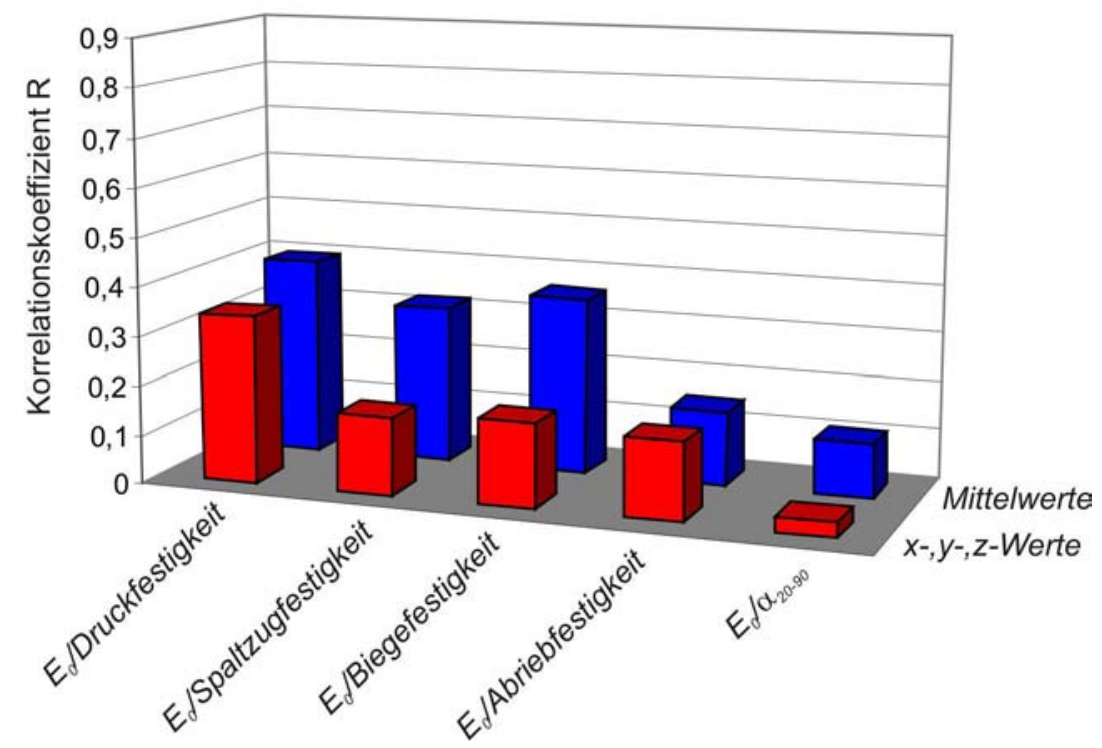

Abb.8-14: Korrelationskoeffizienten (R) für die Beziehung zwischen dem dynamischen E-Modul ( $\left.E_{0}\right)$ und den mechanischen Kennwerten sowie den thermischen Dehnungseigenschaften.

Für die schlechte Korrelation zwischen dem dynamischen E-Modul und den mechanischen Festigkeiten sind eine Reihe von Gründen verantwortlich. Zum ersten hängen die Parameter von unterschiedlichen Gefügekomponenten $\mathrm{ab} . \mathrm{E}_{0}$ wird hauptsächlich von den elastischen Eigenschaften des Mineralbestands $\left(\mathrm{E}_{\mathrm{GVRH}}-\mathrm{Werte}\right)$ und der Rissdichte der Mikrorisse bestimmt, wohingegen für die Festigkeitskennwerte die mechanischen Eigenschaften der Minerale (Härte, $\mathrm{I}_{\mathrm{SPG}}-$ Werte), die Dichte der Mikrorisse und für die Druck-, Spaltzug- und Biegefestigkeiten auch noch die Korngröße ausschlaggebende Kennwerte sind. 

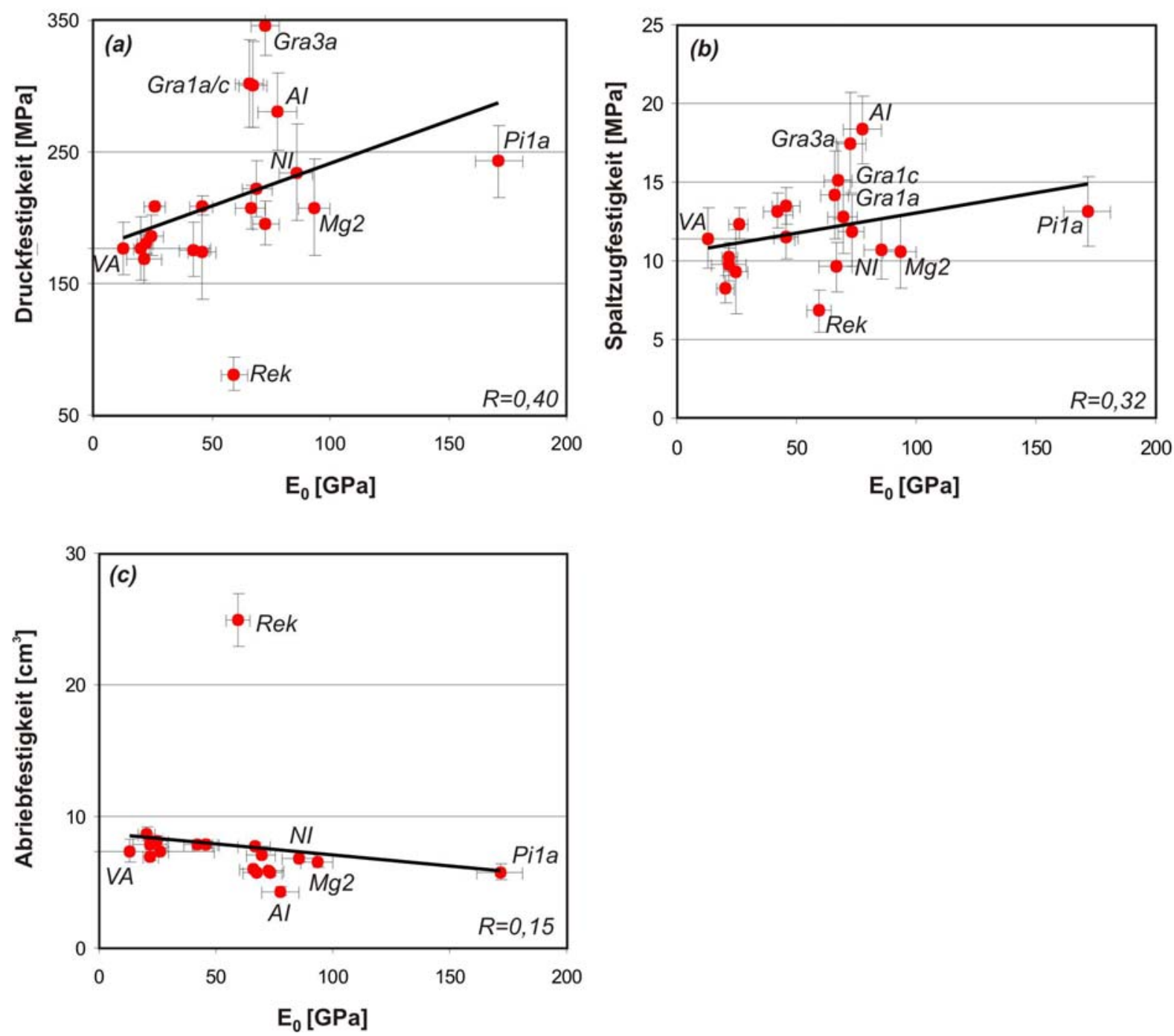

Abb.8-15: Korrelationsdiagramme für die Zusammenhänge zwischen dem dynamischen E-Modul $E_{0}$ und mechanischen Gesteinseigenschaften. (a) $E_{0}$ vs. Druckfestigkeit. (b) $E_{0}$ vs. Spaltzugfestigkeit. (c) $E_{0}$ vs. Abriebfestigkeit. VA - granitischer Gneis. Al - Quarzit. Rek - Kalzit-Marmor. Mg2 - MetaGabbro. Pi1a - Peridotit. Gra1a/1c - granitischer Gneis. Gra3a - granitischer Ultramylonit. NI Gabbro-Norit. Fehlerbalken markieren den 1- $\sigma$-Fehler.

$\mathrm{Da}$ die gesteinsbildenden Minerale bezüglich ihrer mechanischen und elastischen Eigenschaften schlecht korrelieren und die Art und Intensität der Einflussnahme der offenen Mikrorisse deutlich unterschiedlich ist, kann keine gute Korrelation von $\mathrm{E}_{0}$ mit den mechanischen Eigenschaften erwartet werden. Ein weiterer Faktor sind die verschiedenen Prozesse bei den mechanischen und den Ultraschalluntersuchungen. Die Zeit der Krafteinwirkung ist bei der Bestimmung der mechanischen Kennwerte wesentlich höher (Schön 1983) und führt in Ansätzen zur plastischen Verformung und schließlich zum Gesteinsversagen. Dagegen tritt bei der Bestimmung der Ultraschallgeschwindigkeiten ausschließlich elastische Deformation auf, ohne eine bleibende Schädigung der Probe. Auch die Richtungen der Krafteinwirkung sind für die unterschiedlichen Untersuchungsmethoden nicht identisch. Diese Vielzahl von differierenden Faktoren bestimmt die unkonstanten 
Verhältnisse zwischen $\mathrm{E}_{0}$ und den mechanischen Eigenschaften (Abb.8-15). Trotz der multiplen Überlagerung der verschiedenen Einflussgrößen lässt sich einer der Hauptfaktoren für die unkonstanten Verhältnisse feststellen. Mit Sicherheit ist die schlechte Korrelation zwischen $\mathrm{E}_{0}$ und den mechanischen Eigenschaften für den Mineralbestand hier bestimmend (vgl. Kap.4.5) und verhindert eine allgemeingültige Aussage für alle Kristallingesteine (z.B. Kalzitmarmor - Rek und Quarzit - AI in Abb.8-15). Die von Weiss (1992) für Sandsteine festgestellte gute Übereinstimmung von hohen dynamischen E-Moduln und hohen Druckfestigkeiten kann daher für die untersuchten niedrigporösen und bezüglich ihres Mineralbestands sehr variablen Gesteine nicht bestätigt werden.
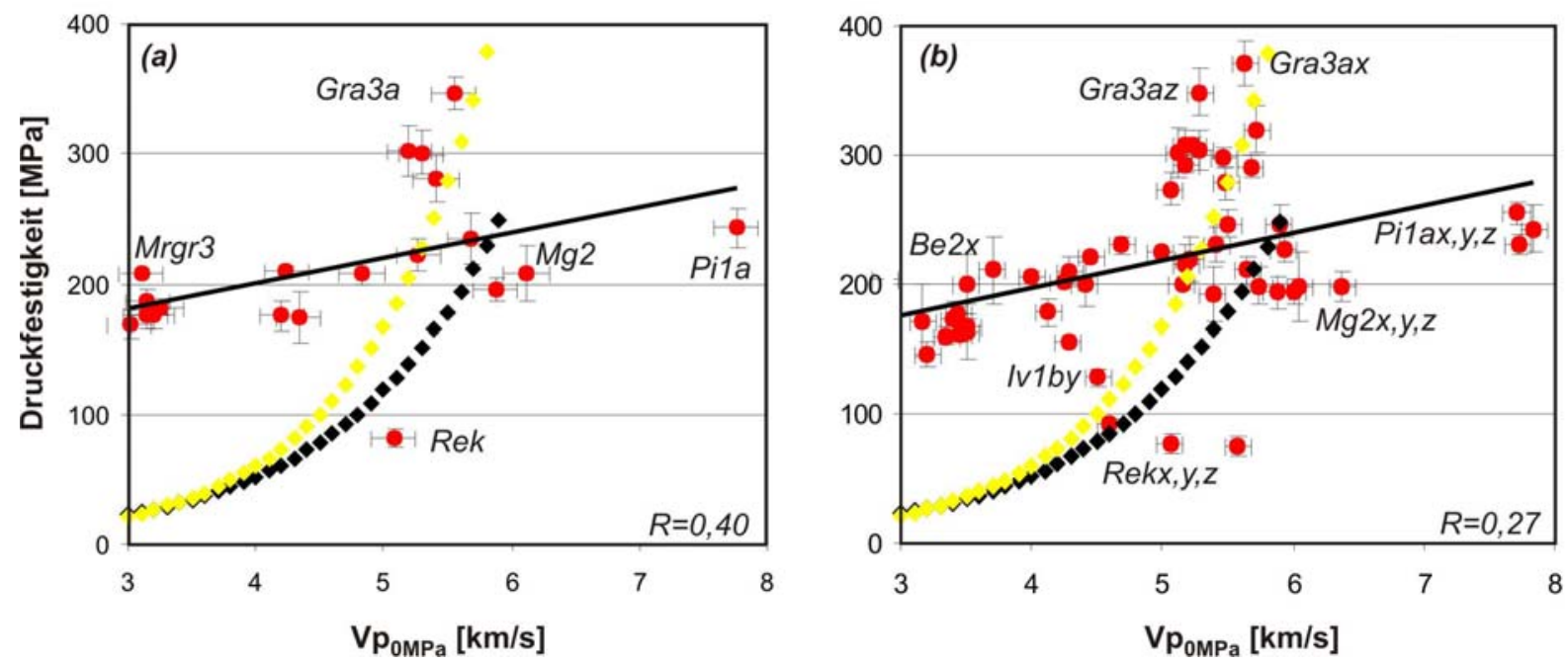

Abb.8-16: Korrelationsdiagramme für die Zusammenhänge zwischen $V p$ (OMPa) und der Druckfestigkeit. Schwarze/gelbe Symbole kennzeichnen den empirischen Zusammenhang für Kalksteine/kristalline Schiefer nach Golubev \& Rabinovic (1976). (a) Mittelwerte. (b) $x$-, $y$-, z-Werte. Mrgr3 - Granit. Rek - Klazit-Marmor. Mg2 - Meta-Gabbro. Pi1a - Peridotit. Gra3a - granitischer Ultramylonit. Iv1b - granodioritischer Mylonit. Fehlerbalken markieren den 1- $\sigma$-Fehler.

Häufig wurden auch Vp-Geschwindigkeiten mit Druckfestigkeiten verglichen und verschiedene empirische Beziehungen aufgestellt (z.B. Freyburg 1972 - Sandstein; Militzer \& Stoll 1973 - Kalkstein; Golubev \& Rabinovic 1976 - Kalkstein und kristalline Schiefer; Gorjainov und Ljachovickij 1979 - sandig tonige Gesteine; Köhler 1991 - Carrara Marmor). Die Zusammenhänge nach Golubev \& Rabinovic (1976) sind in Abb.8-16 zusammen mit den Vp-Werten unter $0 \mathrm{MPa}$ Umschließungsdruck aus den eigenen Ultraschallmessungen dargestellt. Es zeigt sich ähnlich (nicht identisch, weil zwischen den Vp-Werten und den $\mathrm{E}_{0^{-}}$ Werten kein linearer Zusammenhang besteht - vgl. Kap.2.4.5.2) zu den Verhältnissen von $\mathrm{E}_{0}$ zu den Druckfestigkeiten eine schlechte Korrelation von Vp-Werten und den Druckfestigkeiten (Mittelwerte und x-,y-,z-Werte). Außerdem weist keiner der empirischen Zusammenhänge von Golubev \& Rabinovic (1976) eine Übereinstimmung mit den Daten der untersuchten Kristallingesteine auf; noch weniger Gemeinsamkeiten bestehen zu den von 


\section{$\underline{\text { 8. Korrelation der technischen Gesteinseigenschaften }}$}

Köhler (1991) aufgestellten Korrelationen für Marmore. Als einzige Schlussfolgerung gilt, dass empirische Beziehungen, die für eine Gesteinsgruppe mit einer ähnlichen mineralogischen Zusammensetzung und einem ähnlichen Gefügeinventar aufgestellt wurden, nicht auf andere Gesteinsgruppen mit anderen Eigenschaften übertragbar sind. Eine aussagekräftige Gleichung für den Zusammenhang zwischen den dynamischen elastischen Eigenschaften und den Gesteinsfestigkeiten kann im Hinblick auf die wirksamen Gefügeparameter nur dann etabliert werden, wenn sich sowohl die Variationen des Mineralbestands als auch die der Korngröße und der Geometrie der Mikrorisse in einem engen Rahmen bewegen. Zumindest ist in diesem Fall ein auch theoretisch untermauerbarer Ansatz über die mechanische Stabilität des Mineralbestands und die Rissdichte möglich. Sind diese Bedingungen nicht gegeben, so bieten numerische Modellierungen, bei denen die Überlagerung der verschiedenen Einflussgrößen berücksichtigt werden können, eine mögliche Lösung, wobei fundierte Kenntnisse der Gefügeabhängigkeiten und der Abhängigkeiten vom Mineralbestand für die technischen Parameter nötig sind.

Die Prozesse bei der Bestimmung des thermischen Ausdehnungskoeffizienten $\left(\alpha_{20 \mathrm{bis} 90}\right)$ und des dynamischen E-Moduls $\left(\mathrm{E}_{0}\right)$ sind völlig unterschiedlich. Hinzu kommen differierende Abhängigkeiten der Kennwerte von der mineralogischen Zusammensetzung und von den Gefügeelementen (Kap.6.4.1/6.5). Kombiniert mit einer schlechten Korrelation der thermischen Volumenausdehnung und der elastischen Eigenschaften der Minerale ist der nicht signifikante Zusammenhang (Abb.8-14) bezüglich der Gesteinseigenschaften die logische Folge.

\subsubsection{Statischer E-Modul, mechanische Kennwerte, thermische Dehnung}

Beim Vergleich der statischen elastischen Kennwerte $\left(E_{\text {stat50, }}, E_{\text {stats }}\right)$ mit den mechanischen Eigenschaften und der thermischen Dehnung $\left(\alpha_{20 b i s 90}\right)$ fällt folgendes auf:

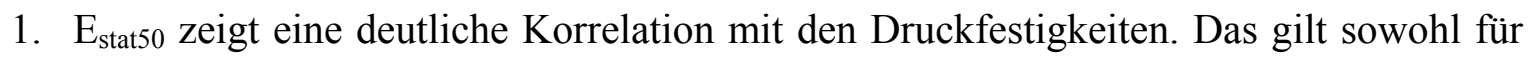
die Mittelwerte, als auch für die x-, y- und z-Werte. Ansonsten sind keine statistisch signifikanten Zusammenhänge zu erkennen (Abb.8-17).

2. $\mathrm{E}_{\text {stats }}$ (Bruchmodul) korreliert gut mit den Druckfestigkeiten (Mittelwerte und x-, y-, zWerte) und deutlich bis gut mit den Mittelwerten der Spaltzug- und Biegefestigkeit (Abb.8-18/Abb.8-19). 


\section{$\underline{\text { 8. Korrelation der technischen Gesteinseigenschaften }}$}

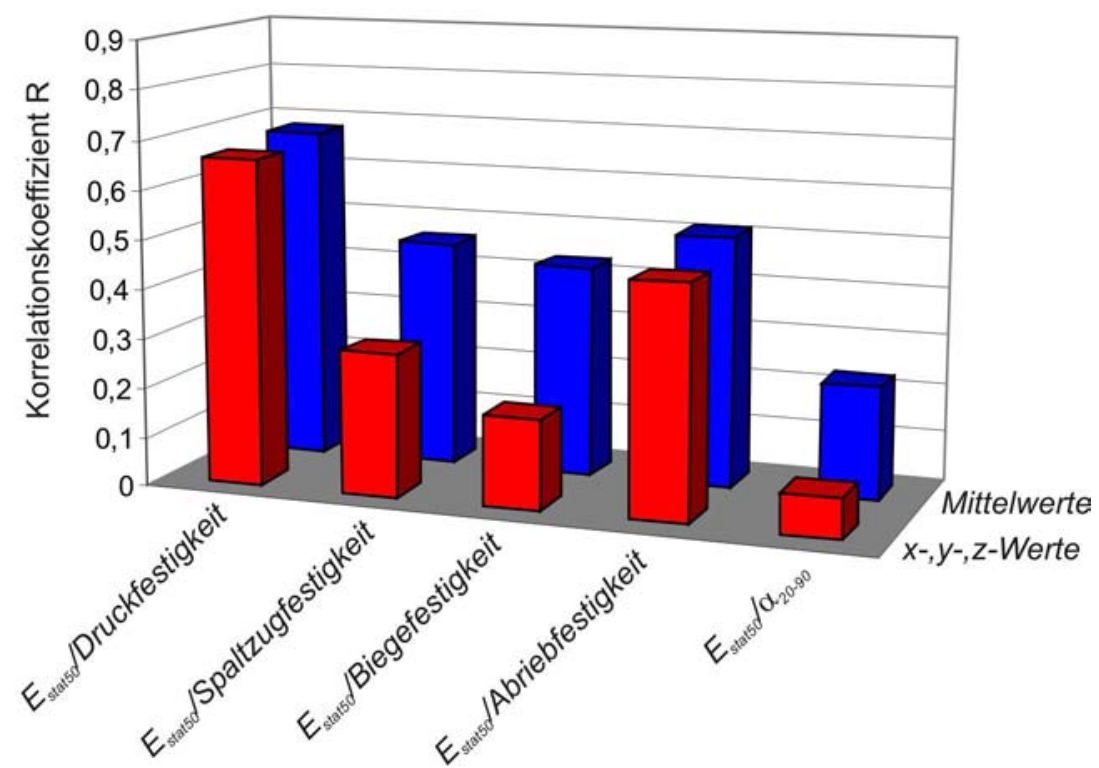

Abb.8-17: Korrelationskoeffizienten (R) für die Zusammenhänge zwischen dem statischen E-Modul $\left(E_{\text {stat5o }}\right)$ und den mechanischen Gesteinseigenschaften und der thermischen Dehnung.

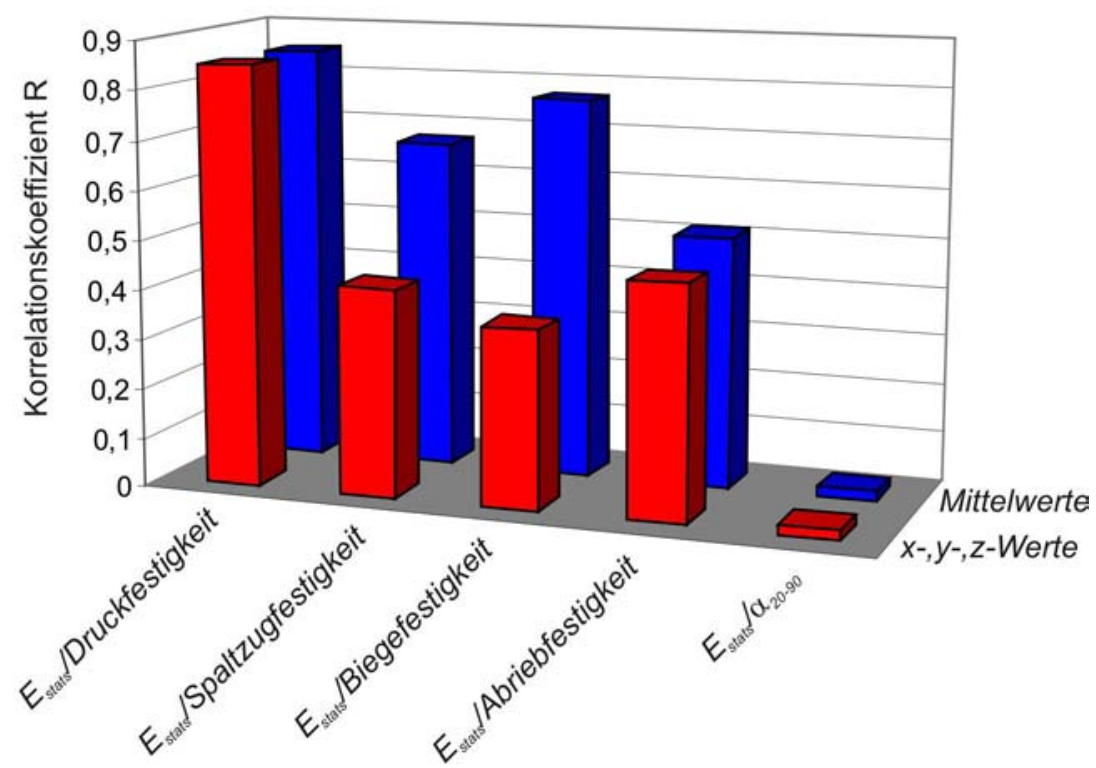

Abb.8-18: Korrelationskoeffizienten (R) für die Zusammenhänge zwischen dem statischen Bruchmodul ( $\left.E_{\text {stats }}\right)$ und den mechanischen Gesteinseigenschaften und der thermischen Dehnung.

Die für $E_{\text {stat50 }}$ und $E_{\text {stats }} \mathrm{zu}$ beobachtende deutliche bzw. gute Korrelation mit der Druckfestigkeit ist nicht unerwartet, werden doch alle drei Parameter aus dem gleichen Versuch heraus ermittelt, so dass die Orientierungen und Beträge der angreifenden Spannungen während des Versuchs identisch sind.

Unterschiedlich sind allerdings die Prozesse: Für die Druckfestigkeit ist das Gesteinsversagen, also der Kohäsionsverlust des Korngefüges entscheidend, wohingegen $\mathrm{E}_{\text {stat50 }}$ und $\mathrm{E}_{\text {stats }}$ das Spannungs-Dehnungs-Verhalten vor dem eigentlichen Bruch beschreiben. Hier sind auch die Unterschiede zwischen den Korrelationen $\mathrm{E}_{\text {stat50 }}$ mit der Druckfestigkeit und $\mathrm{E}_{\text {stats }}$ mit der 
Druckfestigkeit begründet. Die statischen Elastizitätskennwerte werden neben den mechanischen Eigenschaften der Minerale und der Rissdichte der Mikrorisse auch durch das elastische Verhalten des Mineralbestands beeinflusst. Letzterer Faktor ist für $\mathrm{E}_{\text {stat50 }}$ bedeutender als für $\mathrm{E}_{\text {stats, }}$, welcher stärker durch die Mikrorissdichte dominiert wird (vgl. Kap.6.4.2.2).
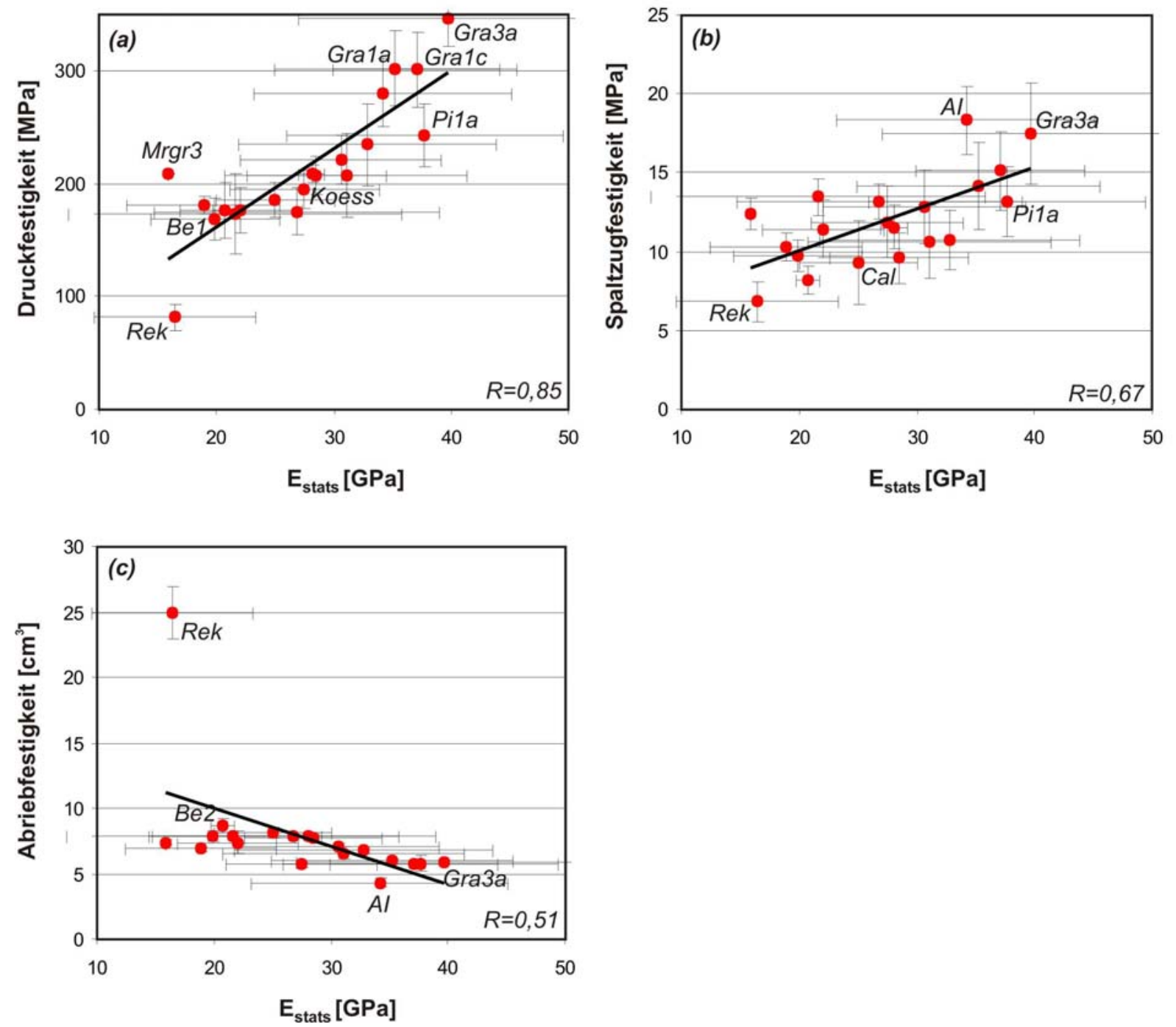

Abb.8-19: Korrelationsdiagramme für die Zusammenhänge zwischen dem Bruchmodul ( $\left.E_{\text {stats }}\right)$ und mechanischen Kennwerten. (a) $E_{\text {stats }}$ vs. Druckfestigkeit. (b) $E_{\text {stats }}$ vs. Spaltzugfestigkeit. (c) $E_{\text {stats }}$ vs. Abriebfestigkeit. Rek - Kalzit-Marmor. Be2 - granitischer Gneis. Be1 - granodioritischer Gneis. Mrgr3 - Granit. Al - Quarzit. Gra3a - granitischer Ultramylonit. Gra1a/1c - granitischer Gneis. Pi1a Peridotit. Cal - granodioritischer Gneis. Fehlerbalken markieren den 1- $\sigma$-Fehler.

Bei einem deutlicheren Einfluss des Mineral-Spannungs-Dehnungs-Verhaltens gewinnt die schlechte Korrelation zwischen den elastischen und mechanischen Eigenschaften der Minerale an Bedeutung und führt zu einer schlechteren Korrelation der Gesteinskennwerte ( $E_{\text {stat50 }} \mathrm{zu}$ Druckfestigkeit). Aus diesem Grund sind auch die Korrelationen der Mittelwerte von Spaltzug-/Biegefestigkeit mit $\mathrm{E}_{\text {stats }}$ besser, als die mit $\mathrm{E}_{\text {stat50. }}$. Dasselbe würde auch für die 
x-, y- und z-Werte von $\mathrm{E}_{\text {stats }}$ und die Spaltzug-/Biegefestigkeit zutreffen, wären da nicht die unterschiedlichen Orientierungen der angreifenden Spannungen während der Experimente.

Die Zusammenhänge zwischen den statischen E-Moduln und der Abriebfestigkeit sind schlecht definiert (Abb.8-19), zudem nichtlinearer Natur und werden mangels eines aussagekräftigen Erklärungsmodells nicht weiter behandelt.

Zwischen der thermischen Dehnung und den statischen E-Moduln wäre dann ein statistisch signifikanter Zusammenhang zu erwarten, wenn der Mineralbestand bezüglich dieser beiden Parameter gut korrelieren würde. Da dies nicht der Fall ist und zudem die Abhängigkeit von den Gefügeelementen unterschiedlich und die Prozesse bei der Ermittlung der Parameter nicht vergleichbar sind, folgt hieraus die bereits festgestellte schlechte Korrelation für die beiden Gesteinseigenschaften (Abb.8-17/Abb.8-18).

\subsection{Zusammenfassung}

Die Qualität der Korrelation und damit auch die Konstanz des Verhältnisses zweier gesteinstechnischer Parameter wird von deren Abhängigkeit vom Mineralbestand und deren Gefügeabhängigkeit bestimmt. Des Weiteren sind die Prozesse während der Ermittlung der Kennwerte und, in Bezug auf die Richtungsabhängigkeit, die Orientierungen der beim Experiment auftretenden Spannungen wichtige Einflussgrößen. Aber auch die Korrelation der betrachteten Eigenschaften für die gesteinsbildenden Minerale ist von Bedeutung. Im Folgenden sind die Korrelationskoeffizienten für die vorgenommenden Korrelationen in Form einer Korrelationsmatrix und die mittleren Verhältnisse der Mittelwerte in einer Verhältnismatrix zusammengefasst. 


\subsubsection{Korrelationsmatrix}

Die Korrelationsmatrix beinhaltet alle Korrelationskoeffizienten (R) für die wechselseitigen Verhältnisse der technischen Gesteinseigenschaften. Es werden die Korrelationen der Mittelwerte (arithmetisches Mittel aus x-, y-, z-Richtung) und der richtungsabhängig ermittelten $\mathrm{x}-$, $\mathrm{y}$ - und z-Werte berücksichtigt.

\begin{tabular}{|c|c|c|c|c|c|c|c|c|c|c|}
\hline & 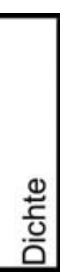 & $\begin{array}{l}: \frac{\pi}{\pi} \\
\frac{\pi}{5} \\
0 \\
0 \\
0\end{array}$ & 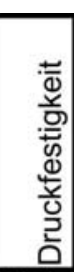 & 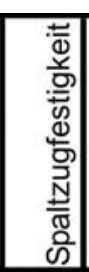 & 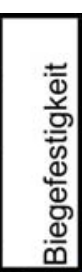 & 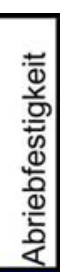 & 울 & 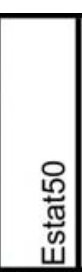 & $\begin{array}{l}\frac{0}{\pi} \\
\frac{\pi}{\omega} \\
w\end{array}$ & \\
\hline Dichte & & 0,54 & 0,07 & 0,08 & 0,15 & & 0,79 & 0,65 & 0,41 & 0,13 \\
\hline Porosität & & & 0,24 & 0,3 & 0,24 & & 0,79 & 0,62 & 0,63 & 0,1 \\
\hline Druckfestigkeit & & & & 0,82 & 0,84 & & 0,4 & 0,68 & 0,85 & 0,2 \\
\hline Spaltzugfestigkeit & & & 0,6 & & 0,87 & & 0,32 & 0,46 & 0,67 & 0 \\
\hline Biegefestigkeit & & & 0,54 & 0,85 & & & 0,36 & 0,44 & 0,77 & 0,2 \\
\hline \multicolumn{11}{|l|}{ Abriebfestigkeit } \\
\hline E0 & & & 0,34 & 0,16 & 0,17 & & & 0,79 & 0,71 & 0,1 \\
\hline Estat50 & & & 0,66 & 0,29 & 0,18 & & 0,74 & & 0,9 & 0,2 \\
\hline Estats & & & 0,85 & 0,42 & 0,36 & & 0,6 & 0,85 & & \\
\hline Alpha20-90 & & & 0,12 & 0,05 & 0,04 & & 0,03 & 0,08 & 0,02 & \\
\hline
\end{tabular}

Abb.8-20: Korrelationskoeffizienten $(R)$ für die Korrelationen der technischen Parameter. Grau Mittelwerte (arithmetisches Mittel aus x-, y-, z-Richtung). Gelb - x-, y-, z-Werte. Blau - nichtlineare Zusammenhänge durch lineare Regression angenähert. Rote Ziffern - statistisch signifikante Korrelation.

\subsubsection{Verhältnismatrix}

In der Verhältnismatrix sind die mittleren Verhältnisse der Mittelwerte (arithmetisches Mittel aus x-, y-, z-Richtung) für die Korrelationen der technischen Gesteinseigenschaften zusammengestellt (Abb.8-21). Die Werte wurden wie folgt ermittelt:

$$
u=\sum_{i=1}^{n} \frac{x_{a i}}{x_{b i}} / n
$$

$u$ - Mittleres Verhältniss der Mittelwerte. $x_{a i}$ - Mittelwert der Eigenschaft a für die i-te Probe. $x_{b i}-$ Mittelwert der Eigenschaft b für die i-te Probe. $n$ - Anzahl der Proben.

Die Verhältnisse sind dimensionslos solange die betrachteten Eigenschaften die gleiche Einheit aufweisen, ansonsten ist die entsprechende Einheit angegeben. Der 1- $\sigma$-Fehler wird aus den Verhältnissen zweier Gesteinsparameter aller 20 Einzelproben berechnet und 
beschreibt die Schwankungsbreite der Verhältnisse innerhalb des Probensatzes.

\begin{tabular}{|c|c|c|c|c|c|c|c|c|c|c|}
\hline & 总 & 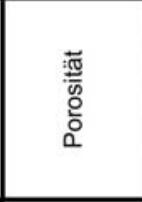 & 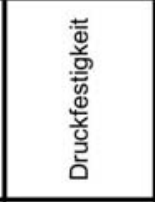 & 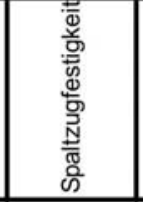 & 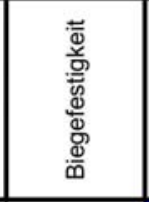 & 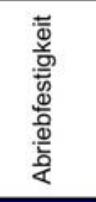 & 요 & 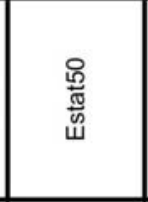 & 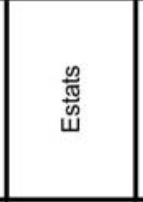 & 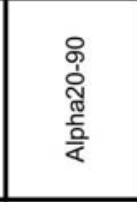 \\
\hline Dichte & & $17,7+-27,0$ & $0,014+-0,01$ & $0,25+-0,06$ & $0,14+-0,04$ & & $0,07+-0,05$ & $0,07+-0,01$ & $0,11+-0,03$ & $0,37+-0,07$ \\
\hline Porosität & $0,17+-0,13$ & & $0,002+-0,004$ & $0,04+-0,04$ & $0,016+-0,02$ & & $0,017+-0,02$ & $0,013+-0,01$ & $0,02+-0,02$ & $0,06+-0,05$ \\
\hline Druckfestigkeit & $78+-22$ & $1346+-2044$ & & $17,8+-3,0$ & $10,6+-2,3$ & & $5,05+-3,10$ & $5,4+-1,1$ & $7,9+-1,6$ & $29,9+-10,6$ \\
\hline Spaltzugfestigkeit & $4,3+-1,1$ & $72,7+-104$ & $0,06+-0,01$ & & $0,58+-0,09$ & & $0,29+-0,19$ & $0,31+-0,07$ & $0,45+-0,11$ & $1,6+-0,43$ \\
\hline Biegefestigkeit & $7,6+-2,5$ & $189+-226$ & $0,1+-0,03$ & $1,76+-0,29$ & & & $0,39+-0,29$ & $0,51+-0,13$ & $0,71+-0,14 \mid$ & $2,86+-0,99$ \\
\hline Abriebfestigkeit & s. & $44,5+-59,9$ & $0,05+-0,06$ & & $0,3+0,37$ & & & $0,22+-0$. & & $1,03+-0$ \\
\hline E0 & $20,4+-11,1$ & $586+-1281$ & $0,28+-0,18$ & $4,89+-2,97$ & $3,4+-1,7$ & $83+$ & & $1,4+-0,7$ & $2,0+-1,0$ & $7,75+-4,90$ \\
\hline Estat50 & $14,1+-2,5$ & $280+-476$ & $0,19+-0,04$ & $3,38+-0,76$ & $2,1+-0,5$ & & $0,92+-0,55$ & & $1,5+-0,2$ & $5,34+-1,76$ \\
\hline Estats & $9,8+-2,5$ & $190+-308$ & $0,13+-0,02$ & $2,32+-0,47$ & $1,5+-0,3$ & & $0,62+-0,35$ & $0,69+-0,08$ & & $3,71+-1,31$ \\
\hline Alpha20-90 & $2,8+-0,5$ & $45,2+-63,7$ & $0,04+-0,02$ & $|0,67+-0,22|$ & $0,38+-0,11$ & & $0,19+-0,14$ & $0,21+-0,06$ & $0,3+-0,1$ & \\
\hline
\end{tabular}

Abb.8-21: Verhältnismatrix für die Verhältnisse der Mittelwerte der technischen Parameter. Grau Verhältnis Zeile/Spalte. Gelb - Verhältnis Spalte/Zeile. Blau - nichtlineare Zusammenhänge durch lineare Regression angenähert. Fehler sind 1- $\sigma$-Fehler und beschreiben die Schwankungsbreite der Verhältnisse. Einheiten: Die Einheiten der Verhältnisse ergeben sich aus den Einheiten der einzelnen Parameter: Dichte $\left[\mathrm{g} / \mathrm{cm}^{3}\right]$, Porosität [\%], Druck-, Spaltzug-, Biegefestigkeit [MPa], Abriebfestigkeit $\left[\mathrm{cm}^{3}\right], E_{0}$, stat50, $E_{\text {stats }}[\mathrm{GPa}], \alpha_{20-90}\left[1 / \mathrm{K}^{*} 10^{6}\right]$. 


\section{Zusammenfassung}

Jedes Einsatzgebiet von Naturwerksteinen, sei es eine Gebäudefassade (Abb.9-1), eine Statue oder ein Bodenbelag stellt spezielle Anforderungen an die technischen Eigenschaften des jeweiligen Gesteins. Die Bearbeitbarkeit kann dabei eine ebenso bedeutende Rolle spielen wie z.B. die Festigkeit oder das Verwitterungsverhalten. Eine Einschätzung der geforderten Eigenschaften erfolgt in der Regel auf der Basis technischer Kennwerte (z.B. Druckfestigkeit, Abriebfestigkeit, w-Wert, E-Modul), die nach geltenden deutschen und europäischen Normen ermittelt werden (DIN EN). Da es sich bei Naturwerksteinen um ein in der Regel anisotropes und inhomogenes Material handelt, ist eine vom Gesteinsgefüge abgekoppelte Betrachtung der technischen Kennwerte in vielen Fällen nicht ausreichend und der falsche Einsatz der Gesteine eine mögliche Folge. Eine detaillierte Gefügeanalyse und damit eine ursächliche Betrachtung und Verknüpfung der verschiedenen Parameter und ihre Relation zum Gesteinsgefüge ist im Rahmen eines europäischen Normenwerks geplant, aber bisher nicht umgesetzt und in der Literatur wenig beschrieben (vgl. Kap.1).

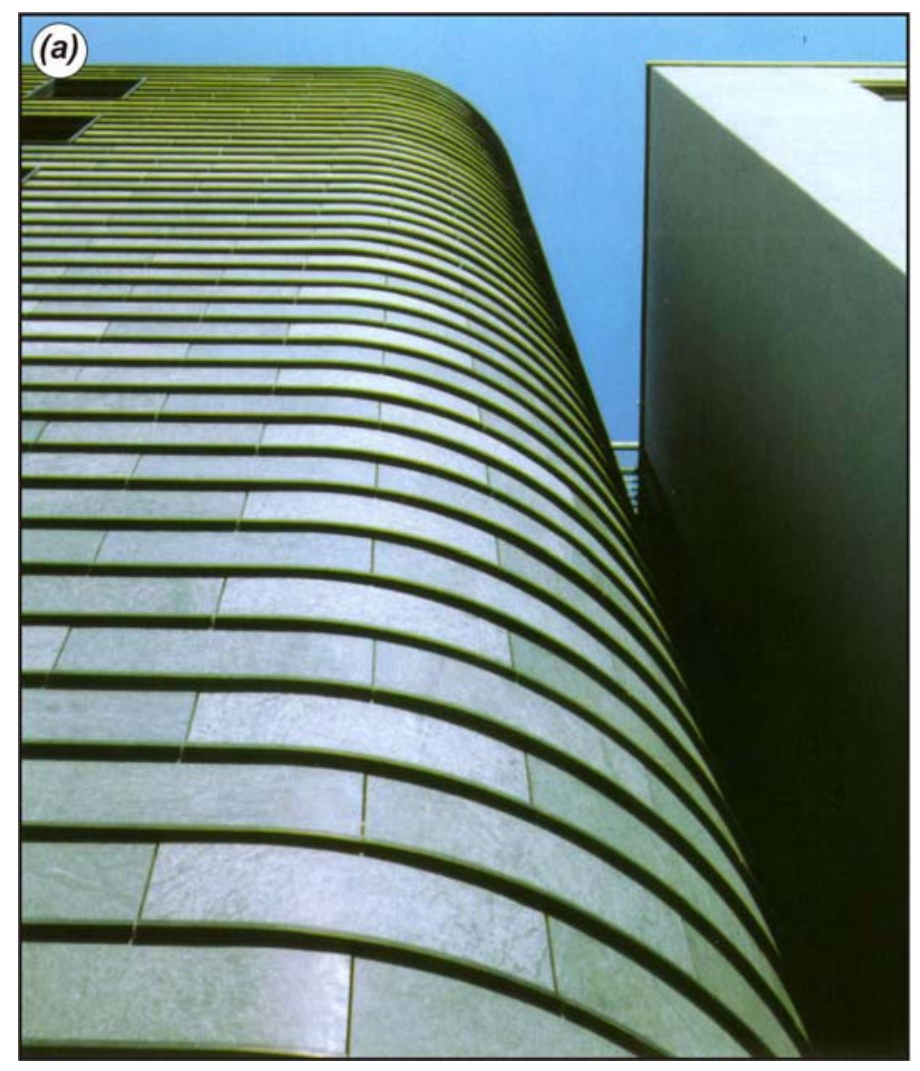

Abb.9-1: (a) Serpentinit-Fassade Ministerialgebäude Dresden (aus Wanetschek \& Wanetschek 2000). (b) Schema zur auf die Fassadenplatte wirkende Windlast (Seitenansicht); Durchbiegung ist überzeichnet dargestellt.

(b) Fassadenplatte

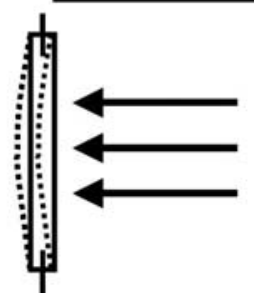

\section{Wind}

Doch gerade die Kenntnis der Gefügeeigenschaften und deren Einfluss liefert wertvolle Informationen zum Verständnis der Anisotropie der technischen Gesteinseigenschaften. Hauptziel der vorliegenden Studie ist daher die Erfassung der Gefügeabhängigkeit der 


\section{Zusammenfassung}

technischen Parameter, aber auch die Bedeutung der mineralogischen Zusammensetzung für die unterschiedlichen Kennwerte wurde in die Betrachtungen einbezogen.

Es wurden 20 niedrigporöse Kristallingesteine verschiedener Zusammensetzung und variierenden Graden der Gefügeanisotropie untersucht. Grundsätzlich sollten Erkenntnisse zu folgenden Kernfragen gewonnen und die praktische Anwendbarkeit der Ergebnisse herausgestellt werden:

1. In welcher Weise hängen die technischen Eigenschaften von der mineralogischen Zusammensetzung ab?

2. In welcher Weise hängen die technischen Eigenschaften vom Gesteinsgefüge ab? Welche Gefügeelemente sind wichtig?

3. Was bestimmt die Anisotropie der technischen Gesteinseigenschaften?

Die Gesteinsproben wurden zunächst auf ihre mineralogische und chemische Zusammensetzung hin untersucht und entsprechend klassifiziert. Zur Erfassung des Gefüges wurde als erstes der makroskopische Gefügeeindruck gewählt und eine Einteilung in Makrogefüge-Gruppen unterschiedlicher Gefügeanisotropie vorgenommen. Zusätzlich konnten für 14 der Gesteine Glimmertexturen bestimmt und ihre maximalen Intensitäten den Makrogefüge-Gruppen zugeordnet werden. Die Texturen nicht glimmerhaltiger Gesteine wurden mittels des am stärksten texturierten Minerals eingeschätzt. Desweiteren wurden die Rissdichte und die bevorzugte Orientierung der offenen Mikrorisse analysiert. Es folgte eine richtungsabhängige und richtungsunabhängige Korrelation der Gesteinscharakteristika (Makrogefüge, Textur der gesteinsbildenden Minerale, offene Mikrorisse, mineralogische Zusammensetzung) mit den technischen Eigenschaften (kapillare Wasseraufnahme, Druck-, Spaltzug-, Biege-, Abriebfestigkeit, dynamischer und statischer E-Modul, thermische Dehnung). Aus der Gefügeabhängigkeit der einzelnen Kennwerte und den Prozessen bei der experimentellen Bestimmung der technischen Eigenschaften konnten allgemeine Grundregeln für die Qualität der Kreuzkorrelationen technischer Parameter abgeleitet werden. 


\section{Zusammenfassung}

\subsection{Charakterisierung und Gruppierung der Proben}

\section{Mineralogische Zusammensetzung, chemische Klassifizierung, Metamorphose,}

\section{Deformation}

Die mineralogische und chemische Charakterisierung dient zum einen der Klassifizierung und Zuordnung der Gesteine im petrographischen Sinne, zum anderen wird die mineralogische Zusammensetzung im Weiteren mit den technischen Parametern in Verbindung gebracht.

Der Probensatz ist hinsichtlich seiner Mineralogie sehr variantenreich und deckt einen großen Bereich der in der Natur verwirklichten Zusammensetzungen kristalliner Gesteine ab. Es kommen 17 verschiedene Minerale mit einem Volumenanteil $>1$ Vol.\% vor. Darunter befinden sich mit Quarz, Plagioklas, Kalifeldspat, Biotit, Muskovit, Pyroxen, Hornblende und Olivin die wichtigsten Vertreter der gesteinsbildenden Minerale, welche mit insgesamt 95\% an der Masse der Erdkruste beteiligt sind (Müller 2001). Nach der mineralogischen Komposition gliedert sich der Probensatz in (vgl. Kap.5.2):

1 Quarzit, 1 Kalzit-Marmor, 1 Peridotit, 1 Gabbro-Norit, 2 Gabbros, 6 Gesteine granodioritischer Zusammensetzung, 8 Gesteine granitischer Zusammensetzung

Der Anteil der mafischen Komponenten liegt für die Gesteine granitischer und granodioritischer Zusammensetzung sowie für den Quarzit unter 35 Vol.\%. Damit sind diese Proben den leukokraten Gesteinen zuzuordnen und stehen im Kontrast zu den melanokraten Gabbros und dem ultramafischen Peridotit. 15 der Proben enthalten Phyllosilikate.

Die chemische Klassifizierung erfolgt gemäß des $\mathrm{SiO}_{2}$-Gehalts. Danach sind 14 der Proben als sauer und je zwei als intermediär, basisch bzw. ultrabasisch einzustufen.

Fünf der Gesteine werden den Magmatiten und 15 den Metamorphiten zugerechnet. Sechs Proben sind Gneise und fünf Proben Mylonite mit einer z.T stark ausgeprägten Gefügeanisotropie; die restlichen neun Gesteine weisen weniger starke Richtungsabhängigkeiten auf. 


\section{Zusammenfassung}

\section{Makrogefüge}

Zum Zweck der primären Erfassung der Gefügeanisotropie wurde der makroskopische Gefügeeindruck gewählt, dessen Eignung als übergeordnetes Element zur Erfassung von Gefügeanisotropien im Rahmen der vorliegenden Arbeit herausgestellt werden konnte. Die Beobachtungskriterien sind die Ausprägung der Foliation, der Lineation, der Kornformregelung und der Materialwechsel (Abb.9-2). Hauptziel war die Schaffung einer Referenzskala von niedrigen zu hohen Gefügeanisotropien, welche die einfache Abschätzung der $\mathrm{zu}$ erwartenden Anisotropie der technischen Gesteinseigenschaften aus dem makroskopischen Gefügeeindruck ermöglicht.

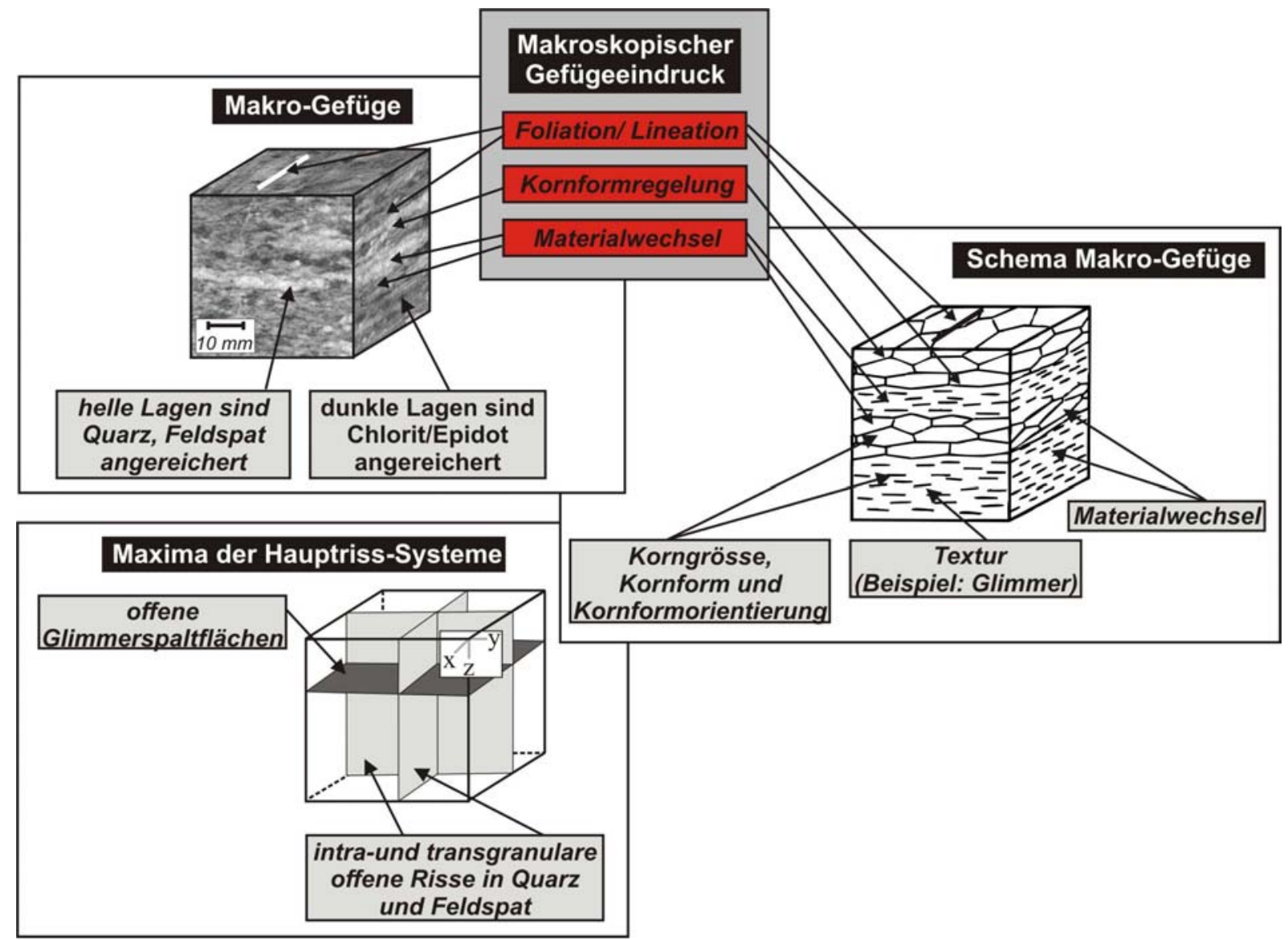

Abb.9-2: Wichtige Gefügemerkmale von Kristallingesteinen am Beispiel eines granodioritischen Mylonits (IV1b). Die für den makroskopischen Gefügeeindruck verwendeten Gefügeelemente sind rot hervorgehoben.

Alle Proben konnten gemäß ihres makroskopischen Erscheinungsbilds nach der zunehmenden Gefügeanisotropie geordnet und in drei Makrogefüge-Gruppen eingeteilt werden (vgl. Kap.5.5; $\boldsymbol{A b b . 9 - 3 ) . ~ D i e ~ G r u p p e ~ I ~ i s t ~ c h a r a k t e r i s i e r t ~ d u r c h ~ e i n ~ r i c h t u n g s l o s e s ~ G e f u ̈ g e ~ b z w . ~}$ ein(e) schwach ausgeprägte(s) Foliation/Linear und eine schwache Kornformregelung in Verbindung mit einem fehlenden Materialwechsel. Sie beinhaltet zwei Granite, einen Granodiorit sowie einen Gabbro-Norit und damit ausnahmslos magmatische Gesteine. 


\section{Zusammenfassung}

Hauptmerkmale der Gruppe II sind ein(e) schwach bis deutlich ausgeprägte(s) Foliation/Linear, eine schwach bis deutlich ausgeprägte Kornformregelung und ein sichtbarer Materialwechsel. Diese Gruppe besteht aus einem Kalzit-Marmor, einem Quarzit, einem granitischen Gneis, zwei Meta-Gabbros, einem Peridotit und einem granodioritischen Protomylonit, ist also im Hinblick auf die mineralogische Komposition sehr inhomogen zusammengesetzt.
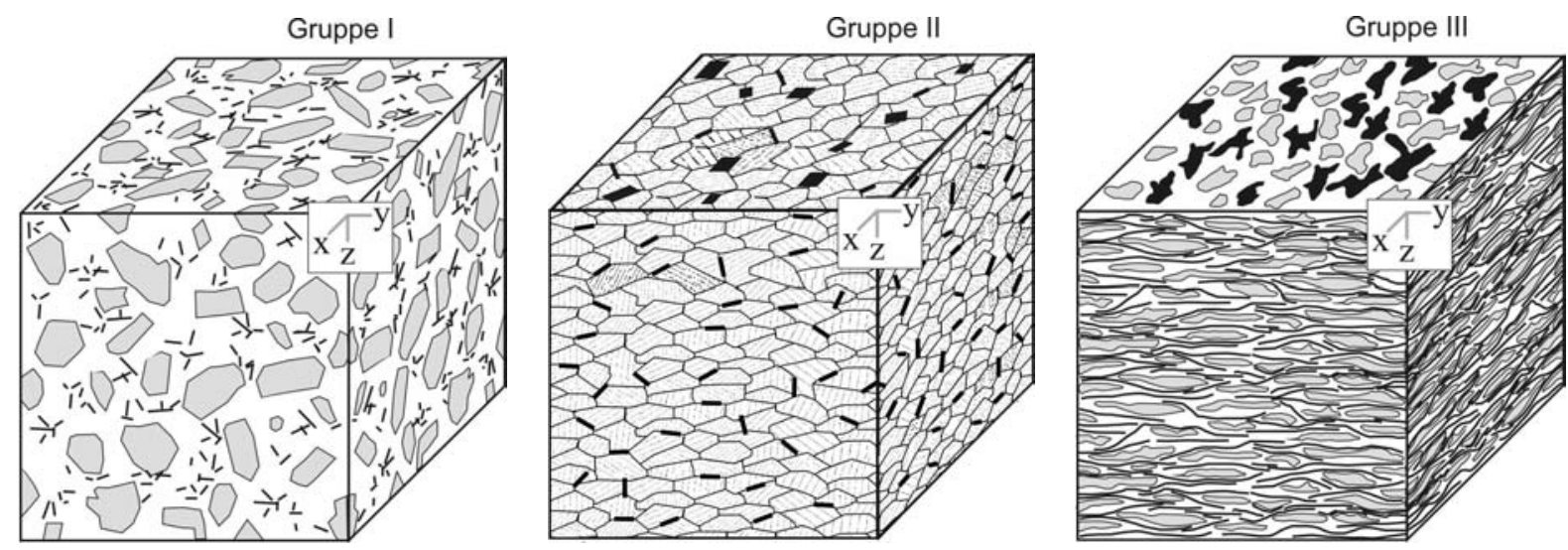

Abb.9-3: Beispielhafte Gefügeschemata zu den Makrogefüge-Gruppen I/ II und III

Gruppe I - isotrope Gesteine

Gruppe II - anisotrope Gesteine

Gruppe III - ausgeprägt anisotrope Gesteine
(2 Granite, 1 Granodiorit, 1 Gabbro-Norit)

(1 Peridotit, 1 Kalzit-Marmor, 1 Quarzit, 2 Meta-Gabbros, 1 granodioritischer Protomylonit, 1 granitischer Gneis)

(1) granitischer Protomylonit, 2 granodioritische Gneise, 1 granodioritischer Mylonit, 3 granitische Gneise, 1 granodioritischer Ultramylonit, 1 granitischer Ultramylonit)

In der Gruppe III befinden sich neun Gneise und Mylonite mit granitischer bzw. granodioritischer Zusammensetzung. Alle hier enthaltenen Proben führen Phyllosilikate und weisen ein(e) das makroskopische Gefügebild dominierende(s) Foliation/Linear, eine starke bis sehr starke Kornformregelung und einen stark ausgeprägten Materialwechsel auf.

\section{$\underline{\text { Textur }}$}

Die Phyllosilikate haben über ihren Volumenanteil, ihre Verteilung im Gesteinsgefüge und ihre bevorzugte Orientierung einen großen Einfluss auf die mechanischen (z.B. Gottschalk et al. 1990; Shea \& Kronenberg 1993) und die elastischen (z.B. Siegesmund 1996) Gesteinseigenschaften. Aus diesem Grund wurden für 14 der glimmerhaltigen Proben die Glimmertexturen bestimmt. Es ergeben sich maximale Intensitäten $\left(I_{\max }\right)$ der (001)-Pole von 


\section{Zusammenfassung}

$\mathrm{I}_{\max }=2,89 \mathrm{mrd}$ bis $\mathrm{I}_{\max }=23,3 \mathrm{mrd}$ und Regelungsschärfen (Woodcock 1977) von $\mathrm{c}_{\mathrm{W}}=0,38$ bis $\mathrm{c}_{\mathrm{W}}=3,73$. Steigende $\mathrm{c}_{\mathrm{W}}$-Werte sind gleichzusetzen mit einer zunehmenden Regelungsschärfe, was prinzipiell im Sinne einer stärkeren Textur, also einer stärker bevorzugten Orientierung der Glimmer (001)-Pole zu verstehen ist. Es besteht eine qualitative Übereinstimmung zwischen zunehmender makroskopischer Gefügeanisotropie und der Glimmertextur, die eine Definition von $\mathrm{c}_{\mathrm{W}}$ - und $\mathrm{I}_{\max }-$ Werten für die Makrogefüge-Gruppen erlaubt (vgl. Kap.5.7):

Gruppe I - isotrope Gesteine

Gruppe II - anisotrope Gesteine

Gruppe III - ausgeprägt anisotrope Gesteine

$$
\begin{array}{ll}
\mathrm{c}_{\mathrm{W}}<1 & \mathrm{I}_{\max }<5 \mathrm{mrd} \\
1 \leq \mathrm{c}_{\mathrm{W}} \leq 2 & 5 \mathrm{mrd} \leq \mathrm{I}_{\max } \leq 10 \mathrm{mrd} \\
\mathrm{c}_{\mathrm{W}}>2 & \mathrm{I}_{\max }>10 \mathrm{mrd}
\end{array}
$$

Die Proben der Makrogefüge-Gruppe I weisen verhältnismäßig schwache Glimmertexturen und die der Gruppe III z.T. extrem starke Glimmertexturen auf. Die Gesteine der Gruppe II liegen mit mittleren maximalen Texturintensitäten und $\mathrm{c}_{\mathrm{W}}$-Werten dazwischen.

Proben, deren Textur nicht über die bevorzugte Orientierung der Glimmer-(001)-Pole charakterisierbar ist, werden mit Hilfe des am stärksten texturierten Minerals beschrieben (vgl. Kap.5.7).

\section{$\underline{\text { Offene Mikrorisse }}$}

Ein weiteres für die gesteinstechnischen Eigenschaften wesentliches Gefügeelement sind die offenen Mikrorisse, deren Geometrie und räumliche Anordnung sowohl die mechanischen (z.B. Sheorey 1997; Stavrogin \& Tarasov 2001) und elastischen (z.B. Siegesmund 1996) Kennwerte, als auch die kapillare Wasseraufnahme maßgeblich beeinflussen. Innerhalb des Probensatzes kommen bevorzugt intra- und transgranulare Risse (auch Spaltflächen-Risse) vor, wohingegen intergranulare (Korngrenzen-)Risse selten anzutreffen sind. Die Rissdichte läßt sich hinreichend mit Hilfe der theoretischen, aus dem Mineralbestand ermittelten Kompressionswellengeschwindigkeit $\left(\mathrm{Vp}_{\mathrm{GVRH}}\right)$ und der $\mathrm{Vp}$ der trockenen Kugelmessung ( $\left.V p_{\text {trockm}}\right)$ quantifizieren. Im Rahmen dieser Arbeit wurde der Rissquantifizierungsparameter $\left(\mathrm{V}_{\mathrm{QRiss}}\right)$ als Differenz dieser beiden Kennwerte abgeleitet (vgl. Kap. 2.4.5.1). Hohe Werte dieses richtungsunabhängigen Parameters können mit relativ höheren Rissdichten und umgekehrt assoziiert werden. Nach der Rissdichte ergeben sich folgende Rissklassen (vgl. Kap.5.9). : 
Rissklasse $\mathrm{A}_{\mathrm{R}}$ - geringe Rissdichte $\quad \underline{\mathrm{Vp}} \underline{\underline{Q R i s s}} \leq 0,8 \mathrm{~km} / \mathrm{s}$

Rissklasse $C_{R}$ - hohe Rissdichte
(1) Granit, 1 Peridotit, 1 granitischer Ultramylonit, 1 KalzitMarmor, 1 Quarzit, 1 Meta-

Gabbro, 1 granitischer Gneis)

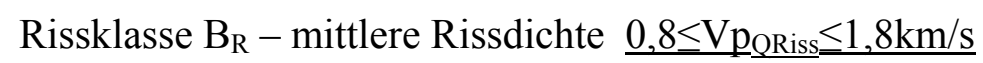

(1 Granodiorit, 1 granitischer Gneis, 1 Gabbro-Norit, 1 MetaGabbro, 1 granodioritischer Protomylonit)

(1 granodioritischer Mylonit, 1 granodioritischer Ultramylonit, 2 granodioritische Gneise, 1 granitischer Protomylonit, 2 granitische Gneise, 1 Granit )

Die offenen Mikrorisse folgen häufig der bevorzugten Orientierung der Glimmer (001)Flächen, so dass ein gute Übereinstimmung der bevorzugten Raumlage der Mikrorisse mit der Glimmertextur und damit auch mit der makroskopischen Gefügeanisotropie zu erwarten wäre. Tatsächlich ist ein solcher Zusammenhang insbesondere bei Proben mit verhältnismäßig hohem Phyllosilikatanteil zu beobachten, lässt sich jedoch nicht verallgemeinern. Ebenfalls besteht keine eindeutiger Zusammenhang zwischen der makroskopischen Gefügeanisotropie und der Rissdichte. Hoch anisotrope Proben der Makrogefüge-Gruppe III weisen nicht zwingend auch hohe Rissdichten auf, so dass eine eindeutige Definition von Rissdichten für die jeweiligen Makrogefüge-Gruppen nicht möglich ist.

\subsection{Technische Gesteinseigenschaften, mineralogische Zusammensetzung und Gefüge}

Die genaue Kenntnis der Zusammenhänge zwischen den technischen Parametern und dem mineralogischen und Gefüge-Inventar ermöglicht einen ganzheitlichen Eindruck des Gesteins in all seinen Anwendungsmöglichkeiten sowie die Einordnung und Bewertung noch unbekannter Proben anhand von einfach zu ermittelnden Gefügecharakteristika. Insbesondere bezüglich der letzteren Applikation werden im Rahmen dieser Arbeit Möglichkeiten und auch Grenzen aufgezeigt.

Die Mittelwerte der technischen Gesteinseigenschaften hängen von der mineralogischen Zusammensetzung und vom Gesteinsgefüge $a b$, deren Anisotropie wird hingegen ausschließlich von der bevorzugten Orientierung der Gefügeelemente bestimmt. Grundsätzlich können nur Gefügeelemente eine Anisotropie der Gesteinseigenschaften verursachen, die selbst gegenüber der entsprechenden Eigenschaft anisotrop sind. Nicht in 


\section{Zusammenfassung}

jedem Fall besteht ein direkter kausaler Zusammenhang, also eine theoretisch untermauerbare Abhängigkeit der technischen Eigenschaft von einem Gefügeparameter. Es geht vielmehr darum, ob bestimmte Gefügeelemente und ihre bevorzugte Orientierung stellvertretend geeignet sind, Aussagen über die zu erwartenden Mittelwerte und die Richtungsabhängigkeit der technischen Kennwerte zu machen. Eine besondere Aufmerksamkeit gilt dabei dem Zusammenhang zwischen dem übergeordneten makroskopischen Erscheinungsbild (bei phyllosilikathaltigen Gesteinen auch Glimmertextur) der Gesteine und der Anisotropie der technischen Parameter.

Die skalaren (richtungsunabhänigigen) Indexeigenschaften Dichte und Porosität hängen für die betrachteten Kristallingesteine von der Dichte des Mineralbestands bzw. von der Mikrorissdichte ab. Bei identischer durchschnittlicher Dichte der gesteinsbildenden Minerale sind die Dichte und die Porosität über die Frequenz der offenen Mikrorisse miteinander verknüpft (vgl. Kap.6.1.1). Zwischen der makroskopischen Gefügeanisotropie und der Dichte/Porosität besteht keine Verbindung.

Die kapillare Wasseraufnahme (w-Wert) ist eine richtungsabhängige Größe, deren Mittelwert vom Volumenanteil der Kapillarporen (Porenradius $\mathrm{r}=0,05 \mu \mathrm{m}$ bis $500 \mu \mathrm{m}$ ) daher einem bestimmten Porenradienbereich des gesamten Porenspektrums beeinflusst wird (vgl. Kap.6.2). Hohe Volumenanteile an Kapillarporen führen zu hohen w-Werten und damit einer hohen kapillaren Saugfähigkeit. Zwischen der Porosität und der kapillaren Wasseraufnahme besteht kein signifikanter Zusammenhang, wenngleich ein Anstieg des Kapillarporenanteils proportional zur Porosität häufig zu beobachten ist. Folglich sind positive Korrelationen von Porosität und kapillare Saugfähigkeit rein zufällig und nicht modellhaft begründbar. Die räumliche Verteilung der offenen Mikrorisse ist für die Anisotropie der Eigenschaft verantwortlich. Die stärkste kapillare Saugfähigkeit weist ein Gestein in der Richtung auf, zu der die Langachsen der Mikrorisse bevorzugt parallel orientiert sind. Eine Übereinstimmung zwischen der Anisotropie der w-Werte und der makroskopischen Gefügeanisotropie ist nicht durchweg gegeben, da die bevorzugte Orientierung der Mikrorisse nicht zwingend mit der makroskopischen Gefügeentwicklung zunimmt.

Die Mittelwerte der mechanischen Gesteinseigenschaften (Druck-, Spaltzug-, Biege- und Abriebfestigkeit) werden durch die mechanische Stabilität des Mineralbestands, die Mikrorissdichte sowie die Korngröße dominiert. Es gilt dabei folgendes Konzept: Jedes 


\section{Zusammenfassung}

Gestein weist eine Basisfestigkeit auf, welche allein auf der mineralogischen Zusammensetzung und den durchschnittlichen mechanischen Eigenschaften der gesteinsbildenden Minerale beruht (rissfreier Zustand). Eine Reduktion dieses Festigkeitswertes erfolgt durch die Anwesenheit von mechanisch schwachen Elementen (z.B. Mikrorisse) und ihrer räumlichen Verteilung im Gesteinsgefüge.

Zur Abschätzung der Resistenz der Minerale und Gesteine gegenüber mechanischer Beanspruchung wurde im Rahmen der vorliegenden Studie der Spaltbarkeitsindex $\left(\mathrm{I}_{\mathrm{Sp}}\right)$ definiert. Er basiert auf der Anzahl und Spaltbarkeit der ausgebildeten Spaltflächen. Ein Mineral mit einer großen Anzahl von Spaltflächen mit einer hohen Spaltbarkeit ist mechanisch schwächer als eines mit einer geringen Anzahl von Spaltflächen einer schlechten Spaltbarkeit. Den der Definition folgend höchsten Spaltbarkeitsindex $\left(\mathrm{I}_{\mathrm{SpM}}=15,0\right)$ hat Kalzit und ist daher das mechanisch instabilste Mineral. Beispiele für mechanische starke Minerale sind Quarz $\left(\mathrm{I}_{\mathrm{SpM}}=1,5\right)$ und Olivin $\left(\mathrm{I}_{\mathrm{SpM}}=5,0\right)$. Der Spaltbarkeitsindex des Gesteins ergibt sich aus der Summe der Spaltbarkeitswerte der Minerale mal deren Volumenanteile; es handelt sich daher um den mittleren Spaltbarkeitsindex der vorkommenden gesteinsbildenden Minerale.

Mit Hilfe des Spaltbarkeitsindex und Festigkeitswerten einer Datenbank (Börner \& Hill 2002) kann für unterschiedliche Gesteinstypen eine erste Einschätzung der zu erwartenden maximalen Festigkeiten erfolgen. Der Gruppe der Marmore als schwächste Gesteinsart mit $\mathrm{I}_{\mathrm{SpG}}$-Werten zwischen 12 und 15 und maximalen Festigkeit von etwa $130 \mathrm{MPa}$ (Druckfestigkeit), $20 \mathrm{MPa}$ (Biegefestigkeit) und $15 \mathrm{~cm}^{3}$ (Abriebfestigkeit) steht dabei die Gruppe der Quarzite mit $\mathrm{I}_{\mathrm{SpG}}$-Werten zwischen 1,5 und 2,5 und maximalen Festigkeiten von $330 \mathrm{MPa}$ (Druckfestigkeit), $37 \mathrm{MPa}$ (Biegefestigkeit) und $4 \mathrm{~cm}^{3}$ (Abriebfestigkeit) gegenüber. Da zur Charakterisierung der mechanischen Gesteinseigenschaften sinnvollerweise nicht die höchsten, sondern die niedrigsten zu erwartenden Festigkeiten anzugeben sind, stellt sich die Frage, welche Gefügeelemente hauptsächlich zur Reduktion der mechanischen Kennwerte beitragen. Hier sind an erster Stelle die offenen Mikrorisse zu nennen. Zum einen weist ein offener Mikroriss ein mechanische Festigkeit von Null auf (keine Kohäsion), zum anderen kommt es an der Riss-Spitze zur Spannungskonzentration, die den Effekt der mechanischen Schwächung noch verstärkt. Die häufig beobachtete Anti-Korrelation von Korngröße und Festigkeitsparametern kann aus der Übereinstimmung von hohen Korngrößen mit hohen Griffith-Risslängen abgeleitet werden (vgl. Kap.6.3.4). Ausgehend von der Basisfestigkeit kann anhand der Mikrorissdichte (quantifiziert durch V $p_{Q R i s s}$ ) und der Korngröße eine 


\section{Zusammenfassung}

Einschätzung der zu erwartenden Festigkeitsreduktion vorgenommen und damit die niedrigste mittlere Festigkeit ermittelt werden.

Aufgrund der zum Teil sehr stark ausgeprägten Richtungsabhängigkeit der mechanischen

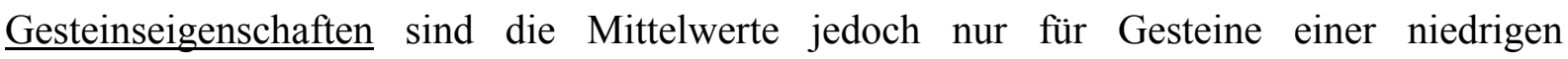
Gefügeanisotropie aussagekräftig. Im Falle einer hohen Gefügeanisotropie können leicht Verhältnisse von maximaler zu minimaler Festigkeit innerhalb eines Gesteins von 3:1 und mehr erreicht werden (z.B. Biegefestigkeit), so dass eine Aussage bezüglich der zu erwartenden Festigkeitsanisotropien dringend erforderlich ist. Wie also lässt sich die Anisotropie der mechanischen Eigenschaften aus der Gefügeanisotropie ableiten? Grundsätzlich spielen hier nur Gefügeelemente eine Rolle, die gegenüber mechanischer Belastung anisotrop sind und damit über eine bevorzugte räumliche Orientierung zu einer Anisotropie der mechanischen Eigenschaften führen können. Beispiele für solche Elemente sind die gut ausgebildeten Spaltflächen von Mineralen einer ausgeprägten Anisotropie der Spaltbarkeit (z.B. Glimmer (001)-Fläche) und offene Mikrorisse. Beide Elemente können zudem penetrative Lagen bilden und damit den Effekt der Anisotropie der Festigkeitskennwerte noch intensivieren. Demgegenüber stehen die Minerale Quarz und Kalzit mit einer schlecht bzw. perfekt ausgeprägten Spaltbarkeit und in beiden Fällen einer niedrigen Spaltbarkeitsanisotropie. Hier ist selbst bei einer starken Textur nur eine geringe resultierende Richtungsabhängigkeit der Gesteinseigenschaften zu erwarten. Der makroskopische Gefügeeindruck umfasst die Foliation/Lineation, die Kornformregelung sowie den Materialwechsel. Damit werden indirekt die Textur der Glimmer (über ihre Einregelung und Verteilung im Gefüge sind sie foliationsbildendes Element) und in Ansätzen auch die offenen Mikrorisse (folgen bevorzugt den Glimmer (001)-Flächen) und damit die wichtigsten Elemente, die eine Anisotropie verursachen können erfasst. Folglich ist die makroskopische Gefügeanisotropie als übergeordneter Parameter geeignet einen Eindruck über die zu erwartende Anisotropie der mechanischen Kennwerte zu erhalten. Für die glimmerhaltigen Gesteine kann aufgrund der guten Korrelation von makroskopischer Gefügeentwicklung mit der Glimmertextur die maximale Intensität der (001)-Flächen verwendet werden (vgl. Kap.6.3.1/6.3.3). Die Proben der Makrogefüge-Gruppe I (isotrope Gesteine) zeigen Anisotropien der mechanischen Festigkeit von durchschnittlich 7,6\% (Druckfestigkeit), $\quad 12,5 \% \quad$ (Spaltzugfestigkeit), $\quad 12,7 \% \quad$ (Biegefestigkeit) und 7,5\% (Abriebfestigkeit). Um ein wesentliches höher sind die zu beobachteten Anisotropiewerte für die Makrogefüge-Gruppe III (ausgeprägt anisotrope Gesteine) von 21,3\% (Druckfestigkeit), 50,4\% (Spaltzugfestigkeit), 77,5\% Biegefestigkeit und 19,5\% (Abriebfestigkeit). Es ist 


\section{Zusammenfassung}

folglich möglich den untersuchten und nach zunehmender makroskopischer Gefügeanisotropie geordneten Probensatz als Referenz-Skala für die schnelle und praktische Abschätzung von Festigkeitsanisotropien zu verwenden. Unbekannte Proben können gemäß ihres makroskopischen Erscheinungsbilds in die Probenserie eingeordnet und entsprechend bewertet werden. Diese Vorgehensweise entbindet jedoch nicht von der Pflicht zusätzlicher mikroskopischer Untersuchungen, um Spezialfälle zu erfassen (vgl. Kap.6.3).

Als eine weitere in der Praxis anwendbare Applikation können Festigkeiten in den unterschiedlichen Raumrichtungen eines Gesteins in eine relative Abfolge gebracht werden. Anhand des Makrogefüges ist es möglich vorherzusagen, welche Richtung die höchsten und welche die niedrigsten Festigkeiten aufweisen wird. Im Falle der Spaltzug- und der Biegefestigkeit (Zugspannungen bei der experimentellen Bestimmung) ist stets die Richtung senkrecht zur Foliation am mechanisch schwächsten, wohingegen die Richtung parallel zum Linear die höchsten Festigkeiten aufweist. Für die Druckfestigkeit (Zug- und Scherspannungen bei der experimentellen Bestimmung) zeigt bei S-Typ-Gesteinen (Foliation ist dominant) die Richtung senkrecht zur Foliation die höchsten und die Richtungen parallel zur Foliation niedrigere Werte. Dahingegen ist bei S-L-Typen und L-Typen (Linear dominant) die Richtung parallel zum Linear jene mit den höchsten Festigkeiten. Für die Abriebfestigkeiten gilt im Grundsatz das selbe wie für die Spaltzug- und Biegefestigkeiten nur das die Anisotropien weniger stark ausgeprägt sind. Sämtliche der beschriebenen Ergebnisse lassen sich theoretisch aus dem Angebot mechanisch schwacher Flächen senkrecht zu den Zug- bzw. parallel zu den Scherspannungen ableiten (vgl. Kap.8.2.1).

Der Mittelwert des dynamischen E-Moduls eines Gesteins, ermittelt mit Hilfe der Ultraschallgeschwindigkeiten von p- und s- Welle, hängt zum einen von den durchschnittlichen elastischen Eigenschaften des Mineralbestands, zum anderen sehr stark von der Mikrorissdichte ab. Für alle Gesteine existiert ein intrinsischer E-Modul, der ausschließlich durch das elastische Verhalten der gesteinsbildenden Minerale definiert ist. Er beschreibt den rissfreien Zustand, der bei Naturwerksteinen nicht vorkommt, welcher aber durch Messungen bei hohem Umschließungsdruck experimentell ermittelt werden kann. Ausgehend von diesem maximalen E-Modul führt die Anwesenheit von offenen Mikrorissen je nach Rissdichte zu einer mehr oder weniger starken Verringerung der Werte. Dieser Effekt kann so stark sein, dass rein rechnerisch negative E-Moduln ermittelt werden. Allerdings haben diese Daten keinerlei Aussagekraft in Bezug auf das tatsächliche Spannungs- 
Dehnungs-Verhalten des Gesteins und deuten darauf hin, dass die Rahmenbedingungen für einen linear elastischen Körper nicht gegeben sind.

Elemente, die das Potential haben eine Anisotropie des E-Moduls zu bewirken sind Minerale mit einer hohen Anisotropie der elastischen Eigenschaften (z.B. Glimmer) und die offenen Mikrorisse. In beiden Fällen kann eine bevorzugte Orientierung zu einer ausgeprägten Richtungsabhängigkeit des E-Moduls führen. Bezüglich der Mikrorisse ist immer die (Durchschallungs-)Richtung parallel zur bevorzugten Orientierung der Risskurzachse am stärksten von der Reduktion des E-Moduls betroffen. Folglich zeigen glimmerhaltige Proben mit einer hohen Rissdichte und einer starken bevorzugten Orientierung der Risse in Kombination mit einer starken Glimmertextur die höchsten Anisotropien des dynamischen EModuls. Dabei weist die Richtung senkrecht zur Foliation in der Regel die niedrigsten und die Richtung parallel zum Linear die höchsten E-Moduln auf. Auch die $\underline{\text { Anisotropie des }}$ dynamischen E-Moduls zeigt eine gute Korrelation zur makroskopischen Gefügeentwicklung. Daher ist der makroskopische Gefügeeindruck geeignet eine Einschätzung über die zu erwartenden Anisotropien zu liefern. Allerdings ist für den dynamischen E-Modul der Einfluss der Mikrorisse stärker als für die mechanischen Eigenschaften. Daher müssen die Mikrorisse und deren Raumlage separat betrachtet werden um eine sichere Aussage in Bezug auf die Anisotropieeigenschaften zu erhalten.

Bei der Verwendung von dynamischen E-Moduln zur Schadensanalyse ist die gefügebedingte Anisotropie des Gesteins stets in die Betrachtungen einzubeziehen, da ansonsten keine sichere Interpretation der Daten möglich ist.

Der statische E-Modul gibt Aufschluss über das Spannungs-Dehnungs-Verhalten bei mechanischer Belastung. Er wird aus dem einaxialen Druckversuch ermittelt und in der Hauptsache durch die durchschnittlichen elastischen und mechanischen Eigenschaften der gesteinsbildenden Minerale und die Rissdichte der offenen Mikrorisse bestimmt. Eine Abgrenzung und Gewichtung des Einflusses der einzelnen Parameter gestaltet sich aufgrund multifaktorieller Überlagerungen schwierig und konnte im Detail nicht vorgenommen werden. Grundsätzlich weisen Gesteine mit einem hohen E-Modul und einer hohen mechanischen Stabilität des Mineralbestands (z.B. Peridotit) auch hohe statische E-Moduln auf. Dahingegen zeichnet Gesteine wie den Kalzit-Marmor mit einem verhältnismäßig niedrigen E-Modul und einer niedrigen mechanischen Festigkeit des Kalzits ein mehr weiches Spannungs-Dehnungs-Verhalten (niedriger statischer E-Modul) aus. Analog zu den Druckfestigkeiten zeigt auch der statische E-Modul eine zunehmende Anisotropie parallel zur 


\section{Zusammenfassung}

makroskopischen Gefügeanisotropie (bei glimmerhaltigen Gesteinen auch zunehmende maximale Intensität der Glimmertextur).

Die Mittelwerte der thermischen Dehnung eines Gesteins, charakterisiert durch den thermischen Ausdehnungskoeffizienten $\alpha$, wird dominiert durch die thermischen Dehnungseigenschaften des Mineralbestands. Andere Gefügeelemente, wie offene Mikrorisse oder die Korngröße spielen lediglich eine untergeordnete Rolle. In niedrigen Temperaturbereichen (bis $40^{\circ} \mathrm{C}$ ) kann es in diesem Zusammenhang zur Risspufferung kommen, was bedeutet, dass sich die Minerale in den präexistierenden Porenraum hineindehnen und die thermischen Ausdehnungskoeffizienten im entsprechenden Temperaturintervall leicht hinter den Erwartungen zurückbleiben. Demgegenüber steht der Prozess der thermischen Rissbildung, welcher innerhalb des Probensatzes nur für den KalzitMarmor beobachtet werden konnte und $\mathrm{zu}$ deutlich höheren thermischen Ausdehnungskoeffizienten führt, als aufgrund des Mineralbestands zu erwarten wäre.

Signifikante Anisotropien der Dehnungseigenschaften können nur durch die Anwesenheit von Mineralen mit einer ausgeprägten Anisotropie der thermischen Dehnung hervorgerufen werden. Weist ein solches Mineral, wie z.B. Kalzit eine deutliche Textur auf, so kommt es gemäß der Vorzugsorientierung der kristallographischen Achsen zu richtungsabhängigen Unterschieden der thermischen Dehnung. Im Fall des Kalzit-Marmors zeigt die Richtung parallel zum Maximum der c-Achsen-Verteilung den höchsten und die Richtung parallel zum Maximum der a-Achsen-Verteilung den niedrigsten thermischen Ausdehnungskoeffizienten. Tritt zusätzlich der Effekt der thermischen Rissbildung auf, so kann dieser parallel zum cAchsen-Maximum am stärksten sein und die Anisotropie der thermischen Dehnung verstärken. Zwischen der makroskopischen Gefügeanisotropie und der Anisotropie der thermischen Dehnung besteht für die untersuchten Proben kein Zusammenhang, was aus der Definition des makroskopischen Gefügeeindrucks resultiert. Hierbei werden Kriterien wie die Foliation, Lineation, die Kornformregelung und der Materialwechsel verwendet, welche für die thermische Dehnung nur eine geringe Bedeutung haben. Umgekehrt gehen die Texturen der hoch anisotropen Minerale (z.B. Kalzit, Feldspat) mit einem entsprechend starken Einfluss auf die Dehnungseigenschaften nicht in die Bewertung der makroskopischen Gefügeanisotropie ein und tragen damit nur gering zum makroskopischen Gefügeeindruck bei. Glimmer, die über ihre hohe Formanisotropie und das damit verbundene charkteristische Regelungs- und Segregationsverhalten das makroskopische Erscheinungsbild stark beeinflussen sind bezüglich ihrer thermischen Dehnungseigenschaften verhältnismäßig 


\section{Zusammenfassung}

niedrig anisotrop. In Kombination mit den Volumenanteilen von $\leq 26 \%$ ist daher selbst bei einer starken Textur keine resultierende Anisotropie der thermischen Dehnung zu erwarten. Will man diese also erfassen, so sind Texturuntersuchungen der hoch anisotropen Minerale mit einem möglichst hohen Volumenanteil unerlässlich.

Als Essenz der Ergebnisse ist folgendes festzustellen:

1. Die Minerale vererben ihre Eigenschaften auf das Gesamtgestein. Das betrifft sowohl den Mittelwert als auch die Anisotropie, sofern das entsprechende Mineral eine deutliche Textur ausgebildet hat. Einzige Ausnahme ist die kapillare Wasseraufnahme, die nicht vom Mineralbestand, sondern ausschließlich von der Beschaffenheit des Porenraums abhängig ist.

2. Offene Mikrorisse schwächen das Gestein bezüglich seiner Festigkeiten und seiner elastischen Eigenschaften; die bevorzugte Orientierung der offenen Mikrorisse kann hier in Verbindung mit einer hohen Rissdichte eine ausgeprägte Anisotropie der gesteinstechnischen Eigenschaften hervorrufen. Bezüglich der thermischen Dehnung gibt es den Effekt der Risspufferung.

3. Der makroskopische Gefügeeindruck und bei den phyllosilikathaltigen Gesteinen die maximale Intensität der Glimmertextur sind als indirekte Parameter geeignet, eine Einschätzung der zu erwartenden Anisotropie der Gesteinseigenschaft zu liefern. Die thermische Dehnung bildet die einzige Ausnahme. Hier dominieren Minerale wie Kalzit mit ihren extremen Dehnungseigenschaften den Ausdehnungskoeffizienten und dessen Richtungsabhängigkeit. 


\section{Zusammenfassung}

\subsection{Kreuzkorrelationen der technischen Kennwerte}

In der Literatur werden häufig Kreuzkorrelationen der technischen Kennwerte angegeben (z.B. Peschel 1983; Köhler 1991; Mirwald 1997), deren Bestimmung in der Hauptsache drei unterschiedlichen Zielrichtungen dient:

1. Die einfach zu bestimmenden Indexeigenschaften Dichte und Porosität werden mit anderen technischen Eigenschaften korreliert, um mit den ermittelten Zusammenhängen eine Einschätzung der technischen Eignung nur aufgrund von Dichte- und Porositäts-Werten vornehmen zu können.

2. Die Verhältnisse der mechanischen Parameter zueinander werden verwendet, um auf der Basis der festgestellten Korrelationen eine mechanische Festigkeit aus einer anderen ableiten zu können ohne sämtliche Gesteinseigenschaften experimentell bestimmen zu müssen.

3. Der dynamische E-Modul (auch Vp-Werte) wird mit zum Zweck der Schadensanalye von Naturwerksteinen mit Festigkeitsparametern verglichen.

Im Rahmen dieser Arbeit wurde die praktische Anwendbarkeit der Korrelationen technischer Eigenschaften für niedrigporöse Kristallingesteine näher beleuchtet.

Zwei Haupteinflussgrößen bestimmen die Qualität der Korrelation zweier technischer Parameter. Auf der einen Seite steht das Gestein mit seinem Gefüge (Gefügeelemente sind z.B. Foliation, Lineation, Textur, Mikrorissverteilung) sowie seiner mineralogischen Zusammensetzung und den daraus resultierenden Gesteinseigenschaften. Auf der anderen Seite befinden sich die experimentellen Rahmenbedingungen bei der Bestimmung der technischen Kennwerte. Hier sind insbesondere die auftretenden Prozesse und die Lage der Prozesszonen zu nennen. Erst wenn beide Seiten in ähnlicher Weise die Eigenschaften (E1 und E2) beeinflussen, kann von einer signifikanten und auch theoretisch ableitbaren Korrelation ausgegangen werden (vgl. Abb.9-4). Konstante Verhältnisse zwischen den Mittelwerten (arithmetisches Mittel aus x-, y-, z-Richtung) zweier technischer Parameter sind zu erwarten, wenn folgende Bedingungen erfüllt sind:

1. Die technischen Kennwerte hängen in möglichst gleicher Weise von den gleichen Gefügemerkmalen ab (vgl. Kap.6). 


\section{Zusammenfassung}

2. Es besteht eine gute Korrelation der technischen Parameter in Bezug auf den Mineralbestand (vgl. Kap.4).

3. Die Prozesse während der experimentellen Ermittlung der technischen Kenngrößen sind entweder für beide Parameter ähnlich (vgl. Kap.8) und/oder für die ermittelten Parameter besteht ein aus physikalischen Gesetzmäßigkeiten ableitbarer Zusammenhang (keine multifaktoriellen und durch Überlagerungen dominierten Verhältnisse).

Bei der richtungsabhängigen Betrachtung ( $\mathrm{x}-$, , y-, z-Richtung) muss für ein repräsentatives Verhältnis zusätzlich ein viertes Kriterium erfüllt sein:

4. Die Raumlage der Prozesszonen bei der experimentellen Ermittlung der technischen Kenngrößen ist für beide Parameter ähnlich (vgl. Kap.8). 


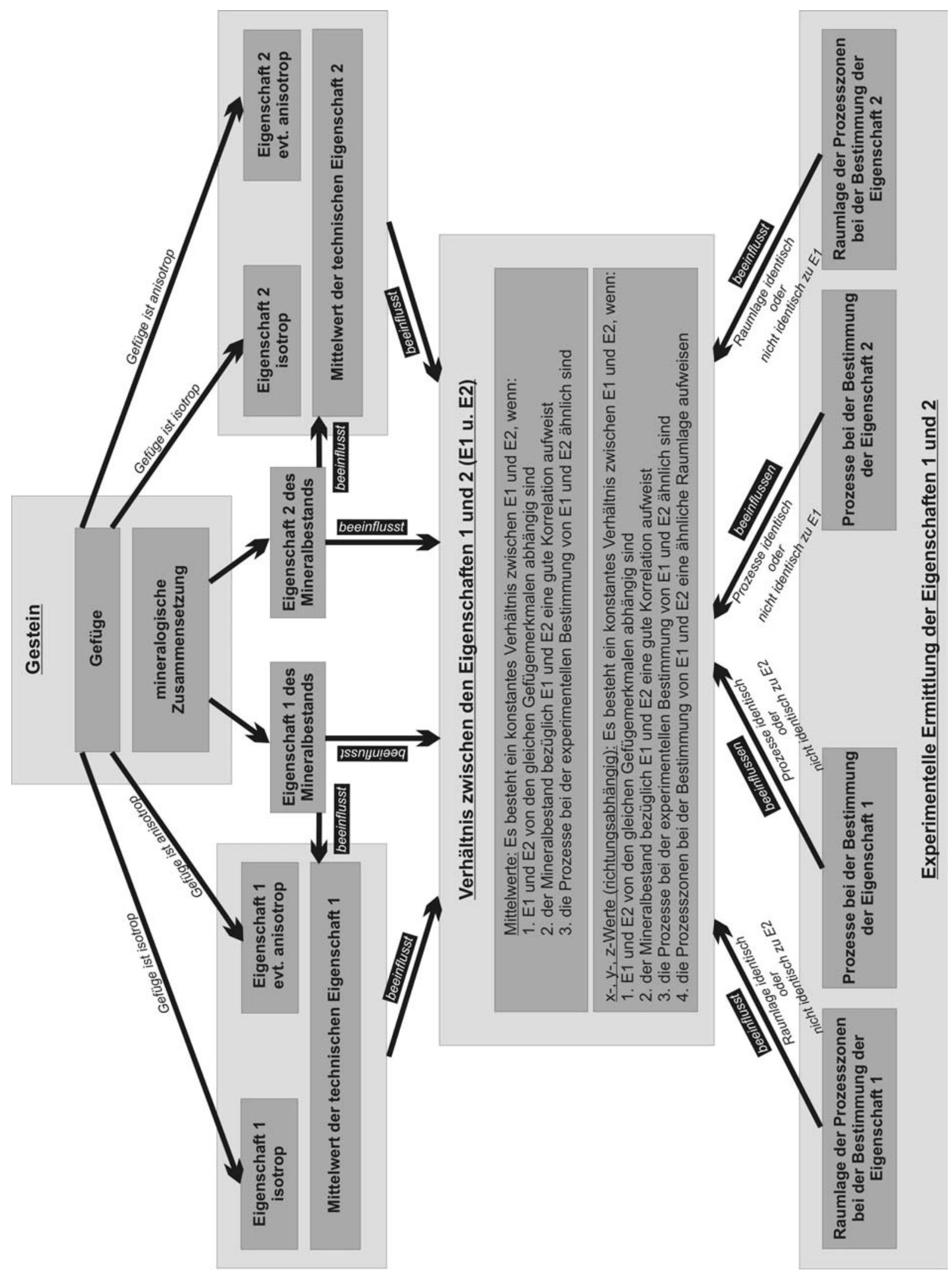

Abb.9-4: Schema zu den Einflussgrößen auf die Kreuzkorrelation gesteinstechnischer Parameter. Für Details siehe Text. 


\section{Zusammenfassung}

Die Anwendung der Kriterien für eine statistisch signifikante Korrelation hat in Einklang mit den experimentellen Daten zu folgenden Ergebnissen geführt:

Die Indexeigenschaften Porosität und Dichte korrelieren generell schlecht mit den technischen Eigenschaften (Druck-, Spaltzug-, Biege-, Abriebfestigkeit, dynamischer und statischer E-Modul, thermische Dehnung), so dass eine Bewertung der technischen Eignung eines Naturwerksteins aus der Porosität und der Dichte nicht möglich ist. Entscheidend für die schlechte Korrelation sind die Abhängigkeit von unterschiedlichen Gefügemerkmalen bzw. die schlecht ausgeprägte Korrelation der Eigenschaften für die gesteinsbildenden Minerale. Am Beispiel der Korrelation Dichte zu den mechanischen Eigenschaften wird dies deutlich (Abb.9-5). Die Dichte des Gesteins hängt bei den innerhalb des Probensatzes vorherrschenden niedrigen Porositäten von der durchschnittlichen Dichte der Minerale ab, wohingegen die mechanischen Eigenschaften von der Mikrorissdichte und den mechanischen Eigenschaften des Mineralbestands sowie der Korngröße dominiert werden. Da zwischen der Dichte der Minerale und deren mechanischer Stabilität gerade für wichtige Gesteinsbildner, wie Quarz und Feldspat kein eindeutiger Zusammenhang besteht, ist die ermittelte schlechte Korrelation die logische Konsequenz.

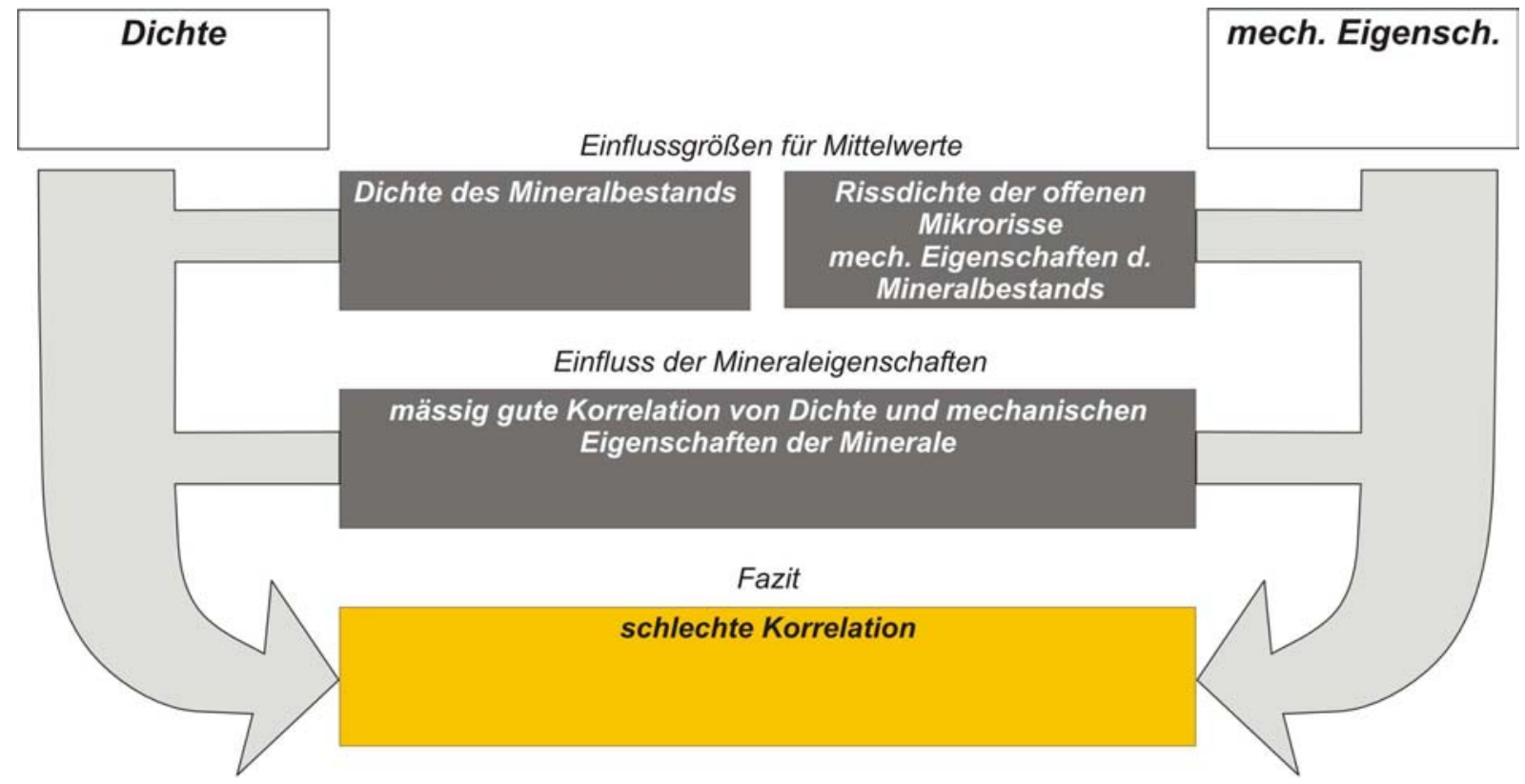

Abb.9-5: Dichte vs. mechanische Eigenschaften. Weitere Korrelationsdiagramme in Anhang III.

Für die mechanischen Eigenschaften untereinander gilt zumindest qualitativ die Abhängigkeit von den gleichen Gesteinsmerkmalen, zudem treten bei der experimentellen Bestimmung ähnliche Prozesse auf. Sowohl die Druck-, Spaltzug-, Biege- als auch die Abriebfestigkeit hängen von der Rissdichte der Mikrorisse, der mechanischen Stabilität der Minerale und der 


\section{Zusammenfassung}

Korngröße (nicht Abriebfestigkeit) ab und bei den Experimenten findet das Gesteinsversagen durch zug- und scherspannungsbedingte Überschreitung der Kohäsion statt. Beides führt zu einer durchweg deutlichen Korrelation der richtungsunabhängigen Mittelwerte. Dabei sind die Korrelationen mit der Abriebfestigkeit nichtlinearer Natur. Entscheidend bei der Bewertung der Verhältnisse der mechanischen Eigenschaften ist die richtungsabhängige Betrachtung. Da die Lage der zum Bruch führenden Spannungen lediglich für die Biege- und die Spaltzugfestigkeit identisch ist, zeigt sich auch nur hier eine gute Korrelation der x-, y-, zWerte (Richtungen parallel und senkrecht zur Foliation) (Abb.9-6). Im Falle der weiteren Verhältnisse der mechanischen Eigenschaften zueinander ist das anders. Es werden zwar die gleichen Gefügeelemente auf ähnliche Art und Weise jedoch in unterschiedlichen Raumlagen aktiviert. In der Praxis führt das zu einer starken Richtungsabhängigkeit der Verhältnisse, deren Ausmaß vom Grad der Gefügeanisotropie abhängt. Je größer die makroskopische Gefügeanisotropie ist desto stärker sind die Schwankungen der Verhältnisse in Abhängigkeit von der Untersuchungsrichtung. Ein standardmäßige Verwendung von Festigkeitsverhältnissen ist für anisotrope Gesteine folglich nicht möglich.

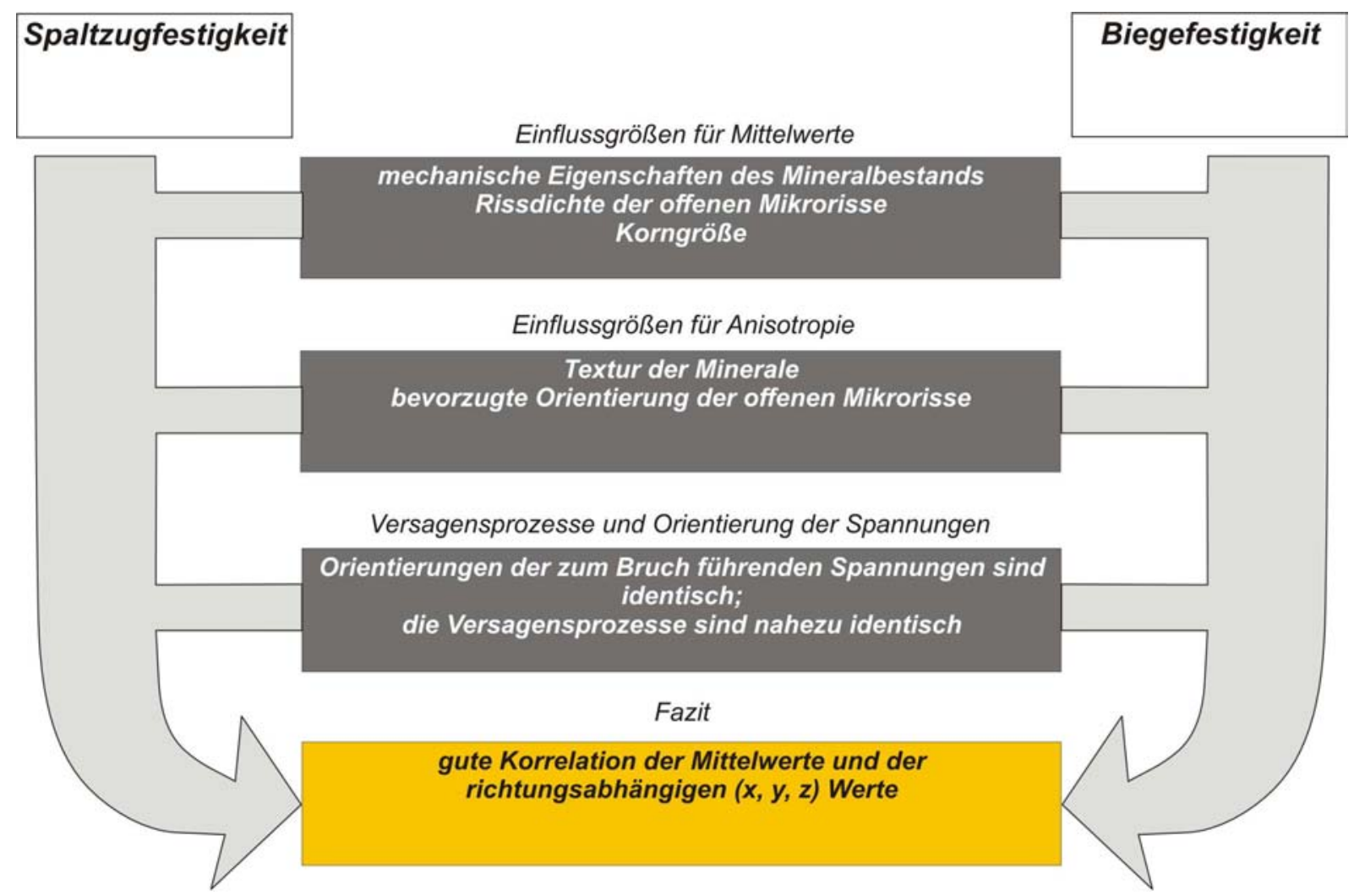

Abb.9-6: Spaltzugfestigkeit vs. Biegefestigkeit. Weitere Korrelationsdiagramme in Anhang III.

In diesem Zusammenhang sind auch die, in der Literatur häufig verwendeten Verhältnisse von Druck- zu Spaltzugfestigkeit von 10-15:1 (z.B. Hirschwald 1912; Peschel 1983; Mirwald 1997) und von Biege- zu Spaltzugfestigkeit von 2-2,5:1 (Peschel 1983) kritisch zu 


\section{Zusammenfassung}

hinterfragen. Im Rahmen dieser Studie wurde ein durchschnittliches Verhältnis von Druckfestigkeit/Spaltzugfestigkeit von 17,8:1 ermittelt, wobei je nach analysierter Richtung auch Ratios von 10,2:1 bzw. 39,3:1 auftreten können (vgl. Kap.8.2). Für das Verhältnis von Biegefestigkeit zu Spaltzugfestigkeit liegt der ermittelte Mittelwert (1,8:1) zwar in der Nähe der Literaturangaben, doch auch hier besteht eine, wenn auch wesentlich geringere Richtungsabhängigkeit. Dadurch bedingt und zusätzlich durch die unterschiedlichen mineralogischen Zusammensetzungen beeinflusst, schwanken die Biege- zu Spaltzugfestigkeits-Verhältnisse zwischen 1,1:1 und 2,6:1 was auch hier eine Verwendung erschwert und im Einzelfall weitergehende Untersuchungen erfordert.

Sowohl die Kompressionswellgeschwindigkeiten (Vp) als auch der dynamische E-Modul werden häufig zur Schadensanalyse von Naturwerksteinen herangezogen.

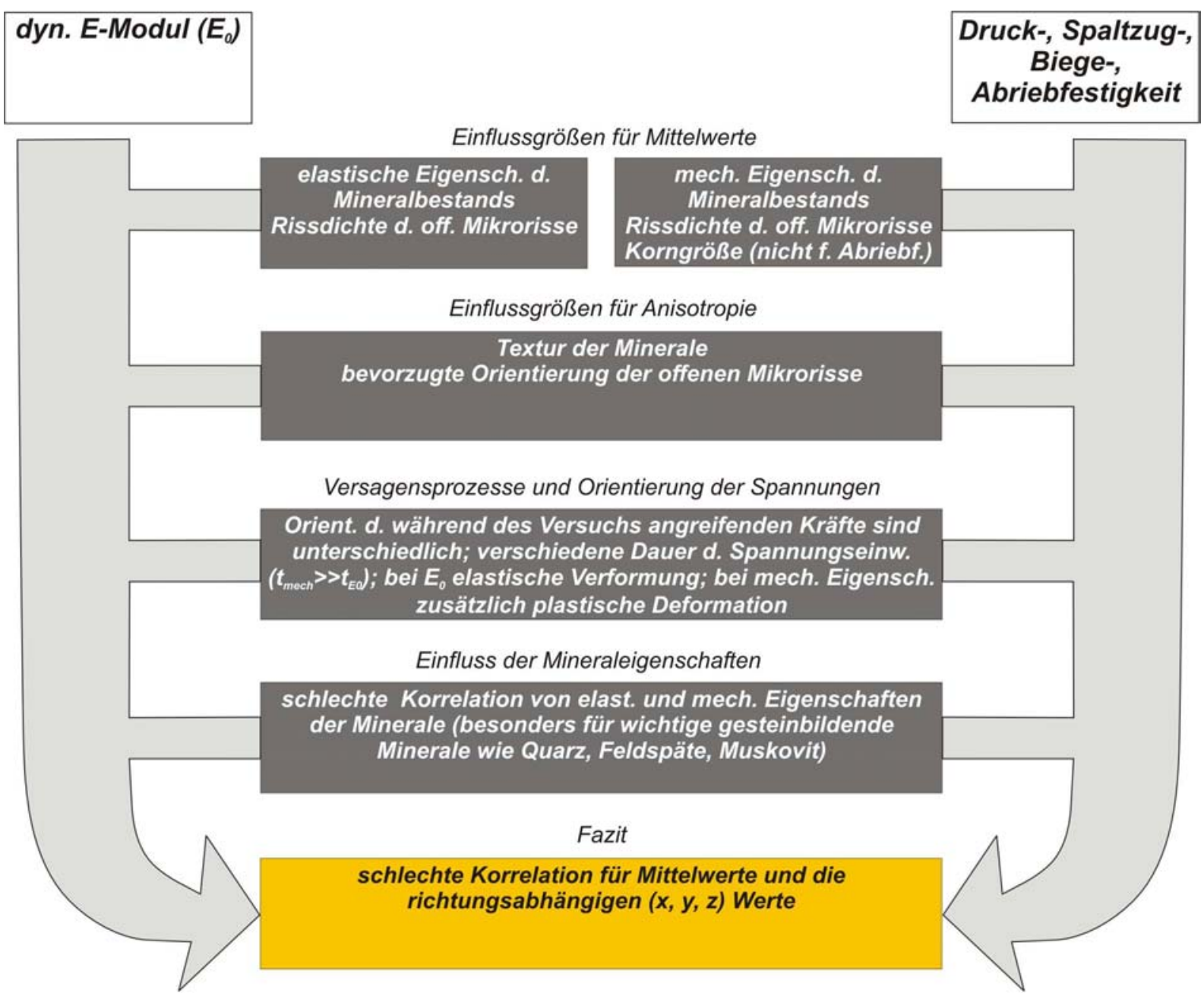

Abb.9-7: Dynamischer E-Modul vs. mechanische Eigenschaften. Weitere Korrelationsdiagramme in Anhang III.

Dahinter verbirgt sich die Absicht aus den elastischen Eigenschaften eine Aussage über die mechanischen Kennwerte zu erlangen. Die zerstörungsfreie Anwendung basiert auf der 


\section{Zusammenfassung}

Annahme, dass, ausgehend von einem intrinsischen (rissfreien) Zustand der E-Modul durch offene Mikrorisse herabgesetzt wird, welche ebenfalls eine Reduktion der Festigkeiten bewirken. Darauf basierend werden hohe E-Moduln (oder Vp) eines Gesteins tendenziell mit hohen Festigkeiten in Verbindung gebracht. Dieses Verfahren wurde bereits von Köhler (1991) und in Ansätzen von Ondrasina et al. (2002) für die Analyse von Marmoren eingesetzt.

Wie die Ergebnisse der vorliegenden Studie zeigen ist eine Erweiterung der Erkenntnisse auf Kristallingesteine im allgemeinen nicht sinnvoll. Hauptursache der schlechten Korrelation zwischen dem dynamischen E-Modul und den mechanischen Eigenschaften sind die Abhängigkeit von unterschiedlichen Gesteinsmerkmalen und die völlig verschiedenen Prozesse bei der experimentellen Bestimmung (Abb.9-7). Hinzu kommt, dass insbesondere für wichtige gesteinsbildende Minerale (Quarz, Feldspat, Muskovit) ein nur schlecht definierter Zusammenhang zwischen den elastischen und den mechanischen Eigenschaften besteht. Damit sind sämtliche der Kriterien für eine signifikante Korrelation (siehe oben) nicht erfüllt und folglich keine stabilen Verhältnisse zwischen den technischen Kennwerten zu erwarten.

Generell erfordert eine Schadensanalyse insbesondere von hoch anisotropen Gesteinen eine detaillierte Einzelfallbetrachtung unter Einbeziehung des gesamten mineralogischen und Gefüge-Inventars. Eine standardisierte Anwendung ist nur bei Gesteinen einer identischen und möglichst monomineralischen Zusammensetzung sowie ähnlicher Gefügemerkmale (Rissdimensionen, Korngröße) denkbar. In diesem Fall kann mit Hilfe von empirischen Untersuchungen eine Referenzskala aufgestellt werden, die über eine gewisse Aussagekraft verfügt und mit deren Hilfe eine grobe Einschätzung der mechanischen Eigenschaften aus dem dynamischen Spannungs-Dehnungs-Verhalten vorgenommen werden kann.

Abschließend lässt sich folgendes zu den Kreuzkorrelationen von technischen Parametern sagen:

Grundsätzlich kommen die Korrelationen der technischen Parameter aufgrund der mannigfaltigen Einflussgrößen und Überlagerungen nicht über die Bedeutung einer „Faustregel“ hinaus. Präzise Ergebnisse sind schon deshalb nicht zu erwarten, weil für die meisten Korrelationen der physikalisch-theoretische Hintergrund für ein stabiles Verhältnis fehlt. Zudem können bei stark anisotropen Gesteinen die richtungsabhängigen Abweichungen vom mittleren Verhältnis sehr groß sein und demzufolge zu einer Fehleinschätzung führen. Insgesamt ist eine standardmäßige Verwendung von Eigenschaftsverhältnissen nur dann in 


\section{Zusammenfassung}

Ansätzen möglich, wenn alle vier Kriterien für eine gute Korrelation erfüllt sind (siehe oben), was allein für die Biege- und die Spaltzugfestigkeit der Fall ist.

\subsection{Verallgemeinerbarkeit der Ergebnisse}

Aufgrund der großen Probenanzahl und der möglichst repräsentativen Auswahl der Gesteine sind die zum Teil empirischen, aber auch modellhaft untermauerten Sachverhalte auf kristalline Gesteine mit einer niedrigen und durch Mikrorisse geprägten Porosität anwendbar. Die Extrapolation der Ergebnisse in Bereiche der technischen Eigenschaften, die innerhalb des Probensatzes nicht auftreten, scheint möglich, zumal viele der ermittelten technischen Kennwerte in der Nähe der oberen und unteren Grenze der für Naturwerksteine verwirklichten Werte liegen. Das betrifft sowohl die gesteinsphysikalischen Eigenschaften mit Ausnahme der kapillaren Wasseraufnahme als auch die Gefügemerkmale. 


\section{Abkürzungsverzeichnis}

\begin{tabular}{|l|l|l|}
\hline Kürzel/Symb. & Beschreibung & Einheit \\
\hline Mech. & & \\
\hline $\mathrm{H}_{\mathrm{G}}$ & Mittlere Härte des Gesteins (aus Mineralbestand) & {$[\mathrm{HV}]$} \\
\hline $\mathrm{H}_{\mathrm{M}}$ & Mittlere Härte des Minerals & {$[\mathrm{HV}]$} \\
\hline $\mathrm{I}_{\mathrm{SPG}}$ & Spaltbarkeitsindex des Gesteins (aus Mineralbestand) & \\
\hline $\mathrm{I}_{\mathrm{SPM}}$ & Spaltbarkeitsindex des Minerals & \\
\hline $\mathrm{A}_{\mathrm{ISPM}}$ & Anisotropie des Spaltbarkeitsindex des Minerals & \\
\hline $\mathrm{R}$ & Druckfestigkeit & {$[\mathrm{MPa}]$} \\
\hline$\beta_{\mathrm{SZ}}$ & Spaltzugfestigkeit & {$[\mathrm{MPa}]$} \\
\hline $\mathrm{R}_{\mathrm{tf}}$ & Biegefestigkeit & {$[\mathrm{MPa}]$} \\
\hline $\mathrm{W}_{\mathrm{V}}$ & Abriebfestigkeit & {$\left[\mathrm{cm}^{3}\right]$} \\
\hline$\gamma$ & Spezifische Bruchflächenenergie & {$\left[\mathrm{J} / \mathrm{m}^{2}\right]$} \\
\hline $\mathrm{K}_{\mathrm{IC}}$ & Bruchzähigkeit (Mode I) & {$\left[\mathrm{MPa}^{*} \mathrm{~m}^{1 / 2}\right]$} \\
\hline $\mathrm{K}_{\mathrm{IIC}}$ & Bruchzähigkeit (Mode II) & {$\left[\mathrm{MPa}^{*} \mathrm{~m}^{1 / 2}\right]$} \\
\hline
\end{tabular}

\begin{tabular}{|c|c|c|}
\hline \multicolumn{3}{|l|}{ Elast. } \\
\hline $\mathrm{Vp}$ & Kompressionswellengeschwindigkeit & {$[\mathrm{km} / \mathrm{s}]$} \\
\hline $\mathrm{Vs}_{1}$ & Geschwindigkeit der schnelleren Scherwelle & {$[\mathrm{km} / \mathrm{s}]$} \\
\hline $\mathrm{Vs}_{2}$ & Geschwindigkeit der langsameren Scherwelle & {$[\mathrm{km} / \mathrm{s}]$} \\
\hline Vp $p_{M V R H}$ & Vp des Minerals (Voigt-Reuss-Hill-Mittel) & {$[\mathrm{km} / \mathrm{s}]$} \\
\hline $\mathrm{Vp}_{\mathrm{GVRH}}$ & Vp des Gesteins (Voigt-Reuss-Hill-Mittel) & {$[\mathrm{km} / \mathrm{s}]$} \\
\hline $\mathrm{E}_{\mathrm{MVRH}}$ & Dyn. E-Modul des Minerals (Voigt-Reuss-Hill-Mittel) & [GPa] \\
\hline $\mathrm{G}_{\mathrm{MVRH}}$ & Dyn. Schermodul des Minerals (Voigt-Reuss-Hill-Mittel) & [GPa] \\
\hline $\mathrm{K}_{\mathrm{MVRH}}$ & Dyn. Schubmodul des Minerals (Voigt-Reuss-Hill-Mittel) & {$[\mathrm{GPa}]$} \\
\hline $\mathrm{E}_{0}$ & Dyn. E-Modul des Gesteins bei 0 MPa Umschließungsdruck & {$[\mathrm{GPa}$} \\
\hline $\mathrm{E}_{400}$ & Dyn. E-Modul d. Gesteins bei $400 \mathrm{MPa}$ Umschließungsdruck & {$[\mathrm{GPa}]$} \\
\hline $\mathrm{E}_{0 \mathrm{~s} 1}$ & Dyn. E-Modul d. Gest. b.0 MPa berechnet mit $\mathrm{Vs}_{1}$ & {$[\mathrm{GPa}]$} \\
\hline $\mathrm{E}_{0 \mathrm{~s} 2}$ & Dyn. E-Modul d. Gest. b.0 MPa berechnet mit $\mathrm{Vs}_{2}$ & {$[\mathrm{GPa}]$} \\
\hline$\Delta \mathrm{E}_{0 \mathrm{~s} 1 / 2}$ & durch Scherwellen-Splitting bedingte rel. Abweichung von $\mathrm{E}_{0}$ & {$[\%]$} \\
\hline $\mathrm{A}_{\mathrm{E} 0}$ & Anisotropie von $\mathrm{E}_{0}$ & {$[\%]$} \\
\hline $\mathrm{A}_{\mathrm{E} 400}$ & Anisotropie von $\mathrm{E}_{400}$ & {$[\%]$} \\
\hline $\mathrm{A}_{\mathrm{E} 0 \mathrm{Gmax}}$ & Maximal mögliche Anisotropie von $\mathrm{E}_{0}$ (aus Mineralbestand) & {$[\%]$} \\
\hline $\mathrm{A}_{\mathrm{VpM}}$ & Anisotropie der Vp des Minerals & \\
\hline$v$ & Poisson-Zahl & \\
\hline$v_{0}$ & Poisson-Zahl bei $0 \mathrm{MPa}$ Umschließungsdruck & \\
\hline$v_{400}$ & Poisson-Zahl bei $400 \mathrm{MPa}$ Umschließungsdruck & \\
\hline $\mathrm{Vp} \mathrm{p}_{\text {trockm }}$ & Mittelwert der Vp aus der Messung d. trockenen Kugel & {$[\mathrm{km} / \mathrm{s}]$} \\
\hline$\Delta \mathrm{Vp}$ & Vp aus der Diff. d. Vp aus Mess. d. wasserges. u. trock. Kugel & {$[\mathrm{km} / \mathrm{s}]$} \\
\hline$\Delta \mathrm{Vp}_{\mathrm{m}}$ & Mittelwert von $\Delta \mathrm{Vp}$ & {$[\mathrm{km} / \mathrm{s}]$} \\
\hline $\mathrm{A}_{\Delta \mathrm{Vp}}$ & Anisotropie von $\Delta \mathrm{Vp}$ & {$[\%]$} \\
\hline$E_{\text {stat }}$ & statischer E-Modul & {$[\mathrm{GPa}]$} \\
\hline $\mathrm{E}_{\text {stat } 10}$ & statischer E-Modul bei $10 \%$ der Bruchlast & {$[\mathrm{GPa}]$} \\
\hline $\mathrm{E}_{\text {stat50 }}$ & statischer E-Modul bei 50\% der Bruchlast & {$[\mathrm{GPa}]$} \\
\hline$E_{\text {stats }}$ & Bruchmodul & {$[\mathrm{GPa}]$} \\
\hline
\end{tabular}




\section{Abkürzungsverzeichis}

\begin{tabular}{|l|l|l|}
\hline Kürzel/Symb. & Beschreibung & Einheit \\
\hline Gefüge & & \\
\hline $\mathrm{S}_{1,2,3}$ & Eigenwerte der Eigenvektoren (Texturen) & \\
\hline $\mathrm{I}_{\max }$ & maximale Intensität der Textur & {$[\mathrm{mrd}]$} \\
\hline $\mathrm{k}_{\mathrm{W}}$ & Symmetrieparameter nach Woodcock & \\
\hline $\mathrm{c}_{\mathrm{W}}$ & Regelungsschärfe nach Woodcock & \\
\hline $\mathrm{T}$ & Formfaktor in Anlehnung an Jelinek & \\
\hline $\mathrm{Vp}_{\mathrm{QRiss}}$ & Rissquantifizierungsparameter & {$[\mathrm{km} / \mathrm{s}]$} \\
\hline $\mathrm{A}_{\text {Riss }}$ & Rissanisotropiefaktor $\left(\mathrm{Vp}_{\mathrm{QRiss}} * \mathrm{~A}_{\Delta \mathrm{Vp}}\right)$ & {$[\mathrm{km} / \mathrm{s} * \%]$} \\
\hline
\end{tabular}

\section{Th. Dehnung}

\begin{tabular}{|l|l|l|}
\hline$\alpha_{20-90}$ & Thermischer Ausdehnungskoeffizient (für $20^{\circ} \mathrm{C} \leq \mathrm{T} \leq 90^{\circ} \mathrm{C}$ ) & {$[1 / \mathrm{K}]$} \\
\hline$\alpha_{\mathrm{VG}}$ & Therm. Volumenausd.koeff. d. Gesteins (für $\left.20^{\circ} \mathrm{C} \leq \mathrm{T} \leq 90^{\circ} \mathrm{C}\right)$ & {$[1 / \mathrm{K}]$} \\
\hline$\alpha_{\mathrm{VM}}$ & Therm. Volumenausd.koeff. d. Minerals (für $20^{\circ} \mathrm{C} \leq \mathrm{T} \leq 90^{\circ} \mathrm{C}$ ) & {$[1 / \mathrm{K}]$} \\
\hline$\alpha_{\mathrm{V}}$ & Thermischer Volumenausdehnungskoeffizient & {$[1 / \mathrm{K}]$} \\
\hline$\alpha_{\mathrm{VGT}}$ & Therm. Volumenausd.koeff. d. Gesteins (theor. aus Min.best) & {$[1 / \mathrm{K}]$} \\
\hline$\Delta \alpha_{\mathrm{V}}$ & $\alpha_{\mathrm{VGT}}-\alpha_{\mathrm{VG}}$ & {$[1 / \mathrm{K}]$} \\
\hline $\mathrm{A}_{\alpha \mathrm{Gmax}}$ & Max. mögliche Anisotr. d. therm. Ausd.koeff. (aus Min.best.) & {$[\%]$} \\
\hline $\mathrm{A}_{\alpha \mathrm{M}}$ & Anisotropie des therm. Ausdehnungskoeff. d. Minerals & {$[\%]$} \\
\hline $\mathrm{A}_{\alpha \mathrm{G}}$ & Anisotropie des therm. Ausdehnungskoeff. des Gesteins & {$[\%]$} \\
\hline$\Delta_{\mathrm{VRiss}}$ & Relat. Zunahme des Gesteinsv. durch therm. Rissbildung & {$[\mathrm{Vol} \%]$} \\
\hline
\end{tabular}

\begin{tabular}{|l|l|l|}
\hline Sonstige & & \\
\hline $\mathrm{k}_{\mathrm{G}}$ & Kationenpackungsindex des Gesteins & {$\left[\mathrm{mol} / \mathrm{cm}^{3}\right]$} \\
\hline $\mathrm{k}_{\mathrm{M}}$ & Kationenpackungsindex des Minerals & {$\left[\mathrm{mol} / \mathrm{cm}^{3}\right]$} \\
\hline $\mathrm{M}_{\mathrm{G}}$ & Mittleres Atomgewicht des Gesteins & \\
\hline $\mathrm{V}_{\mathrm{M}}$ & Molares Volumen & {$\left[\mathrm{cm}^{3}\right]$} \\
\hline$\rho_{\mathrm{M}}$ & Matrixdichte & {$\left[\mathrm{g} / \mathrm{cm}^{3}\right]$} \\
\hline$\rho_{\mathrm{Roh}}$ & Rohdichte & {$\left[\mathrm{g} / \mathrm{cm}^{3}\right]$} \\
\hline$\phi$ & Effektive Porosität & {$[\mathrm{Vol} . \%]$} \\
\hline$\phi_{\mathrm{rkap}}$ & Volumenanteil der Kapillarporen am Gesteinsvolumen & {$[\mathrm{Vol} \%]$} \\
\hline $\mathrm{W}$ & Wasseraufnahmekoeffizient & {$[\mathrm{kg} / \mathrm{m} 2 * \sqrt{\mathrm{t}}]$} \\
\hline $\mathrm{r}_{\mathrm{m}}$ & Mittlerer Porenradius & {$[\mu \mathrm{m}]$} \\
\hline $\mathrm{S}_{\mathrm{Kap}}$ & Sättigungsgrad der Kapillarporen & {$[\%]$} \\
\hline $\mathrm{r}_{\text {Kapm }}$ & Mittlerer Radius der Kapillarporen & {$[\mu \mathrm{m}]$} \\
\hline $\mathrm{a} 1, \mathrm{a} 2, \mathrm{~b} 3$ & Mikrorissachsen & {$[\mathrm{mm}]$} \\
\hline
\end{tabular}

\begin{tabular}{|l|l|l|}
\hline Minerale & & \\
\hline $\mathrm{Ab}$ & Albit & \\
\hline $\mathrm{An}$ & Anorthit & \\
\hline $\mathrm{Apa}$ & Apatit & \\
\hline $\mathrm{Bt}$ & Biotit & \\
\hline $\mathrm{Cc}$ & Kalzit & \\
\hline $\mathrm{Chl}$ & Chlorit & \\
\hline $\mathrm{Cpx}$ & Klinopyroxen & \\
\hline $\mathrm{Crd}$ & Cordierit & \\
\hline Dio & Diopsid & \\
\hline Dum & Dumortierit & \\
\hline En & Enstatit & \\
\hline Ep & Epidot & \\
\hline
\end{tabular}




\section{$\underline{\text { 10. Abkürzungsverzeichis }}$}

\begin{tabular}{|l|l|l|}
\hline Kürzel/Symb. Beschreibung & Einheit \\
\hline Fs & Feldspat & \\
\hline Grs & Grossular & \\
\hline Gt & Granat & \\
\hline Häm & Hämatit & \\
\hline Hbl & Hornblende & \\
\hline Hed & Hedenbergit & \\
\hline Ilm & Ilmenit & \\
\hline Kfs & Kalifeldspat & \\
\hline Ky & Kyanit & \\
\hline Kzo & Klinozoisit & \\
\hline MA & Mikrokristallines Aggregat & \\
\hline Mag & Magnetit & \\
\hline Ms & Muskovit & \\
\hline Ol & Olivin & \\
\hline Opx & Orthopyroxen & \\
\hline Pl & Plagioklas & \\
\hline Pyr & Pyrop & \\
\hline Pyx & Pyroxen & \\
\hline Qz & Quarz & \\
\hline Rt & Rutil & \\
\hline Ser & Serizit & \\
\hline Sil & Sillimanit & \\
\hline Spi & Spinell & \\
\hline Tit & Titanit & \\
\hline Turm & Turmalin & \\
\hline Zr & Zirkon & \\
\hline & & \\
\hline
\end{tabular}

\begin{tabular}{|l|l|l|}
\hline Proben & & \\
\hline AI & Quarzit (Azul Imperial) & \\
\hline Be1 & Granodioritischer Gneis (Serizzo Monte Rosa) & \\
\hline Be2 & Granitischer Gneis (Serizzo Monte Rosa) & \\
\hline Cal & Granodioritischer Gneis (Calanca) & \\
\hline Gra1a & Granitischer Gneis & \\
\hline Gra1c & Granitischer Gneis & \\
\hline Gra3a & Granitischer Ultramylonit & \\
\hline Iv1 & Granodioritischer Protomylonit & \\
\hline Iv1b & Granodioritischer Mylonit & \\
\hline Iv2 & Granodioritischer Ultramylonit & \\
\hline Koess & Granit (Kösseine) & \\
\hline Mg1b & Meta-Gabbro & \\
\hline Mg2 & Meta-Gabbro & \\
\hline Mrgr3 & Granit & \\
\hline Mrmy5 & Granitischer Protomylonit & \\
\hline NI & Gabbro-Norit (Nero Impala) & \\
\hline PDD & Granodiorit (Padang dunkel) & \\
\hline Pi1a & Peridotit & \\
\hline Rek & Kalzit-Marmor (Rosa Estremoz) & \\
\hline VA & Granitischer Gneis (Verde Andeer) & \\
\hline
\end{tabular}




\section{$\underline{\text { 11. Literaturverzeichnis }}$}

\section{Literaturverzeichnis}

Adam, J. F. 1989. Methoden und Algorithmen zur Verwaltung und Analyse axialer 3-DRichtungsdaten und ihrer Belegungsdichten. Göttinger Arb. Geol. Paläont. 40, 100.

Aleksandrov, K. S., Alchikov, U. V., Belikov, B. P., Zaslavskii, B. I. \& Krupnyi, A. I. 1974. Velocities of elastic waves in minerals at atmospheric pressure and increasing precision of elastic constants by means of EVM (in russian). Izv. Acad. Sci. UDSSR Geol. Ser. 10, 15-24.

Aleksandrov, K. S. \& Ryzhova, T. V. 1961. The elastic properties of rock forming minerals II: layered silicates. Izv. Acad. Sci. UDSSR Geophys. Phys. Solid Earth, 11651168.

Al-Lahyani, K., Shehata, W. M. \& Sabtan, A. A. 1995. Effect of microfissures and the engeneering properties of the marble at Wadi Lisb, Saudi Arabia. Bulletin of the Int. Ass. of Engeneering Geology 52, 33-37.

Alm, O., Jaktlund, L. L. \& Kou, S. Q. 1985. The influence of microcrack density on the elastic and fracture mechanical properities of Stripa granite. Physics of the Earth and Planetary Interiors 40, 161-179.

Assad, J. M., Tatham, R. \& McDonald, J. A. 1992. A physical study of microcrack-induced anisotropy. Geophysics 57, 1562-1570.

Atkinson, B. K. 1979. Pure and Applied Geophysics 117, 1011-1024.

Atkinson, B. K. \& Avdis, V. 1980. International Journal of Rock Mechanics and Mining Science 17, 383-386.

Atkinson, B. K. \& Meredith, P. G. 1987. Experimental fracture mechanics data for rocks and minerals. In: Fracture mechanics of rocks (edited by Atkinson, B. K.). Academic Press, London, 477-425.

Azzoni, A., Bailo, F., Rondena, E. \& Zaninetti, A. 1996. Assessment of texture coefficient for different rock types and correlation with uniaxial compressive strength and rock weathering. Rock Mechanics and Rock Engineering 29(1), 39-46.

Barron, T. H. K. 1998. Generalized theory of thermal expansion of solids. In: Thermal expansion of solids (edited by Taylor, R. E.). Cindas Data Series on Material Properties I-4. ASM International, Materials Park, 1-108.

Barton, N. \& Choubey, V. 1977. The shear strength of rock joints in theory and practice. Rock Mechanics 10, 1-54.

Bass, J. D. 1995. Elasticity of Minerals, Glasses, Melts. In: Mineral Physics and Crystallography-A Handbook of Physikal Constants (edited by Ahrens, T. J.) 2. American Geophysical Union, Washington, 354-379.

Battaglia, S., Franzini, M. \& Mango, F. 1993. High sensitivity apparatus for measuring linear thermal expansion: preliminary results on the response of marbles. Il Nuovo Cimento 16, 453-461.

Bauer, S. J. \& Johnson, B. 1979. Effects of slow uniform heating on the physical properties of Westerly and Charocoal granites. Proceedings of the 20th Symposium for Rock Mechanics, 7-18.

Bergmann, M. 1995. Schülerduden: Die Physik. Duden Verlag, Mannheim.

Birch, F. 1960. The velocity of compressional waves in rocks up to 10 kilobars; part 1. Journal of Geophysical Research 65, 1083-1102.

Birch, F. 1961. The velocity of compressional waves in rocks up to 10 kilobars; part 2. Journal of Geophysical Research 66, 2199-2224.

Blenkinsop, T. 2000. Deformation Microstructures and Mechanics in Minerals and Rocks. Kluwer Academic Publishers, Dordrecht. 


\section{$\underline{\text { 11. Literaturverzeichnis }}$}

Bonczar, L. J., Graham, E. K. \& Wang, H. 1977. The pressure and temperature dependence of the elastic constants of pyrope garnet. Journal of Geophysical Research 82/17, 2529-2534.

Börner, K. \& Hill, D. 2002. Große Enzyklopädie der Steine 2002. Abraxas-Verlag, Hasede.

Brace, W. F. \& Walsh, J. B. 1962. American Mineralogist 47, 1111-1122.

Brosch, F. J., Schachner, K., Bluemel, M. \& Fasching, A. 2000. Preliminary investigation results on fabrics and related mechanical properties of an anisotropic gneiss. Journal of Structural Geology 22, 1773-1787.

Brown, E. T. 1981. Rock characterization, testing and monitoring: ISRM suggested methods. Pergamon Press, Oxford.

Bunge, H. J. 1985. Representation of preferred orientations. In: Preferred orientation in deformed metals and rocks: an introduction to modern texture analysis (edited by Wenk, H. R.). Academic Press, Orlando, 73-108.

Bunge, H. J. 1987. Theoretical methods in texture analysis. DMG Informationsgesellschaft, Oberursel.

Buntebarth, G. 1982. Density and seismic velocity in relation to mineralogical constitution based on an ionic model for minerals. Earth and Planetary Science Letters 57(2), 358-366.

Christensen, N. I. 1984. The magnitude, symmetry and origin of upper mantle anisotropy based on fabric analysis of ultramafic tectonites. Geophys. J. R. Astr. Soc. 76, 89111.

Christensen, N. I. \& Fountain, D. 1975. Constitution of the lower continental crust based on experimental studies of seismic velocities in granulite. Bull. Soc. Am. 86, 227-236.

Christoffersen, R. \& Kronenberg, A. K. 1993. Dislocation interactions in experimentally deformed biotite. In: Microstructures and rheology of rocks and rock-forming minerals; a collection of papers in honor of John Christie's 60th birthday. (edited by Kirby Stephen, H., Sylvester, A. G., Tullis, J., Wenk, H. R. \& Treagus, S. H.). Journal of Structural Geology 15; 9-10. Pergamon, Oxford-New York, International, 1077-1095.

Correns, C. W. 1968. Einführung in die Mineralogie. Springer-Verlag, Berlin.

Crampin, S. 1987. The geological and industrial implications of extensive-dilatancy anisotropy. Nature 328, 491-496.

Czichos, H. 1991. Hütte - Die Grundlagen der Ingenieurwissenschaften. Springer-Verlag, Berlin.

Dandekar, D. P. 1968. Variation in the elastic constants of calcite with pressure. $A G U$ Transactions 49/1, 323.

Deer, W. A., Howie, R. A. \& Zussman, J. 1992. An introduction to the rock forming minerals. Longman, Hong Kong.

Dietrich, D. 1986. Calcite fabrics around folds as indicators of deformation history. Journal of Structural Geology 8, 655-668.

Dornbusch, H.-J. 1995. Gefüge-, Mikrostruktur- und Texturuntersuchungen an Hochtemperatur-Scherzonen in granulitfaziellen Metabasiten der Ivrea-Zone. In: Geotektonische Forschungen 83, 94.

Doveton, J. H. 1987. Log analysis of petrofacies and lithofacies. GFZ Logging Course. Geoforschungszentrum Potsdam.

Duerrast, H., Rasolofosaon, P. N. J. \& Siegesmund, S. 2002. P-wave velocity and permeability distribution of sandstones from a fractured tight gas reservoir. Geophysics 67/1, 241-253.

Duerrast, H., Siegesmund, S. \& Prasad, M. 1999. Die Schadensanalyse von Naturwerksteinen mittels Ultraschalldiagnostik: Moeglichkeiten und Grenzen. Zt. d. dt. Geol. Ges. 150(2), 359-374. 


\section{$\underline{\text { 11. Literaturverzeichnis }}$}

Duyster, J. 1991. Strukturgeologische Untersuchungen im Moldanubikum (Waldviertel, Österreich) und methodische Untersuchungen zur bildanalytischen Gefügequantifizierung von Gneisen.

Eisbacher, G. H. 1996. Einführung in die Tektonik. Ferdinand Enke Verlag, Stuttgart.

Erskine, B. G., Heidelbach, F. \& Wenk, H.-R. 1993. Lattice preferred orientation and microstructures of deformed Cordilleran marbles: correlation of shear indicators and determination of strain path. Journal of Structural Geology 15, 1189-1205.

Etheridge, M. A. \& Hobbs, B. E. 1974. Chemical and deformation controls on recrystallization of mica. Contrib. Mineral. Petrol. 19, 111-124.

Fahy, M. P. \& Guccione, M. J. 1979. Estimating the strength of sandstone using petrographic thin section data. Bulletin of the International Association of Engineering Geologists 16(4), 467-485.

Falls, S. D., Young, R. P., Carlson, S. R. \& Chow, T. 1992. Ultrasonic tomography and acoustic emission in hydraulically fractured Lac du Bonnet grey granite. Journal of Geophysical Research, B, Solid Earth and Planets 97(5), 6867-6884.

Fei, Y. 1995. Thermal Expansion. In: Mineral Physics and Crystallography-A Handbook of Physikal Constants (edited by Ahrens, T. J.) 2. American Geophysical Union, Washington, 354.

Feldmann, K. 1989. Texture investigations by neutron time-of-flight diffraction. Textures and Microstructures 10, 309-323.

Ferrero, A. M. \& Marini, P. 2001. Experimental studies on the mechanical behaviour of two thermal cracked marbles. Rock Mechanics and Rock Engineering 34/1, 57-66.

Fitzner, B. 1988. Untersuchungen der Zusammenhänge zwischen dem Hohlraumgefüge von Natursteinen und physikalischen Verwitterungsvorgängen. Geol. Institut RWTH, Aachen.

Fitzner, B. \& Snethlage, R. 1982. Einfluß der Porenradienverteilung auf das Verwitterungsverhalten ausgewählter Sandsteine. Bautenschutz und Bausanierung 3, 97-103.

Franzini, M. 1995. Stones in monuments: natural and anthropogenic deterioation of marble artifacts. Eur. J. Mineral. 7, 735-743.

Freyburg, E. 1972. Der untere und mittlere Buntsandstein SW-Thüringens in seinen gesteinstechnischen Eigenschaften. Berichte der deutsche Gesellschaft der geologischen Wissenschaften 17, 911-919.

Frisillo, A. L. \& Barsch, G. R. 1972. Measurement of single-crystal elastic constants of bronzite as a function of pressure and temperature. Journal of Geophysical Research 77, 6360-6383.

Gaal, P. S. 1998. Pushrod Dilatometers. In: Thermal expansion of solids (edited by Taylor, R. E.). Cindas Data Series on Material Properties I-4. ASM International, Materials Park, 165-179.

Gartung, E. 1985. Empfehlung Nr. 10 des Arbeitskreises 19 "Versuchstechnik Fels" der deutschen Gesellschaft für Erd- und Grundbau e. V. - Indirekter Zugversuch an Gesteinsproben - Spaltzugversuch. Bautechnik 6, 197-199.

Gebrande, H. 1982. Elastic wave velocities and constants of elasticity at normal conditions. In: Physical properties of rocks (edited by Angenheister, G.). Landolt-BörnsteinNumerical Data and Functional Relationships in Science and Technology GroupV: Geophysics and Space Research. Volume1b. Springer-Verlag, Berlin, 8-35.

Glover, P. W., Baud, P., Darot, M., Meredith, P. G., Boon, S. A., LeRavalac, M., Zoussi, S. \& Reuschle. 1995. Alpha/beta phase transition in quartz monitored using acoustic emissions. Geophysical Journal International 120, 775-782. 


\section{Literaturverzeichnis}

Golubev, A. A. \& Rabinovic, G. J. 1976. Resultaty primenenia apparatury akusticeskogo karotasa dlja opredelenia proenostych svoistv gornych porod na mestorosdeniach tverdych iskopaemych. Pridkladnaja Geofizika 73, 109-116.

Gorjainov, N. N. \& Ljachovickij, F. M. 1979. Seismiceskie metody v insenernoi geologii. Izdat. Nedra, Moskva.

Gottschalk, R. R., Kronenberg, A. K., Russell, J. E. \& Handin, J. 1990. Mechanical anisotropy of gneiss; failure criterion and textural sources of directional behavior. Journal of Geophysical Research, B, Solid Earth and Planets 95(13), 21,61321,634 .

Grasselli, G. \& Egger, P. 2003. Constitutive law for the shear strength of rock joints based on three-dimensional surface parameters. International Journal of Rock Mechanics and Mining Science 40, 25-40.

Griffith, A. A. 1924. The theory of rupture. In: 1st International Congress Applied Mechanics, Delft, 55-63.

Grimm, W. D. 1999. Beobachtungen und Ueberlegungen zur Verformung von Marmorobjekten durch Gefuegeauflockerung. Zt. d. dt. Geol. Ges. 150(2), 195236.

Gudmundsson, A. 1999. Fracture dimensions, displacements and fluid transport. Journal of Structural Geology 22(9), 1221-1231.

Gupta, A. S. \& Seshagiri, R. K. 2000. Weathering effects on the strength and deformational behaviour of crystalline rocks under uniaxial compression state. Engineering Geology 56(3-4), 257-274.

Haberfield, C. M. \& Johnston, I. W. 1994. A mechanistically-based model for rough rock joint. International Journal of Rock Mechanics and Mining Science 31, 279-292.

Hahn, T. 1998. Thermal expansion measurements using optical interferometry. In: Thermal expansion of solids (edited by Taylor, R. E.). Cindas Data Series on Material Properties I-4. ASM International, Materials Park, 181-192.

Hall, A. 1996. Igneous Petrology. Longman Group Limited, Essex.

Haney, M. G. \& Shakoor, A. 1994. The relationship between tensile and compressive strength of selected sandstones as influenced by index properties and petrographic characteristics. In: 7th International IAEG Congress Sept. 5-9 2, Lisbon/Portugal, 493-500.

Harte, B. 1983. Mantle peridotites and processes - the kimberlite sample. In: Continental basalts and mantle xenoliths (edited by Hawkesworth, C. J. \& Norry, M. J.). Shiva, Nantwich, 46-91.

Hartley, N. E. W. \& Wilshaw, T. R. 1973. Journal of Material Science 8, 265-278.

Heinrichs, H. \& Herrmann, A. G. 1990. Praktikum der Analytischen Geochemie. SpringerVerlag, Berlin.

Heinzler, M., Kilgus, R., Näher, F., Paetzold, H., Röhrer, W. \& Schilling, K. 1997. Tabellenbuch Metall. Verlag Europa-Lehrmittel, Haan-Gruiten.

Hill, R. W. 1952. The elastic constants for anisotropic behaviour of a crystalline aggregate. Proc. Phys. Soc. London A65, 349.

Hirschwald, J. 1912. Handbuch der bautechnischen Gesteinsprüfung. Verlag Gebrüder Bornträger, Berlin.

Holbrook, W. S., Gajewski, D. \& Podehl, C. 1987. Shear wave velocity and Poissons ratio structure of the upper lithosphere in Southwest Germany. Geophysical Research Letters 14, 231-234.

Hollemann, A. E. \& Wiberg, N. 1995. Lehrbuch der anorganischen Chemie. Walter de Gruyter, Berlin. 


\section{Literaturverzeichnis}

Howarth, D. F. \& Rowlands, J. C. 1987. Quantitative assessment of rock texture and correlation with drillability and strength properties. Rock Mechanics and Rock Engineering 20(1), 57-85.

Hudson. 1981. Wave speeds and attenuation of elastic waves in material containing cracks. Geophys. J. R. Astr. Soc. 64, 133-150.

Hugman, R. H. H. \& Friedman, M. 1979. Effects of texture and composition on mechanical behaviour of experimentally deformed carbonate rocks. The American Association of Petroleum Geologists Bulletin 63(9), 1478-1489.

Ide, J. M. 1937. The velocity of sound in rocks and glasses as a function of temperature. Journal of Geology 45, 689-716.

Isaak, D. G. 1992. High temperature elasticity of iron-bearing olivines. Journal of Geophysical Research 97/B5, 1871-1885.

Jahns, E. 1995. Quantifizierte seismische Anisotropie: Ein skalenübergreifender Vergleich am Beispiel des KTB. Cuvillier Verlag, Göttingen.

Jelinek, V. 1981. Characterization of the magnetic fabric of rocks. Tectonophysics 79(3-4), T63-T67.

Ji, S. \& Mainprice, D. 1988. Natural deformation fabrics of plagioclase: implications for slip systems and seismic anisotropy. Tectonophysics 147, 145-163.

Juckenack, C. 1990. Beitrag der Anisotropie der magnetischen Suszeptibilität (AMS) für Struktur- und Gefügeuntersuchungen von Metamorphiten: Einzelbeispiele und regionale Anwendungen im Spessart-Kristallin. Dissertation, Universität Göttingen, 151.

Kelsall, P. C., Watters, R. J. \& Franzone, J. G. 1986. Engineering characterization of fissured, weathered dolerite and vesicular basalt. In: 27th U.S. Symposium on Rock mechanics - rock mechanics: key to energy production, Tuscaloosa/ USA, 77-84.

Kern, H. 1982. P-and s-wave velocities in crustal and mantle rocks under the simultaneous action of high confining pressure and high temperature and the effect of rock microstructure. In: High pressure researches in geoscience (edited by Schreyer, W.). Schweizerbart, Stuttgart, 15-45.

Kleber, W. 1990. Einführung in die Kristallographie. Verlag Technik GmbH, Berlin.

Klopfer, H. 1985. Feuchte. In: Lehrbuch der Bauphysik. Teubner Verlag, Stuttgart, 265-434.

Koch, A. \& Siegesmund, S. 2001. Gesteinstechnische Eigenschaften ausgewählter Bausandsteine. Zeitschrift der deutschen geologischen Gesellschaft 152/2-4, 681700.

Koch, A. \& Siegesmund, S. 2002. Bowing of marble panels: on-site damage analysis from the Oeconomicum building at Göttingen (Germany). In: Natural Stone, Weathering Phenomena, Conservation Strategies and Case Studies (edited by Siegesmund, S., Weiss, T. \& Vollbrecht, A.). Geological Society of London Special Publication 205. Geological Society of London, London, 299-314.

Köhler, W. 1991. Untersuchungen zu Verwitterungsvorgängen an Carrara-Marmor in Potsdam-Sansssouci. Berichte zu Forschung und Praxis der Denkmalpflege in Deutschland, Steinschäden-Steinkonservierung 2, 50-55.

Koroneos, E. G., Tassojannopoulos, A. I. \& Diamantopoulou, A. 1980. On the mechanical and physical properties of ten Hellenic marbles. Engineering Geology 16(3-4), 263-290.

Kownatzki, R. 1997. Verwitterungszustandserfassung von Naturbausteinwerken unter besonderer Berücksichtigung phänomenologischer Verfahren, Aachen.

Kranz, R. L. 1983. Microcracks in rocks; a review. In: Continental tectonics; structure, kinematics and dynamics. (edited by Friedman, M. \& Toksoez, M. N.). Tectonophysics 100; 1-3. Elsevier, Amsterdam, Netherlands, 449-480. 


\section{Literaturverzeichnis}

Kronenberg, A. K., Kirby, S. H. \& Pinkston, J. 1990. Basal slip and mechanical anisotropy of biotite. Journal of Geophysical Research, B, Solid Earth and Planets 95(12), 19,257-19,278.

Kruhl, H. J. 1987. Preferred lattice orientation of plagioclase from amphibolite and greenschist facies rocks near the insubric line (Western Alps). Tectonophysics 135, 233-242.

Laffitte, P. 1953. Etude de la precision des analyses de roche. Bull. Soc. Geol. France 6(3), 723-745.

Leiss, B., Gröger, H. R., Ullemeyer, K. \& Lebit, H. 2002. Textures and microstructures of naturally deformed amphibolites from the northern Cascades, NW USA: methodology and regional aspects. In: Deformation Mechanisms, Rheology and Tectonics: Current Status and Future Perspectives (edited by De Meer, S., Drury, M. R., De Bresser, J. H. P. \& Pennock, G. M.) 200. Geological Society of London, London, 219-238.

Leiss, B. \& Ullemeyer, K. 1999. Texture characterisation of carbonate rocks and some implications for the modelling of physical anisotropies, derived from idealized texture types. Zt. d. dt. Geol. Ges. 150(2), 259-274.

Levien, L., Weidner, D. J. \& Prewitt, C. T. 1979. Elasticity of diopside. Physics and Chemistry of Minerals 4/2, 105-113.

Li, C., Prikryl, R. \& Nordlund, E. 1998. The stress-strain behaviour of rock material related to fracture under compression. In: Computational methods in engineering geology. (edited by Pusch, R.). Engineering Geology 49; 3-4. Elsevier, Amsterdam, Netherlands, 293-302.

Lu, C. \& Jackson, I. 1998. Seismic-frequency laboratory measurements of shear mode viscoelasticity in crustal rocks; II, Thermally stressed quartzite and granite. Pure and Applied Geophysics 153(2-4), 441-473.

Maaloe, S. \& Aoki, K. 1977. The mayor element composition of the upper mantle estimated from the composition of lherzolites. Contrib. Mineral. Petrol. 63, 161-173.

Mainprice, D., L., B. J., Blumenfeld, P. \& Tubia, J. P. 1986. Dominant c-slip in naturally deformed quartz: implications for dramatic plastic softening at high temperatures. Geology 14, 819-822.

Mancktelow, N. S. 1987. Quartz textures from the Simplon fault zone, Southwest Switzerland and North Italy. In: Tectonic and structural processes on a macro-, meso- and micro-scale. (edited by Zwart, H. J., Martens, M., van der Molen, I., Passchier, C. W., Spiers, C. \& Vissers, R. L. M.). Tectonophysics 135; 1-3. Elsevier, Amsterdam, Netherlands, 133-153.

Mares, V. M. \& Kronenberg, A. K. 1993. Experimental deformation of muscovite. In: Microstructures and rheology of rocks and rock-forming minerals; a collection of papers in honor of John Christie's 60th birthday. (edited by Kirby Stephen, H., Sylvester, A. G., Tullis, J., Wenk, H. R. \& Treagus, S. H.). Journal of Structural Geology 15; 9-10. Pergamon, Oxford-New York, International, 1061-1075.

McKinstry, H. A., Huang, C. Y. \& McKinstry, S. T. 1998. Thermal expansion by x-ray diffraction. In: Thermal expansion of solids (edited by Taylor, R. E.). Cindas Data Series on Material Properties I-4. ASM International, Materials Park, 193-206.

McSkimin, H. J., Anreatch, P. \& Thurston, R. N. 1965. Elastic moduli of quartz versus hydrostatic pressure at $25{ }^{\circ} \mathrm{C}$ and $-195.8^{\circ} \mathrm{C}$. Journal of Applied Geophysics 36, 1624-1632.

Means, W. D., Williams, P. F. \& Hobbs, B. E. 1984. Incremental deformation and fabric development in KCl/mica mixture. Journal of Structural Geology 6, 391-398.

Meglis, I. L., Greenfield, R. J., Engelder, T. \& Graham, E. K. 1996. Pressure dependence of velocity and attenuation and its relationship to crack closure in crystalline rocks. 


\section{$\underline{\text { 11. Literaturverzeichnis }}$}

Journal of Geophysical Research, B, Solid Earth and Planets 101(8), 1752317533.

Meredith, P. G. \& Atkinson, B. K. 1982. Earthquake Predict. Res. 1, 377-391.

Metz, F. 1992. Zur Charakterisierung von Porenraum und ausgewählten Gebrauchseigenschaften verschiedener Natursteine. Hochschul-Verlag, Hannover.

Metzner, C. \& Grimmeisen, W. 1990. Mona: a user-friendly computer program for calculating the modal mineralogy of rocks from chemical analysis. Eur. J. Mineral. 2, 735-738.

Militzer, H. \& Stoll, R. 1973. Einige Beiträge der Geophysik zur Primärdatenerfassung im Bergbau. Neue Bergbautechnik 3, 21-25.

Mirwald, P. W. 1997. Physikalische Eigenschaften der Gesteine. In: Naturwerksteine und Umweltschutz in der Denkmalpflege. Ebner-Verlag, Ulm.

Monicard, R. P. 1980. Properties of reservoir rocks: Core analysis. Edition Technip.

Moon, V. G. 1993. Geotechnical characteristics of ignimbrite; a soft pyroclastic rock type. Engineering Geology 35(1-2), 33-48.

Müller, F. M. 2001. Gesteinskunde. Ebner Verlag, Ulm.

Murawski, H. 1992. Geologisches Wörterbuch. Ferdinand Enke Verlag, Stuttgart.

Norton, M. G. \& Atkinson, B. K. 1981. Tectonophysics 77, 283-295.

Nur, A. \& Simmons, G. 1969. The effect of saturation on velocity in low porosity rocks. Earth and Planetary Science Letters 7, 183-193.

O'Connell, R. J. \& Budianski, B. 1974. Seismic velocities in dry and saturated cracked solids. Journal of Geophysical Research 82, 5719-5735.

Olsson, W. A. 1974. Microfracturing and faulting in a limestone. Tectonophysics 24, 277-285.

Ondrasina, J., Kirchner, D. \& Siegesmund, S. 2002. Freeze-thaw cycles and their influence on marble deterioation: a long term experiment. In: Natural Stone, Weathering Phenomena, Conservation Strategies and Case Studies (edited by Siegesmund, S., Weiss, T. \& Vollbrecht, A.). Geological Society of London Special Publication 205. Geological Society of London, London, 9-18.

Passchier, C. W. \& Trouw, R. A. J. 1998. Microtectonics. Springer-Verlag, Berlin.

Peck, L., Barton, C. C. \& Gordon, R. B. 1985. Microstructure and the resistance of rock to tensile fracture. JGR. Journal of Geophysical Research. B 90(13), 11,533-11,546.

Peschel, A. 1983. Natursteine. VEB Deutscher Verlag für Grundstoffindustrie, Leipzig.

Popp, T. 1994. Der Einfluss von Gesteinsmatrix, Mikrorissgefügen und intergranularen Fluiden auf die elastischen Wellengeschwindigkeiten und die elektrische Leitfähigkeit krustenrelevanter Gesteine unter PT-Bedingungen. Dissertation, Universität Kiel, 167.

Poschlod, K. 1989. Das Wasser im Porenraum kristalliner Naturwerksteine und sein Einfluß auf die Verwitterung. Münchner Geowissenschaftliche Abhandlungen 7, 62.

Prikryl, R. 1998. The effect of rock fabric on some mechanical properties of rocks: an example of granites. Dissertation, Universität Prag, 154.

Prikryl, R. 2001. Some microstructural aspects of strength variation in rocks. International Journal of Rock Mechanics and Mining Sciences (1997) 38(5), 671-682.

Prinz, H. 1997. Abriß der Ingenieurgeologie. Ferdinand Enke Verlag, Stuttgart.

Purtak, F. 2001. Tragfähigkeit von schlankem Quadermauerwerk aus Naturstein, Technische Universität.

Ramamurthy, T. 1993. Strength and modulus responses of anisotropic rocks. In: Comprehensive rock engineering: principles, practice and projects (edited by Brown, E. T.) 1. Pergamon Press, Oxford, 313-329. 


\section{Literaturverzeichnis}

Rasolofosaon, P. N. J., Rabbel, W., Siegesmund, S. \& Vollbrecht, A. 2000. Characterization of crack distribution; fabric analysis versus ultrasonic inversion. Geophysical Journal International 141(2), 413-434.

Rathore, J. S., Fjaer, E., Holt, M. R. \& Renlie, L. 1991. Experimental versus theoretical acoustic anisotropy in controlled cracked synthetics. In: 61st Annual International Meeting, SEG, 687-690.

Reinsch, D. 1991. Natursteinkunde. Ferdinand Enke Verlag, Stuttgart.

Richter, D. \& Simmons, G. 1974. Thermal expansion behaviour of igneous rocks. International Journal of Rock Mechanics and Mining Sciences and Geomechanical Abstracts 11, 403-411.

Rohowski, H. 2001. Druckfestigkeit und Ausbruchlast neu geregelt. Naturstein 3, 88-92.

Rösler, H. J. 1991. Lehrbuch der Mineralogie. Deutscher Verlag für Grundstoffindustrie, Leipzig.

Rüdrich, J., Weiss, T. \& Siegesmund, S. 2001. Deterioation characteristics of marbles from the Marmorpalais Potsdam (Germany): a compilation. Zeitschrift der deutschen geologischen Gesellschaft 152/ 2-4, 637-663.

Rüdrich, J., Weiss, T. \& Siegesmund, S. 2002. Thermal behaviour of weathered and consolidated marbles. In: Natural Stone, Weathering Phenomena, Conservation Strategies and Case Studies (edited by Siegesmund, S., Weiss, T. \& Vollbrecht, A.). Geological Society of London Special Publication 205. Geological Society of London, London, 255-272.

Rüdrich, J. M. 2003. Gefügekontrollierte Verwitterung natürlicher und konservierter Marmore. Unpublished Dissertation thesis, Georg-August Universität.

Ruffino, G. 1998. Historical perspective. In: Thermal expansion of solids (edited by Taylor, R. E.). Cindas Data Series on Material Properties I-4. ASM International, Materials Park, 135-156.

Rummel, F. 1982. Fracture and Flow of rocks and minerals. In: Physical properties of rocks (edited by Angenheister, G.). Landolt-Börnstein-Numerical Data and Functional Relationships in Science and Technology Group V: Geophysics and Space Research. Volume1b. Springer-Verlag, Berlin, 141-238.

Rybach, L. \& Buntebarth, G. 1982. Relationships between the petrophysical properties density, seismic velocity, heat generation, and mineralogical constitution. Earth and Planetary Science Letters 57(2), 367-376.

Schild, M., Siegesmund, S., Vollbrecht, A. \& Mazurek, M. 2001. Characterization of granite matrix porosity and pore-space geometry by in situ and laboratory methods. Geophysical Journal International 146(1), 111-125.

Schön, J. 1983. Petrophysik. Akademie-Verlag, Berlin.

Schuh, H. 1987. Physikalische Eigenschaften von Sandsteinen und ihren verwitterten Oberflächen. TU München, München.

Segall, P. \& Pollard, D. D. 1980. Mechanics of discontinuous faults. Journal of Geophysical Research 85, 4337-4350.

Shea, W. T., Jr. \& Kronenberg, A. K. 1993. Strength and anisotropy of foliated rocks with varied mica contents. In: Microstructures and rheology of rocks and rock-forming minerals; a collection of papers in honor of John Christie's 60th birthday. (edited by Kirby Stephen, H., Sylvester, A. G., Tullis, J., Wenk, H. R. \& Treagus, S. H.). Journal of Structural Geology 15; 9-10. Pergamon, Oxford-New York, International, 1097-1121.

Shelton, G. L. 1981. Experimental deformation of single and polyphase crustal rocks at high temperatures and pressures, Brown University.

Sheorey, P. R. 1997. Empirical Rock Failure Criteria. A. A. Balkema Publishers, Rotterdam. 


\section{$\underline{\text { 11. Literaturverzeichnis }}$}

Siegesmund, S. 1989. Texturelle und strukturelle Eigenschaften mylonitischer Gesteine der Insubrischen Linie (Ivrea-Zone, Italien) und ihr Einfluss auf die elastischen Gesteinseigenschaften. Dissertation, Universität Kiel, 172.

Siegesmund, S. 1996. The significance of rock fabrics for the geological interpretation of geophysical anisotropies. Geotektonische Forschungen 85, 1-123.

Siegesmund, S. \& Kern, H. 1990. Velocity anisotropy and shear-wave splitting in rocks from the mylonite belt along the Insubric Line (Ivrea Zone, Italy). Earth and Planetary Science Letters 99(1-2), 29-47.

Siegesmund, S., Kern, H. \& Vollbrecht, A. 1991. The effect of oriented microcracks on seismic velocities in an ultramylonite. Tectonophysics 186(3-4), 241-251.

Siegesmund, S., Ullemeyer, K. \& Dahms, M. 1995. Control of magnetic rock fabrics by mica preferred orientation; a quantitative approach. Journal of Structural Geology 17(11), 1601-1613.

Siegesmund, S., Ullemeyer, K., Weiss, T. \& Tschegg, E. K. 2000. Physical weathering of marble caused by anisotropic thermal expansion. Int. J. Earth Sci. 89, 170-182.

Siegesmund, S. \& Vollbrecht, A. 1991. Complete seismic properties obtained from microcrack fabric and textures in an amphibolite from the Ivrea zone, Western Alps, Italy. Tectonophysics 199, 13-24.

Siegesmund, S., Vollbrecht, A. \& Pros, Z. 1993. Fabric changes and their influence on pwave velocity patterns; examples from a polyphase deformed orthogneiss. Tectonophysics 225(4), 477-492.

Siegesmund, S., Weiss, T., Vollbrecht, A. \& Ullemeyer, K. 1999. Marble as a natural building stone: rock fabrics, physical and mechanical properties. Zt. d. dt. geol. Ges. 150(2), 237-258.

Simmons, G. \& Cooper, H. W. 1978. Thermal cycling cracks in three igneous rocks. International Journal of Rock Mechanics and Mining Sciences and Geomechanical Abstracts 15, 145-148.

Skrotzki, W., Weber, K. \& Wedel, A. 1992. The geological significance of microstructures in crystalline rocks. Geotektonische Forschungen 78, 125.

Smith, V. J. \& Brown, W. L. 1988. Feldspar Minerals. Springer-Verlag, Berlin.

Smorodinov, M. I., Motovilov, E. A. \& Volkov, V. A. 1970. Determinations of correlation relationships between strength and some characteristics of rocks. In: 2nd Congress for the International Society for Rock Mechanics 2, Beograd (Yugoslavia), 3.

Smyth, J. R. \& McCormick, T. C. 1995. Crystallographic Data For Minerals. In: Mineral Physics and Crystallography-A Handbook of Physikal Constants (edited by Ahrens, T. J.) 2. American Geophysical Union, Washington, 354.

Snethlage, R. 1984. Natursteinkonservierung: Forschungsprogramm des Zentrallabors für Denkmalpflege, 1979-1983. Bayerisches Landesamt für Denkmalpflege, München.

Snethlage, R. 1985. Natursteinkonservierung: Internationales Kolloquium, München. Bayerisches Landesamt für Denkmalpflege, München.

Stavrogin, A. N. \& Tarasov, B. G. 2001. Experimental Physics and Rock Mechanics-Results of Laboratory Study. A. A. Balkema Publishers, Lissw.

Stephenson, D. E. \& Triandafilidis, G. E. 1974. Influence of Specimen Size and Geometry of Uniaxial Compressive Strength of Rock. Bulletin of the Association of Engineering Geologists 11(1), 29-47.

Streckeisen, A. 1974. Classification and nomenclature of igneous rocks. Geologische Rundschau 63, 773-786.

Streckeisen, A. 1980. Classification and nomenclature of igneous rocks. Geologische Rundschau 69, 194-207. 


\section{$\underline{\text { 11. Literaturverzeichnis }}$}

Strohmeyer, D. \& Siegesmund, S. 2002. Anisotropic technical properties of building stones and their development due to fabric changes. In: Natural Stone, Weathering Phenomena, Conservation Strategies and Case Studies (edited by Siegesmund, S., Weiss, T. \& Vollbrecht, A.). Geological Society of London Special Publication 205. Geological Society of London, London, 107-127.

Strohmeyer, D., Weiss, T., Siegesmund, S., Ondrasina, J. \& Zeisig, A. 2001. Natural building stones: Fabric induced anisotropy and its influence on mechanical properties. Acta Universitatis Carolinae Geologica 45/1, 47.

Swenson, C. A. 1998. High sensitivity measurement techniques. In: Thermal expansion of solids (edited by Taylor, R. E.). Cindas Data Series on Material Properties I-4. ASM International, Materials Park, 207-224.

Tapponnier, P. \& Brace, W. F. 1976. Development of stress-induced microcracks in Westerly Granite. International Journal of Rock Mechanics and Mining Science 13, 103112.

Tatham, R. H. 1985. Shear waves and lithology. In: Seismic shear waves Part B: applications (edited by Dohr, G. P.). Geophysical Press, London, 87-133.

Taylor, R. E., Tothrock, B. D. \& Kirby, R. K. 1998. Twin telescope methods. In: Thermal expansion of solids (edited by Taylor, R. E.). Cindas Data Series on Material Properties I-4. ASM International, Materials Park, 225-242.

Techmer, K. S. 1996. Element mobilization in a ductile shear zone from the Sesia-Lanzo zone (northern Italy): a microstructural, petrographical and chemical study. Z. geol. Wiss. 24 (5/6), 699-713.

Tertsch, H. 1949. Die Festigkeitserscheinungen der Kristalle. J. Springer Verlag, Wien.

Tröger, W. E. 1982. Optische Bestimmung der gesteinsbildenden Minerale. E. Schweizerbartsche Verlagsbuchhandlung, Stuttgart.

Tschegg, K. E., Widhalm, C. \& Eppensteiner, W. 1999. Ursachen mangelnder Formbeständigkeit von Marmorplatten. Zt. d. dt. Geol. Ges. 150(2), 283-297.

Tullis, J. 1977. Preferred orientation of quartz produced by slip during plain strain. Tectonophysics 39, 87-102.

Tullis, T. E., Horowitz, F. G. \& Tullis, J. 1991. Flow laws of polyphase aggregates from endmember flow laws. Journal of Geophysical Research, B, Solid Earth and Planets 96(5), 8081-8096.

Twiss, R. J. \& Moores, E. M. 1997. Structural Geology. W.H. Freeman and Company, New York.

Ullemeyer, K. 1992. Methodische Untersuchungen zur Röntgentexturanalyse und Gefügeuntersuchungen an Granuliten der Südlichen Böhmischen Mass. Dissertation, Universität Göttingen, 104.

Ullemeyer, K., Spalthoff, P., Heinitz, J., Isakov, N. N., Nikitin, A. N. \& Weber, K. 1998. The SKAT texture diffractometer at the pulsed reactor IBR-2 at Dubna: experimental layout and first measurements. Nuclear Instruments and Methods in Physics research $A$ 412, 80-88.

Ullemeyer, K. \& Weber, K. 1999. Latice preferred orientation as an indicator of a complex deformation history of rocks. Textures and Microstructures 33, 45-60.

Vaughan, M. T. \& Guggenheim, S. 1986. Elasticity of muscovite and its relationship to crystal structure. Journal of Geophysical Research 91, 4657-4664.

Vogel, H. 1999. Gerthsen Physik. Springer Verlag, Berlin.

Vollbrecht, A., Duerrast, H., Kraus, J. \& Weber, K. 1994. Paleostress directions deduced from microcrack fabrics in KTB core samples and granites from the surrounding area. Scientific Drilling 4(5-6), 233-241. 


\section{$\underline{\text { 11. Literaturverzeichnis }}$}

Vollbrecht, A., Rust, S. \& Weber, K. 1991. Development of microcracks in granites during cooling and uplift: examples from the Variscan basement in NE Bavaria, Germany. Journal of Structural Geology 13, 787-799.

Wanetschek, M. \& Wanetschek, H. 2000: Naturstein und Architektur. Callwey-Verlag München.

Weiss, G. 1992. Die Eis- und Salzkristallisation im Porenraum von Sandsteinen und ihre Auswirkung auf das Gefüge unter besonderer Berücksichtigung gesteinsspezifischer Parameter. TU München, München.

Weiss, T. 1998. Gefügeanisotropie und ihre Auswirkung auf das seismische Erscheinungsbild: Fallbeispiele aus der Lithosphäre Süddeutschlands. Geotektonische Forschungen 91, 156.

Weiss, T., Rasolofosaon, P. N. J. \& Siegesmund, S. 2002b. Ultrasonic wave velocities as a diagnostic tool for the quality assessment of marble. In: Natural Stone, Weathering Phenomena, Conservation Strategies and Case Studies (edited by Siegesmund, S., Weiss, T. \& Vollbrecht, A.). Geological Society of London Special Publication 205. Geological Society of London, London, 149-164.

Weiss, T., Siegesmund, S. \& Fuller, R. F. J. 2002. Thermal stresses in calcite and dolomite marbles via finite element modelling. In: Natural Stone, Weathering Phenomena, Conservation Strategies and Case Studies (edited by Siegesmund, S., Weiss, T. \& Vollbrecht, A.). Geological Society of London Special Publication 205. Geological Society of London, London, 89-102.

Wenk, E. \& Pannetier, J. 1990. Texture developed in deformed granodiorites from Santa Rosa mylonite zone, Southern California. Journal of Structural Geology 12(2), 177184.

Widhalm, C., Tschegg, E. \& Eppensteiner, W. 1996. Anisotropic thermal expansion causes deformation of marble caldding. J. Perf. Constr. Facil. ASCE 10, 5-10.

Wilkens, R. H., Fryer, G. J. \& Karsten, J. L. 1991. Evolution of porosity and seismic structure of upper oceanic crust: importance of aspect ratio. Journal of Geophysical Research 96, 17981-17995.

Winkler, E. M. 1994. Stone in Architecture: Properties and Durability. Springer-Verlag, Berlin.

Wise, D. U., Dunn, D. E., Engelder, J. T., Geisser, P. A., Hatcher, R. D., Kish, S. H., Odom, A. L. \& Schamel, S. 1984. Fault-related rocks: suggestions for terminology. Geology 12, 391-394.

Wittke, W. 1984. Felsmechanik. Springer Verlag, Berlin.

Wong, T. f. \& Biegel, R. 1985. Effects of pressure on the micromechanics of faulting in San Marcos gabbro. Journal of Structural Geology 7, 737-749.

Woodcock, N. H. 1977. Specification of fabric shapes using an eigenvalue method. Geol. Soc. America Bull. 88, 1231-1236.

Young, R. A. e. 1995. The rietveld method. Oxford University Press, Oxford.

Zeisig, A., Siegesmund, S. \& Weiss, T. 2002. Thermal expansion and its control on the durability of marbles. In: Natural Stone, Weathering Phenomena, Conservation Strategies and Case Studies (edited by Siegesmund, S., Weiss, T. \& Vollbrecht, A.). Geological Society of London Special Publication 205. Geological Society of London, London, 65-80.

Zimmer, U. 2001. Quantitative Untersuchungen zur Mikrorissigkeit aus akustischen Gesteinseigenschaften am Beispiel Steinsalz und Anhydrit. Dissertation, Universität Berlin, 189. 


\section{Literaturverzeichnis}

Zucali, M., Chateigner, M., Dugnani, M., Lutterotti, L. \& Ouladdiaf, B. 2002. Quantitative texture analysis of glaucophanite deformed under eclogite facies conditions (Sesia-Lanzo Zone, Western Alps): comparison between x-ray and neutron diffraction analysis. In: Deformation Mechanisms, Rheology and Tectonics: Current Status and Future Perspectives (edited by De Meer, S., Drury, M. R., De Bresser, J. H. P. \& Pennock, G. M.). Geological Society of London Special Publication 200. Geological Society of London, London, 239-253.

\section{Normen}

DIN 52108, 1988. Prüfung anorganischer nichtmetallischer Werkstoffe; Verschleißprüfung mit der Schleifscheibe nach Boehme. Beuth Verlag, Berlin.

DIN EN 1926, 1999. Prüfverfahren für Naturstein: Bestimmung der Druckfestigkeit. Beuth Verlag, Berlin.

DIN 22024, 1989. Rohstoffuntersuchungen im Steinkohlenbergbau: Bestimmung der Spaltzugfestigkeit von Festgesteinen. Beuth Verlag, Berlin.

prDIN EN 1925, 1995. Bestimmung des Wasseraufnahmekoeffizienten infolge Kapillarwirkung. Beuth Verlag, Berlin.

DIN EN 12372, 1999. Bestimmung der Biegefestigkeit unter Mittellinienlast. Beuth Verlag, Berlin.

DIN 18516-3, 1999. Außenwandbekleidungen, hinterlüftet. Teil 3: Naturwerkstein Anforderungen, Bemessung. Beuth Verlag, Berlin.

DIN EN 1341, 2000. Platten aus Naturstein für Außenbereiche, Anforderungen und Prüfverfahren. Beuth Verlag, Berlin.

DIN EN 1342, 2000. Pflastersteine aus Naturstein für Außenbereiche, Anforderungen und Prüfverfahren. Beuth Verlag, Berlin.

DIN EN 1936, 1999. Prüfung von Naturstein - Bestimmung der Reindichte, der Rohdichte, der offenen Porosität und der Gesamtporosität. Beuth Verlag, Berlin.

DIN EN 771-6, 2001. Festlegungen für Mauersteine - Teil 6: Natursteine. Beuth Verlag, Berlin.

prEN 14146, 2001. Natural stone test methods - Determination of the dynamic modulus of elasticity (by measuring the fundamental resonance frequency). CEN, Brüssel.

prEN 14580, 2002. Natural stone test methods - Determination of static elastic modulus. CEN, Brüssel.

\section{Internetressourcen}

www.uni-marburg.de/geowissenschaften/mo-desc.htm

www.webmineral.com 
12. Anhang

12. Anhang 


\section{Anhang l.1: Chemische Zusammensetzung und mittlere Atommasse}

\begin{tabular}{|c|c|c|c|c|c|c|c|c|c|c|c|c|c|c|c|}
\hline & Probe & $\begin{array}{c}\mathrm{SiO}_{2} \\
(\mathrm{Gew} . \\
\%)\end{array}$ & $\begin{array}{c}\mathrm{TiO}_{2} \\
\text { (Gew. } \\
\%)\end{array}$ & $\begin{array}{c}\mathrm{Al}_{2} \mathrm{O}_{3} \\
(\mathrm{Gew} . \\
\%)\end{array}$ & $\begin{array}{c}\mathrm{Fe}_{2} \mathrm{O}_{3} \\
(\mathrm{Gew} . \\
\%)\end{array}$ & $\begin{array}{c}\mathrm{FeO} \\
(\mathrm{Gew} . \\
\%)\end{array}$ & $\begin{array}{c}\text { MnO } \\
\text { (Gew. } \\
\%)\end{array}$ & $\begin{array}{c}\text { MgO } \\
\text { (Gew. } \\
\%)\end{array}$ & $\begin{array}{c}\mathrm{CaO} \\
\text { (Gew. } \\
\%)\end{array}$ & $\begin{array}{c}\mathrm{Na}_{2} \mathrm{O} \\
(\mathrm{Gew} . \\
\%)\end{array}$ & $\begin{array}{c}\mathrm{K}_{2} \mathrm{O} \\
(\mathrm{Gew} . \\
\%)\end{array}$ & $\begin{array}{c}\mathrm{P}_{2} \mathrm{O}_{5} \\
(\mathrm{Gew} . \\
\%)\end{array}$ & $\begin{array}{c}\mathrm{CO}_{2} \\
(\mathrm{Gew} . \\
\%)\end{array}$ & $\begin{array}{c}\text { Summe } \\
\text { (Gew. } \\
\%)\end{array}$ & $\begin{array}{c}\text { M } \\
*\end{array}$ \\
\hline \multirow{21}{*}{ 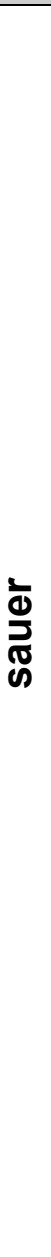 } & Quarzit (Al) & 91,4 & 0,054 & 6,1 & 0,37 & n.b. & 0,12 & 0,05 & 0,01 & 0,03 & 1,14 & 0,12 & & 99,29 & 20,31 \\
\hline & granitischer Gneis (Be2) & 73,2 & 0,262 & 13,7 & 0,29 & 1,57 & 0,05 & 0,51 & 1,17 & 3,20 & 4,51 & 0,15 & & 98,60 & 20,81 \\
\hline & Granit (MrGr3) & 73,1 & 0,209 & 14,1 & 0,30 & 1,28 & 0,03 & 0,3 & 0,76 & 3,05 & 5,21 & 0,22 & & 98,55 & 20,86 \\
\hline & $\begin{array}{c}\text { granitischer Protomylonit } \\
\text { (MrMy5) }\end{array}$ & 72,7 & 0,191 & 14,3 & 0,19 & 1,40 & 0,05 & 0,32 & 0,89 & 3,10 & 5,05 & 0,23 & & 98,42 & 20,87 \\
\hline & $\begin{array}{l}\text { granitischer Orthogneis } \\
\text { (VA)-1 }\end{array}$ & 72,4 & 0,33 & 13,4 & 0,74 & 1,41 & 0,04 & 0,61 & 1,03 & 2,82 & 5,30 & 0,15 & & 98,24 & 21,02 \\
\hline & $\begin{array}{l}\text { granitischer Orthogneis } \\
\text { (VA)-2 }\end{array}$ & 72,8 & 0,33 & 13,7 & 0,76 & 1,41 & 0,04 & 0,62 & 1,04 & 2,82 & 5,34 & 0,15 & & 99,01 & 20,86 \\
\hline & VA Mittelwert & 72,6 & 0,33 & 13,55 & 0,75 & 1,41 & 0,04 & 0,62 & 1,04 & 2,82 & 5,32 & 0,15 & & & 20,94 \\
\hline & VA Standardabw.(1s) & 0,3 & 0,00 & 0,21 & 0,01 & 0,00 & 0,00 & 0,01 & 0,01 & 0,00 & 0,03 & 0,00 & & & 0,11 \\
\hline & VA relativer Fehler (\%) & 0,39 & 0,21 & 1,57 & 1,88 & 0,00 & 0,00 & 1,15 & 0,68 & 0,00 & 0,53 & 1,40 & & & 0,55 \\
\hline & $\begin{array}{c}\text { granitischer Gneis } \\
\text { (Gra1a)-1 }\end{array}$ & 72,7 & 0,36 & 14,2 & 0,09 & 2,56 & 0,05 & 0,84 & 1,64 & 3,10 & 4,50 & 0,17 & & 100,21 & 20,45 \\
\hline & $\begin{array}{c}\text { granitischer Gneis } \\
\text { (Gra1a)-2 }\end{array}$ & 72,7 & 0,36 & 14,2 & 0,02 & 2,56 & 0,04 & 0,85 & 1,64 & 3,08 & 4,46 & 0,17 & & 100,08 & 20,46 \\
\hline & Gra1a Mittelwert & 72,7 & 0,36 & 14,2 & 0,06 & 2,56 & 0,05 & 0,85 & 1,64 & 3,09 & 4,48 & 0,17 & & & 20,45 \\
\hline & Gra1a Standardabw.(1s) & 0,0 & 0,00 & 0,0 & 0,05 & 0,00 & 0,01 & 0,01 & 0,00 & 0,01 & 0,03 & 0,00 & & & 0,01 \\
\hline & Gra1a relativer Fehler (\%) & 0,0 & 0,00 & 0,0 & 85,07 & 0,00 & 14,0 & 0,84 & 0,00 & 0,46 & 0,63 & 0,42 & & & 0,04 \\
\hline & granitischer Gneis (Gra1c) & 73,3 & 0,28 & 13,8 & 0,01 & 2,30 & 0,04 & 0,63 & 1,65 & 3,30 & 4,03 & 0,07 & & 99,48 & 20,55 \\
\hline & $\begin{array}{l}\text { granitischer Ultramylonit } \\
\text { (Gra3a)-1 }\end{array}$ & 71,1 & 0,47 & 14,8 & 0,33 & 2,52 & 0,04 & 0,83 & 1,57 & 2,90 & 5,03 & 0,22 & & 99,81 & 20,61 \\
\hline & $\begin{array}{l}\text { granitischer Ultramylonit } \\
\text { (Gra3a)-2 }\end{array}$ & 71,3 & 0,47 & 14,8 & 0,33 & 2,52 & 0,05 & 0,83 & 1,57 & 2,90 & 5,05 & 0,21 & & 100,03 & 20,57 \\
\hline & Gra3a Mittelwert & 71,2 & 0,47 & 14,8 & 0,33 & 2,52 & 0,04 & 0,83 & 1,57 & 2,90 & 5,04 & 0,22 & & & 20,59 \\
\hline & Gra3a Standardabw.(1s) & 0,14 & 0,00 & 0,0 & 0,00 & 0,00 & 0,00 & 0,00 & 0,00 & 0,00 & 0,01 & 0,00 & & & 0,03 \\
\hline & Gra3a relativer Fehler (\%) & 0,20 & 0,00 & 0,0 & 0,00 & 0,00 & 4,77 & 0,00 & 0,00 & 0,00 & 0,28 & 1,63 & & & 0,15 \\
\hline & Granit (Koess) & 70,7 & 0,54 & 14,3 & 0,41 & 2,95 & 0,04 & 0,62 & 1,39 & 2,60 & 5,23 & 0,28 & & 99,06 & 20,77 \\
\hline
\end{tabular}




\begin{tabular}{|c|c|c|c|c|c|c|c|c|c|c|c|c|c|c|c|}
\hline & Probe & $\begin{array}{c}\mathrm{SiO}_{2} \\
(\mathrm{Gew} \\
\%)\end{array}$ & $\begin{array}{c}\mathrm{TiO}_{2} \\
\text { (Gew. } \\
\%)\end{array}$ & $\begin{array}{c}\mathrm{Al}_{2} \mathrm{O}_{3} \\
(\mathrm{Gew} . \\
\%)\end{array}$ & $\begin{array}{c}\mathrm{Fe}_{2} \mathrm{O}_{3} \\
(\mathrm{Gew} \\
\%)\end{array}$ & $\begin{array}{c}\text { FeO } \\
\text { (Gew. } \\
\% \text { ) }\end{array}$ & $\begin{array}{c}\text { MnO } \\
\text { (Gew. } \\
\% \text { ) }\end{array}$ & $\begin{array}{c}\text { MgO } \\
\text { (Gew. } \\
\% \text { ) }\end{array}$ & $\begin{array}{c}\text { CaO } \\
\text { (Gew. } \\
\% \text { ) }\end{array}$ & $\begin{array}{c}\mathrm{Na}_{2} \mathrm{O} \\
(\mathrm{Gew} . \\
\%)\end{array}$ & $\begin{array}{c}\mathrm{K}_{2} \mathrm{O} \\
(\mathrm{Gew} . \\
\%)\end{array}$ & $\begin{array}{c}\mathrm{P}_{2} \mathrm{O}_{5} \\
\text { (Gew. } \\
\%)\end{array}$ & $\begin{array}{c}\mathrm{CO}_{2} \\
(\mathrm{Gew} . \\
\%)\end{array}$ & $\begin{array}{c}\text { Summe } \\
\text { (Gew. } \\
\%)\end{array}$ & $\underset{*}{M}$ \\
\hline & $\begin{array}{l}\text { granodioritischer } \\
\text { Protomylonit (Iv1) }\end{array}$ & 66,7 & 0,51 & 15,6 & 4,14 & n.b. & 0,08 & 1,19 & 3,39 & 3,56 & 3,14 & 0,13 & & 98,44 & 21,25 \\
\hline & $\begin{array}{c}\text { granodioritischer Mylonit } \\
\text { (Iv1b) }\end{array}$ & 69,4 & 0,38 & 14,6 & 3,03 & n.b. & 0,06 & 0,79 & 2,8 & 3,07 & 3,71 & 0,09 & & 97,93 & 21,26 \\
\hline & $\begin{array}{l}\text { granodioritischer } \\
\text { Ultramylonit (Iv2)-1 }\end{array}$ & 64,4 & 0,60 & 16,2 & 4,72 & n.b. & 0,09 & 1,57 & 3,77 & 3,49 & 2,96 & 0,15 & & 97,94 & 21,43 \\
\hline & $\begin{array}{l}\text { granodioritischer } \\
\text { Ultramylonit (Iv2)-2 }\end{array}$ & 65,4 & 0,57 & 15,9 & 4,56 & n.b. & 0,08 & 1,37 & 3,86 & 3,51 & 2,74 & 0,14 & & 98,14 & 21,36 \\
\hline & Iv2 Mittelwert & 64,9 & 0,58 & 16,1 & 4,64 & n.b. & 0,08 & 1,47 & 3,82 & 3,50 & 2,85 & 0,15 & & & 21,39 \\
\hline & Iv2 Standardabw.(1s) & 0,70 & 0,02 & 0,21 & 0,11 & n.b. & 0,00 & 0,14 & 0,06 & 0,01 & 0,16 & 0,01 & & & 0,05 \\
\hline & Iv2 relativer Fehler (\%) & 1,1 & 3,40 & 1,32 & 2,44 & n.b. & 0,80 & 9,62 & 1,67 & 0,40 & 5,46 & 3,82 & & & 0,24 \\
\hline & $\begin{array}{c}\text { granodioritischer Gneis } \\
\text { (Be1) }\end{array}$ & 66,5 & 0,53 & 15,7 & 0,93 & 2,49 & 0,06 & 1,25 & 3,38 & 3,73 & 3,28 & 0,19 & & 98,04 & 21,08 \\
\hline & $\begin{array}{l}\text { granodioritischer } \\
\text { Paragneis (Cal)-1 }\end{array}$ & 66,5 & 0,67 & 15,7 & 0,69 & 3,95 & 0,07 & 1,68 & 3,70 & 2,95 & 3,28 & 0,21 & & 99,39 & 20,72 \\
\hline & $\begin{array}{l}\text { granodioritischer } \\
\text { Paragneis (Cal)-2 }\end{array}$ & 66,5 & 0,66 & 15,6 & 0,66 & 3,95 & 0,07 & 1,65 & 3,69 & 2,95 & 3,27 & 0,21 & & 99,20 & 20,76 \\
\hline & Cal Mittelwert & 66,5 & 0,66 & 15,7 & 0,67 & 3,95 & 0,07 & 1,67 & 3,70 & 2,95 & 3,28 & 0,21 & & & 20,74 \\
\hline & Cal Standardabw.(1s) & 0,0 & 0,01 & 0,1 & 0,02 & 0,00 & 0,00 & 0,02 & 0,01 & 0,00 & 0,01 & 0,00 & & & 0,02 \\
\hline & Cal relativer Fehler (\%) & 0,0 & 0,85 & 0,45 & 3,16 & 0,00 & 2,92 & 1,27 & 0,19 & 0,00 & 0,22 & 0,34 & & & 0,11 \\
\hline \multirow{2}{*}{ 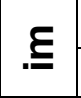 } & Granodiorit (PDD) & 60,0 & 0,80 & 16,5 & 2,07 & 4,59 & 0,12 & 3,51 & 6,28 & 3,04 & 2,71 & 0,19 & & 99,81 & 20,95 \\
\hline & Gabbro-Norit (NI) & 53,2 & 0,14 & 19,4 & 0,70 & 5,64 & 0,15 & 6,43 & 11,86 & 2,75 & 0,22 & 0,01 & & 100,50 & 20,69 \\
\hline \multirow{6}{*}{$\begin{array}{l}\frac{c}{0} \\
\frac{0}{0} \\
\frac{0}{8} \\
0\end{array}$} & Meta-Gabbro (MG1b) & 48,1 & 0,94 & 16,6 & 10,18 & n.b. & 0,17 & 9,09 & 12,51 & 2,35 & 0,15 & 0,07 & & 100,17 & 21,77 \\
\hline & Meta-Gabbro (MG2)-1 & 44,9 & 1,39 & 17,5 & 13,76 & n.b. & 0,22 & 6,73 & 12,16 & 2,90 & 0,71 & 0,11 & & 100,34 & 22,10 \\
\hline & Meta-Gabbro (MG2)-2 & 44,8 & 1,39 & 17,5 & 13,76 & n.b. & 0,22 & 6,76 & 12,17 & 2,90 & 0,70 & 0,11 & & 100,3 & 22,12 \\
\hline & MG2 Mittelwert & 44,9 & 1,39 & 17,5 & 13,76 & n.b. & 0,22 & 6,75 & 12,17 & 2,90 & 0,71 & 0,11 & & & 22,11 \\
\hline & MG2 Standardabw.(1s) & 0,01 & 0,00 & 0,0 & 0,00 & n.b. & 0,00 & 0,02 & 0,01 & 0,00 & 0,01 & 0,00 & & & 0,01 \\
\hline & MG2 relativer Fehler (\%) & 0,2 & 0,36 & 0,0 & 0,00 & n.b. & 0,3 & 0,31 & 0,06 & 0,00 & 1,00 & 0,66 & & & 0,06 \\
\hline \multirow{2}{*}{ 음 } & Peridotit (Pi1a) & 44,8 & 0,09 & 2,9 & 0,98 & 7,45 & 0,14 & 40,49 & 2,69 & 0,02 & 0,01 & 0,01 & & 99,57 & 20,28 \\
\hline & Marmor (Rek) & 0,4 & 0,02 & 0,1 & 0,12 & n.b. & 0,00 & 0,65 & 55,26 & 0,04 & 0,00 & 0,01 & $43,4^{* *}$ & 98,24 & 20,03 \\
\hline-0 & Tonschiefer (Std.)-1 & 64,0 & 0,67 & 14,7 & 5,22 & n.b. & 0,075 & 2,49 & 1,86 & 4,00 & 2,24 & 0,15 & & 95,40 & n.b. \\
\hline
\end{tabular}




\begin{tabular}{|c|c|c|c|c|c|c|c|c|c|c|c|c|c|c|}
\hline Probe & $\begin{array}{c}\mathrm{SiO}_{2} \\
(\mathrm{Gew} . \\
\%)\end{array}$ & $\begin{array}{c}\mathrm{TiO}_{2} \\
\text { (Gew. } \\
\%)\end{array}$ & $\begin{array}{c}\mathrm{Al}_{2} \mathrm{O}_{3} \\
\text { (Gew. } \\
\%)\end{array}$ & $\begin{array}{c}\mathrm{Fe}_{2} \mathrm{O}_{3} \\
(\mathrm{Gew} . \\
\%)\end{array}$ & $\begin{array}{c}\text { FeO } \\
\text { (Gew. } \\
\%)\end{array}$ & $\begin{array}{c}\text { MnO } \\
\text { (Gew. } \\
\%)\end{array}$ & $\begin{array}{c}\text { MgO } \\
\text { (Gew. } \\
\% \text { ) }\end{array}$ & $\begin{array}{c}\text { CaO } \\
\text { (Gew. } \\
\% \text { ) }\end{array}$ & $\begin{array}{c}\mathrm{Na}_{2} \mathrm{O} \\
(\mathrm{Gew} . \\
\%)\end{array}$ & $\begin{array}{c}\mathrm{K}_{2} \mathrm{O} \\
(\mathrm{Gew} . \\
\%)\end{array}$ & $\begin{array}{c}\mathrm{P}_{2} \mathrm{O}_{5} \\
(\mathrm{Gew} \\
\%)\end{array}$ & $\begin{array}{c}\mathrm{CO}_{2} \\
(\mathrm{Gew} . \\
\%)\end{array}$ & $\begin{array}{c}\text { Summe } \\
\text { (Gew. } \\
\%)\end{array}$ & $\underset{*}{\mathbf{M}}$ \\
\hline Tonschiefer (Std.)-2 & 64,1 & 0,67 & 14,7 & 5,24 & n.b. & 0,074 & 2,48 & 1,88 & 4,00 & 2,24 & 0,15 & & 95,53 & n.b. \\
\hline Tonschiefer (Std.)-3 & 64,6 & 0,67 & 14,8 & 5,24 & n.b. & 0,076 & 2,49 & 1,85 & 4,00 & 2,26 & 0,15 & & 96,13 & n.b. \\
\hline Tonschiefer (Std.)-4 & 64,4 & 0,67 & 14,8 & 5,23 & n.b. & 0,075 & 2,49 & 1,84 & 4,00 & 2,25 & 0,15 & & 95,91 & n.b. \\
\hline Std. Mittelwert & 64,28 & 0,067 & 14,8 & 5,23 & n.b. & 0,075 & 2,49 & 1,86 & 4,00 & 2,25 & 0,15 & & & \\
\hline Std. Standardabw.(1s) & 0,28 & 0,00 & 0,06 & 0,01 & n.b. & 0,00 & 0,01 & 0,02 & 0,00 & 0,01 & 0,00 & & & \\
\hline Std. relativer Fehler (\%) & 0,43 & 0,44 & 0,39 & 0,18 & n.b. & 1,09 & 0,2 & 0,92 & 0,00 & 0,43 & 1,28 & & & \\
\hline
\end{tabular}

Daten sind nach dem $\mathrm{SiO}_{2}$-Gehalt sortiert, Probenserien (Iv1-Iv2;Gra1a-Gra3a;MG1b-MG2) wurden zusammengelassen und nach dem mittleren SiO ${ }_{2}-$ Gehalt eingeordnet, Standardmessungen sind ausgenommen; im - intermediär; ub - ultrabasisch;Std. - Standard * mittlere Atommasse , die chemischen Analysen

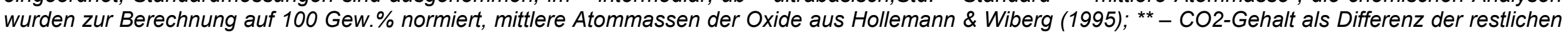
Elementoxide zu 100\%; Standardabweichung (1s) ausgehend von einer Stichprobe. 


\section{Anhang l.2: Mineralogische Zusammensetzung und Kationenpackungsindex}

\begin{tabular}{|c|c|c|c|c|c|c|c|c|c|}
\hline & Probe & $\begin{array}{c}\text { Qz } \\
\text { (Vol.\%) }\end{array}$ & $\begin{array}{c}\text { Kfs } \\
\text { (Vol.\%) }\end{array}$ & $\begin{array}{c}\mathrm{PI} \\
\text { (Vol.\%) }\end{array}$ & $\begin{array}{c}\text { Ms } \\
\text { (Vol.\%) }\end{array}$ & $\begin{array}{c}\mathrm{Bt} \\
\text { (Vol.\%) }\end{array}$ & $\begin{array}{c}\text { Chl } \\
\text { (Vol.\%) }\end{array}$ & $\begin{array}{c}\text { Dum } \\
\text { (Vol.\%) }\end{array}$ & $\begin{array}{c}\mathbf{k}_{\mathrm{G}} \\
\left(\mathrm{mol} / \mathrm{cm}^{3}\right)^{-2}\end{array}$ \\
\hline \multirow{16}{*}{ 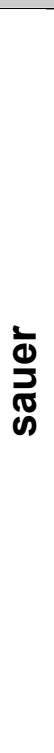 } & Quarzit (Al) * & 87 & & & 9 & & & 4 & 4,50 \\
\hline & granitischer Gneis (Be2) * & 38 & 19 & 28 & 7 & 7 & 1 & & 4,70 \\
\hline & Granit (MrGr3) ** & $33+-1$ & $25+-2$ & $24+-1$ & $15+-1$ & $3+-2$ & & & 4,70 \\
\hline & granitischer Protomylonit (MrMy5) * & 36 & 22 & 25 & 10 & 6 & 1 & & 4,69 \\
\hline & granitischer Orthogneis (VA) ** & $34+-1$ & $19+-1$ & $21+-1$ & $22+-2$ & & $4+-1$ & & 4,64 \\
\hline & Probe & Qz & Kfs & PI & Ms & Bt & Chl & Gt & \\
\hline & granitischer Gneis (Gra1a) ** & $33+-1$ & $32+-1$ & $28+-1$ & & $4+-1$ & & $2+-0,5$ & 4,67 \\
\hline & granitischer Gneis (Gra1c) ** & $34+-1$ & $28+-1$ & $30+-1$ & & $5+-1$ & & $3+-0,5$ & 4,75 \\
\hline & granitischer Ultramylonit (Gra3a) ** & $30+-1$ & $34+-1$ & $26+-1$ & & $7+-1$ & & $3+-0,5$ & 4,76 \\
\hline & Granit (Koess) ** & $33+-1$ & $26+-2$ & $30+-1$ & & $10+-1$ & $1+-0,5$ & & 4,71 \\
\hline & Probe & Qz & Kfs & PI & Ms & Chl & Gt & Ep & \\
\hline & $\underset{* * *}{\text { granodioritischer Protomylonit (Iv1) }}$ & 36 & 5 & 28 & 15 & 6 & 4 & 6 & 4,79 \\
\hline & granodioritischer Mylonit (Iv1b) *** & 36 & 5 & 28 & 15 & 6 & 4 & 6 & 4,79 \\
\hline & $\underset{* * *}{\text { granodioritischer Ultramylonit (Iv2) }}$ & 36 & 5 & 28 & 15 & 6 & 4 & 6 & 4,79 \\
\hline & granodioritischer Gneis (Be1) * & 32 & 11 & 38 & 4 & $13(\mathrm{Bt})$ & 1 (Chl) & & 4,73 \\
\hline & granodioritischer Paragneis (Cal) * & 34 & 9 & 38 & $18(\mathrm{Bt})$ & 1 (Chl) & & & 4,80 \\
\hline \multirow{3}{*}{$\underline{E}$} & Probe & Qz & Kfs & PI & Bt & Cpx & Opx & Mag & \\
\hline & Granodiorit (PDD) ** & $19+-1$ & $10+-2$ & $51+-1$ & $11+-2$ & $7+-1$ & & $2+-0,5$ & 4,98 \\
\hline & Gabbro-Norit (NI) ** & $1+-0,5$ & & $74+-1$ & & $13+-1$ & $12+-1$ & & 5,28 \\
\hline \multirow{3}{*}{ 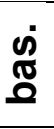 } & Probe & $\mathbf{P I}$ & Cc & $\mathrm{Hbl}$ & Cpx & Opx & Ol & Spi & \\
\hline & Meta-Gabbro (MG1b) **** & 35 & & 44 & 16 & 5 & & & 5,59 \\
\hline & Meta-Gabbro (MG2) **** & 35 & & 44 & 16 & 5 & & & 5,59 \\
\hline \multirow{3}{*}{ مْ } & Peridotit (Pi1a) ** & & & & $13+-1$ & $26+-1$ & $59+-1$ & $2+-0,5$ & 6,61 \\
\hline & Probe & Cc & Häm & & & & & & \\
\hline & Marmor (Rek) * & 98 & 2 & & & & & & 5,44 \\
\hline
\end{tabular}

Daten sind nach dem $\mathrm{SiO}_{2}$-Gehalt sortiert, Probenserien (Iv1-Iv2;Gra1a-Gra3a;MG1b-MG2) wurden zusammengelassen und nach dem mittleren SiO 2 -Gehalt eingeordnet; alle Fehler sind absolute Fehler. für Modalbestandswerte ohne Angabe eines Fehler gilt F=+-2\% absolut* - Modalbestand berechnet mit Mona-vgl.

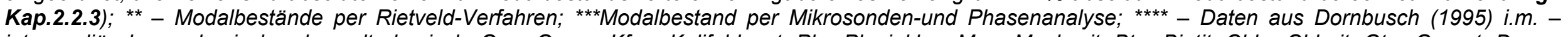
intermediär; bas. - basisch; u.b. - ultrabasisch; Qz - Quarz; Kfs - Kalifeldspat; Pl - Plagioklas; Ms - Muskovit; Bt - Biotit; Chl - Chlorit; Gt - Granat; Dum -

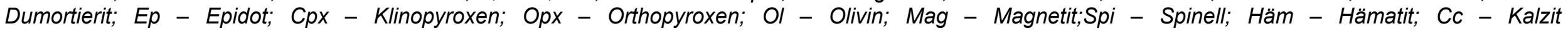


Anhang I.3: Texturen und Mikrorissquantifizierung

\begin{tabular}{|c|c|c|c|c|c|c|c|c|c|}
\hline Proben Gruppe I & Mineral & $\begin{array}{c}\text { max. } \\
\text { Intensität } \\
{[\mathrm{mrd}]}\end{array}$ & $k_{w}$ & $c_{w}$ & $\mathbf{T}$ & $\begin{array}{l}\text { Vp } p_{Q R i s s} \\
{[\mathrm{~km} / \mathrm{s}]}\end{array}$ & $\begin{array}{c}A_{\Delta V p} \\
{[\%]}\end{array}$ & $\begin{array}{c}\Delta \mathbf{V p}_{\mathrm{m}} \\
{[\mathrm{km} / \mathrm{s}]}\end{array}$ & $\begin{array}{c}\mathrm{Vp}_{\mathrm{QRiss}}- \\
\Delta \mathrm{p}_{\mathrm{m}} \\
{[\mathrm{km} / \mathrm{s}]}\end{array}$ \\
\hline Granit (Koess) & Biotit (001-Pole) & 2,89 & 0,56 & 0,38 & $-0,28$ & $0,28+-0,18$ & 87 & 0,12 & 0,16 \\
\hline Granodiorit (PDD) & Biotit (001-Pole) & 3,11 & 0,32 & 0,53 & $-0,51$ & $0,86+-0,23$ & 30 & 0,66 & 0,20 \\
\hline Gabbro-Norit (NI) & --- & n.b. & n.b. & n.b. & n.b. & $1,17+-0,17$ & 30 & 0,89 & 0,28 \\
\hline Granit (MrGr3) & Biotit/Muskovit(001-Pole) & 4,10 & 12,21 & 0,77 & 0,85 & $2,92+-0,20$ & 42 & 1,75 & 1,17 \\
\hline \multicolumn{10}{|l|}{ Proben Gruppe II } \\
\hline Peridotit (Pi1a) & Olivin (100)-Pole & 5,33 & n.b. & n.b. & n.b. & $0,36+-0,18$ & 250 & 0,01 & 0,35 \\
\hline Marmor (Rek) & Kalzit (c-Achse) & 2,88 & n.b. & n.b. & n.b. & $0,48+-0,22$ & 53 & 0,37 & 0,11 \\
\hline Quarzit (Al) & Quarz (c-Achse) & 1,38 & n.b. & n.b. & n.b. & $0,55+-0,24$ & 100 & 0,19 & 0,36 \\
\hline Meta-Gabbro (MG1b) & Hornblende (001-Pole) & 7,79 & n.b. & n.b. & n.b. & $1,68+-0,31$ & 21 & 1,43 & 0,25 \\
\hline Meta-Gabbro (MG2) & Hornblende (001-Pole) & 7,79 & n.b. & n.b. & n.b. & $0,56+-0,31$ & 60 & 0,33 & 0,23 \\
\hline granodioritischer Protomylonit (Iv1) & Muskovit (001-Pole) & 5,54 & 0,06 & 1,24 & $-0,89$ & $1,69+-0,36$ & 59 & 0,91 & 0,78 \\
\hline granitischer Gneis (Gra1a) & Biotit (001-Pole) & 5,71 & 8,62 & 1,26 & 0,79 & $0,77+-0,16$ & 44 & 0,36 & 0,41 \\
\hline \multicolumn{10}{|l|}{ Proben Gruppe III } \\
\hline granitischer Protomylonit (MrMy5) & Biotit/Muskovit (001-Pole) & 11,07 & 10,33 & 2,44 & 0,82 & $2,58+-0,31$ & 41 & 1,57 & 1,01 \\
\hline granodioritischer Gneis (Be1) & Biotit (001-Pole) & 15,48 & 4,79 & 2,75 & 0,65 & $2,61+-0,31$ & 44 & 1,80 & 0,81 \\
\hline granodioritischer Mylonit (Iv1b) & Muskovit (001-Pole) & 12,25 & 1,01 & 2,91 & 0,00 & $1,84+-0,36$ & 50 & 1,13 & 0,71 \\
\hline granodioritischer Paragneis (Cal) & Biotit (001-Pole) & 16,05 & 1,65 & 3,04 & 0,25 & $2,51+-0,29$ & 58 & 1,61 & 0,90 \\
\hline granitischer Orthogneis (VA) & Muskovit (001-Pole) & 15,89 & 8,65 & 2,44 & 0,79 & $2,54+-0,20$ & 49 & 1,53 & 1,01 \\
\hline granitischer Gneis (Gra1c) & Biotit (001-Pole) & 8,61 & 8,62 & 1,26 & 0,79 & $0,93+-0,16$ & 51 & 0,50 & 0,43 \\
\hline granitischer Gneis (Be2) & Biotit/Muskovit (001-Pole) & 16,12 & 0,43 & 3,67 & $-0,40$ & $3,27+-0,31$ & 52 & 1,90 & 1,37 \\
\hline granodioritischer Ultramylonit (Iv2) & Muskovit (001-Pole) & 17,56 & 1,62 & 2,80 & 0,24 & $2,05+-0,36$ & 61 & 1,25 & 0,80 \\
\hline granitischer Ultramylonit (Gra3a) & Biotit (001-Pole) & 23,30 & 1,92 & 3,73 & 0,31 & $0,41+-0,16$ & 100 & 0,07 & 0,34 \\
\hline
\end{tabular}

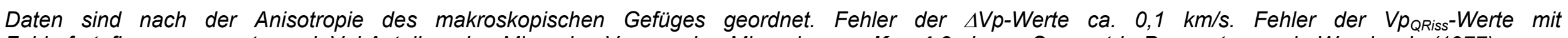

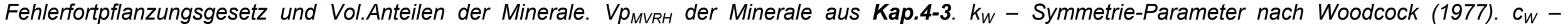

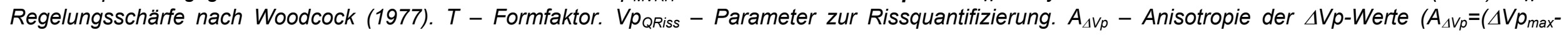
$\left.\left.\Delta V p_{\text {min }}\right) / \Delta V p_{\text {max }}{ }^{*} 100\right)$. $\Delta V p_{m}-$ Mittlere $V p$ aus dem Differenzisolinien-Plot der Ultraschall-Kugelmessung. 
Anhang I.4: Dichte, Porosität, kapillare Wasseraufnahme

\begin{tabular}{|c|c|c|c|c|c|c|}
\hline & $\begin{array}{c}\rho_{\mathrm{M}} \\
{\left[\mathrm{g} / \mathrm{cm}^{3}\right]^{*}}\end{array}$ & $\begin{array}{c}\rho_{\text {roh }} \\
{\left[\mathrm{g} / \mathrm{cm}^{3}\right]^{* *}}\end{array}$ & $\Phi[\underset{* * *}{*} \%]$ & $\begin{array}{c}\mathbf{r}_{\mathrm{m}} \\
{[\mu \mathrm{m}]^{* * * *}}\end{array}$ & $\begin{array}{c}\Phi_{\text {rkap }} \\
{\left[\text { [Vl. \% }_{* * * *}^{*}\right.}\end{array}$ & $\underset{\left[\mathrm{kg} / \mathrm{m}^{2 *} \sqrt{\mathrm{t}}\right]}{\mathrm{w}}$ \\
\hline \multicolumn{7}{|l|}{ Proben Gruppe I } \\
\hline Granit (Koess) x-Richtung & & & & & & 0,003 \\
\hline y-Richtung & & & & & & n.b. \\
\hline z-Richtung & & & & & & n.b. \\
\hline Mittelwert & 2,68 & 2,67 & 0,11 & 0,02 & 0,02 & 0,003 \\
\hline Anisotropie [\%] & & & & & & n.b. \\
\hline Granodiorit (PDD) x-Richtung & & & & & & 0,008 \\
\hline$y$-Richtung & & & & & & n.b. \\
\hline z-Richtung & & & & & & n.b. \\
\hline Mittelwert & 2,80 & 2,80 & 0,25 & 0,03 & 0 & 0,008 \\
\hline Anisotropie [\%] & & & & & & n.b. \\
\hline Gabbro-Norit (NI) x-Richtung & & & & & & $<0,06$ \\
\hline y-Richtung & & & & & & $<0,06$ \\
\hline z-Richtung & & & & & & $<0,06$ \\
\hline Mittelwert & 2,92 & 2,92 & 0,04 & n.b. & n.b. & n.b. \\
\hline Anisotropie [\%] & & & & & & n.b. \\
\hline Granit (MrGr3) x-Richtung & & & & & & 0,18 \\
\hline y-Richtung & & & & & & 0,18 \\
\hline z-Richtung & & & & & & n.b. \\
\hline Mittelwert & 2,67 & 2,65 & 1,03 & 0,09 & 0,68 & $0,18+-0,10$ \\
\hline Anisotropie [\%] & & & & & & $1+-46$ \\
\hline \multicolumn{7}{|l|}{ Proben Gruppe II } \\
\hline Peridotit (Pi1a) x-Richtung & & & & & & 0,03 \\
\hline$y$-Richtung & & & & & & n.b. \\
\hline z-Richtung & & & & & & n.b. \\
\hline Mittelwert & 3,34 & 3,34 & 0,03 & n.b. & n.b. & 0,03 \\
\hline Anisotropie [\%] & & & & & & n.b. \\
\hline Marmor (Rek) x-Richtung & & & & & & 0,01 \\
\hline y-Richtung & & & & & & n.b. \\
\hline z-Richtung & & & & & & 0,03 \\
\hline Mittelwert & 2,72 & 2,71 & 0,14 & 0,06 & 0,08 & 0,02 \\
\hline Anisotropie [\%] & & & & & & n.b. \\
\hline Quarzit (AI) x-Richtung & & & & & & 0,01 \\
\hline y-Richtung & & & & & & n.b. \\
\hline z-Richtung & & & & & & 0,03 \\
\hline Mittelwert & 2,69 & 2,69 & 0,18 & 0,01 & 0 & 0,02 \\
\hline Anisotropie [\%] & & & & & & n.b. \\
\hline $\begin{array}{c}\text { Meta-Gabbro (MG1b) x- } \\
\text { Richtung }\end{array}$ & & & & & & 0,02 \\
\hline y-Richtung & & & & & & n.b. \\
\hline z-Richtung & & & & & & 0,01 \\
\hline Mittelwert & 3,06 & 3,05 & 0,31 & 0,10 & 0,22 & 0,02 \\
\hline Anisotropie [\%] & & & & & & n.b. \\
\hline $\begin{array}{c}\text { Meta-Gabbro (MG2) X- } \\
\text { Richtung }\end{array}$ & & & & & & 0,01 \\
\hline y-Richtung & & & & & & n.b. \\
\hline z-Richtung & & & & & & 0,01 \\
\hline Mittelwert & 3,09 & 3,09 & 0,13 & n.b. & n.b. & 0,02 \\
\hline Anisotropie [\%] & & & & & & n.b. \\
\hline $\begin{array}{c}\text { granod. Protomylonit (Iv1) x- } \\
\text { Richtung }\end{array}$ & & & & & & 0,08 \\
\hline y-Richtung & & & & & & 0,07 \\
\hline z-Richtung & & & & & & 0,07 \\
\hline
\end{tabular}




\section{Anhang}

\begin{tabular}{|c|c|c|c|c|c|c|}
\hline & $\begin{array}{c}\rho_{\mathrm{M}} \\
{\left[\mathbf{g} / \mathrm{cm}^{3}\right]^{*}}\end{array}$ & $\begin{array}{c}\rho_{\text {roh }} \\
{\left[\mathrm{g} / \mathrm{cm}^{3}\right]^{* *}}\end{array}$ & $\Phi[\underset{* * *}{[\mathrm{Vol} \%]}$ & $\begin{array}{c}\mathbf{r}_{\mathbf{m}} \\
{[\mu \mathrm{m}]^{* * * *}}\end{array}$ & $\begin{array}{c}\Phi_{\text {rkap }} \\
{\left[\text { [Vol.\%] }^{*}{ }^{* * * *}\right.}\end{array}$ & $\underset{\left[\mathrm{kg} / \mathrm{m}^{2 *} \sqrt{\mathrm{t}}\right]}{\mathrm{w}]}$ \\
\hline Mittelwert & 2,79 & 2,78 & 0,47 & 0,08 & 0,21 & $0,07+-0,10$ \\
\hline Anisotropie [\%] & & & & & & $12+-99$ \\
\hline $\begin{array}{l}\text { granit. Gneis (Gra1a) x- } \\
\text { Richtung }\end{array}$ & & & & & & 0,05 \\
\hline$y$-Richtung & & & & & & n.b. \\
\hline z-Richtung & & & & & & 0,02 \\
\hline Mittelwert & 2,71 & 2,69 & 0,55 & 0,04 & 0,21 & 0,04 \\
\hline Anisotropie [\%] & & & & & & n.b. \\
\hline Proben Gruppe III & & & & & & \\
\hline $\begin{array}{c}\text { granit. Protomylonit (MrMy5) } \\
\text { x-Richtung }\end{array}$ & & & & & & 0,18 \\
\hline y-Richtung & & & & & & n.b. \\
\hline z-Richtung & & & & & & 0,14 \\
\hline Mittelwert & 2,67 & 2,64 & 0,94 & 0,09 & 0,59 & $0,16+-0,10$ \\
\hline Anisotropie [\%] & & & & & & $20+-43$ \\
\hline $\begin{array}{c}\text { granod. Gneis (Be1) x- } \\
\text { Richtung }\end{array}$ & & & & & & 0,17 \\
\hline$y$-Richtung & & & & & & 0,12 \\
\hline z-Richtung & & & & & & 0,14 \\
\hline Mittelwert & 2,72 & 2,69 & 0,87 & 0,14 & 0,66 & $0,14+-0,10$ \\
\hline Anisotropie [\%] & & & & & & $29+-43$ \\
\hline $\begin{array}{c}\text { granod. Mylonit (Iv1b) x- } \\
\text { Richtung }\end{array}$ & & & & & & 0,08 \\
\hline$y$-Richtung & & & & & & n.b. \\
\hline z-Richtung & & & & & & 0,08 \\
\hline Mittelwert & 2,74 & 2,73 & 0,39 & 0,08 & 0,16 & $0,08+-0,10$ \\
\hline Anisotropie [\%] & & & & & & $2+-75$ \\
\hline $\begin{array}{c}\text { granod. Paragneis (Cal) } x- \\
\text { Richtung }\end{array}$ & & & & & & 0,12 \\
\hline$y$-Richtung & & & & & & n.b. \\
\hline z-Richtung & & & & & & 0,10 \\
\hline Mittelwert & 2,73 & 2,71 & 0,73 & 0,16 & 0,56 & $0,11+-0,10$ \\
\hline Anisotropie [\%] & & & & & & $15+-66$ \\
\hline $\begin{array}{l}\text { granit. Orthogneis (VA) } x- \\
\text { Richtung }\end{array}$ & & & & & & 0,15 \\
\hline y-Richtung & & & & & & n.b. \\
\hline z-Richtung & & & & & & 0,11 \\
\hline Mittelwert & 2,71 & 2,68 & 0,89 & 0,08 & 0,52 & $0,13+-0,10$ \\
\hline Anisotropie [\%] & & & & & & $26+-49$ \\
\hline $\begin{array}{l}\text { granit. Gneis (Gra1c) x- } \\
\text { Richtung }\end{array}$ & & & & & & $<0,06$ \\
\hline$y$-Richtung & & & & & & $<0,06$ \\
\hline z-Richtung & & & & & & $<0,06$ \\
\hline Mittelwert & 2,69 & 2,68 & 0,46 & 0,01 & 0 & n.b. \\
\hline Anisotropie [\%] & & & & & & n.b. \\
\hline $\begin{array}{c}\text { granit. Gneis (Be2) x- } \\
\text { Richtung }\end{array}$ & & & & & & 0,27 \\
\hline$y$-Richtung & & & & & & n.b. \\
\hline z-Richtung & & & & & & 0,17 \\
\hline Mittelwert & 2,67 & 2,64 & 1,01 & 0,16 & 0,87 & $0,22+-0,10$ \\
\hline Anisotropie [\%] & & & & & & 39 \\
\hline $\begin{array}{l}\text { granod. Ultramylonit (Iv2) x- } \\
\text { Richtung }\end{array}$ & & & & & & 0,07 \\
\hline y-Richtung & & & & & & n.b. \\
\hline z-Richtung & & & & & & 0,09 \\
\hline Mittelwert & 2,75 & 2,74 & 0,32 & 0,07 & 0,13 & $0,08+-0,10$ \\
\hline
\end{tabular}




\section{Anhang}

\begin{tabular}{|c|c|c|c|c|c|c|}
\hline & $\begin{array}{c}\rho_{\mathrm{M}} \\
{\left[\mathrm{g} / \mathrm{cm}^{3}\right]^{*}}\end{array}$ & $\begin{array}{c}\rho_{\text {roh }} \\
{\left[\mathrm{g} / \mathrm{cm}^{3}\right]^{* *}}\end{array}$ & $\Phi[\underset{* * *}{\operatorname{Vol} . \%]}$ & $\begin{array}{c}r_{m} \\
{[\mu m]^{* * * *}}\end{array}$ & $\begin{array}{c}\Phi_{\text {rkap }} \\
{\left[\text { Vol.\%] }_{* * * *}^{*}\right.}\end{array}$ & $\underset{\left[\mathrm{kg} / \mathrm{m}^{2 *} \sqrt{\mathrm{t}} \mathrm{t}\right]}{\mathrm{w}}$ \\
\hline Anisotropie [\%] & & & & & & $22+-85$ \\
\hline $\begin{array}{l}\text { granit. Ultramylonit (Gra3a) x- } \\
\text { Richtung }\end{array}$ & & & & & & $<0,06$ \\
\hline$y$-Richtung & & & & & & $<0,06$ \\
\hline z-Richtung & & & & & & $<0,06$ \\
\hline Mittelwert & 2,69 & 2,68 & 0,18 & 0,01 & 0 & n.b. \\
\hline Anisotropie [\%] & & & & & & n.b. \\
\hline
\end{tabular}

Daten sind nach der Anisotropie des makroskopischen Gefüges geordnet. ${ }^{*} \rho_{M}-$ Matrixdichte $\left[\mathrm{g} / \mathrm{cm}^{3}\right]$. ${ }^{* *} \rho_{\text {roh }}-$ Rohdichte $\left[\mathrm{g} / \mathrm{cm}^{3}\right] .{ }^{* * *} \Phi-$ Porosität [Vol.\%]. ${ }^{* * * *} r_{m}-$ mittlerer Porenradius [ $\left.\mu \mathrm{m}\right] .{ }^{* * * *} \Phi_{r k a p}-$ Volumenanteil der Kapillarporen [Vol.\%]. ${ }^{* * * * *} w-$ Wasseraufnahmekoeffizient $\left[\mathrm{kg} / \mathrm{m}^{2 *} \mathrm{vt}\right]$. n.b. - nicht bestimmt. Fehler: Für $\rho_{M}$ und $\rho_{\text {roh }}$ ergibt sich aufgrund von Wägefehlern ein $1 \sigma$-Fehler von $<0,01 \mathrm{~g} / \mathrm{cm} 3$. $\Phi$ ist mit einem $1 \sigma$-Fehler von <0,01 Vol.\% behaftet. Für $r_{m}$ und $\Phi_{\text {rkap }}$ kann der $1 \sigma$ - Fehler

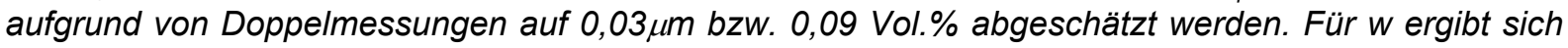
anhand von Mehrfachmessungen an Dummy-Proben ein gerätebedingter $1 \sigma$-Fehler von 0,06 $\mathrm{kg} / \mathrm{m}^{2 *} \gamma_{t}$. Für Die Fehler der Anisotropien und der Mittelwerte (arithmetisches Mittel aus den Richtungen $x, y, z)$ ergeben sich mit Hilfe der Fehlerfortpflanzung aus den Fehlern der Einzelrichtungen $(x, y, z)$ und den Berechnungsgrundlagen (vgl. Kap.2.4.1/2.4.2). 
Anhang I.5: Mechanische Eigenschaften

\begin{tabular}{|c|c|c|c|c|c|c|c|c|c|c|}
\hline & $\mathbf{R}[\mathrm{MPa}]^{*}$ & $\mathrm{n}^{* *}$ & $\begin{array}{c}\beta_{\mathrm{Sz}} \\
\mathrm{[MPa}^{* * *}\end{array}$ & $\mathbf{n}$ & $\mathbf{R}_{\mathrm{tf}}[\mathrm{MPa}]^{\star \star \star *}$ & $\mathbf{n}$ & $\mathbf{W}_{\mathrm{v}}\left[\mathrm{cm}^{3}\right]^{\star \star * \star * *}$ & $\mathbf{n}$ & $\mathrm{H}_{\mathrm{VG}}{ }^{* * * * * *}$ & $\mathrm{I}_{\mathrm{SPG}}{ }^{* * * * * * *}$ \\
\hline \multicolumn{11}{|l|}{ Proben Gruppe I } \\
\hline Granit (Koess) x-Richtung & $194+-11$ & 10 & $12,2+-1,2$ & 8 & $18,0+-1,1$ & 10 & $5,8+-0,2$ & 2 & & \\
\hline y-Richtung & $194+-10$ & 6 & $10,9+-1,3$ & 6 & $19,7+-1,1$ & 5 & $5,7+-0,1$ & 2 & & \\
\hline z-Richtung & $199+-8$ & 6 & $12,5+-1,5$ & 8 & $22,6+-0,8$ & 7 & $5,6+-0,1$ & 2 & & \\
\hline Mittelwert & $195+-17$ & & $11,9+-2,3$ & & $20,1+-1,7$ & & $5,7+-0,2$ & & $646+-41$ & $6,1+-0,2$ \\
\hline Anisotropie [\%] & $2,6+-6,3$ & & $12,7+-14,6$ & & $20,3+-5,6$ & & $2,8+-3,3$ & & & \\
\hline Granodiorit (PDD) x-Richtung & $231+-15$ & 5 & $13,0+-1,0$ & 10 & $19,7+-0,4$ & 8 & $7,2+-0,1$ & 2 & & \\
\hline y-Richtung & $219+-10$ & 6 & $13,3+-1,5$ & 8 & $22,4+-0,9$ & 8 & $7,0+-0,2$ & 2 & & \\
\hline z-Richtung & $216+-12$ & 6 & $12,1+-1,5$ & 8 & $21,0+-0,8$ & 10 & $7,2+-0,1$ & 2 & & \\
\hline Mittelwert & $222+-22$ & & $12,8+-2,3$ & & $21,0+-1,3$ & & $7,1+-0,2$ & & $686+-20$ & $6,9+-0,2$ \\
\hline Anisotropie [\%] & $6,5+-8,0$ & & $8,9+-15,2$ & & $11,9+-3,9$ & & $3,6+-2,5$ & & & \\
\hline Gabbro-Norit (NI) x-Richtung & $211+-14$ & 5 & $10,3+-0,9$ & 10 & $17,3+-0,3$ & 6 & $6,8+-0,2$ & 2 & & \\
\hline y-Richtung & $247+-26$ & 4 & $11,2+-1,0$ & 10 & $17,5+-0,6$ & 7 & $6,3+-0,1$ & 2 & & \\
\hline z-Richtung & $246+-21$ & 6 & $10,6+-1,3$ & 10 & $16,5+-0,6$ & 8 & $7,2+-0,1$ & 2 & & \\
\hline Mittelwert & $235+-36$ & & $10,7+-1,9$ & & $17,1+-0,9$ & & $6,8+-0,2$ & & $663+-11$ & $8,2+-0,1$ \\
\hline Anisotropie [\%] & 14,5 & & 8,0 & & 5,9 & & 12,9 & & & \\
\hline Granit (MrGr3) x-Richtung & 200 & 1 & $13,6+-0,7$ & 7 & n.b. & & $7,0+-0,0$ & 2 & & \\
\hline$y$-Richtung & 214 & 1 & $10,8+-0,5$ & 4 & n.b. & & $7,8+-0,1$ & 2 & & \\
\hline z-Richtung & 211 & 1 & $12,7+-0,5$ & 8 & n.b. & & $7,3+-0,1$ & 2 & & \\
\hline Mittelwert & 208 & & $12,4+-1,0$ & & n.b. & & $7,4+-0,2$ & & $667+-18$ & $5,9+-0,2$ \\
\hline Anisotropie [\%] & 6,5 & & $20,3+-5,2$ & & n.b. & & $10,7+-1,1$ & & & \\
\hline \multicolumn{11}{|l|}{ Proben Gruppe II } \\
\hline Peridotit (Pi1a) x-Richtung & $243+-+12$ & 4 & $13,4+-1,4$ & 6 & $30,3+-1,3$ & 4 & $5,5+-0,3$ & 2 & & \\
\hline y-Richtung & $255+-18$ & 6 & $12,3+-1,0$ & 8 & $22,3+-0,7$ & 3 & $6,1+-0,2$ & 2 & & \\
\hline z-Richtung & $231+-17$ & 6 & $13,7+-1,4$ & 8 & $21,3+-1,3$ & 4 & $5,7+-0,5$ & 2 & & \\
\hline Mittelwert & $243+-28$ & & $13,1+-2,2$ & & $24,6+-1,9$ & & $5,8+-0,6$ & & $750+-13$ & $5,9+-0,1$ \\
\hline Anisotropie [\%] & 9,4 & & 10,0 & & 29,7 & & 10,5 & & & \\
\hline Marmor (Rek) x-Richtung & $77+-7$ & 6 & $7,2+-0,9$ & 10 & $16,6+-0,8$ & 6 & $25,2+-0,4$ & 2 & & \\
\hline y-Richtung & $75+-6$ & 6 & $7,3+-0,5$ & 10 & $19,3+-1,5$ & 6 & $23,8+-0,5$ & 2 & & \\
\hline Mittelwert & $81+-12$ & & $6,8+-1,3$ & & $16,4+-2,0$ & & $24,9+-2,0$ & & $87+-10$ & $14,7+-0,3$ \\
\hline Anisotropie [\%] & 18,5 & & 20,0 & & 31,0 & & 7,4 & & & \\
\hline
\end{tabular}




\begin{tabular}{|c|c|c|c|c|c|c|c|c|c|c|}
\hline & $\mathrm{R}$ [MPa] ${ }^{*}$ & $\mathrm{n}^{* *}$ & $\begin{array}{c}\beta_{\mathrm{Sz}} \\
\text { [MPa] }^{* * *}\end{array}$ & $\mathbf{n}$ & $\mathbf{R}_{\mathrm{tf}}[\mathrm{MPa}]^{* * * *}$ & $\mathbf{n}$ & $\mathbf{W}_{\mathrm{v}}\left[\mathrm{cm}^{3}\right]^{\star \star \star \star \star}$ & $\mathbf{n}$ & $\mathrm{H}_{\mathrm{VG}}{ }^{* * * * * *}$ & $\mathrm{I}_{\mathrm{SPG}}{ }^{* * * * * *}$ \\
\hline Quarzit (Al) x-Richtung & $274+-15$ & 6 & $17,6+-1,4$ & 5 & $21,0+-1,4$ & 3 & $4,5+-0,2$ & 2 & & \\
\hline y-Richtung & $278+-19$ & 4 & $16,4+-1,2$ & 10 & $28,1+-1,6$ & 5 & $4,4+-0,2$ & 2 & & \\
\hline z-Richtung & $291+-17$ & 5 & $21,0+-1,1$ & 6 & $36,2+-0$ & 2 & $4,1+-0,2$ & 2 & & \\
\hline Mittelwert & $281+-30$ & & $18,3+-2,2$ & & $28,4+-2,1$ & & $4,3+-0,3$ & & $946+-41$ & $2,1+-0,2$ \\
\hline Anisotropie [\%] & 5,8 & & 21,9 & & 41,9 & & 8,5 & & & \\
\hline Meta-Gabbro (MG1b) x-Richtung & $193+-8$ & 6 & $11,4+-0,8$ & 10 & $18,1+-0,4$ & 4 & $7,0+-0,2$ & 2 & & \\
\hline y-Richtung & $200+-8$ & 6 & $9,2+-1,0$ & 9 & $16,4+-1,5$ & 5 & $7,6+-0,1$ & 2 & & \\
\hline z-Richtung & $230+-12$ & 6 & $8,3+-0,9$ & 10 & $10,3+-0,8$ & 4 & $8,8+-0,1$ & 2 & & \\
\hline Mittelwert & $208+-16$ & & $9,6+-1,6$ & & $14,9+-1,7$ & & $7,8+-0,2$ & & $714+-18$ & $8,4+-0,3$ \\
\hline Anisotropie [\%] & 16,1 & & 27,9 & & 43,1 & & 20,7 & & & \\
\hline Meta-Gabbro (MG2) x-Richtung & $198+-22$ & 6 & $13,0+-1,5$ & 7 & $26,2+-2,8$ & 3 & $6,5+-0,1$ & 2 & & \\
\hline y-Richtung & $198+-20$ & 6 & $9,2+-1,2$ & 7 & $18,7+-3,9$ & 2 & $5,9+-0,4$ & 2 & & \\
\hline z-Richtung & $227+-22$ & 9 & $9,7+-1,4$ & 6 & $13,2+-2,2$ & 3 & $7,4+-0,3$ & 2 & & \\
\hline Mittelwert & $208+-37$ & & $10,6+-2,4$ & & $19,4+-5,3$ & & $6,6+-0,5$ & & $714+-18$ & $8,4+-0,3$ \\
\hline Anisotropie [\%] & 12,8 & & 29,3 & & 49,6 & & 20,5 & & & \\
\hline granod. Protomylonit (Iv1) x-Richtung & $221+-6$ & 5 & $13,5+-0,8$ & 6 & n.b. & & $7,4+-0,2$ & 2 & & \\
\hline y-Richtung & $201+-4$ & 5 & $11,1+-0,9$ & 5 & n.b. & & $7,6+-0,1$ & 2 & & \\
\hline z-Richtung & $206+-2$ & 5 & $10,0+-0,7$ & 8 & n.b. & & $8,6+-0,1$ & 3 & & \\
\hline Mittelwert & $209+-7$ & & $11,5+-1,4$ & & n.b. & & $7,8+-0,2$ & & $682+-37$ & $5,4+-0,3$ \\
\hline Anisotropie [\%] & $9,0+-3,1$ & & $26,0+-6,5$ & & n.b. & & $14,2+-2,2$ & & & \\
\hline granit. Gneis (Gra1a) x-Richtung & $307+-19$ & 6 & $14,5+-1,9$ & 6 & $31,7+-1,1$ & 5 & $5,9+-0,2$ & 2 & & \\
\hline y-Richtung & $307+-18$ & 6 & $14,0+-1,3$ & 8 & $32,0+-0,5$ & 2 & $6,0+-0,0$ & 2 & & \\
\hline z-Richtung & $293+-21$ & 4 & $14,1+-1,4$ & 8 & $27,8+-2,2$ & 5 & $6,1+-0,1$ & 2 & & \\
\hline Mittelwert & $302+-34$ & & $14,2+-2,8$ & & $30,5+-2,5$ & & $6,0+-0,2$ & & $753+-15$ & $5,9+-0,1$ \\
\hline Anisotropie [\%] & $19,2+-9,0$ & & $3,9+-15,6$ & & $13,3+-7,0$ & & $3,4+-3,2$ & & & \\
\hline \multicolumn{11}{|l|}{ Proben Gruppe III } \\
\hline $\begin{array}{l}\text { granit. Protomylonit (MrMy5) x- } \\
\text { Richtung }\end{array}$ & $177+-3$ & 2 & $12,1+-0,6$ & 10 & n.b. & & $6,7+-0,1$ & 2 & & \\
\hline y-Richtung & 161 & 1 & $11,6+-0,5$ & 5 & n.b. & & $6,3+-0,1$ & 2 & & \\
\hline z-Richtung & $204+-7$ & 4 & $7,2+-0,5$ & 4 & n.b. & & $7,6+-0,1$ & 2 & & \\
\hline Mittelwert & $181+-8$ & & $10,3+-0,9$ & & n.b. & & $6,9+-0,2$ & & $685+-28$ & $5,8+-0,3$ \\
\hline Anisotropie [\%] & $21,1+-2,7$ & & $40,3+-4,9$ & & n.b. & & $17,1+-2,5$ & & & \\
\hline granod. Gneis (Be1) x-Richtung & $174+-13$ & 6 & $12,8+-0,6$ & 7 & n.b. & & $7,4+-0,2$ & 2 & & \\
\hline y-Richtung & $160+-7$ & 4 & $10,5+-0,7$ & 7 & n.b. & & $7,7+-0,4$ & 2 & & \\
\hline z-Richtung & $172+-12$ & 4 & $5,9+-0,5$ & 12 & n.b. & & $8,6+-0,0$ & 2 & & \\
\hline
\end{tabular}




\begin{tabular}{|c|c|c|c|c|c|c|c|c|c|c|}
\hline & $\mathbf{R}[\mathrm{MPa}]^{*}$ & $\mathrm{n}^{* *}$ & $\begin{array}{c}\beta_{\mathrm{sz}} \\
{[\mathrm{MPa}]^{* * *}}\end{array}$ & $\mathbf{n}$ & $\mathbf{R}_{\mathrm{tf}}[\mathrm{MPa}]^{\star \star * *}$ & $\mathbf{n}$ & $\mathbf{W}_{\mathrm{v}}\left[\mathrm{cm}^{3}\right]^{\star * * * *}$ & $\mathbf{n}$ & $\mathrm{H}_{\mathrm{VG}}{ }^{* * * * * *}$ & $\mathrm{I}_{\mathrm{SPG}}{ }^{* * * * * * *}$ \\
\hline Mittelwert & $169+-19$ & & $9,8+-1,0$ & & n.b. & & $7,9+-0,4$ & & $670+-28$ & $6,0+-0,3$ \\
\hline Anisotropie [\%] & $8,0+-8,0$ & & $54,1+-4,4$ & & n.b. & & $13,7+-2,1$ & & & \\
\hline granod. Mylonit (Iv1b) x-Richtung & $225+-28$ & 9 & $18,3+-0,4$ & 5 & n.b. & & $6,8+-0,1$ & 2 & & \\
\hline y-Richtung & $128+-13$ & 5 & $12,6+-0,8$ & 9 & n.b. & & $7,9+-0,2$ & 2 & & \\
\hline z-Richtung & $168+-18$ & 5 & $9,5+-0,8$ & 18 & n.b. & & $8,9+-0,1$ & 2 & & \\
\hline Mittelwert & $174+-36$ & & $13,5+-1,2$ & & n.b. & & $7,9+-0,2$ & & $682+-37$ & $5,4+-0,3$ \\
\hline Anisotropie [\%] & $43,1+-9,1$ & & $48,1+-4,3$ & & n.b. & & $23,2+-1,7$ & & & \\
\hline granod. Paragneis (Cal) $x$-Richtung & $211+-8$ & 6 & $12,5+-1,7$ & 8 & $22,5+-0,7$ & 11 & $7,4+-0,1$ & 2 & & \\
\hline y-Richtung & $164+-5$ & 6 & $9,7+-1,9$ & 10 & $17,8+-0,8$ & 3 & $8,2+-0,4$ & 2 & & \\
\hline z-Richtung & $184+-12$ & 6 & $5,7+-0,7$ & 10 & $6,0+-0,4$ & 10 & $8,8+-0,0$ & 2 & & \\
\hline xz45-Richtung & $110+-6$ & 3 & & & & & & & & \\
\hline yz45-Richtung & $108+-5$ & 4 & & & & & & & & \\
\hline Mittelwert & $186+-15$ & & $9,3+-2,6$ & & $15,4+-1,1$ & & $8,1+-0,4$ & & $679+-28$ & $6,0+-0,3$ \\
\hline Anisotropie [\%] & $22,3+-3,8$ & & $54,8+-8,2$ & & $73,4+-2,0$ & & $15,9+-1,5$ & & & \\
\hline granit. Orthogneis (VA) $x$-Richtung & $178+-13$ & 5 & $14,8+-1,6$ & 6 & $24,3+-0,5$ & 5 & $6,6+-0,2$ & 2 & & \\
\hline y-Richtung & $147+-9$ & 6 & $14,1+-0,8$ & 6 & $15,1+-1,1$ & 7 & $7,1+-0,1$ & 2 & & \\
\hline z-Richtung & $204+-12$ & 6 & $5,5+-0,5$ & 8 & $6,7+-0,2$ & 6 & $8,5+-0,8$ & 2 & & \\
\hline xz45-Richtung & $113+-7$ & 6 & & & & & & & & \\
\hline Mittelwert & $176+-20$ & & $11,4+-1,9$ & & $15,3+-1,2$ & & $7,4+-0,8$ & & $621+-14$ & $5,7+-0,2$ \\
\hline Anisotropie [\%] & $28,0+-6,1$ & & $62,8+-5,4$ & & $72,6+-1,0$ & & $22,9+-7,5$ & & & \\
\hline granit. Gneis (Gra1c) x-Richtung & $298+-18$ & 2 & $14,5+-1,5$ & 5 & n.b. & & $5,7+-0,1$ & 2 & & \\
\hline$y$-Richtung & $304+-7$ & 5 & $17,3+-1,2$ & 10 & n.b. & & $5,9+-0,0$ & 2 & & \\
\hline z-Richtung & $302+-27$ & 3 & $13,6+-1,6$ & 6 & n.b. & & $5,7+-0,1$ & 2 & & \\
\hline Mittelwert & $301+-33$ & & $15,1+-2,5$ & & n.b. & & $5,8+-0,1$ & & $763+-15$ & $5,8+-0,1$ \\
\hline Anisotropie [\%] & $2,0+-6,3$ & & $21,1+-10,8$ & & n.b. & & $4,7+-1,8$ & & & \\
\hline granit. Gneis (Be2) x-Richtung & $210+-23$ & 3 & $12,3+-0,7$ & 9 & n.b. & & $7,3+-0,4$ & 2 & & \\
\hline y-Richtung & $145+-5$ & 4 & $6,6+-0,3$ & 11 & n.b. & & $9,3+-0,1$ & 2 & & \\
\hline z-Richtung & $174+-6$ & 4 & $5,8+-0,4$ & 6 & n.b. & & $9,3+-0,3$ & 3 & & \\
\hline Mittelwert & $176+-24$ & & $8,2+-0,9$ & & n.b. & & $8,6+-0,5$ & & $707+-28$ & $5,7+-0,3$ \\
\hline Anisotropie [\%] & $31,0+-7,9$ & & $52,5+-4,5$ & & n.b. & & $21,6+-0,5$ & & & \\
\hline granod. Ultramylonit (Iv2) x-Richtung & $200+-14$ & 7 & $19,3+-0,6$ & 6 & n.b. & & $6,4+-0,1$ & 3 & & \\
\hline y-Richtung & $155+-11$ & 5 & $12,5+-0,7$ & 17 & n.b. & & $7,9+-0,1$ & 3 & & \\
\hline z-Richtung & $172+-10$ & 8 & $7,7+-0,6$ & 14 & n.b. & & $9,1+-0,1$ & 2 & & \\
\hline Mittelwert & $176+-20$ & & $13,2+-1,1$ & & n.b. & & $7,8+-0,2$ & & $682+-37$ & $5,4+-0,3$ \\
\hline Anisotropie [\%] & $22,5+-7,7$ & & $60,1+-3,1$ & & n.b. & & $30,0+-1,1$ & & & \\
\hline
\end{tabular}




\section{Anhang}

\begin{tabular}{|c|c|c|c|c|c|c|c|c|c|c|}
\hline & $\mathrm{R}[\mathrm{MPa}]^{*}$ & $\mathbf{n}^{* *}$ & $\begin{array}{c}\beta \mathrm{Sz} \\
{[\mathrm{MPa}]^{\star * *}}\end{array}$ & $\mathbf{n}$ & $R_{\mathrm{tf}}[\mathrm{MPa}]^{\star \star \star * *}$ & $\mathbf{n}$ & $W_{v}\left[\mathrm{~cm}^{3}\right]^{\star \star \star \star * *}$ & $\mathbf{n}$ & $\mathrm{H}_{\mathrm{VG}}{ }^{* * * * * *}$ & $I_{\mathrm{SPG}}{ }^{\star \star \star \star \star \star *}$ \\
\hline granit. Ultramylonit (Gra3a) x-Richtung & $371+-12$ & 5 & $21,45+-1,8$ & 5 & $48,4+-2,7$ & 4 & $5,1+-0,0$ & 2 & & \\
\hline$y$-Richtung & $320+-10$ & 5 & $22,1+-2,4$ & 8 & $43,1+-1,5$ & 5 & $5,5+-0,1$ & 2 & & \\
\hline z-Richtung & $349+-18$ & 4 & $8,9+-1,2$ & 8 & 6,8 & 1 & $7,0+-0,0$ & 2 & & \\
\hline Mittelwert & $347+-24$ & & $17,5+-3,2$ & & $32,8+-3,1$ & & $5,9+-0,1$ & & $734+-15$ & $6,0+-0,1$ \\
\hline Anisotropie [\%] & $13,7+-3,9$ & & $59,7+-7$ & & $85,9+-0,8$ & & $26,7+-0,4$ & & & \\
\hline
\end{tabular}

Daten sind nach der Anisotropie des makroskopischen Gefüges geordnet. Die Fehler der Anisotropien und der Mittelwerte (arithmetisches Mittel aus den

Richtungen $x, y, z)$. ergeben sich mit Hilfe der Fehlerfortpflanzung aus den Fehlern der Einzelrichtungen $(x, y, z)$ und den Berechnungsgrundlagen (vgl.

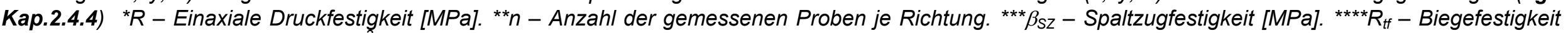
[MPa]. ${ }^{* * * *} W v$-Abriebfestigkeit $\left[\mathrm{cm}^{3}\right] .{ }^{* * * * * *} H_{V G}$ - mittlere Härte des Gesteins (aus den Härten der Minerale und der mineralogischen Zusammensetzung. ${ }^{* * * * * *} I_{S P G}-$ mittlere Spaltbarkeit des Gesteins (aus den Spaltbarkeiten der Minerale und der mineralogischen Zusammensetzung. 


\section{Anhang I.6: Dynamische elastische Eigenschaften}

\begin{tabular}{|c|c|c|c|c|c|c|c|c|c|c|c|c|c|c|c|c|c|c|c|}
\hline & $\begin{array}{c}\mathrm{Vp}_{0}(0,5 \\
\mathrm{MHz}) \\
{[\mathrm{km} / \mathrm{s}]} \\
\mathrm{a}\end{array}$ & $\begin{array}{c}\mathrm{Vp}_{0} \\
(\mathrm{Ku}) \\
{[\mathrm{km} / \mathrm{s}]} \\
\mathrm{b}\end{array}$ & $\begin{array}{c}\mathrm{Vp}_{0}(1 \\
\mathrm{MHz}) \\
{[\mathrm{km} / \mathrm{s}]} \\
\mathrm{c}\end{array}$ & $\begin{array}{c}\mathbf{V s}_{0 / 1}(0,5 \\
\mathrm{MHz}) \\
{[\mathrm{km} / \mathrm{s}]} \\
\mathrm{d}\end{array}$ & $\begin{array}{c}\mathbf{V s}_{0 / 2}(0,5 \\
\mathrm{MHz}) \\
{[\mathrm{km} / \mathrm{s}]} \\
\mathrm{e}\end{array}$ & $\begin{array}{c}\mathbf{V s}_{0 / 1}(1 \\
\mathrm{MHz}) \\
{[\mathrm{km} / \mathrm{s}]} \\
\mathrm{f}\end{array}$ & $\begin{array}{c}\mathbf{V s}_{0 / 2}(1 \\
\mathrm{MHz}) \\
{[\mathrm{km} / \mathrm{s}]} \\
\mathrm{g} \\
\end{array}$ & $\begin{array}{c}V_{p_{400}} \\
{[\mathrm{~km} / \mathrm{s}]} \\
\mathrm{h}\end{array}$ & $\begin{array}{l}\mathbf{V s}_{400 / 1} \\
{[\mathbf{k m} / \mathbf{s}]} \\
\mathbf{i}\end{array}$ & $\begin{array}{c}\mathbf{V s} \mathbf{s}_{400 / 2} \\
{[\mathbf{k m} / \mathbf{s}]} \\
\mathbf{j}\end{array}$ & $\begin{array}{c}\mathrm{E}_{01 / 2}(0,5 \\
\mathrm{MHz}) \\
{[\mathrm{GPa}]} \\
\mathrm{k}\end{array}$ & $\begin{array}{c}\mathrm{E}_{0 / 1}(1 \\
\mathrm{MHz}) \\
{[\mathrm{GPa}]} \\
\mathrm{I}\end{array}$ & $\begin{array}{c}\mathrm{E}_{0 / 2}(1 \\
\mathrm{MHz}) \\
{[\mathrm{GPa}]} \\
\mathrm{m}\end{array}$ & $\begin{array}{c}\mathrm{E}_{01 / 2(1}(1 \\
\mathrm{MHz}) \\
{[\mathrm{GPa}]} \\
\mathrm{n}\end{array}$ & $\begin{array}{c}\mathrm{E}_{400} 1 / 2 \\
{[\mathrm{GPa}]} \\
0\end{array}$ & $\begin{array}{c}v_{01 / 2} \\
(0,5 \\
M H z) \\
p\end{array}$ & $\begin{array}{c}v_{01 / 2} \\
(1 \mathrm{MHz}) \\
q\end{array}$ & $\begin{array}{l}v_{400} \frac{1 / 2}{r} \\
r\end{array}$ & $\begin{array}{c}\mathrm{E}_{\mathrm{VRH}} \\
{[\mathrm{GPa}]} \\
\mathbf{s}\end{array}$ \\
\hline \multicolumn{20}{|l|}{$\begin{array}{l}\text { Proben } \\
\text { Gruppe I }\end{array}$} \\
\hline $\begin{array}{c}\text { Granit } \\
\text { (Koess) x- } \\
\text { Richtung }\end{array}$ & 5,90 & 5,96 & 6,00 & 3,44 & 3,43 & 3,43 & 3,38 & 6,59 & 3,85 & 3,68 & $\begin{array}{c}78,7+- \\
8,0\end{array}$ & 79,1 & 77,4 & $\begin{array}{c}78,5+- \\
3,3 \\
\end{array}$ & $\begin{array}{c}95,6+- \\
3,7 \\
\end{array}$ & $\begin{array}{c}0,24+- \\
0,10 \\
\end{array}$ & $\begin{array}{c}0,26+- \\
0,03 \\
\end{array}$ & $\begin{array}{c}0,26+- \\
0,03 \\
\end{array}$ & \\
\hline y-Richtung & 5,79 & 5,77 & 5,88 & 3,38 & 3,36 & 3,35 & 3,12 & 6,44 & 3,74 & 3,35 & $\begin{array}{c}75,7+- \\
7,8\end{array}$ & 75,6 & 67,9 & $\begin{array}{c}72,0+- \\
3,1\end{array}$ & $\begin{array}{c}86,4+- \\
3,4\end{array}$ & $\begin{array}{c}0,24+- \\
0,10\end{array}$ & $\begin{array}{c}0,28+- \\
0,03\end{array}$ & $\begin{array}{c}0,28+- \\
0,03\end{array}$ & \\
\hline z-Richtung & 5,80 & 5,94 & 5,74 & 3,46 & 3,42 & 3,35 & 2,95 & 6,59 & 3,80 & 3,36 & $\begin{array}{c}77,9+- \\
8,1 \\
\end{array}$ & 74,5 & 61,5 & $\begin{array}{c}68,3+- \\
3,0 \\
\end{array}$ & $\begin{array}{c}88,7+- \\
3,4 \\
\end{array}$ & $\begin{array}{c}0,23+- \\
0,10 \\
\end{array}$ & $\begin{array}{c}0,28+- \\
0,04 \\
\end{array}$ & $\begin{array}{c}0,29+- \\
0,03 \\
\end{array}$ & \\
\hline Mittelwert & 5,83 & 5,89 & 5,87 & 3,42 & 3,40 & 3,38 & 3,15 & 6,54 & 3,80 & 3,46 & 77,4 & 76,4 & 69,9 & $\begin{array}{c}72,9+- \\
5,5 \\
\end{array}$ & $\begin{array}{l}90,21 \\
+-6,1 \\
\end{array}$ & 0,24 & 0,28 & 0,28 & $\begin{array}{c}86,9+- \\
2,2\end{array}$ \\
\hline $\begin{array}{c}\text { Anisotropie } \\
{[\%]}\end{array}$ & 1,9 & 3,2 & 4,3 & 2,3 & 2,0 & 2,3 & 12,7 & 2,3 & 2,9 & 9,0 & 3,7 & 5,8 & 20,3 & $\begin{array}{c}12,9+- \\
6,5 \\
\end{array}$ & 9,6 & 6,2 & 7,8 & 11,3 & \\
\hline $\begin{array}{c}\text { Granodiorit } \\
\text { (PDD) x- } \\
\text { Richtung } \\
\end{array}$ & 5,49 & 5,40 & 5,41 & 3,27 & 3,24 & 3,29 & 3,03 & 6,53 & 3,74 & 3,68 & $\begin{array}{c}72,9+- \\
8,1\end{array}$ & 73,0 & 65,3 & $\begin{array}{c}69,4+- \\
3,3\end{array}$ & $\begin{array}{c}97,2+- \\
3,8\end{array}$ & $\begin{array}{c}0,23+- \\
0,10\end{array}$ & $\begin{array}{c}0,24+- \\
0,04\end{array}$ & $\begin{array}{c}0,26+- \\
0,03\end{array}$ & \\
\hline$y$-Richtung & 5,60 & 5,56 & 5,21 & 3,30 & 3,27 & 3,35 & 3,24 & 6,54 & 4,02 & 3,67 & $\begin{array}{c}74,8+- \\
8,1\end{array}$ & 72,0 & 69,5 & $\begin{array}{c}70,9+- \\
3,8\end{array}$ & \begin{tabular}{l|}
102,3 \\
$+-4,1$ \\
\end{tabular} & $\begin{array}{c}0,24+- \\
0,10\end{array}$ & $\begin{array}{c}0,17+- \\
0,04\end{array}$ & $\begin{array}{c}0,24+- \\
0,03\end{array}$ & \\
\hline z-Richtung & 5,50 & 5,47 & 5,18 & 3,29 & 3,29 & 3,25 & 3,08 & 6,48 & 4,00 & 3,70 & $\begin{array}{c}74,0+- \\
8,2 \\
\end{array}$ & 69,4 & 65,1 & $\begin{array}{c}67,4+- \\
3,5 \\
\end{array}$ & $\begin{array}{l}101,9 \\
+-4,1 \\
\end{array}$ & $\begin{array}{c}0,22+- \\
0,11 \\
\end{array}$ & $\begin{array}{c}0,20+- \\
0,04 \\
\end{array}$ & $\begin{array}{c}0,23+- \\
0,03 \\
\end{array}$ & \\
\hline Mittelwert & 5,53 & 5,48 & 5,27 & 3,29 & 3,27 & 3,30 & 3,12 & 6,52 & 3,92 & 3,68 & 73,9 & 71,5 & 66,6 & $\begin{array}{c}69,3+- \\
6,1 \\
\end{array}$ & $\begin{array}{c}100,48 \\
+-6,9 \\
\end{array}$ & 0,23 & 0,20 & 0,24 & $\begin{array}{c}95,2+- \\
3,2 \\
\end{array}$ \\
\hline $\begin{array}{c}\text { Anisotropie } \\
{[\%]}\end{array}$ & 2,0 & 2,9 & 4,3 & 0,9 & 1,5 & 3,0 & 6,5 & 0,9 & 7,0 & 0,8 & 2,5 & 4,9 & 6,3 & $\begin{array}{c}4,9+- \\
6,8 \\
\end{array}$ & 5,0 & 6,8 & 30,9 & 13,1 & \\
\hline $\begin{array}{c}\text { Gabbro-Norit } \\
\text { (NI) x- } \\
\text { Richtung }\end{array}$ & 5,60 & 5,80 & 5,65 & 3,42 & 3,36 & 3,65 & 3,44 & 7,18 & 3,98 & 3,95 & $\begin{array}{c}81,3+- \\
9,0\end{array}$ & 88,8 & 83,3 & $\begin{array}{c}86,3+- \\
4,2\end{array}$ & $\begin{array}{l}117,6 \\
+-4,2\end{array}$ & $\begin{array}{c}0,21+- \\
0,10 \\
\end{array}$ & $\begin{array}{c}0,18+- \\
0,04 \\
\end{array}$ & $\begin{array}{c}0,28+- \\
0,03\end{array}$ & \\
\hline y-Richtung & 5,45 & 5,61 & 5,50 & 3,38 & 3,36 & 3,55 & 3,51 & 7,13 & 4,04 & 4,01 & $\begin{array}{c}79,0+- \\
9,1 \\
\end{array}$ & 84,1 & 83,2 & $\begin{array}{c}83,7+- \\
4,3 \\
\end{array}$ & \begin{tabular}{l|}
119,8 \\
$+-4,3$ \\
\end{tabular} & $\begin{array}{c}0,19+- \\
0,11 \\
\end{array}$ & $\begin{array}{c}0,15+- \\
0,04\end{array}$ & $\begin{array}{c}0,27+- \\
0,03\end{array}$ & \\
\hline z-Richtung & 5,87 & 6,14 & 5,89 & 3,44 & 3,38 & 3,57 & 3,40 & 7,36 & 4,17 & 4,01 & $\begin{array}{c}84,6+- \\
8,6 \\
\end{array}$ & 90,0 & 84,4 & $\begin{array}{c}87,3+- \\
3,9 \\
\end{array}$ & $\begin{array}{r}124,7 \\
+-4,3 \\
\end{array}$ & $\begin{array}{c}0,25+- \\
0,10 \\
\end{array}$ & $\begin{array}{c}0,23+- \\
0,04 \\
\end{array}$ & $\begin{array}{c}0,28+- \\
0,03 \\
\end{array}$ & \\
\hline Mittelwert & 5,64 & 5,85 & 5,68 & 3,41 & 3,37 & 3,59 & 3,45 & 7,22 & 4,06 & 3,99 & 81,6 & 87,6 & 83,6 & $\begin{array}{c}85,7+- \\
7,1 \\
\end{array}$ & $\begin{array}{l}120,7 \\
+-7,4 \\
\end{array}$ & 0,22 & 0,19 & 0,27 & $\begin{array}{l}109,0 \\
+-2,6 \\
\end{array}$ \\
\hline $\begin{array}{c}\text { Anisotropie } \\
{[\%]}\end{array}$ & 7,2 & 8,6 & 6,6 & 1,7 & 0,6 & 2,7 & 3,1 & 3,1 & 4,1 & 4,0 & 6,6 & 6,6 & 1,4 & 4,1 & 5,7 & 22,4 & 35,1 & 5,2 & \\
\hline Richtung & & & & & & & & & & & 4,9 & & & 2,0 & 3,4 & 0,17 & 0,06 & 0,03 & \\
\hline
\end{tabular}




\begin{tabular}{|c|c|c|c|c|c|c|c|c|c|c|c|c|c|c|c|c|c|c|c|}
\hline & $\begin{array}{c}\mathrm{Vp}_{0}(0,5 \\
\mathrm{MHz}) \\
{[\mathrm{km} / \mathrm{s}]} \\
\mathrm{a} \\
\end{array}$ & $\begin{array}{c}\mathrm{Vp}_{0} \\
(\mathrm{Ku}) \\
{[\mathrm{km} / \mathrm{s}]} \\
\mathbf{b}\end{array}$ & $\begin{array}{c}\mathrm{Vp}_{0}(1 \\
\mathrm{MHz}) \\
{[\mathrm{km} / \mathrm{s}]} \\
\mathrm{c}\end{array}$ & $\begin{array}{c}\mathbf{V s}_{0 / 1}(0,5 \\
\mathrm{MHz}) \\
{[\mathrm{km} / \mathrm{s}]} \\
\mathrm{d}\end{array}$ & $\begin{array}{c}s_{0 / 2}(0,5 \\
M H z) \\
{[k m / s]} \\
e\end{array}$ & $\begin{array}{c}\mathbf{V s}_{0 / 1}(1 \\
\mathrm{MHz}) \\
{[\mathrm{km} / \mathrm{s}]} \\
\mathrm{f}\end{array}$ & $\begin{array}{c}\mathbf{V s}_{0 / 2}(1 \\
\mathrm{MHz}) \\
{[\mathrm{km} / \mathrm{s}]} \\
\mathrm{g}\end{array}$ & $\begin{array}{c}V p_{400} \\
{[\mathrm{~km} / \mathrm{s}]} \\
\mathrm{h}\end{array}$ & $\begin{array}{c}\mathbf{V s _ { 4 0 0 / 1 }} \\
{[\mathbf{k m} / \mathbf{s}]} \\
\mathbf{i}\end{array}$ & $\begin{array}{c}V_{s_{400 / 2}} \\
{[\mathrm{~km} / \mathrm{s}]} \\
j\end{array}$ & $\begin{array}{c}\mathrm{E}_{01 / 2}(0,5 \\
\mathrm{MHz}) \\
{[\mathrm{GPa}]} \\
\mathrm{k}\end{array}$ & $\begin{array}{c}\mathrm{E}_{0 / 1}(1 \\
\mathrm{MHz}) \\
{[\mathrm{GPa}]} \\
\mathrm{I}\end{array}$ & $\begin{array}{c}\mathrm{E}_{0 / 2}(1 \\
\mathrm{MHz}) \\
{[\mathrm{GPa}]} \\
\mathrm{m}\end{array}$ & $\begin{array}{c}\mathrm{E}_{01 / 2}(1 \\
\mathrm{MHz}) \\
{[\mathrm{GPa}]} \\
\mathrm{n}\end{array}$ & $\begin{array}{c}\mathrm{E}_{400} 1 / 2 \\
{[\mathrm{GPa}]} \\
0\end{array}$ & $\begin{array}{c}v_{01 / 2} \\
(0,5 \\
M H z) \\
p\end{array}$ & $\begin{array}{c}v_{01 / 2} \\
\left(\begin{array}{c}1 \mathrm{MHz} \\
q\end{array}\right)\end{array}$ & $v_{4001 / 2}$ & $\begin{array}{c}E_{\mathrm{VRH}} \\
{[\mathrm{GPa}]} \\
\mathbf{s}\end{array}$ \\
\hline$y$-Richtung & 2,38 & 2,54 & 2,42 & n.b. & n.b. & 1,82 & 1,82 & 6,13 & 3,69 & 3,68 & n.b. & 14,9 & 14,9 & \begin{tabular}{|c|}
$23,0+-$ \\
2,9 \\
\end{tabular} & $\begin{array}{c}88,3+- \\
3,8 \\
\end{array}$ & n.b. & $\begin{array}{c}0,30+- \\
0,14 \\
\end{array}$ & $\begin{array}{c}0,22+- \\
0,03 \\
\end{array}$ & \\
\hline z-Richtung & 3,25 & 3,44 & 3,40 & n.b. & n.b. & 2,32 & 1,85 & 6,02 & 3,64 & 3,51 & n.b. & 30,3 & 23,4 & \begin{tabular}{|c|}
$27,8+-$ \\
2,2 \\
\end{tabular} & $\begin{array}{c}83,8+- \\
3,6\end{array}$ & n.b. & $\begin{array}{c}0,20+- \\
0,07 \\
\end{array}$ & $\begin{array}{c}0,23+- \\
0,04\end{array}$ & \\
\hline Mittelwert & 3,03 & 3,21 & 3,11 & 2,23 & 1,9 & 2,1 & 1,84 & 6,12 & 3,67 & 3,51 & n.b. & 24,9 & 20,7 & $\begin{array}{c}25,9+- \\
4,1 \\
\end{array}$ & $\begin{array}{c}85,0+- \\
6,2\end{array}$ & n.b. & 0,25 & 0,24 & $\begin{array}{c}86,9+- \\
2,3\end{array}$ \\
\hline $\begin{array}{c}\text { Anisotropie } \\
{[\%]}\end{array}$ & 31,0 & 30,6 & 30,9 & n.b. & n.b. & 21,6 & 1,6 & 3,1 & 1,4 & 9,2 & n.b. & 50,8 & 37,1 & \begin{tabular}{|c|}
$17,2+-$ \\
12,1 \\
\end{tabular} & 5,9 & n.b. & 34,3 & 18,5 & \\
\hline \multicolumn{20}{|l|}{$\begin{array}{l}\text { Proben } \\
\text { Gruppe II }\end{array}$} \\
\hline $\begin{array}{l}\text { Peridotit } \\
\text { (Pi1a) x- } \\
\text { Richtung }\end{array}$ & 8,03 & 8,14 & 7,84 & 4,68 & 4,61 & 4,60 & 4,50 & 8,44 & 4,83 & 4,76 & $\begin{array}{c}180,0+- \\
13,4\end{array}$ & 174,7 & 169,4 & $\begin{array}{c}172,3+ \\
-5,7\end{array}$ & $\begin{array}{l}193,8 \\
+-5,9\end{array}$ & $\begin{array}{c}0,25+- \\
0,07\end{array}$ & $\begin{array}{c}0,25+- \\
0,03\end{array}$ & $\begin{array}{c}0,26+- \\
0,02\end{array}$ & \\
\hline y-Richtung & 7,67 & 7,78 & 7,71 & 4,65 & 4,55 & 4,68 & 4,45 & 8,23 & 4,81 & 4,66 & $\begin{array}{c}172,3+- \\
13,8\end{array}$ & 176,5 & 165,2 & \begin{tabular}{|c|}
$171,2+$ \\
$-5,8$
\end{tabular} & $\begin{array}{l}187,6 \\
+-5,9\end{array}$ & $\begin{array}{c}0,22+- \\
0,08\end{array}$ & $\begin{array}{c}0,23+- \\
0,03\end{array}$ & $\begin{array}{c}0,25+- \\
0,02\end{array}$ & \\
\hline z-Richtung & 7,82 & 7,84 & 7,72 & 4,74 & 4,58 & 4,60 & 4,50 & 8,19 & 4,85 & 4,70 & $\begin{array}{c}177,65+- \\
13,9 \\
\end{array}$ & 172,9 & 167,9 & $\begin{array}{c}170,6+ \\
-5,8 \\
\end{array}$ & $\begin{array}{l}189,2 \\
+-6,0 \\
\end{array}$ & $\begin{array}{c}0,22+- \\
0,07\end{array}$ & $\begin{array}{c}0,23+- \\
0,03 \\
\end{array}$ & $\begin{array}{c}0,24+- \\
0,03\end{array}$ & \\
\hline Mittelwert & 7,84 & 7,92 & 7,76 & 4,69 & 4,58 & 4,63 & 4,48 & 8,29 & 4,83 & 4,71 & 176,6 & 174,7 & 167,5 & \begin{tabular}{|c|}
$171,4+$ \\
$-10,0$ \\
\end{tabular} & $\begin{array}{r}190,2 \\
+-10,3 \\
\end{array}$ & 0,23 & 0,24 & 0,25 & $\begin{array}{r}190,4 \\
+-3,4 \\
\end{array}$ \\
\hline $\begin{array}{c}\text { Anisotropie } \\
{[\%]}\end{array}$ & 4,5 & 4,4 & 1,7 & 1,9 & 1,3 & 1,7 & 1,1 & 3,0 & 0,8 & 2,1 & 4,2 & 2,0 & 2,5 & 1,0 & 3,2 & 11,8 & 6,5 & & \\
\hline $\begin{array}{c}\text { Marmor (Rek) } \\
\text { x-Richtung }\end{array}$ & 5,56 & 6,1 & 5,06 & 3,22 & 3,05 & 3,05 & 2,80 & 6,88 & 3,69 & 3,68 & $\begin{array}{c}67,7+- \\
7,2\end{array}$ & 61,3 & 54,5 & \begin{tabular}{|c|}
$58,1+-$ \\
3,0 \\
\end{tabular} & $\begin{array}{c}96,0+- \\
3,5\end{array}$ & $\begin{array}{c}0,27+- \\
0,10 \\
\end{array}$ & $\begin{array}{c}0,25+- \\
0,04 \\
\end{array}$ & $\begin{array}{c}0,30+- \\
0,03\end{array}$ & \\
\hline$y$-Richtung & 5,69 & 6,26 & 5,58 & 3,21 & 3,04 & 3,09 & 3,03 & 7,02 & 3,75 & 3,60 & $\begin{array}{c}68,2+- \\
7,0 \\
\end{array}$ & 66,3 & 64,3 & \begin{tabular}{|c|}
$65,5+-$ \\
3,0 \\
\end{tabular} & $\begin{array}{c}96,3+- \\
3,5\end{array}$ & $\begin{array}{c}0,28+- \\
0,10 \\
\end{array}$ & $\begin{array}{c}0,28+- \\
0,04 \\
\end{array}$ & $\begin{array}{c}0,31+- \\
0,03\end{array}$ & \\
\hline z-Richtung & 5,21 & 5,61 & 4,60 & 3,06 & 3,05 & 2,97 & 2,97 & 6,60 & 3,64 & 3,53 & $\begin{array}{c}62,9+- \\
7,2 \\
\end{array}$ & 54,7 & 54,7 & $\begin{array}{c}54,8+- \\
3,4 \\
\end{array}$ & $\begin{array}{c}90,2+- \\
3,5\end{array}$ & $\begin{array}{c}0,24+- \\
0,11 \\
\end{array}$ & $\begin{array}{c}0,14+- \\
0,05 \\
\end{array}$ & $\begin{array}{c}0,29+- \\
0,03\end{array}$ & \\
\hline Mittelwert & 5,49 & 5,99 & 5,08 & 3,16 & 3,05 & 3,04 & 2,93 & 6,83 & 3,69 & 3,60 & 66,3 & 60,8 & 57,8 & \begin{tabular}{|c}
$59,5+-$ \\
5,4
\end{tabular} & $\begin{array}{c}94,2+- \\
6,0\end{array}$ & 0,27 & 0,23 & 0,30 & $\begin{array}{c}84,3+- \\
3,4\end{array}$ \\
\hline $\begin{array}{c}\text { Anisotropie } \\
{[\%]}\end{array}$ & 8,4 & 10,4 & 17,6 & 5,0 & 0,3 & 3,9 & 7,6 & 6,0 & 2,9 & 4,1 & 7,9 & 17,5 & 15,2 & \begin{tabular}{|c|}
$16,2+-$ \\
5,9
\end{tabular} & 6,3 & 16,2 & 50,0 & 6,6 & \\
\hline $\begin{array}{l}\text { Quarzit (Al) } \\
\text { x-Richtung }\end{array}$ & 5,18 & 5,34 & 5,06 & 3,72 & 3,60 & 3,74 & 3,61 & 6,16 & 4,04 & 4,02 & $\begin{array}{c}72,2+- \\
11,5\end{array}$ & 67,5 & 68,7 & \begin{tabular}{|c|}
$68,4+-$ \\
5,2 \\
\end{tabular} & $\begin{array}{c}98,0+- \\
4,6\end{array}$ & $\begin{array}{c}0,00+- \\
0,14 \\
\end{array}$ & $\begin{array}{c}-0,05+- \\
0,06 \\
\end{array}$ & $\begin{array}{c}0,12+- \\
0,04\end{array}$ & \\
\hline$y$-Richtung & 5,55 & 5,59 & 5,49 & 3,87 & 3,70 & 3,82 & 3,65 & 6,18 & 4,13 & 4,05 & $\begin{array}{c}82,1+- \\
11,0 \\
\end{array}$ & 80,8 & 79,0 & \begin{tabular}{|c|}
$80,2+-$ \\
4,5 \\
\end{tabular} & $\begin{array}{c}99,1+- \\
4,8 \\
\end{array}$ & $\begin{array}{c}0,06+- \\
0,12 \\
\end{array}$ & $\begin{array}{c}0,06+- \\
0,05 \\
\end{array}$ & $\begin{array}{c}0,10+- \\
0,04 \\
\end{array}$ & \\
\hline z-Richtung & 5,67 & 5,68 & 5,68 & 3,90 & 3,76 & 3,79 & 3,66 & 6,25 & 4,13 & 4,07 & $\begin{array}{c}85,3+- \\
10,9 \\
\end{array}$ & 85,4 & 82,8 & \begin{tabular}{|c|}
$83,8+-$ \\
4,3 \\
\end{tabular} & $\begin{array}{c}99,5+- \\
4,7 \\
\end{array}$ & $\begin{array}{c}0,08+- \\
0,12 \\
\end{array}$ & $\begin{array}{c}0,12+- \\
0,04 \\
\end{array}$ & $\begin{array}{c}0,10+- \\
0,04 \\
\end{array}$ & \\
\hline Mittelwert & 5,47 & 5,54 & 5,41 & 3,83 & 3,67 & 3,78 & 3,64 & 6,20 & 4,10 & 4,05 & 79,9 & 77,9 & 76,8 & \begin{tabular}{|c|}
$77,5+-$ \\
8,1 \\
\end{tabular} & $\begin{array}{c}98,9+- \\
8,1 \\
\end{array}$ & 0,05 & 0,04 & 0,11 & $\begin{array}{c}97,2+- \\
3,9 \\
\end{array}$ \\
\hline $\begin{array}{c}\begin{array}{c}\text { Anisotropie } \\
{[\%]}\end{array} \\
\end{array}$ & 8,6 & 6,0 & 10,9 & 4,6 & 4,3 & 2,1 & 1,4 & 1,4 & 2,2 & 1,2 & 15,3 & 21,0 & 17,0 & $\begin{array}{c}18,3+- \\
7,5 \\
\end{array}$ & 1,5 & 98,1 & 147,4 & 17,4 & \\
\hline
\end{tabular}




\begin{tabular}{|c|c|c|c|c|c|c|c|c|c|c|c|c|c|c|c|c|c|c|c|}
\hline & $\begin{array}{c}\mathrm{V} p_{0}(0,5 \\
\mathrm{MHz}) \\
{[\mathrm{km} / \mathrm{s}]} \\
\mathrm{a}\end{array}$ & $\begin{array}{c}\mathrm{Vp}_{0} \\
(\mathrm{Ku}) \\
{[\mathrm{km} / \mathrm{s}]} \\
\mathrm{b}\end{array}$ & $\begin{array}{c}\mathrm{Vp}_{0}(1 \\
\mathrm{MHz}) \\
{[\mathrm{km} / \mathrm{s}]} \\
\mathrm{c}\end{array}$ & $\begin{array}{c}\mathbf{V s}_{0 / 1}(0,5 \\
\mathrm{MHz}) \\
{[\mathrm{km} / \mathrm{s}]} \\
\mathrm{d}\end{array}$ & $\begin{array}{c}\mathbf{V s}_{0 / 2}(0,5 \\
\mathrm{MHz}) \\
{[\mathrm{km} / \mathrm{s}]} \\
e\end{array}$ & $\begin{array}{c}\mathrm{Vs}_{0 / 1}(1 \\
\mathrm{MHz}) \\
{[\mathrm{km} / \mathrm{s}]} \\
\mathrm{f}\end{array}$ & $\begin{array}{c}\mathrm{Vs}_{0 / 2}(1 \\
\mathrm{MHz}) \\
{[\mathrm{km} / \mathrm{s}]} \\
\mathrm{g}\end{array}$ & $\begin{array}{c}\mathrm{Vp}_{400} \\
{[\mathrm{~km} / \mathrm{s}]} \\
\mathrm{h}\end{array}$ & $\begin{array}{l}V_{\mathbf{s}_{400 / 1}} \\
{[\mathrm{~km} / \mathrm{s}]} \\
\mathbf{i}\end{array}$ & $\begin{array}{l}V_{s_{400 / 2}} \\
{[\mathrm{~km} / \mathrm{s}]}\end{array}$ & $\begin{array}{c}\mathrm{E}_{01 / 2}(0,5 \\
\mathrm{MHz}) \\
{[\mathrm{GPa}]} \\
\mathrm{k}\end{array}$ & $\begin{array}{c}\mathrm{E}_{0 / 1}(1 \\
\mathrm{MHz}) \\
{[\mathrm{GPa}]} \\
\mathrm{I}\end{array}$ & $\begin{array}{c}\mathrm{E}_{0 / 2}(1 \\
\mathrm{MHz}) \\
{[\mathrm{GPa}]} \\
\mathrm{m}\end{array}$ & $\begin{array}{c}\mathrm{E}_{01 / 2}(1 \\
\mathrm{MHz}) \\
{[\mathrm{GPa}]} \\
\mathrm{n}\end{array}$ & $\begin{array}{c}\mathrm{E}_{400} 1 / 2 \\
{[\mathrm{GPa}]} \\
0\end{array}$ & $\begin{array}{c}v_{01 / 2} \\
(0,5 \\
M H z) \\
p\end{array}$ & $\begin{array}{c}v_{01 / 2} \\
(1 \mathrm{MHz}) \\
q\end{array}$ & $\begin{array}{c}v_{400} \frac{1 / 2}{r} \\
r\end{array}$ & $\begin{array}{c}E_{\mathrm{VRH}} \\
{[\mathrm{GPa}]} \\
\mathbf{s}\end{array}$ \\
\hline $\begin{array}{l}\text { Meta-Gabbro } \\
\text { (MG1b) x- } \\
\text { Richtung }\end{array}$ & 5,69 & 5,38 & 5,39 & 3,35 & 3,30 & 3,28 & 3,22 & 7,59 & 4,25 & 4,20 & $\begin{array}{c}83,9+- \\
8,9\end{array}$ & 79,1 & 77,3 & $\begin{array}{c}78,5+- \\
3,8\end{array}$ & $\begin{array}{l}139,3 \\
+-4,7\end{array}$ & $\begin{array}{c}0,24+- \\
0,10\end{array}$ & $\begin{array}{c}0,21+- \\
0,04\end{array}$ & $\begin{array}{c}0,28+- \\
0,03\end{array}$ & \\
\hline y-Richtung & 4,72 & 5,11 & 4,41 & 3,23 & 3,03 & 3,00 & 2,78 & 7,22 & 4,07 & 3,79 & $\begin{array}{c}66,4+- \\
9,8 \\
\end{array}$ & 58,7 & 55,1 & \begin{tabular}{|c|}
$57,4+-$ \\
3,8 \\
\end{tabular} & $\begin{array}{l}121,9 \\
+-4,3 \\
\end{array}$ & $\begin{array}{c}0,11+- \\
0,14 \\
\end{array}$ & $\begin{array}{c}0,12+- \\
0,06 \\
\end{array}$ & $\begin{array}{c}0,29+- \\
0,03 \\
\end{array}$ & \\
\hline z-Richtung & 4,92 & 5,18 & 4,69 & 3,30 & 3,03 & 3,13 & 2,82 & 7,14 & 4,15 & 3,98 & $\begin{array}{c}70,3+- \\
9,5\end{array}$ & 65,6 & 59,0 & $\begin{array}{c}63,0+- \\
3,7\end{array}$ & $\begin{array}{l}127,4 \\
+-4,6\end{array}$ & $\begin{array}{c}0,15+- \\
0,13\end{array}$ & $\begin{array}{c}0,16+- \\
0,05\end{array}$ & $\begin{array}{c}0,26+- \\
0,03\end{array}$ & \\
\hline Mittelwert & 5,11 & 5,22 & 4,83 & 3,29 & 3,12 & 3,14 & 2,94 & 7,32 & 4,16 & 3,99 & 73,6 & 67,8 & 63,8 & $\begin{array}{c}66,3+- \\
6,5\end{array}$ & $\begin{array}{l}129,6 \\
+-7,8\end{array}$ & 0,17 & 0,17 & 0,28 & $\begin{array}{l}120,6 \\
+-5,8\end{array}$ \\
\hline $\begin{array}{c}\text { Anisotropie } \\
{[\%]}\end{array}$ & 17,0 & 5,0 & 18,1 & 3,6 & 8,2 & 8,5 & 13,7 & 5,9 & 4,2 & 9,8 & 21,0 & 25,8 & 28,7 & \begin{tabular}{|c|}
$26,9+-$ \\
5,9 \\
\end{tabular} & 12,5 & 55,3 & 42,3 & 10,1 & \\
\hline $\begin{array}{l}\text { Meta-Gabbro } \\
\text { (MG2) x- } \\
\text { Richtung }\end{array}$ & 6,64 & 6,55 & 6,05 & 3,82 & 3,65 & 3,58 & 3,21 & 6,16 & 4,55 & 3,98 & $\begin{array}{c}109,4+- \\
9,7\end{array}$ & 97,6 & 83,2 & $\begin{array}{c}90,5+- \\
3,8\end{array}$ & $\begin{array}{l}140,6 \\
+-6,2\end{array}$ & $\begin{array}{c}0,27+- \\
0,08\end{array}$ & $\begin{array}{c}0,27+- \\
0,03\end{array}$ & $\begin{array}{c}0,25+- \\
0,04\end{array}$ & \\
\hline y-Richtung & 6,42 & 6,25 & 6,37 & 3,76 & 3,60 & 3,80 & 3,37 & 6,43 & 4,60 & 4,07 & $\begin{array}{c}105,1+- \\
9,7\end{array}$ & 109,4 & 91,8 & $\begin{array}{c}100,7+ \\
-4,0 \\
\end{array}$ & $\begin{array}{l}141,6 \\
+-6,0 \\
\end{array}$ & $\begin{array}{c}0,26+- \\
0,09 \\
\end{array}$ & $\begin{array}{c}0,27+- \\
0,03\end{array}$ & $\begin{array}{c}0,22+- \\
0,04\end{array}$ & \\
\hline z-Richtung & 6,10 & 6,15 & 5,93 & 3,63 & 3,60 & 3,40 & 3,33 & 6,01 & 3,94 & 3,92 & $\begin{array}{c}99,3+- \\
9,9\end{array}$ & 89,8 & 87,2 & $\begin{array}{c}88,3+- \\
3,8 \\
\end{array}$ & $\begin{array}{l}119,8 \\
+-5,2\end{array}$ & $\begin{array}{c}0,23+- \\
0,09\end{array}$ & $\begin{array}{c}0,26+- \\
0,03\end{array}$ & $\begin{array}{c}0,25+- \\
0,04\end{array}$ & \\
\hline Mittelwert & 6,39 & 6,32 & 6,12 & 3,74 & 3,62 & 3,59 & 3,30 & 6,20 & 4,36 & 3,99 & 104,6 & 98,9 & 87,4 & $\begin{array}{c}93,2+- \\
6,8\end{array}$ & $\begin{array}{l}134,0 \\
+-7,8\end{array}$ & 0,25 & 0,27 & 0,24 & $\begin{array}{l}120,6 \\
+-5,8\end{array}$ \\
\hline $\begin{array}{c}\text { Anisotropie } \\
{[\%]}\end{array}$ & 8,1 & 6,1 & 6,9 & 5,0 & 1,4 & 10,5 & 4,7 & 6,5 & 14,3 & 3,7 & 9,2 & 17,9 & 9,4 & \begin{tabular}{|c|}
$12,3+-$ \\
5,2 \\
\end{tabular} & 15,4 & 14,6 & 2,8 & 13,9 & \\
\hline $\begin{array}{l}\text { granod. } \\
\text { Protomylonit } \\
\text { (Iv1) x- } \\
\text { Richtung } \\
\end{array}$ & 4,96 & 4,89 & 4,45 & 2,89 & 2,88 & 2,61 & 2,61 & 6,42 & 4,05 & 3,91 & $\begin{array}{c}53,5+- \\
6,7\end{array}$ & 46,8 & 46,8 & $\begin{array}{c}47,1+- \\
2,7\end{array}$ & $\begin{array}{l}105,0 \\
+-4,4\end{array}$ & $\begin{array}{c}0,24+- \\
0,11\end{array}$ & $\begin{array}{c}0,24+- \\
0,05\end{array}$ & $\begin{array}{c}0,19+- \\
0,04\end{array}$ & \\
\hline y-Richtung & 4,37 & 4,18 & 4,00 & 2,92 & 2,82 & 2,60 & 2,48 & 6,24 & 3,96 & 3,96 & $\begin{array}{c}49,9+- \\
7,9\end{array}$ & 42,6 & 40,6 & \begin{tabular}{|c|}
$48,2+-$ \\
3,0
\end{tabular} & $\begin{array}{c}99,4+- \\
4,2\end{array}$ & $\begin{array}{c}0,12+- \\
0,15\end{array}$ & $\begin{array}{c}0,14+- \\
0,05\end{array}$ & $\begin{array}{c}0,20+- \\
0,04\end{array}$ & \\
\hline z-Richtung & 4,30 & 4,28 & 4,25 & 2,89 & 2,76 & 2,75 & 2,62 & 6,31 & 3,91 & 3,78 & $\begin{array}{c}51,5+- \\
8,0\end{array}$ & 47,9 & 45,5 & \begin{tabular}{|c|}
$41,8+-$ \\
2,9 \\
\end{tabular} & $\begin{array}{l}101,8 \\
+-4,5 \\
\end{array}$ & $\begin{array}{c}0,12+- \\
0,15 \\
\end{array}$ & $\begin{array}{c}0,16+- \\
0,06\end{array}$ & $\begin{array}{c}0,16+- \\
0,04\end{array}$ & \\
\hline Mittelwert & 4,54 & 4,45 & 4,23 & 2,90 & 2,82 & 2,65 & 2,57 & 6,32 & 3,97 & 3,65 & 53,1 & 45,8 & 44,3 & \begin{tabular}{|c|}
$45,7+-$ \\
5,0 \\
\end{tabular} & $\begin{array}{l}102,0 \\
+-7,6 \\
\end{array}$ & 0,16 & 0,18 & 0,19 & $\begin{array}{c}95,3+- \\
6,8 \\
\end{array}$ \\
\hline $\begin{array}{c}\text { Anisotropie } \\
{[\%]}\end{array}$ & 13,3 & 14,5 & 10,1 & 1,0 & 4,2 & 5,5 & 5,3 & 2,8 & 3,5 & 0,8 & 13,7 & 11,1 & 13,2 & \begin{tabular}{|c|}
$13,2+-$ \\
8,8 \\
\end{tabular} & 5,3 & 50,8 & 42,1 & 20,4 & \\
\hline $\begin{array}{c}\text { granit. Gneis } \\
\text { (Gra1a) x- } \\
\text { Richtung } \\
\end{array}$ & 5,12 & 5,25 & 5,18 & 3,21 & 3,17 & 3,17 & 3,15 & 6,16 & 3,63 & 3,63 & $\begin{array}{c}65,2+- \\
8,0\end{array}$ & 68,1 & 65,4 & $\begin{array}{c}65,1+- \\
3,3\end{array}$ & $\begin{array}{c}88,1+- \\
3,7\end{array}$ & $\begin{array}{c}0,18+- \\
0,12\end{array}$ & $\begin{array}{c}0,20+- \\
0,04\end{array}$ & $\begin{array}{c}0,23+- \\
0,03\end{array}$ & \\
\hline$y$-Richtung & 5,25 & 5,46 & 5,23 & 3,24 & 3,16 & 3,28 & 3,19 & 6,18 & 3,62 & 3,58 & $\begin{array}{c}66,9+- \\
7,9 \\
\end{array}$ & 68,1 & 65,9 & \begin{tabular}{|c|}
$67,5+-$ \\
3,5 \\
\end{tabular} & $\begin{array}{c}87,3+- \\
3,6\end{array}$ & $\begin{array}{c}0,20+- \\
0,11 \\
\end{array}$ & $\begin{array}{c}0,19+- \\
0,04\end{array}$ & $\begin{array}{c}0,24+- \\
0,03\end{array}$ & \\
\hline & & & & & & & & & & & $65,5+-$ & & & $65,2+-$ & $88,4+-$ & $0,18+-$ & $0,20+-$ & $0,25+-$ & \\
\hline Mittelwert & 5,16 & 5,32 & 5,20 & 3,22 & 3,17 & 3,21 & 3,17 & 6,20 & 3,62 & 3,61 & 65,9 & 67,0 & 65,3 & $\begin{array}{c}66,0+- \\
5,8\end{array}$ & $\begin{array}{c}88,0+- \\
6,3\end{array}$ & 0,19 & 0,20 & 0,24 & $\begin{array}{c}88,6+- \\
2,1\end{array}$ \\
\hline
\end{tabular}




\begin{tabular}{|c|c|c|c|c|c|c|c|c|c|c|c|c|c|c|c|c|c|c|c|}
\hline & $\begin{array}{c}\mathrm{V} p_{0}(0,5 \\
\mathrm{MHz}) \\
{[\mathrm{km} / \mathrm{s}]} \\
\mathrm{a}\end{array}$ & $\begin{array}{c}\mathrm{Vp}_{0} \\
(\mathrm{Ku}) \\
{[\mathrm{km} / \mathrm{s}]} \\
\mathrm{b}\end{array}$ & $\begin{array}{c}\mathrm{Vp}_{0}(1 \\
\mathrm{MHz}) \\
{[\mathrm{km} / \mathrm{s}]} \\
\mathrm{c}\end{array}$ & $\begin{array}{c}\mathbf{S s}_{0 / 1}(0,5 \\
\mathrm{MHz}) \\
{[\mathrm{km} / \mathrm{s}]} \\
\mathrm{d}\end{array}$ & $\begin{array}{c}\mathrm{Vs}_{0 / 2}(0,5 \\
\mathrm{MHz}) \\
{[\mathrm{km} / \mathrm{s}]} \\
\mathrm{e}\end{array}$ & $\begin{array}{c}\mathbf{V s}_{0 / 1}(1 \\
\mathrm{MHz}) \\
{[\mathrm{km} / \mathrm{s}]} \\
\mathrm{f}\end{array}$ & $\begin{array}{c}\mathrm{Vs}_{0 / 2}(1 \\
\mathrm{MHz}) \\
{[\mathrm{km} / \mathrm{s}]} \\
\mathrm{g}\end{array}$ & $\begin{array}{l}\mathrm{Vp}_{400} \\
{[\mathrm{~km} / \mathrm{s}]} \\
\mathrm{h}\end{array}$ & $\begin{array}{l}V_{\mathbf{s}_{400 / 1}} \\
{[\mathrm{~km} / \mathbf{s}]} \\
\mathbf{i}\end{array}$ & $\begin{array}{l}\mathrm{Vs}_{400 / 2} \\
{[\mathrm{~km} / \mathrm{s}]} \\
\mathbf{j}\end{array}$ & $\begin{array}{c}\mathrm{E}_{01 / 2}(0,5 \\
\mathrm{MHz}) \\
{[\mathrm{GPa}]} \\
\mathrm{k}\end{array}$ & $\begin{array}{c}\mathrm{E}_{0 / 1}(1 \\
\mathrm{MHz}) \\
{[\mathrm{GPa}]} \\
\mathrm{I}\end{array}$ & $\begin{array}{c}\mathrm{E}_{0 / 2}(1 \\
\mathrm{MHz}) \\
{[\mathrm{GPa}]} \\
\mathrm{m}\end{array}$ & $\begin{array}{c}\mathrm{E}_{01 / 2}(1 \\
\mathrm{MHz}) \\
{[\mathrm{GPa}]} \\
\mathrm{n}\end{array}$ & $\begin{array}{c}\mathrm{E}_{400} 1 / 2 \\
{[\mathrm{GPa}]} \\
0\end{array}$ & $\begin{array}{c}v_{01 / 2} \\
(\mathbf{0}, 5 \\
\mathbf{M H z}) \\
\mathbf{p}\end{array}$ & $\begin{array}{c}v_{01 / 2} \\
(1 \mathrm{MHz}) \\
q\end{array}$ & $v_{4001 / 2}$ & $\begin{array}{c}E_{\mathrm{VRH}} \\
{[\mathrm{GPa}]} \\
\mathbf{s}\end{array}$ \\
\hline $\begin{array}{c}\text { Anisotropie } \\
{[\%]}\end{array}$ & 2,5 & 3,8 & 1,0 & 0,9 & 0,9 & 3,4 & 1,3 & 1,4 & 0,3 & 1,4 & 2,4 & 4,7 & 1,8 & $\begin{array}{c}3,5+- \\
7,2 \\
\end{array}$ & 1,3 & 12,2 & 6,6 & 5,9 & \\
\hline \multicolumn{20}{|l|}{$\begin{array}{c}\text { Proben } \\
\text { Gruppe III }\end{array}$} \\
\hline $\begin{array}{c}\text { granit. } \\
\text { Protomylonit } \\
\text { (MrMy5) x- } \\
\text { Richtung }\end{array}$ & 3,78 & 3,95 & 3,44 & 2,31 & 2,08 & 1,99 & 1,99 & 6,32 & 3,69 & 3,59 & $\begin{array}{c}32,0+- \\
5,0\end{array}$ & 26,1 & 26,1 & $\begin{array}{c}26,4+- \\
2,0\end{array}$ & $\begin{array}{c}88,6+- \\
3,6\end{array}$ & $\begin{array}{c}0,25+- \\
0,15\end{array}$ & $\begin{array}{c}0,25+- \\
0,06\end{array}$ & $\begin{array}{c}0,25+- \\
0,03\end{array}$ & \\
\hline y-Richtung & 3,53 & 3,58 & 3,45 & 2,27 & 2,02 & 1,26 & 1,91 & 6,32 & 3,80 & 3,58 & $\begin{array}{c}30,0+- \\
5,1 \\
\end{array}$ & 30,3 & 24,7 & \begin{tabular}{|c|}
$18,3+-$ \\
1,4 \\
\end{tabular} & $\begin{array}{c}90,3+- \\
3,7 \\
\end{array}$ & $\begin{array}{c}0,21+- \\
0,17 \\
\end{array}$ & $\begin{array}{c}0,37+- \\
0,05 \\
\end{array}$ & $\begin{array}{c}0,24+- \\
0,03 \\
\end{array}$ & \\
\hline z-Richtung & 2,99 & 2,98 & 2,88 & 2,09 & 1,95 & 1,95 & 1,80 & 6,07 & 3,74 & 3,62 & $\begin{array}{c}23,5+- \\
5,6\end{array}$ & 21,6 & 20,2 & $\begin{array}{c}21,3+- \\
2,1 \\
\end{array}$ & $\begin{array}{c}87,5+- \\
3,8\end{array}$ & $\begin{array}{c}0,08+- \\
0,22 \\
\end{array}$ & $\begin{array}{c}0,13+- \\
0,08\end{array}$ & $\begin{array}{c}0,21+- \\
0,04\end{array}$ & \\
\hline Mittelwert & 3,43 & 3,50 & 3,26 & 3,22 & 2,02 & 1,73 & 1,9 & 6,24 & 3,74 & 3,60 & 28,4 & 26,0 & 23,7 & $\begin{array}{c}22,0+- \\
3,2\end{array}$ & $\begin{array}{c}88,8+- \\
6,4\end{array}$ & 0,18 & 0,25 & 0,23 & $\begin{array}{c}87,5+- \\
4,2\end{array}$ \\
\hline $\begin{array}{c}\text { Anisotropie } \\
{[\%]}\end{array}$ & 20,9 & 24,6 & 16,5 & 9,5 & 6,3 & 36,7 & 9,5 & 4,0 & 2,9 & 1,1 & 26,6 & 28,7 & 22,6 & \begin{tabular}{|c|}
$30,6+-$ \\
10,7 \\
\end{tabular} & 3,1 & 67,4 & 63,9 & 16,8 & \\
\hline $\begin{array}{c}\text { granod. } \\
\text { Gneis (Be1) } \\
\text { x-Richtung }\end{array}$ & 3,83 & 3,94 & 3,40 & 2,34 & 2,03 & 2,38 & 2,06 & 6,43 & 3,77 & 3,47 & $\begin{array}{c}32,7+- \\
5,1\end{array}$ & 31,1 & 27,7 & $\begin{array}{c}30,3+- \\
2,5\end{array}$ & $\begin{array}{c}90,4+- \\
3,6\end{array}$ & $\begin{array}{c}0,26+- \\
0,15\end{array}$ & $\begin{array}{c}0,13+- \\
0,07\end{array}$ & $\begin{array}{c}0,27+- \\
0,03\end{array}$ & \\
\hline$y$-Richtung & 3,40 & 3,65 & 3,35 & 2,35 & 2,04 & 2,10 & 1,95 & 6,41 & 3,82 & 3,39 & $\begin{array}{c}29,9+- \\
5,9 \\
\end{array}$ & 27,9 & 25,5 & \begin{tabular}{|c|}
$27,0+-$ \\
2,1 \\
\end{tabular} & $\begin{array}{c}89,7+- \\
3,6 \\
\end{array}$ & $\begin{array}{c}0,14+- \\
0,18 \\
\end{array}$ & $\begin{array}{c}0,21+- \\
0,07 \\
\end{array}$ & $\begin{array}{c}0,27+- \\
0,03 \\
\end{array}$ & \\
\hline z-Richtung & 2,58 & 2,81 & 2,30 & n.b. & n.b. & 1,93 & 1,91 & 6,17 & 3,60 & 3,51 & n.b. & 6,2 & 7,7 & $\begin{array}{c}7,0+- \\
6,4\end{array}$ & $\begin{array}{c}86,0+- \\
3,6\end{array}$ & n.b. & $\begin{array}{c}-0,64+- \\
0,31\end{array}$ & $\begin{array}{c}0,25+- \\
0,03\end{array}$ & \\
\hline Mittelwert & 3,27 & 3,47 & 3,02 & 2,35 & 2,03 & 2,14 & 1,96 & 6,34 & 3,73 & 3,46 & n.b. & 21,63 & 20,3 & $\begin{array}{c}21,4+- \\
7,2\end{array}$ & $\begin{array}{c}88,7+- \\
6,2\end{array}$ & n.b. & $-0,10$ & 0,26 & $\begin{array}{c}87,7+- \\
4,2\end{array}$ \\
\hline $\begin{array}{c}\text { Anisotropie } \\
{[\%]} \\
\end{array}$ & 32,6 & 28,7 & 32,4 & 0,4 & 0,5 & 18,9 & 5,9 & 4,0 & 5,8 & 3,4 & n.b. & 80,1 & 72,2 & \begin{tabular}{|c|}
$76,8+-$ \\
10,3 \\
\end{tabular} & 4,8 & n.b. & 406,2 & 6,4 & \\
\hline $\begin{array}{c}\text { granod. } \\
\text { Mylonit (Iv1b) } \\
\text { x-Richtung }\end{array}$ & 4,80 & 4,81 & 4,99 & 2,90 & 2,71 & 2,90 & 2,68 & 6,67 & 3,86 & 3,64 & $\begin{array}{c}53,5+- \\
6,7\end{array}$ & 57,2 & 50,9 & $\begin{array}{c}54,3+- \\
2,8\end{array}$ & $\begin{array}{c}97,8+- \\
3,7\end{array}$ & $\begin{array}{c}0,24+- \\
0,12\end{array}$ & $\begin{array}{c}0,27+- \\
0,04\end{array}$ & $\begin{array}{c}0,27+- \\
0,03\end{array}$ & \\
\hline$y$-Richtung & 4,33 & 4,50 & 4,51 & 2,89 & 2,62 & 2,89 & 2,66 & 6,30 & 3,82 & 3,66 & $\begin{array}{c}48,2+- \\
7,2 \\
\end{array}$ & 52,5 & 47,6 & \begin{tabular}{|c|}
$50,4+-$ \\
3,0 \\
\end{tabular} & $\begin{array}{c}94,1+- \\
3,9 \\
\end{array}$ & $\begin{array}{c}0,16+- \\
0,14 \\
\end{array}$ & $\begin{array}{c}0,20+- \\
0,05 \\
\end{array}$ & $\begin{array}{c}0,23+- \\
0,03 \\
\end{array}$ & \\
\hline z-Richtung & 3,60 & 3,53 & 3,51 & 2,62 & 2,61 & 2,73 & 2,55 & 6,11 & 3,68 & 3,64 & $\begin{array}{c}35,3+- \\
9,0\end{array}$ & 29,8 & 33,4 & \begin{tabular}{|c|}
$32,4+-$ \\
4,3 \\
\end{tabular} & $\begin{array}{c}89,6+- \\
3,8\end{array}$ & $\begin{array}{c}-0,05+- \\
0,21 \\
\end{array}$ & $\begin{array}{c}-0,15+- \\
0,10\end{array}$ & $\begin{array}{c}0,22+- \\
0,04\end{array}$ & \\
\hline Mittelwert & 4,24 & 4,28 & 4,34 & 2,80 & 2,65 & 2,84 & 2,63 & 6,36 & 3,79 & 3,65 & 46,7 & 46,5 & 44,0 & $\begin{array}{c}45,7+- \\
5,9\end{array}$ & $\begin{array}{c}93,8+- \\
6,6\end{array}$ & 0,11 & 0,11 & 0,24 & $\begin{array}{c}95,3+- \\
6,8\end{array}$ \\
\hline $\begin{array}{c}\text { Anisotropie } \\
{[\%]}\end{array}$ & 25,0 & 26,6 & 29,7 & 9,7 & 3,7 & 5,9 & 4,9 & 8,4 & 4,7 & 0,5 & 34,0 & 47,9 & 34,4 & $\begin{array}{c}40,3+- \\
7,3 \\
\end{array}$ & 8,4 & 124,3 & 155,0 & 18,1 & \\
\hline $\begin{array}{l}\text { granod. } \\
\text { Paragneis } \\
\text { (Cal) x- } \\
\text { Richtung }\end{array}$ & 4,19 & 4,52 & 3,70 & 2,53 & 1,93 & 2,50 & 1,95 & 6,56 & 3,91 & 3,41 & $\begin{array}{c}35,4+- \\
4,9\end{array}$ & 36,7 & 27,0 & $\begin{array}{c}32,9+- \\
2,3\end{array}$ & $\begin{array}{c}93,2+- \\
3,6\end{array}$ & $\begin{array}{c}0,30+- \\
0,13\end{array}$ & $\begin{array}{c}0,22+- \\
0,06\end{array}$ & $\begin{array}{c}0,27+- \\
0,03\end{array}$ & \\
\hline$y$-Richtung & 3,81 & 3,80 & 3,50 & 2,57 & 1,89 & 2,42 & 1,82 & 6,41 & 3,84 & 3,42 & $\begin{array}{c}33,7+- \\
5,3\end{array}$ & 33,1 & 23,6 & \begin{tabular}{|c|}
$29,7+-$ \\
2,2
\end{tabular} & $\begin{array}{c}88,7+- \\
3,6\end{array}$ & $\begin{array}{c}0,24+- \\
0,15\end{array}$ & $\begin{array}{c}0,21+- \\
0,06\end{array}$ & $\begin{array}{c}0,28+- \\
0,03\end{array}$ & \\
\hline
\end{tabular}




\begin{tabular}{|c|c|c|c|c|c|c|c|c|c|c|c|c|c|c|c|c|c|c|c|}
\hline & $\begin{array}{c}\mathrm{V} p_{0}(0,5 \\
\mathrm{MHz}) \\
{[\mathrm{km} / \mathrm{s}]} \\
\mathrm{a}\end{array}$ & $\begin{array}{c}\mathbf{V p}_{0} \\
(\mathrm{Ku}) \\
{[\mathrm{km} / \mathrm{s}]} \\
\mathbf{b}\end{array}$ & $\begin{array}{c}\mathrm{Vp}_{0}(1 \\
\mathrm{MHz}) \\
{[\mathrm{km} / \mathrm{s}]} \\
\mathrm{c}\end{array}$ & $\begin{array}{c}\mathbf{V s}_{0 / 1}(0,5 \\
\mathrm{MHz}) \\
{[\mathrm{km} / \mathrm{s}]} \\
\mathrm{d}\end{array}$ & $\begin{array}{c}\mathbf{V s}_{0 / 2}(0,5 \\
\mathrm{MHz}) \\
{[\mathrm{km} / \mathrm{s}]} \\
e\end{array}$ & $\begin{array}{c}\mathrm{Vs}_{0 / 1}(1 \\
\mathrm{MHz}) \\
{[\mathrm{km} / \mathrm{s}]} \\
\mathrm{f}\end{array}$ & $\begin{array}{c}\mathrm{Vs}_{0 / 2}(1 \\
\mathrm{MHz}) \\
{[\mathrm{km} / \mathrm{s}]} \\
\mathrm{g}\end{array}$ & $\begin{array}{c}V p_{400} \\
{[\mathrm{~km} / \mathrm{s}]} \\
\mathrm{h}\end{array}$ & $\begin{array}{c}V_{s_{400 / 1}} \\
{[\mathbf{k m} / \mathbf{s}]} \\
\mathbf{i}\end{array}$ & $\begin{array}{l}\mathrm{Vs}_{400 / 2} \\
{[\mathrm{~km} / \mathrm{s}]} \\
\mathrm{j}\end{array}$ & $\begin{array}{c}\mathrm{E}_{01 / 2}(0,5 \\
\mathrm{MHz}) \\
{[\mathrm{GPa}]} \\
\mathrm{k}\end{array}$ & $\begin{array}{c}\mathrm{E}_{0 / 1}(1 \\
\mathrm{MHz}) \\
{[\mathrm{GPa}]} \\
\mathrm{I}\end{array}$ & $\begin{array}{c}\mathrm{E}_{0 / 2}(1 \\
\mathrm{MHz}) \\
{[\mathrm{GPa}]} \\
\mathrm{m}\end{array}$ & $\begin{array}{c}\mathrm{E}_{01 / 2}(1 \\
\mathrm{MHz}) \\
{[\mathrm{GPa}]} \\
\mathrm{n}\end{array}$ & $\begin{array}{c}\mathrm{E}_{400} 1 / 2 \\
{[\mathrm{GPa}]} \\
0\end{array}$ & $\begin{array}{c}v_{01 / 2} \\
(0,5 \\
M H z) \\
p\end{array}$ & $\begin{array}{c}v_{01 / 2} \\
(1 \mathrm{MHz}) \\
q\end{array}$ & $v_{4001 / 2}$ & $\begin{array}{c}E_{\mathrm{VRH}} \\
{[\mathrm{GPa}]} \\
\mathbf{s}\end{array}$ \\
\hline z-Richtung & 2,04 & 2,26 & 2,25 & n.b. & n.b. & 1,81 & 1,74 & 5,89 & 3,41 & 3,36 & n.b. & 10,4 & 12,4 & $\begin{array}{c}11,6+- \\
3,6\end{array}$ & $\begin{array}{c}78,4+- \\
3,4\end{array}$ & n.b. & $\begin{array}{c}-0,32+- \\
0,20\end{array}$ & $\begin{array}{c}0,25+- \\
0,04\end{array}$ & \\
\hline Mittelwert & 3,35 & 3,53 & 3,15 & 2,55 & 1,91 & 2,24 & 1,84 & 6,29 & 3,72 & 3,40 & n.b. & 26,7 & 21,0 & \begin{tabular}{|c|}
$24,7+-$ \\
4,9 \\
\end{tabular} & $\begin{array}{c}86,8+- \\
6,1 \\
\end{array}$ & n.b. & 0,03 & 0,27 & $\begin{array}{c}89,0+- \\
3,8 \\
\end{array}$ \\
\hline $\begin{array}{c}\text { Anisotropie } \\
{[\%]}\end{array}$ & 51,3 & 50,0 & 39,2 & 1,6 & 2,1 & 27,6 & 10,8 & 10,2 & 12,8 & 1,8 & n.b. & 71,7 & 54,1 & \begin{tabular}{|c|}
$64,6+-$ \\
9,3 \\
\end{tabular} & 15,6 & n.b. & 249,5 & 7,9 & \\
\hline $\begin{array}{l}\text { granit. } \\
\text { Orthogneis } \\
\text { (VA) x- } \\
\text { Richtung }\end{array}$ & 4,12 & 4,21 & 4,13 & 2,44 & 2,10 & 2,44 & 2,21 & 6,34 & 3,80 & 3,51 & $\begin{array}{c}35,8+- \\
5,0\end{array}$ & 39,4 & 34,1 & $\begin{array}{c}37,2+- \\
2,3\end{array}$ & $\begin{array}{c}90,6+- \\
3,6\end{array}$ & $\begin{array}{c}0,28+- \\
0,13\end{array}$ & $\begin{array}{c}0,26+- \\
0,05\end{array}$ & $\begin{array}{c}0,25+- \\
0,03\end{array}$ & \\
\hline$y$-Richtung & 3,33 & 3,38 & 3,20 & 2,45 & 1,97 & 2,45 & 1,95 & 6,25 & 3,81 & 3,53 & $\begin{array}{c}29,3+- \\
6,1\end{array}$ & 25,5 & 24,6 & \begin{tabular}{|c|}
$27,6+-$ \\
2,7 \\
\end{tabular} & $\begin{array}{c}90,3+- \\
3,7\end{array}$ & $\begin{array}{c}0,11+- \\
0,19 \\
\end{array}$ & $\begin{array}{c}0,05+- \\
0,08\end{array}$ & $\begin{array}{c}0,24+- \\
0,03\end{array}$ & \\
\hline z-Richtung & 2,46 & 3,00 & 2,10 & 2,15 & 2,11 & 2,02 & 1,85 & 5,98 & 3,57 & 3,55 & $\begin{array}{l}0,1+- \\
29,93\end{array}$ & $-102,7$ & $-4,3$ & $\begin{array}{c}-26,6+- \\
36,5\end{array}$ & $\begin{array}{c}84,2+- \\
3,6\end{array}$ & $\begin{array}{c}-1,0+- \\
1,22\end{array}$ & $\begin{array}{c}-2,31+- \\
1,81\end{array}$ & $\begin{array}{c}0,23+- \\
0,04\end{array}$ & \\
\hline Mittelwert & 3,30 & 3,53 & 3,14 & 2,35 & 2,06 & 2,30 & 2,00 & 6,19 & 3,73 & 3,53 & 21,8 & $-12,6$ & 18,1 & \begin{tabular}{|c|}
$12,7+-$ \\
36,7
\end{tabular} & $\begin{array}{c}88,4+- \\
6,3\end{array}$ & $-0,20$ & $-0,66$ & 0,24 & $\begin{array}{c}87,5+- \\
2,4\end{array}$ \\
\hline $\begin{array}{c}\text { Anisotropie } \\
{[\%]}\end{array}$ & 40,3 & 28,7 & 49,2 & 12,2 & 6,6 & 17,6 & 16,3 & 5,7 & 6,3 & 1,1 & 99,8 & 360,7 & 112,6 & 171,7 & 7,1 & 453,7 & 962,6 & 10,2 & \\
\hline $\begin{array}{c}\text { granit. Gneis } \\
\text { (Gra1c) x- } \\
\text { Richtung }\end{array}$ & 5,25 & 5,37 & 5,47 & 3,26 & 3,19 & 3,30 & 3,25 & 6,14 & 3,63 & 3,63 & $\begin{array}{c}67,0+- \\
8,0\end{array}$ & 70,8 & 69,5 & $\begin{array}{c}70,4+- \\
3,4\end{array}$ & $\begin{array}{c}87,3+- \\
3,7\end{array}$ & $\begin{array}{c}0,20+- \\
0,11\end{array}$ & $\begin{array}{c}0,22+- \\
0,04\end{array}$ & $\begin{array}{c}0,23+- \\
0,03\end{array}$ & \\
\hline y-Richtung & 5,17 & 5,13 & 5,29 & 3,26 & 3,18 & 3,28 & 3,22 & 6,12 & 3,67 & 3,66 & $\begin{array}{c}66,0+- \\
8,1\end{array}$ & 66,5 & 65,3 & $\begin{array}{c}68+- \\
3,4\end{array}$ & $\begin{array}{c}88,2+- \\
3,8\end{array}$ & $\begin{array}{c}0,18+- \\
0,12\end{array}$ & $\begin{array}{c}0,20+- \\
0,04\end{array}$ & $\begin{array}{c}0,22+- \\
0,03\end{array}$ & \\
\hline z-Richtung & 5,07 & 5,09 & 5,12 & 3,24 & 3,22 & 3,11 & 3,14 & 6,17 & 3,66 & 3,66 & $\begin{array}{c}65,0+- \\
8,3 \\
\end{array}$ & 62,6 & 63,3 & \begin{tabular}{|c|}
$63,2+-$ \\
3,3 \\
\end{tabular} & $\begin{array}{c}88,5+- \\
3,7\end{array}$ & $\begin{array}{c}0,15+- \\
0,12\end{array}$ & $\begin{array}{c}0,20+- \\
0,04\end{array}$ & $\begin{array}{c}0,23+- \\
0,03\end{array}$ & \\
\hline Mittelwert & 5,16 & 5,20 & 5,29 & 3,25 & 3,20 & 3,23 & 3,20 & 6,20 & 3,65 & 3,65 & 66,0 & 66,6 & 66,0 & \begin{tabular}{|c|}
$67,2+-$ \\
5,8
\end{tabular} & $\begin{array}{c}88,0+- \\
6,4\end{array}$ & 0,18 & 0,21 & 0,23 & $\begin{array}{c}91,6+- \\
2,1\end{array}$ \\
\hline $\begin{array}{c}\text { Anisotropie } \\
{[\%]}\end{array}$ & 3,4 & 5,2 & 6,4 & 0,6 & 1,2 & 5,8 & 3,4 & 1,4 & 1,1 & 0,8 & 2,9 & 11,6 & 8,9 & $\begin{array}{c}10,3+- \\
7,2 \\
\end{array}$ & 1,4 & 19,6 & 10,8 & 4,7 & \\
\hline $\begin{array}{c}\text { granit. Gneis } \\
\text { (Be2) x- } \\
\text { Richtung }\end{array}$ & 3,88 & 3,98 & 4,29 & 2,13 & 1,75 & 1,88 & 1,56 & 6,18 & 3,31 & 3,15 & $\begin{array}{c}26,8+- \\
4,0\end{array}$ & 25,8 & 18,3 & $\begin{array}{c}22,2+- \\
1,4\end{array}$ & $\begin{array}{c}73,1+- \\
3,0\end{array}$ & $\begin{array}{c}0,33+- \\
0,13\end{array}$ & $\begin{array}{c}0,40+- \\
0,04\end{array}$ & $\begin{array}{c}0,31+- \\
0,03\end{array}$ & \\
\hline$y$-Richtung & 2,95 & 2,84 & 3,14 & n.b. & n.b. & 2,28 & 1,95 & 6,15 & 3,76 & 3,54 & n.b. & 25,9 & 23,4 & $\begin{array}{c}25,9+- \\
2,5 \\
\end{array}$ & $\begin{array}{c}87,4+- \\
3,7\end{array}$ & n.b. & $\begin{array}{c}0,08+- \\
0,08\end{array}$ & $\begin{array}{c}0,23+- \\
0,04\end{array}$ & \\
\hline z-Richtung & 2,05 & 1,91 & 2,15 & n.b. & n.b. & 1,60 & 1,41 & 5,89 & 3,48 & 3,48 & n.b. & 11,9 & 11,8 & $\begin{array}{c}12,3+- \\
1,9 \\
\end{array}$ & $\begin{array}{c}79,7+- \\
3,5\end{array}$ & n.b. & $\begin{array}{c}0,019+- \\
0,13\end{array}$ & $\begin{array}{c}0,23+- \\
0,04\end{array}$ & \\
\hline Mittelwert & 2,96 & 2,91 & 3,19 & 2,13 & 1,75 & 1,92 & 1,64 & 6,07 & 3,52 & 3,39 & n.b. & 21,2 & 17,8 & \begin{tabular}{|c|}
$20,1+-$ \\
3,4
\end{tabular} & $\begin{array}{c}80,0+- \\
5,9\end{array}$ & n.b. & 0,17 & 0,26 & $\begin{array}{c}88,1+- \\
4,2\end{array}$ \\
\hline $\begin{array}{c}\text { Anisotropie } \\
{[\%]}\end{array}$ & 47,2 & 52,0 & 49,9 & n.b. & n.b. & 29,8 & 27,7 & 4,7 & 12,0 & 11,0 & n.b. & 54,1 & 49,6 & $\begin{array}{c}52,4+- \\
13,5 \\
\end{array}$ & 16,3 & n.b. & 95,1 & 26,9 & \\
\hline $\begin{array}{l}\text { granod. } \\
\text { Ultramylonit } \\
\text { (Iv2) x- } \\
\text { Richtung }\end{array}$ & 4,98 & 4,93 & 5,15 & 2,98 & 2,63 & 3,10 & 2,60 & 6,69 & 3,82 & 3,58 & $\begin{array}{c}54,9+- \\
6,5\end{array}$ & 64,0 & 49,2 & $\begin{array}{c}57,2+- \\
2,8\end{array}$ & $\begin{array}{c}96,4+- \\
3,7\end{array}$ & $\begin{array}{c}0,27+- \\
0,11\end{array}$ & $\begin{array}{c}0,28+- \\
0,04\end{array}$ & $\begin{array}{c}0,28+- \\
0,03\end{array}$ & \\
\hline
\end{tabular}




\begin{tabular}{|c|c|c|c|c|c|c|c|c|c|c|c|c|c|c|c|c|c|c|c|}
\hline & $\begin{array}{c}\mathrm{Vp}_{0}(0,5 \\
\mathrm{MHz}) \\
{[\mathrm{km} / \mathrm{s}]} \\
\mathrm{a} \\
\end{array}$ & $\begin{array}{c}\mathrm{Vp}_{0} \\
(\mathrm{Ku}) \\
{[\mathrm{km} / \mathrm{s}]} \\
\mathrm{b}\end{array}$ & $\begin{array}{c}\mathrm{Vp}_{0}(1 \\
\mathrm{MHz}) \\
{[\mathrm{km} / \mathrm{s}]} \\
\mathrm{c}\end{array}$ & $\begin{array}{c}\mathbf{V s}_{0 / 1}(0,5 \\
\mathrm{MHz}) \\
{[\mathrm{km} / \mathrm{s}]} \\
\mathrm{d}\end{array}$ & $\begin{array}{c}\mathrm{Vs}_{0 / 2}(0,5 \\
\mathrm{MHz}) \\
{[\mathrm{km} / \mathrm{s}]} \\
\mathrm{e}\end{array}$ & $\begin{array}{c}\mathbf{V s}_{0 / 1}(1 \\
\mathrm{MHz}) \\
{[\mathrm{km} / \mathrm{s}]} \\
\mathrm{f}\end{array}$ & $\begin{array}{c}\mathbf{V s}_{0 / 2}(1 \\
\mathrm{MHz}) \\
{[\mathrm{km} / \mathrm{s}]} \\
\mathrm{g}\end{array}$ & $\begin{array}{c}\mathrm{Vp}_{400} \\
{[\mathrm{~km} / \mathrm{s}]} \\
\mathrm{h}\end{array}$ & $\begin{array}{c}\mathbf{V s}_{400 / 1} \\
{[\mathbf{k m} / \mathbf{s}]} \\
\mathbf{i}\end{array}$ & $\begin{array}{c}\mathbf{V s _ { 4 0 0 / 2 }} \\
{[\mathbf{k m} / \mathbf{s}]} \\
\mathbf{j}\end{array}$ & $\begin{array}{c}\mathrm{E}_{01 / 2}(0,5 \\
\mathrm{MHz}) \\
{[\mathrm{GPa}]} \\
\mathrm{k}\end{array}$ & $\begin{array}{c}\mathrm{E}_{0 / 1}(1 \\
\mathrm{MHz}) \\
{[\mathrm{GPa}]} \\
\mathrm{I}\end{array}$ & $\begin{array}{c}\mathrm{E}_{0 / 2}(1 \\
\mathrm{MHz}) \\
{[\mathrm{GPa}]} \\
\mathrm{m}\end{array}$ & $\begin{array}{c}\mathrm{E}_{01 / 2}(1 \\
\mathrm{MHz}) \\
{[\mathrm{GPa}]} \\
\mathrm{n}\end{array}$ & $\begin{array}{c}E_{4001 / 2} \\
{\left[\begin{array}{c}G P a \\
0\end{array}\right]} \\
0\end{array}$ & $\begin{array}{c}v_{01 / 2} \\
(0,5 \\
M H z) \\
p\end{array}$ & $\begin{array}{c}v_{01 / 2} \\
(1 \mathrm{MHz}) \\
q\end{array}$ & $\begin{array}{l}v_{400} \frac{1}{2} \\
r\end{array}$ & $\begin{array}{c}E_{\mathrm{VRH}} \\
\text { [GPa] } \\
\mathbf{s}\end{array}$ \\
\hline$y$-Richtung & 4,34 & 4,27 & 4,28 & 2,97 & 2,51 & 2,75 & 2,50 & 6,33 & 3,84 & 3,56 & $\begin{array}{c}48,3+- \\
7,1 \\
\end{array}$ & 47,6 & 42,5 & $\begin{array}{c}45,4+- \\
2,8 \\
\end{array}$ & $\begin{array}{c}93,4+- \\
3,8 \\
\end{array}$ & $\begin{array}{c}0,17+- \\
0,14 \\
\end{array}$ & $\begin{array}{c}0,20+- \\
0,05 \\
\end{array}$ & $\begin{array}{c}0,24+- \\
0,03 \\
\end{array}$ & \\
\hline z-Richtung & 3,36 & 3,17 & 3,17 & 2,58 & 2,50 & 2,47 & 2,47 & 6,05 & 3,60 & 3,59 & $\begin{array}{c}29,6+- \\
10,2 \\
\end{array}$ & 24,3 & 24,3 & $\begin{array}{c}24,4+- \\
4,7 \\
\end{array}$ & $\begin{array}{c}87,2+- \\
3,8 \\
\end{array}$ & $\begin{array}{c}-0,17+- \\
0,26 \\
\end{array}$ & $\begin{array}{c}0,27+- \\
0,13 \\
\end{array}$ & $\begin{array}{c}0,23+- \\
0,04 \\
\end{array}$ & \\
\hline Mittelwert & 4,23 & 3,17 & 3,17 & 2,58 & 2,50 & 2,47 & 2,47 & 6,05 & 3,60 & 3,58 & 44,2 & 45,3 & 38,7 & $\begin{array}{c}42,4+- \\
6,2 \\
\end{array}$ & $\begin{array}{c}92,3+- \\
6,5 \\
\end{array}$ & 0,09 & 0,07 & 0,25 & $\begin{array}{c}95,3+- \\
6,8\end{array}$ \\
\hline $\begin{array}{c}\text { Anisotropie } \\
{[\%]}\end{array}$ & 32,5 & 35,7 & 38,5 & 13,4 & 4,9 & 20,3 & 5,0 & 9,6 & 6,3 & 0,8 & 46,1 & 62,0 & 50,6 & $\begin{array}{c}57,3+- \\
6,3 \\
\end{array}$ & 9,5 & 162,3 & 197,6 & 18,8 & \\
\hline $\begin{array}{c}\text { granit. } \\
\text { Ultramylonit } \\
\text { (Gra3a) x- } \\
\text { Richtung }\end{array}$ & 5,66 & 5,67 & 5,63 & 3,44 & 3,34 & 3,46 & 3,28 & 6,09 & 3,59 & 3,50 & $\begin{array}{c}75,4+- \\
8,1\end{array}$ & 76,8 & 71,8 & $\begin{array}{c}74,6+- \\
3,5\end{array}$ & $\begin{array}{c}84,1+- \\
3,6\end{array}$ & $\begin{array}{c}0,22+- \\
0,10\end{array}$ & $\begin{array}{c}0,20+- \\
0,04\end{array}$ & $\begin{array}{c}0,24+- \\
0,03\end{array}$ & \\
\hline y-Richtung & 5,78 & 5,73 & 5,72 & 3,48 & 3,36 & 3,45 & 3,28 & 6,15 & 3,59 & 3,51 & $\begin{array}{c}77,4+- \\
8,1 \\
\end{array}$ & 77,5 & 72,5 & $\begin{array}{c}75,3+- \\
3,4 \\
\end{array}$ & $\begin{array}{c}84,8+- \\
3,6 \\
\end{array}$ & $\begin{array}{c}0,23+- \\
0,10 \\
\end{array}$ & $\begin{array}{c}0,22+- \\
0,04 \\
\end{array}$ & $\begin{array}{c}0,25+- \\
0,03\end{array}$ & \\
\hline z-Richtung & 5,44 & 5,40 & 5,29 & 3,36 & 3,33 & 3,30 & 3,21 & 6,06 & 3,54 & 3,49 & $\begin{array}{c}72,0+- \\
8,3 \\
\end{array}$ & 69,0 & 66,8 & $\begin{array}{c}68,1+- \\
3,5 \\
\end{array}$ & $\begin{array}{c}82,9+- \\
3,5 \\
\end{array}$ & $\begin{array}{c}0,20+- \\
0,11 \\
\end{array}$ & $\begin{array}{c}0,24+- \\
0,04 \\
\end{array}$ & \begin{tabular}{c|}
$0,25+-$ \\
0,03 \\
\end{tabular} & \\
\hline Mittelwert & 5,63 & 5,60 & 5,55 & 3,43 & 3,34 & 3,40 & 3,26 & 6,10 & 3,57 & 3,50 & 75,0 & 74,4 & 70,4 & $\begin{array}{c}72,7+- \\
6 \\
\end{array}$ & $\begin{array}{c}83,9+- \\
6,2\end{array}$ & 0,22 & 0,22 & 0,25 & $\begin{array}{c}90,4+- \\
2,1\end{array}$ \\
\hline $\begin{array}{c}\text { Anisotropie } \\
{[\%]}\end{array}$ & 5,9 & 5,8 & 7,5 & 3,4 & 0,9 & 4,6 & 2,1 & 1,5 & 1,4 & 0,60 & 7,0 & 11,0 & 7,86 & $\begin{array}{c}9,5+- \\
6,6 \\
\end{array}$ & 2,2 & 15,0 & 17,0 & 2,6 & \\
\hline
\end{tabular}

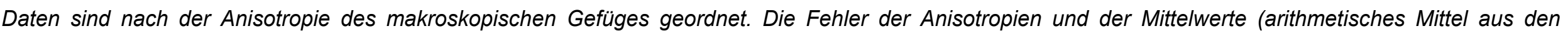

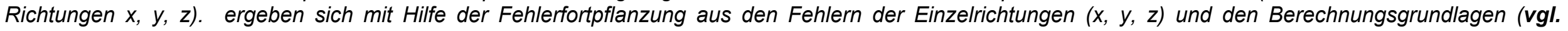
Kap.2.4.5.2) a - Kompressionswellengeschwindigkeit bei $0 \mathrm{MPa}$ Umschließungsdruck [km/s]. b-Kompressionswellengeschwindigkeit aus der Kugelmessung

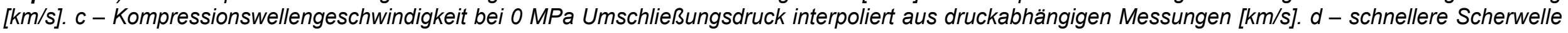
bei $0 \mathrm{MPa}$ Umschließungsdruck [km/s]. e - langsamere Scherwelle bei O MPa Umschließungsdruck [Km/s]. $f$ - schnellere Schwerwelle bei 0 MPa

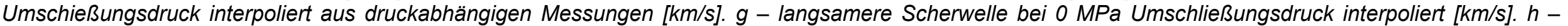

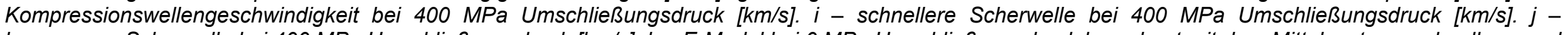
langsamere Scherwelle bei 400 MPa Umschließungsdruck [km/s]. $k$ - E-Modul bei 0 MPa Umschließungsdruck berechnet mit dem Mittelwert aus schnellerer und langsamerer Scherwelle [GPa]. I - E-Modul bei 0 MPa Umschließungsdruck berechnet mit schnellerer Scherwelle; interpoliert [GPa]. $m$ - E-Modul bei 0 MPa

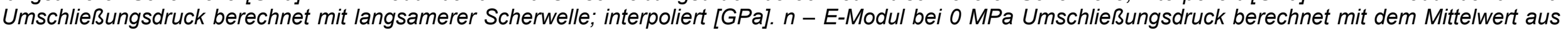
schnellerer und langsamerer Scherwelle; interpoliert [GPa]. o - E-Modul bei $400 \mathrm{MPa}$ Umschließungsdruck berechnet mit dem Mittelwert aus schnellerer und langsamerer Scherwelle [GPa]. p - Poisson-Zahl bei 0 MPa Umschießungsdruck berechnet mit dem Mittelwert aus schnellerer und langsamerer Scherwelle. $q$ -

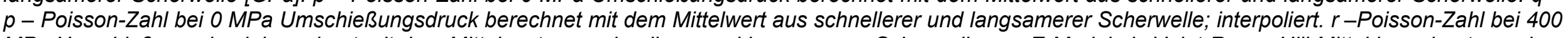

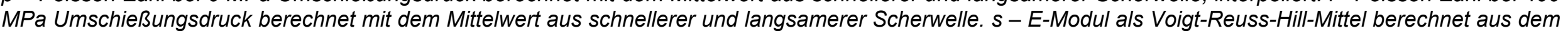
Mineralbestand und den Einkristalleigenschaften [GPa]. 


\section{Anhang I.7: Statische elastische Eigenschaften}

\begin{tabular}{|c|c|c|c|c|c|c|c|}
\hline & $\mathrm{E}_{\text {stat10 }}[\mathrm{GPa}]$ & $\mathrm{E}_{\text {stat30 }}[\mathrm{GPa}]$ & $\mathrm{E}_{\text {stat50 }}$ [GPa] & $\mathbf{E}_{\text {stat70 }}[\mathrm{GPa}]$ & $\mathrm{E}_{\text {stat90 }}$ [GPa] & 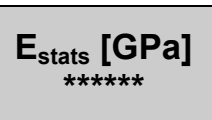 & $\begin{array}{c}\mathrm{E}_{\text {stat50 }} / \mathrm{E}_{\text {stat10 }} \\
{[\mathrm{GPa}]} \\
* * * * * * *\end{array}$ \\
\hline \multicolumn{8}{|l|}{ Proben Gruppe I } \\
\hline Granit (Koess) X-Richtung & $19,7+-6,6$ & $32,7+-3,5$ & $36,9+-1,9$ & $35,8+-1,4$ & $29,2+-5,6$ & $24,1+-2,6$ & $1,87+-0,63$ \\
\hline y-Richtung & $30,0+-12,5$ & $38,7+-9,2$ & $39,6+-5,7$ & $38,5+-4,1$ & $31,2+-5,3$ & $29,3+-4,4$ & $1,32+-0,58$ \\
\hline z-Richtung & $30,9+-9,4$ & $37,1+-6,5$ & $37,1+-3,8$ & $37,0+-4,5$ & $31,2+-5,1$ & $29,1+-3,9$ & $1,20+-0,39$ \\
\hline Mittelwert & 26,9 & 36,2 & 37,9 & 37,1 & 30,5 & 27,5 & 1,46 \\
\hline Anisotropie [\%] & 36,2 & 15,5 & 6,8 & 7,0 & 6,4 & 17,8 & 35,9 \\
\hline Granodiorit (PDD) x-Richtung & $32,3+-5,8$ & $42,7+-2,4$ & $45,1+-2,4$ & $46,1+-4,8$ & $36,9+-11,0$ & $34,7+-6,24$ & $1,39+-0,26$ \\
\hline y-Richtung & $20,4+-4,9$ & $38,0+-2,9$ & $44,9+-2,1$ & $44,1+-1,7$ & $31,4+-4,7$ & $28,0+-3,4$ & $2,20+-0,54$ \\
\hline z-Richtung & $26,0+-12,4$ & $38,4+-6,6$ & $42,7+-4,5$ & $43,8+-4,0$ & $30,4+-8,2$ & $29,2+-4,8$ & $1,64+-0,80$ \\
\hline Mittelwert & 26,3 & 39,7 & 44,2 & 44,7 & 32,9 & 30,6 & 1,74 \\
\hline Anisotropie [\%] & 36,8 & 11,0 & 5,3 & 5,0 & 17,6 & 19,3 & 36,6 \\
\hline Gabbro-Norit (NI) x-Richtung & $22,6+-6,7$ & $42,2+-3,4$ & $47,9+-3,2$ & $43,7+-5,8$ & $20,7+-8,1$ & $24,4+-3,4$ & $2,11+-0,64$ \\
\hline y-Richtung & $37,7+-5,9$ & $49,6+-2,7$ & $55,4+-2,7$ & $61,1+-5,2$ & $53,7+-6,3$ & $37,7+-5,8$ & $1,47+-0,24$ \\
\hline z-Richtung & $40,3+-10,5$ & $51,3+-4,9$ & $53,2+-2,8$ & $54,7+-7,6$ & $37,6+-13,7$ & $36,3+-8,7$ & $1,32+-0,35$ \\
\hline Mittelwert & 33,5 & 47,7 & 52,2 & 53,2 & 37,3 & 32,8 & 1,64 \\
\hline Anisotropie [\%] & 43,9 & 17,8 & 13,5 & 28,5 & 61,5 & 35,3 & 37,7 \\
\hline Granit (MrGr3) x-Richtung & n.b. & n.b. & n.b. & n.b. & n.b. & n.b. & n.b. \\
\hline y-Richtung & 8,7 & 20,4 & 28,7 & 34,0 & 34,4 & 14,8 & 3,30 \\
\hline z-Richtung & 10,0 & 23,9 & 28,6 & 27,8 & 21,7 & 16,9 & 2,86 \\
\hline Mittelwert & 9,35 & 22,2 & 28,7 & 30,9 & 28,1 & 15,9 & n.b. \\
\hline Anisotropie [\%] & n.b. & n.b. & n.b. & n.b. & n.b. & n.b. & n.b. \\
\hline \multicolumn{8}{|l|}{ Proben Gruppe II } \\
\hline Peridotit (Pi1a) x-Richtung & $44,4+-5,2$ & $59,7+-2,4$ & $62,9+-4,0$ & $65,3+-10,0$ & $40,0+-16,7$ & $41,9+-5,9$ & $1,41+-0,19$ \\
\hline y-Richtung & $33,9+-16,9$ & $47,5+-15,3$ & $53,9+-8,2$ & $58,7+-9,6$ & $45,6+-13,6$ & $36,6+-8,9$ & $1,58+-0,83$ \\
\hline z-Richtung & $25,3+-5,3$ & $46,4+-3,1$ & $55,7+-2,2$ & $55,7+-5,8$ & $35,9+-14,4$ & $34,6+-5,0$ & $2,20+-0,46$ \\
\hline Mittelwert & 34,5 & 51,2 & 57,5 & 59,9 & 40,5 & 37,7 & 1,73 \\
\hline Anisotropie [\%] & 43,0 & 22,3 & 14,3 & 14,7 & 21,3 & 17,4 & 35,7 \\
\hline Marmor (Rek) x-Richtung & $12,0+-4,5$ & $23,3+-8,8$ & $25,4+-10,1$ & $22,1+-7,7$ & $12,5+-3,2$ & $16,3+-5,0$ & $2,12+-1,16$ \\
\hline z-Richtung & n.b. & n.b. & n.b. & n.b. & n.b. & n.b. & n.b. \\
\hline Mittelwert & 11,8 & 22,7 & 25,4 & 22,3 & 12,4 & 16,4 & n.b. \\
\hline
\end{tabular}




\begin{tabular}{|c|c|c|c|c|c|c|c|}
\hline & $\mathrm{E}_{\text {stat10 }}[\mathrm{GPa}]$ & $\mathrm{E}_{\text {stat } 30}[\mathrm{GPa}]$ & $\mathrm{E}_{\text {stat50 }}[\mathrm{GPa}]$ & $\mathrm{E}_{\text {stat70 }}[\mathrm{GPa}]$ & $\mathrm{E}_{\text {stat90 }}[\mathrm{GPa}]$ & $\underset{\text { stats }}{\mathbf{E}_{* \star * * * *}[\mathrm{GPa}]}$ & $\begin{array}{c}\mathrm{E}_{\text {stat50 }} / \mathrm{E}_{\text {stat10 }} \\
{[\mathrm{GPa}]} \\
* * \star * * * *\end{array}$ \\
\hline Anisotropie [\%] & n.b. & n.b. & n.b. & n.b. & n.b. & n.b. & n.b. \\
\hline Quarzit (Al) x-Richtung & $38,1+-9,1$ & $40,5+-1,8$ & $41,6+-2,7$ & $46,7+-3,8$ & $33,4+-7,8$ & $37,7+-2,6$ & $1,07+-0,27$ \\
\hline y-Richtung & $27,2+-1,7$ & $39,7+-0,4$ & $44,3+-0,5$ & $45,4+-3,0$ & $41,5+-2,3$ & $32,3+-2,3$ & $1,63+-0,10$ \\
\hline z-Richtung & $23,6+-12,7$ & $35,6+-12,2$ & $43,8+-8,4$ & $48,0+-6,8$ & $36,0+-11,9$ & $32,6+-10,4$ & $1,85+-1,06$ \\
\hline Mittelwert & 29,6 & 38,6 & 43,2 & 49,7 & 37,0 & 34,2 & 1,53 \\
\hline Anisotropie [\%] & 38,1 & 12,1 & 6,1 & 5,4 & 19,5 & 14,3 & 41,2 \\
\hline Meta-Gabbro (MG1b) x-Richtung & $25,2+-1,9$ & $40,7+-2,8$ & $43,8+-2,7$ & $38,1+-2,3$ & $21,8+-2,2$ & $26,4+-28,2$ & $1,74+-0,17$ \\
\hline y-Richtung & $20,5+-3,3$ & $36,1+-1,4$ & $42,3+-1,5$ & $40,9+-4,3$ & $29,4+-7,9$ & $25,6+-3,2$ & $2,06+-0,34$ \\
\hline z-Richtung & $26,9+-9,4$ & $38,8+-2,8$ & $44,5+-2,2$ & $48,3+-1,1$ & $42,0+-3,1$ & $33,5+-4,1$ & $1,65+-0,58$ \\
\hline Mittelwert & 24,2 & 38,5 & 43,5 & 42,4 & 31,1 & 28,5 & 1,81 \\
\hline Anisotropie [\%] & 23,8 & 11,3 & 4,9 & 21,1 & 48,1 & 23,6 & 19,8 \\
\hline Meta-Gabbro (MG2) x-Richtung & $29,6+-10,8$ & $40,2+-6,7$ & $42,1+-7,2$ & $41,2+-12,2$ & $27,0+-14,4$ & $27,2+-6,7$ & $1,42+-0,57$ \\
\hline y-Richtung & $35,5+-5,3$ & $44,3+-2,7$ & $41,6+-2,4$ & $39,1+-6,4$ & $27,6+-11,5$ & $30,9+-4,2$ & $1,17+-0,19$ \\
\hline z-Richtung & $31,9+-8,6$ & $45,0+-3,7$ & $49,5+-4,3$ & $52,3+-4,3$ & $42,1+-4,1$ & $35,1+-6,6$ & $1,55+-0,44$ \\
\hline Mittelwert & 32,3 & 43,2 & 44,4 & 44,2 & 32,2 & 31,1 & 1,38 \\
\hline Anisotropie [\%] & 16,6 & 10,7 & 16,0 & 25,2 & 35,9 & 22,5 & 24,5 \\
\hline $\begin{array}{l}\text { granod. Protomylonit (Iv1) x- } \\
\text { Richtung }\end{array}$ & 24,2 & 35,2 & 37,2 & 34,6 & 22,4 & 30,6 & 1,54 \\
\hline y-Richtung & $28,6+-1,8$ & $33,2+-1,8$ & $35,0+-3,7$ & $37,1+-3,1$ & $23,9+-2,2$ & $28,1+-1,0$ & $1,33+-0,21$ \\
\hline z-Richtung & $26,3+-4,1$ & $34,1+-1,7$ & $35,0+-0,2$ & $36,4+-0,6$ & $30,1+-1,2$ & $25,6+-0,5$ & $1,22+-0,15$ \\
\hline Mittelwert & 26,4 & 34,2 & 35,7 & 36,0 & 25,5 & 28,1 & 1,36 \\
\hline Anisotropie [\%] & 15,4 & 5,7 & 5,9 & 6,7 & 25,6 & 16,3 & 20,4 \\
\hline granit. Gneis (Gra1a) x-Richtung & $29,5+-3,9$ & $38,7+-2,4$ & $45,1+-3,4$ & $50,2+-3,1$ & $44,4+-4,5$ & $34,1+-4,0$ & $1,53+-0,23$ \\
\hline$y$-Richtung & $33,3+-9,1$ & $41,3+-5,3$ & $46,0+-3,6$ & $50,7+-3,5$ & $44,6+-3,3$ & $35,7+-5,5$ & $1,38+-0,39$ \\
\hline z-Richtung & $27,6+-9,2$ & $39,2+-4,9$ & $47,4+-4,6$ & $52,3+-5,4$ & $44,7+-3,1$ & $35,9+-7,8$ & $1,72+-0,60$ \\
\hline Mittelwert & 30,1 & 39,7 & 46,2 & 51,1 & 44,6 & 35,2 & 1,54 \\
\hline Anisotropie [\%] & 17,1 & 6,3 & 4,9 & 4,0 & 0,7 & 5,0 & 19,6 \\
\hline \multicolumn{8}{|l|}{ Proben Gruppe III } \\
\hline $\begin{array}{l}\text { granit. Protomylonit (MrMy5) x- } \\
\text { Richtung }\end{array}$ & $13,9+-4,9$ & $25,9+-2,4$ & $29,6+-4,5$ & $26,6+-9,2$ & $21,3+-8,1$ & $20,6+-5,6$ & $2,13+-0,82$ \\
\hline y-Richtung & 10,8 & 20,8 & 24,6 & 21,7 & 13,0 & 13,9 & 2,28 \\
\hline Mittelwert & 13,0 & 24,0 & 29,0 & 27,5 & 20,3 & 18,9 & 2,23 \\
\hline Anisotropie [\%] & 25,0 & 19,7 & 25,0 & 36,6 & 51,0 & 37,1 & 6,5 \\
\hline
\end{tabular}




\begin{tabular}{|c|c|c|c|c|c|c|c|}
\hline & $\mathrm{E}_{\text {stat10 }}[\mathrm{GPa}]$ & $\mathrm{E}_{\text {stat30 }}[\mathrm{GPa}]$ & $\mathrm{E}_{\text {stat50 }}[\mathrm{GPa}]$ & $\mathrm{E}_{\text {stat70 }}[\mathrm{GPa}]$ & $\mathbf{E}_{\text {stat90 }}[\mathrm{GPa}]$ & $\underset{* * * * * *}{\mathbf{E}_{\text {stats }}[\mathrm{GPa}]}$ & $\begin{array}{c}\mathrm{E}_{\text {stat50 }} / \mathrm{E}_{\text {stat10 }} \\
{[\mathrm{GPa}]} \\
* * \star * * * *\end{array}$ \\
\hline granod. Gneis (Be1) x-Richtung & $13,4+-1,5$ & $30,3+-7,1$ & $37,1+-6,8$ & $35,3+-1,7$ & $19,2+-8,5$ & $18,8+-2,3$ & $2,76+-0,59$ \\
\hline y-Richtung & $12,7+-8,0$ & $24,8+-6,6$ & $29,2+-4,2$ & $26,4+-2,4$ & $15,9+-4,1$ & $17,6+-4,3$ & $2,30+-1,49$ \\
\hline z-Richtung & $20,4+-9,7$ & $33,5+-9,5$ & $36,8+-4,4$ & $36,1+-2,6$ & $26,6+-7,8$ & $23,2+-2,5$ & $1,80+-0,88$ \\
\hline Mittelwert & 15,5 & 29,5 & 34,4 & 32,6 & 20,6 & 19,9 & 2,29 \\
\hline Anisotropie [\%] & 37,7 & 26,0 & 21,3 & 27,9 & 40,2 & 24,1 & 34,8 \\
\hline granod. Mylonit (Iv1b) x-Richtung & $22,8+-7,8$ & $32,0+-7,7$ & $37,9+-10,0$ & $40,8+-15,6$ & $30,9+-17,7$ & $28,5+-11,8$ & $1,66+-0,72$ \\
\hline y-Richtung & $15,0+-7,3$ & $22,1+-7,6$ & $24,0+-6,6$ & $23,1+-6,6$ & $15,3+-5,9$ & $17,0+-7,4$ & $1,60+-0,89$ \\
\hline z-Richtung & $15,3+-3,6$ & $24,7+-2,4$ & $29,7+-1,7$ & $28,8+-1,4$ & $17,1+-5,1$ & $19,3+-2,6$ & $1,94+-0,47$ \\
\hline Mittelwert & 17,7 & 26,3 & 30,5 & 30,9 & 21,1 & 21,6 & 1,73 \\
\hline Anisotropie [\%] & 34,2 & 30,9 & 36,7 & 43,4 & 50,5 & 40,1 & 17,6 \\
\hline $\begin{array}{l}\text { granod. Paragneis (Cal) } \mathrm{x}- \\
\text { Richtung }\end{array}$ & $27,3+-4,0$ & $36,3+-1,9$ & $39,8+-2,1$ & $40,3+-5,2$ & $27,1+-7,7$ & $26,8+-2,3$ & $1,46+-0,23$ \\
\hline y-Richtung & $22,9+-3,7$ & $33,2+-0,4$ & $36,7+-0,5$ & $32,7+-3,8$ & $18,2+-4,8$ & $24,3+-3,0$ & $1,60+-0,26$ \\
\hline z-Richtung & $16,6+-2,5$ & $27,4+-5,7$ & $32,2+-1,2$ & $32,1+-3,0$ & $26,3+-7,3$ & $23,9+-3,3$ & $1,94+-0,30$ \\
\hline xz45-Richtung & $17,3+-9,7$ & $20,5+-6,4$ & $25,0+-5,1$ & $27,6+-7,7$ & $26,7+-13,9$ & $14,3+-0,3$ & n.b. \\
\hline yz45-Richtung & $7,6+-1,5$ & $14,2+-0,6$ & $18,3+-2,0$ & $19,3+-3,6$ & $14,9+-2,66$ & $12,5+-1,4$ & n.b. \\
\hline Mittelwert & 22,3 & 32,3 & 36,2 & 35,0 & 23,9 & 25,0 & 1,67 \\
\hline Anisotropie [\%] & 39,2 & 24,5 & 19,1 & 20,3 & 32,8 & 10,8 & 24,8 \\
\hline granit. Orthogneis (VA) x-Richtung & $17,0+-4,4$ & $30,9+-1,5$ & $35,5+-1,9$ & $34,4+-4,4$ & $21,9+-9,0$ & $22,7+-4,4$ & $2,09+-0,55$ \\
\hline y-Richtung & $12,2+-1,6$ & $22,5+-0,6$ & $30,0+-0,4$ & $31,6+-1,9$ & $23,5+-7,1$ & $20,9+-2,3$ & $2,46+-0,32$ \\
\hline z-Richtung & $15,7+-1,6$ & $27,4+-1,3$ & $32,8+-0,7$ & $33,8+-1,2$ & $28,8+-3,7$ & $22,6+-1,4$ & $2,09+-0,22$ \\
\hline xz45-Richtung & $8,9+-2,3$ & $14,4+-4,0$ & $20,2+-3,1$ & $21,9+-2,7$ & $16,9+-2,7$ & $13,3+-1,5$ & n.b. \\
\hline Mittelwert & 15,0 & 26,9 & 32,8 & 33,3 & 24,6 & 22,1 & 2,21 \\
\hline Anisotropie [\%] & 28,2 & 27,2 & 15,5 & 8,1 & 22,6 & 7,9 & 15,1 \\
\hline granit. Gneis (Gra1c) x-Richtung & $34,8+-10,8$ & $45,0+-11,3$ & $51,1+-11,2$ & $55,2+-10,8$ & $47,2+-15,1$ & $37,6+-5,2$ & $1,47+-0,56$ \\
\hline y-Richtung & $28,7+-3,4$ & $37,3+-3,0$ & $42,7+-4,3$ & $47,5+-4,1$ & $44,0+-2,9$ & $34,9+-2,4$ & $1,49+-0,23$ \\
\hline z-Richtung & $32,2+-6,2$ & $40,9+-3,9$ & $45,2+-3,3$ & $50,4+-3,9$ & $47,8+-2,9$ & $38,6+-4,3$ & $1,40+-0,29$ \\
\hline Mittelwert & 31,9 & 41,1 & 46,3 & 51,0 & 46,3 & 37,0 & 1,45 \\
\hline Anisotropie [\%] & 17,5 & 17,1 & 16,4 & 14,0 & 8,0 & 9,6 & 5,7 \\
\hline granit. Gneis (Be2) x-Richtung & $15,5+-1,5$ & $31,0+-7,2$ & $36,2+-6,2$ & $38,3+-7,8$ & $30,7+-10,7$ & $20,4+-1,0$ & $2,34+-0,46$ \\
\hline y-Richtung & $15,7+-0,4$ & $27,2+-0,9$ & $32,8+-0,0$ & $32,6+-3,21$ & $20,0+-0,4$ & $21,6+-0,0$ & $2,09+-0,05$ \\
\hline Mittelwert & 14,3 & 27,3 & 33,1 & 34,1 & 24,2 & 20,7 & 2,34 \\
\hline Anisotropie [\%] & 26,1 & 23,2 & 16,6 & 17,8 & 34,9 & 6,5 & 19,8 \\
\hline
\end{tabular}




\begin{tabular}{|c|c|c|c|c|c|c|c|}
\hline & $\mathrm{E}_{\text {stat10 }}[\mathrm{GPa}]$ & $\mathrm{E}_{\text {stat30 }}[\mathrm{GPa}]$ & $\mathrm{E}_{\text {stat50 }}[\mathrm{GPa}]$ & $\mathrm{E}_{\text {stat70 }}[\mathrm{GPa}]$ & $\mathrm{E}_{\text {stat90 }}[\mathrm{GPa}]$ & $\underset{\text { stats }}{\mathrm{E}_{* * * * * *}}$ [GPa] & $\begin{array}{c}\mathrm{E}_{\text {stat50 }} / \mathrm{E}_{\text {stat10 }} \\
{[\mathrm{GPa}]} \\
* * * * * *\end{array}$ \\
\hline $\begin{array}{l}\text { granod. Ultramylonit (Iv2) x- } \\
\text { Richtung }\end{array}$ & $15,6+-6,8$ & $37,2+-4,6$ & $47,1+-7,0$ & $48,0+-11,8$ & $36,0+-21,1$ & $25,7+-10,3$ & $3,02+-1,39$ \\
\hline y-Richtung & $23,9+-6,7$ & $38,2+-9,1$ & $43,9+-9,0$ & $43,9+-6,9$ & $21,5+-2,2$ & $30,9+-6,3$ & $1,83+-0,64$ \\
\hline z-Richtung & $19,4+-2,0$ & $30,5+-2,5$ & $35,7+-2,8$ & $35,3+-5,1$ & $26,4+-9,1$ & $23,9+-1,5$ & $1,84+-0,24$ \\
\hline Mittelwert & 19,6 & 35,3 & 42,2 & 42,4 & 28,0 & 26,8 & 2,23 \\
\hline Anisotropie [\%] & 34,7 & 20,2 & 24,2 & 26,5 & 40,3 & 22,7 & 39,2 \\
\hline $\begin{array}{l}\text { granit. Ultramylonit (Gra3a) x- } \\
\text { Richtung }\end{array}$ & $35,9+-11,6$ & $42,6+-4,7$ & $47,3+-2,2$ & $51,8+-1,5$ & $40,0+-4,8$ & $41,3+-7,8$ & $1,32+-0,43$ \\
\hline y-Richtung & $32,7+-10,4$ & $39,2+-6,6$ & $43,9+-3,8$ & $49,0+-3,9$ & $43,9+-4,2$ & $38,3+-8,7$ & $1,34+-0,44$ \\
\hline z-Richtung & $31,5+-4,9$ & $40,0+-1,5$ & $45,3+-2,4$ & $50,3+-3,0$ & $47,7+-3,4$ & $39,6+-5,0$ & $1,44+-0,24$ \\
\hline Mittelwert & 33,4 & 40,6 & 45,5 & 50,4 & 43,9 & 39,7 & 1,37 \\
\hline Anisotropie [\%] & 12,2 & 8,0 & 7,2 & 5,4 & 16,1 & 7,3 & 8,4 \\
\hline
\end{tabular}

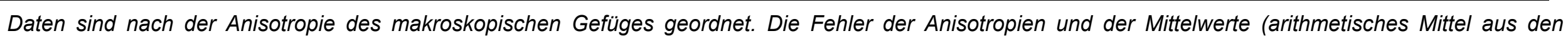

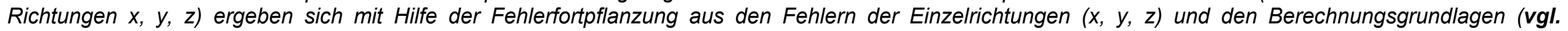

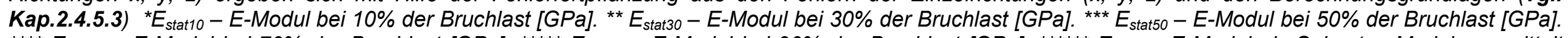

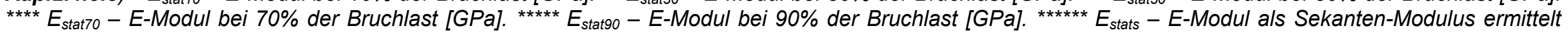
[GPa].. ${ }^{* * * * * *} E_{\text {stat } 50} / E_{\text {stat } 10}-$ Quotient aus E-Modul bei $50 \%$ Bruchlast und $10 \%$ Bruchlast. n.b. - nicht bestimmt. 


\section{Anhang I.8: Thermische Dehnung}

\begin{tabular}{|c|c|c|c|c|c|c|c|c|}
\hline & $\alpha\left[1 / K^{*} 10^{-6}\right]$ & $\begin{array}{c}\text { Mittelwert* } \\
{\left[1 / K^{*} 10^{-6}\right]}\end{array}$ & $\mathbf{n}$ & $\mathrm{A}_{\alpha \mathrm{G}}[\%]^{\star *}$ & $\begin{array}{l}\mathbf{A}_{\alpha \mathrm{Gmax}} \\
{[\%]^{\star * *}}\end{array}$ & $\alpha_{\mathrm{VGT}_{-6}}\left[1 / \mathrm{K}^{* * * *} 10\right.$ & $\alpha_{\mathrm{VG}_{6}}\left[1 / \mathrm{K}^{* * * *} 10^{-}\right.$ & $\begin{array}{c}a_{\mathrm{VGT}^{-}} \\
\mathrm{a}_{\mathrm{VG}}\left[1 / \mathrm{K}^{*} 10^{-}\right.\end{array}$ \\
\hline \multicolumn{9}{|l|}{ Proben Gruppe I } \\
\hline Granit (Koess) x-Richtung $\left(\alpha_{\mathrm{x}}\right)$ & $9,37+-0,33$ & & 3 & & & & & \\
\hline y-Richtung $\left(\alpha_{y}\right)$ & $8,12+-0,49$ & & 3 & & & & & \\
\hline z-Richtung $\left(\alpha_{z} ; \alpha ; A_{\alpha G} ; A_{\alpha G m a x} ; \alpha_{V G T} ; \alpha_{V G} ; \alpha_{V G T}-\alpha_{V G}\right)$ & $10,2+-1,08$ & 9,22 & 3 & $20,0+-9,8$ & $69,7+-2,2$ & $23,0+-0,60$ & $27,6+-1,23$ & $-4,65+-1,37$ \\
\hline Granodiorit (PDD) x-Richtung $\left(\alpha_{x}\right)$ & $6,73+-0,29$ & & 2 & & & & & \\
\hline y-Richtung $\left(\alpha_{y}\right)$ & $6,99+-0,26$ & & 2 & & & & & \\
\hline z-Richtung $\left(\alpha_{z} ; \alpha ; A_{\alpha G} ; A_{\alpha G m a x} ; \alpha_{V G T} ; \alpha_{V G} ; \alpha_{V G T}-\alpha_{V G}\right)$ & $8,32+-0,33$ & 7,35 & 2 & $19,0+-4,8$ & $75,3+-2,4$ & $21,7+-0,90$ & $22,0+-0,51$ & $-0,37+-1,04$ \\
\hline Gabbro-Norit (NI) x-Richtung $\left(\alpha_{\mathrm{x}}\right)$ & $5,14+-0,24$ & & 3 & & & & & \\
\hline y-Richtung $\left(\alpha_{y}\right)$ & $4,68+-0,32$ & & 3 & & & & & \\
\hline z-Richtung $\left(\alpha_{z} ; \alpha ; A_{\alpha G} ; \mathbf{A}_{\alpha G m a x} ; \alpha_{V G T} ; \alpha_{V G} ; \alpha_{V G T}-\alpha_{V G}\right)$ & $5,23+-0,27$ & 5,02 & 3 & $10,5+-7,6$ & $81,1+-1,2$ & $17,6+-0,46$ & $15,1+-0,48$ & $2,55+-1,17$ \\
\hline Granit (MrGr3) x-Richtung $\left(\alpha_{x}\right)$ & $7,02+-0,11$ & & 2 & & & & & \\
\hline y-Richtung $\left(\alpha_{\mathrm{y}}\right)$ & $6,70+-0,08$ & & 2 & & & & & \\
\hline $\begin{array}{c}\text { z-Richtung }\left(\alpha_{\mathrm{z}} ; \alpha ; \mathbf{A}_{\alpha \mathrm{G}} ; \mathbf{A}_{\alpha \mathrm{Gmax}} ; \alpha_{\mathrm{VGT}} ; \alpha_{\mathrm{VG}} ; \alpha_{\mathrm{VGT}}-\alpha_{\mathrm{VG}}\right) \\
\text { Proben Gruppe II }\end{array}$ & $6,68+-0,01$ & 6,80 & 2 & $4,8+-1,6$ & $64,6+-2,2$ & $24,5+-0,67$ & $20,4+-0,14$ & $4,15+-0,69$ \\
\hline Peridotit (Pi1a) $x$-Richtung $\left(\alpha_{x}\right)$ & $8,16+-0,18$ & & 2 & & & & & \\
\hline y-Richtung $\left(\alpha_{y}\right)$ & $8,69+-0,53$ & & 2 & & & & & \\
\hline z-Richtung $\left(\alpha_{z} ; \alpha ; A_{\alpha G} ; A_{\alpha G \max } ; \alpha_{V G T} ; \alpha_{V G} ; \alpha_{V G T}-\alpha_{V G}\right)$ & $8,74+-0,37$ & 8,53 & 2 & $6,7+-4,4$ & $32,7+-0,8$ & $27,4+-0,51$ & $25,6+-0,67$ & $1,83+-0,84$ \\
\hline Marmor (Rek) x-Richtung $\left(\alpha_{x}\right)$ & $7,61+-0,35$ & & 3 & & & & & \\
\hline y-Richtung $\left(\alpha_{y}\right)$ & $4,24+-0,31$ & & 2 & & & & & \\
\hline z-Richtung $\left(\alpha_{z} ; \alpha ; A_{\alpha G} ; A_{\alpha G m a x} ; \alpha_{V G T} ; \alpha_{V G} ; \alpha_{V G T}-\alpha_{V G}\right)$ & $16,4+-0,5$ & 9,43 & 3 & $74,2+-2,6$ & $118,7+-2,6$ & $6,70+-0,50$ & $28,3+-1,25$ & $-21,6+-1,34$ \\
\hline Quarzit $(\mathrm{Al}) \mathrm{x}$-Richtung $\left(\alpha_{\mathrm{x}}\right)$ & $11,4+-0,08$ & & 2 & & & & & \\
\hline y-Richtung $\left(\alpha_{y}\right)$ & $11,5+-0,17$ & & 2 & & & & & \\
\hline z-Richtung $\left(\alpha_{z} ; \alpha ; \mathbf{A}_{\alpha \mathrm{G}} ; \mathbf{A}_{\alpha \mathrm{Gmax}} ; \alpha_{\mathrm{VGT}} ; \alpha_{\mathrm{VG}} ; \alpha_{\mathrm{VGT}}-\alpha_{\mathrm{VG}}\right)$ & $11,1+-0,36$ & 11,3 & 2 & $3,1+-3,4$ & $36,0+-1,4$ & $36,4+-1,13$ & $34,0+-0,40$ & $2,37+-1,20$ \\
\hline Meta-Gabbro (MG1b) x-Richtung $\left(\alpha_{\mathrm{x}}\right)$ & $6,0+-0,27$ & & 2 & & & & & \\
\hline y-Richtung $\left(\alpha_{y}\right)$ & $6,56+-0,32$ & & 2 & & & & & \\
\hline z-Richtung $\left(\alpha_{z} ; \alpha ; A_{\alpha G} ; A_{\alpha G m a x} ; \alpha_{V G T} ; \alpha_{V G} ; \alpha_{V G T}-\alpha_{V G}\right)$ & $6,09+-0,46$ & 6,22 & 2 & $8,6+-6,1$ & $68,5+-2,6$ & $22,9+-1,00$ & $18,6+-0,62$ & $4,21+-1,18$ \\
\hline Meta-Gabbro (MG2) x-Richtung $\left(\alpha_{\mathrm{x}}\right)$ & $6,89+-0,11$ & & 2 & & & & & \\
\hline y-Richtung $\left(\alpha_{y}\right)$ & $7,23+-0,26$ & & 2 & & & & & \\
\hline z-Richtung $\left(\alpha_{z} ; \alpha ; A_{\alpha G} ; A_{\alpha G m a x} ; \alpha_{V G T} ; \alpha_{V G} ; \alpha_{V G T}-\alpha_{V G}\right)$ & $8,02+-0,14$ & 7,38 & 3 & $14,0+-2,0$ & $68,5+-2,6$ & $22,9+-1,00$ & $22,1+-0,32$ & $7,18+-1,05$ \\
\hline
\end{tabular}




\begin{tabular}{|c|c|c|c|c|c|c|c|c|}
\hline & $\alpha\left[1 / K^{*} 10^{-6}\right]$ & $\begin{array}{c}\text { Mittelwert* } \\
{\left[1 / K^{*} 10^{-6}\right]}\end{array}$ & $\mathbf{n}$ & $\mathbf{A}_{\alpha \mathrm{G}}[\%]^{* *}$ & $\begin{array}{l}\mathrm{A}_{\alpha \mathrm{Gmax}} \\
{[\%]^{\star \star *}}\end{array}$ & $\alpha_{\mathrm{VGT}_{-6}}\left[1 / \mathrm{K}^{* * * *} 10\right.$ & $\alpha_{\mathrm{VG}_{6}}\left[1 / \mathrm{K}^{* * * *} 10^{-}\right.$ & $\begin{array}{c}a_{\mathrm{VGT}} \\
a_{\mathrm{VG}}\left[1 / \mathrm{K}^{*} 10^{-}\right. \\
]\end{array}$ \\
\hline granod. Protomylonit (Iv1) x-Richtung $\left(\alpha_{x}\right)$ & $8,93+-0,14$ & & 2 & & & & & \\
\hline y-Richtung $\left(\alpha_{y}\right)$ & $9,00+-0,05$ & & 2 & & & & & \\
\hline z-Richtung $\left(\alpha_{z} ; \alpha ; A_{\alpha G} ; A_{\alpha G m a x} ; \alpha_{V G T} ; \alpha_{V G} ; \alpha_{V G T}-\alpha_{V G}\right)$ & $8,32+-0,17$ & 8,75 & 2 & $7,6+-1,9$ & $54,1+-3,1$ & $28,2+-1,43$ & $26,2+-0,22$ & $1,92+-1,45$ \\
\hline granit. Gneis (Gra1a) x-Richtung $\left(\alpha_{\mathrm{x}}\right)$ & $7,46+-0,06$ & & 2 & & & & & \\
\hline$y$-Richtung $\left(\alpha_{\mathrm{y}}\right)$ & $6,97+-0,07$ & & 2 & & & & & \\
\hline z-Richtung $\left(\alpha_{\mathrm{z}} ; \alpha ; \mathbf{A}_{\alpha \mathrm{G}} ; \mathbf{A}_{\alpha \mathrm{Gmax}} ; \alpha_{\mathrm{VGT}} ; \alpha_{\mathrm{VG}} ; \alpha_{\mathrm{VGT}}-\alpha_{\mathrm{VG}}\right)$ & $6,54+-0,21$ & 6,99 & 2 & $12,3+-2,9$ & $71,3+-1,5$ & $21,2+-0,55$ & $21,0+-0,23$ & $0,25+-0,59$ \\
\hline \multicolumn{9}{|l|}{ Proben Gruppe III } \\
\hline granit. Protomylonit (MrMy5) x-Richtung $\left(\alpha_{x}\right)$ & $7,80+-0,04$ & & 2 & & & & & \\
\hline y-Richtung $\left(\alpha_{\mathrm{y}}\right)$ & $7,91+-0,07$ & & 2 & & & & & \\
\hline z-Richtung $\left(\alpha_{z} ; \alpha ; A_{\alpha G} ; A_{\alpha G m a x} ; \alpha_{V G T} ; \alpha_{V G} ; \alpha_{V G T}-\alpha_{V G}\right)$ & $8,24+-0,07$ & 7,98 & 2 & $5,3+-1,0$ & $63,5+-3,0$ & $25,1+-1,47$ & $23,9+-0,11$ & $1,17+-1,48$ \\
\hline granod. Gneis (Be1) $x$-Richtung $\left(\alpha_{x}\right)$ & $7,66+-0,05$ & & 2 & & & & & \\
\hline y-Richtung $\left(\alpha_{\mathrm{y}}\right)$ & $7,35+-0,02$ & & 2 & & & & & \\
\hline z-Richtung $\left(\alpha_{\mathrm{z}} ; \alpha ; \mathbf{A}_{\alpha \mathrm{G}} ; \mathbf{A}_{\alpha \mathrm{Gmax}} ; \alpha_{\mathrm{VGT}} ; \alpha_{\mathrm{VG}} ; \alpha_{\mathrm{VGT}}-\alpha_{\mathrm{VG}}\right)$ & $6,95+-0,08$ & 7,32 & 2 & $9,3+-1,3$ & $64,6+-3,0$ & $24,6+-1,47$ & $22,0+-0,10$ & $2,63+-1,48$ \\
\hline granod. Mylonit (Iv1b) x-Richtung $\left(\alpha_{x}\right)$ & $8,08+-0,08$ & & 2 & & & & & \\
\hline$y$-Richtung $\left(\alpha_{y}\right)$ & $7,46+-0,30$ & & 2 & & & & & \\
\hline z-Richtung $\left(\alpha_{z} ; \alpha ; A_{\alpha G} ; A_{\alpha G m a x} ; \alpha_{V G T} ; \alpha_{V G} ; \alpha_{V G T}-\alpha_{V G}\right)$ & $8,11+-0,43$ & 7,89 & 5 & $8,0+-6,2$ & $54,1+-3,1$ & $28,2+-1,43$ & $23,7+-0,54$ & $4,51+-1,53$ \\
\hline granod. Paragneis (Cal) x-Richtung $\left(\alpha_{x}\right)$ & $6,93+-0,09$ & & 2 & & & & & \\
\hline y-Richtung $\left(\alpha_{y}\right)$ & $6,54+-0,25$ & & 2 & & & & & \\
\hline z-Richtung $\left(\alpha_{\mathrm{z}} ; \alpha ; \mathbf{A}_{\alpha \mathrm{G}} ; \mathbf{A}_{\alpha \mathrm{Gmax}} ; \alpha_{\mathrm{VGT}} ; \alpha_{\mathrm{VG}} ; \alpha_{\mathrm{VGT}}-\alpha_{\mathrm{VG}}\right)$ & $6,81+-0,30$ & 6,76 & 2 & $5,6+-3,9$ & $63,8+-3,0$ & $25,5+-1,29$ & $20,3+-0,41$ & $5,20+-1,36$ \\
\hline granit. Orthogneis (VA) $x$-Richtung $\left(\alpha_{x}\right)$ & $7,63+-0,57$ & & 3 & & & & & \\
\hline y-Richtung $\left(\alpha_{y}\right)$ & $6,41+-0,23$ & & 3 & & & & & \\
\hline z-Richtung $\left(\alpha_{z} ; \alpha ; A_{\alpha G} ; A_{\alpha G m a x} ; \alpha_{V G T} ; \alpha_{V G} ; \alpha_{V G T}-\alpha_{V G}\right)$ & $8,97+-1,34$ & 7,67 & 3 & $28,6+-11$ & $58,3+-1,6$ & $26,7+-0,89$ & $23,0+-1,48$ & $3,68+-1,73$ \\
\hline granit. Gneis (Gra1c) x-Richtung $\left(\alpha_{x}\right)$ & $7,39+-0,08$ & & 2 & & & & & \\
\hline y-Richtung $\left(\alpha_{\mathrm{y}}\right)$ & $7,23+-0,09$ & & 2 & & & & & \\
\hline z-Richtung $\left(\alpha_{\mathrm{z}} ; \alpha ; \mathbf{A}_{\alpha \mathrm{G}} ; \mathbf{A}_{\alpha \mathrm{Gmax}} ; \alpha_{\mathrm{VGT}} ; \alpha_{\mathrm{VG}} ; \alpha_{\mathrm{VGT}}-\alpha_{\mathrm{VG}}\right)$ & $6,18+-0,12$ & 6,93 & 2 & $16,3+-1,9$ & $70,1+-1,45$ & $22,0+-0,55$ & $20,8+-0,18$ & $1,17+-0,58$ \\
\hline granit. Gneis (Be2) $x$-Richtung $\left(\alpha_{x}\right)$ & $7,65+-0,03$ & & 2 & & & & & \\
\hline y-Richtung $\left(\alpha_{y}\right)$ & $6,87+-0,13$ & & 2 & & & & & \\
\hline z-Richtung $\left(\alpha_{\mathrm{z}} ; \alpha ; \mathbf{A}_{\alpha \mathrm{G}} ; \mathbf{A}_{\alpha \mathrm{Gmax}} ; \alpha_{\mathrm{VGT}} ; \alpha_{\mathrm{VG}} ; \alpha_{\mathrm{VGT}}-\alpha_{\mathrm{VG}}\right)$ & $7,88+-0,00$ & 7,47 & 2 & $12,9+-1,6$ & $63,7+-3,0$ & $25,2+-1,47$ & $22,4+-0,13$ & $2,84+-1,48$ \\
\hline granod. Ultramylonit (Iv2) x-Richtung $\left(\alpha_{x}\right)$ & $8,05+-0,21$ & & 2 & & & & & \\
\hline y-Richtung $\left(\alpha_{y}\right)$ & $8,05+-0,09$ & & 2 & & & & & \\
\hline z-Richtung $\left(\alpha_{z} ; \alpha ; A_{\alpha G} ; A_{\alpha G m a x} ; \alpha_{V G T} ; \alpha_{V G} ; \alpha_{V G T}-\alpha_{V G}\right)$ & $8,80+-0,33$ & 8,30 & 2 & $8,5+-4,1$ & $54,1+-3,1$ & $28,2+-1,43$ & $24,9+-0,40$ & $3,26+-1,49$ \\
\hline granit. Ultramylonit (Gra3a) x-Richtung $\left(\alpha_{x}\right)$ & $7,02+-0,32$ & & 2 & & & & & \\
\hline
\end{tabular}




\begin{tabular}{|c|c|c|c|c|c|c|c|c|}
\hline & $\alpha\left[1 / K^{*} 10^{-6}\right]$ & $\begin{array}{l}\text { Mittelwert } \\
{\left[1 / K^{*} 10^{-6}\right]}\end{array}$ & $\mathbf{n}$ & $A_{\alpha G}[\%]^{\star *}$ & $\begin{array}{l}A_{\alpha G \max } \\
{[\%]^{\star \star \star}}\end{array}$ & $\alpha_{\mathrm{VGG}_{-6}}\left[1 / \mathrm{K}^{* * * *}\right.$ & $\underset{\mathrm{VGG}_{6}\left[1 / \mathrm{K}^{* * * *} 10^{-}\right.}{ }$ & $\begin{array}{c}a_{\mathrm{VGT}^{-}} \\
\mathrm{a}_{\mathrm{VG}}\left[1 / \mathrm{K}^{*} 10^{-}\right. \\
]\end{array}$ \\
\hline$y$-Richtung $\left(\alpha_{y}\right)$ & $5,92+-0,25$ & & 2 & & & & & \\
\hline$z-$ Richtung $\left(\alpha_{z} ; \alpha ; A_{\alpha G} ; A_{\alpha G \max } ; \alpha_{V G T} ; \alpha_{V G} ; \alpha_{V G T}-\alpha_{V G}\right)$ & $6,37+-0,10$ & 6,44 & 2 & $15,7+-5,3$ & $71,2+-1,45$ & $21,3+-0,55$ & $19,3+-0,42$ & $1,97+-0,69$ \\
\hline quarzit. Ultramylonit (QM) $x$-Richtung $\left(\alpha_{x}\right)$ & $14,4+-0,03$ & & 2 & & & & & \\
\hline$y$-Richtung $\left(\alpha_{y}\right)$ & $11,3+-0,24$ & & 2 & & & & & \\
\hline z-Richtung $\left(\alpha_{z} ; \alpha ; \mathbf{A}_{\alpha G} ; \mathbf{A}_{\alpha G \max } ; \alpha_{V G T} ; \alpha_{V G} ; \alpha_{V G T}-\alpha_{V G}\right)$ & $11,9+-0,25$ & 12,5 & 2 & $21,9+-1,7$ & $36+-0,7$ & $37,0+-0,74$ & $37,6+-0,35$ & n.b. \\
\hline
\end{tabular}

Daten sind nach der Anisotropie des makroskopischen Gefüges geordnet. $n$ - Anzahl der gemessenen Proben. * arithmetisches Mittel aus den a-Werten der Richtungen $x, y, z .{ }^{* *}$ Anisotropie der thermischen Dehnung nach $\left(A_{\alpha G}=\alpha_{\max }-\alpha_{\min } / \alpha_{\max }{ }^{*} 100\right)$. ${ }^{* * *}$ maximal mögliche Anisotropie der thermischen Dehnung aus mineralogischer Zusammensetzung und Einkristalldaten. ${ }^{* * *}$ aus der mineralogischen Zusammensetzung und den Einkristalldaten berechneter Volumenausdehnungskoeffizient ( $\alpha_{V G T}$ ) des Gesteins. ${ }^{* * * * *}$ Volumenausdehnungskoeffizient berechnet nach $\alpha_{V G}=\alpha_{x}+\alpha_{y}+\alpha_{z}$. 


\section{Anhang I.9: Meta-Gabbros}

\begin{tabular}{|c|c|c|c|c|c|c|c|c|c|c|c|c|c|c|c|c|c|c|c|c|c|c|c|c|c|c|}
\hline Probe $/ \beta_{\mathrm{sz}}$ * & $0^{\circ}(z)$ & $\mathbf{n}$ & $15^{\circ}$ & $\mathbf{n}$ & $30^{\circ}$ & $\mathbf{n}$ & $45^{\circ}$ & $\mathbf{n}$ & $60^{\circ}$ & $n$ & $75^{\circ}$ & $\mathbf{n}$ & $90^{\circ}(-x)$ & $n$ & $105^{\circ}$ & $\mathbf{r}$ & $120^{\circ}$ & $\mathbf{n}$ & $135^{\circ}$ & $\mathbf{n}$ & $150^{\circ}$ & $\mathbf{n}$ & $165^{\circ}$ & $n$ & $\begin{array}{c}180^{\circ} \\
(-z)\end{array}$ & $\mathbf{n}$ \\
\hline Mg1b & $\begin{array}{c}8,2+- \\
0,9 \\
\end{array}$ & 3 & $\begin{array}{c}8,0+- \\
0,6 \\
\end{array}$ & 3 & $\begin{array}{c}7,0+- \\
0,7\end{array}$ & 3 & $\begin{array}{c}8,3+- \\
0,6\end{array}$ & 3 & $\begin{array}{c}10,0+ \\
-1,8\end{array}$ & 3 & $\begin{array}{c}10,2+ \\
-0,9\end{array}$ & 3 & $\begin{array}{c}11,4+- \\
0,8\end{array}$ & 3 & $\begin{array}{l}10,7+ \\
+-1,0\end{array}$ & 3 & $\begin{array}{c}8,3+- \\
0,7\end{array}$ & 3 & $\begin{array}{c}9,7+- \\
0,5\end{array}$ & 3 & $\begin{array}{c}9,5+- \\
0,4\end{array}$ & 3 & $\begin{array}{c}7,7+- \\
0,6\end{array}$ & 3 & $\begin{array}{c}8,2+- \\
0,9\end{array}$ & 3 \\
\hline Mg2 & $\begin{array}{c}9,7+- \\
1,4\end{array}$ & 3 & $\begin{array}{c}9,0+- \\
0,3\end{array}$ & 3 & $\begin{array}{c}9,0+- \\
0,8\end{array}$ & 3 & $\begin{array}{c}10,1+ \\
-1,5\end{array}$ & 3 & $\begin{array}{c}10,2+ \\
-1,8\end{array}$ & 3 & $\begin{array}{c}11,6+ \\
-3,7\end{array}$ & 3 & $\begin{array}{c}13,0+- \\
1,5\end{array}$ & 3 & $\begin{array}{c}12,4+ \\
-1,0\end{array}$ & 3 & $\begin{array}{c}13,5+ \\
-1,6\end{array}$ & 3 & $\begin{array}{c}12,2+- \\
0,9\end{array}$ & 3 & $\begin{array}{c}8,2+- \\
1,0\end{array}$ & 3 & $\begin{array}{r}7,28 \\
+-0,9\end{array}$ & 3 & $\begin{array}{c}9,7+- \\
1,4\end{array}$ & 3 \\
\hline Probe $/ \beta_{\mathrm{sz}}{ }^{*}$ & $0^{\circ}(y)$ & $\mathbf{n}$ & $15^{\circ}$ & $\mathbf{n}$ & $30^{\circ}$ & $\mathbf{n}$ & $45^{\circ}$ & $\mathbf{n}$ & $60^{\circ}$ & $n$ & $75^{\circ}$ & $\mathbf{n}$ & $90^{\circ}(-z)$ & $n$ & $105^{\circ}$ & $\mathbf{r}$ & $120^{\circ}$ & $\mathbf{n}$ & $135^{\circ}$ & $\mathbf{n}$ & $150^{\circ}$ & $\mathbf{n}$ & $165^{\circ}$ & $n$ & $\begin{array}{c}180^{\circ} \\
(-y)\end{array}$ & $n$ \\
\hline Mg1b & $\begin{array}{c}9,2+- \\
1,0 \\
\end{array}$ & 3 & $\begin{array}{c}9,0+- \\
0,8\end{array}$ & 3 & $\begin{array}{c}9,2+- \\
1,8 \\
\end{array}$ & 3 & $\begin{array}{c}10,0+ \\
-1,0 \\
\end{array}$ & 3 & $\begin{array}{c}8,1+- \\
0,7\end{array}$ & 3 & $\begin{array}{c}8,2+- \\
0,1 \\
\end{array}$ & 3 & $8,2+-0,9$ & 3 & $\begin{array}{c}9,2+- \\
1,1 \\
\end{array}$ & 3 & $\begin{array}{c}9,6+- \\
1,2 \\
\end{array}$ & 3 & $\begin{array}{c}8,5+- \\
0,2 \\
\end{array}$ & 3 & $\begin{array}{c}8,7+- \\
1,4 \\
\end{array}$ & 3 & $\begin{array}{c}8,5+- \\
0,9\end{array}$ & 3 & $\begin{array}{c}9,2+- \\
1,0 \\
\end{array}$ & 3 \\
\hline Mg2 & $\begin{array}{c}9,2+- \\
1,2\end{array}$ & 3 & $\begin{array}{c}10,7+- \\
0,7\end{array}$ & 3 & $\begin{array}{c}8,5+- \\
1,5\end{array}$ & 3 & $\begin{array}{c}9,9+- \\
2,6\end{array}$ & 3 & $\begin{array}{c}8,1+- \\
1,3\end{array}$ & 3 & $\begin{array}{c}9,5+- \\
2,3\end{array}$ & 3 & $9,7+-1,4$ & 3 & $\begin{array}{c}7,1+- \\
0,9\end{array}$ & $\approx$ & $\begin{array}{c}8,8+- \\
2,6\end{array}$ & 3 & $\begin{array}{c}7,4+- \\
0,5\end{array}$ & 3 & $\begin{array}{c}8,9+- \\
0,6\end{array}$ & 3 & $\begin{array}{c}8,4+- \\
2,7\end{array}$ & 3 & $\begin{array}{c}9,2+- \\
1,2\end{array}$ & 3 \\
\hline $\begin{array}{c}\text { Probel } \\
\text { Hbl }(110)^{* *}\end{array}$ & $0^{\circ}(z)$ & $\mathbf{n}$ & $15^{\circ}$ & $\mathbf{n}$ & $30^{\circ}$ & $\mathbf{n}$ & $45^{\circ}$ & $\mathbf{n}$ & $60^{\circ}$ & $n$ & $75^{\circ}$ & $\mathbf{n}$ & $90^{\circ}(-x)$ & $n$ & $105^{\circ}$ & $\mathbf{r}$ & $120^{\circ}$ & $\mathbf{n}$ & $135^{\circ}$ & $\mathbf{n}$ & $150^{\circ}$ & $\mathbf{n}$ & $165^{\circ}$ & $n$ & $\begin{array}{c}180^{\circ} \\
(-z)\end{array}$ & $\mathbf{n}$ \\
\hline $\mathrm{Mg1b} / 2$ & 1,94 & & 1,43 & & 0,98 & & 0,60 & & 0,59 & & 0,46 & & 0,37 & & 0,44 & & 0,57 & & 0,57 & & 0,87 & & 1,61 & & 1,94 & \\
\hline $\begin{array}{c}\text { Probe/ } \\
\text { Hbl(110)** }\end{array}$ & $0^{\circ}(y)$ & $\mathbf{n}$ & $15^{\circ}$ & $\mathbf{n}$ & $30^{\circ}$ & $\mathbf{n}$ & $45^{\circ}$ & $\mathbf{n}$ & $60^{\circ}$ & $n$ & $75^{\circ}$ & $\mathbf{n}$ & $90^{\circ}(-z)$ & $n$ & $105^{\circ}$ & $r$ & $120^{\circ}$ & $\mathbf{n}$ & $135^{\circ}$ & $\mathbf{n}$ & $150^{\circ}$ & $\mathbf{n}$ & $165^{\circ}$ & $n$ & $\begin{array}{l}180^{\circ} \\
(-y)\end{array}$ & $n$ \\
\hline $\mathrm{Mg} 1 \mathrm{~b} / 2$ & 1,65 & & 1,84 & & 1,87 & & 1,77 & & 1,83 & & 1,89 & & 1,94 & & 1,62 & & 1,65 & & 1,5 & & 1,49 & & 1,68 & & 1,65 & \\
\hline $\begin{array}{c}\text { Probel } \\
\text { Dio(110)*** }\end{array}$ & $0^{\circ}(z)$ & $\mathbf{n}$ & $15^{\circ}$ & $\mathbf{n}$ & $30^{\circ}$ & $\mathbf{n}$ & $45^{\circ}$ & $\mathbf{n}$ & $60^{\circ}$ & $n$ & $75^{\circ}$ & $\mathbf{n}$ & $90^{\circ}(-x)$ & $n$ & $105^{\circ}$ & $r$ & $120^{\circ}$ & $\mathbf{n}$ & $135^{\circ}$ & $\mathbf{n}$ & $150^{\circ}$ & $\mathbf{n}$ & $165^{\circ}$ & $n$ & $\begin{array}{c}180^{\circ} \\
(-z)\end{array}$ & $n$ \\
\hline $\mathrm{Mg} 1 \mathrm{~b} / 2$ & 0,85 & & 1,04 & & 1,07 & & 1,09 & & 1,03 & & 1,02 & & 1,06 & & 0,91 & & 0,68 & & 0,78 & & 0,79 & & 0,85 & & 0,85 & \\
\hline $\begin{array}{c}\text { Probel } \\
\text { Dio }(110)^{* * *}\end{array}$ & $0^{\circ}(y)$ & $\mathbf{n}$ & $15^{\circ}$ & $\mathbf{n}$ & $30^{\circ}$ & $\mathbf{n}$ & $45^{\circ}$ & $\mathbf{n}$ & $60^{\circ}$ & $n$ & $75^{\circ}$ & $\mathbf{n}$ & $90^{\circ}(-z)$ & $n$ & $105^{\circ}$ & $r$ & $120^{\circ}$ & $\mathbf{n}$ & $135^{\circ}$ & $\mathbf{n}$ & $150^{\circ}$ & $\mathbf{n}$ & $165^{\circ}$ & $n$ & $\begin{array}{c}180^{\circ} \\
(-y)\end{array}$ & $n$ \\
\hline $\mathrm{Mg} 1 \mathrm{~b} / 2$ & 1,01 & & 1,02 & & 1,04 & & 1,07 & & 1,09 & & 1,02 & & 0,85 & & 0,99 & & 0,91 & & 0,84 & & 0,84 & & 0,90 & & 1,01 & \\
\hline Probel $\Delta \mathbf{V} p^{\star * \star *}$ & $0^{\circ}(z)$ & $\mathbf{n}$ & $15^{\circ}$ & $\mathbf{n}$ & $30^{\circ}$ & $n$ & $45^{\circ}$ & $\mathbf{n}$ & $60^{\circ}$ & $n$ & $75^{\circ}$ & $\mathbf{n}$ & $90^{\circ}(-x)$ & $n$ & $105^{\circ}$ & $r$ & $120^{\circ}$ & $\mathbf{n}$ & $135^{\circ}$ & $\mathbf{n}$ & $150^{\circ}$ & $\mathbf{n}$ & $165^{\circ}$ & $n$ & $\begin{array}{c}180^{\circ} \\
(-z)\end{array}$ & $n$ \\
\hline Mg1b & 1,41 & & 1,38 & & 1,26 & & 1,27 & & 1,33 & & 1,54 & & 1,48 & & 1,43 & & 1,50 & & 1,49 & & 1,49 & & 1,45 & & 1,41 & \\
\hline Mg2 & 0,33 & & 0,38 & & 0,39 & & 0,37 & & 0,33 & & 0,31 & & 0,25 & & 0,31 & & 0,28 & & 0,22 & & 0,21 & & 0,26 & & 0,33 & \\
\hline Probe $/ \Delta \mathbf{V p}^{\star \star \star \star *}$ & $0^{\circ}(y)$ & $\mathbf{n}$ & $15^{\circ}$ & $\mathbf{n}$ & $30^{\circ}$ & $n$ & $45^{\circ}$ & $\mathbf{n}$ & $60^{\circ}$ & $n$ & $75^{\circ}$ & $\mathbf{n}$ & $90^{\circ}(-z)$ & $n$ & $105^{\circ}$ & $\mathbf{r}$ & $120^{\circ}$ & $\mathbf{n}$ & $135^{\circ}$ & $\mathbf{n}$ & $150^{\circ}$ & $\mathbf{n}$ & $165^{\circ}$ & $n$ & $\begin{array}{c}180^{\circ} \\
(-y)\end{array}$ & $\mathbf{n}$ \\
\hline Mg1b & 1,42 & & 1,38 & & 1,35 & & 1,34 & & 1,34 & & 1,38 & & 1,41 & & 1,45 & & 1,54 & & 1,54 & & 1,51 & & 1,37 & & 1,42 & \\
\hline
\end{tabular}

${ }^{*}$ Spaltzugfestigkeiten $\beta_{S z}\left[\mathrm{MPa}\right.$ ]. ${ }^{* *}$ Intensität der Hornblende (110)-Flächen [mrd]. ***Intensität der Diopsid (110)-Flächen [mrd]. **** $\Delta$ Vp-Werte aus dem

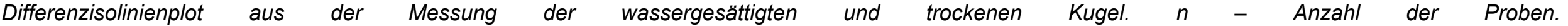




\section{Anhang II.1: Datenblatt Kösseine Granit (Koess)}
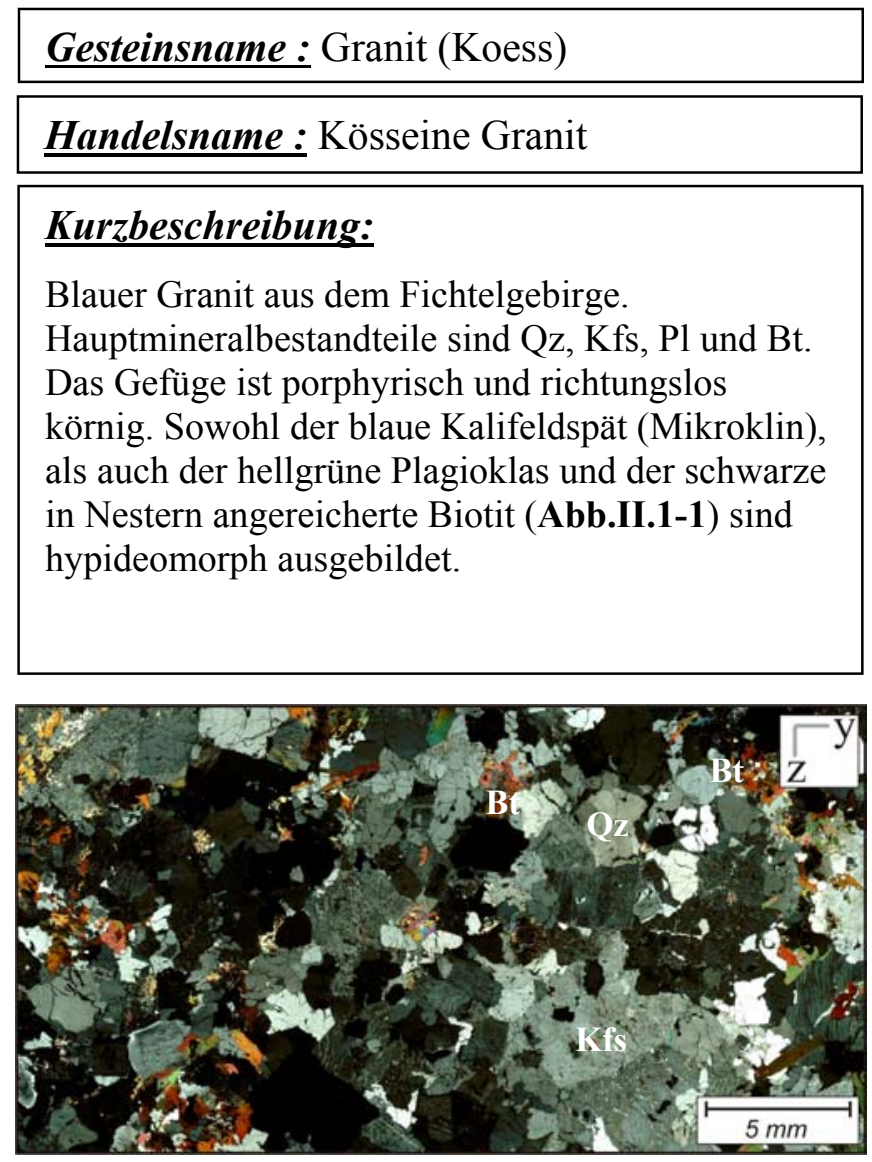

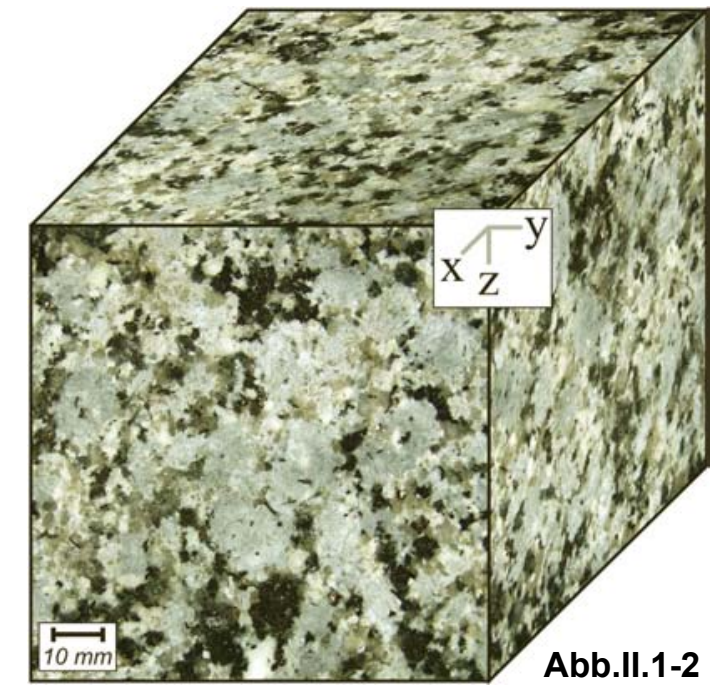

Kfs weist Einschlüsse von $\mathrm{Pl}$ und $\mathrm{Bt}$ auf und zeigt häufig perthitische

Entmischung. Plagioklas ist durchweg polysynthetisch nach dem Albitgesetz verzwillingt. Die Korngrenzen sind gerade(Abb.II-2), geschlossen und teilweise belegt (Fluideinschlüsse).

Abb.II.1-2

Mineralbestand (Vol.\%):

\begin{tabular}{|c|c|c|c|}
\hline Quarz & Kalifeldspat & Plagioklas & Biotit \\
\hline $33+-1$ & $26+-2$ & $30+-1$ & $10+-1$ \\
\hline Chlorit & Erz, Turm, Chl & \multicolumn{2}{|c}{} \\
\cline { 1 - 2 } $1+-0,5$ & $<1$ & &
\end{tabular}

\section{Korngröße:}

\begin{tabular}{|c|c|}
\hline grobkörnig & $5-10 \mathrm{~mm}$ \\
\hline Korngefüge: inequigranular-polygonal
\end{tabular}

Textur maximale Intensität (mrd)/Regelungsschärfe $c_{w}$ /Formfaktor T:

\begin{tabular}{|c|c|c|c|}
\hline Biotit (001)-Pole & Kalifeldspat & Plagioklas & Quarz \\
\hline $2,89 / 0,38 /$ n.b. & n.b. & n.b. & n.b. \\
\hline
\end{tabular}

Risse:

\begin{tabular}{|c|c|c|c|}
\hline Quarz & Kalifeldspat & Plagioklas & Biotit \\
\hline intra off/verh & intra off & $\begin{array}{c}\text { intra (auch Spalt) } \\
\text { off }\end{array}$ & intra (Spalt) off \\
\hline
\end{tabular}

sonstige: Korngrenzen sehr selten offen, teilweise belegt

\section{Referenzen:---}


Anhang II.2: Datenblatt Padang dunkel (PDD)

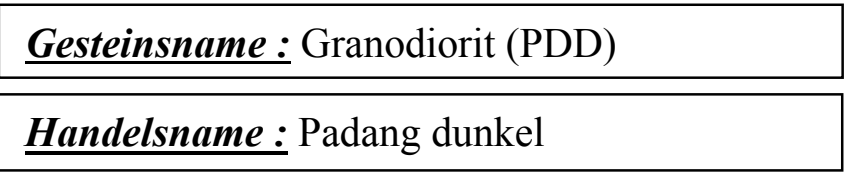

\section{Kurzbeschreibung:}

Granodiorit aus China. Hauptmineralbestandteile sind Pl, Qz, Bt, Kfs und Cpx. Magnetit kommt in geringeren Mengen vor. Das Gefüge ist richtungslos körnig (Abb.II.2-1). Kfs weist Einschlüsse von $\mathrm{Pl}$, Cpx und Bt auf, zudem sind an der Phasengrenze Kfs/Pl mürmekitische

Verwachsungen zu beobachten. Die

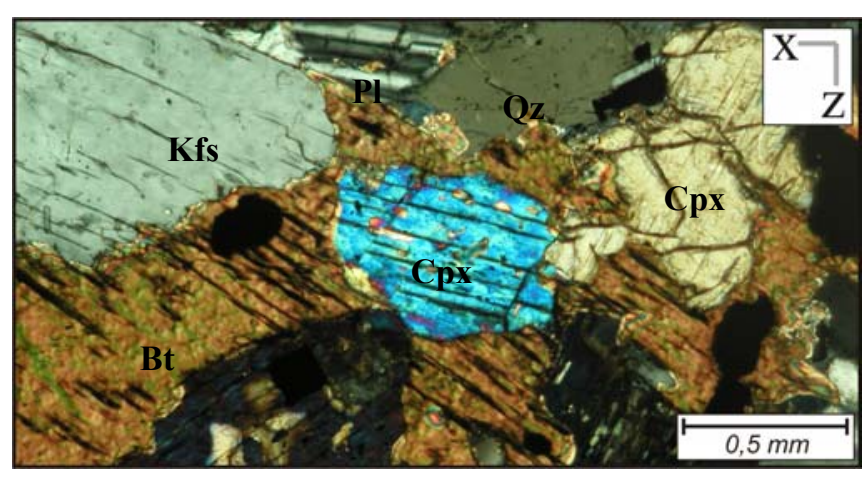

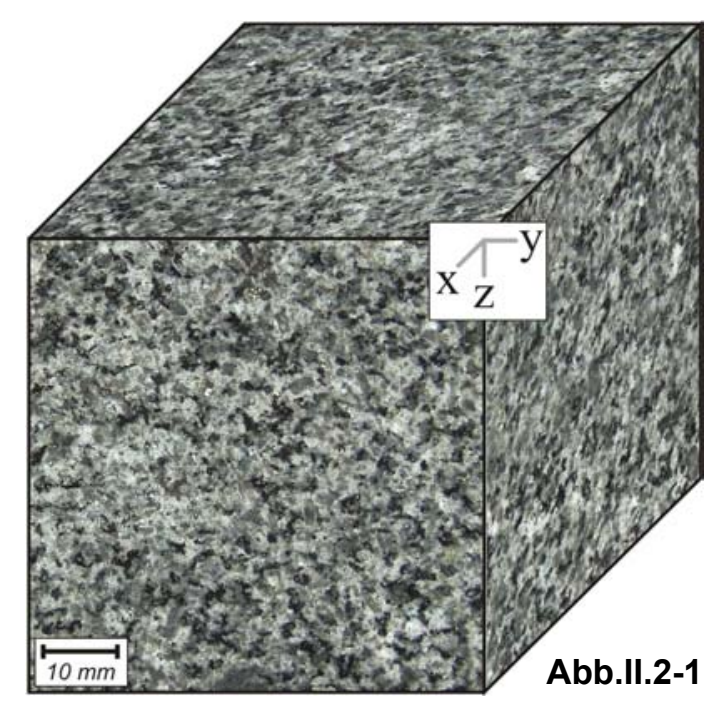

leistenförmig bis kurzprismatisch ausgebildeten P1 sind gelegentlich zoniert und zeigen Einschlüsse von Cpx. Qz löscht gerade bis leicht undulös aus. Alle Komponenten haben gerade bzw. leicht verzahnte Korngrenzen (Abb.II.2-2).

Abb.ll.2-2

Mineralbestand (Vol.\%):

\begin{tabular}{|c|c|c|c|}
\hline Quarz & Kalifeldspat & Plagioklas & Biotit \\
\hline $19+-1$ & $10+-2$ & $51+-1$ & $11+-2$ \\
\hline Klinopyroxen & Magnetit & Chl & \multirow{2}{*}{} \\
\hline $7+-1$ & $2+-0,5$ & $<1$ & \multicolumn{1}{|c}{} \\
\cline { 1 - 3 } & &
\end{tabular}

\section{Korngröße:}

\begin{tabular}{|c|c|}
\hline mittelkörnig & $2-5 \mathrm{~mm}$ \\
\hline
\end{tabular}

Korngefüge: inequigranular-polygonal/interlobat

\section{Textur maximale Intensität (mrd)/Regelungsschärfe $c_{w}$ /Formfaktor T:}

\begin{tabular}{|c|c|c|}
\hline Biotit (001)-Pole & Klinopyroxen & Orthopyroxen \\
\hline $3,11 / 0,53 /-0,51$ & n.b. & n.b. \\
\hline
\end{tabular}

\section{Risse:}

\begin{tabular}{|c|c|c|c|c|}
\hline Quarz & Kalifeldspat & Plagioklas & Biotit & Klinopyroxen \\
\hline $\begin{array}{c}\text { intra/trans } \\
\text { off/verh }\end{array}$ & $\begin{array}{c}\text { intra/trans } \\
\text { off/verh }\end{array}$ & intra/trans off & $\begin{array}{c}\text { intra(Spalt) } \\
\text { off }\end{array}$ & $\begin{array}{c}\text { intra(auch Spalt) } \\
\text { off }\end{array}$ \\
\hline
\end{tabular}

sonstige: Korngrenzen teilweise offen, durchweg belegt (Fluideinschlüsse) 


\section{Anhang}

\section{Anhang II.3: Datenblatt Nero Impala (NI)}

\begin{tabular}{|l|}
\hline Gesteinsname : Gabbro-Norit (NI) \\
\hline \hline Handelsname : Nero Impala \\
\hline
\end{tabular}

\section{Kurzbeschreibung:}

Dunkel grau-grüner Gabbro-Norit aus Südafrika (Abb.II.3-1). Hauptmineralbestand-teile sind Pl, Cpx und Opx. Die makroskopisch nur schwach sichtbare Foliation wird hauptsächlich durch die Kornformanisotropie und Kornformregelung der leistenförmigen bis kurzprismatischen Pl definiert. Die häufig hypideomorph ausgebildeten Cpx und Opx

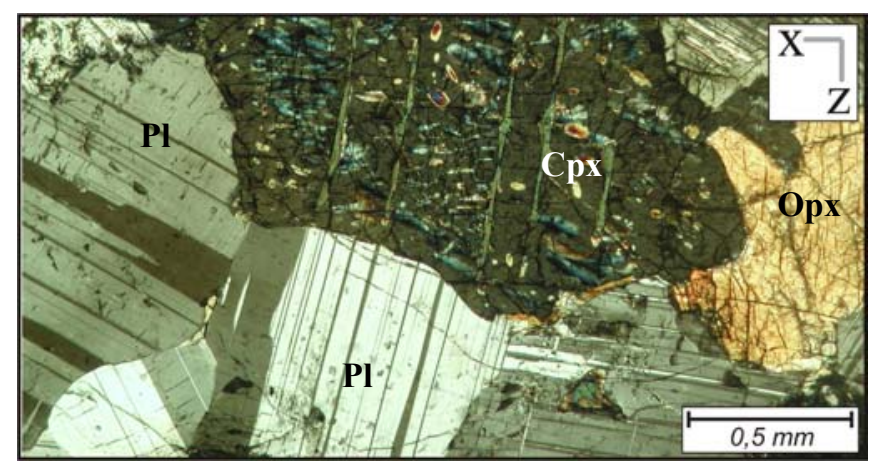

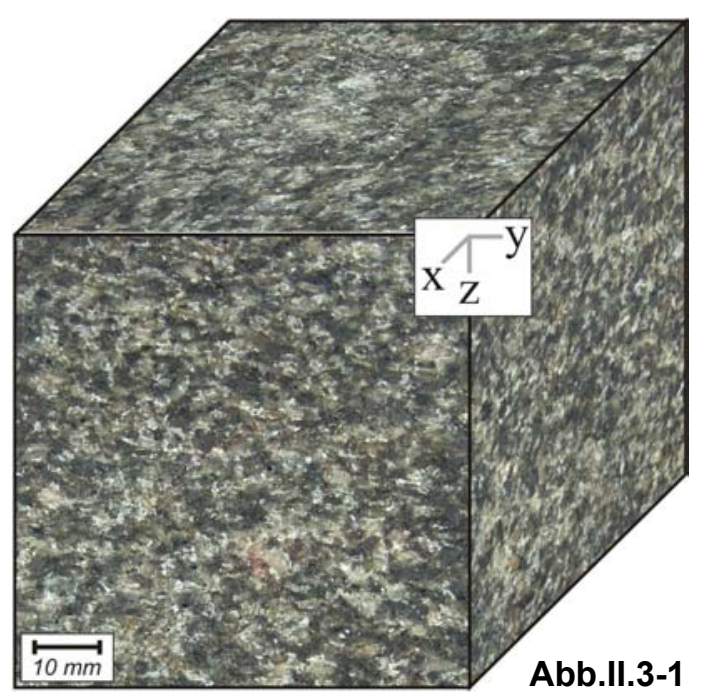

zeigen eine geringe Kornform-regelung. Die Pl sind durchweg verzwillingt und weisen gelegentlich Einschlüsse von Cpx und anderen Pl auf. Die Cpx sind häufig nach Opx entmischt (Abb.II.32). Die Korngrenzen sind gerade und belegt (Fluideinschlüsse).

Abb.II.3-2

Mineralbestand (Vol.\%):

\begin{tabular}{|c|c|c|c|}
\hline Quarz & Plagioklas & Klinopyroxen & Orthopyroxen \\
\hline $1+-0,5$ & $74+-1$ & $13+-1$ & $12+-1$ \\
\hline Akz. & \multicolumn{3}{|l}{} \\
\cline { 1 - 3 }$<1$ &
\end{tabular}

\section{Korngröße:}

\begin{tabular}{|c|c|}
\hline mittelkörnig & $2-5 \mathrm{~mm}$ \\
\hline
\end{tabular}

Korngefüge: equigranular-polygonal

Textur maximale Intensität (mrd)/ Regelungsschärfe $c_{w} /$ Formfaktor T:

\begin{tabular}{|c|c|c|}
\hline Plagioklas & Klinopyroxen & Orthopyroxen \\
\hline n.b. & n.b. & n.b. \\
\hline
\end{tabular}

Risse:

\begin{tabular}{|c|c|c|}
\hline Plagioklas & Klinopyroxen & Orthopyroxen \\
\hline intra/trans off/verh & intra(auch Spalt) off & intra(auch Spalt) off \\
\hline
\end{tabular}

sonstige: Korngrenzen selten offen, belegt

\section{Referenzen: ----}




\section{Anhang II.4: Datenblatt Granit (Mrgr3)}

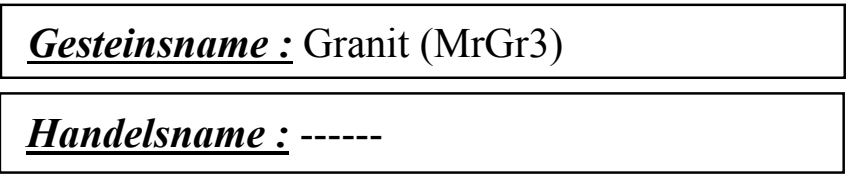

\section{Kurzbeschreibung:}

Feinkörniger Granit vom Lago d’Antrona/Norditalien (Abb.II.4-1).

Hauptmineralbestandteile sind Qz, Kfs, Pl, Ms und Bt. Das porphyrische Gefüge zeigt eine Fluidaltextur. Größere Kfs-Kristalle, welche sowohl Karlsbader Zwillinge als auch perthitische Entmischung aufweisen, schwimmen in einer feinkörnigen Matrix aus

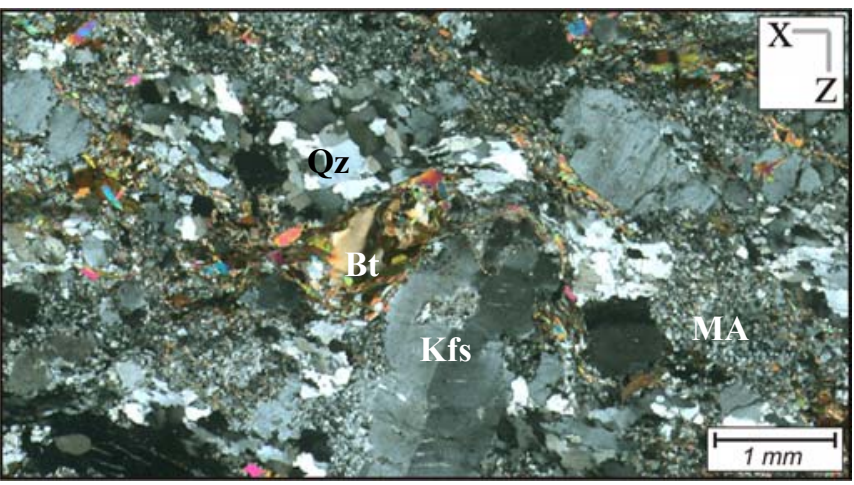

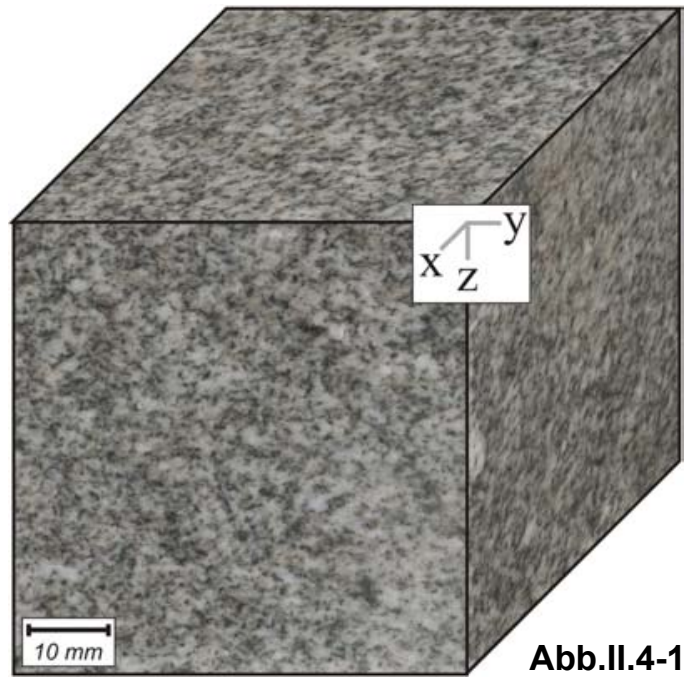

aus Qz, Ms, Pl. Bt ist in Nestern angereichert. Qz löscht gerade bis leicht undulös aus. Die Korngrenzen sind gerade bis gebogen und gelegentlich offen (Abb.II.4-2).

Abb.II.4-2

Mineralbestand (Vol.\%):

\begin{tabular}{|c|c|c|c|}
\hline Quarz & Kalifeldspat & Plagioklas & Muskovit \\
\hline $33+-1$ & $25+-2$ & $24+-1$ & $15+-1$ \\
\hline Biotit & Zr & \multicolumn{2}{|c}{} \\
\cline { 1 - 2 } $3+-2$ & $<1$ &
\end{tabular}

\section{Korngröße:}

feinkörnig

$0,5-2 \mathrm{~mm}$

Korngefüge: inequigranular-polygonal/interlobat

Textur maximale Intensität (mrd)/ Regelungsschärfe $c_{w} /$ Formfaktor T:

\begin{tabular}{|c|c|c|}
\hline Muskovit/Biotit (001)-Pole & Quarz (c-Achse) & Plagioklas (001)-Pole \\
\hline $4,10 / 0,77 / 0,85$ & 1,46 & 1,07 \\
\hline
\end{tabular}

\section{Risse:}

\begin{tabular}{|c|c|c|c|}
\hline Quarz & Kalifeldspat & Plagioklas & Muskovit \\
\hline intra off & intra verh & intra off & intra(Spalt) off \\
\hline
\end{tabular}

sonstige: Korngrenzen teilweise offen 


\section{Anhang II.5: Datenblatt Peridotit (Pi1a)}

\section{Gesteinsname : Peridotit (Pila)}

\section{Handelsname :}

Kurzbeschreibung:

Dunkelgrüner Peridotit (Lherzolith an der Grenze zum Harzburgit) aus Balmuccia/ Norditalien (Abb.II.5-1). Haupmineralbestand ist Ol, Opx und Cpx. In geringeren Mengen kommt Spinell vor. Die Foliation und das deutliche Linear werden durch die Kornformanisotropie und die Kornformregelung von Ol, Opx und Cpx

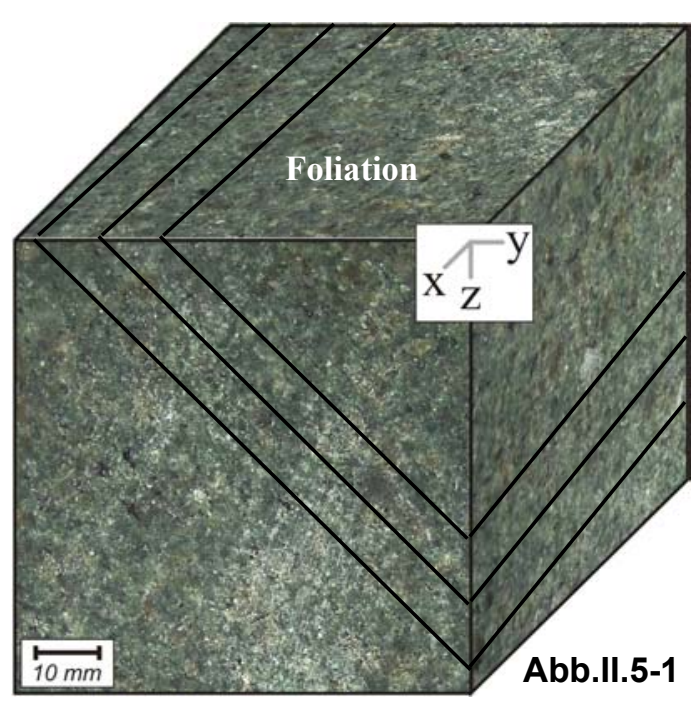

definiert. Die Kornlangachsen sind parallel zur Foliation eingeregelt. Das Gefüge ist vollständig rekristallisiert (equillibriert). Cpx steht immer im Kontakt mit Opx. Die Korngrenzen sind gerade bis gebogen, meist geschlossen und belegt (Abb.II.5-2).

Abb.II.5-2

Mineralbestand (Vol.\%):

\begin{tabular}{|c|c|c|c|}
\hline Klinopyroxen & Orthopyroxen & Olivin & Spinell \\
\hline $13+-1$ & $26+-1$ & $59+-1$ & $2+-0,5$ \\
\hline Akz. & \multicolumn{3}{|l}{} \\
\cline { 1 - 3 }$<1$ & \multicolumn{3}{|l}{}
\end{tabular}

Korngröße:

\begin{tabular}{|c|c|}
\hline mittelkörnig & $2-5 \mathrm{~mm}$ \\
\hline
\end{tabular}

Korngefüge: equi/inequigranular-polygonal

Textur maximale Intensität (mrd)/ Regelungsschärfe $c_{w} /$ Formfaktor T:

\begin{tabular}{|c|c|c|}
\hline Klinopyroxen & Orthopyroxen (001)-Pole & Olivin (100)-Pole \\
\hline n.b. & $1,70 /$ n.b./n.b. & $5,33 /$ n.b./n.b. \\
\hline
\end{tabular}

Risse:

\begin{tabular}{|c|c|c|}
\hline Klinopyroxen & Orthopyroxen & Olivin \\
\hline $\begin{array}{c}\text { intra (auch Spalt)/trans } \\
\text { verh }\end{array}$ & $\begin{array}{c}\text { intra (auch Spalt)/trans } \\
\text { verh }\end{array}$ & intra /trans verh \\
\hline
\end{tabular}

sonstige: Korngrenzen sehr selten offen, meist belegt

Referenzen: Skrotzki et al. (1992) 
Anhang II.6: Datenblatt Rosa Estremoz (Rek)

Gesteinsname : Marmor (Rek)

Handelsname : Rosa Estremoz

\section{Kurzbeschreibung:}

Rosafarbender Kalzit-Marmor aus Portugal (Abb.II.6-1). Das Gestein besteht zu 98\% aus Kalzit. Die rosa bis rötliche Farbe entsteht durch feinkörnige dispers verteilte, zuweilen in Schlieren angereichte Hämatitschuppen. Die Foliation wird durch die Kornformanisotropie und Kornformregelung von Kalzit bestimmt und durch Hämatitschlieren

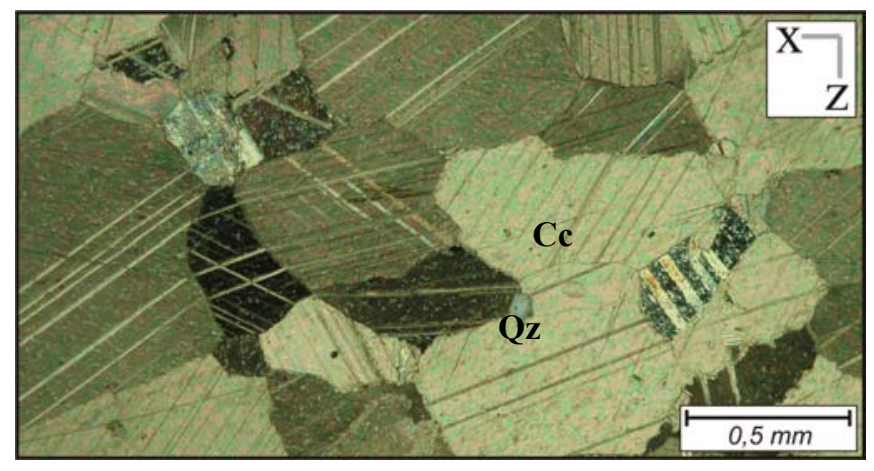

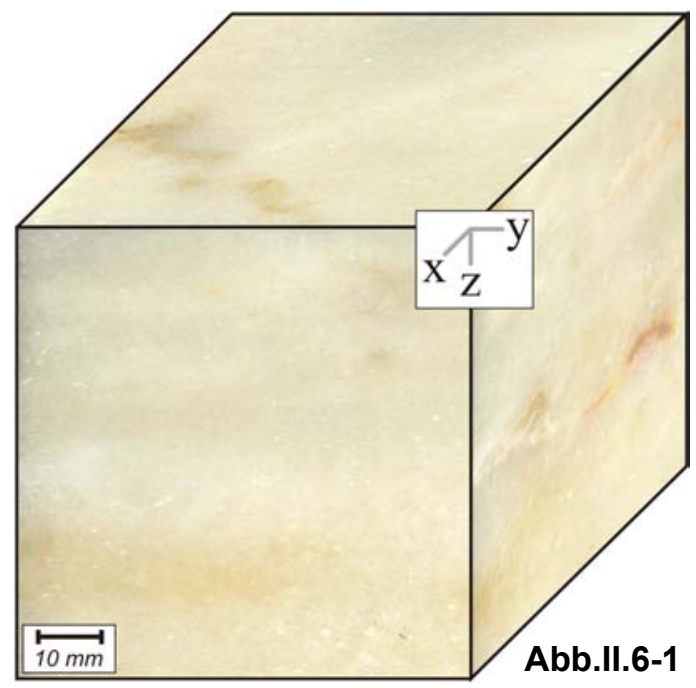

nachgezeichnet. Kalzit ist durchweg verzwillingt. Die Korngrenzen sind auf den ersten Blick gerade (Abb.II.6-2) weisen jedoch eine Mikroverzahnung auf.

\section{Abb.II.6-2}

Mineralbestand (Vol.\%):

\begin{tabular}{|c|c|c|}
\hline Kalzit & Hämatit & Qz, Kfs \\
\hline 98 & 2 & $<1$ \\
\hline
\end{tabular}

\section{Korngröße:}

\begin{tabular}{|c|c|}
\hline feinkörnig & $0,5-2 \mathrm{~mm}$ \\
\hline
\end{tabular}

Korngefüge: seriat-polygonal

Textur maximale Intensität (mrd)/ Regelungsschärfe $c_{w} /$ Formfaktor T:

\begin{tabular}{|c|}
\hline Kalzit (c-Achse) \\
\hline $2,88 /$ n.b./n.b. \\
\hline
\end{tabular}

\section{Risse:}

\begin{tabular}{|c|}
\hline Kalzit \\
\hline intra off \\
\hline
\end{tabular}

sonstige: Korngrenzen selten offen, mikroverzahnt

\section{Referenzen:----}




\section{Anhang}

\section{Anhang II.7: Datenblatt Azul Imperial (AI)}

\section{Gesteinsname : Quarzit (AI)}

Handelsname : Azul Imperial

\section{Kurzbeschreibung :}

Schwach metamorpher und wenig deformierter, blauer Quarzit aus Brasilien mit reliktischem sedimentären Lagenbau (Abb.II.7-1). Das Korngefüge ist inequigranular, wobei der Quarz (graue IF) eine größere und Sericit und Dumortierit (max. blaue IF) kleinere Korngrößen haben (Abb. II.7-2).

Die Korngrenzen sind zum Teil leicht

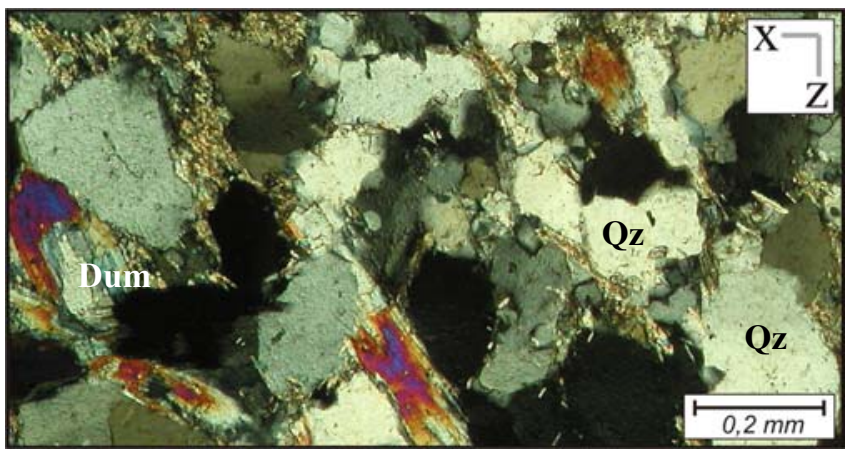

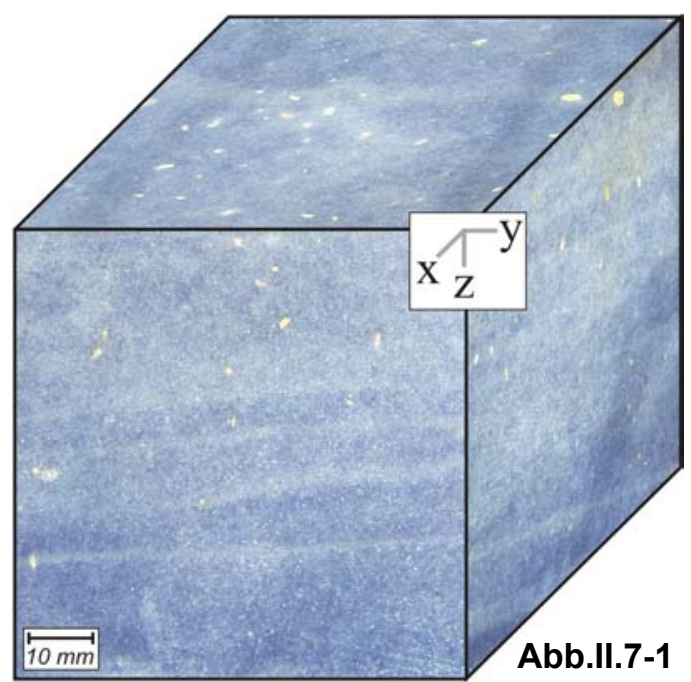

verzahnt.Quarz zeigt eine deutliche Kornformregelung. Entlang der Korngrenzen hydrothermal induzierte Serizitisierung des Quarz und Bildung von Dumortierit; letzeres verleiht dem Gestein die blaue Farbe.

Abb. II.7-2

Mineralbestand (Vol.\%):

\begin{tabular}{|c|c|c|}
\hline Quarz & Muskovit (Serizit) & Dumortierit \\
\hline 87 & 9 & 4 \\
\hline Akz. & \multicolumn{2}{|l}{} \\
\cline { 1 - 1 }$<1$ & \multicolumn{2}{|l}{} \\
\cline { 1 - 1 } & &
\end{tabular}

\section{Korngröße:}

sehr feinkörnig $0,2-0,5 \mathrm{~mm}$

Korngefüge: seriat-polygonal

Textur maximale Intensität (mrd)/Regelungsschärfe $c_{w} /$ Formfaktor T:

Quarz (c-Achse)

1,38/n.b./n.b.

Risse:

\begin{tabular}{|c|c|c|}
\hline Quarz & Muskovit (Serizit) & Dumortierit \\
\hline intra off & intra(Spalt) off & intra (Spalt) off \\
\hline
\end{tabular}

sonstige: Korngrenzen serizitisiert, selten offen

\section{Referenzen: ---}




\section{Anhang II.8: Datenblatt Meta-Gabbro (Mg1b)}

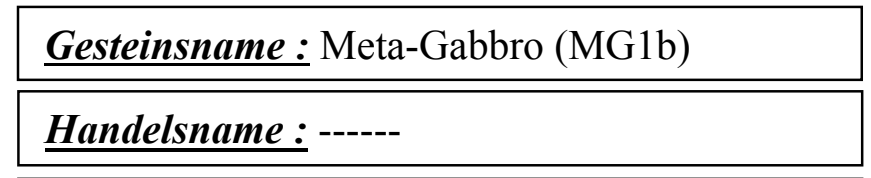

Kurzbeschreibung(Probenserie MG1b/MG2):

Granulitfaziell überprägter und durchgreifend equilibrierter Gabbro aus Anzola/ Italien; das Gestein stammt aus dem Randbereich einer Hochtemperatur-Scherzone. Die Hauptmineralbestandteile sind $\mathrm{Pl}, \mathrm{Hbl}$ und $\mathrm{Cpx}$. Opx kommt in geringeren Mengen vor. Das dominate Kornstreckungslinear (Abb.II.8-1) wird durch die Formanisotropie und die

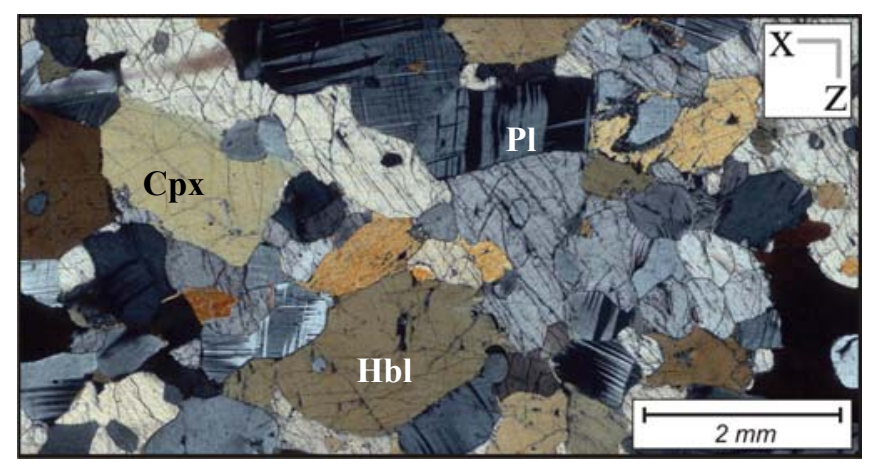

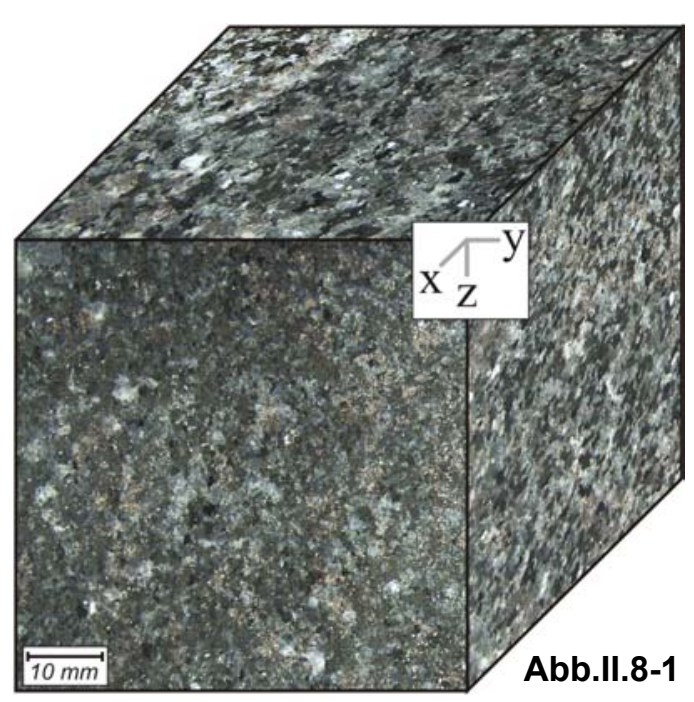

Kornformregelung von hypidiomorph ausgebildeten $\mathrm{Pl}, \mathrm{Hbl}$ und $\mathrm{Cpx}$ bestimmt. Die gelegentlich offenen Korngrenzen sind gerade und treffen vielfach im $120^{\circ}$-Winkel aufeinander (granoblastisches Gefüge--Abb.II.8-2).

Abb.II.8-2

Mineralbestand (Vol.\%):

\begin{tabular}{|c|c|c|c|}
\hline Plagioklas & Hornblende & Klinopyroxen & Orthpyroxen \\
\hline 35 & 44 & 16 & 5 \\
\hline Gt, Ilm, Bt, Tit & \multicolumn{3}{|l}{} \\
\cline { 1 - 1 }$<1$ & \multicolumn{3}{|l}{} \\
\cline { 1 - 2 }
\end{tabular}

\section{Korngröße:}

\begin{tabular}{|c|c|}
\hline Korngroßße: \\
\hline mittelkörnig & $2-5 \mathrm{~mm}$ \\
\hline
\end{tabular}

Korngefüge: equi/inequigranular-polygonal

Textur maximale Intensität (mrd)/Regelungsschärfe $c_{w}$ /Formfaktor T:

\begin{tabular}{|c|c|c|}
\hline Hornblende (001-Pole) & Klinopyroxen (001-Pole) & Plagioklas (001-Pole) \\
\hline $7,79 /$ n.b./n.b. & $1,36 /$ n.b./n.b. & $1,10 /$ n.b./n.b. \\
\hline
\end{tabular}

Risse:

\begin{tabular}{|c|c|c|}
\hline Plagioklas & Hornblende & Klinopyroxen \\
\hline intra/trans off/verh & $\begin{array}{c}\text { intra (Spalt)/trans } \\
\text { off }\end{array}$ & $\begin{array}{c}\text { intra (Spalt)/trans } \\
\text { off } / \text { verh }\end{array}$ \\
\hline
\end{tabular}

sonstige: Korngrenzen teilweise offen und belegt, teilweise geschlossen und belegt

Referenzen: Dornbusch (1995) 


\section{Anhang II.9: Datenblatt Meta-Gabbro (Mg2)}

\section{Gesteinsname : Meta-Gabbro (MG2)}

Handelsname : ------

Kurzbeschreibung(Probenserie MG1b/MG2):

Granulitfaziell überprägter und durchgreifend equilibrierter Gabbro aus Anzola/ Italien; das Gestein stammt aus dem Randbereich einer Hochtemperatur-Scherzone. Die Probe MG2 ist mineralogisch identisch mit der bereits beschriebenen Probe MG1b. Im Unterschied zu letzterer weist MG2 eine undeutliche Foliation auf (Abb.II.9-1), welche durch

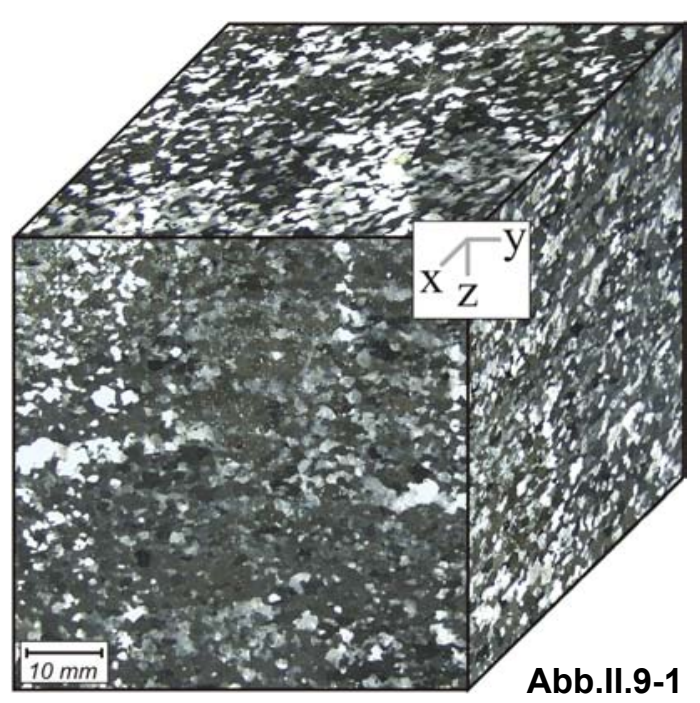

Plagioklas bzw. Hornblende/ Klinopyroxen angereicherte Lagen definiert wird. Des Weiteren zeigt MG2 eine Tendenz zu größeren Korngrößen bezüglich aller Komponenten. Die Korngrenzen sind durchweg geschlossen und gerade (Abb.II.9-2).

Abb.II.9-2

Mineralbestand (Vol.\%):

\begin{tabular}{|c|c|c|c|}
\hline Plagioklas & Hornblende & Klinopyroxen & Orthopyroxen \\
\hline 35 & 44 & 16 & 5 \\
\hline Gt, Ilm, Bt, Tit & \multicolumn{3}{|l}{} \\
\cline { 1 - 3 }$<1$ & \multicolumn{3}{|l}{}
\end{tabular}

\section{Korngröße:}

\begin{tabular}{|c|c|}
\hline mittelkörnig & $2-5 \mathrm{~mm}$ \\
\hline
\end{tabular}

Korngefüge: equi/inequigranular-polygonal

Textur maximale Intensität (mrd)/Regelungsschärfe $c_{w} /$ Formfaktor T:

\begin{tabular}{|c|c|c|}
\hline Hornblende (001-Pole) & Klinopyroxen (001-Pole) & Plagioklas (001-Pole) \\
\hline $7,79 /$ n.b./n.b. & $1,36 /$ n.b./n.b. & $1,10 /$ n.b./n.b. \\
\hline
\end{tabular}

Risse:

\begin{tabular}{|c|c|c|}
\hline Plagioklas & Hornblende & Klinopyroxen \\
\hline intra/trans off/verh & $\begin{array}{c}\text { intra (Spalt)/trans } \\
\text { off }\end{array}$ & $\begin{array}{c}\text { intra (Spalt)/trans } \\
\text { off/verh }\end{array}$ \\
\hline
\end{tabular}

sonstige: selten offene Korngrenzen

Referenzen: Dornbusch (1995) 
Anhang II.10: Datenblatt granodioritischer Protomylonit (Iv1)
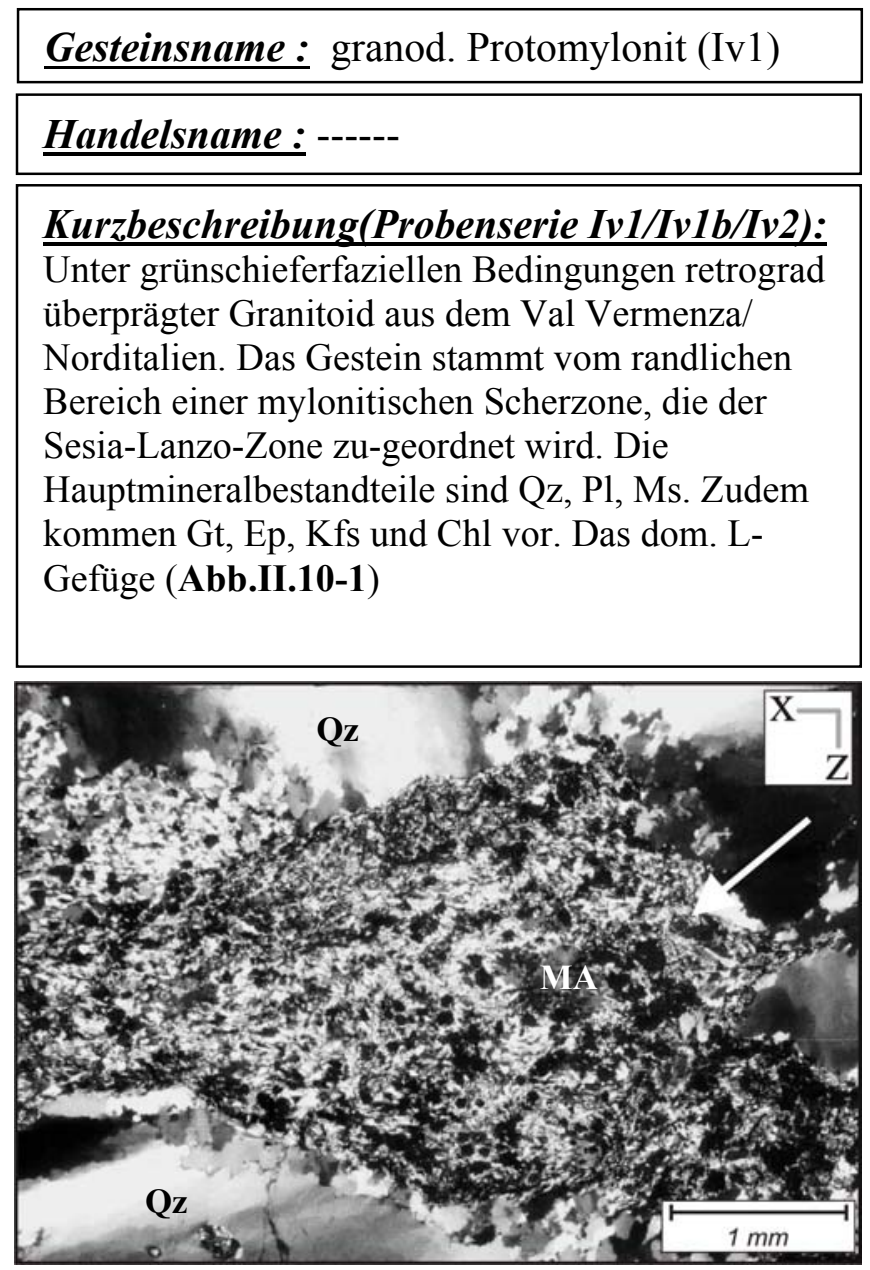

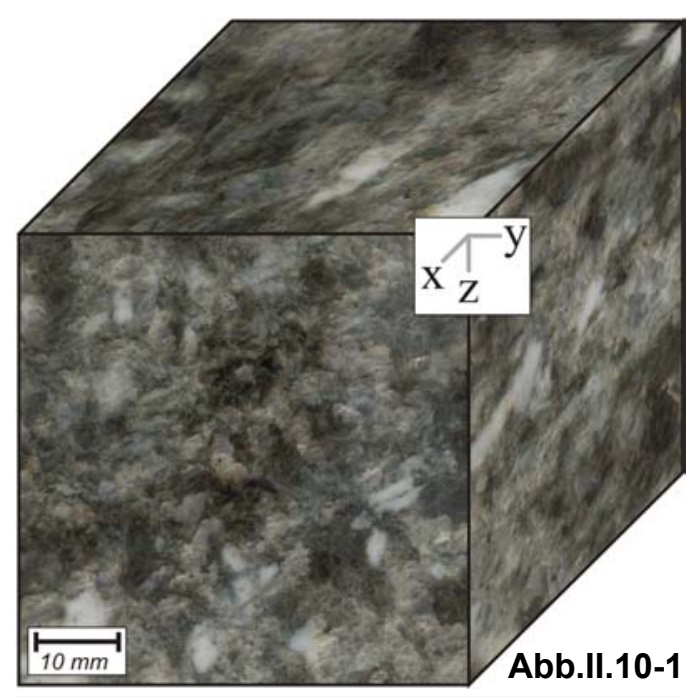

wird von großen, gelängten QzKristallen gebildet, die neben KfsKlasten von mikrokristallinen, grünen Aggregaten (MA) umgeben sind. Diese sind mit Ep, Gt und Chl angereichert. Qz zeigt randliche Subkornbildung und damit Indizien für eine beginnende Mylonitisierung (Abb.II.10-2).

Abb.II.10-2

Mineralbestand (Vol.\%):

\begin{tabular}{|c|c|c|c|}
\hline Quarz & Kalifeldspat & Plagioklas & Muskovit \\
\hline 36 & 5 & 28 & 15 \\
\hline Chlorit & Granat & Klinozoisit/Epidot & Apa, Rt, Zr \\
\hline 6 & 4 & 6 & $<1$ \\
\hline
\end{tabular}

\section{Korngröße:}

\begin{tabular}{|c|c|}
\hline mittelkörnig & $2-5 \mathrm{~mm}$ \\
\hline
\end{tabular}

Korngefüge: inequigranular-interlobat

Textur maximale Intensität (mrd)/Regelungsschärfe $c_{w} /$ Formfaktor T:

\begin{tabular}{|c|c|c|}
\hline Muskovit (001)-Pole & Quarz (c-Achse) & Plagioklas (001-pole) \\
\hline $5,54 / 1,24 /-0,89$ & $1,7 /$ n.b./n.b. & $1,08 /$ n.b./n.b. \\
\hline
\end{tabular}

Risse:

\begin{tabular}{|c|c|c|c|}
\hline Quarz & Kalifeldspat & Plagioklas & Muskovit \\
\hline intra verh & intra off/verh & intra off/verh & intra (Spalt) off \\
\hline
\end{tabular}

sonstige: Korngrenzen selten offen

Referenzen: Juckenack (1990); Techmer (1996); Strohmeyer \& Siegesmund (2002) 
Anhang II.11: Datenblatt granitischer Gneis (Gra1a)

\begin{tabular}{|l|}
\hline Gesteinsname : granitischer Gneis (Grala) \\
\hline Handelsname : ----- \\
\hline Kurzbeschreibung: \\
Gneis aus dem Waldviertel im Nieder- \\
österreichischen Maidling. Das Gestein wird den \\
"Granuliten“ der Gföhler Einheit zugeordnet. \\
Hauptmineralbestandteile sind Quarz, Kalifeldspat, \\
Plagioklas; Biotit und Granat kommen in \\
geringeren Mengen vor. Die makroskopisch \\
deutliche Foliation wird durch lagige Variation des \\
Mineralbestands,
\end{tabular}
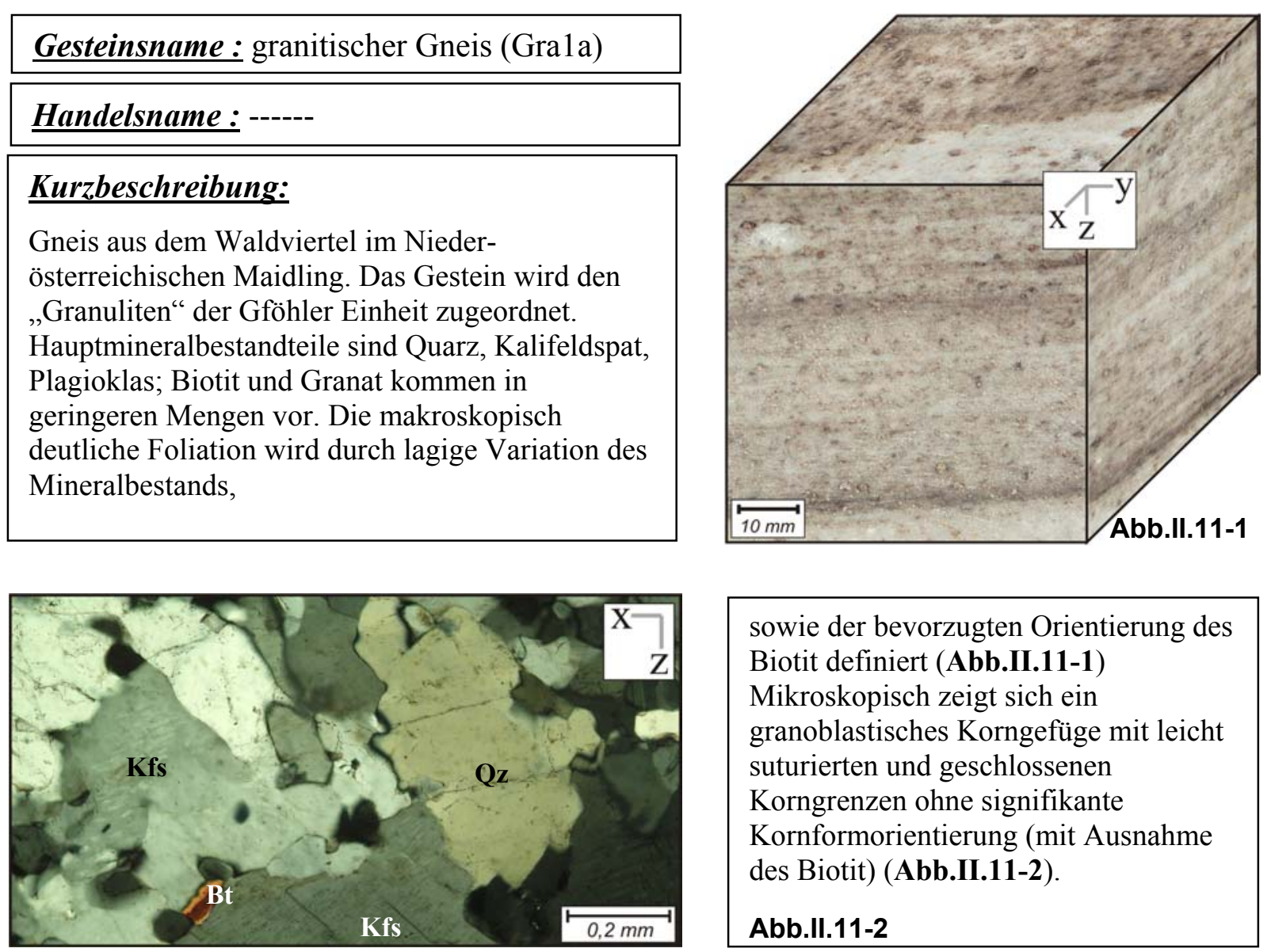

sowie der bevorzugten Orientierung des Biotit definiert (Abb.II.11-1)

Mikroskopisch zeigt sich ein granoblastisches Korngefüge mit leicht suturierten und geschlossenen

Korngrenzen ohne signifikante Kornformorientierung (mit Ausnahme des Biotit) (Abb.II.11-2).

Abb.II.11-2

Mineralbestand (Vol.\%):

\begin{tabular}{|c|c|c|c|}
\hline Quarz & Kalifeldspat & Plagioklas & Biotit \\
\hline $33+-1$ & $32+-1$ & $28+-1$ & $4+-1$ \\
\hline Granat & Ky, Sil, Ru & \multicolumn{2}{|c}{} \\
\hline $3+-0,5$ & $<1$ & \multicolumn{2}{|c}{} \\
\cline { 1 - 2 } & &
\end{tabular}

\section{Korngröße:}

\begin{tabular}{l|l} 
sehr feinkörnig & $0,2-0,5 \mathrm{~mm}$ \\
\hline
\end{tabular}

Korngefüge: inequigranular-polygonal/interlobat

Textur maximale Intensität (mrd)/Regelungsschärfe $c_{w}$ /Formfaktor T:

\begin{tabular}{|c|c|c|}
\hline Biotit (001)-Pole & Quarz (c-Achse) & Plagioklas (001)-Pole \\
\hline $5,71 / 1,26 / 0,79$ & 1,37 & 1,42 \\
\hline
\end{tabular}

Risse:

\begin{tabular}{|c|c|c|c|}
\hline Quarz & Kalifeldspat & Plagioklas & Biotit \\
\hline intra verh & intra verh/off & intra verh/off & Intra (Spalt) off \\
\hline
\end{tabular}

sonstige: Korngrenzen selten offen, teilweise belegt

Referenzen: Duyster (1991); Ullemeyer (1992) 
Anhang II.12: Datenblatt granitischer Protomylonit (Mrmy5)

\begin{tabular}{|l|}
\hline Gesteinsname : granit. Protomylonit (MrMy5) \\
\hline \hline Handelsname :------ \\
\hline \hline Kurzbeschreibung: \\
Heller mylonitisierter Gneis vom \\
Mattmarkstausee/Schweiz (Abb.II.12-1). \\
Hauptmineralbestandteile sind Qz, Pl, Kfs, Ms und \\
Bt. Das porphyroklastische Gefüge wird durch \\
große Kfs-Kristalle bestimmt, die in einer \\
feinkörnigen Matrix aus Qz, Pl und Ms \\
schwimmen. Alternierende Ms/Bt- und Qz,Pl- \\
angereicherte Lagen bilden die makros- und
\end{tabular}
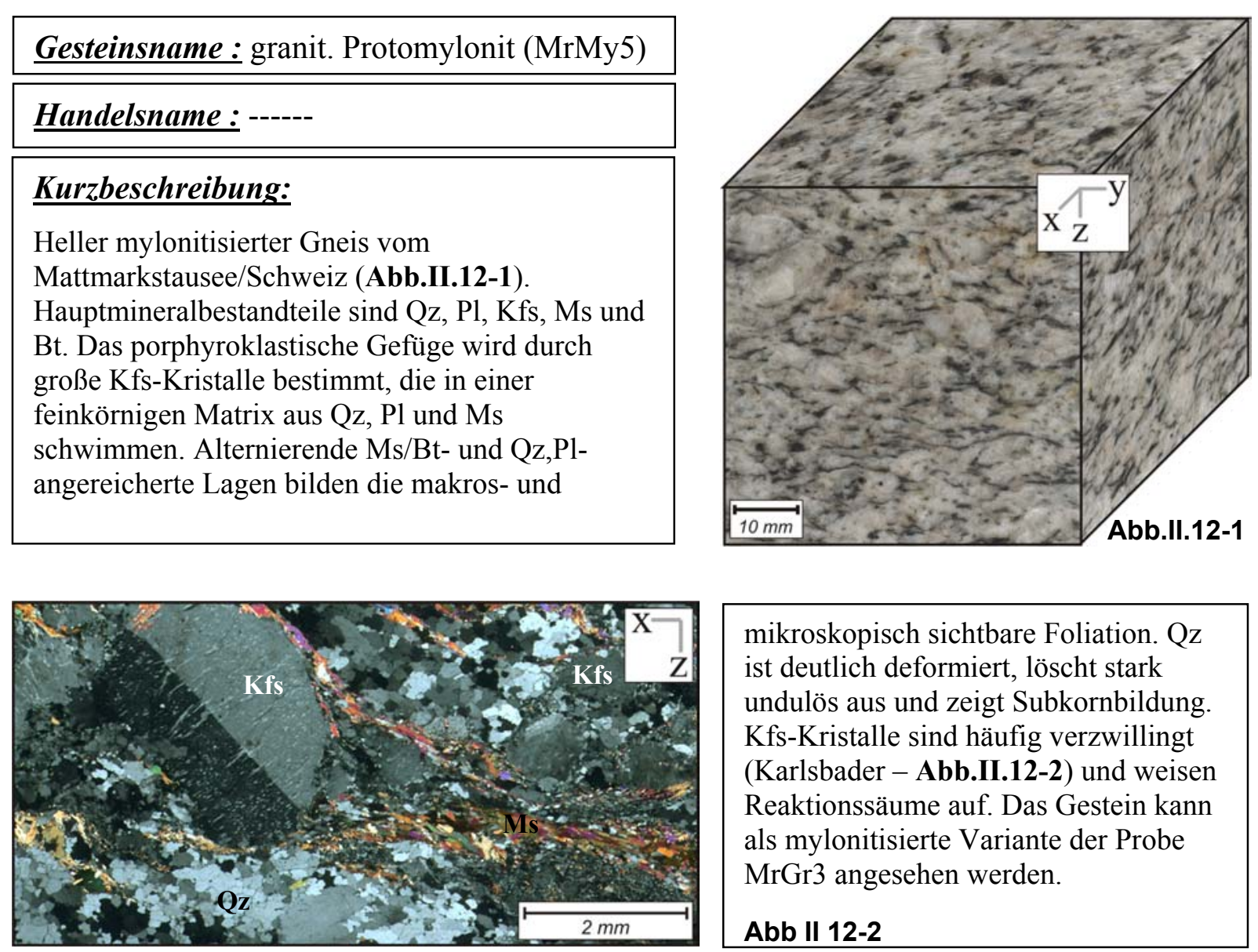

mikroskopisch sichtbare Foliation. Qz ist deutlich deformiert, löscht stark undulös aus und zeigt Subkornbildung. Kfs-Kristalle sind häufig verzwillingt (Karlsbader - Abb.II.12-2) und weisen Reaktionssäume auf. Das Gestein kann als mylonitisierte Variante der Probe $\mathrm{MrGr} 3$ angesehen werden.

\section{Abb II 12-2}

Mineralbestand (Vol.\%):

\begin{tabular}{|c|c|c|c|}
\hline Quarz & Kalifeldspat & Plagioklas & Muskovit \\
\hline 36 & 22 & 25 & 10 \\
\hline Biotit & Chlorit & Akz. & \multirow{2}{*}{} \\
\hline 6 & 1 & $<1$ & \multicolumn{1}{|c}{} \\
\cline { 1 - 3 } & \multicolumn{2}{|c}{} \\
\cline { 1 - 3 } & &
\end{tabular}

\section{Korngröße:}

\begin{tabular}{|c|c|}
\hline mittelkörnig & $2-5 \mathrm{~mm}$ \\
\hline
\end{tabular}

Korngefüge: inequigranular-interlobat

Textur maximale Intensität (mrd)/Regelungsschärfe $c_{w}$ /Formfaktor T:

\begin{tabular}{|c|c|c|}
\hline Biotit/Muskovit (001-Pole) & Quarz (c-Achse) & Plagioklas (001-Pole) \\
\hline $11,07 / 2,44 / 0,82$ & 1,93 & 1,12 \\
\hline
\end{tabular}

Risse:

\begin{tabular}{|c|c|c|c|c|}
\hline Quarz & Kalifeldspat & Plagioklas & Muskovit & Biotit \\
\hline intra/trans off & intra off/verh & intra off & intra(Spalt) off & intra(Spalt) off \\
\hline
\end{tabular}

sonstige: Korngrenzen teilweise offen

\section{Referenzen: ----}




\section{Anhang II.13: Datenblatt granodioritischer Gneis (Be1)}

Gesteinsname : granitischer Gneis (Be1)

Handelsname : Serizzo Monte Rosa

\section{Kurzbeschreibung:}

Schwarz-weißer Augengneis aus

Beura/Norditalien. Hauptmineralbestandteile sind Qz, Pl, Kfs, Bt und Ms. Chlorit kommt als retrograde Bildung vor. Das Gestein hat ein SGefüge mit großen Kfs-Augen. Die Foliation wird durch lagig angereicherte $\mathrm{Bt} / \mathrm{Ms}$ bestimmt, welche mit Qz/Pl-angereicherten Lagen alternieren (Abb.II.13-1). Ein Linear ist nur schwach

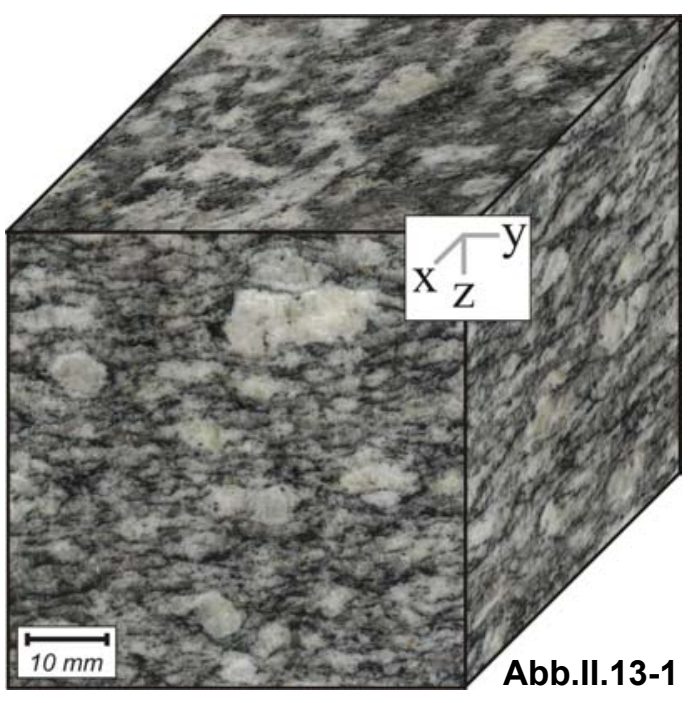

ausgebildet und in Form eines

Kornstreckungslinears von $\mathrm{Kfs}$ und $\mathrm{Bt}$ auf der Foliation zu beobachten. Die Kfs zeigen durchweg Reaktionssäume, viele kleine Einschlüsse und teilweise perthitische Entmischung. Pl ist teilweise verzwillingt (Abb.II.13-2).

Abb.II.13-2

Mineralbestand (Vol.\%):

\begin{tabular}{|c|c|c|c|}
\hline Quarz & Kalifeldspat & Plagioklas & Muskovit \\
\hline 32 & 11 & 38 & 4 \\
\hline Biotit & Chlorit & Ep, Turm & \multicolumn{1}{|}{} \\
\hline 13 & 1 & $<1$ & \multicolumn{1}{|c}{} \\
\cline { 1 - 3 } & \multicolumn{2}{|c|}{}
\end{tabular}

\section{Korngröße:}

\begin{tabular}{l|l} 
mittelkörnig & $2-5 \mathrm{~mm}$ \\
\hline
\end{tabular}

Korngefüge: inequigranular-polygonal/interlobat

Textur maximale Intensität (mrd)/ Regelungsschärfe $c_{w} /$ Formfaktor T:

\begin{tabular}{|c|c|c|}
\hline Biotit/ Muskovit (001-Pole) & Quarz (c-Achse) & Plagioklas (001)-Pole \\
\hline $15,48 / 2,75 / 0,65$ (oblat) & 2,23 & 1,42 \\
\hline
\end{tabular}

Risse:

\begin{tabular}{|c|c|c|c|}
\hline Quarz & Kalifeldspat & Plagioklas & Biotit \\
\hline intra off/verh & intra off/verh & intra off & intra(Spalt) off \\
\hline
\end{tabular}

sonstige: Korngrenzen selten offen, teilweise belegt (Fluideinschlüsse)

\section{Referenzen: ----}


Anhang II.14: Datenblatt granodioritischer Mylonit (Iv1b)
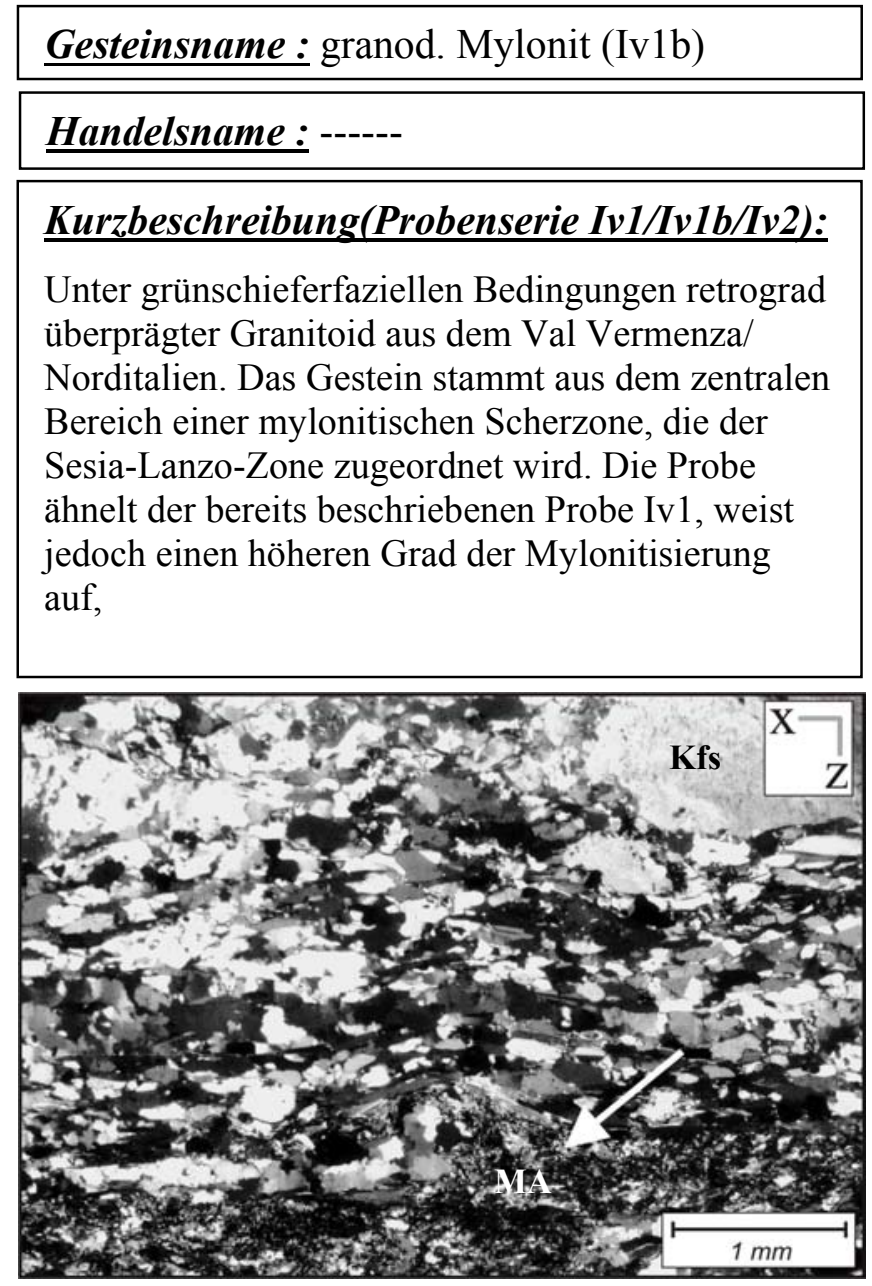

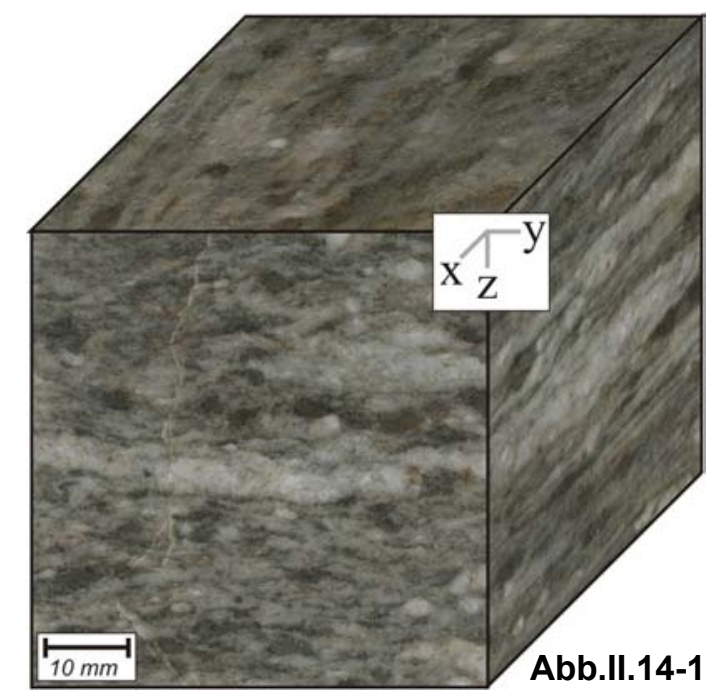

was die Umwandlung von einem LGefüge zu einem L-S-Gefüge zur Folge hat und von einer deutlicheren Segregation des Mineralbestands in Qz/Pl(hell) und Ep/Chl(dunkel) angereichterte Lagen begleitet wird (Abb.II.14-1). Zudem verringern sich die Korngrössen von Qz und Kfs.

Abb.II.14-2

Mineralbestand (Vol.\%):
\begin{tabular}{|c|c|c|c|}
\hline Quarz & Kalifeldspat & Plagioklas & Muskovit \\
\hline 36 & 5 & 28 & 15 \\
\hline Chlorit & Granat & Klinozoisit/Epidot & Apa, Rt, Zr \\
\hline 6 & 4 & 6 & $<1$ \\
\hline
\end{tabular}

\section{Korngröße:}

\begin{tabular}{l|l} 
feinkörnig & $0,5-2 \mathrm{~mm}$ \\
\hline
\end{tabular}

Korngefüge: inequigranular-interlobat

Textur maximale Intensität (mrd)/Regelungsschärfe $c_{w} /$ Formfaktor T:

\begin{tabular}{|c|c|c|}
\hline Muskovit (001)-Pole & Quarz (c-Achse) & Plagioklas (001)-Pole \\
\hline $12,25 / 2,91 / 0,00$ & $1,56 /$ n.b./n.b. & $1,08 /$ n.b./n.b. \\
\hline
\end{tabular}

Risse:

\begin{tabular}{|c|c|c|c|}
\hline Quarz & Kalifeldspat & Plagioklas & Muskovit \\
\hline intra verh & intra off/verh & intra off/verh & intra (Spalt) off \\
\hline
\end{tabular}

sonstige: Korngrenzen selten offen

Referenzen: Juckenack (1990); Techmer (1996); Strohmeyer \& Siegesmund (2002) 
Anhang II.15: Datenblatt Calanca (Cal)

Gesteinsname : granod. Paragneis (Cal)

Handelsname : Calanca

\section{Kurzbeschreibung:}

Schwarz-weißer Paragneis mit flaseriger Foliation aus dem Tessin/Norditalien (Abb.II.15-1).

Hauptmineralbestandteile sind Qz, Pl, Bt und Kfs. In geringen Mengen kommt Chlorit vor. Das makroskopisch beobachtbare S-L-Gefüge wird durch alternierende Qz/ Fs und Bt- Lagen und die Kornformanisotropie und Kornformregelung der Hauptkomponenten

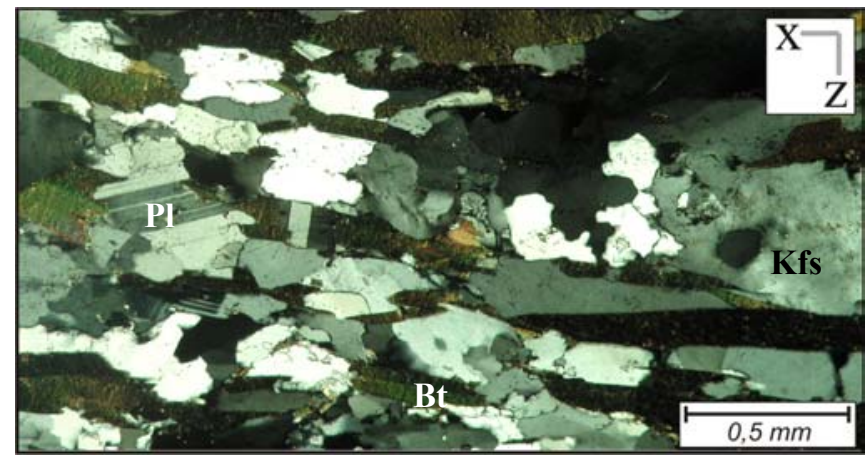

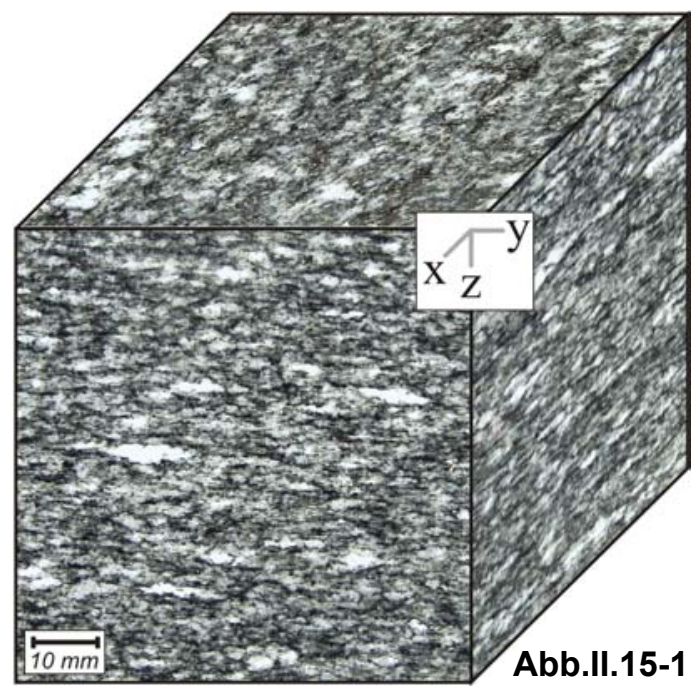

bestimmt. Das Kornstreckungslinear wird von gelängten Fs und langgestreckten Qz-Aggregaten gebildet. Qz löscht leicht undulös aus, $\mathrm{Pl}$ ist häufig verzwillingt (Abb.II.15-2) und an der Phasengrenze von Kfs und $\mathrm{Pl}$ sind gelegentlich mürmekitische Verwachsungen zu beobachten.

Abb.II.15-2

Mineralbestand (Vol.\%):

\begin{tabular}{|c|c|c|c|}
\hline Quarz & Kalifeldspat & Plagioklas & Biotit \\
\hline 34 & 9 & 38 & 18 \\
\hline Chlorit & Ep & \multicolumn{2}{|}{} \\
\cline { 1 - 2 } 1 & $<1$ & \multicolumn{2}{c}{} \\
\cline { 1 - 2 } & &
\end{tabular}

\section{Korngröße:}

\begin{tabular}{|c|c|}
\hline feinkörnig & $0,5-2 \mathrm{~mm}$ \\
\hline
\end{tabular}

Korngefüge: seriat-polygonal/interlobat

Textur maximale Intensität (mrd)/ Regelungsschärfe $c_{w} /$ Formfaktor T:

\begin{tabular}{|c|c|c|}
\hline Biotit (001)-Pole & Quarz (c-Achse) & Plagioklas (001-Pole) \\
\hline $16,65 / 3,04 / 0,25$ & 2,08 & 1,53 \\
\hline
\end{tabular}

Risse:

\begin{tabular}{|c|c|c|c|}
\hline Quarz & Kalifeldspat & Plagioklas & Biotit \\
\hline intra off/verh & intra off/verh & intra off/verh & intra (Spalt) off \\
\hline
\end{tabular}

sonstige: Korngrenzen selten offen, gelegentlich belegt (Fluideinschlüsse)

\section{Referenzen: ----}


Anhang II.16: Datenblatt Verde Andeer (VA)

Gesteinsname : granitischer Orthogneis (VA)

Handelsname : Verde Andeer

\section{Kurzbeschreibung:}

Grüner Orthogneis aus Graubünden/Schweiz

(Abb.II.16-1). Hauptmineralbestandteile sind Qz, $\mathrm{Pl}$, Kfs und Ms (Phengit). Große Kfs-Klasten sowie stark deformierte, undulös auslöschende Qz bilden die Augen des makroskopischen S-Gefüges und schwimmen in einer Matrix aus mikrokristallinem Qz, Pl, Ms und Chl (Abb.II.16-2). Die Foliation wird durch

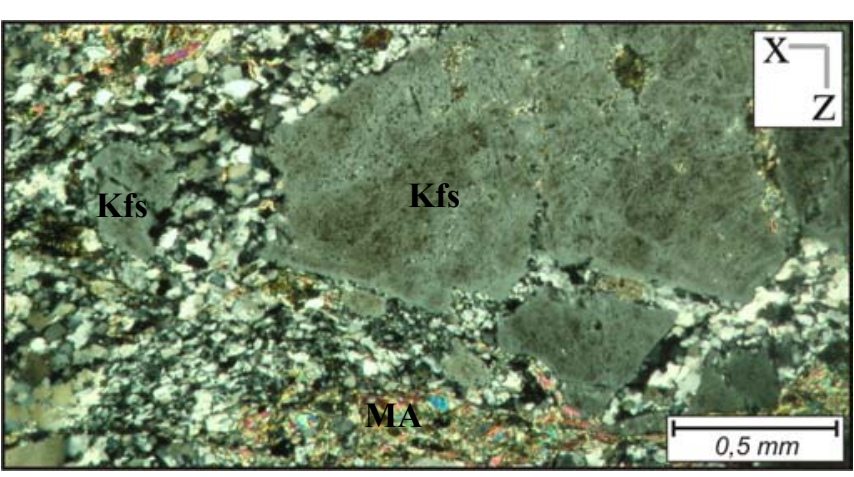

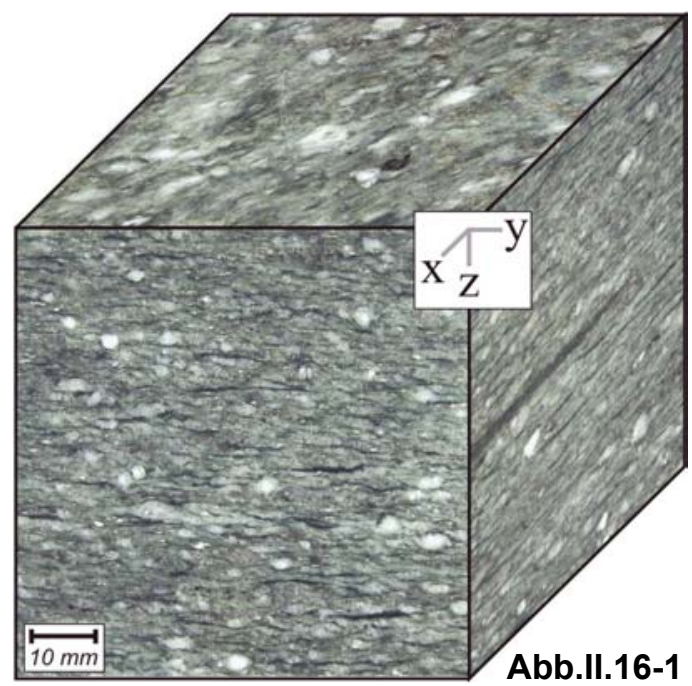

alternierende_Ms/Chl- und Qz/Plangereicherte Lagen bestimmt. Das Kornstreckungslinear ist nur schwach ausgebildet und wird hauptsächlich durch die Form-regelung der KfsKlasten definiert. Pl ist selten verzwillingt und Qz zeigt Anzeichen dynamischer Rekristallisation.

Abb.ll.16-2

Mineralbestand (Vol.\%):

\begin{tabular}{|c|c|c|c|}
\hline Quarz & Kalifeldspat & Plagioklas & Phengit \\
\hline $34+-1$ & $19+-1$ & $21+-1$ & $22+-2$ \\
\hline Chlorit & Turm, Gt, Ep & \multicolumn{2}{|c}{} \\
\cline { 1 - 2 } $4+-1$ & $<1$ &
\end{tabular}

\section{Korngröße:}

\begin{tabular}{l|r} 
feinkörnig & $0,5-2 \mathrm{~mm}$ \\
\hline
\end{tabular}

Korngefüge: inequigranular-polygonal/interlobat

Textur maximale Intensität (mrd)/ Regelungsschärfe $c_{w} /$ Formfaktor T:

\begin{tabular}{|c|c|c|}
\hline Phengit/Muskovit (001-Pole) & Quarz (c-Achse) & Plagioklas \\
\hline $15,89 / 2,44 / 0,79$ & 1,44 & n.b. \\
\hline
\end{tabular}

Risse:

\begin{tabular}{|c|c|c|c|}
\hline Quarz & Kalifeldspat & Plagioklas & Phengit \\
\hline intra verh/min & intra off/verh/min & intra off/verh & intra(Spalt) off \\
\hline
\end{tabular}

sonstige: Korngrenzen teilweise offen, insgesamt wenige Risse im mikrokristallinen Bereich

\section{Referenzen: ----}


Anhang II.17: Datenblatt granitischer Gneis (Gra1c)

Gesteinsname : granitischer Gneis (Gra1c)

Handelsname : ------

Kurzbeschreibung:

Gneis aus dem Waldviertel im Niederösterreichischen Maidling. Das Gestein wird den „Granuliten“ der Gföhler Einheit zugeordnet. Hauptmineralbestandteile sind Quarz, Kalifeldspat, Plagioklas; Biotit und Granat kommen in geringeren Mengen vor. Die Probe Gra1c unterscheidet sich von der bereits beschriebenen Probe Gra1a lediglich
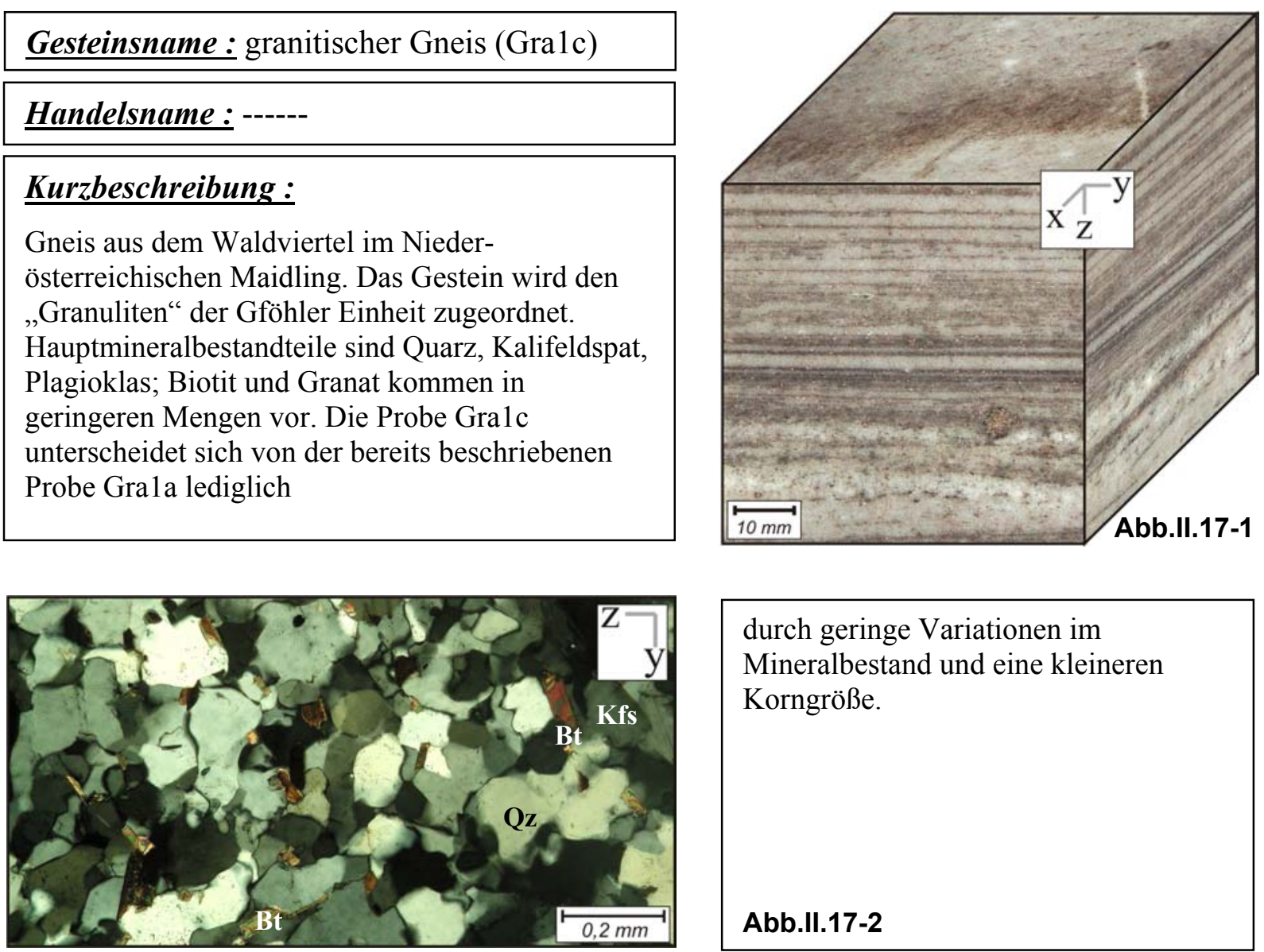

durch geringe Variationen im Mineralbestand und eine kleineren Korngröße.

Abb.II.17-2

Mineralbestand (Vol.\%):

\begin{tabular}{|c|c|c|c|}
\hline Quarz & Kalifeldspat & Plagioklas & Biotit \\
\hline $34+-1$ & $28+-1$ & $30+-1$ & $5+-1$ \\
\hline Granat & $\mathrm{Ky}, \mathrm{Sil}, \mathrm{Ru}$ & \multicolumn{2}{|c}{} \\
\cline { 1 - 2 } $3+-0,5$ & $<1$ & \multicolumn{2}{|c}{} \\
\cline { 1 - 2 } & \multicolumn{2}{|c|}{}
\end{tabular}

\section{Korngröße:}

sehr feinkörnig

$0,2-0,5 \mathrm{~mm}$

Korngefüge: inequigranular-polygonal/interlobat

Textur maximale Intensität (mrd)/ Regelungsschärfe $c_{w} /$ Formfaktor T:

\begin{tabular}{|c|c|c|}
\hline Biotit (001)-Pole & Quarz & Plagioklas \\
\hline $8,61 / 1,26 / 0,79$ & n.b. & n.b. \\
\hline
\end{tabular}

Risse:

\begin{tabular}{|c|c|c|c|}
\hline Quarz & Kalifeldspat & Plagioklas & Biotit \\
\hline intra verh & intra verh/off & intra verh/off & intra (Spalt) off \\
\hline
\end{tabular}

sonstige: Korngrenzen selten offen, teilweise belegt

Referenzen: Duyster (1991); Ullemeyer (1992) 
Anhang II.18: Datenblatt granitischer Gneis (Be2)

\begin{tabular}{|l|}
\hline Gesteinsname : granitischer Gneis (Be2) \\
\hline Handelsname : Serizzo Monte Rosa \\
\hline Kurzbeschreibung: \\
Schwarz-weißer, stengeliger Gneis aus \\
Beura/Norditalien (Abb.II.18-1). \\
Hauptmineralbestandteile sind Qz, P1, Kfs, Ms und \\
Bt. Als retrogrades Unwandlungsprodukt kommt \\
Chl vor. Das makroskopische Erscheinungsbild \\
wird durch ein stark ausgebildetes Linear \\
(langgestreckte Qz/Fs-Aggregate) und rotational \\
angeordnete Bt und \\
\hline
\end{tabular}
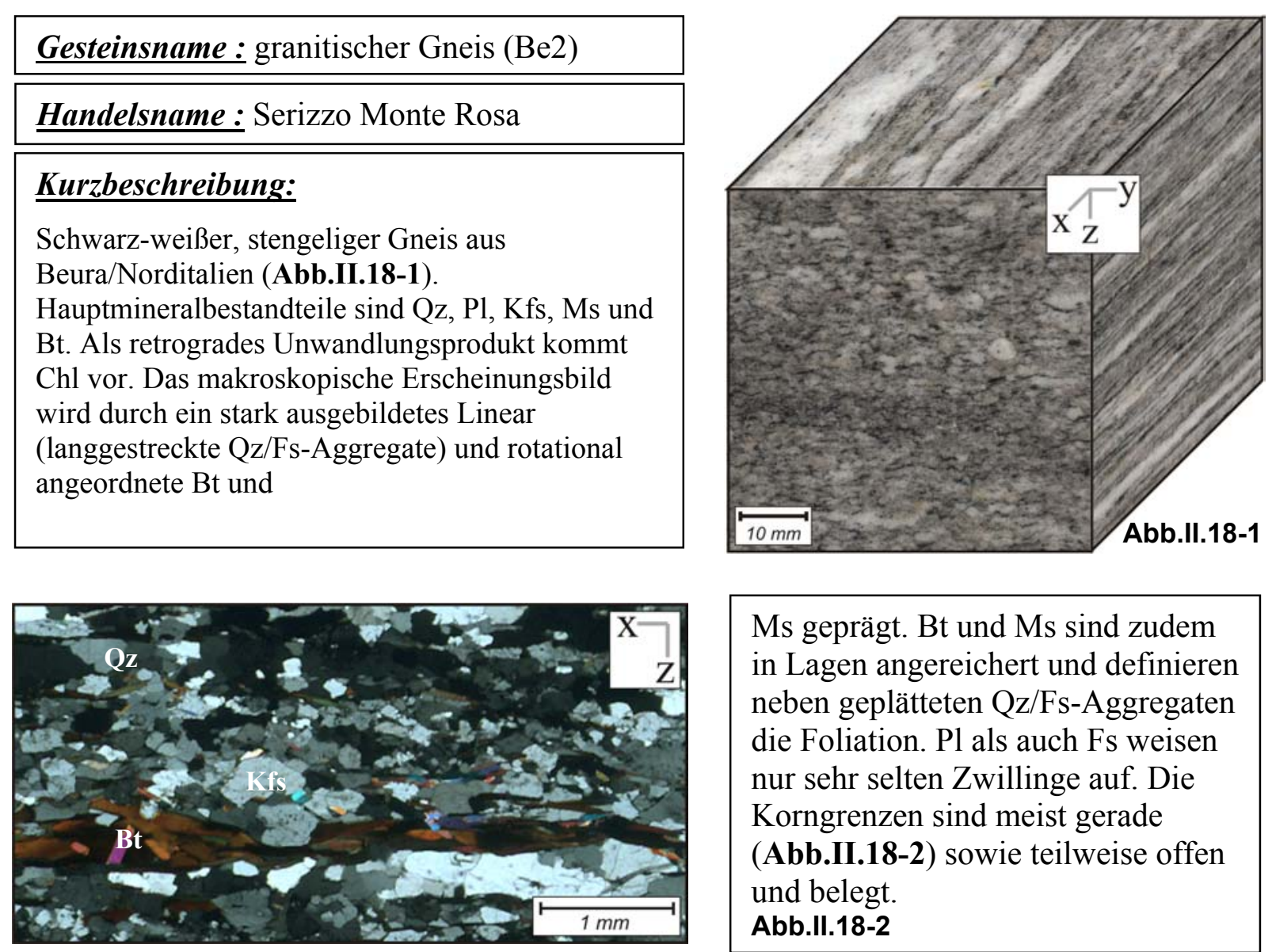

Ms geprägt. Bt und Ms sind zudem in Lagen angereichert und definieren neben geplätteten Qz/Fs-Aggregaten die Foliation. Pl als auch Fs weisen nur sehr selten Zwillinge auf. Die Korngrenzen sind meist gerade (Abb.II.18-2) sowie teilweise offen und belegt.

Abb.II.18-2

Mineralbestand (Vol.\%):

\begin{tabular}{|c|c|c|c|}
\hline Quarz & Kalifeldspat & Plagioklas & Muskovit \\
\hline 38 & 19 & 28 & 7 \\
\hline Biotit & Chlorit & $\mathrm{Zr}$ & \multicolumn{1}{|}{} \\
\cline { 1 - 3 } & 1 & $<1$ & \multicolumn{1}{|c}{} \\
\cline { 1 - 3 } & &
\end{tabular}

\section{Korngröße:}

\begin{tabular}{|l|l|}
\hline feinkörnig & $0,5-2 \mathrm{~mm}$ \\
\hline
\end{tabular}

Korngefüge: inequigranular-polygonal

Textur maximale Intensität (mrd)/Regelungsschärfe $c_{w}$ /Formfaktor T:

\begin{tabular}{|c|c|c|}
\hline Biotit/ Muskovit (001)-Pole & Quarz (c-Achse) & Plagioklas (001)-Pole \\
\hline $16,12 / 3,67 /-0,40$ & 4,95 & 1,26 \\
\hline
\end{tabular}

Risse:

\begin{tabular}{|c|c|c|c|c|}
\hline Quarz & Kalifeldspat & Plagioklas & Muskovit & Biotit \\
\hline intra off & intra off & intra off & intra(Spalt) off & intra(Spalt) off \\
\hline
\end{tabular}

sonstige: Korngrenzen teilweise offen, belegt

\section{Referenzen: ----}


Anhang II.19: Datenblatt granodioritischer Ultramylonit (Iv2)

Gesteinsname : granod. Ultramylonit (Iv2)

Handelsname : ------

\section{Kurzbeschreibung(Probenserie Iv1//v1b/Iv2):}

Unter grünschieferfaziellen Bedingungen retrograd überprägter Granitoid aus dem Val Vermenza/ Norditalien. Das Gestein stammt aus dem zentralen Bereich einer mylonitischen Scherzone, die der Sesia-Lanzo-Zone zugeordnet wird. Die Probe ähnelt den bereits beschriebenen Proben Iv1 und Ivlb. Sie zeigt den höchsten Grad der Mylonitisierung

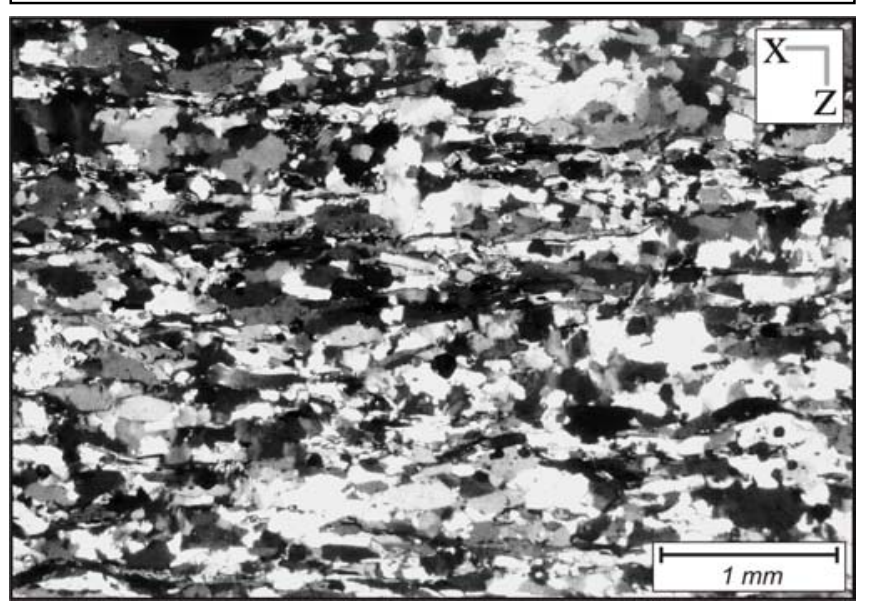

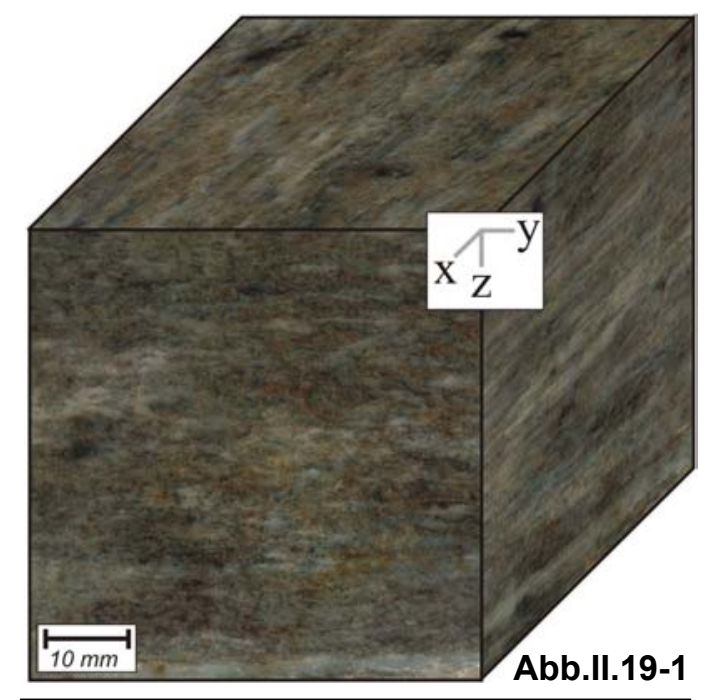

innerhalb dieser Probenserie. Das makroskopische L-S-Gefüge mit einer straff ausgebildeten und engständigen Foliation (Abb.II.19-1) ist durchgreifend rekristallisiert und annähernd equigranular(Abb. II.19-2). Sowohl Qz als auch die Fs und Ms zeigen eine bevorzugte Kornformorientierung.

Abb. II.19-2

Mineralbestand (Vol.\%):

\begin{tabular}{|c|c|c|c|}
\hline Quarz & Kalifeldspat & Plagioklas & Muskovit \\
\hline 36 & 5 & 28 & 15 \\
\hline Chlorit & Granat & Klinozoisit/Epidot & Apa, Rt, Zr \\
\hline 6 & 4 & 6 & $<1$ \\
\hline
\end{tabular}

\section{Korngröße:}

\begin{tabular}{|c|c|}
\hline feinkörnig & $0,5-2 \mathrm{~mm}$ \\
\hline
\end{tabular}

Korngefüge: inequigranular-polygonal

Textur maximale Intensität (mrd)/Regelungsschärfe $c_{w} /$ Formfaktor T:

\begin{tabular}{|c|c|c|}
\hline Muskovit (001)-Pole & Quarz (c-Achse) & Plagioklas (001)-Pole \\
\hline $17,56 / 2,80 / 0,24$ & $1,82 /$ n.b./n.b. & $1,02 /$ n.b./n.b. \\
\hline
\end{tabular}

Risse:

\begin{tabular}{|c|c|c|c|}
\hline Quarz & Kalifeldspat & Plagioklas & Muskovit \\
\hline intra verh & intra off/verh & intra off/verh & intra (Spalt) off \\
\hline
\end{tabular}

sonstige: Korngrenzen sehr selten offen

Referenzen: Juckenack (1990); Techmer (1996); Strohmeyer \& Siegesmund (2002) 
Anhang II.20: Datenblatt granitischer Ultramylonit (Gra3a)

Gesteinsname : granit. Ultramylonit (Gra3a)

Handelsname :

\section{Kurzbeschreibung :}

Gneis aus dem Waldviertel im Nieder-

österreichischen Krug. Das Gestein wird den „Granuliten“ der Gföhler Einheit, im spe-ziellen dem St. Leonharder Granulit-Komplex zugeordnet. Hauptmineralbestandteile sind Qz, Kfs, Plagioklas; $\mathrm{Bt}$ und Gt kommen in geringeren Mengen vor. Die Foliation wird durch die Kornformorientierung des Quarz und die
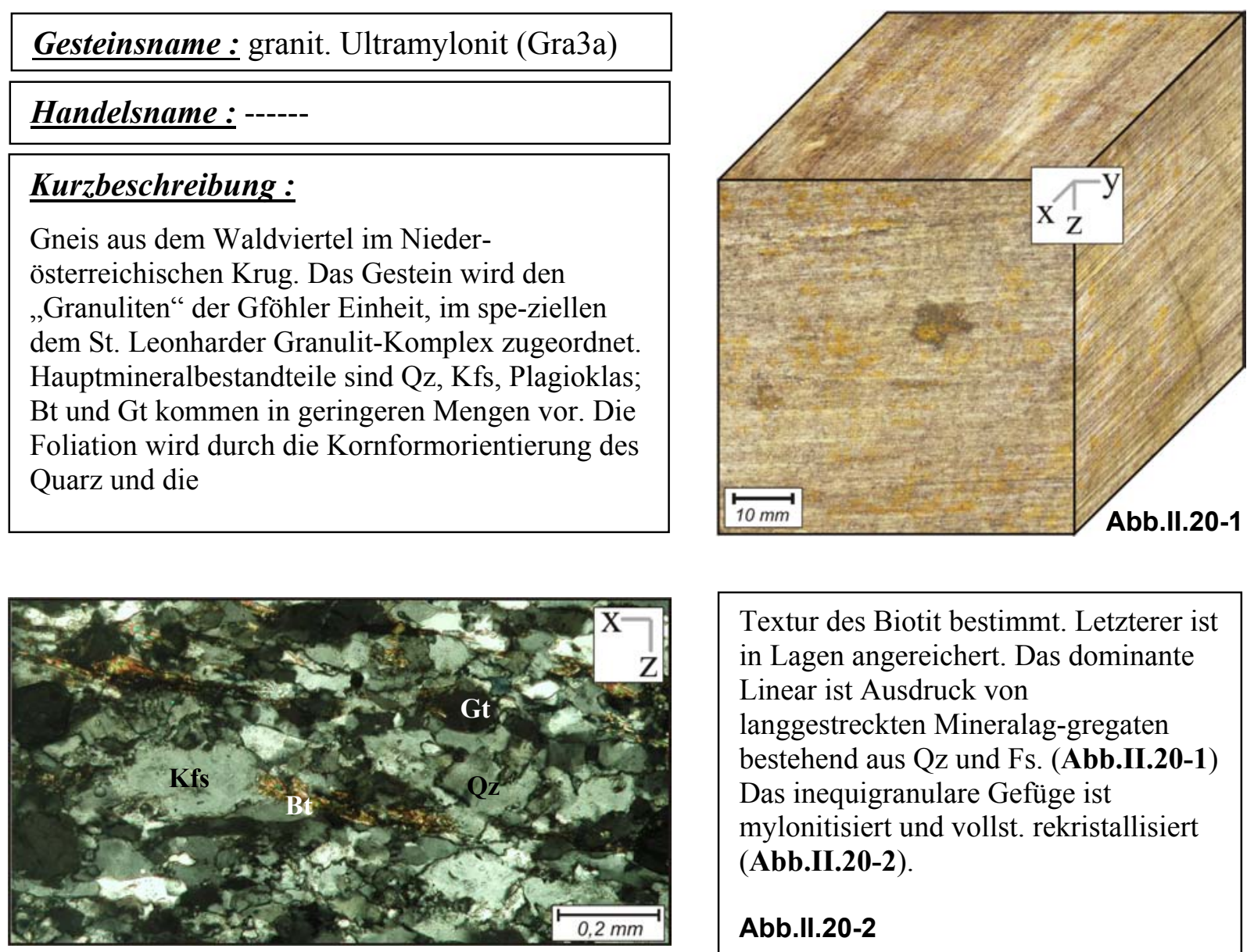

Textur des Biotit bestimmt. Letzterer ist in Lagen angereichert. Das dominante Linear ist Ausdruck von langgestreckten Mineralag-gregaten bestehend aus Qz und Fs. (Abb.II.20-1) Das inequigranulare Gefüge ist mylonitisiert und vollst. rekristallisiert (Abb.II.20-2).

Abb.II.20-2

Mineralbestand (Vol.\%):

\begin{tabular}{|l|l|l|l|}
\hline Quarz & Kalifeldspat & Plagioklas & Biotit \\
\hline $30+-1$ & $34+-1$ & $26+-1$ & $7+-1$ \\
\hline Granat & $\mathrm{Ky}, \mathrm{Sil}, \mathrm{Ru}$ & \multicolumn{2}{|l}{} \\
\cline { 1 - 2 } $3+-0,5$ & $<1$ & & \\
\cline { 1 - 2 } & &
\end{tabular}

\section{Korngröße:}

\begin{tabular}{|l|l|}
\hline extrem feinkörnig & $<0,2 \mathrm{~mm}$ \\
\hline
\end{tabular}

Korngefüge: equi/inequigranular-interlobat

Textur maximale Intensität (mrd)/Regelungsschärfe $c_{w}$ /Formfaktor T:

\begin{tabular}{|c|c|c|}
\hline Biotit (001)-Pole & Quarz (c-Achse) & Plagioklas (001)-Pole \\
\hline $23,30 / 3,73 / 0,31$ & $3,53(8,1)^{*}$ & 1,27 \\
\hline
\end{tabular}

Risse:

\begin{tabular}{|l|l|l|l|}
\hline Quarz & Kalifeldspat & Plagioklas & Biotit \\
\hline intra verh & intra verh/off & intra verh/off & intra (Spalt) off \\
\hline
\end{tabular}

sonstige: Korngrenzen sehr selten offen, teilweise belegt

Referenzen: Duyster (1991);*Ullemeyer (1992) 


\section{Anhang III: Korrelationsdiagramme}

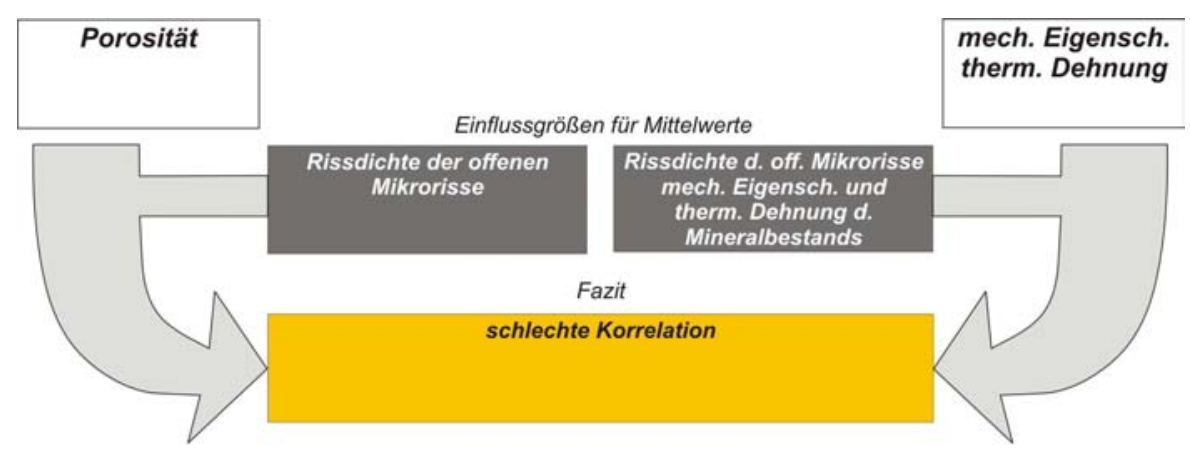

Porosität vs. mechanische Eigenschaften und thermische Dehnung.

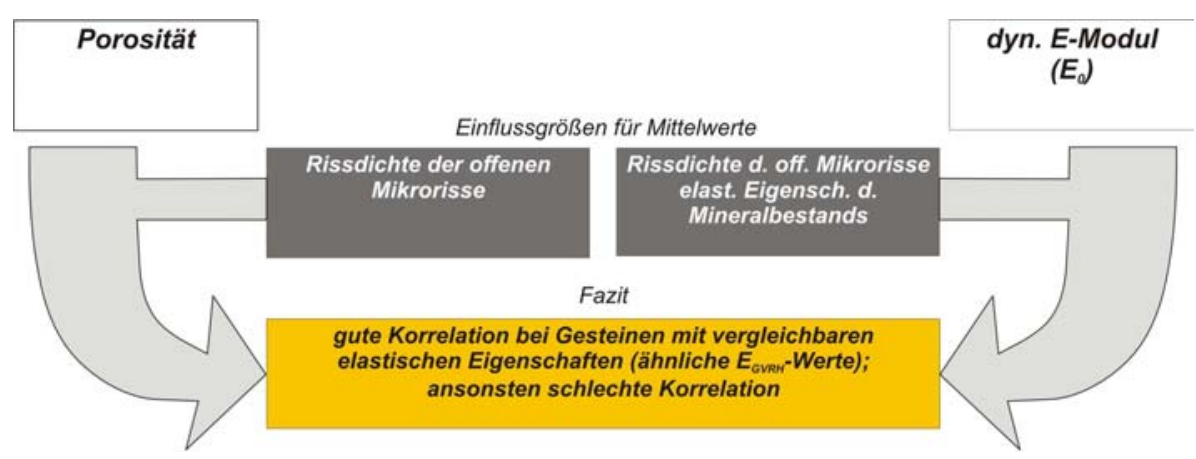

Porosität vs. dynamischer E-Modul.

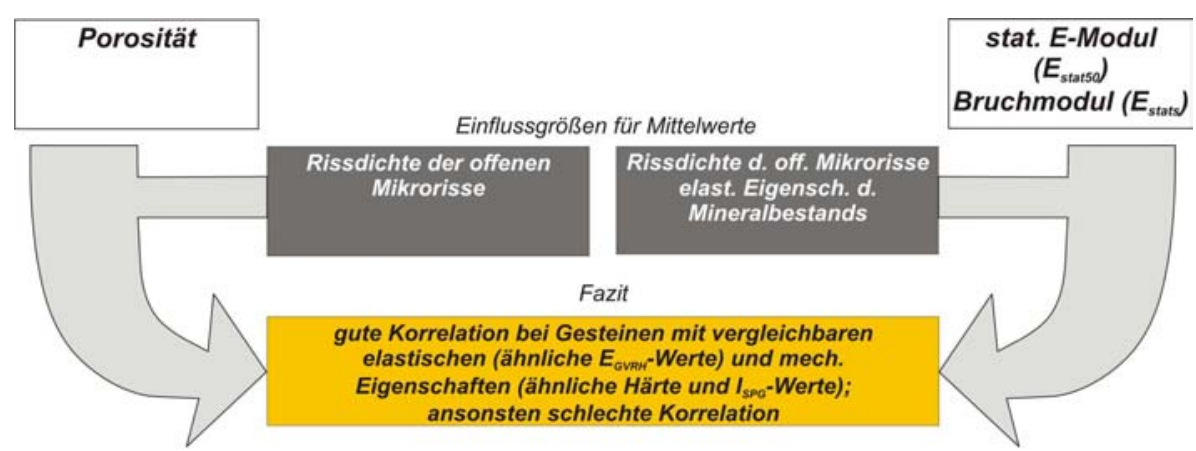

Porosität vs. statischer (bei 50\% Bruchlast) E-Modul und Bruchmodul. 


\section{Anhang}

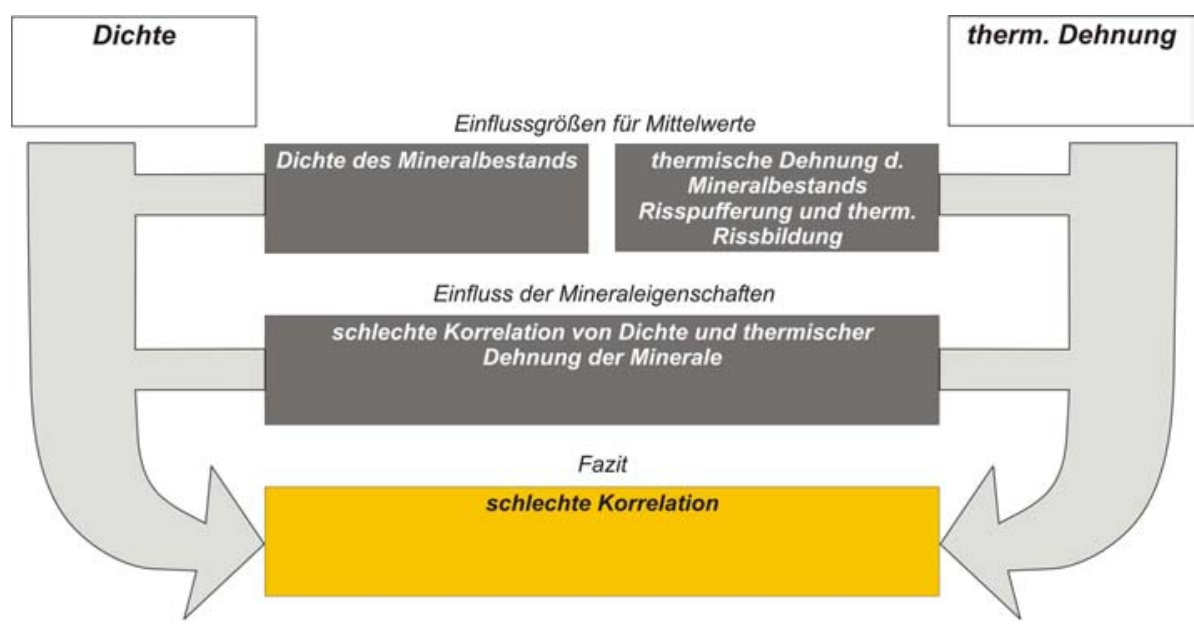

Dichte vs. thermische Dehnung.

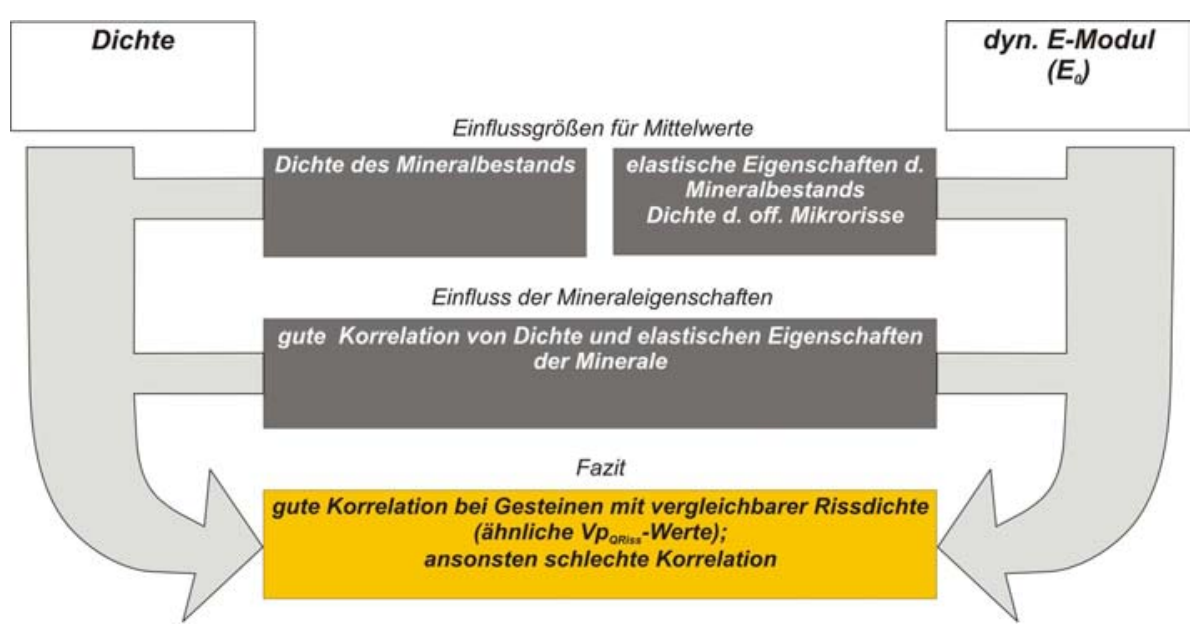

Dichte vs. dynamischer E-Modul.

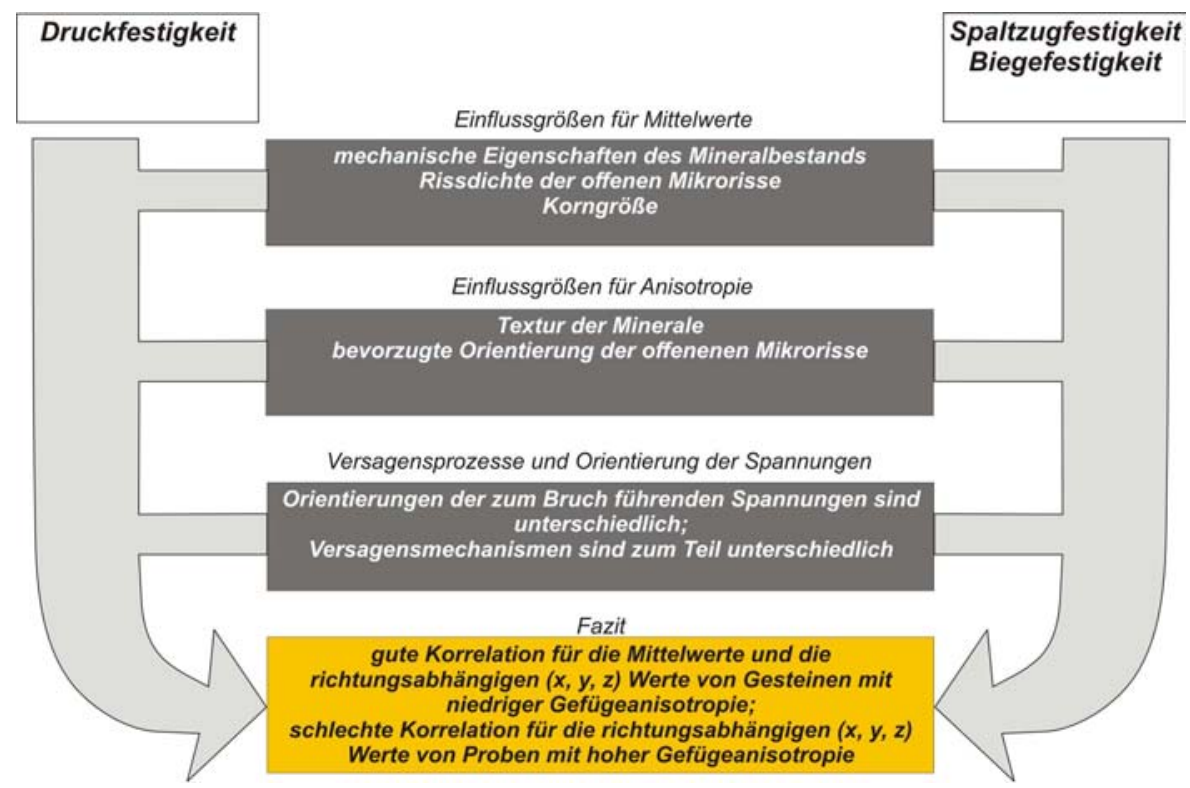

Druckfestigkeit vs. Spaltzug- und Biegefestigkeit. 


\section{Anhang}

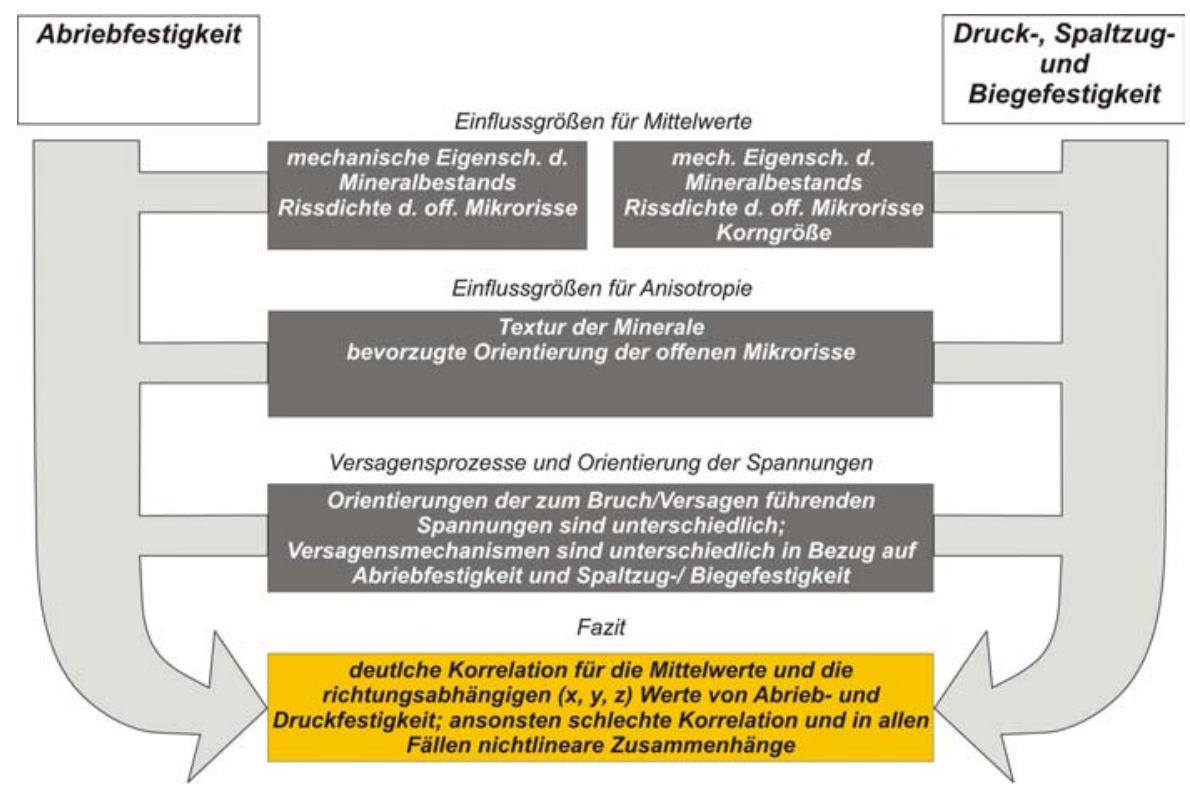

Abriebfestigkeit vs. Druck-, Spaltzug-, Biegefestigkeit.

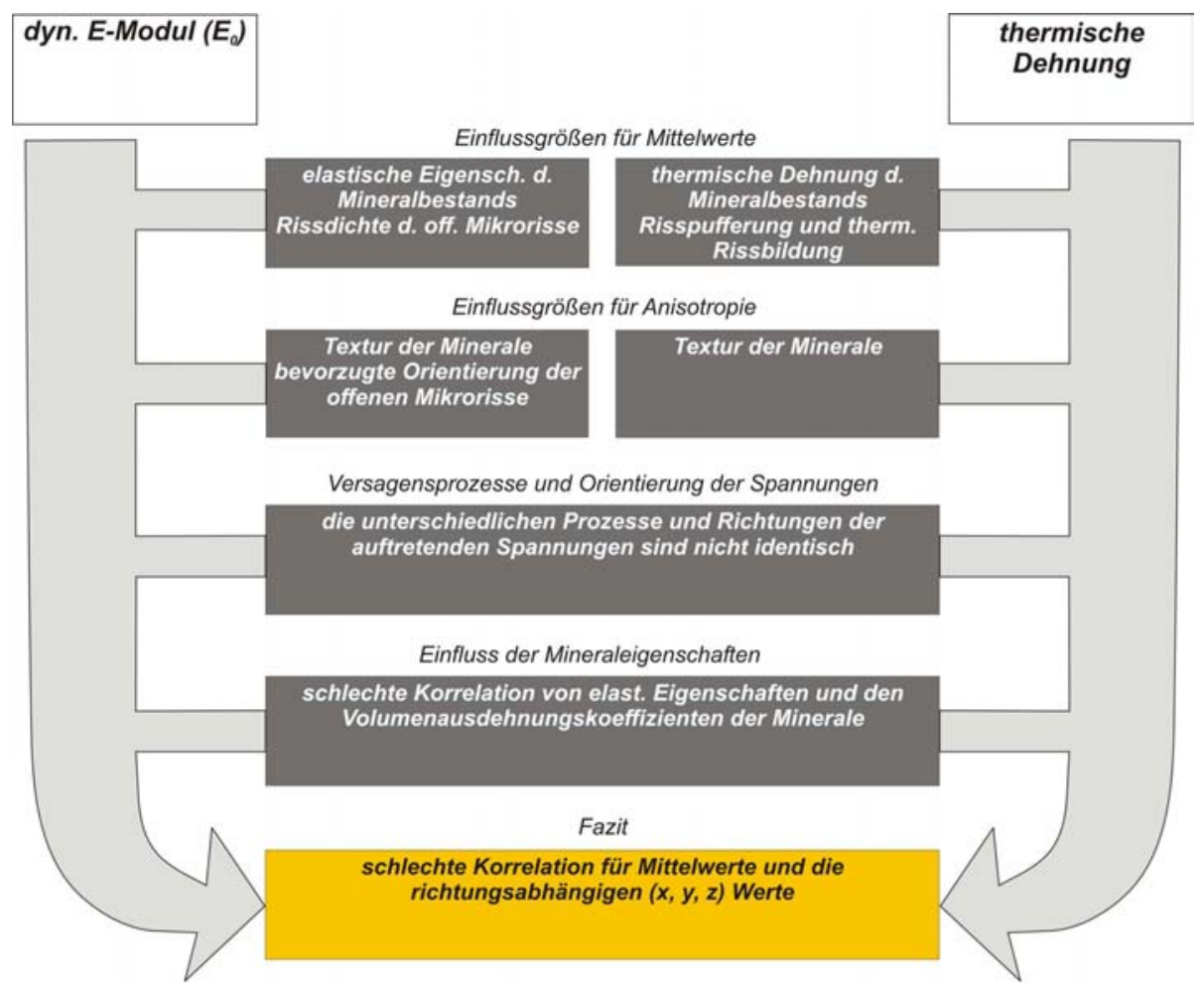

Dynamischer E-Modul vs. thermische Dehnung. 


\section{Anhang}

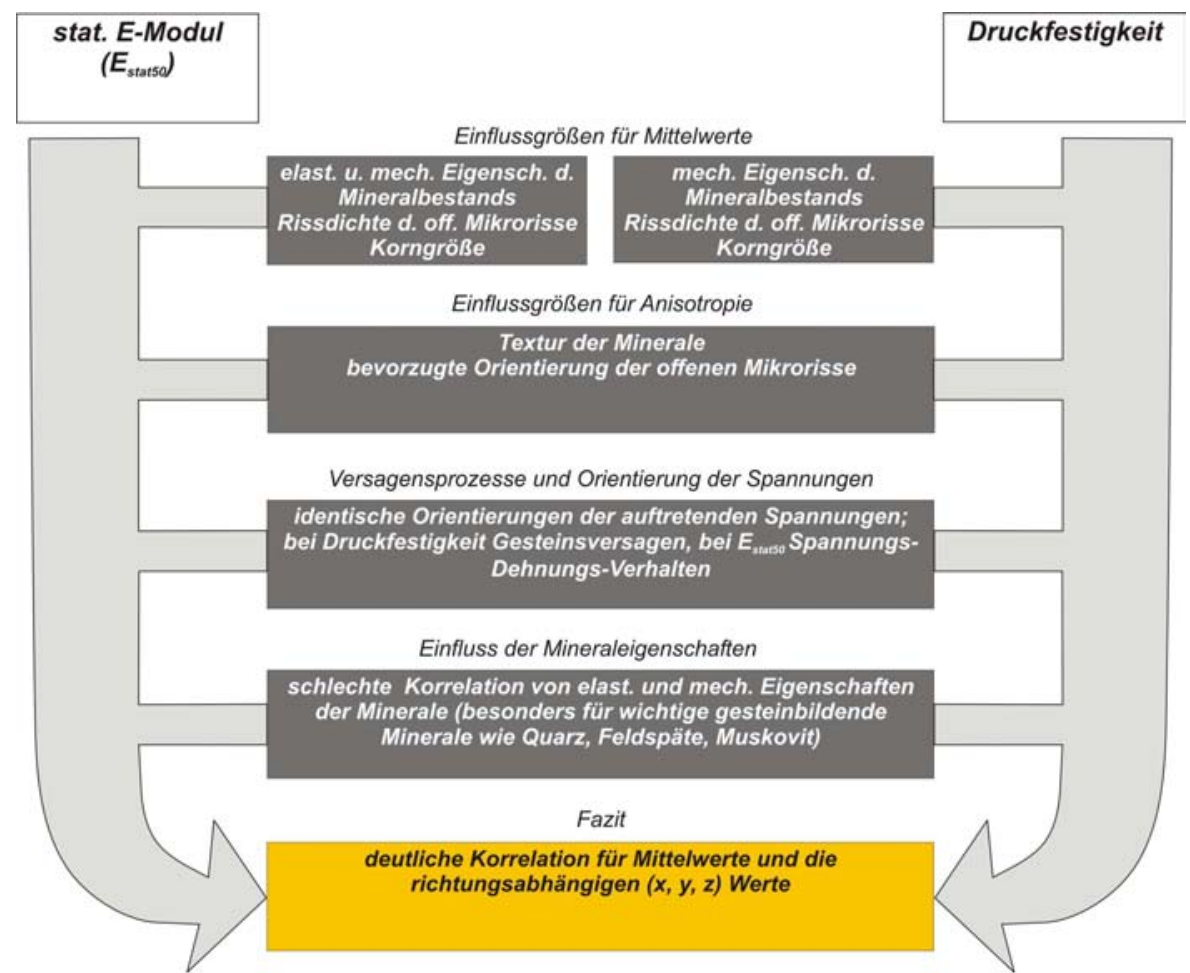

Statischer E-Modul (bei 50\% Bruchlast) vs. Druckfestigkeit.

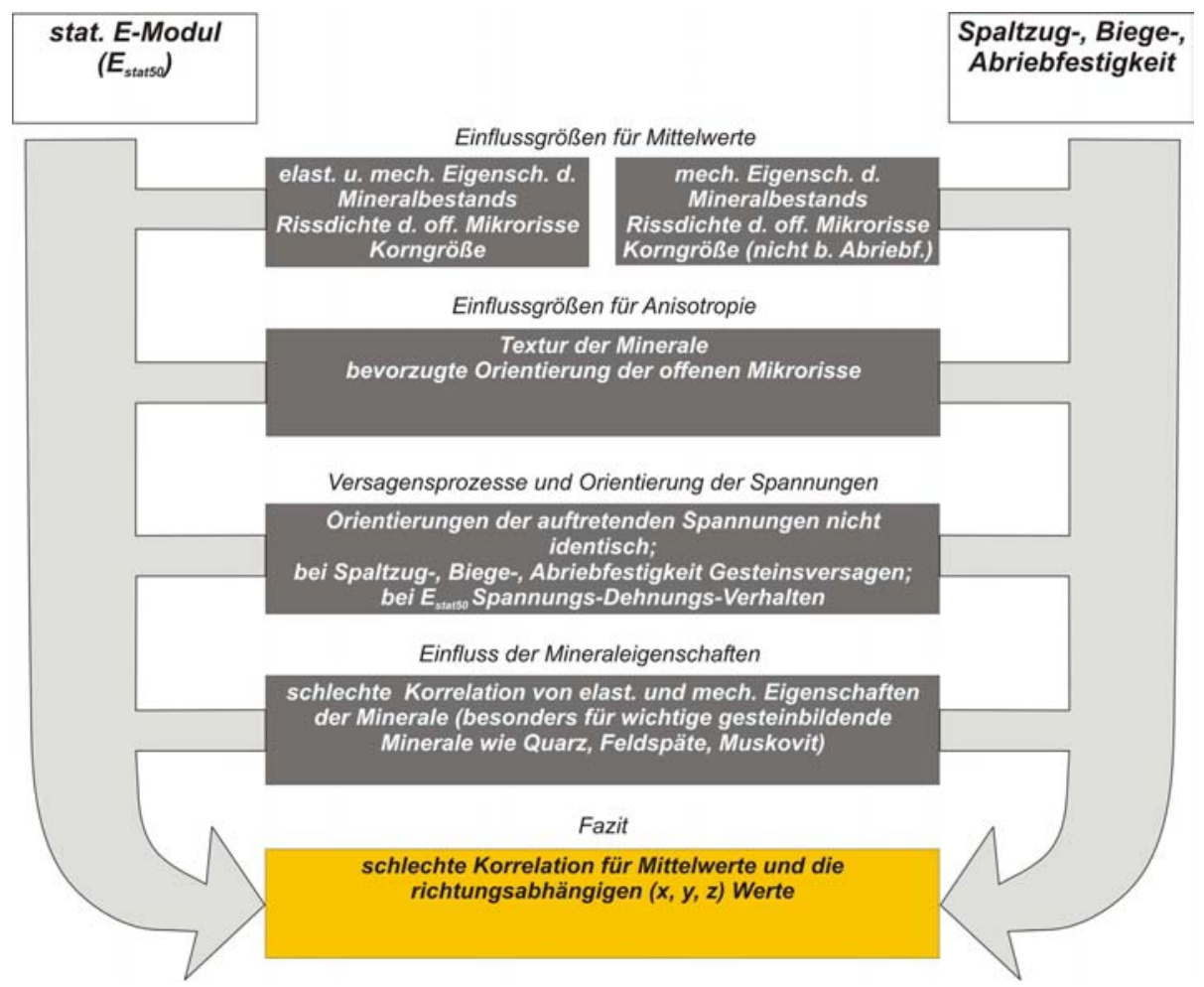

Statischer E-Modul (bei 50\% Bruchlast) vs. Spaltzug-, Biege-, Abriebfestigkeit. 


\section{Anhang}

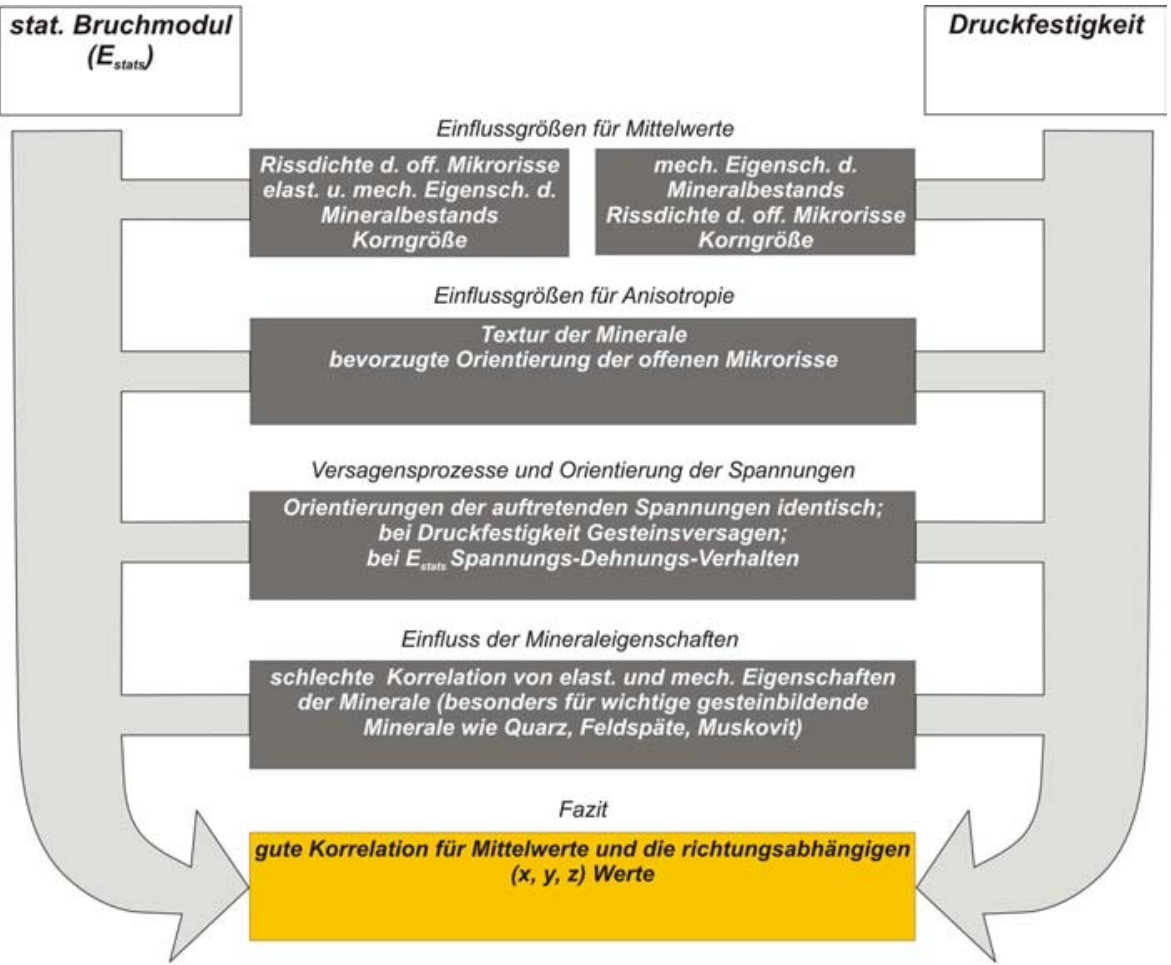

Bruchmodul vs. Druckfestigkeit.

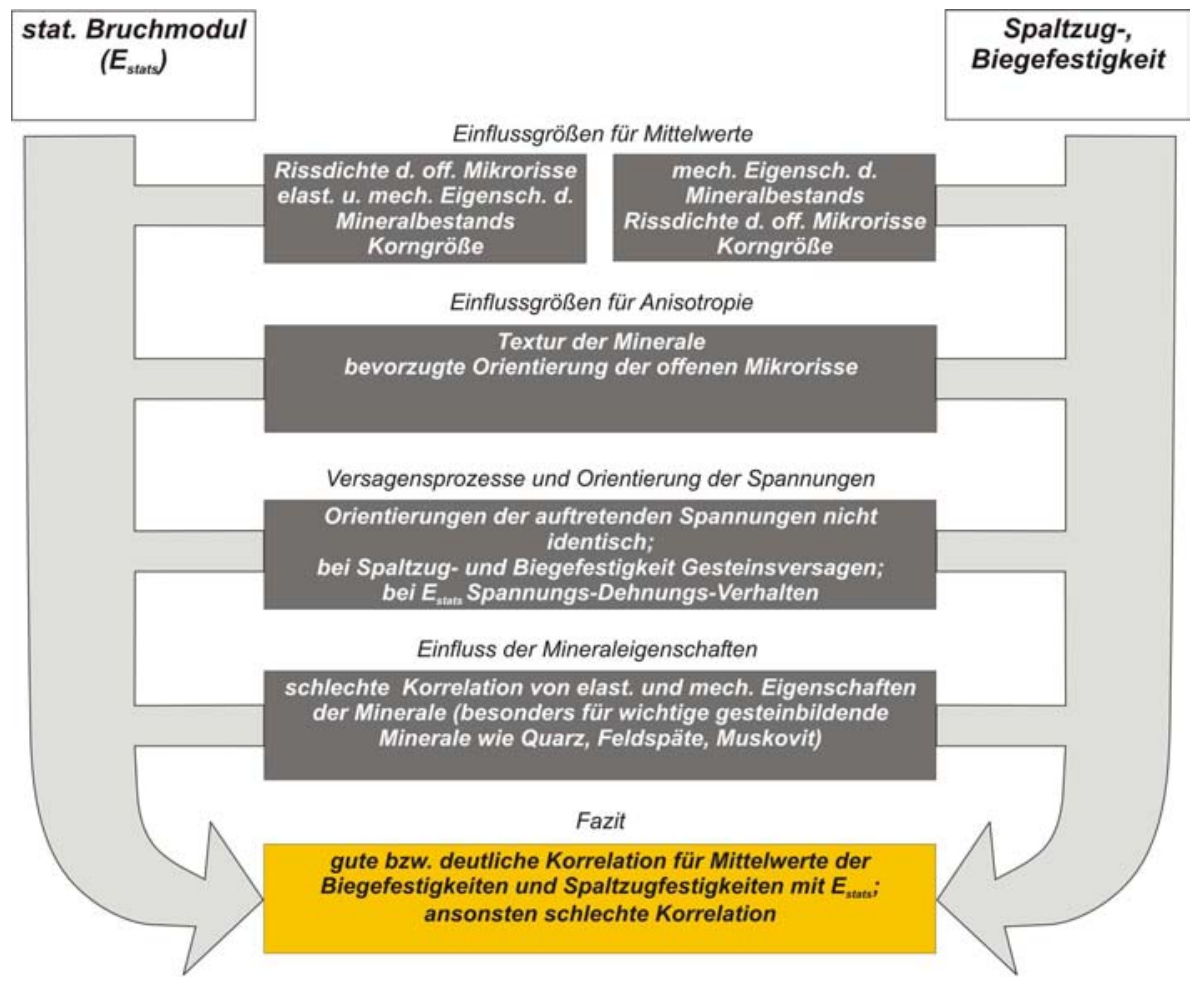

Bruchmodul vs. Spaltzug-, Biegefestigkeit. 


\section{Anhang}

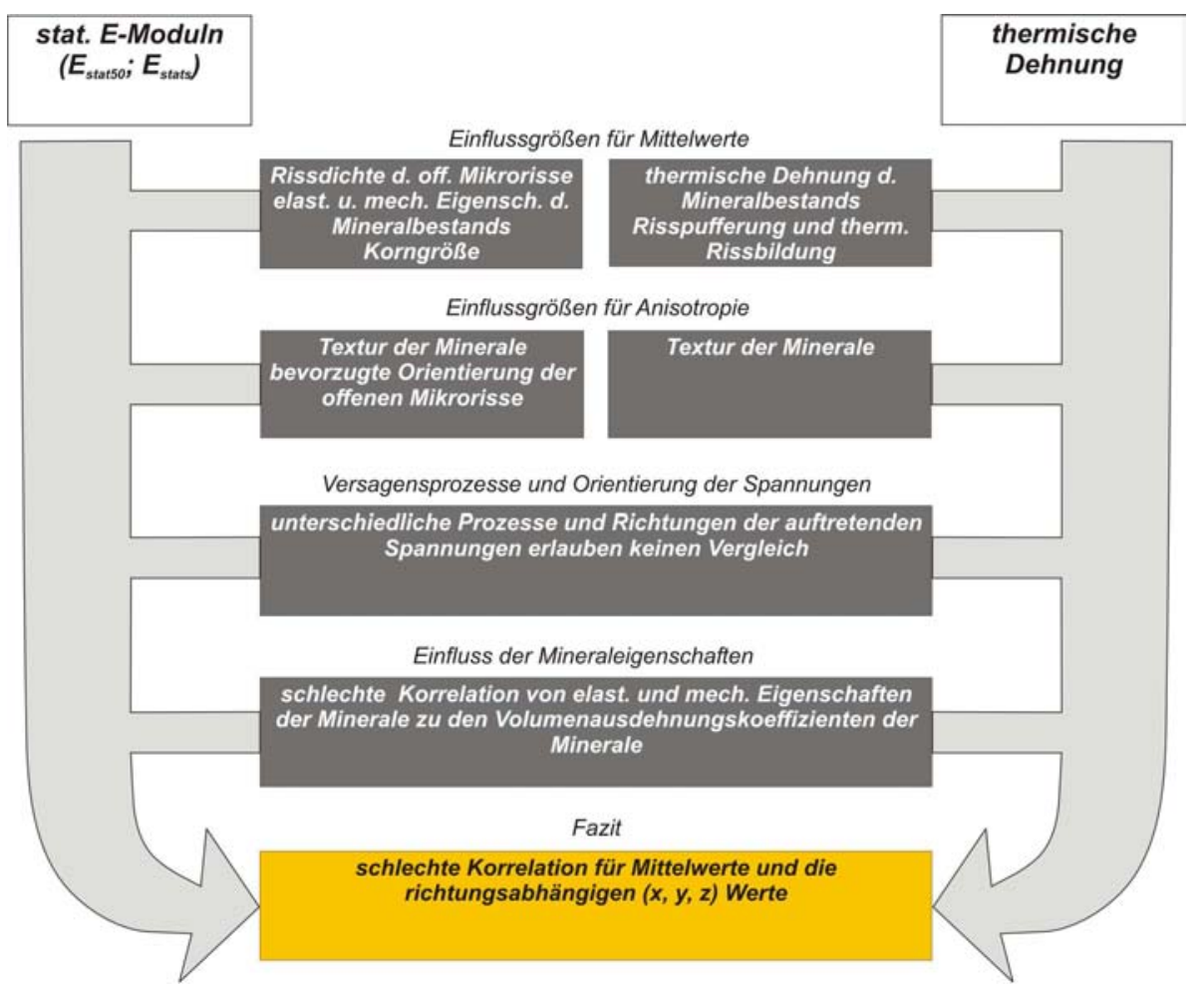

Statischer E-Modul (bei 50\% Bruchlast) und Bruchmodul vs. thermische Dehnung. 


\section{Danksagung}

Die vorliegende Forschungsarbeit entstand im Rahmen des vom Bundesministerium für Bildung und Forschung geförderten Projekts: „Naturwerksteine: Gefüge und technische Gesteinseigenschaften“ (Förderkennzeichen: 03DU0GO3/3). Für die Projektleitung, die Betreuung der Arbeit und die damit verbundenen hervorragenden Arbeitsbedingungen danke ich Herrn Prof. Siegfried Siegesmund.

Herrn Prof. K.-H. Nitsch danke ich für das Interesse am Dissertationsthema und die Übernahme des Korreferats.

Des Weiteren gilt mein Dank:

$+\quad$ Meinen Arbeitskollegen Dr. Thomas Weiss, Jörg Rüdrich und Andreas Koch, die über eine angenehme Arbeitsatmosphäre und eine immerwährende Diskussionsbereitschaft maßgeblich zum Gelingen der Arbeit beigetragen haben

$+\quad$ Lothar Laake, Herrn Schubert und Andreas Hampe, die alle technischen Probleme bei der Konstruktion des Dilatometers gelöst haben

+ Michael Sosnitza, Günther Tondock und Holger Schwanke für die immer auch spontane Hilfe bei allen technischen Problemen des Laboralltags und der Probennahme

$+\quad$ Dr. Klaus Ullemeyer und Peter Spalthoff für die Neutronenbeugungs-Messungen und die Unterstützung bei der quantitativen Texturanalyse

$+\quad$ Dr. Till Heinrichs und Dr. Andreas Kronz für die Unterstützung am FE-REM bzw. an der Elektronenstrahl-Mikrosonde

$+\quad$ Karl-Jochen Stein, der mir alle Fragen aus dem Sektor der Naturwerksteine beantworten konnte

$+\quad$ Rudolf Naumann für Durchführung der quantitativen Phasenanalyse

$+\quad$ Dr. Axel Vollbrecht für sein umfassendes Wissen über Mikrorisse

$+\quad$ Thomas Busche für die geographische Sicht der Dinge und die Hilfe bei einigen Applikationen

Für die Unterstützung im Kampf gegen die „Fehlerteufel“ und den beginnenden Wahnsinn danke ich Joseph Ondrasina, Monica Axmann und Sabine Sommer. alles ! 


\section{CURRICULUM VITAE}

\section{Berufserfahrung}

\section{Wissenschaftlicher Mitarbeiter}

Universität Göttingen, Institut für Strukturgeologie und Geodynamik

Organisation und Durchführung der Forschungsarbeit zur Gefügeabhängigkeit petrophysikalischer Parameter Eine Publikation sowie sechs Vorträge und Poster auf internationalen Konferenzen

\section{Laborleiter}

Universität Göttingen, Institut für Strukturgeologie und Geodynamik, Tektoniklabor

Entwicklung und Verbesserung von Messgeräten Betreuung von Kunden und Studenten

Labor- und Netzwerkadministration

Studentische Hilfskraft im Mikrosondenlabor Universität Göttingen, Geochemisches Institut

Elektronenstrahlmikrosonde und Rasterelektronenmikroskop (Jeol JXA 8900):

Kalibration, Messungen, trouble-shooting, Einweisung und Beratung von Kunden und Studenten, Aufbau und Verbesserung des Labors

\section{Hochschulpraktikum}

Firma Wille Geotechnik $\mathrm{GmbH}$

Anwendung sowie Bau von geotechnischen Geräten zur Gesteins-und Bodenmechanik

Kundenbetreuung

Messevorbereitungen
Daniel Strohmeyer geb. am 22.07.1972 in Walsrode Staatsangeh.: deutsch

2000-2002

2000-2002

1998-1999

1997-1998 


\section{CURRICULUM VITAE}

\section{Bildungshintergrund}

\section{Doktorand}

Universität Göttingen, Institut für Strukturgeologie und Geodynamik

Projekt: Naturwerksteine: Gefüge und technische Eigenschaften (finanziert vom Bundesministerium für Bildung und Forschung)

Schwerpunkte: Gesteinsphysik, Strukturgeologie, technische Mineralogie

\section{Diplomand}

Universität Göttingen, Geochemisches Institut

1999-2000

Diplomarbeit Teil 1: Sr- und Nd-Isotopie als Tracer für Sedimentherkunft und Sedimentationsprozesse im zentralen Arktischen Ozean während des späten Quartär

Schwerpunkte: Paläoklimatologie, Isotopensysteme, Geologie der Arktisregion

Diplomarbeit Teil 2: Kartierbericht zur geologischen

Kartierung auf Blatt 178 Hopfgarten (Osttirol/

Österreich)

Schwerpunkte: Kristallingeologie, Strukturgeologie, Geologie der Alpen

\section{Student der Geologie/Paläontologie}

Universität Göttingen

$1995-1999$

Schwerpunkte: Geochemie, Strukturgeologie, Methoden

Ausbildung zum Werkzeugmechaniker

Mercedes-Benz AG Werk Bremen

Zivildienst

Jugendzentrum "Die Scheune e.V." Worpswede

Betreuung von Jugendlichen, Jugendfreizeiten,

Reparaturen

Schule/Abitur

Gymnasium Osterholz-Scharmbeck

bis 1992

Leistungskurse: Mathematik, Physik

Prüfungskurse: Deutsch, Erdkunde 